BNL-94667-2011

\title{
30th Anniversary Symposium OF THE US/JAPAN COLLABORATION IN HIGH ENERGY PHYSICS
}

\section{高エネルギ一物理学日米科学技術協力事業 30 周年記念シンポジウム}

OCTOBER 20-21, 2010

HAWAII, U.S.A. 


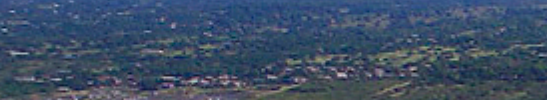

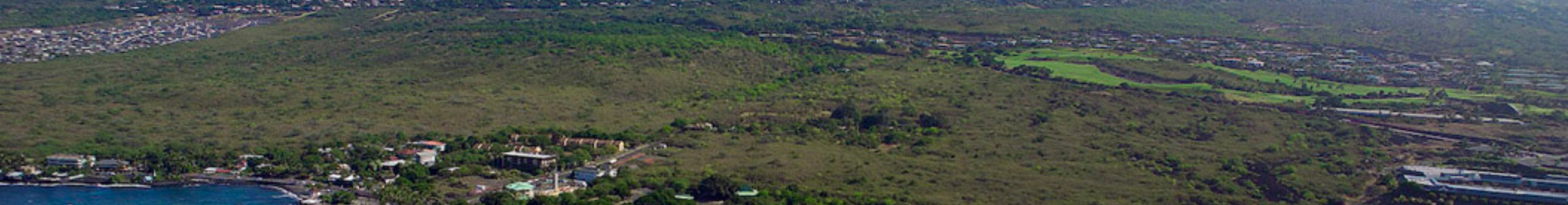

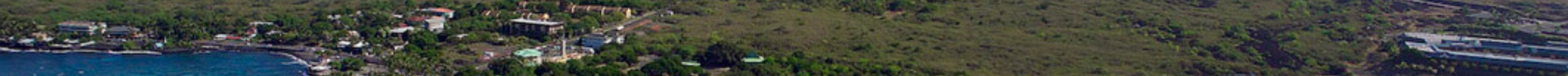

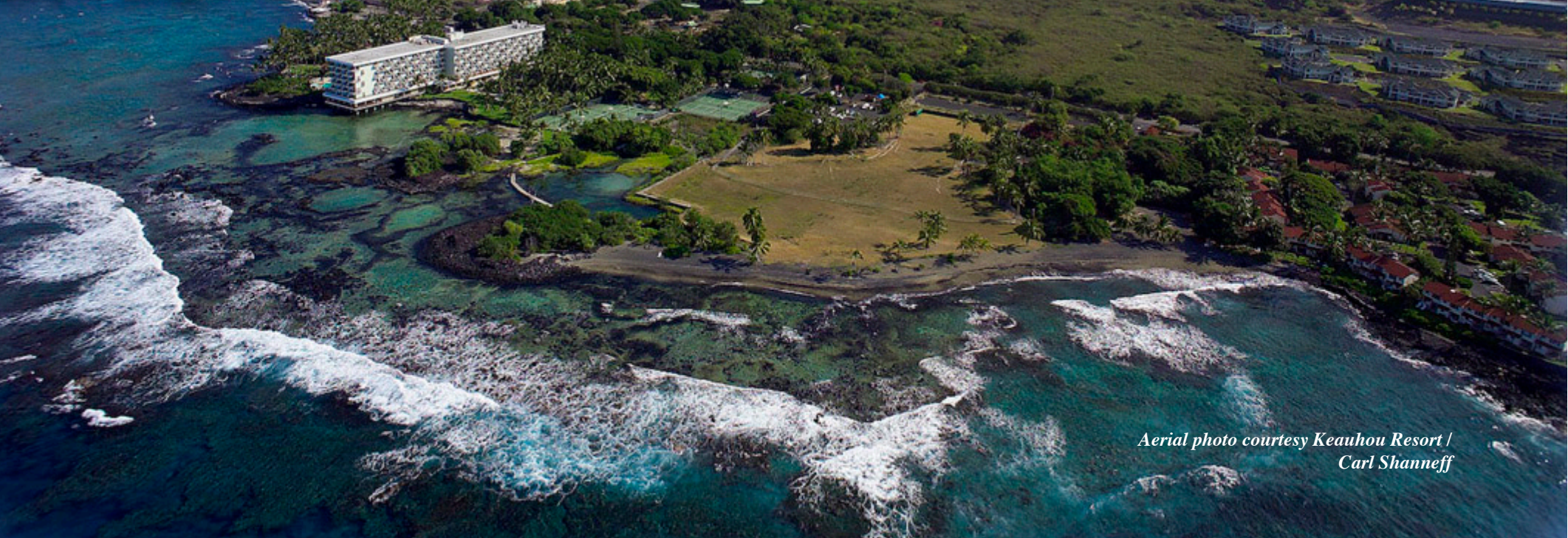




\title{
The US/Japan HEP Collaboration 30th Anniversary Symposium
}

\author{
高エネルギー物理学日米科学技術協力事業 \\ 30 周年記念シンポジウム
}

October 20 - 21, 2010

平成22年10月20 - 21 日

Keauhou Beach Resort

Kailua-Kona, Hawaii, U.S.A.

Organized by the US/Japan Joint Committee for the HEP Collaboration Jointly supported by DOE (USA) and MEXT (Japan)

Local Support by the College of Natural Sciences, University of Hawaii at Manoa and the University of Hawaii System

\section{主催：高エネルギ一物理学日米科学技術協力事業合同委員会} 後援：文部科学省 (日本) 及びエネルギー省 (米国)

現地後援: ハワイ大学マノア分校自然科学カレッジ及びハワイ大学システム

Edited by Satoshi Ozaki

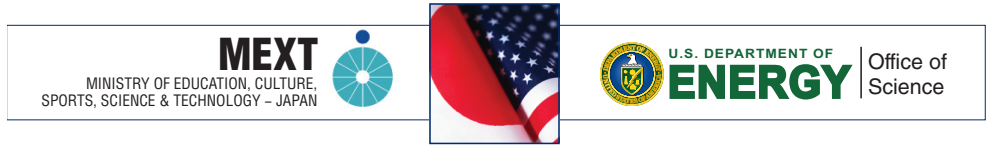




\section{DISCLAIMER}

This report was prepared as an account of a conference sponsored by an agency of the United States Government. Neither the United States Government nor any agency thereof, nor any of their employees, nor any of their contractors, subcontractors or their employees, makes any warranty, express or implied, or assumes any legal liability or responsibility for the accuracy, completeness, or any third party's use or the results of such use of any information, apparatus, product, or process disclosed, or represents that its use would not infringe privately owned rights. Reference herein to any specific commercial product, process, or service by trade name, trademark, manufacturer, or otherwise, does not necessarily constitute or imply its endorsement, recommendation, or favoring by the United States Government or any agency thereof or its contractors or subcontractors. The views and opinions of authors expressed herein do not necessarily state or reflect those of the United States Government or any agency thereof.

\section{NOTICE}

These proceedings have been prepared by employees of Brookhaven Science Associates, LLC under Contract No. DE-AC02-98CH10886 with the U.S. Department of Energy. The United States Government retains a non-exclusive, paid-up, irrevocable, world-wide license to publish or reproduce the published form of this manuscript, or allow others to do so, for United States Government purposes. 


\section{PREFACE}

This Symposium celebrating the 30th Anniversary of the formal collaboration between Japan and the United States of America on High Energy Physics took place on October 20 and 21, 2010 at the Keauhou Beach Resort in Kailua Kona, Hawaii. This High Energy Physics collaboration was established with the signing of the implementing arrangement in November 1979 at SLAC, which established a Joint Committee to guide the collaboration program and provided a framework within which the collaboration was to be carried forward. This implementing arrangement was established under an Agreement between the Government of Japan and the Government of the United States of America on Cooperation in Research and Development in Energy and Related Fields, and was signed by K. Shinozawa, representing the Ministry of Education Science and Culture of Japan, and by J. E. Leiss, representing the Department of Energy of the United States of America.

This Symposium was organized by the Joint Committee, which gave an approval at its meeting on April 26-27, 2010 in Matsushima, Miyagi, Japan. The goals of this Symposium were to reflect on the past activities and accomplishments of the collaboration, discuss the ongoing programs, and look forward to future prospects. With these goals in mind the scientific program of the Symposium was developed by a Joint Organizing Group consisting of M. Nozaki (KEK), K. Kondo (Waseda), T. Yamanaka (Osaka), and S. Kim (Tsukuba) on the Japanese side, and G. Loew (SLAC), S. Ozaki (BNL) and R. Rubinstein (Fermilab) on the U.S. side. Because the scope of this collaboration over the past 30 years encompasses a vast number of experiments and projects, we regrettably had to limit the presentations for the experimental and R\&D programs at the Symposium to highlights on selected topics. The Symposium participants included 46 people from the Japanese side, and 31 people from the US side.

On behalf of the Joint Organizing Group, I sincerely thank the Japanese Ministry of Education, Culture, Sports, Science and Technology (MEXT), and the U.S. Department of Energy (DOE) for their strong support of the Symposium. We are grateful for the logisticand financial-support of the local co-sponsors of the Symposium, the College of Natural Sciences of the University of Hawaii, and the University of Hawaii
System. In particular, we express our gratitude to Vice President J. Gains, Dean A. Teramura, and Professor T. Browder who helped us make this Symposium a memorable one. We also acknowledge the sponsorship of the High Energy Accelerator Research Organization (KEK), Brookhaven National Laboratory (BNL), Fermi National Accelerator Laboratory (Fermilab), Lawrence Berkeley National Laboratory (LBNL), and SLAC National Accelerator Laboratory (SLAC), all being major players in this Collaboration, and, more importantly, contributors in making this Symposium a successful one.

We are especially grateful for the skill and dedication of the conference secretary, Maria Anzaldi, who supported me in preparing for the Symposium and the publication of these proceedings. Barbara Moebes, Misa Miyai, and Masami Yokoyama provided vital support at the Symposium venue. We acknowledge with thanks Akira Yamaguchi who organized arrangements for the participants from Japan, and coordinated with the symposium secretariat with regard to the Symposium arrangements. I would like to extend our gratitude to Patricia Yalden for designing and producing the very attractive poster and the proceedings, and Avril Woodhead for editing the contributed abstracts.

The generous support of Dean A. Teramura, College of Natural Sciences, and the warm welcome by Director Nishimura and Professor Hayashi, Subaru Telescope Observatory, gave a wonderful opportunity to many participants at the Symposium to enjoy an excursion to the Summit of Mauna Kea at an altitude of about 14,000 feet, and a visit to the Subaru Telescope and the University of Hawaii Telescope. This spectacular trip was particularly meaningful now that the boundaries between high-energy physics and astrophysics are coming closer together.

About eight weeks after the Symposium, we received the sad news of the death of Professor Tetsuji Nishikawa, the former Director General of KEK, on December 15, 2010 at the age of 84. As W. Wallenmeyer, B. Hildebrand, and K. Kondo described in their talks, Professor Nishikawa was instrumental in initiating the US/Japan Collaboration in HEP, and greatly contributed to the 30-years of success, which are detailed in these proceedings. We respectfully dedicate these proceedings to the memory of late Dr. Tetsuji Nishikawa, the father of this successful Collaboration.

On behalf of the Joint Organizing Group

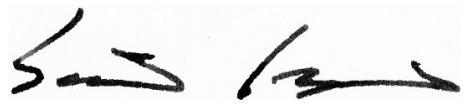

Satoshi Ozaki 



\section{DEDICATION}

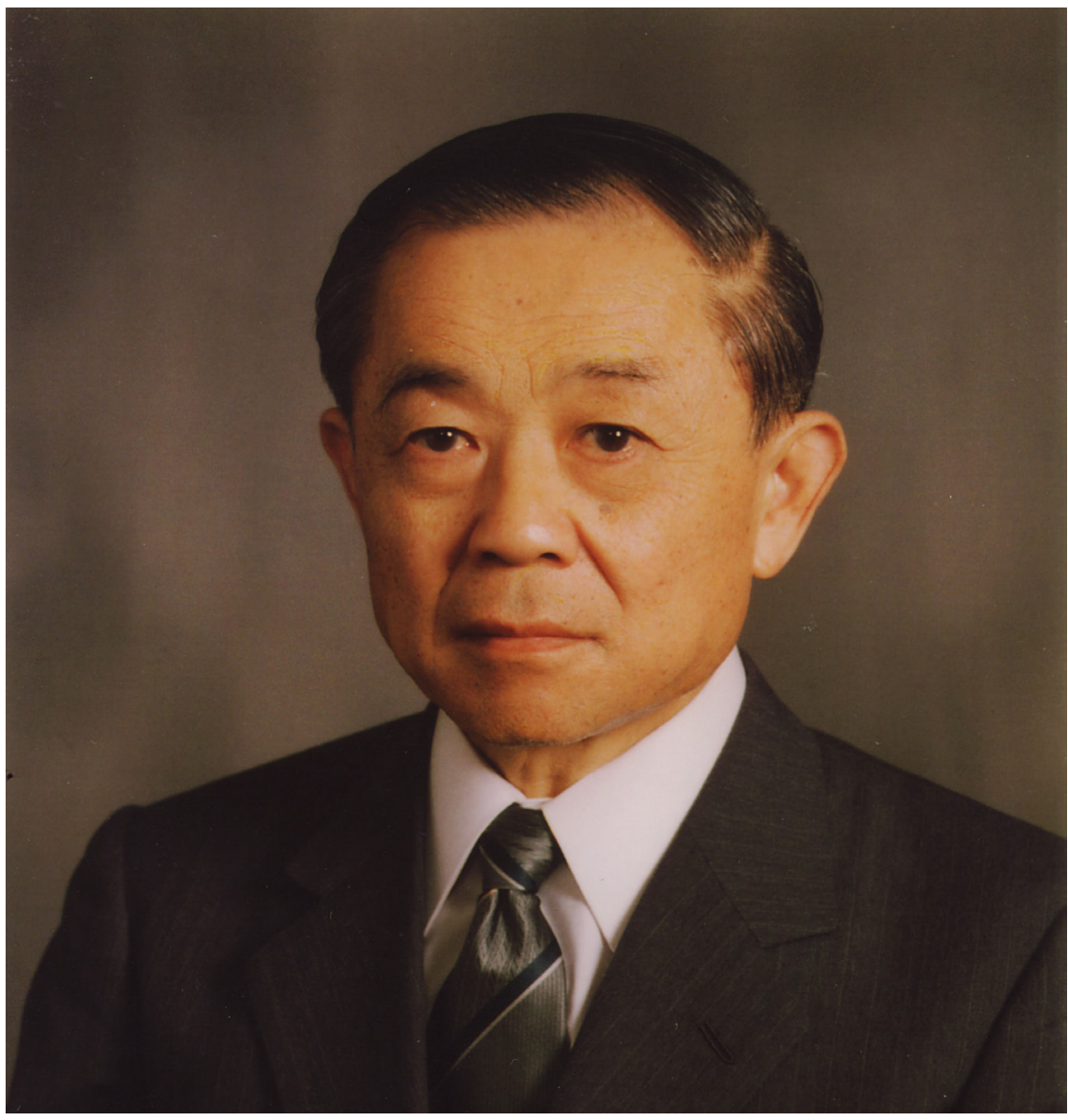

Dr. Tetsuji Nishikawa

The late Dr. Tetsuji Nishikawa was one of the founding fathers of Japanese Particle Accelerator Science after World War II. He played a key role in establishing the National Laboratory for High Energy Physics in Tsukuba (now the High Energy Accelerator Research Organization - KEK), and thereafter served as its Director General from 1977 to 1989. Several innovative accelerators were built at KEK under his leadership: The $12-\mathrm{GeV}$ proton synchrotron and its $500-\mathrm{MeV}$ booster proton synchrotron which was used for neutron-science, muon-science and for cancer therapy; the 2.5-GeV electron LINAC currently operating at $8 \mathrm{GeV}$; the TRISTAN electron-positron collider; and the Photon Factories with the $2.5 \mathrm{GeV}$ - and $6.5 \mathrm{GeV}$-electron synchrotrons.

He was the person who recognized the importance of scientific cooperation between Japan and the United States. He discussed his concept with the officials in the funding agencies of both countries, and the leaders of the major particle-physics laboratories in the United States and ultimately forged the agreement of the US-Japan Scientific Cooperation Program in the Field of High Energy Physics in 1979, the $30^{\text {th }}$ anniversary of which we celebrated with a Symposium in Hawaii. This program has operated very successfully throughout the past three decades.

After retiring from KEK, the late Dr. Nishikawa served as the President of the Tokyo University of Science from 1990 to 2001, making a notable contribution in the field of education in Japanese Universities. 



\section{CONTENTS}

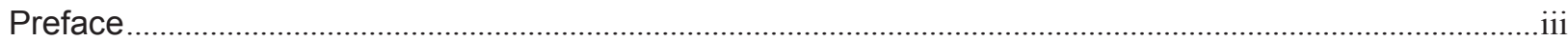

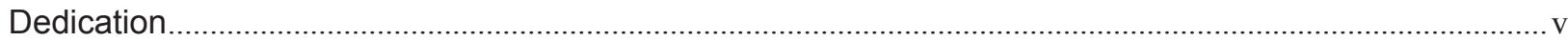

- Session 1: Opening Session

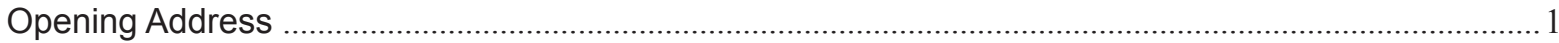

Fumihiko Takasaki

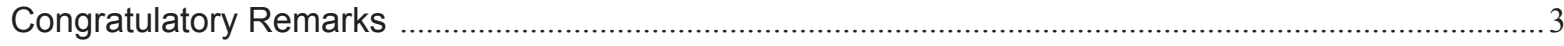

Yoshihiko Kamo

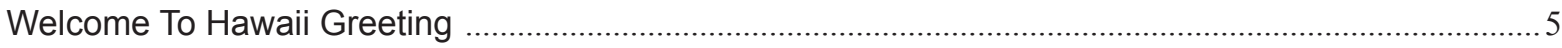

Alan Teramura

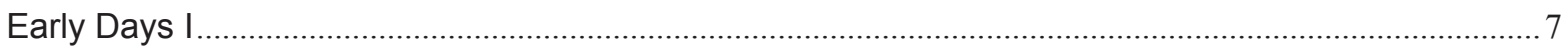

Bernard Hildebrand

Early Days II

William Wallenmeyer

Prehistoric Anecdotes

Kunitaka Kondo

Japanese Physics in 1970's

Hirotaka Sugawara

State of Physics in U.S. in the Late 1970s and CDF as a Pillar of the Collaboration

Alvin Tollestrup

The Japan/US HEP Collaboration: Program and Funding History

Kasuke Takahashi

- Session 2: Science Highlight I (Completed Experiments)

PEP4 Program at LBNL and SLAC

Hiroaki Aihara

Neutrino Scattering Experiment at BNL

Yorikiyo Nagashima

To and From Tau Neutrino Experiment at Fermilab

Mitsuhiro Nakamura

The "LASS" Program - The LASS Experiment and the SLD Experiment at SLAC

David Leith

US-Japan and the KTeV Research Program at Fermilab

Robert Tschirhart

The Rare K-decay Experiment at BNL and Its Accomplishments .65

Laurence Littenberg

The AMY Experiment at TRISTAN, KEK .71

Stephen Olsen 


\section{- Session 3: Science Highlight II (On-going Experiments)}

PHENIX Past, Present, and Future: QCD at large T and $\alpha_{s}$

Barbara Jacak and Kyoichiro Ozawa

CDF Experiment at Fermilab

Fumihiko Ukegawa

Exciting High Pt Physics Results and Opportunities in Run II

Robert Roser

Fermi Large Area Telescope

Tsuneyoshi (Tune) Kamae

Belle and Belle-II

Thomas Browder

Super-Kamiokande

Yoichiro Suzuki

The Super-Kamiokande Collaboration

Henry Sobel

KamLAND

Kunio Inoue

- Session 4: Science Highlight III (R\&D on Acc. \& Det.)

U.S./Japan Collaborative Accelerator R\&D: JLC/NLC/ILC

Gregory Loew

Accelerator R\&D for LC - Activities on ATF \& STF

Seiya Yamaguchi

Development of Superconducting Magnets for Particle Accelerators and Detectors

Takakazu Shintomi and Akira Yamamoto

R\&D on the Detector Technology: Last Decade And Future

Junji Haba

- Session 5: Future Prospects for High Energy Physics and Accelerators

Perspective

Hitoshi Murayama

The Future of the US Programs

Pier Oddone

Future Accelerators - ICFA Chair's View

Atsuto Suzuki

- Session 6: Future Plan of Laboratories

Future Plans at SLAC 165

David B. MacFarlane

Future Plans at Brookhaven National Laboratory

Samuel Aronson

Fermilab: Now and Future

Young-Kee Kim 
Future Plans at KEK

Masanori Yamauchi

Future Plan at J-PARC

Koichiro Nishikawa

- Session 7: Research Topics for Future

Proposal for Japan-US Collaboration on New Test Facility For Novel Accelerator R\&D FACET at KEK

Mitsuhiro Yoshida, et al.

Laser Plasma Accelerators For Future Colliders And Light Sources

Wim Leemans

Advanced and Innovative Accelerators

Mitsuru Uesaka

Proposal for US-Japan Collaboration for Precision Muon Physics g-2 and

EDM Measurements at J-PARC and Fermilab

Naohito Saito

Muon Collider: R\&D Status

Robert Palmer

- Session 8: Closing Session

Value of the US/Japan Collaboration in the Past and Future: A Summary of the Round Table

Discussions

Kohei Yorita

Value of the US-Japan Collaboration in the Past and Future: Closing Address

Takayuki Fujiyoshi

US-Japan HEP Collaboration 30th Anniversary Symposium: Closing Address

Dennis Kovar

- Session 8: Closing Session

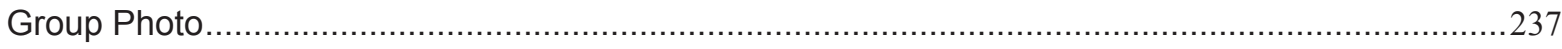

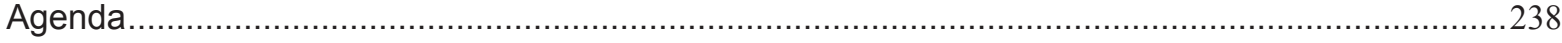

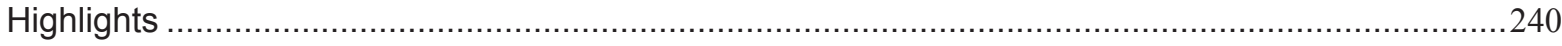

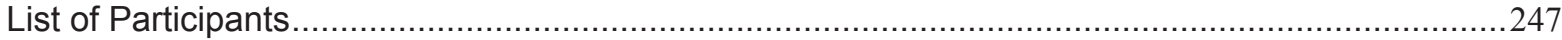





\title{
Opening Address
}

\author{
Fumihiko Takasaki, KEK
}

Good morning, distinguished guests, ladies and gentlemen.

On behalf of the Joint Committee of the Japan-US Scientific Cooperation Program in the Field of the High Energy Physics, we would like to welcome all of you to the symposium celebrating the $30^{\text {th }}$ anniversary of this cooperation program. Dr. Denis Kovar of DOE and myself are currently serving as the co-chairs of the joint committee.

It was 31 years ago when this cooperation Program was founded and it has been providing an excellent opportunity for physicists from two countries to work together. It has served as the framework for many Japanese to challenge their ambition in the High Energy Physics. For the junior Japanese fellow, it served to become familiar with the most advanced High Energy Physics experiments in the world and to know physicists in the US labs. In fact, there are many Japanese who got training in this program as the graduate students or young post-docs and became leaders of High Energy Physics in Japan later.

It is a really remarkable fact that this cooperation has been successfully executed for so many years. The success is primary due to the strong desire and ambition of physicists who wanted to realize their dream at the most competitive and challenging facilities and with the most experienced people.

I would say that this success is only possible by the quite generous support and help extended to the Japanese researchers by the US government, US labs leadership, officials and scientists in the US institutions. Of course, we know that it was impossible to carry out this project without the deep understanding and the support of the Japanese government, Japan Science Promotion Society, and many officials in Japan.

At this occasion of the $30^{\text {th }}$ anniversary, we would like to thank many people who contributed to the establishment of this program, especially Mrs. Saito, Shinozawa, Drs Nishikawa, Kikuchi, Panofsky, Lederman, Wallenmeyer and Hildebrand.

I would like to thank also Drs. Ozaki and Sugawara who made indispensable contribution to the successful operation of this program. They have been always earnest and enthusiastic promoters and supporters of this program from the beginning until today.

Now, I believe that research in the High Energy Physics will further disclose many more mysteries of the universe and provide us with many surprises in the coming decades. The Japanese and US people will be front runners in this research and therefore the cooperation between two people will become more and more important and this framework of the cooperation will serve as the basis for the ambitious endeavor of the next generation.

We hope that this symposium provides us with an opportunity to look back what we did in the past, to listen to what we are doing now and to think about our future of this program. We also hope that you enjoy the reunion with our old friends and interactions with the people of new generation at this opportunity.

Finally, we thank Dr. Ozaki and BNL people for their many works for preparing this symposium. We thank also people of the University Hawaii who kindly hosted this symposium including the tour to the SUBARU observatory.

Thank you very much for your attention. 



\title{
Congratulatory Remarks
}

\author{
Yoshihiko Kamo, Consulate General of Japan at Honolulu, Honolulu, HI
}

Good morning everybody, I will introduce myself - I am Yoshihiko Kamo, Consul-General of Japan in Honolulu.

Well, Distinguished Delegates, Ladies and Gentlemen I would like to extend my heart-felt welcome to the members of the Japan/US Collaborative Program in the Field of High Energy Physics to Hawaii. It's an honor and a privilege for me to be with you here today, and I hope that all of you will enjoy your stay here on the big island which is blessed with abundant natural beauty. I also wish everyone meaningful and fruitful discussions that will deepen friendships and cooperation.

Many scientists and researchers from all over Japan and the United States, along with researchers from the University of Hawaii have assembled here today to celebrate the $30^{\text {th }}$ anniversary of the Japan/US Collaborative Program in the field of high energy physics established as part of the Japan/US Cooperation Agreement on Research and Development in Energy and Related Fields in 1979. It's an excellent occasion to sum up the experience of the past 30 years and look ahead and discuss the future activities of the program.

I have heard that the Japan/US Collaborative Program in the Field of High Energy Physics has made many significant achievements throughout the years, including training and educating skilled researchers with international perspectives. I hope today's Symposium will serve as an opportunity to further advance the programs activities.

Japanese immigration to Hawaii is one of the most important events that founded the strong relationship between Japan and The United States, and an even stronger one between Japan and Hawaii. Immigrants from Japan began arriving in Hawaii in 1868. Government-contracted laborers from Japan began arriving in large numbers after Japan and Hawaii signed an immigration agreement in 1885. This year marks the 125 anniversary of Japan's government sponsored immigration. By 1924, the number of immigrants from Japan was estimated to have reached 200,000. Today the people of Japanese descent have a very significant presence in Hawaii as there are many prominent and influential Japanese Americans in the political, economic and educational fields.

As for the economic relations, Japanese investments poured into Hawaii in the 1980s. These investments helped create important infrastructures in Hawaii. In addition, many Japanese travel and retail companies opened offices in Hawaii in which it led to an influx of Japanese visitors. To this day, the Japanese visitor count continues to be the largest among international visitors. The pleasant and beautiful land of Hawaii where traditional Japanese culture remains strong is a treasure for the Japanese people. Moreover, the national astronomical observatory of Japan operates the Subaru Telescope on the summit of Mauna Kea which is located at the 4,200 meters above sea level of this island. 
With regards to the recent developments in the US/Japan Collaboration projects, following the 2009 Japan/US national agreement on clean energy development, both countries made a commitment to collaborate on clean energy projects in Okinawa and Hawaii which share similarities such as energy structure, industrial structure and population size. The United States and Japan are currently discussing the specific areas in which they can collaborate. In addition, after Japan hosts the 2010 APEC Summit in Yokohama, the United States is set to host the 2011 APEC Summit in Honolulu next November.

These things that I mentioned are just a few examples of the relationships between Japan and Hawaii or for that matter the United States. It is very timely and meaningful to have this important Symposium in Hawaii which has such strong historical ties with Japan. It is my sincere hope that the Symposium will score a big success and partnership among members of the Japan/US Collaborative Program in the Field of High Energy Physics will grow even stronger in the years to come.

In closing, I would like to convey my best wishes to all of you gathered here today, and to the further development of the Japan/US partnership in the Field of High Energy Physics.

Thank you very much for your time. 


\section{Welcome To Hawaii Greeting}

\section{A. Teramura, University of Hawaii}

Good morning and Aloha. So, by raising your hand, how many people is this your first time in Hawaii? So there are quite a few people. Let me tell you this, that there are two words that I want to acquaint you with. One is Aloha. Aloha can be used both as a greeting "hello", as well as a salutation like "goodbye", and when someone says Aloha, then to be polite you're supposed to respond back with Aloha. So, let's try this again. Good morning and Aloha. "Aloha" - very good - excellent. Okay, the second word that I think that you'll find very useful and something you'll use often, while you're here is the word Mahalo, and despite the fact that the word Mahalo is written on most of the trash cans, in public places, Mahalo does not mean trash can. Mahalo actually means thank you, and so you'll hear that word Mahalo being used often so Aloha and Mahalo I think are two important words for you to know.

Certainly is my great privilege and honor to welcome you all here to Hawaii, to our beautiful garden state. I thought I just might tell you a little bit about the University of Hawaii. We are actually a system. We have 10 campuses located on four different islands. Seven of those campuses are community colleges, and three of them offer at least a bachelorette degree. One of those campuses, the University of Hawaii at Manoa, from which I belong, actually is the flagship campus. We offer the graduate degrees in the system, and that includes not only the Liberal Arts Degrees, MA, MS and PhD Degrees, but also a number of professional degrees such as in Engineering, Medicine, Law, Nursing, Education, etc. So, the system is actually quite spread out across the state. The system has about 58,000 students. Manoa itself has roughly about 22,000 students, and about 6,000 of those are graduate students.

We like to say that actually we have no minority students, and the reason is because there is no one group that is the majority. That's an interesting way of thinking of it. About $20 \%$ or so of our student population really are what you might call Caucasians, about $20 \%$ are Japanese Americans, about $20 \%$ are Korean, and about $20 \%$ are Native Hawaiians, and then we have a whole host of other types of cultures. So, really one of the real unique and beneficial parts of coming to the University of Hawaii, is we really celebrate the great diversity that we have at our university culturally, and that's really a real unique experience for our students.

Well, this is a research conference, and I probably need to tell you that the University of Hawaii is not just a fun place to come to, to go surfing and be in a beautiful location. Actually, last year we had a research grant volume in excess of $\$ 450$ million dollars, which probably places us in the top 10 public research institutions in the United States, and we're very proud of that fact, and it's all due to the fact that we have some outstanding world class faculty at our campuses and some of those are hear in attendance today.

The University Administration really is pleased to be able to be co-sponsoring this group here for the next few days, and we certainly want to thank you for choosing Hawaii as a meeting place. I know you have a very packed schedule, and I look forward to listening to some of the talks, and I'll see some of you on top of the Summit Mauna Kea at 4,000 meters. Mahalo and Aloha. 



\title{
Early Days I
}

\author{
Bernard Hildebrand \\ Department of Energy (Retired), Bethesda, Maryland, 20814
}

The High Energy Physics (HEP) programs have had a long history of open international collaboration with scientists and engineers from many nations including Japan. HEP has enjoyed the participation of theoreticians, experimentalists, engineers, and technicians, all having a high interest in the field. This joint, as well as individual efforts, has led to many successes, discoveries, and advances in HEP. The goals of a deeper understanding of the nature of forces, matter and energy have been universal factors in attracting outstanding participants. Complexity and size have made collaboration, an extension of the old traditions of sharing, a requirement for many HEP activities.

Early contacts on US/Japan possibilities in joint energy research programs were initiated by the Japanese in the spring of 1978. In March of that year, Mr. Azuma from the Bank of Tokyo visited the US Department of Energy and the DOE accelerator laboratories to explore energy collaboration. He met with John Deutsch and scientists. It seemed that Japan with a large trade unbalance was accumulating dollars, and feared that this would have an adverse effect on the yen/dollar ratio. Additionally, Japan was intrinsically interested in the development of modern modes of energy production. It was believed that investment in collaborative long-term projects would be positive and would not affect the yen/dollar ratio.

The concept of US/Japan Collaboration in developing new energy sources was agreed to at the highest political level at the summit meeting between President Carter and Premier Fukuda in Washington in May 1978. The understanding was reinforced at the time of the major international economic summit at Bonn in July 1978.

The US/Japan Collaboration in HEP, as part of an energy agreement, was endorsed at the meeting of the HEP Advisory Panel at Stanford in August 1978.

The $19^{\text {th }}$ International High Energy Physics Conference in Tokyo was also the opportunity to exchange information on concrete collaboration possibilities relative to experiments and R\&D.

The Energy Meeting at the Okura, chaired by Deutsch and Miyazaki, was held immediately following the International HEP Conference. Joint working sub-panels, for each of the research areas were established to consider possible collaboration activities. The US members of this HEP joint working group were; Lederman, Ozaki, and Hildebrand, while the Japanese members were Shinozawa, Saito, Nishikawa, and Yamaguchi. While Wallenmeyer would ordinarily be on the sub-panel, he had previously accepted an invitation to visit China following the HEP International Conference. Fortunately, the US had two important strengths on the Sub-panel: they were Lederman who had been busy writing a draft HEP Cooperating Agreement which we edited the night before the Sub-panel met, and Ozaki who also translated and clarified any Japanese/English translation problems. 
The US/Japan HEP Sub-group concluded that, HEP was an essential complement to the program of developing alternative sources of energy for the needs of the world well into the $21^{\text {st }}$ century, and that new knowledge must be vigorously pursued. In addition, the Sub-panel members exchanged views on a well-defined joint program of research accelerator and instrumental R\&D, as well as construction possibilities. Joint use of present and anticipated facilities at BNL, Fermilab, KEK, and SLAC were to carry out unique experiments. The estimated level of funding implied a US/Japan contribution of \$10-14 m (US) per year for each country. Also, considered were joint studies of accelerator technology, instrumentation, and computer technologies. The funding level for these works was estimated $\$ 3-5 \mathrm{~m}$ for each country. To increase the scope and maximize the productivity of accelerators under design and construction at collaborating laboratories it was proposed to have an estimated schedule of funding of $\$ 3 \mathrm{~m}$ per year for each country increasing to $\$ 5 \mathrm{~m}$ over a five-year period.

The Japanese delegation reported on the work of the US/Japan HEP sub-group at the plenary session of the Energy Meeting and submitted the written report. This report carefully noted that the Sub-group was generally encouraged relative to the scale and scope of tasks related to joint use of HEP facilities and accelerator and instrumental R\&D. The attachment to the report notes that funding estimates are not to be construed as a commitment on either side.

Letter of appreciation to Professor Nishikawa from B. Hildebrand and W. Wallenmeyer can be found on page 11. 


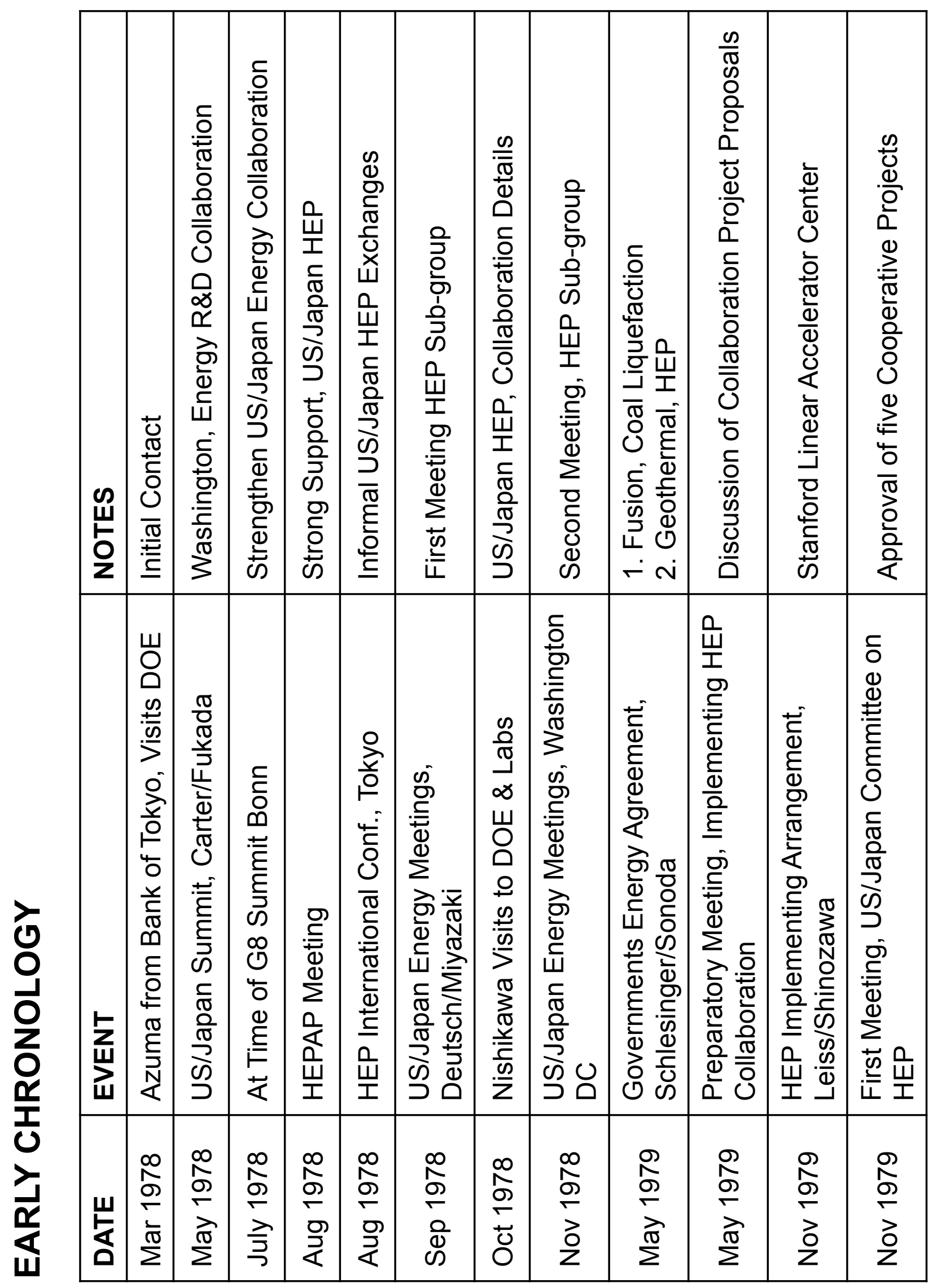


I. Joint Japan-U.S. Collabozation in High Energy Physics

II. Objectives and Rationale

High Energy Physics is an exploratory field at the frontier of basic research and directed towards an understanding of the basic laws which determine the proparties of energy, mass and forces of nature. He believe this subject to be an essehtial complement to the prograin of developing alternative sources of energy for the needs of the vorld well into the 2 Ist century. The history of technology is very clear: all the presently discussed sources of energy vere discovered as a byproduct of basic scientific resezrch. To insure the objectives of this Japan-U.S. collaboration, new krowledge pust be vigorously pursued.

III. We have exchanged views regarding a well defined program of joint collaboration in high energy physics. We have been encouraged in our discussions of scale and scope of tasies related to "Joint Use of Existing High Energy Facilities"(A) and "Joint Program of Accelerator and Instrumental Research and Development"(B). Furthermore ideas have been excharged as to the "future contributions to the construction of accelerators and expansion of auxiliary equipment"(c).

September 5, 1978 Tokyo, Japan
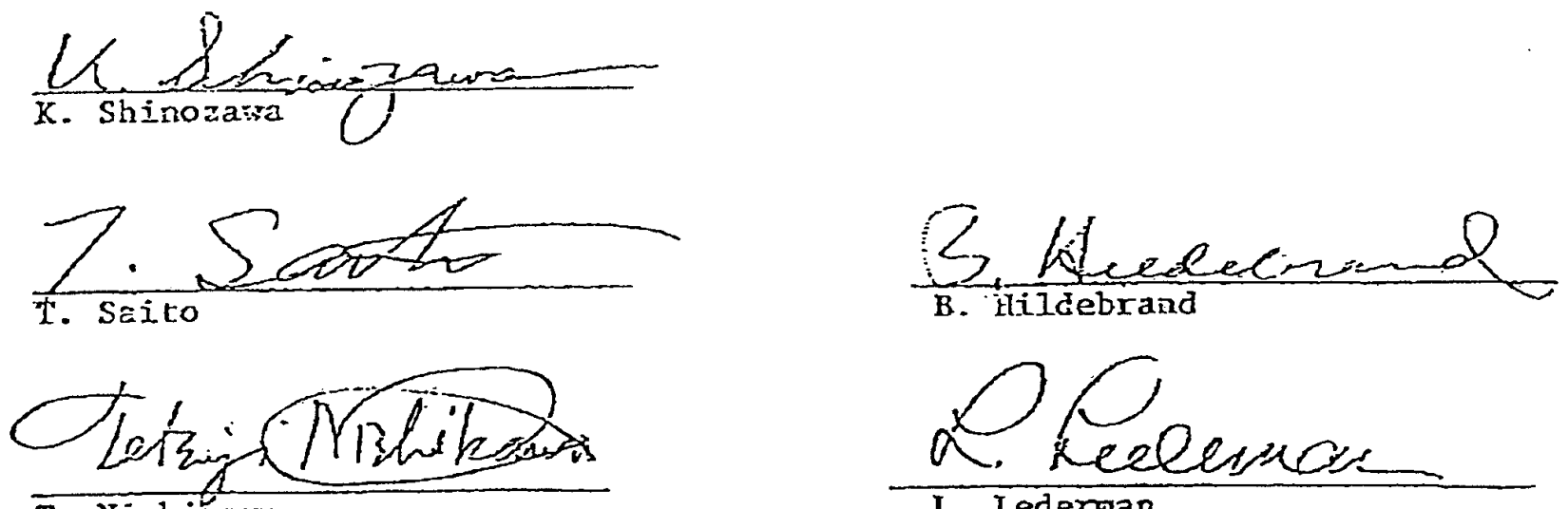

T. Nishikawa

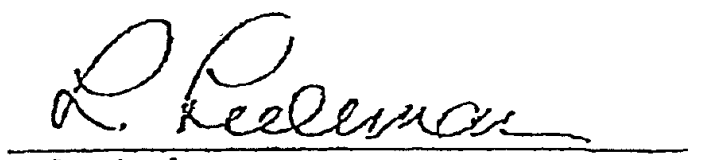

L. Lederman
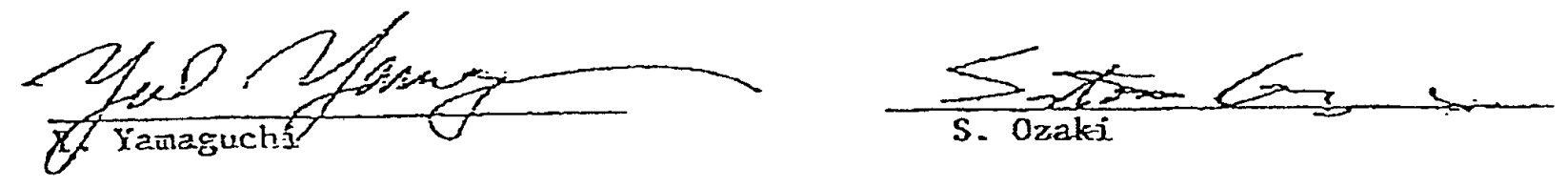

S. Ozaki 
SEPTEMBER 27, 2010

BERNIE HILDEBRAND

BILL WALLENTEYER

5225 POOKS HILL ROAD.

\$1613 SOUTH

BETHESDA, MARYLAND 20814

USA

DEAR PROFESSOR NISHIKAWA,

WE WERE DISAPPOINTED TO LEARNTHMT YOW WILL NOT BE PARTICIPATING IN THE 30 Th RNNIVERSARY MEETINE UN JAPAN/CS COOPERATION IN HIBH ENERCY PHYSICS. IT WOULD HAVE BEEN WONDERFUL TOEXCHANGE HELLOS ANO STURIES ON OUR NUMEROUS MEETINGS TOGETHER TO IRONOUT OWR COLLABORATIVE FROGRAMS FOR EACH YEAR.

IT HAS BEEN BOTH AN HONOR AND A PLEASURE TO TO WORK WITM YOU IAND YTUR LEADERSHIP IN MAKING THE JAPAN/US COLLABORATION IN HIGHENERGYPATSICS A Suctess.

\footnotetext{
WTH BESTIWISHES, SIDEERCLY, Brimire the delazano Bill Wallemmeyes
} 



\title{
Early Days II
}

\author{
William Wallenmeyer \\ Department of Energy (Retired), Rockville, MD 20850
}

Continuation of historical events leading up to the formation and first meeting of the Japan/US Committee on High-Energy Physics.

In the two and a half months following the September 1978 meeting of the HEP Working Subgroup, much work was completed in preparing for the second meeting in November 1978. This activity included T. Nishikawa's journey to the United States in October 1978 for discussions at the Department of Energy and the accelerator laboratories about a joint collaborative effort in High Energy Physics.

Secretary of Energy Schlesinger ${ }^{1}$ met in Japan on November 6, 1978 with Prime Minister Fukuda. One subject they discussed was the proposed Japan/US Collaboration in Research and Development on New Energy Sources and Related Fields; including High Energy Physics.

The second set of meetings of the joint Working Group on Research and Development on New Energy Sources and Related Fields and its Sub-groups was held in Washington on November 14-16, 1978. The Japan membership on the HEP Subgroup included T. Saito, MOE, and T. Nishikawa, KEK. The US membership included L. Lederman, FNAL; S. Ozaki, BNL; B. Hildebrand, DOE, and new members Jack Sandweiss, Yale University and W. Wallenmeyer, DOE. The Co-Chairs were Saito and Wallenmeyer. The Subgroup reached agreement on: 1. Objectives and procedures for the HEP collaboration; 2. Scale of a viable program on joint use of existing and new HEP facilities (Program A) and a joint program of accelerator and detector instrumentation research and development (Program B), estimated overall at an average cost of about $\$ 13$ to $\$ 19$ million per year from each country; 3. Specific examples of projects for Program A and Program B ready to be considered for implementation by April 1979 and soon thereafter; and, 4. The need for a program of joint participation in the construction and subsequent use of new forefront facilities (Program C), with a prior need for further study of Program C. The Subgroup recommended an early formation of the Japan-US Coordinating Committee for HEP to implement Programs A and B by April 1979; and the formulation of an Implementing Agreement based on the Subgroup's report and to be agreed to by the Sub-group. Unfortunately, the Ministry of Finance was not yet prepared to make a full commitment to HEP

${ }^{1}$ President Carter considered the energy crisis the Nation's greatest challenge, except for preventing war. On the day after his inauguration in January 1977, he named James Schlesinger as his personal representative to work on an immediate energy problem. On February 2, Carter proclaimed a national emergency on energy. In the first 90 days of Carter's presidency, Schlesinger developed the administration's basic energy-reorganization plans, including a new Cabinet Level Department of Energy, and new energy policy strategies. Legislative action creating the Department of Energy was completed by August 3, 1977, Carter signed the Bill into law on August 4, and the next day named Schlesinger as the first Secretary of Energy. The Department was officially activated on October 1, 1977. 
under the Fukuda initiative. However, the MOESC was planning to commit new funds for HEP cooperative projects starting in April 1979. Dr. Deutch stated in his closing remarks as US CoChair of the overall negotiations that the United States was prepared to proceed immediately with the joint US/Japan high energy physics program as soon as the Japanese agreed to participate. I personally indicated to Saito and Nishikawa that the position of the DOE highenergy physics program was:

"The US is prepared to proceed immediately with a joint Japan/US high energy physics program on an equitable basis -- up to the full level indicated in the subgroup report -- as soon as high energy physics is included in the joint agreement. Funds currently in the DOE high energy physics funding budget for research, operations and equipment, are adequate to initiate a full program of collaborative projects."

It is important to note that there was a successful informal Japan/US collaborative effort underway in high-energy physics at this time which had started in the late 1950s, with impressive scientific results that earned mutual trust, respect and warmth for the collaborative efforts. It also provided an experience and knowledge base and helped lead to strong support among the scientists, including the HEP Advisory Panel (HEPAP), for the formal collaborative effort in high-energy physics indicated in the Fukuda energy initiative.

On May 2, 1979 in Washington, DC, during the recently elected Prime Minister Ohira's visit to the United States, an "AGREEMENT BETWEEN THE GOVERNMENT OF THE UNITED STATES OF AMERICA AND THE GOVERNMENT OF JAPAN ON COOPERATION IN RESEARCH AND DEVELOPMENT IN ENERGY AND RELATED FIELDS" was signed for the Government of the United States by Secretary of Energy, James Schlesinger, and for the Government of Japan by Foreign Minister Sunao Sonoda. The Agreement included high-energy physics as one of the areas for cooperation. It was directed that implementing arrangements, specifying the details and procedures of cooperative activities in each area, should be made between the two Governments or their agencies, whichever was appropriate.

On May 28 and $29^{\text {th }}, 1979$ a preparatory Japan/US Meeting on High Energy Physics was held in Washington, DC. Japan participants included H. Ueki, MOE; T. Nishikawa, KEK; H. Nagasue, KEK; T. Fujii, Tokyo University; and, T. Kitagaki, Tohoku University. US participants were J. Leiss, DOE; J. Ballam, SLAC; R. Birge, LBL; B. Hildebrand, DOE; L. Lederman, Fermilab; S. Ozaki, BNL; R. Rau, BNL; J. Sandweiss, Yale University; A. Tollestrup, Fermilab; and W. Wallenmeyer, DOE. Also present from the DOE were J. Metzler, International Affairs, J. Stekert, Planning and Evaluation, and E. Summers, Office of Energy Research. The Japanese proposals for experimental research and proposals for accelerator and instrumentation research and development were discussed, including input from the accelerator laboratory participants on the status for those at their laboratory. Participants from both countries agreed that the proposed joint experimental research, and research and development programs were viable, scientifically excellent and timely, and that the FY1979 planned research program should start as soon as possible. The participants had preliminary discussions of an implementing arrangement for the collaboration and agreed as to its general principles. It was also agreed that drafts of an implementing arrangement would be exchanged, with the goal of signing such an agreement 
soon. It was recommended that a Japan/US Coordinating Committee be established as soon as possible after the signing.

Nishikawa called at 9:30 am on Nov. $6^{\text {th }}$ to inform us that clearance had been obtained for the implementing arrangement from the Ministry of Finance, and that there would be no more changes. Therefore we could continue with earlier scheduled plans to have the signing between the Japan MOE and US DOE at SLAC on November $11^{\text {th }}$.

On Sunday November 11, 1979, a group at SLAC witnessed the signing of the "IMPLEMENTING ARRANGEMENT BETWEEN THE DEPARTMENT OF ENERGY OF THE UNITED STATES OF AMERICA AND THE MINISTRY OF EDUCATION, SCIENCE AND CULTURE OF JAPAN ON COOPERATION IN THE FIELD OF HIGH ENERGY PHYSICS". This Arrangement was under the US/Japan Agreement on Cooperation in Research and Development in Energy and Related Fields. The group shown in the picture included, among others, the six Japanese and six Americans who were the first members of the U.S.-Japan Committee on High Energy Physics, that was established by the signing about to take place. The Implementing Arrangement was signed for the Japan Ministry of Education, Science and Culture (MOE) by Mr. Kohei Shinogawa, Director-General Bureau of Science and International Affairs, and for the United States Department of Energy by Dr James Leiss, Associate Director, Office of Energy Research, for High Energy and Nuclear Physics.

On November 12, 1979, the US-Japan Committee on High Energy Physics held at SLAC their first meeting. J. Leiss, DOE and Tetsuji Nishikawa, Director-General National Laboratory for High Energy Physics (KEK) were co-chairpersons. There were five other Japanese members: K. Shinozawa, MOE; T. Fujii, University of Tokyo; K. Kikuchi, KEK; T. Kitagaki, Tohoku University; and G. Takeda, Tohoku University; and, five other US members: R. Birge, LBL; L. Lederman, Fermilab; W. Panofsky, SLAC; R. Rau, BNL; and J. Sandweiss, Yale University. Other participants included K. Haga and N. Higuchi, KEK; G. Shigeto, MOE; S. Ozaki, BNL; G. Rickansrud, SLAC; W. Wallenmeyer, B Hildebrand, J. Metzler, and S. Stamp, DOE; and K. Nomura, Interpreter.

Thus was formally begun the Japan-US Cooperative Program in High Energy Physics of which we celebrate with this symposium, the very successful first 30 years of activity! 
Second Meeting of HEP Working Subgroup for JAPAN-US ENERGY MEETINGS

Washington, DC on November 14-16, 1978

\section{Members}

- US

- William Wallenmeyer, DOE

- Leon Lederman, Fermilab

- Satoshi Ozaki, BNL

- Jack Sandweiss, Yale University

- Bernard Hildebrand, DOE

\section{PREPARATORY MEETING Meeting for The JAPAN/US Collaboration on HIGH ENERGY PHYSICS on MAY 28-29, 1979 in WASHINGTON, DC}

\section{From Japan}

T. Nishikawa, Director-General, KEK

H. Ueki, Director, Science Division, MOESC

H. Nagasue, Director, Research Cooperation Division, KEK.

T. Fujii; Professor, Tohoku University.

T. Kitagaki, Professor, Tohoku University.

U.S

J. Leiss, Associate Director, Office of Energy Research, for HE and NP, DOE.

J. Ballam, Associate Director for Research Division, SLAC.

R. Birge, Associate Director for Physics Division, LBL.

B. Hildebrand, Branch Chief for Physics Research, Division of High Energy Physics, DOE.

L. Lederman, Director-Designate, Fermilab

S. Ozaki, Senior Physicist, Group Leader, BNL

R. Rau; Associate Director for High Energy Physics, BNL

J. Sandweiss, Chairman Department of Physics, Yale University

A. Tollestrup; Head, Colliding Beam Detector Group, Fermilab

W. Wallenmeyer, Director, Division of High Energy Physics, DOE

J. Metzler, International Affairs, DOE

J. Stekert, Planning and Evaluation, DOE

R. Summers, Office of Energy Research, DOE 


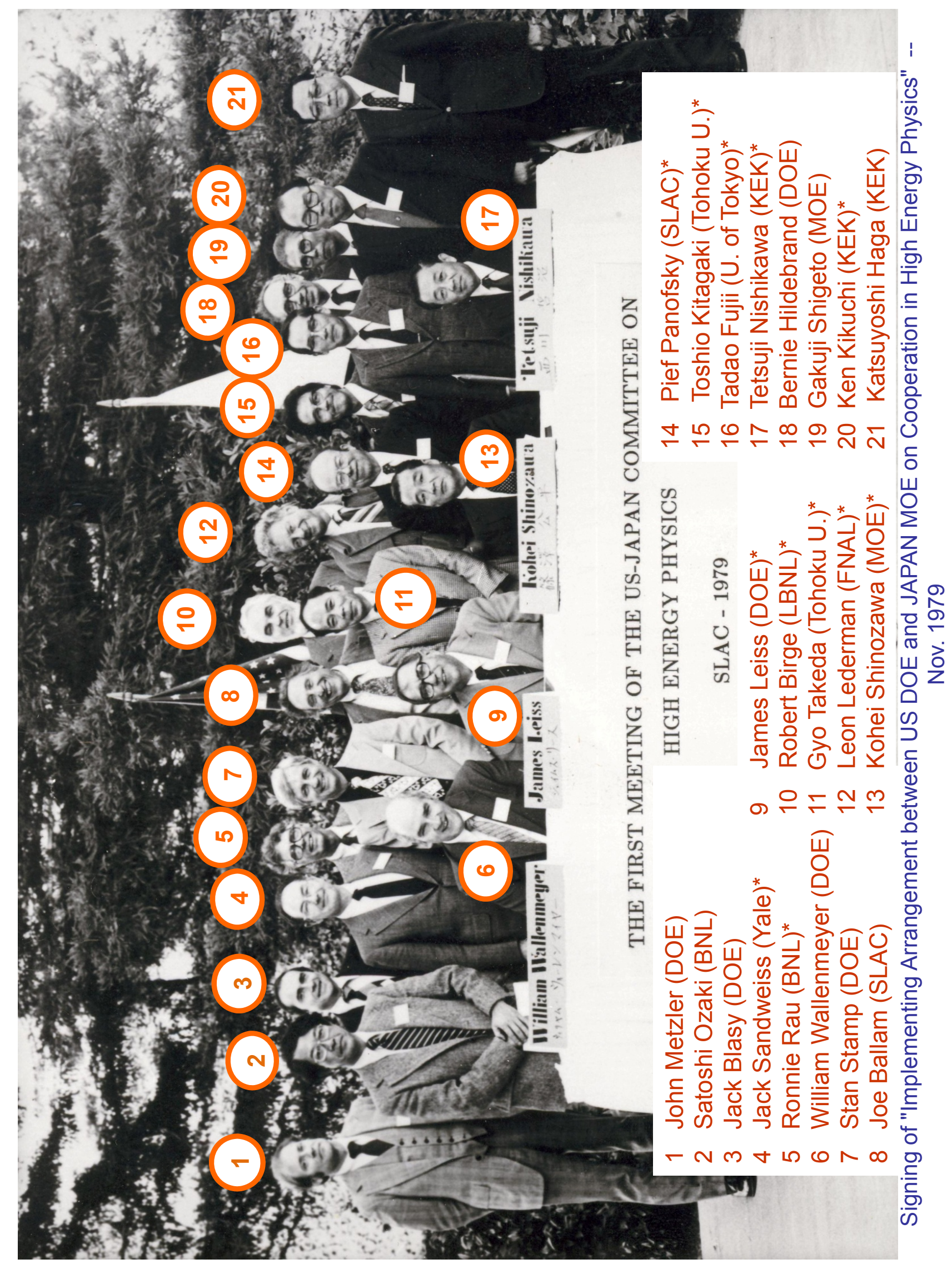





\title{
Prehistoric Anecdotes
}

\author{
Kunitaka Kondo \\ University of Tsukuba/Waseda University
}

I spoke about how the US-Japan (USJ) collaboration in High Energy Physics (HEP) was brought about within my personal knowledge and experiences. To initiate USJ collaboration in HEP, we had two great leaders, Dr. Wolfgang K.H. Panofsky (Pief) and Dr. Tetsuji Nishikawa, directors of SLAC and KEK, respectively, during that period. They had family roots and their own experiences in understanding each other countries and to be motivated for the collaboration.

A small-scale USJ collaboration in HEP, officially supported by Japan Society for Promotion of Science (JSPS), was carried out between Dr. V.W. Hughes (Yale) and K.K. (Univ. of Tokyo) at BNL and SLAC during 1973 and 1980. M. Mishina and K.K. who worked on this project wished to move to the higher energies.

The major step for the current USJ collaboration was taken by the $3^{\text {rd }}$ Conference between Prime Minister T. Fukuda and President J. Carter in 1978, where they agreed upon the USJ collaboration in energy researches including HEP.

By the communication between directors of BNL, FNAL, SLAC and KEK, the first round experimental subjects and people to work on individual subjects were defined before the USJ Signing Ceremony at SLAC in November 1978. 


\section{Two Great Leaders}

\section{Dr. Panofsky and Japan:}

- Dr. Albert Mosse: D. Panofsky's grandfather, Preussen lawyer.

1887 (Meiji 20) Prime Minister, Hirobumi Itoh, set up "Law Examination Committee". It consisted of 3
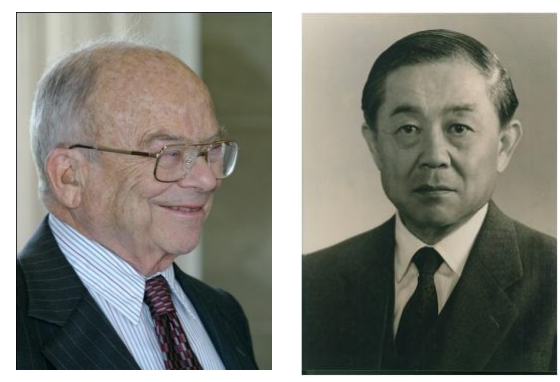

Japanese politicians \& 3 foreign lawyers including Dr. Mosse.

They worked on the draft of Meiji Constitution and for amendment of Unequal Treaties.

- Dr. W.K.H.Panofsky:

1977 Centennial celebration of the Physical Society of Japan:

Dr. Panofsky and Dr. Menon(India) were invited to give special talks.

\section{Dr. Nishikawa and US:}

- Dr. Shoji Nishikawa(1884-1954): Tetuji Nishikawa.'s father, Professor of Physics at U. of Tokyo \& Chief Researcher at RIKEN, X-ray crystallography: X-ray diffraction from cellose, spinel structure of crystals, application of the group theory to crystal structure analysis: 1917 : Invited to US for 3 months at Stanford U., for 18 months at Cornell U.

- Dr. Tetsuji Nishikawa:

1965 67: Invited to US to work at Accelerator Division of BNL.

Dr. Mosse's family in front of the Huge Statue of Buddha at Kamakura.

From left:

Pief's grandmother, Calorine Mosse, mother, Dora, as a child and other family members with maids.

By the courtesy of Adele (Mrs. Panofsky) and Dr. G.Loew(SLAC) .

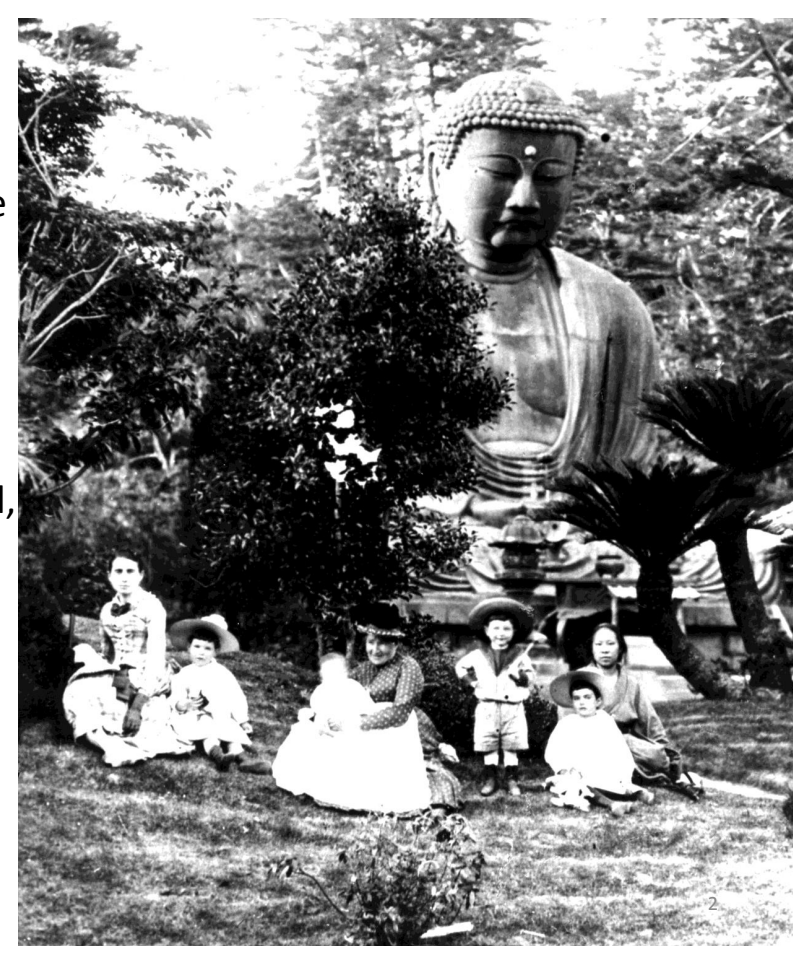




\section{Coherent Bremsstrahlung from Si Single Crystal}

T.Nishikawa and K.K. worked on this experiment at INS:

S.Kato, et. al., J.Phys.Soc.Jpn. 20. (1965),303.

The polarized photon beam was used for a search of an exotic quark state with a negative result.

T.Nishikawa, et. al.

Phys.Rev.Lett.21, 1288 (1968).

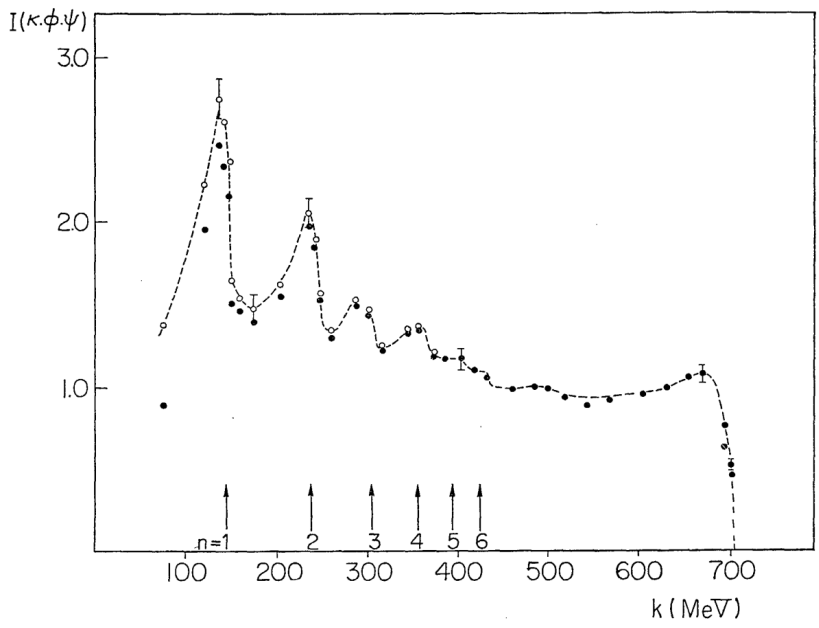

W.K.H.Panofsky cited this figure in his talk "Experimental Techniques" at International Symposium on Electron and Photon Interactions at High Energies, DESY, June 8-12, 1965.

\section{A USJ Collaboration before 1979}

1973 1980: First USJ collaboration in HEP experiments officially funded (JSPS) from Japan.

INS(U. of Tokyo; K.Kondo) and Yale U (V.W.Hughes*).

1. Scattering of $K$ and Pbar by polarized proton @BNL:

Yale; V.Hughes, M.Zeller,..., Japan; M.Mishina, I.Nakano

2. Scattering of polarized electron by polarized proton

E80,E130 @SLAC

US: Yale; V.Hughes..., SLAC; D.Coward...,

Germany: Bielefeld; W.Raith,..., Japan: K.Kondo, N.Sasao.

*V.W.Hughes later proposed BNL g-2 experiment as a USJ project: J contribution; S.Kurokawa, A.Yamamoto,.... for the muon ring. 


\section{Internal Spin Structure of Proton (SLAC E-80)}

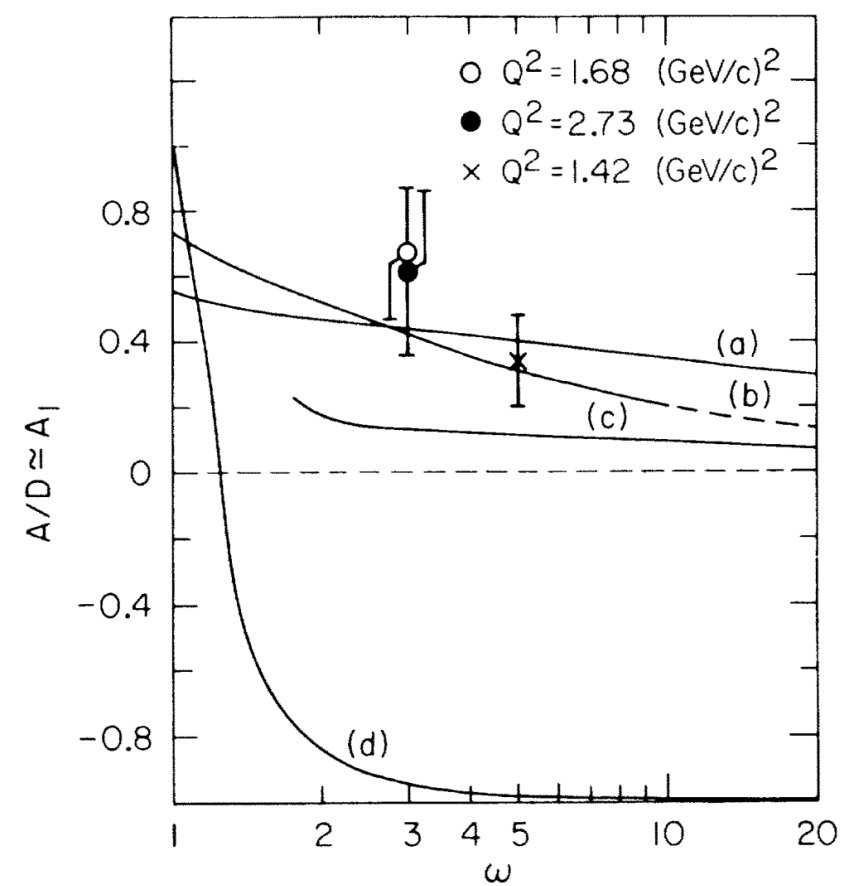

Phys.Rev.Lett.37,1261

(1976)

M.J.Alguard et al

"Deep Inelastic Scattering

of Polarized Electrons by

Polarized Protons":

Theoretical predictions:

(a)J.Kuti \& V.F.Weisskopf

(b)F.Close

(c) G.Domokos et al

(d)S.D.Drell \& T.D.Lee

\section{Final Steps to the USJ Collaboraion in HEP}

\section{Toward the Energy Frontier:}

1977. ISABELLE Workshop @ BNL.

K.K. met with Dr. A. Tollestrup, and visited FNAL on his way back.

- 1978 . "International Conference on High Energy Physics" at Tokyo:

Dr. L. Lederman encouraged K.K. to work on the PbarP collider experiment at Fermilab.

May 3 ${ }^{\text {rd }}$ 1978: 3rd T.Fukuda \& J.Carter Conf. @Washington D.C.:

- US-J Collaborations in Energy Researches; 1. Coal gasification, 2. Plasma fusion, 3. Photosynthesis, 4. Nuclear energy, 5. High energy physics (Japan initiative) were agreed upon.

The support by Drs. W.Wallenmyer and Hildeband (DOE) was crucial.

\section{Materialization of the Accord:}

- T.Nishikawa made close communications with US lab. directors about subjects for the HEP collaboration. Subjects and people to work on each subject were well defined before the Signing Ceremony on Nov.11, 1979 at SLAC. 


\title{
Japanese Physics in the 1970s
}

\author{
Hirotaka Sugawara, JSPS, Washington Office
}

Experimental high-energy physics was dominated by US researchers in the 1970s. Their discoveries included charm quarks (fig1), bottom quarks (fig2), and tau leptons (fig3) among others. At that time, Japanese high-energy physics stood poised to take off with the creation of $\mathrm{KEK}$, and the start up of its first major accelerator, a $12 \mathrm{GeV}$ proton synchrotron. Its construction began in 1969 and was completed in 1974. The beam's intensity quickly reached its design value (fig4) although the energy limitation of $12 \mathrm{GeV}$ was rather restrictive for undertaking world-class experiments using this machine. It was much later in the 1990s that Japanese scientists were able to complete the world's first long-baseline neutrino experiment by sending a neutrino beam into the Kamioka mine.

Let me quote from the executive summary of the report of the 2003 committee assessing the performance of the $12 \mathrm{GeV}$ KEK-PS. This committee, formed before the shutting down of the KEK-PS, and facing the starting of J-PARC synchrotron, was chaired by Satoshi Ozaki.

\section{Executive Summary}

The KEK 12 GeV Proton Synchrotron (PS) Program since 1976:

Since its commissioning in 1976, the $12 \mathrm{GeV} P S$ at KEK has successfully supported a highly productive physics program in Japan. For more than 25 years, the PS has operated well with good reliability in spite of the constraint of having limited aperture. This limited aperture has prevented the PS from taking advantage of the steadily increased booster intensity over the years. With many innovative steps the $12 \mathrm{GeV}$ PS operations group recently succeeded in increasing the average intensity for the fast extraction from $4 \times 10^{12} \mathrm{ppp}$ to $6 \times 10^{12} \mathrm{ppp}$, the improvement that was essential for the successful run of the $K 2 \mathrm{~K}$ experiment.

The experimental program of the $12 \mathrm{GeV}$ PS can be divided into three periods, namely 1977 1984 (before TRISTAN), 1985 - 1998 (after the start of TRISTAN and before K2K), and the period since 1999 (after the start of the K2K experiment). During the first period, the PS was the only high-energy accelerator in Japan, and as such, it had supported a wide range of particle and nuclear physics experiments. Notable accomplishments in this period are an early determination of the upper limit for $K+\rightarrow \pi^{+} v v$-bar and the rejection of the existence of barionium states.

Japanese researchers in theoretical high-energy physics were active throughout the entire 1970s. Let me mention a few examples from this period:

\section{Kobayashi-Maskawa Model, 1973}

The Nagoya group was flexible about the number of quarks because of the signal of "new quark" in the Niu'cosmic ray experiment that led to considerable unique work at this time. Much later, the model was confirmed by the KEK and SLAC B-factories; Kobayashi and Maskawa were awarded the Nobel Prize for their model in 2008.

\section{Supersymmetry}

In 1966, Miyazawa first proposed supersymmetry algebra in the context of hadron physics based on his work with Sugawara in 1965. Supersymmetry became the central theme of high-energy theory although it has yet to be confirmed experimentally. 


\section{Proton Decay}

In 1978, Yoshimura made a proposal to apply the grand unified theory to understanding the baryon number of the universe. Proton decay is the essential parameter in this theory. We still are awaiting the discovery of proton decay.

\section{Neutrino Mixing (Must have a title about the work, as in 1,2 and 3.}

Maki-Nakagawa-Sakata contemplated possible neutrino mixing as early as 1962 . It was only after observations on solar- and atmospheric-neutrino that their insight was confirmed as being right.

\section{5. "Color Change"}

Y. Nambu, a Japanese-born American physicist, has made enormous contributions to high-energy theories, including his major works on "color change" in quantum chromodynamics, spontaneous symmetry breaking in particle physics, and he was one of the first proponents of the string theory.

The US-Japan collaboration in high-energy physics started in 1979 with much effort by both USand Japanese-physicists including T. Nishikawa who then was the Director General of KEK. This project particularly was effective in training Japanese experimentalists in Japanese universities by sending them to various laboratories in the United States conducting research in high-energy physics; KEK's physicists were busy with the KEK-PS experiments, and later in the 1980s with the TRISTAN. Physicists from several Japanese universities also participated closely in TRISTAN, and this commitment prevented them taking leadership roles in some experiments done in the US high-energy laboratories, with some notable exceptions. The situation was summarized in the report of the 1986 Evaluation Committee of US-Japan Project chaired by the late Professor Hayakawa of Nagoya University, and consisting of Tomoo Ishihara, Hirotaka Sugawara, Yoshio Yamaguchi, and Toshimitu Yamazaki.

The role played by the US-Japan collaboration in high-energy physics in the early 1980s.

1. The only high-energy facilities available in Japan for Japanese researchers in this field at the start of this project were the $1.3 \mathrm{Gev}$ electron-synchrotron in INS, and the $12 \mathrm{GeV}$ proton-synchrotrons in KEK. The handicap facing them in accessing high-energy accelerators, especially colliders, was taken into account when their role in the project was evaluated.

2. The committee also considered the effects of the start-up of the TRISTAN project soon after this collaboration began. . The anticipated number of participants in the US-Japan project fell, as some scientists transferred to work on TRISTAN. Nevertheless, we realized that the experience learned in US-Japan project was useful in conducting research with the TRISTAN accelerator.

The annual budget for the collaboration of 15 oku-yen is as large as one "tokokutei kenkyu", but the number of participating groups only is about 1/10 of a regular "tokutei kenkyu". The travel money expended is comparable to the entire travel budget of Science Council of Japan (Gakujutsu kaigi). These figures demonstrate that the project is far better financed than others. Undoubtedly, this is because it represents the largest international collaboration that Japan ever undertook in the field of basic science.

Therefore, some may expect the project should produce the best possible results, both in scientific outputs, and in the leadership of Japanese scientists. However, considering the smallness of Japanese budget compared with the large investment by the United States in 
constructing and operating the accelerators, we may not have anticipated the best output. Nevertheless, we found that most experiments accumulated invaluable, reliable data that contributed greatly to the development of physics. The committee recognized that the processes foot printed by this project were a necessary step for the development of Japan as a leader in the field of high-energy physics. The committee also realized that Japanese researchers learned great lessons that were useful in the TRISTAN project, and that their training was undertaken in an international context, giving them an understanding different lives and cultures. Generically, the committee highly appreciated the value of this project, but nevertheless felt obliged to offer the following critique:

Although we understand the handicap facing Japanese researchers in speaking a foreign language and living a different life style, we point out that only few Japanese researchers are playing a leading role in the experiments.

\section{Conclusion}

The US-Japan collaboration in high-energy physics from the end of 1970s to the early 1980s played a very important role, especially for those researchers in Japanese universities partly because the period overlapped the construction stage of the KEK. 
High Energy Physics in 1970's was dominated by US

B.Richter etal. 1974

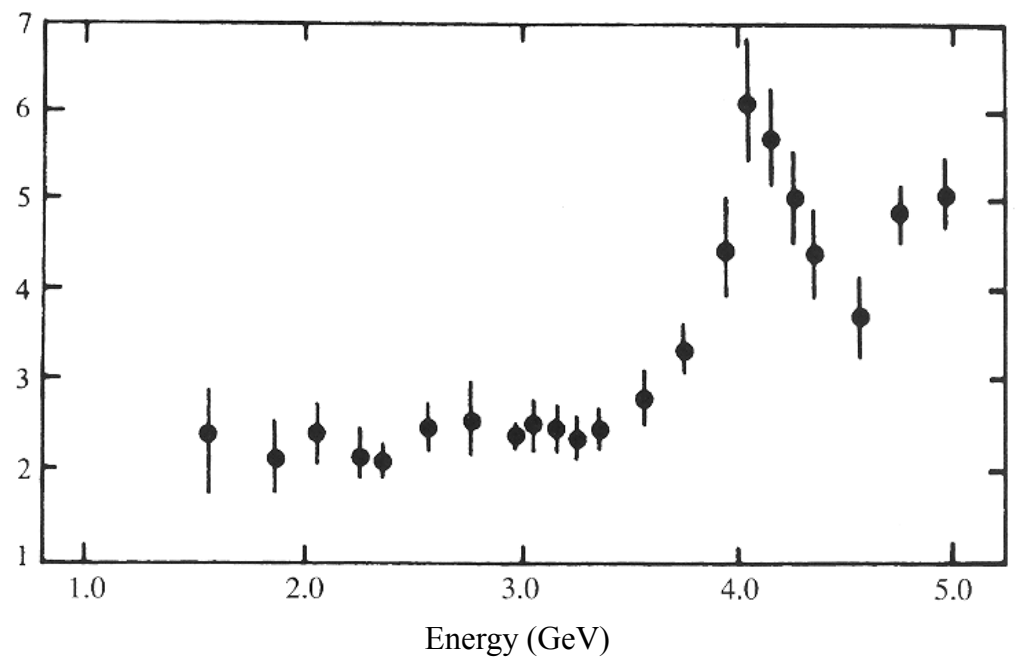

Fig. 1 Discovery of Charm Quark

L. Lederman etal. 1977

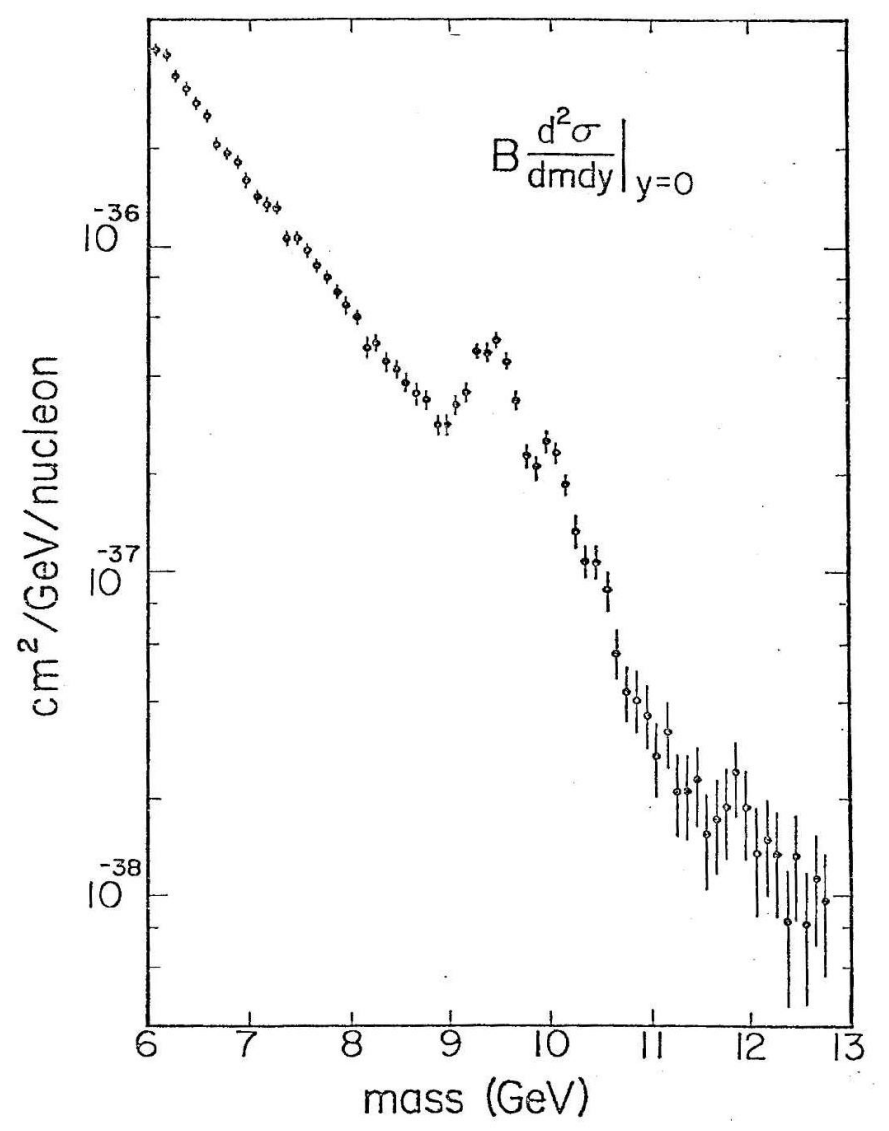

Fig. 2 Discovery of Bottom Quark 


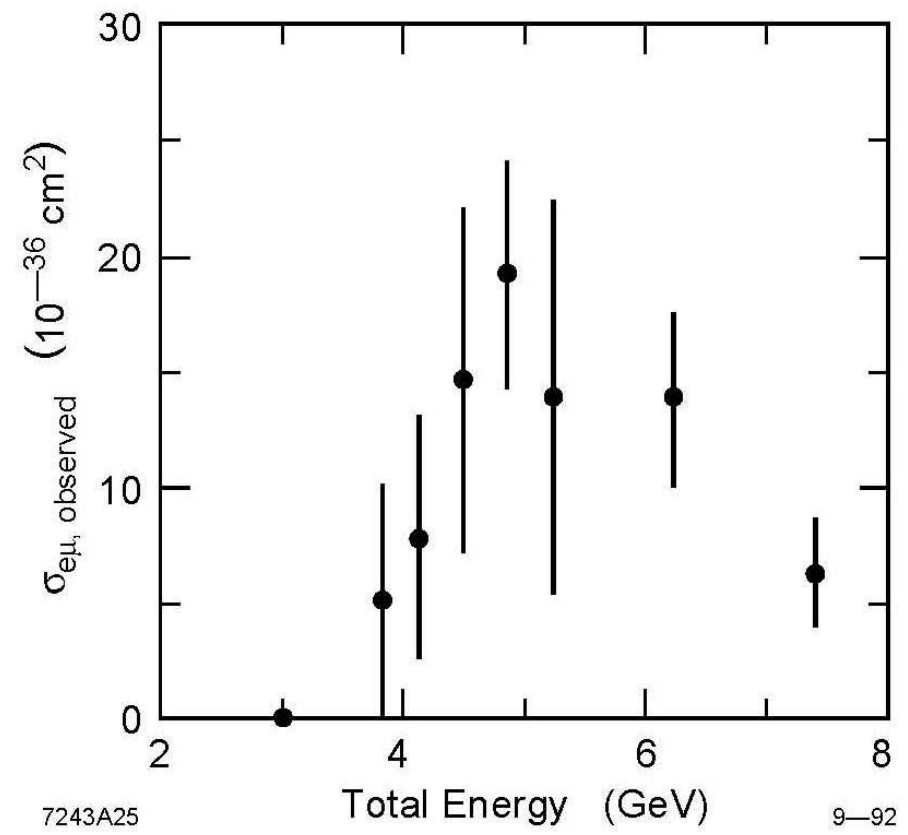

Fig. 3 The observed cross section for the signature e- $\mu$ events from the SLAC-LBL experiment at SPEAR. This observed cross section is not corrected for acceptance. There are 86 events with a calculated background of 22 events. ${ }^{33}$

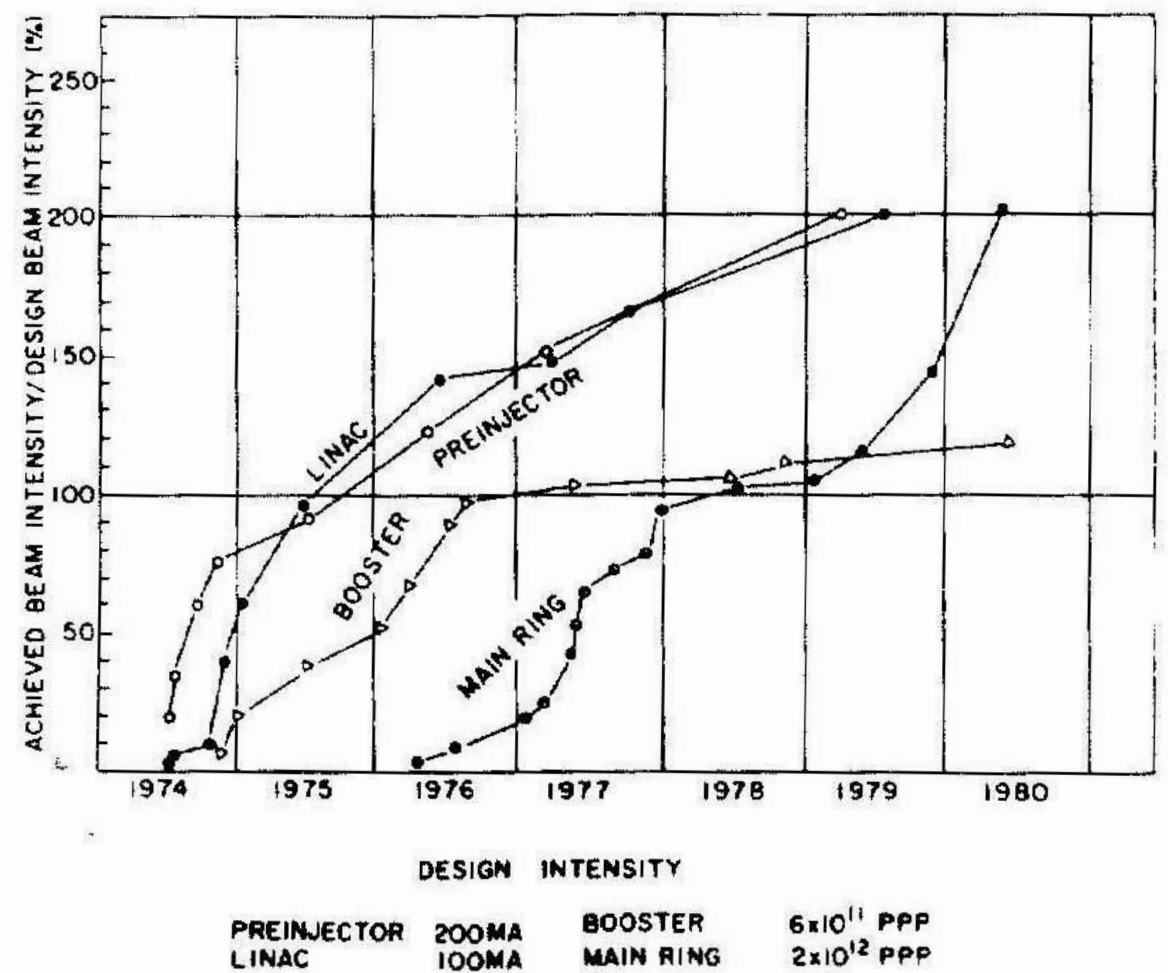

Fig. 4 Growth of the beam intensity. 

State of Physics in U.S. in the Late 1970s

And

CDF as A Pillar of the Collaboration

Alvin Tollestrup, Fermilab, Batavia, IL 

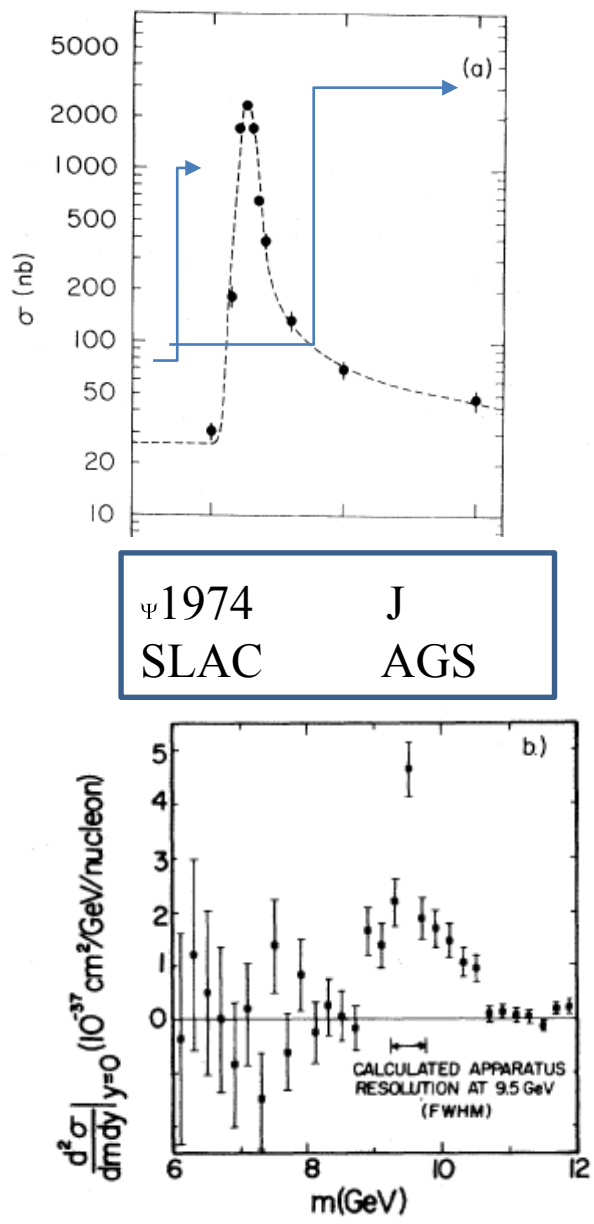

Y FNAL 1977
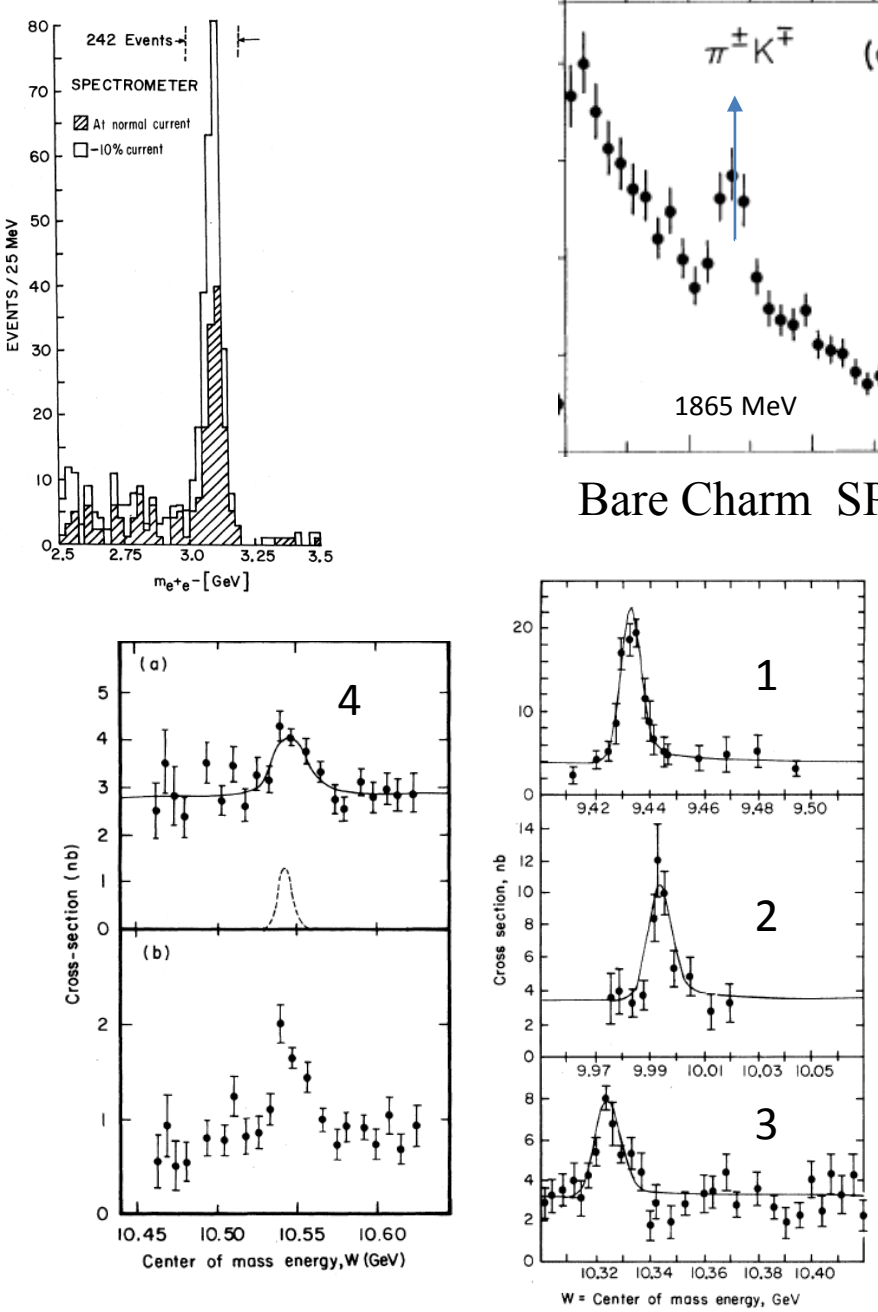

CLEO 1980

Y $1,2,3,4$

\section{THE AGREEMENT 1978-1979}

May 1978 Summit meeting. Prime Minister Fukuda and Pres. J. Carter.

May 2, 1979 the "Agreement" was signed by Schlesinger, Sec. Energy and Sonoda, Minister of Foreign Affairs. Covered a long list of items. HEP not explicitly mentioned!

But the HEP community had been very busy! Even in late 1978 a plan was being discussed for cooperation between US and Japan HEP communities.

May 28, 1979 Meeting in DC to discuss the 1979 effort and layout the 1980 plan.

\begin{tabular}{|lll|}
\multicolumn{1}{c}{ U.S. } & JAPAN \\
\hline Birge & Sandweiss & Nishikawa \\
Lederman & Hildebrand & Fujii \\
Ozaki & Leiss & Kitgaki \\
Pief & Wallenmeyer & Ueki \\
Rau & Metzler & Nagasue \\
\hline
\end{tabular}




\section{Some Pictures}

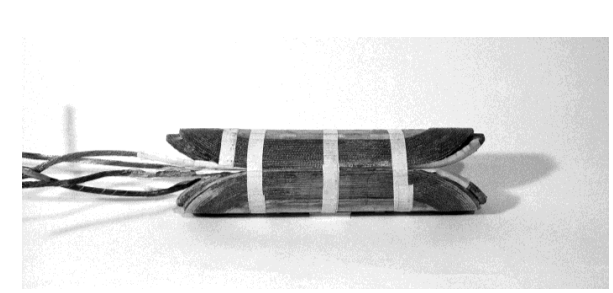

1976 model magnet

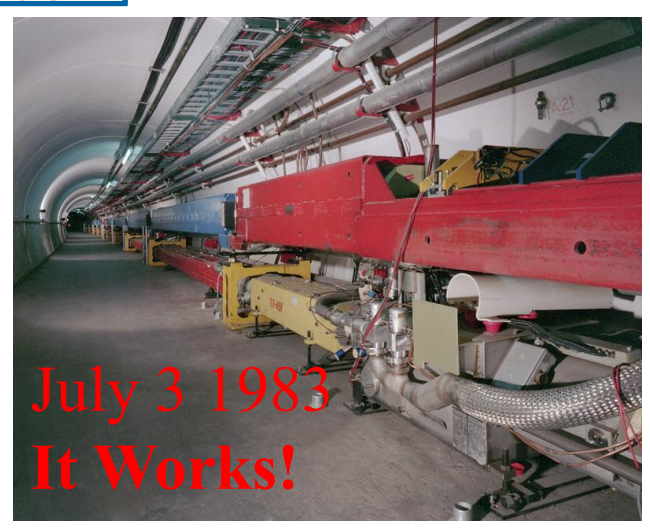

1976: Director winding model magnet

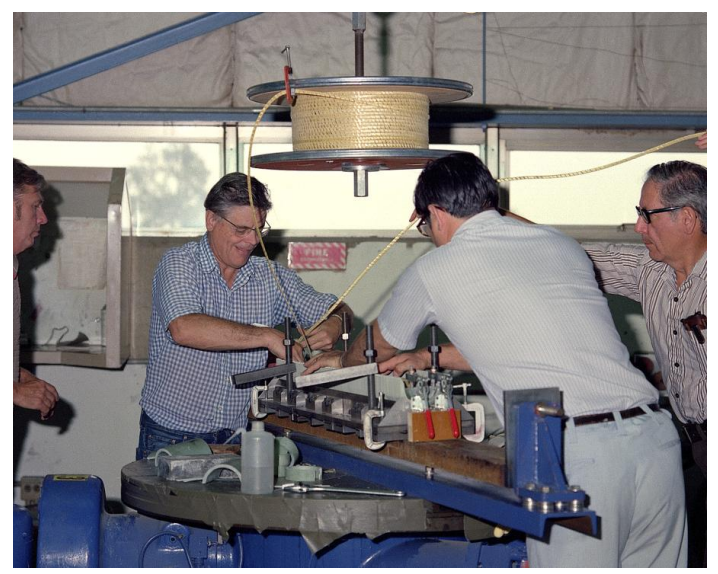

1983 Real

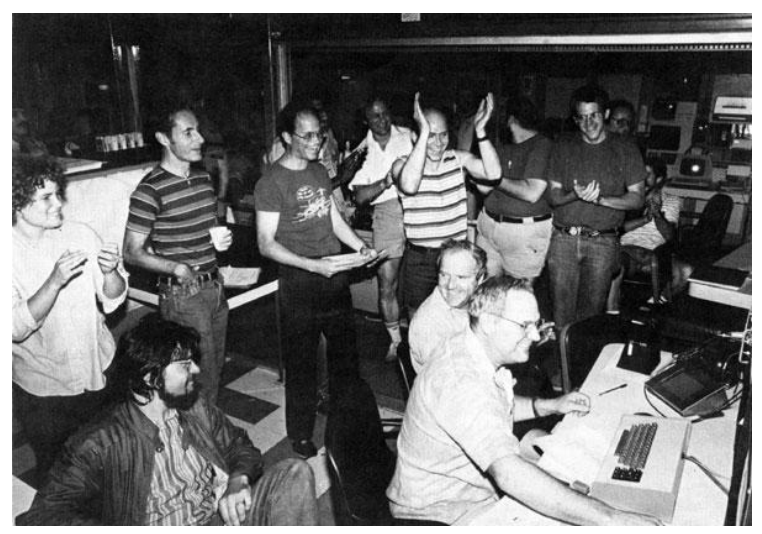

\section{TEVATRON AUTHORIZED JULY 1979}

1979: Sho Ohnuma and R. Yamada indicated that K. Kondo and several other Japanese we in the U. S. looking for ways to collaborate in HEP. I met them at a Conference at BNL and told them about our plans for a colliding beams experiment at FNAL. They came to visit and met with Lederman. The collaboration with CDF was finalized at the end of the year!

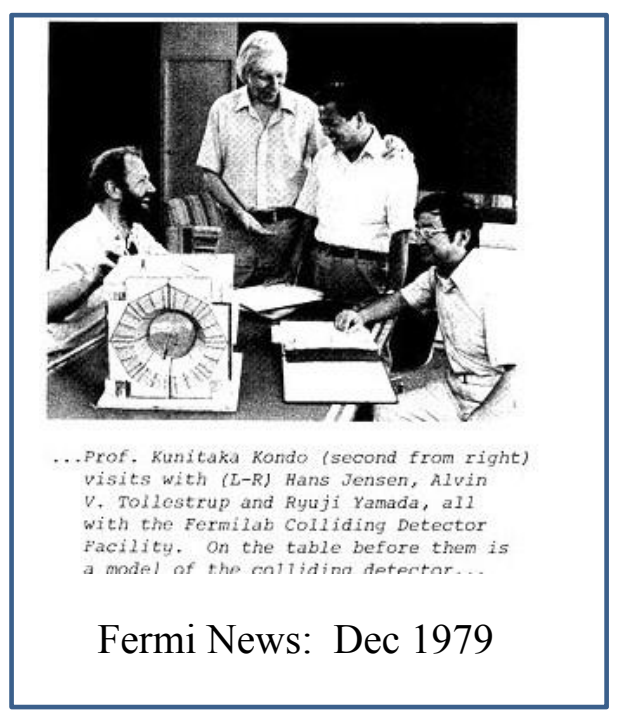

1980: Italians joined CDF

1981: Conceptual Design Document

1982, July 1. CDF Construction Begins! Start construction of pbar source.

1983, July 3. First Beam in Tevatron!

1985, Oct. 13. First Collisions at CDF 


\section{CDF Central Tracker The heart of precision spectroscopy at CDF}

The solenoid was constructed in Hitachi under supervision of Shigeki Mori/Tsukuba University. The design was unique:

. Constructed in 1983 it was the largest solenoid built- $-3.0 \mathrm{~m} \times 5.0 \mathrm{~m}$ long, $1.5 \mathrm{~T}$. It stores about $30 \mathrm{MJ}$.

- Indirectly cooled outer shell of aluminum

- New conductor developed. $3 \mathrm{~mm}$ x $20 \mathrm{~mm} \mathrm{Al} \mathrm{co-extruded} \mathrm{around} \mathrm{NbTi} \mathrm{SC.}$

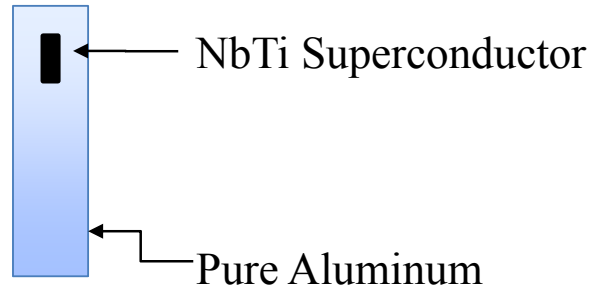

. Thin in Xo leads to better particle identification.

- This was only one example where a new technology was brought into CDF by a Collaborator resulting in a major upgrade to the properties of the detector.

\section{CDF Solenoid Hitachi 1983}
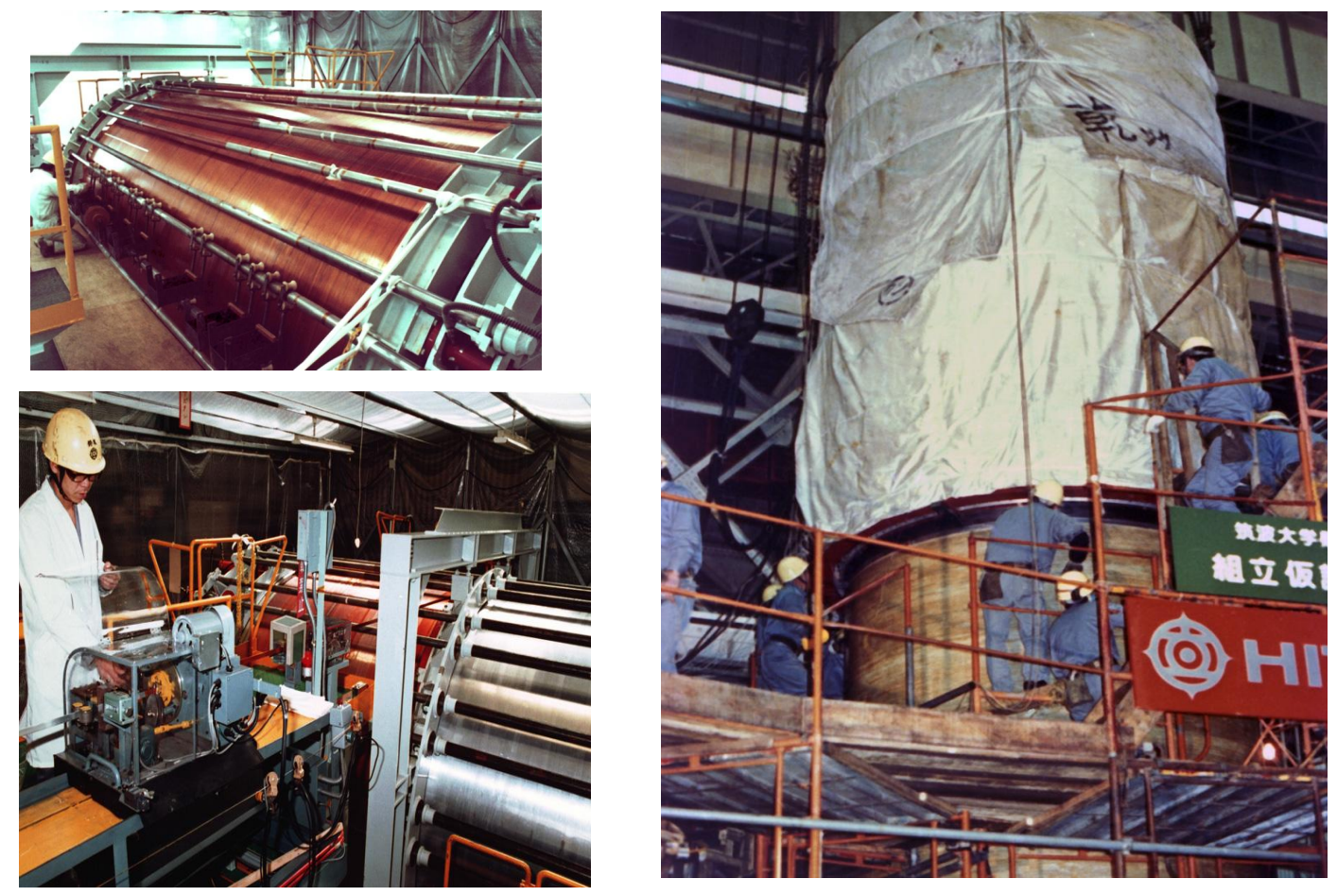


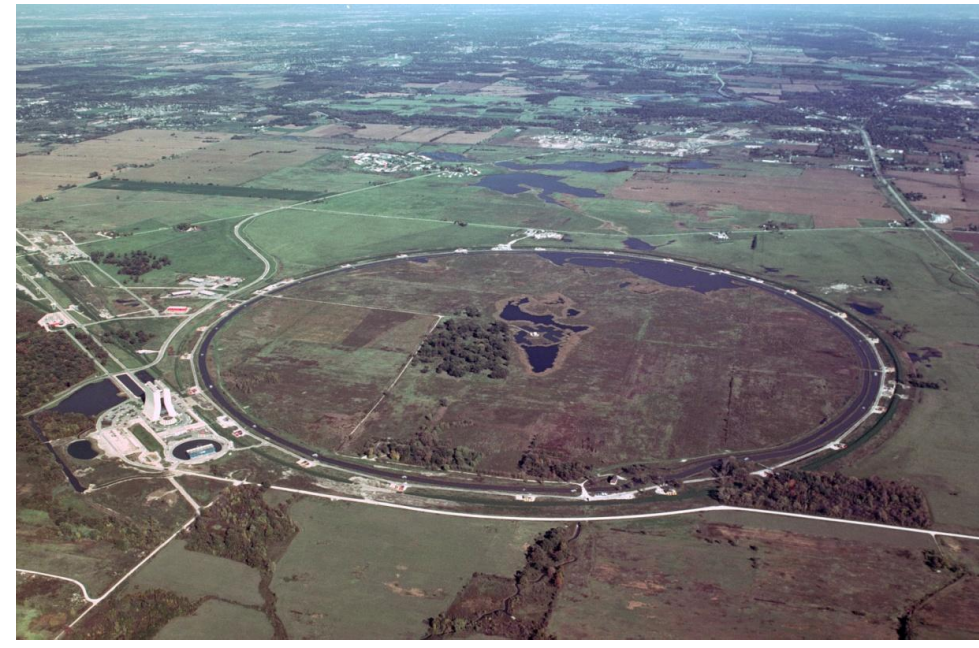

1981

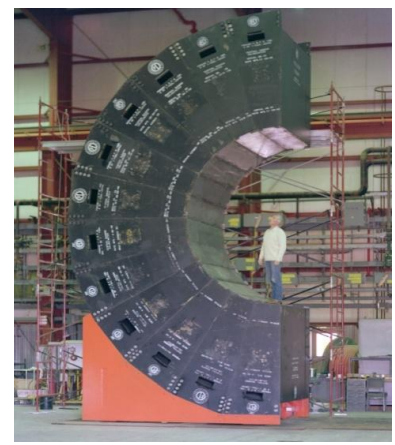

1982

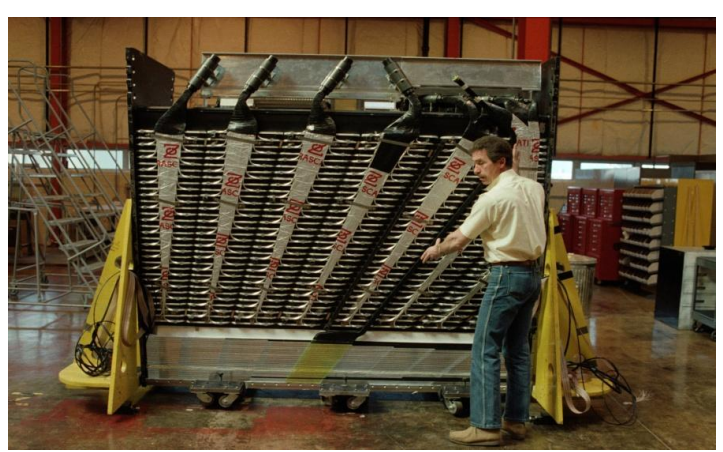

1982

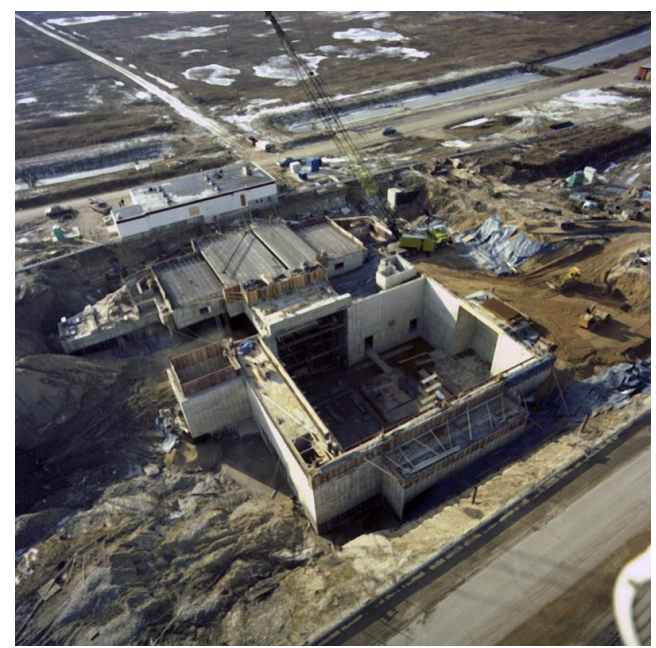

1982

\section{First Collisions: October 13, 1985}

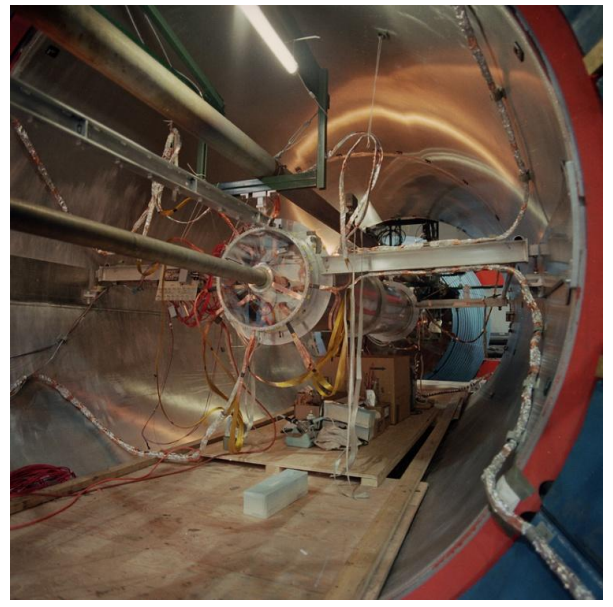

"First Collisions" 20 events! 1987: 29 nb-1. First physics! 88-89: 4 pb-1. W \& Z mass, sin2qw Evidence for top, Start on B physics

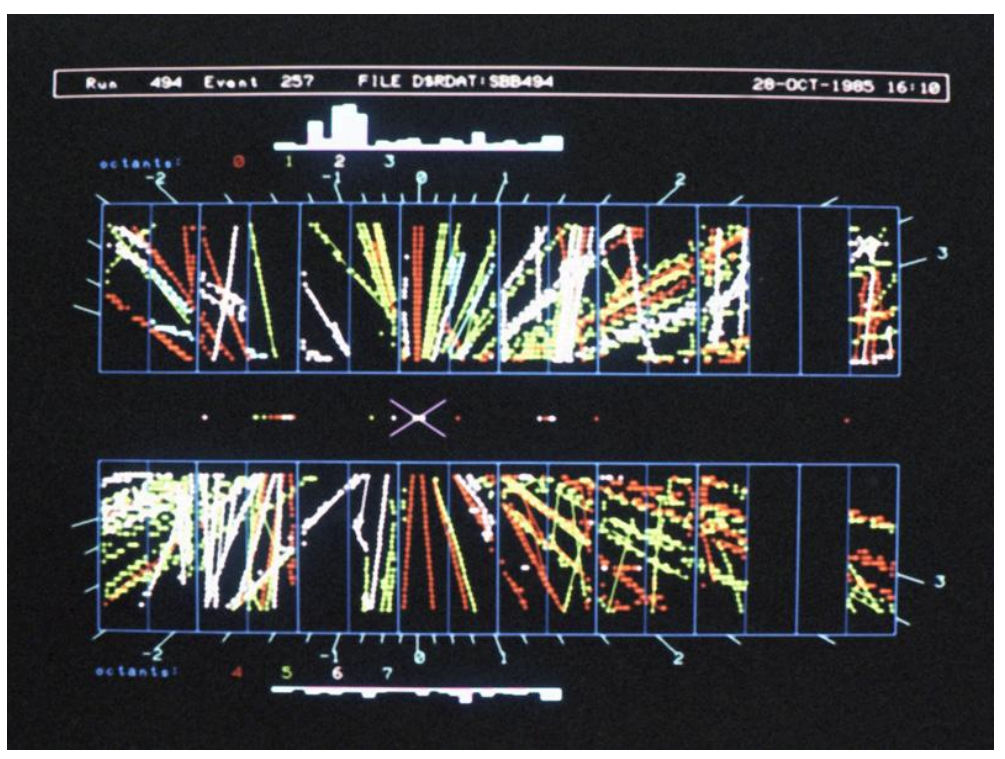

Sequence of pictures showing progress in the construction of the collider.

1. Before construction.

2. Collision Hall CDF.

3. Central hadron Cal Shell.

4. Wedge, CEM,CHA cal. 


\section{Top Evidence 1994}

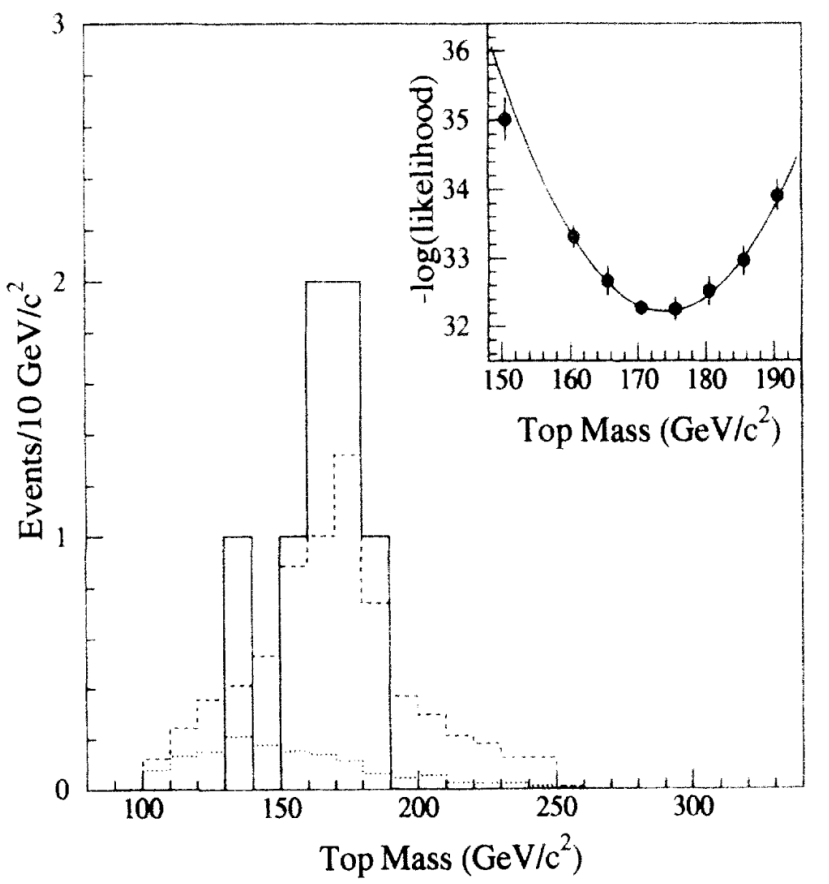

PRL 73 225(1994) : "Evidence for Top Quark Production in pp Collision at $\operatorname{sqrt}(\mathrm{s})=1.8 \mathrm{TeV}^{\prime \prime}$ $174 \pm 10_{-12}{ }^{+13} \mathrm{GeV} / \mathrm{c}^{2}$

\section{Japanese Graduates from CDF 1984 - March 1999}

\begin{abstract}
Hitoshi Miyata
Yoshio Hayashide

Teruki Kamon

Masayuki Shibata

Akihiro Yamashita

Masaki Sekiguchi

Youhei Morita

Shun-ichi Kanda

Toshihiro Mimashi

Fumihiko Ukegawa

Mariko Ninomiya

Yoshihiro Seiya

Satoru Ogawa

Mikio Takano

Takashi Ion

Takeshi Chikamatsu

Ryutaro Oishi

Hisafumi Mitsushio

Takashi Asakawa

Yukihiro Kato

Ei-ichiro Hayashi

Shin Aota

Makoto Shimojima

Tomoko Kuwabara

Hiroyuki Sato

Takeshi Takano

Jun-ichi Suzuki

Masakazu Okabe

Takanobu Handa

Hirofumi Ikeda
\end{abstract}

\begin{abstract}
University of Tsukuba
University of Tsukuba

University of Tsukuba

University of Tsukuba

University of Tsukuba

University of Tsukuba

University of Tsukuba

University of Tsukuba

University of Tsukuba

University of Tsukuba

University of Tsukuba

University of Tsukuba

University of Tsukuba

University of Tsukuba

University of Tsukuba

University of Tsukuba

University of Tsukuba

University of Tsukuba

University of Tsukuba

Osaka City University

University of Tsukuba

University of Tsukuba

University of Tsukuba

University of Tsukuba

University of Tsukuba

University of Tsukuba

University of Tsukuba

University of Tsukuba

Hiroshima

University of Tsukuba
\end{abstract}

June 1984

February 1986

June 1986

June 1988

June 1988

October 1988

January 1989

June 1990

September 1990

September 1991

January 1992

January 1992

January 1992

June 1994

June 1996

April 1994

January 1995

January 1996

February 1996

June 1996

September 1996

January 1997

January 1997

June 1997

June 1997

September 1997

January 1998

January 1998

January 1999

March 1999
Professor, Nigata University

Professor, Texas A\&M

Physicist, Spring-8 Photon Factory, RIKEN

Associate Professor, KEK, Public Relations Dept.

Assistant Professor, KEK Accelerator Laboratory

Professor, University of Tsukuba

Professor, Osaka City University

Professor, Toho University

Assistant Professor, KEK

Professor, Miyagi Gakuin Women's Unversity

Associate Professor, Kinki University

Professor, Nagasaki Institute of Applied Science

See R. Roser's talk for remaining 30 students 


\title{
The Japan/US HEP Collaboration: Program and Funding History
}

\author{
Kasuke Takahashi, KEK, Japan
}

As you have already seen and heard through the talks by the preceding speakers, we now know that tremendously fruitful results and outputs have been obtained through this Japan/US HEP Collaboration project. That is really the thing with which I feel very happy to remember.

So, here, I would only talk briefly that who and what sorts of things had really involved heavily in the beginning of these most fruitful collaboration efforts. As you can see from the photo-picture taken at the occasion of Implementation Sign Ceremony for the collaboration, held at SLAC in November 11, 1979, the collaboration had formally started with the very diligent efforts taken by those people you can see in the photo-picture shown here, such as those physicists like Dr.'s T.Nishikawa, T.Kitagaki, T.Fujii and K.Kikuchi from Japan, and Dr.'s Pief Panofsky, Leon Lederman, Joe Ballam, Rony Rau, Jack Sandweiss and Satoshi Ozaki from U.S.

In fact, since the establishment of KEK in 1971, HEP activities in Japan had started very urgently and actually very wide-variety efforts had been taken individually through the kind supports by people in US-HEP laboratories such as in BNL, Fermilab, LBL and SLAC. Since no-mention was made here until now about bubble chamber physics, I would like to say about the activities related to this. In particular, the bubble chamber film-analysis group lead by Prof.T.Kitagaki has made very active works by using bubble chamber films taken at the U.S. laboratories such as BNL, Fermilab and SLAC, since early 1960's and hereafter. Through these varieties of efforts at many Japanese institutions, HEP students and young physicists have been trained and experienced on HEP works, and at the sametime very deep friendships among physicists in both US and Japan, have been brought up widely. One big epoch to Japanese HEP community was the 1978(12-th) International HEP Conference held in Tokyo in 1978 for the first time in the Asian Area. Through this sort of a big event, the Japanese Government and also the general Japanese community or society have gradually understood what sorts of good effects and benefits would come out into the society by these kinds of such pure scientific collaborative efforts as HEP activity.

With these kinds of understanding and the strong supports from the other fields of science and the Governmental offices, about 1.3 Billion Yen sort of yearly annual funding had been created and given to KEK under the name of the Japan/US HEP Collaboration activity. The Japanese Government, in particular, the ministry of Education, Science, and Culture (Monbu-shyo in Japanese), has been quite supportive for this collaboration activities, and has been always generous enough for keeping this amount of annual budgetal support to KEK and the HEP community in Japan for these thirty years or so.

Of course, I wish this sort of extremely good and healthy collaboration would be continued steady for number of years to come. And I would like to say my sincere thanks to my many US friends not only seen here today, but also to other people missing here in this time of an occasion.

And finally, thank you again to the organizing committee-members of this very important and memorable symposium for me to participate in the meeting, and Good Luck for the future. 


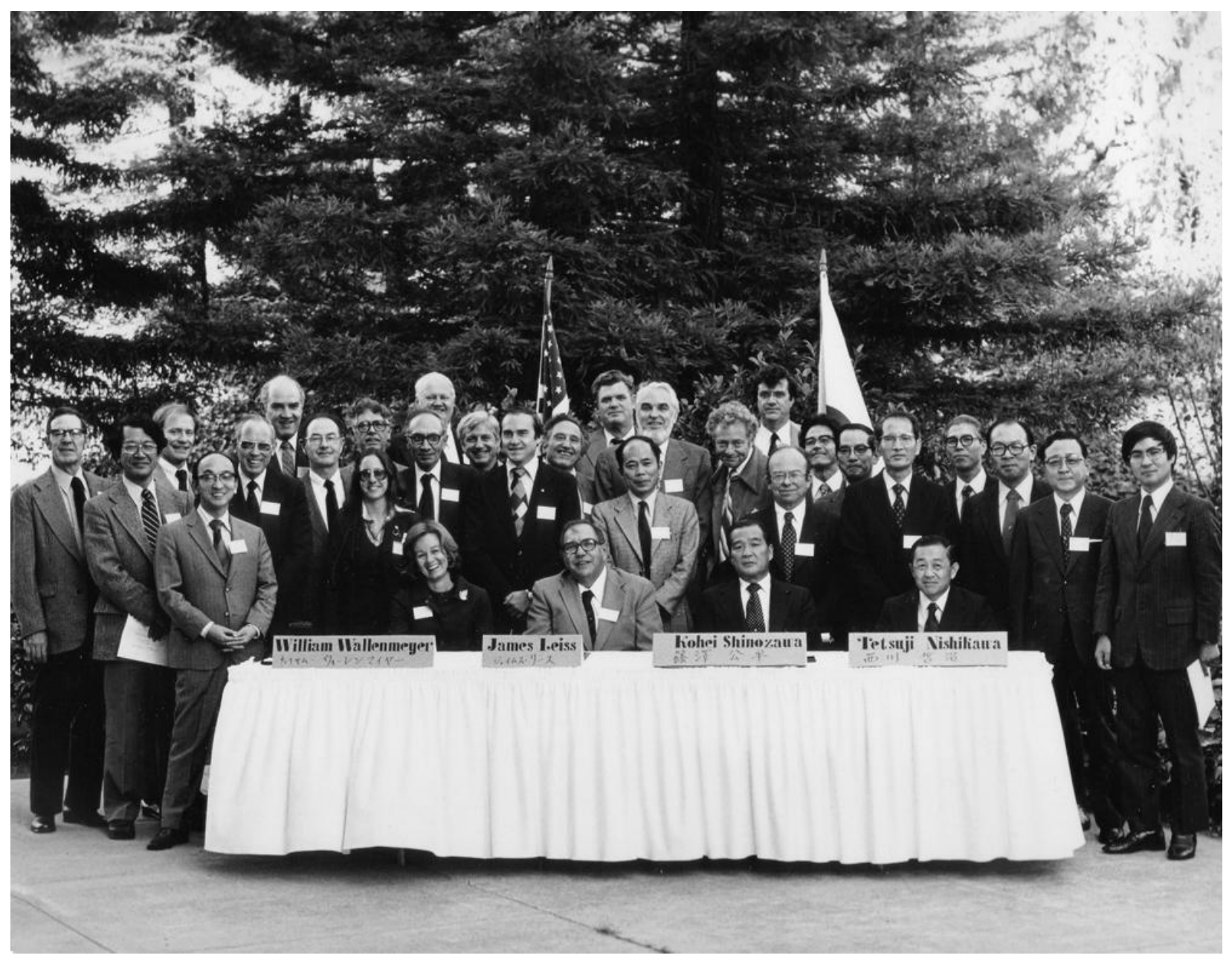

Figure Caption

A photo-picture showing the Implementation Sign Ceremony on "Joint Japan/US Collaboration in High Energy Physics", held at SLAC on November 11, 1979. 


\title{
PEP4 program at LBNL and SLAC
}

\author{
Hiroaki Aihara \\ The University of Tokyo, Japan
}

The PEP4/TPC detector was completed and started taking data at the SLAC Positron Electron Project (PEP) accelerator in 1982. The central part of this detector is the Time Projection Chamber (TPC) invented by Dr. David Nygren of LBNL. TPC is a 3D-imaging detector for charged particles, simultaneously measuring their momenta (in a magnetic field) and ionization energy loss (dE/dX) and therefore providing particle identification. The PEP4/TPC is the first large-scale TPC that was used in the collider experiment. In the following years many siblings of this TPC were constructed for a variety of accelerator (collider and fixed-target) and non-accelerator experiments.

The TPC was immersed in an axial magnetic field provided by a $4 \mathrm{~T}$ warm solenoid for the initial data taking period and then by a thin $1.5 \mathrm{~T}$ superconducting solenoid. Again this thin large superconducting solenoid coil is the first of its kind. Outside the solenoid coil there were six modules of lead-MWPC sandwich calorimeter operated in the Geiger discharge mode. This calorimeter measures the energy of electromagnetic showers by counting the number of fired Geiger cells rather than integrating charge (analog signal) produced in MWPC. It is the first digital calorimeter used in the collider environment. The University of Tokyo group lead by Professor Tuneyoshi Kamae shared the responsibility of design, construction and operation of this Geigermode digital electromagnetic calorimeter with LBNL physicists.

A large number of high quality publications were produced by the PEP4/TPC collaboration. In particular, the University of Tokyo group made significant contributions to heavy (charm and beauty) quark physics and the detailed study on hadronization/fragmentation process of quarks.

In conclusion, the PEP4/TPC experiment pioneered many advanced detector technologies, such as TPC with CCD readout, a thin superconducting solenoid and a digital calorimeter. They are now widely in use in many particle/nuclear physics experiments. Through the pioneering work on the detector R\&D and many superb physics analyses, the experiment nurtured many leaders of our field and produced a large number of outstanding PhDs. PEP4/TPC program is a great success story in the long history of US-Japan collaboration. 
Participation in the Electron-Positron Colliding Experiment (PEP-4) at SLAC-PEP and Development of New Detection and Data Handling Technology

Spokesman

JAPAN:

UNITED STATES:

Participating Groups JAPAN:

UNITED STATES:
T. Kamae, Univ. of Tokyo Dr. R. Nygren, LBL, Univ. of California

Univ. of Tokyo and INS

(Univ. of Tokyo)

LBL (Univ. of California), UCLA, Yale, UC Riverside, Johns Hopkins and others.
HEAVY QUARK PRODUCTION IN $\mathrm{e}^{+} \mathrm{e}^{-}$ANNIHILATION AT $29 \mathrm{GeV}$

$$
\text { Hiroaki Aihara }
$$

University of Tokyo, Tokyo 113, Japan

(Representing PEP-4 TPC Collaboration)

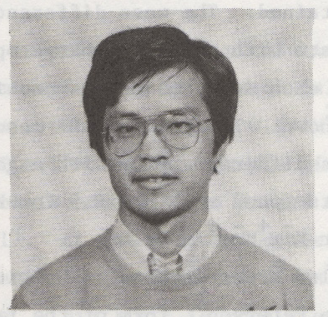

We present comprehensive studies on heavy quark production in $e^{+} e^{-}$annihilations using the PEP-4 TPC detector. The results include the measurements of $b$ and_c quark fragmentation functions and of the forward-backward asymmetries in $\mathrm{e}^{+} \mathrm{e}^{-} \rightarrow c \bar{c}$ and $\mathrm{b} \overline{\mathrm{c}}$, based on $\mathrm{D}^{*}$ and prompt lepton events. We have observed $\mathrm{F}^{*}$ meson via its radiative decay $F^{\star} \rightarrow \gamma F$ followed by $F \rightarrow K K \pi$. Gluon emission from $b$ quark is investigated using $\mathrm{b}$ quark events tagged by prompt leptons.
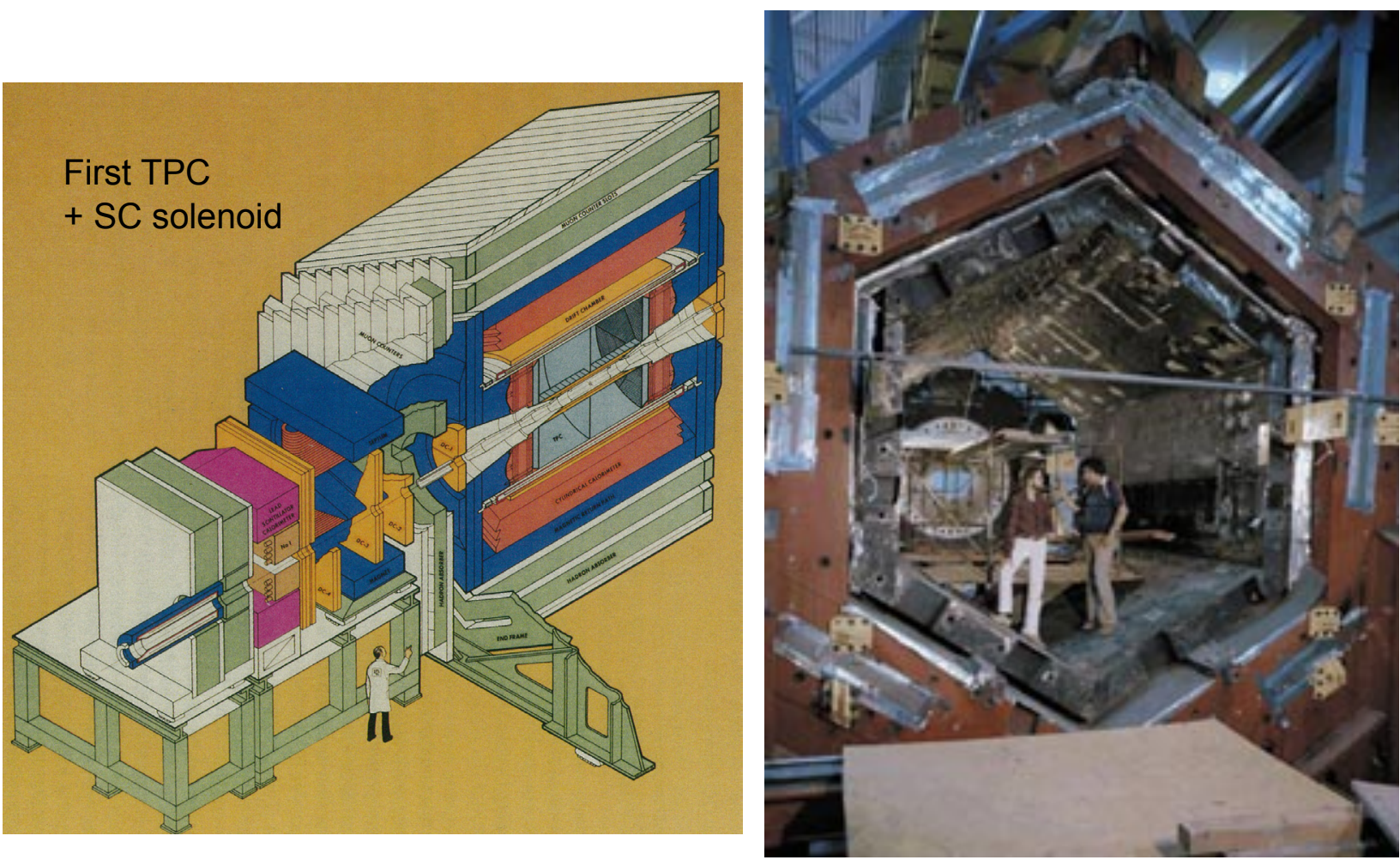

Dave Nygren (LBL) \& Fred Catania (SLAC) 
PEP4-TPC energy-deposit measurements (185 samples @ 8.5 atm Ar-CH4 80-20\%)

\section{Electrons reach a Fermi} plateau value of 1.4 times min. ionization. Muons from pion decays are separated from pions at low momentum; $\underline{\pi / \mathrm{K}}$ are separated over all momenta except in the cross-over region. (Low-momentum protons and deuterons originate from hadron-nucleus collisions in inner materials such as the beam pipe.)

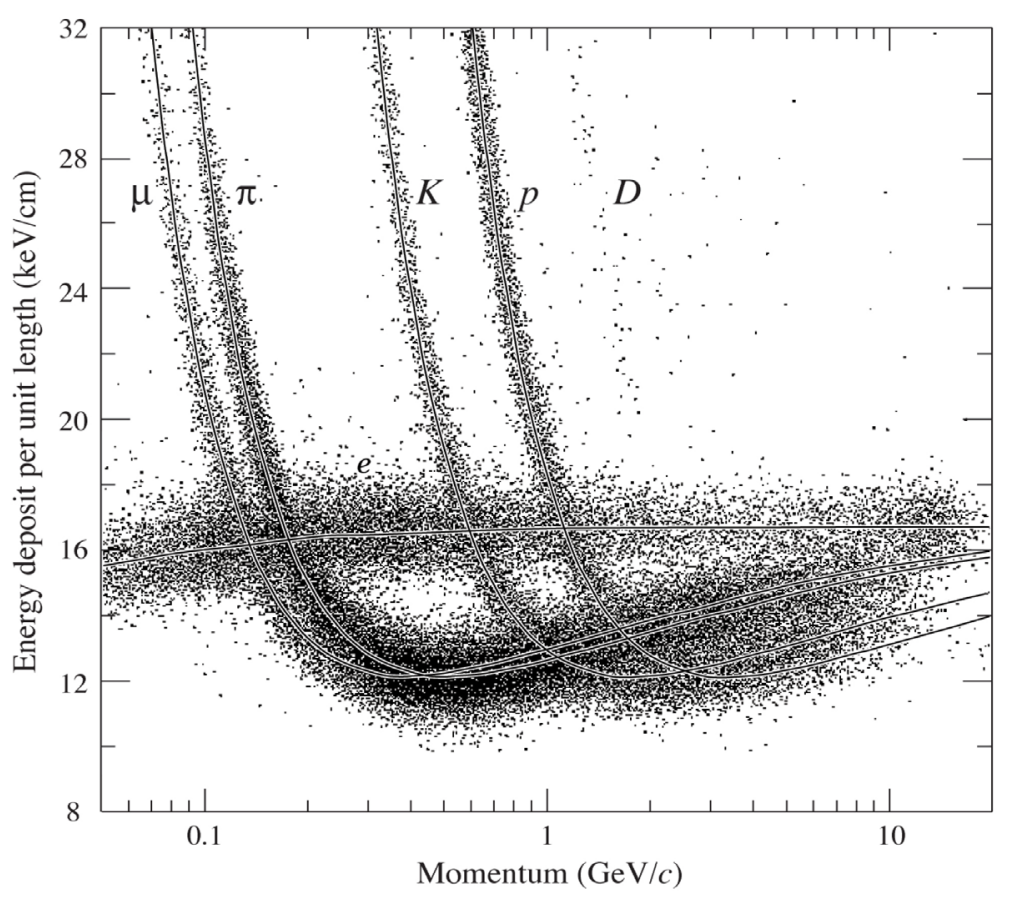

$\mathrm{dE} / \mathrm{dE}$ resolution $\sim 4 \%$

\section{First Digital EM Calorimeter based on Geiger-mode chamber}

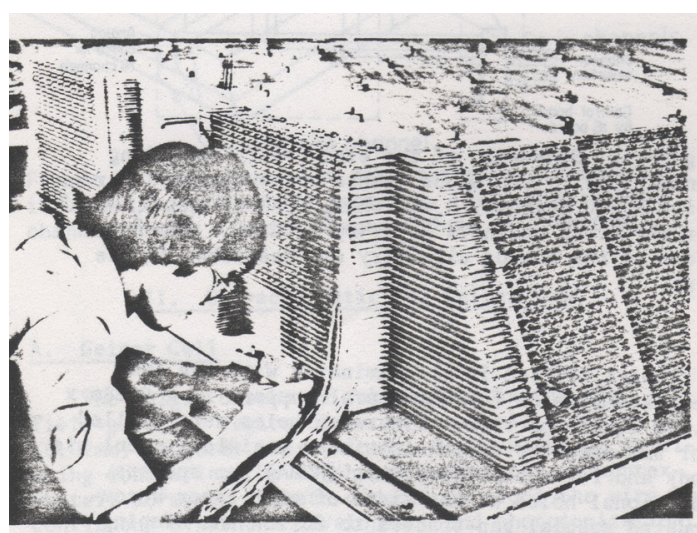

Figure 7. Photograph of partially assembled module showing laminated structure, tie rods and wiring. Details are given in the text.

After all layers were stacked the tie rods were inserted and tightened. Internal wiring for both sense wire and cathode strip channels (Figure 7) was carried out using wire-wrap techniques. Short cables bring all channel signals to the end bulkheads, where gas-tight connectors in slots provide for external gas-tight connectors in slots provide for external
high voltage and signal-cable connections to the module. 3mm-thick gasketed aluminum panels on the sides and top complete the gas enclosure.

\section{E. Electronics}

Figure 6 shows schematically the analog electronics and the electrical connections to the sense wire channels. For the cathode strip channels the connections are direct, and trimming capacitors are needed tions a the $50 \mathrm{nhm}$ distribution of a typical anode channel triggered on almost anything. The spacing of successive numbers of cell discharges determines relative values for the low energy slope of the energy scale.

For the cathode-strip channels discrete pulse heights are not available. Instead, relative channel sensitivities are inferred by relating measured average pulse heights to those of the calibrated anode channels looking at the same events. Figure 9 shows preliminary measurements illustrating the accuracy with which this can be done. The very broad pulse height spectrum for cosmic rays with a wide angular distribution gives an anode-cathode pulse height difference which is sharply peaked, a conse-

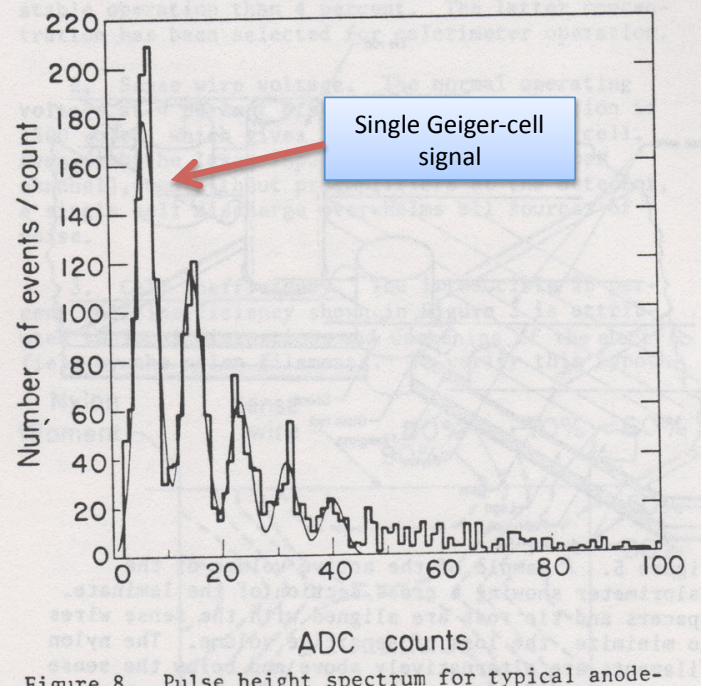




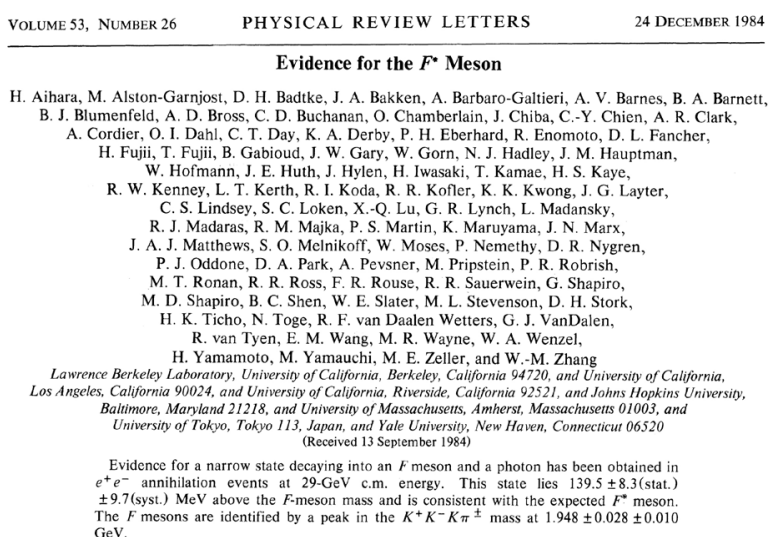

The quark model predicts four ground-state given by $(d p / p)^{2} \simeq(0.06)^{2}+(0.035 p)^{2} \quad(p$ in
charmed mesonst: D, $D^{*}, F$, and $F^{*}$. The first $\left.\mathrm{GeV} / c\right)$ for tracks in hadronic jets. charmed mesons ${ }^{1}: D, D^{*}, F$, and $F^{*}$. The first three of these are now well established, ${ }^{2-4}$ and
searches for the $F^{*}$ have been conducted by several experiments, with the first candidates reported rethe $F$ are isosinglets and the $F^{*} \rightarrow F \pi^{0}$ decay is forbidden leaving the radiative decay to the $F$ as the only significant mode.

We report evidence for a narrow state that decays to an $F$ meson and a monochromatic photon usin PEP storage ring at SLAC OCr detservation the based on 29095 hadronic events (corresponding to an integrated luminosity of $77 \mathrm{pb}^{-1}$ ) produced in $e^{+} e^{-}$annihilation at $E_{\mathrm{crn},}=29 \mathrm{GeV}$. mentum and ionization loss $(d E / d x)$ of charged particles are measure in a time projection chamber (TPC). In the TPC, the pulse heights measured by anode wires of multiwire proportional chambers.
resolution is $3.9 \%$ The average $d E / d x$ resolution is $3.9 \%$ for tracks with at least thirty ion-
zation samples. zation samples. At a momentum of $5 \mathrm{GeV} /$, typi-
cal for this analysis, the measured $\pi \cdot K$ separation
in $d E / d x$ is $14 \%$, (C) 1984 The American Physical Society Photons are detected either by the hexagonal Geiger-mode calorimeter, or by the TPC as $e^{+} e^{-}$ $16 \% / \sqrt{ } E$ (rms whoton energy is measured to about nearby pairs of photons are resolved if the angle between them is greater than $60 \mathrm{mrad}$. Photons are accepted if their polar angle $\theta$ is between $50^{\circ}$ and
$130^{\circ}$ and if their energy is greater than $400 \mathrm{MeV}$ in the calorimeter. Photons of any energy are accepted if they convert in front of the TPC, and the conversion pair is unambiguously identified. Of photons contained in the solid angle and energy range defined by the selection criteria, $41 \%$ are detected
in the calorimeter and $8 \%$ are detected in the TPC as conversion pairs

With the use of the measured momentum and electron $\left(x^{2}\right)$ are calculated for each track to be an on $\left(\chi_{p}^{2}\right)$. A particle is the "iden $\left(x_{k}^{2}\right)$, or a proif (a) the number of good $d E / d x$ sample points is 30 r more, (b) $\chi_{k}^{2}<2.7$ (for one degree of freedom),
nd (c) $\chi_{k}^{2}<\chi_{e}^{2}, \chi_{n}^{2}, X_{p}^{2}$ if $p<2 \mathrm{GeV}$ or $x_{k}^{2}<\chi_{e}^{2}$, The $d E / d x$ resolution is $3.5-4 \%$ depending on the
number of $d E / d x$ samples available. Based on the

Volume 53, NUmber 23 PHYSICAL REVIEW LETTERS 3 December 1984 Observation of Strangeness Correlations in $e^{+} e^{-}$Annihilation at $\sqrt{s}=29 \mathrm{GeV}$ H. Aihara, M. Alston-Garnjost, D. H. Badtke, J. A. Bakken, A. Barbaro-Galtieri, A. V. Barnes, B. A. Barnett,
H. U. Bengtsson, B. J. Blumenfeld, A. D. Bross, C. D. Buchanan, O. Chamberlain, J. Chiba, C.-Y. Chien, A. R. Clark, A. Cordier, O. I. Dahl, C. T. Day, K. A. Derby, P. H. Eberhard, D. L. Fancher, H. Fujii,

T. Fujii, B. Gabioud, J. W. Gary, W. Gorn, N. J. Hadley, J. M. Hauptman, H. J. Hilke, W. Hofmann,
J. E. Huth, J. Hylen, H. Iwasaki, T. Kamae, H. S. Kaye, R. W. Kenney, L. T. Kerth, R. I. Koda, R. R. Kofler,

K. K. Kwong, J. G. Layter, C. S. Lindsey, S. C. Loken, X.-Q. Lu, G. R. Lynch, L. Madansky, S. O. Melnikoff, W. Moses, P. Nemethy, D. R. Nygren, P. J. Oddone, D. A. Park,

M. Pevsner, M. Pripstein, P. R. Robrish, M. T. Ronan, R. R. Ross,
A.

A. Pevsner, M. Pripstein, P. R. Robrish, M. T. Ronan, R. R. Ross,
F. R. Rouse, R. R. Sauerwein, G. Shapiro, M. D. Shapiro,

B. C. Shen, W. E. Slater, M. L. Stevenson, D. H. Stork, H. K. Ticho

N. Toge, R. F. van Daalen Wetters, G. J. VanDalen,
R. van Tyen, H. Videau, M. R. Wayne,

R. van Tyen, H. Videau, M. R. Wayne,
W. A. Wenzel, H. Yamamoto, M. Yamauchi,

E. M. Wang, and W.-M. Zhang
Lawrence Berkeley Laboratory, University of California, Berkeley, California 94720, and University of California, Los Angeles,
California 90024, and Universily of Callf fornia, Riverside, Callfornia 92521 , and Johns Hopkins University, 218 , and University of M Massachusetts, , Amherst,
and University of Tokyo, Tokyo 113, Japan
(Received 27 August 1984)

Correlations in rapidity space are presented for identified $\pi \pm$ and $K^{ \pm}$in $e^{+} e^{-}$annihilation at 29-GeV c.m. energy. Short-range $K K$ correlations indicate local flavor compensation in the hadronization process. Long-range $K K$ and $\pi \pi$ correlations prove that the initial par-
tons carry flavor. In addition, we observe significant $K \pi$ correlations as a result of heavyquark decays.

PACS numbers: $13.65++$

In standard models of hadronization the process $e^{+} e^{-} \rightarrow$ hadrons is governed by two distinct time scales. At early times, the production of a parton system is described by perturbative heory. On a teractions transform these partons into hadrons. The large momentum scale involved in the creation of the initial $q \bar{q}$ pair results in a large separation in phase space of hadrons containing those partons. Since the two primary quarks in an annihilation event carry opposite quantum numbers, this process will give rise to long-range flavor correlations
(LRC). Such studies by previous experiments have indeed provided evidence that the priments have indeed provided evidence that the primary partons
are charged ${ }^{1.2}$ On the other hand, the final formation of hadrons is usually assumed to be governed by small- $Q^{2}$ phenomena which generate local, short-range correlations (SRC) (see Fig. 1). In this paper we examine these two regimes by studying flavor correlation in multihadron events, with special emphasis on the investigation of number of strange auarks produced in a typical number of strange quarks cleaner in a typical specific $q \bar{q}$ pair than, for example, charge.

The data used in this analysis were collected by the time-projection-chamber (TPC) detector at the
PEP storage ring and consist of 23650 annihilation PEP storage ring and consist of 23650 annihilation
events, representing $68 \mathrm{pb}^{-1}$ at $29-\mathrm{GeV}$ c.m. energy. The PEP4 facility and the multihadronic event selection have been described previously. ${ }^{3}$ The TPC provides both tracking information and
charged-particle identification. The detector has a

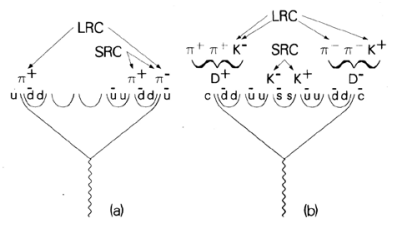

FIG. 1. The mechanisms responsible for long- and short-range flavor corres
between kaons and pions.

Evidence of soft and collinear gluon emission in $e^{+} e^{-}$hadronic events TPC/Two-Gamma Collaboration

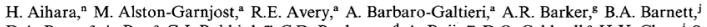
D.A. Bauer, ${ }^{,}$A. Bay, ${ }^{a}$ G.J. Bobbink, ${ }^{m}$ C.D. Buchanan, ${ }^{d}$ A. Buijs, ${ }^{\text {, }}$ D.O. Caldwell, ${ }^{8}$ H-Y. Chao, ${ }^{i}$ S-B. Chun, ${ }^{d}$

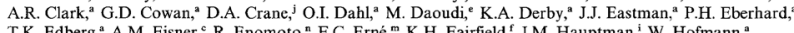

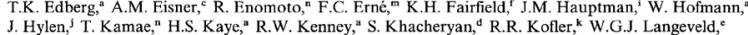

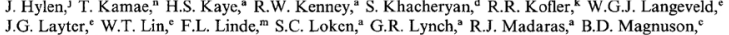
J.G. Layter, ${ }^{,}$W.T. Lin, ${ }^{,}$F.L. Linde, ${ }^{\text {m }}$ S.C. Loken, ${ }^{2}$ G.R. Lynch, ${ }^{2}$ R.J. Madaras, ${ }^{2}$ B.D. Magnuson, ${ }^{e}$
G.E. Masek, ${ }^{,}$L G. Mathis, ${ }^{2}$ J.A.J. Matthews, ${ }^{,}$S.J. Maxfield, ${ }^{,}$E.S. Miller, ${ }^{f}$ W. Moses,, D. R. Nygren,

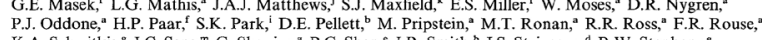
P.J. Oddone, ${ }^{,}$H.P. Paar,
K.A. Schwitkis, ${ }^{\text {, }}$ J.C. Sens, ${ }^{\text {m }}$ G. Shapiro, ${ }^{a}$ B.C. Shen, ${ }^{,}$J.R. Smith, ${ }^{b}$ J.S. Steinman, ${ }^{d}$ R.W. R. Stephens,

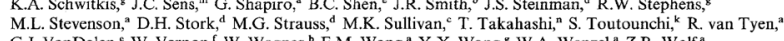

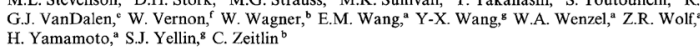

- Lawrence Berkeley Laboratory, University of California, Berkeley, Calif, USA
o University of California, Davis, Calif, US A A

: University of California, Davis, Calif, USA

c. University of California Intercampus Institutc for

' University of California, Riverside, Calir,, USA

- University of Califiornia, Santa Barbara, Calif, USA

Ames Laboratory, Iowa. State University, Ames, IA

"University of Massachusetts, Amherst, Mass., USA

National Institute for Nuclcar and High Energy Physics, Amsterdam, The Netherlands

Received 24 April 1989

Abstract. In the study of rapidity distributions of charged pions produced in $e^{+} e^{-}$annihilation at
$29 \mathrm{GeV}$, we observe a significant dip in the rapidity $29 \mathrm{GeV}$, we observe a significant dip in the rapidity
distributions at $y \approx 0$ for events with low sphericity. distributions at $y \approx 0$ for events with low sphericity.
A comparison of data with several QCD-based phen

A comparison of data with several QCD-based phen-
omenological models strongly suggests that this structure is related to the emission of multiple soft and/or collinear gluons.

1 Introduction

Although quantum chromodynamics (QCD) is becesed to be the fundamental theory of hadronic pro$9 \begin{aligned} & \text { physical hadronic amplitudes. Comparisons of theo- } \\ & \text { retical predictions and experimental measurements }\end{aligned}$ usually involve phenomenological models of $\mathrm{QCD}$ [1]. In these models, an initial parton configuration
is created by the $e^{+} e^{-}$annihilation photon. These partons, which typically are initially highly virtual (i.e. far off their mass shell), cascade down in mass vi successive emission of gluons, initiating a quark-gluon
shower analogous to an electromagnetic shower. $\mathrm{Fi}$ nally, the partons in the shower rearrange somehow to form color singlet hadrons. The actual models are formulated in two steps: first, a configuration of partons is determined based on QCD perturbation theory; next, the formation of hadrons from those partons is described by a phenomenological model The two regimes are separated by a rather arbitrary mum two-parton invariant mass. wo alternative schemes for the perturbative QCD 


\title{
Neutrino Scattering Experiment at BNL
}

\author{
Yorikiyo Nagashima, Osaka University, Japan
}

The Standard Model was proposed between 1961 and 1967, but it was not recognized as such until 1978, when at ICHEP78 in Tokyo, R. Taylor from SLAC presented the asymmetry of the polarized electron Deuteron scattering data and singled it out from other experimentally equally viable models. C. Baltay from Yale, in the same conference, demonstrated that all the Weinberg angles measured in variety of experiments more or less agreed. The ICHEP 78 was also a scene when the first scientific results of the $12 \mathrm{GeV}$ Proton Synchrotron at KEK was presented. This marked the Japanese debut to the world high energy society.

The next year, the Japan-US collaboration project began, and BNL E734 was among the first approved programs. An urgent topic at that time was to measure the Weinberg angle in the pure leptonic reaction and confirm if it was the universal constant. The reaction is free from QCD correction and was considered the most fundamental test of the model. The detector (fig. 3) was a 170 ton liquid scintillator/proportional tube electromagnetic calorimeter followed by a gamma catcher and muon detector. As the electron energy $\mathrm{E}$ and its scattering angle was constrained by $\mathrm{E} \theta^{2}<2 \mathrm{~m}_{\mathrm{e}}$, the signal was a single forward going shower. The obtained data and their theoretical expression are given in fig.4. From the measured data, values of the neutral coupling constants $\mathrm{g}_{\mathrm{V}}$ and $\mathrm{g}_{\mathrm{A}}$ which contain the Weinberg angle are determined with four-fold ambiguity. It was resolved by combining data from the reactor neutrino and the muon pair production by electron-positron annihilation (fig 5, right). The measured value was

$$
\sin ^{2} \theta_{\mathrm{W}}=0.199 \pm 0.018 \text { (stat) } \pm 0.013 \text { (syst) }
$$

and confirmed the validity of the Standard Model in the pure leptonic sector. Similar results were obtained by the CHARM/CERN experiment.

The Weinberg angle was also determined in the elastic $v(v b a r)-p$ scattering and confirmed the Standard Model in the semi-leptonic sector. The process is parity violating and the cross section includes the axial vector form factor whose mass parameter was determined as $\mathrm{m}_{\mathrm{A}}=1.06 \pm 0.05$ $\mathrm{GeV} / \mathrm{c}^{2}$. Another result was the upper limit of the second-class current which is forbidden by the Standard Model. It is complementary to that obtained by the nuclear beta decay as the process is at much higher value of the momentum transfer $\mathrm{Q}^{2}$.

The third topic was the neutrino oscillation $v_{\mu}->v_{\mathrm{e}}$ (fig.6). The excluded region was comparable to the present values at large mixing angle and about a few factors above the LSND/MiniBooNE results. Note the data were obtained in the 1980s. 


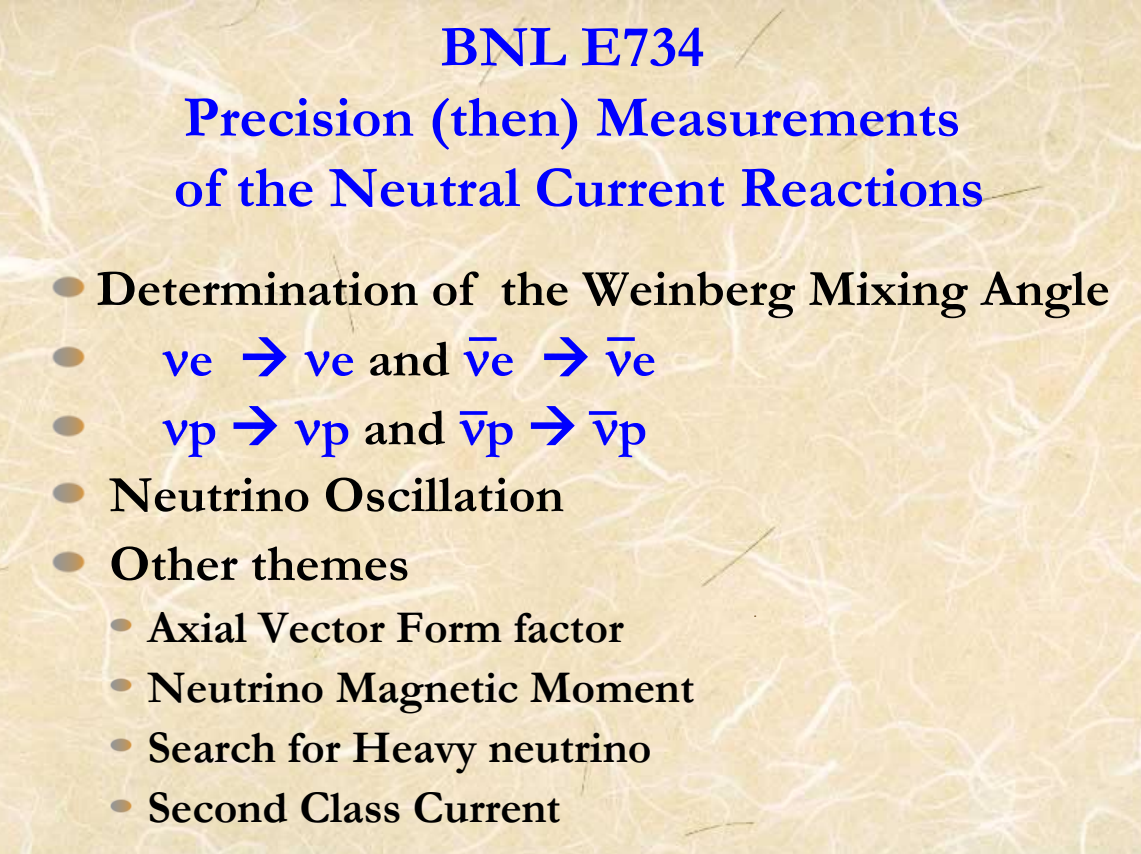

Figure 1: Topics of this talk

\section{Determination of electroweak parameters from the elastic scattering} of muon neutrinos and antineutrinos on electrons

L. A. Ahrens, S. H. Aronson, P. L. Connolly, ${ }^{(a)}$ B. G. Gibbard, M. J. Murtagh, S. J. Murtagh, ${ }^{(b)}$ S. Terada, ${ }^{(c)}$ and D. H. White ${ }^{(d)}$

Physics Department, Brookhaven National Laboratory, Upton, New York 11973

J. L. Callas, ${ }^{(\mathrm{e})}$ D. Cutts, M. V. Diwan, ${ }^{(\mathrm{f})}$ J. S. Hoftun, B. W. Hughlock, ${ }^{(\mathrm{g})}$ R. E. Lanou, and T. Shinkawa ${ }^{(\mathrm{h})}$

Department of Physics, Brown University, Providence, Rhode Island 02912

Y. Kurihara ${ }^{(\mathrm{h})}$

Department of Physics, Hiroshima University, Hiroshima 730, Japan

K. Amako and S. Kabe

National Laboratory for High Energy Physics (KEK), Ibaraki-Ken 305, Japan

Y. Nagashima, Y. Suzuki, ${ }^{(i)}$ S. Tatsumi, ${ }^{(j)}$ and Y. Yamaguchi ${ }^{(\mathbf{k})}$

Physics Department, Osaka University, Toyanaka, Osaka 560, Japan

K. Abe, ${ }^{(h)}$ E. W. Beier, D. C. Doughty, ${ }^{(1)}$ L. S. Durkin, ${ }^{(m)}$ S. M. Heagy, ${ }^{(n)}$ M. Hurley, ${ }^{(0)}$ A. K. Mann, F. M. Newcomer, H. H. Williams, and T. York ${ }^{(p)}$

Department of Physics, University of Pennsylvania, Philadelphia, Pennsylvania 19104

D. Hedin, ${ }^{(\mathrm{q})}$ M. D. Marx, and E. Stern ${ }^{(\mathrm{r})}$

Department of Physics, State University of New York, Stony Brook, New York, 11794 (Received 27 October 1989)

Figure 2: List of E734 participants: Figures 2, 4, 5;left taken from L. A. Ahrens et al, Phys. Rev. D41 (1990) 3297, http://link.aps.org/abstract/PRD/v41/p3297. Copyright (1990) by the American Physical Society. 


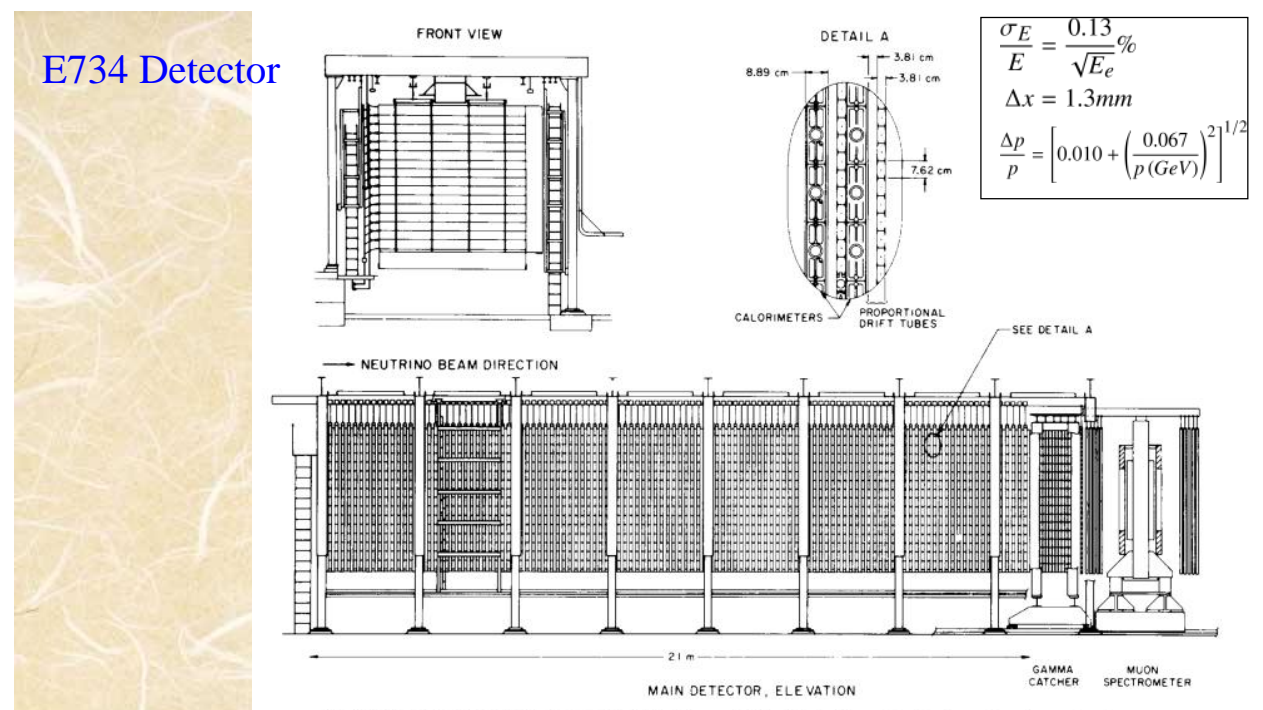

Fig. 2. Schematic drawing of the complete BNL-Brown-KEK-Osaka-Pennsylvania-Stony Brook neutrino detector.

Liquid Scintillator Calorimetor:

$4 \mathrm{~m} \times 4 \mathrm{~m} \times 0.1 \mathrm{~m}$ (16 cells), 112 modules, total 170 tons, $\left(\sim 16 \lambda, 25 \mathrm{X}_{0}\right)$

+ Proportinal Drift Tubes

$\sim 2 v_{\mu}$ interactions/burst (1.4 s)

Figure 3: Layout of E734 Detector: Figure from L.A.Ahrens et al.:Phys. Rev. D34 (1986) 75,

http://link.aps.org/abstract/PRD/v34/p75. Copyright (1986) by the American Physical Society.

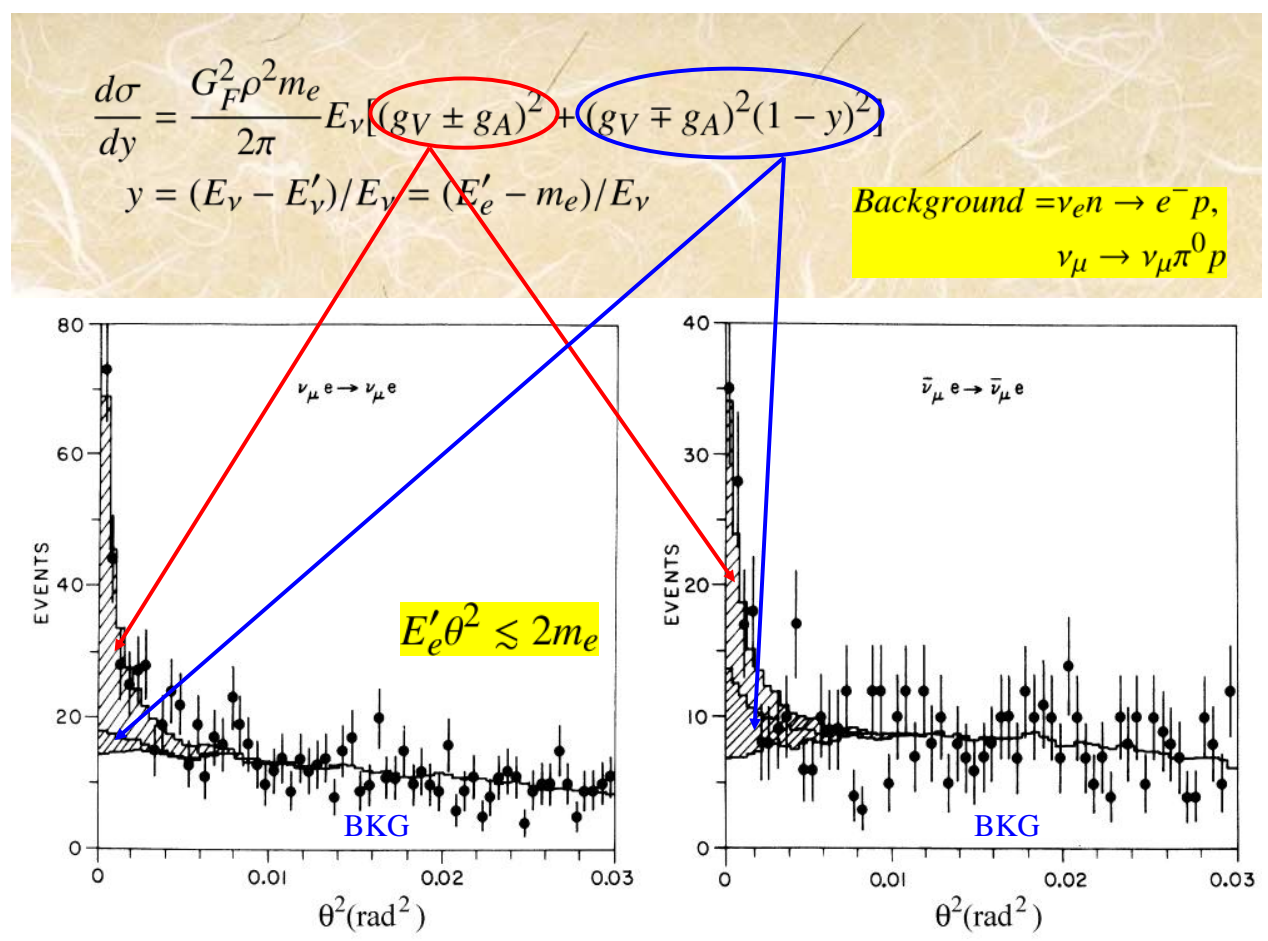

Figure 4: Differential distributions in $\theta^{2}$ for the neutrino and antineutrino scattering with electrons. Data are points with error bars.

Figure from L.A.Ahrens et al.: Phys. Rev. D41 (1990) 3297. 


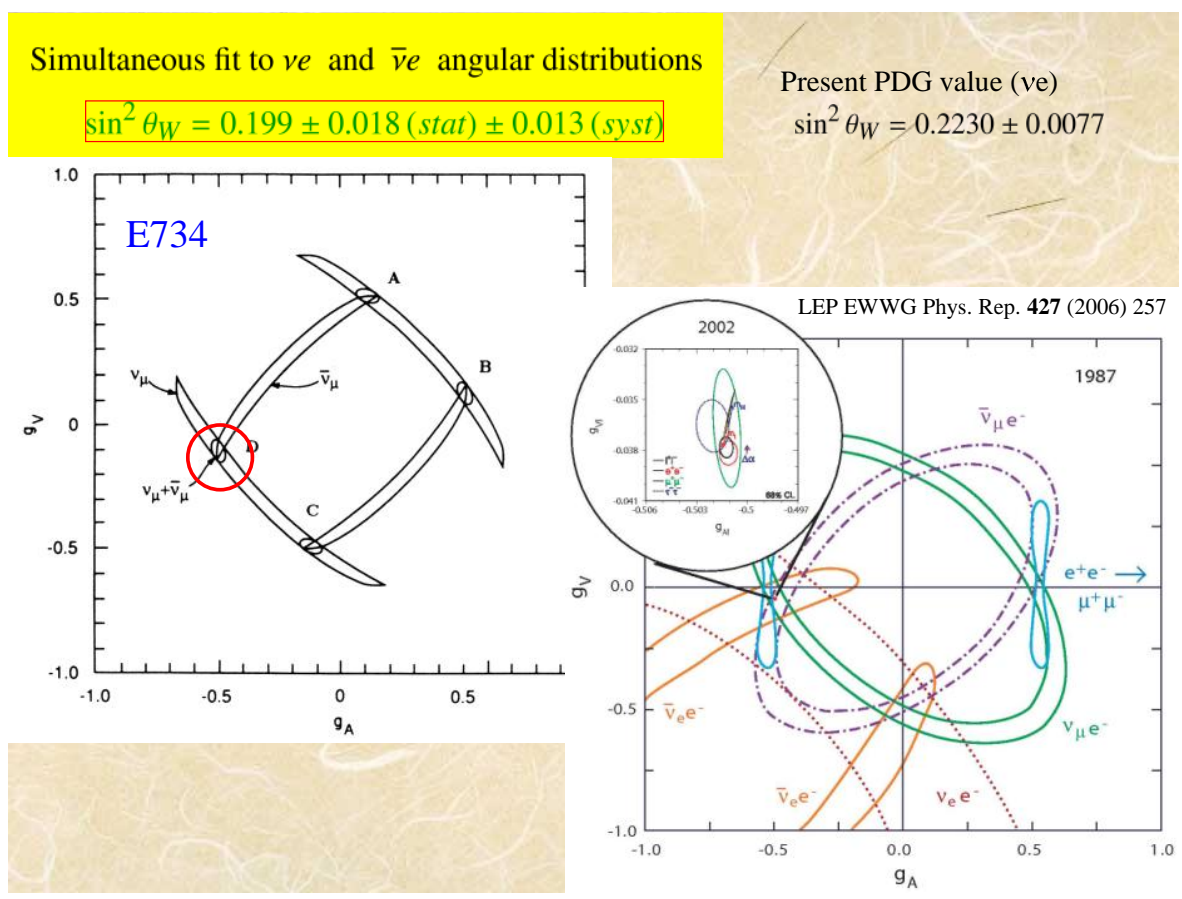

Figure 5: Left: Values of $g_{A}$ and $g_{V}$ with four fold ambiguity obtained from E734. L.A.Ahrens et al, Phys. Rev. D41 (1990) 3297. Right: constraints from world experiments on $\mathrm{g}_{\mathrm{A}}-\mathrm{g}_{\mathrm{V}}$ circa 1987 and 2002 (insert). LEP Electroweak working group, Phys. Rep, 427 (2006) 257. Copyright (2006) by the Elsevier.
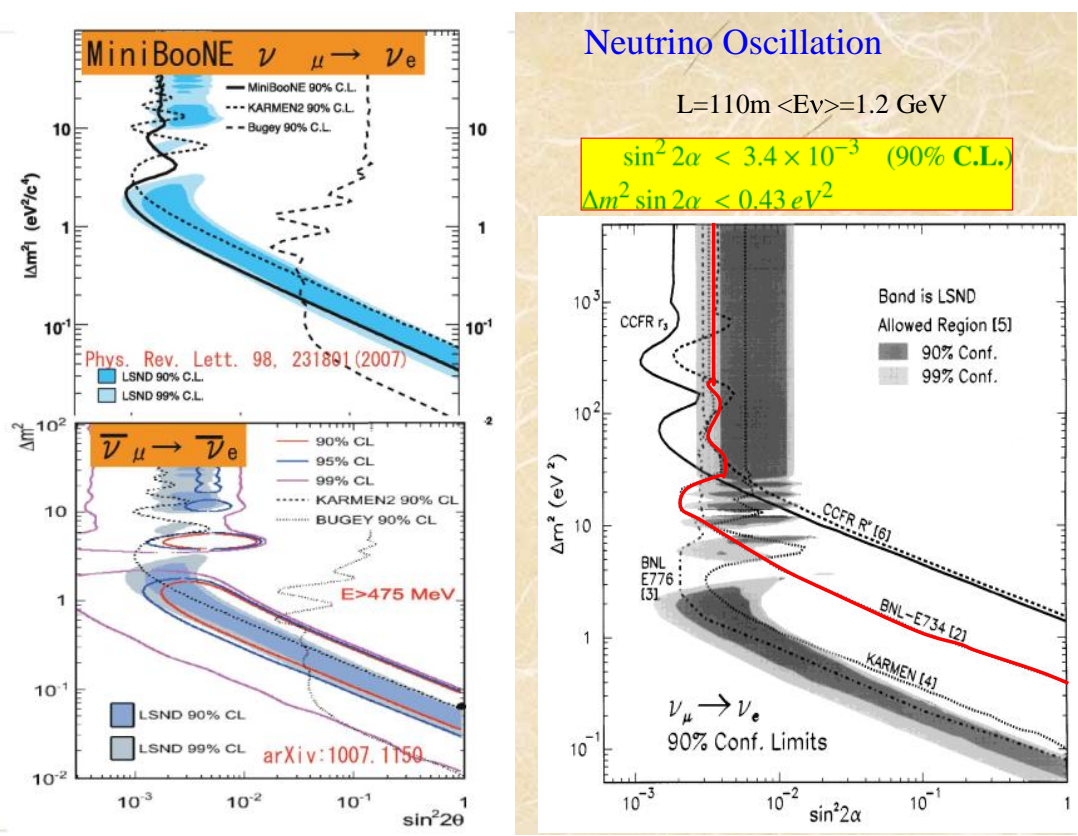

Figure 6: constraints obtained by E734 and other experiments. Bands are finite results from LSND and Mini-BooNE. A.A.Aguilar-Arevalo et al, Phys. Rev. Lett. 98 (2007) 231801, ibid., 105 (2010) 181801.

http://link.aps.org/abstract/PRD/v105/p181801. Copyright (2010) by the American Physical Society. 


\title{
To and from Tau neutrino Experiment at Fermilab
}

\author{
Mitsuhiro Nakamura, Nagoya University, Japan
}

The Japan-US collaborative work using nuclear emulsion was triggered by the discovery of charmed particles by K.Niu in 1971[1]. To study the new particles, an emulsion -counter hybrid experiment Fermilab E531 was performed with the collaboration between Japanese Emulsion groups and US counter group. In this experiment, by measuring hundred of charmed particles, the lifetime difference between charged and neutral charmed particles, which was discovered in cosmic ray experiments, was confirmed. Adding to the initially aimed physics, an upper limit of neutrino oscillation (Muon neutrino to Tau neutrino) was set[2]. This is the start point of the succeeding neutrino oscillation study by using nuclear emulsion, Fermilab E531, CERN WA75(CHORUS) and CERN/LNGS CNGS1(OPERA).

In $1990 \bowtie$, Tau neutrino was listed as a strong candidate of dark matter in the universe. In order to study its mass, to utilize neutrino oscillation phenomena, predicted by Maki, Nakagawa and Sakata, was proposed. A short base-line neutrino oscillation experiment CERN WA95(CHORUS) was organized to investigate this possibility. In parallel, an experiment aim to detect Tau neutrinos among prompt neutrino beam was performed as Fermilab E872 DONUT and succeeded to detect Tau neutrinos in 1998 [3].

The essential tool to lead this experiment successful is the automated nuclear emulsion read-out system proposed and realized by K.Niwa [4]. The automated emulsion read-out system is playing essential roles in CHORUS and OPERA.

We reported the first Tau neutrino event in Takayama in 1998, where Super Kamiokande group confirmed the deficit of atmospheric Muon neutrinos discovered by Kamiokande and proposed that neutrino oscillation from muon neutrino to tau neutrino is the origin of this deficit. A long base line neutrino oscillation experiment OPERA aiming to detect Tau neutrino appearance was organized. The scale of OPERA is about 3000 times larger than DONUT. The nuclear emulsion film of $9.3 \mathrm{M}$ was developed and produced by Fujifilm. OPERA reported its first candidate in this year [5]. Data taking will continue to 2013 and will confirm the existence of Neutrino oscillation by unambiguous way. This will be the first clear observation of Lepton Flavor Violation phenomena.

From the beginning of 2010, R\&D and production of Nuclear emulsion has been started in Nagoya. We succeeded to develop high sensitivity emulsion, about two times higher sensitivity than commercially available one, and fine grain nuclear emulsion of $40 \mathrm{~nm}$ size $\mathrm{AgBr}$ grains, which can be used to detect the short trajectory of recoil atoms kicked by dark matter in the universe, i.e. dark matter directional detection[6].

Nuclear emulsion technology developed in Japan and polished up by Japan-US collaboration plays unique and essential role in neutrino physics, i.e. discovery of Tau neutrino and confirmation of neutrino oscillation by detecting Tau neutrino Appearance. We will continue the $R \& D$ work to activate the latent talent of nuclear emulsions. 
$\underline{\text { References }}$

[1] K.Niu et al., Prog.Theor.Phys.46:1644-1646,1971.

[2] E531 Collab., Phys.Rev.Lett.57:2897-2900,1986.

[3] DONUT Collab., Phys.Lett.B504:218-224,2001.

[4] K.Niwa et al., Nucl.Instrum.Meth.B51:466-472,1990.

[5] OPERA Collab.,Phys.Lett.B691:138-145,2010.

[6] T.Naka et al.,Nucl.Instr.Meth.A581:761-764,2007. 


\section{Nuclear Emulsion Exp. in JP-US collab.}

1975

Fermilab Emulsion Exposure to $205 \mathrm{GeV}$ Proton

: Charm Hadro-production

1977-1984 Fermilab E531: Charm production in Neutrino int oscillation search

1984-1989 Fermilab E653: Hadro-production of B \& C 1995-2007 Fermilab E872 (DONUT):

Direct observation of Tau Neutrino

2005-2006 Fermilab T952(PEANUT):

Test Experiment for OPERA at NuMI

A number of Physics Output \& Technical developments

\section{Fermilab E531}

1) Lifetime measurement of

Neutral \& Charged

Charmed hadrons

( confirm lifetime difference observed in Cosmic-ray

exp.) (1980)

2) muon neutrino $\rightarrow$ tau neutrino oscillation upper limit (1981, 1986)

Start point of Neutrino

Oscillation Study

by Nuclear Emulsion

E531 $\rightarrow$ CHORUS $\rightarrow$ OPERA

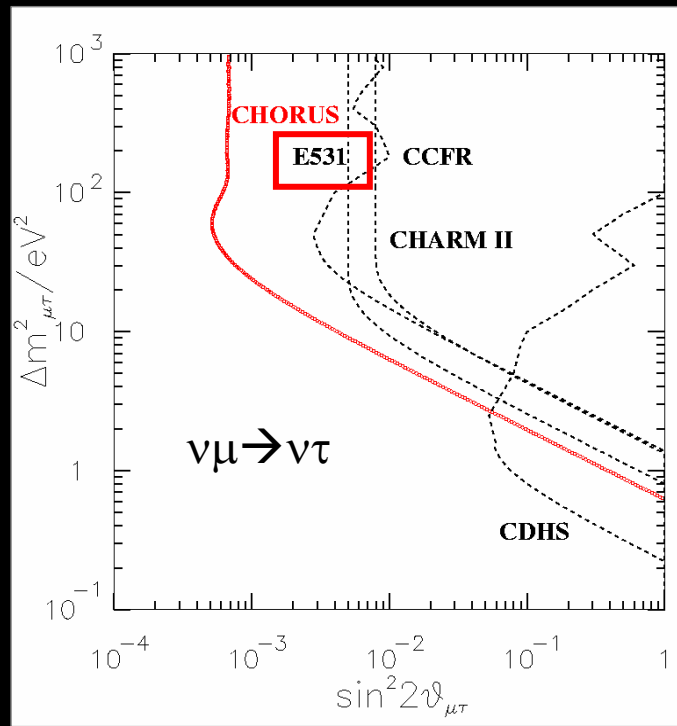

Fermilab CERN CNGS 


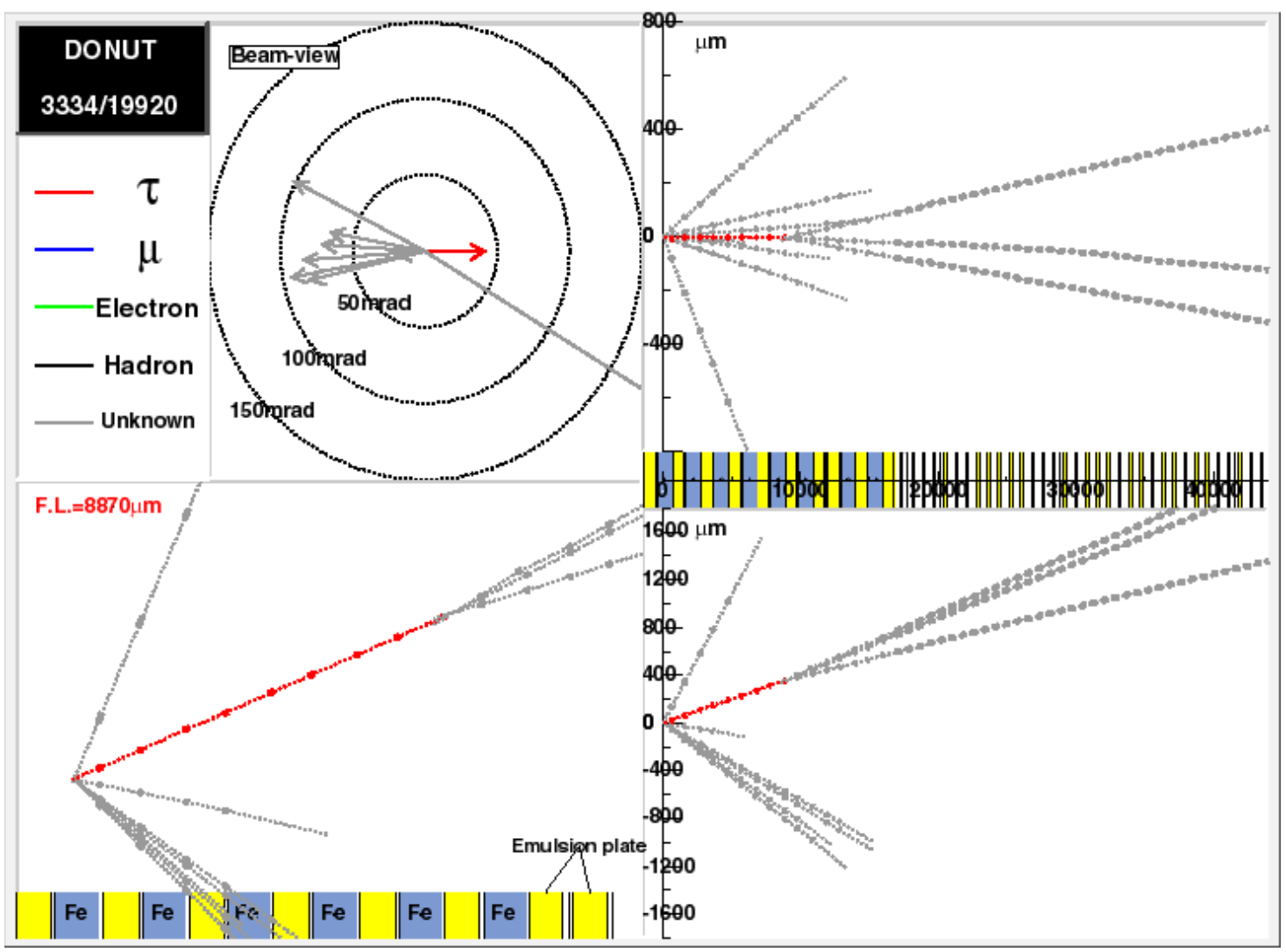

\section{ECC}

- ECC : Developed in cosmic-ray experiments by Japanese researchers

- Sandwich Structure Material + Emulsion Target Tracker

- P measurement by Multiple Coulomb Scattering.

- Electromagnetic component: ID and Energy measurement by Shower counting

Discovery of Naked Charmed particles by K.Niu in 1971

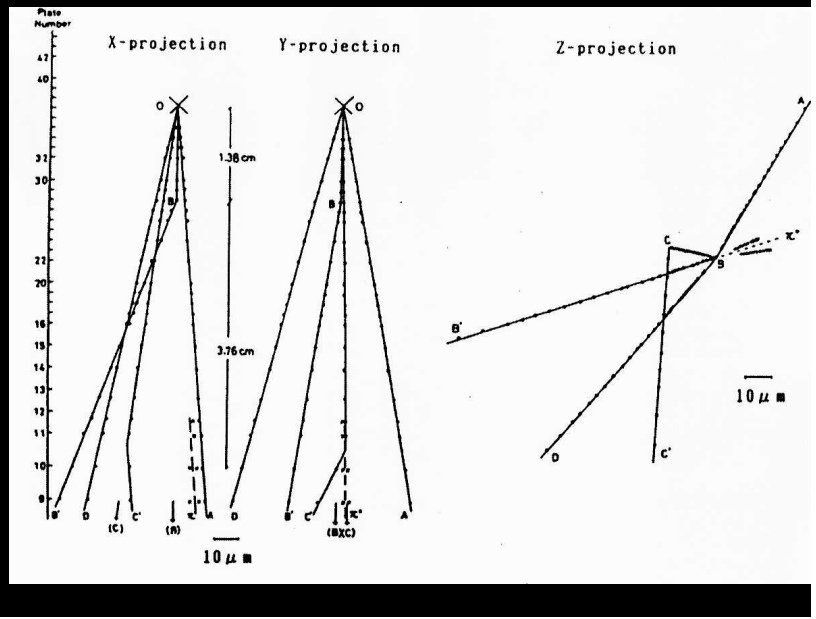




\section{Automated Emulsion Read-out system}

- Principle was proposed by K. Niwa in mid. 70 s.

- First system construction in 1983.

- First application to real experiment in 1994. CHORUS (CERN WA95)

- Advanced version was developed by T.Nakano and applied to DONUT, OPERA.

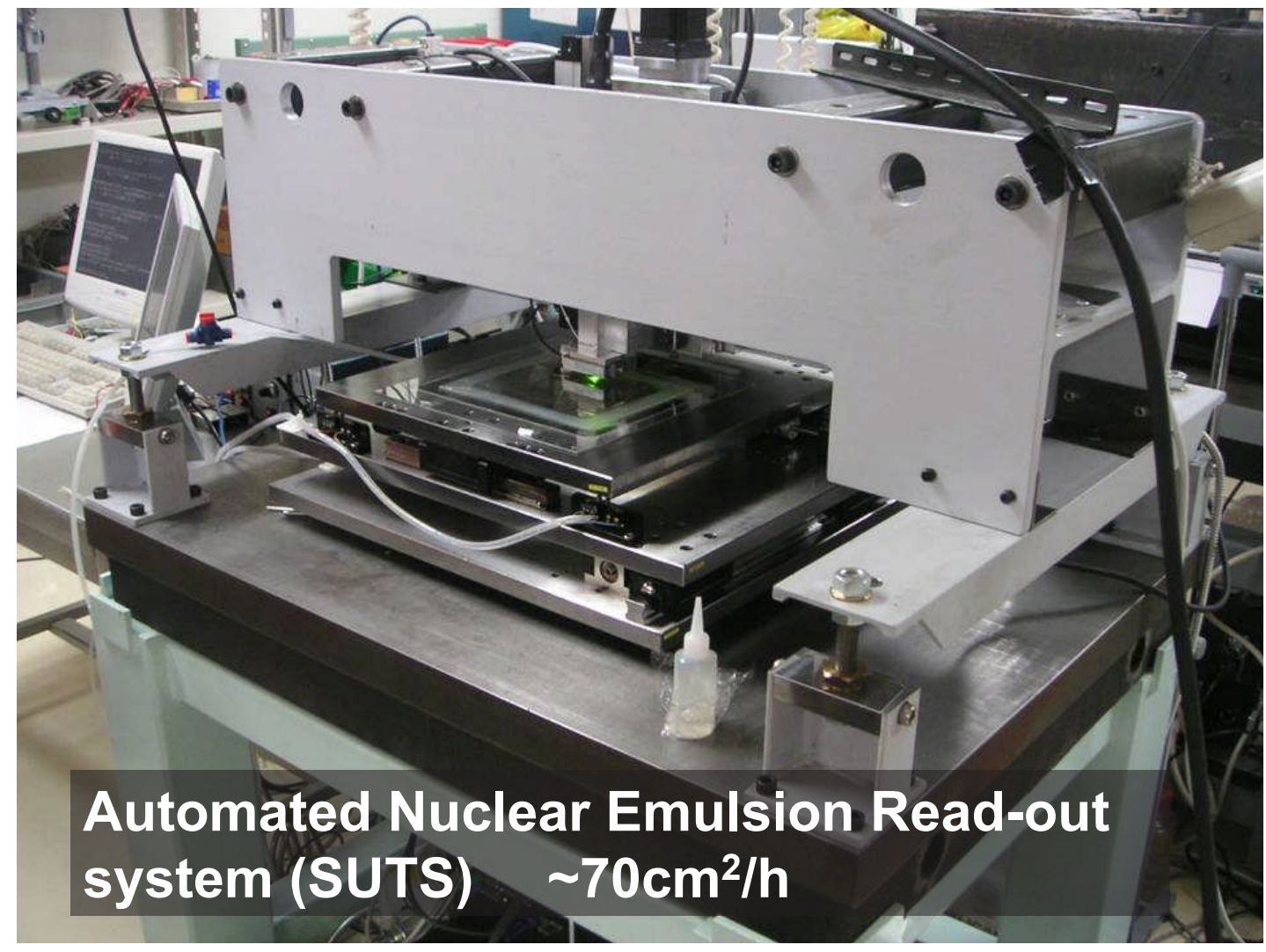




\section{OPERA}

- Tau Neutrino Appearance Detection to conclude the existence of Neutrino Oscillation.

- Conceptual design in 1995 stimulated by Kamiokande result

+ Experiences in DONUT (1998).

Official proposal in 2000.

Physics Run : 2008,2009,2010,2011,2013(plan)

First Tau Neutrino event in 2010 (using part of 2008 \& 2009 data.)

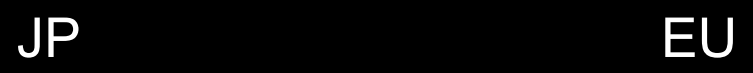

K.Niwa $\rightarrow$ M.Nakamura - Y.Declais(Lyon) $\rightarrow$ A.Ereditate(Bern)

Emulsion+Scanning Beam + Counter + Scanning

\section{OPERA First Tau Neutrino Event}

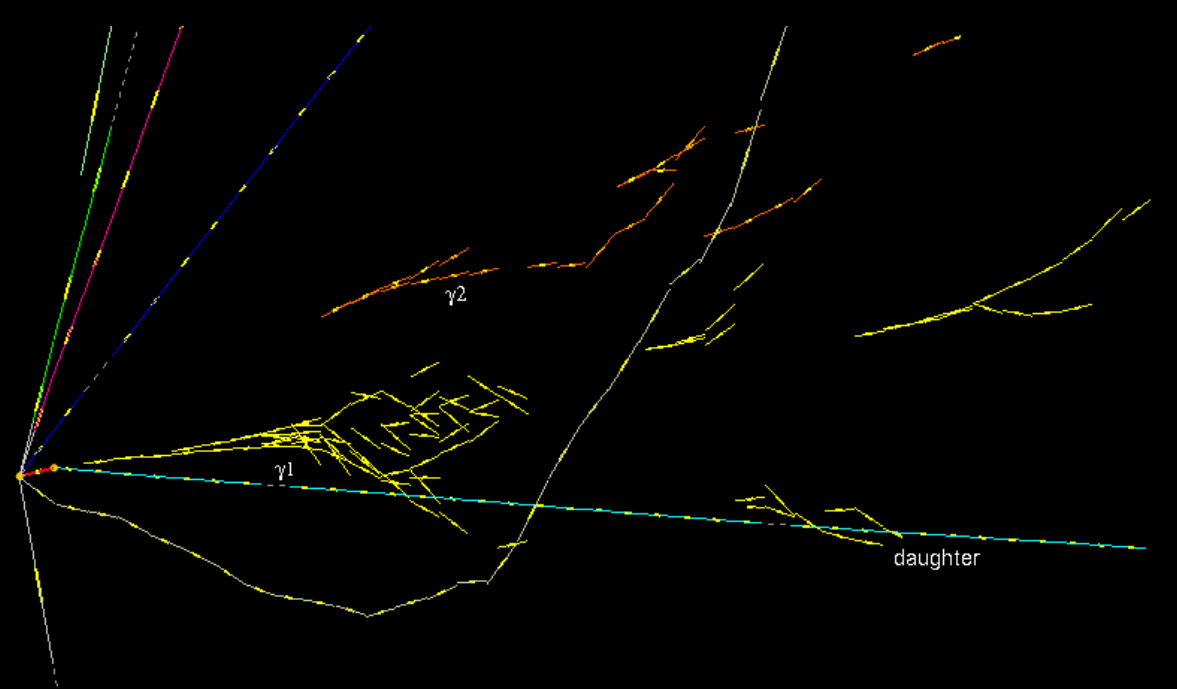




\section{For Future Application of Emulsion}

R\&D of Key Technologies (2010 )

1) Nuclear Emulsion Gel R\&D and production in Nagoya with a help of retired Photographic Engineers of Fujifilm.

2) R\&D of new read-out system 100 times faster than SUTS.

Physics Target

1) Neutrino Physics ( NuMI, Neutrino Factory)

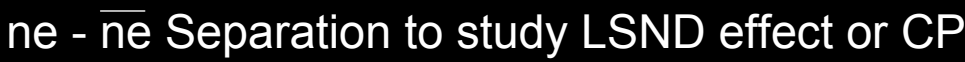

2) Dark Matter directional detection

3) Muon-radiography : Structure Investigation of Volcano, Iron furnace etc.

4) Neutron radiography : directional detection under high gamma BG.

\section{Summary}

- Fruitful outputs in Physics and Technology from Japan-US Emulsion-counter collaboration.

- Nuclear Emulsion technology polished in Japan-US collaboration plays a key role in OPERA Tau Neutrino Appearance detection experiment to conclude the existence of Neutrino Oscillation (proposed by Maki-Nakagawa-Sakata \& detected by Kamiokande).

- Continue to explore New frontier by advanced Nuclear Emulsion Technology. 



\title{
The "LASS" Program - the LASS Experiment and the SLD Experiment at SLAC
}

\author{
David Leith, SLAC, Menlo Park, CA
}

Our Japanese saga started in 1975/6 with meetings with Prof. Ryo Kajikawa in Nagoya University, and the beginning of a long personal friendship, and a very productive scientific collaboration. Nagoya University applied successfully to the JSPS for support, and thus began the US/Japan collaboration for the "LASS" program. Prof. Nishikawa worked hard for the formation of the Japan/US collaboration in HEP, and supported the Nagoya group's role on the LASS experiment as part of that partnership. Initially, Seigi Iwata came to SLAC as the senior resident staff member, but soon thereafter Shiro Suzuki took over as the KEK/Mombusho ACO, and resident leader of the Japanese group. At the start of the Japan/US program, SLAC focused on two research areas; a series of bubble chamber experiments, and the LASS experiment.

Early in the program, Joe Ballam made a very wise, generous, and impactful decision, viz., to hire a series of young Japanese scientists under short-term appointments at SLAC, using laboratory resources offset by the Japan/US investment. The LASS program had over 30 Japanese colleagues working on this experiment from 1979 to 1999; eighteen post docs and graduate students worked long-term at SLAC in these visiting appointments. It was not until around 1990 that such temporary professional positions were established in Japan, facilitated by the success at SLAC. The collage of photographs in Fig.1 captures the Japanese membership of the collaboration, and more fully, in the table of Fig. 2. By the 1980s, the group from Nagoya University had been joined by Prof. Haruo Yuta's group from Tohoku University, now were working on building the SLD experiment, while still participating in analyzing the LASS data. The "Ballam plan" still continued to support a fraction of the resident Japanese scientists. The impressive table in Fig. 2 captures the role they played in the activities, displays both the pastand the current-institutions, and records those still active in Japan HEP.

Within the Japan/US program, the LASS team designed and built a multi-particle spectrometer with full geometric coverage (Fig 3). The spectrometer operated in a radio-frequency-separated hadron beam at SLAC. The analyses of the experimental findings, shown in Figs. 4 and 5, established the full excitation-spectrum of light quark meson states for both the radial- and the orbital-quantum numbers; this was accomplished most completely for the strange quark states, but also effectively for the strangeonium spectrum. Fig. 6 shows the full expected spectrum of light-quark states as dashed lines, and those observed in the LASS experiment as solid lines or boxes. The work on the SLD experiment involved helping to design and build the CRID particleidentification system, and on the important CCD vertex detector of the SLD detector, running the experiment through data taking, and participating in data analysis. The SLD ran at the first linear electron-positron collider, the SLC, and acquired data with a polarized electron beam at the $\mathrm{Z}$ boson mass. The research involved precise measurements of various important asymmetries, $[A(b), R(B), A(L R)]$, a measurement of alpha-s, and precision studies of $b$ quark fragmentation, both at the $\mathrm{Z}$ mass. These achievements all were covered in highly cited publications.

In conclusion, the very strong, productive science program of the LASS collaboration offered 'hands-on' learning experience in designing and building detector instrumentation, running large 
HEP experiments '24/7', whilst paying attention to developing the software to support both production of data, and its analyses. The results from LASS provided a broad, empirical foundation on the detailed spectroscopy of light-quark meson systems, particularly emphasizing the strange quark. The SLD experiment introduced new kinds of detector technology, and, with the polarized beams of the high-energy collider, generated precision measurements that challenged the SM story for heavy quark couplings, and effectively complimented the four LEP experiments at CERN. Undoubtedly, the development of the Japanese human resources that now are an important part of ongoing basic research in Japan is yet another invaluable deliverable from this program. Finally, I am deeply thankful for the rich set of very important friends that came into my life only through this Japan/US program.

I acknowledge invaluable technical help from Terry Anderson, SLAC Technical Illustrations, on creating the collage of photographs of Japanese colleagues, the help of Jean Dekin, the SLAC Archivist, in resurrecting photographs of long gone Japanese colleagues from the lab archives, and Nobu Toge for his help in gathering photographs from far-flung colleagues throughout Japan. Credit Figure 3: Walter Zawojski, SLAC. 


\section{Figure Captions}

Fig. 1 A collage of photographs of the Japanese scientists who worked on the LASS program.

Fig. 2 A table of the full Japanese team from the LASS program, showing their institutional affiliation, length of stay, and whether they are active in Japan HEP today.

Fig. 3 A drawing of the LASS spectrometer - a complete geometrical coverage, multi-particle detector installed in a separated pi-meson and K-meson beam at SLAC. It proved to be an effective "digital bubble chamber".

Fig. 4 A Dalitz Plot of three-quarters of a million events from the reaction $\mathrm{K}$ $\mathrm{P} \rightarrow \mathrm{K}$ pi $\mathrm{n}$ at $11 \mathrm{GeV} / \mathrm{c}$ showing clear resonance bands for both $\mathrm{K}$ * and $\mathrm{N}^{*}$ states, and also demonstrating the essentially full geometric coverage of the decay angular distributions.

Fig. 5 Displays the S-wave, and P-wave scattering amplitudes,a result of the partial wave analysis of the data shown in Fig. 4 above. Three spin 1 resonances can be clearly observed in the P-wave amplitude and phase variations, and a spin 0 state in the S-wave behavior.

Fig. 6 The level scheme for quark anti-quark bound states - a Grotrian plot with the full spectrum of orbital and radial angular momentum quantum numbers plotted. Dashed lines represent expected states, while full lines, or boxes, indicate the experimentally observed states. Essentially most of the expected states have been identified for the strange quark mesons, and large fraction of the strangeonium states, too. 


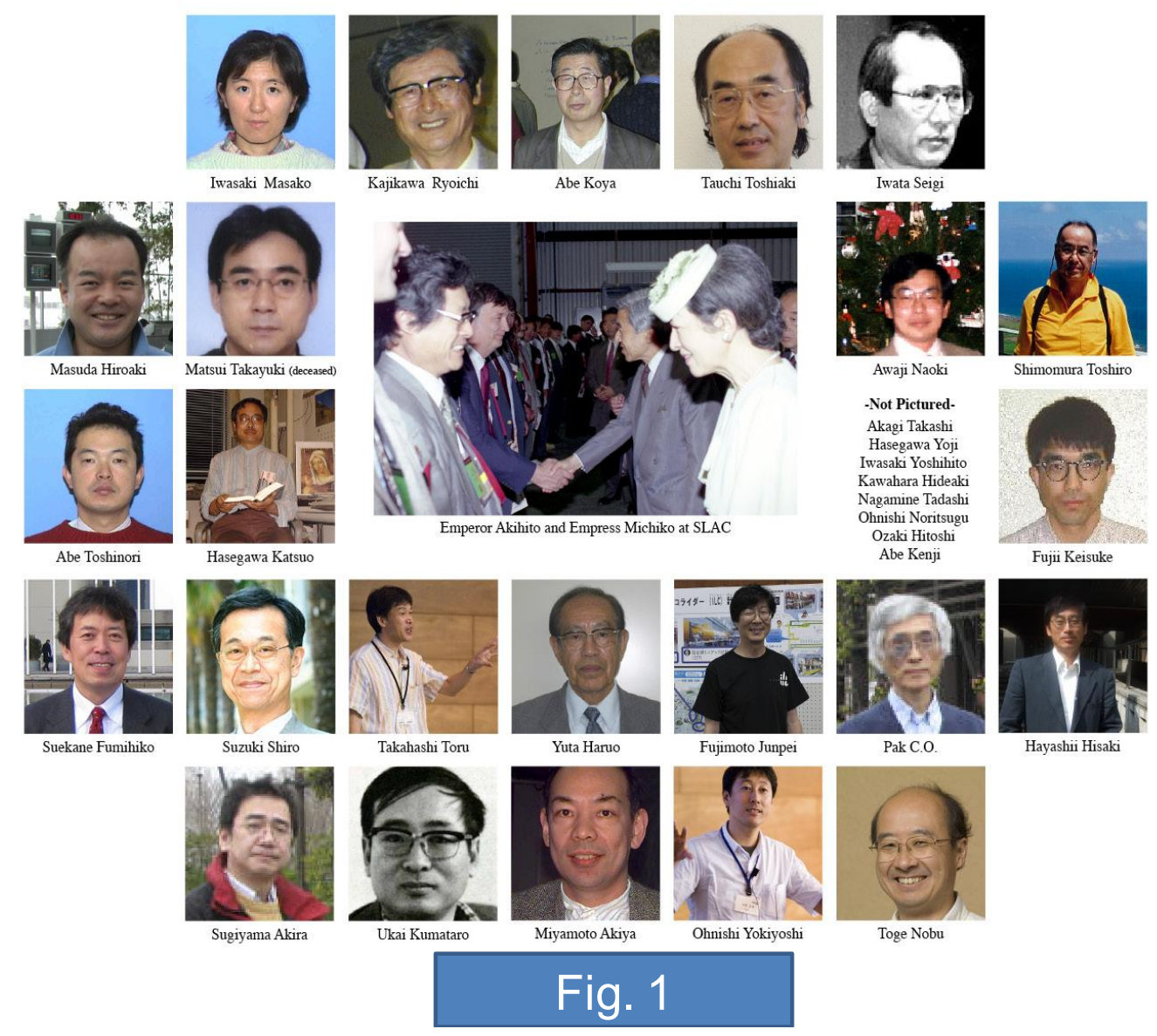

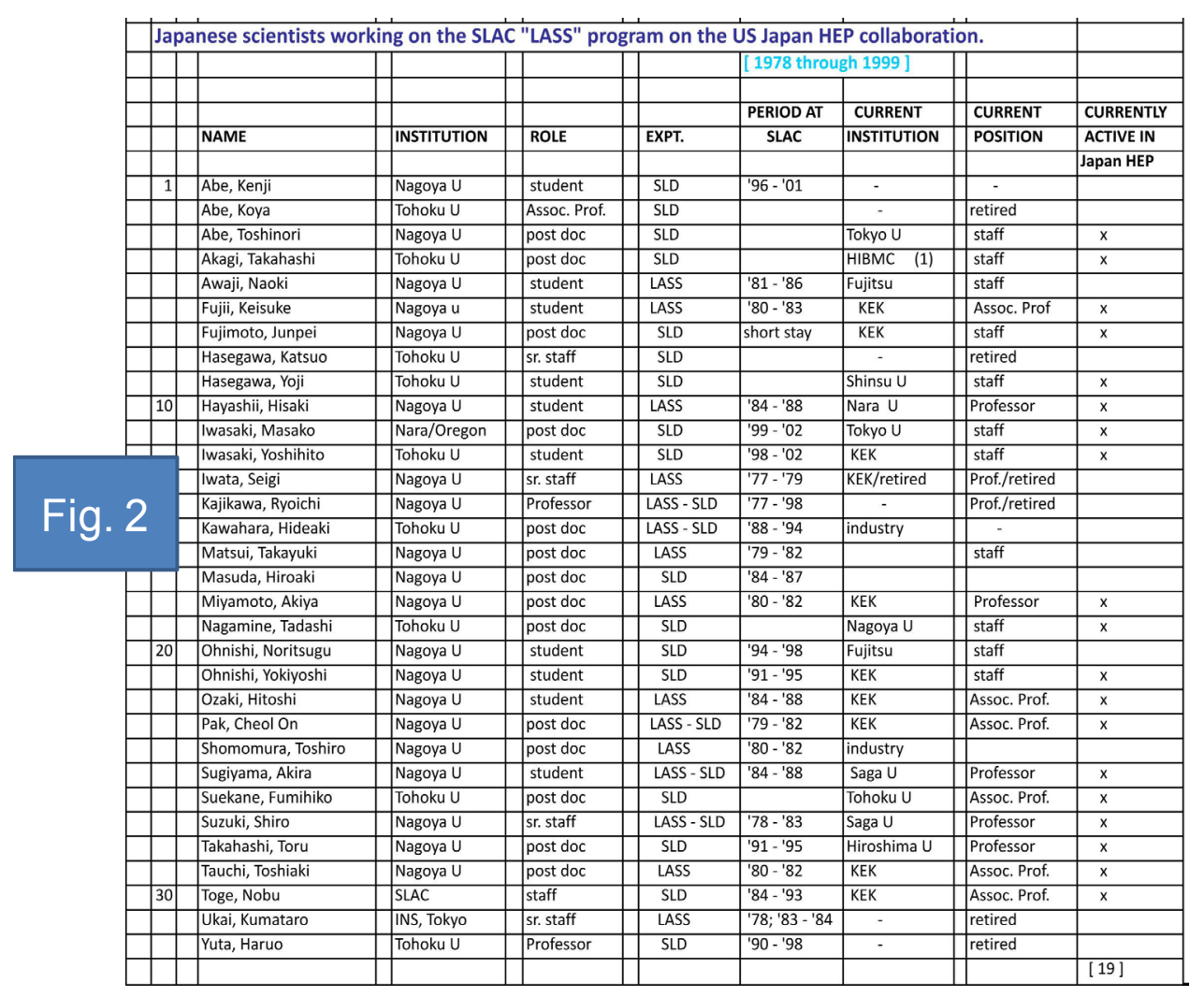




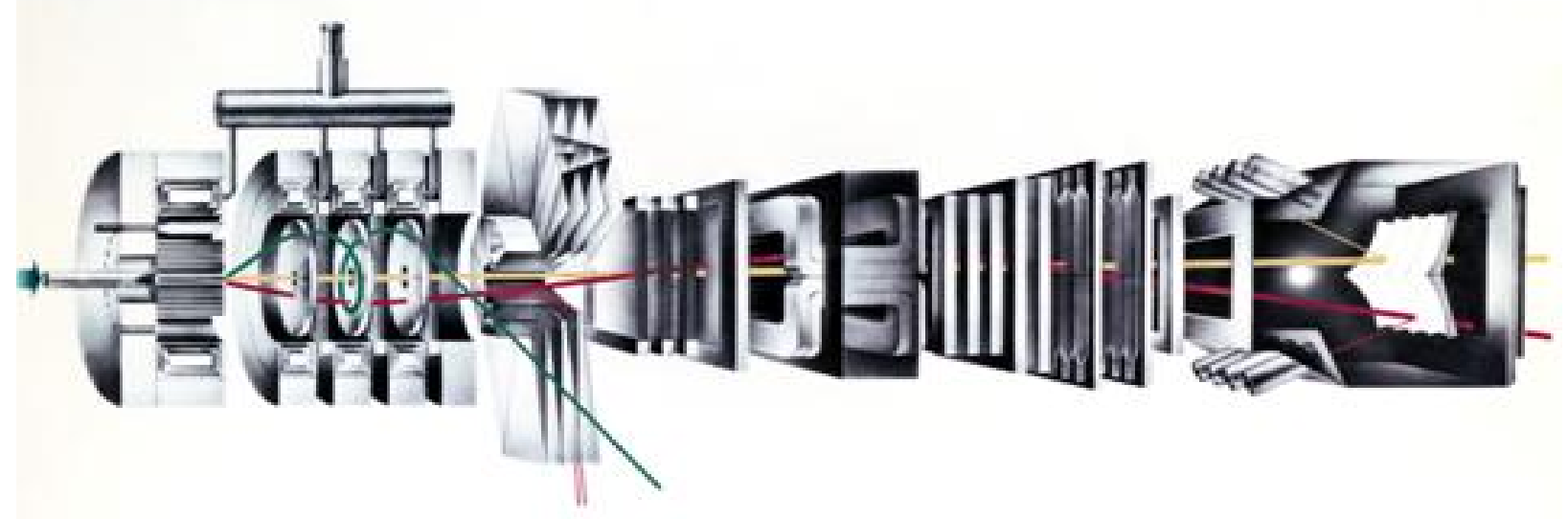

Fig. 3

$\mathrm{K}^{-} \pi^{+}$Elastic Scattering from K- $\mathrm{p} \rightarrow \mathrm{K}^{-} \pi^{+} \mathrm{n}$ at $11 \mathrm{GeV} / \mathrm{c}$ \{ NPB 296 (1988) 493 ; Naoki Awaji, Ph.D Thesis, Nagoya (1986) \}
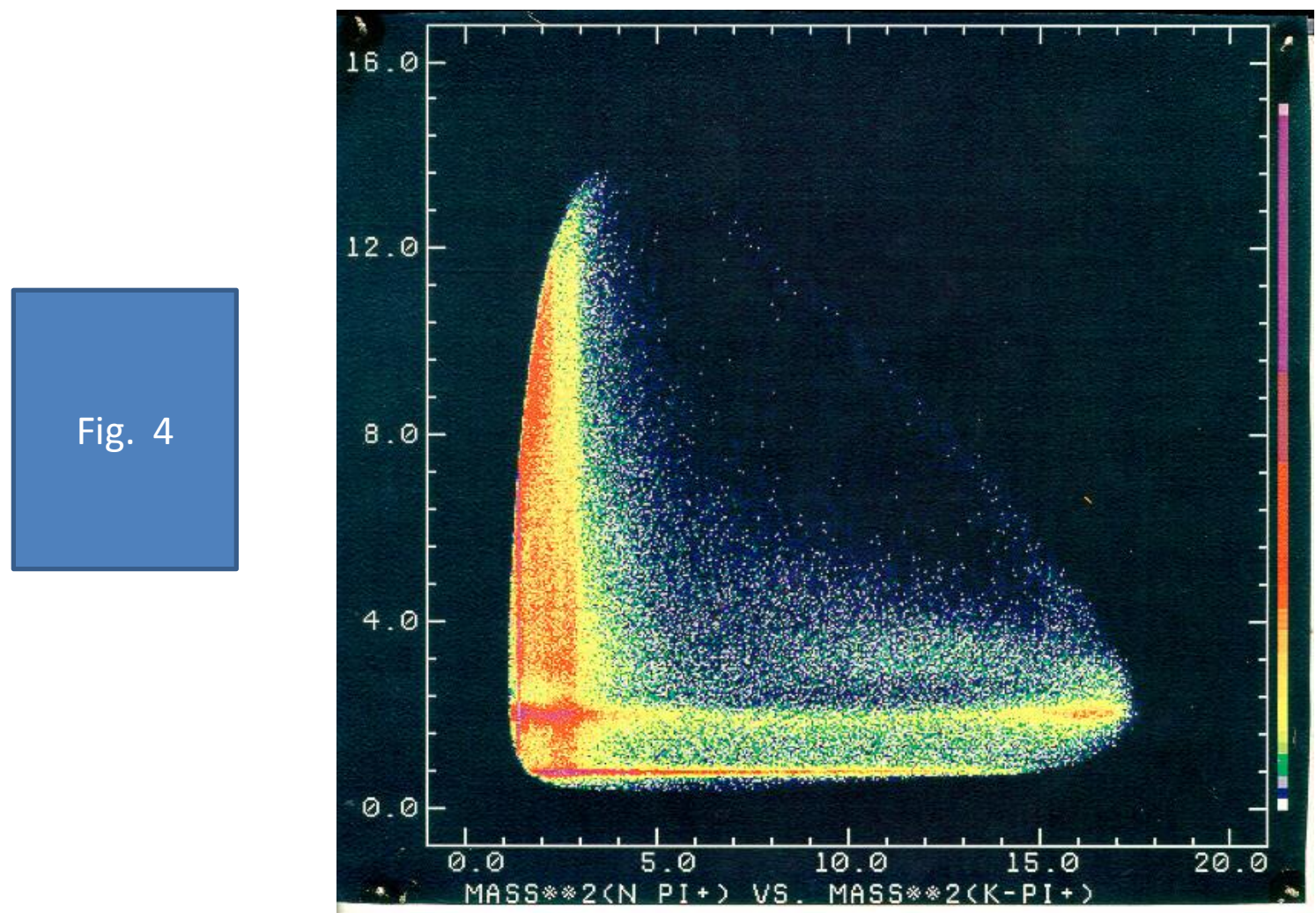

$730 \mathrm{k}$ events 
The magnitude, (a), and the phase, (b), of the S-wave and $\mathrm{P}$-wave amplitudes in the mass region below

\section{$1.84 \mathrm{GeV} / \mathrm{c} 2$.}

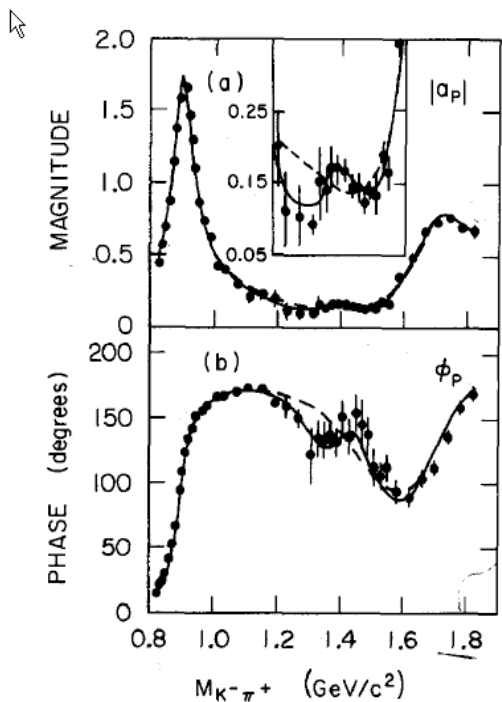

D. Aston et al. / $\mathrm{K}^{-} \pi^{+}$scattering

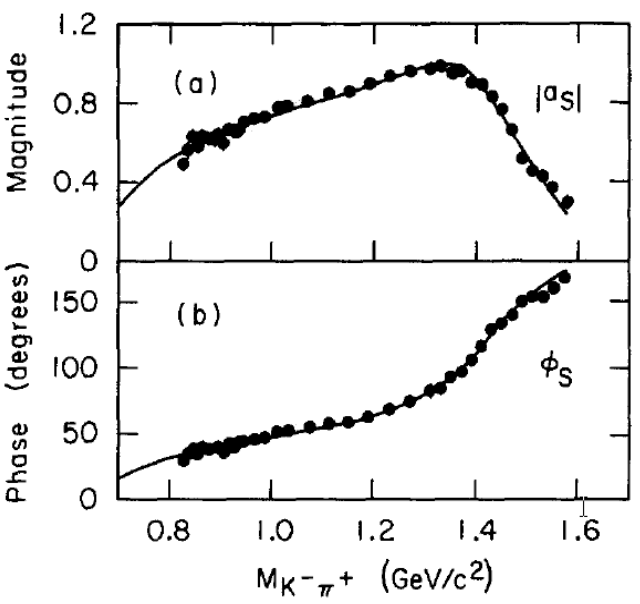

Fig. 5

Strange Meson Level Scheme

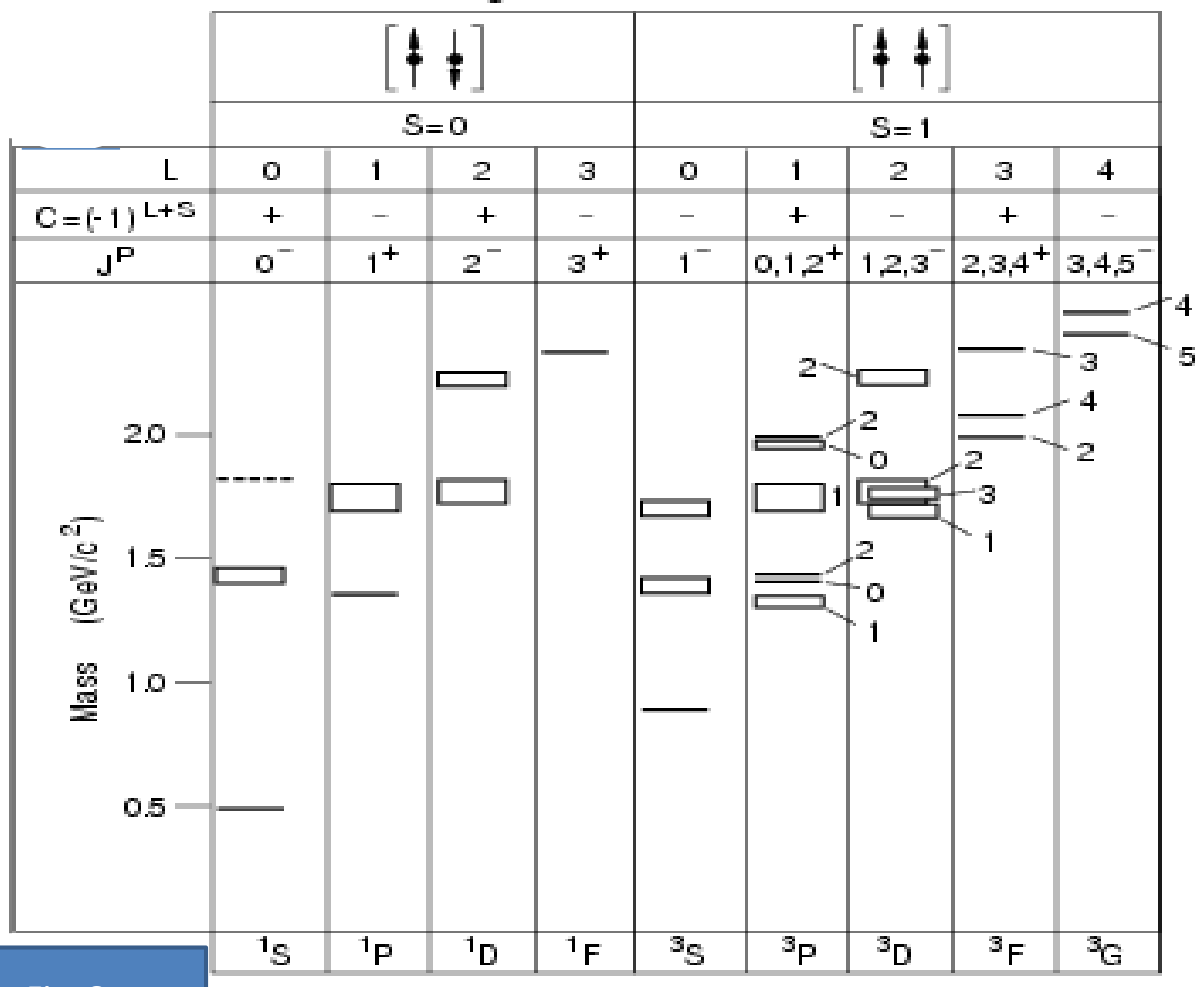

Fig. 6 


\title{
US-Japan and the KTeV Research Program at Fermilab
}

\author{
R. Tschirhart, \\ Fermi National Accelerator Laboratory, Batavia, IL
}

The $\mathrm{KTeV}$ experiment at Fermilab primarily was a comprehensive study of neutral kaon decays wherein we collected data from 1996 to 2000. The research program yielded $32 \mathrm{PhD}$ theses and 50 publications in physics journals, most notably establishing matter/antimatter asymmetry in particle-decay amplitudes through measuring the $\operatorname{Re}\left(\varepsilon^{\prime} / \varepsilon\right)$ parameter. This rich research program was fueled by intense beams of in-flight high-energy $(50 \mathrm{GeV})$ neutral kaons produced by 800 $\mathrm{GeV}$ protons extracted from Fermilab's Tevatron impinging on a beryllium-oxide target.

The subsequent kaon decays were reconstructed with a high-precision spectrometer and cesiumiodide calorimeter systems. The performance attained in resolving the photon energy of kaon decay products is unprecedented in this field $(\sim 1 \%)$. US-Japan funded the custom photomultiplier tubes that instrumented the cesium-iodide calorimeter and other important calorimeter sub-systems. US-Japan also funded the visionary data-acquisition system that recorded data from the experiment. This system was designed by $T$. Yamanaka and T. Nakaya and was the first to filter events by fully reconstructing them in real-time, whilst rejecting most of the reconstructed events in pursuit of the desired ones. Their technique of aggressively filtering data based on full software reconstruction now is the standard in modern high-energyphysics experiments. 


\section{Context and Prejudice:}

\section{Flavor Physics in the early 1990's}

- Neutrino mixing out of reach??

- What is CP violation? Why is it so small? A new super-weak force??

- The top quark is elusive.....very high mass; does it even exist?

- If the top quark exists and is heavy...new high mass particles can compete in virtual loops in flavor changing neutral currents.

- But...B meson's are reconstructed only by the handful.

- $\mathrm{K}$ mesons can be produced in large numbers, but how can we possibly measure the rare $\mathrm{K}_{\mathrm{L}} \mathrm{gp}^{0} \mathrm{p}^{0}$ neutral mode decay with sufficient precision to search for direct matter antimatter asymmetries??

\section{Kaons at the TeVatron}

- The KTeV experiment was primarily a comprehensive study of neutral kaon decays, data collected 1996-2000.

- The research program yielded $32 \mathrm{PhD}$ theses and 50 physics publications, most notably the establishment of matter/antimatter asymmetry in particle decay amplitudes.

- This rich research program was largely driven by intense beams of inflight neutral kaon decays reconstructed with a high-speed, high-resolution spectrometer.

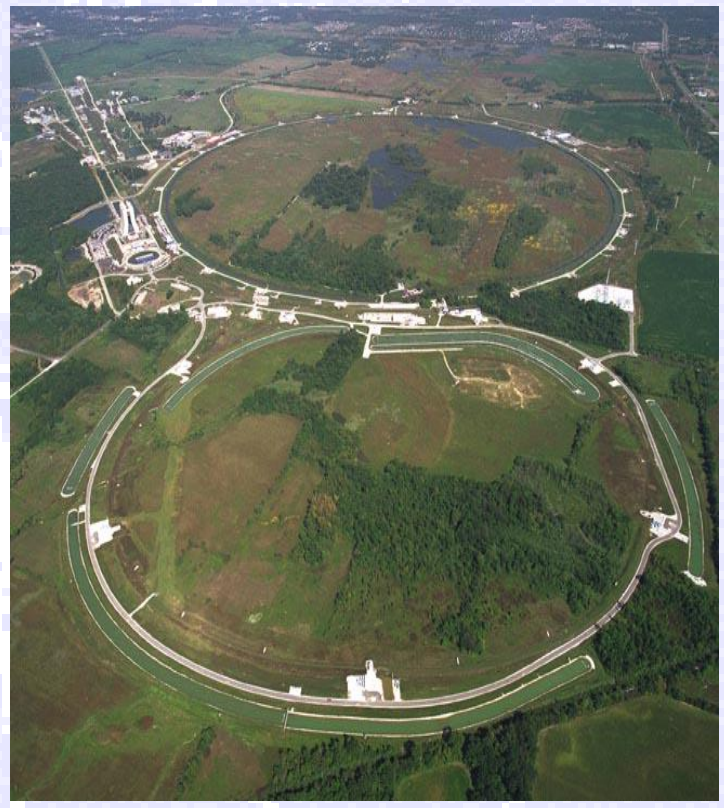




\section{Fast forward... Highlights of KTeV Science...}

- $\operatorname{Re}\left(\varepsilon^{\prime} / \varepsilon\right)=$

$(1.66 \pm 0.26) \times 10^{-3^{*}}$

$\frac{\Gamma\left(\mathbf{K}^{0} \rightarrow \pi^{+} \pi^{-}\right)-\Gamma\left(\mathbf{K}^{0} \rightarrow \pi^{+} \pi^{-}\right)}{\Gamma\left(\mathbf{K}^{0} \rightarrow \pi^{+} \pi^{-}\right)+\Gamma\left(\mathbf{K}^{0} \rightarrow \pi^{+} \pi^{-}\right)}=(5.04 \pm 0.22) \times 10^{-6}$ **

Matter/Antimatter asymmetry in a decay amplitude established, Superweak model excluded.

Large component of this asymmetry might arise from New Physics.

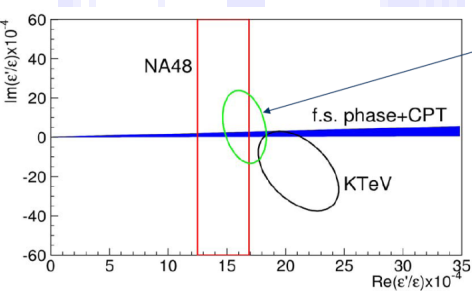

$\mathrm{KTeV}+\mathrm{NA} 48$

- $\mathrm{K}_{\mathrm{L}} \rightarrow \pi^{0} \mathrm{ee}, \pi^{0} \mu \mu$

$K T e V$ limited these rates to $\times 8$ and $\times 25$ the Standard model rates (10-11 level) for the ee and $\mu \mu$ modes... fertile hunting ground for new physics models.

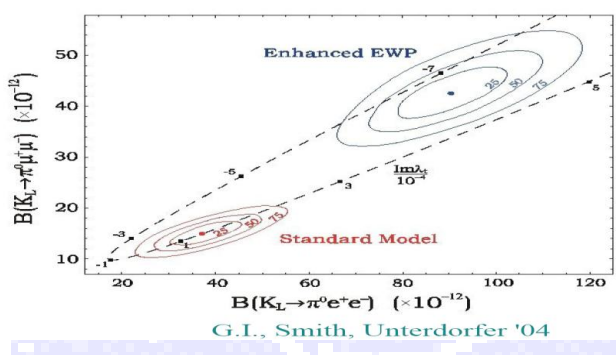

*PDG, **J Imazoto

US-Japan 30th Anniversary Symposium October 20th 2010

R. Tschirhart - Fermilab

\section{US-Japan Contributions to KTeV: Unprecedented photon calorimetry and Trigger \& DAQ}
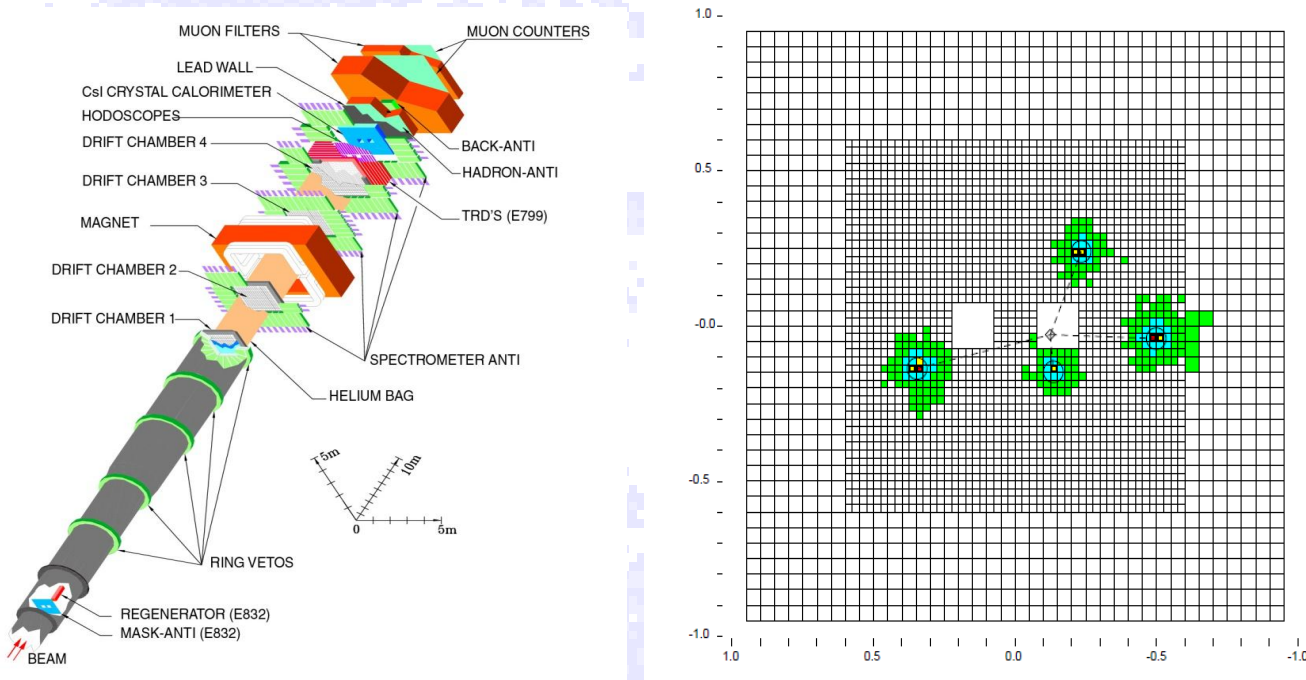

Beam view of $\mathrm{K}^{0} \rightarrow \pi^{0} \pi^{0}$ in CsI calorimeter 


\section{Delivered EM-calorimetry performance better than $1 \%$ resolution over full physics energy range: Best in the field}
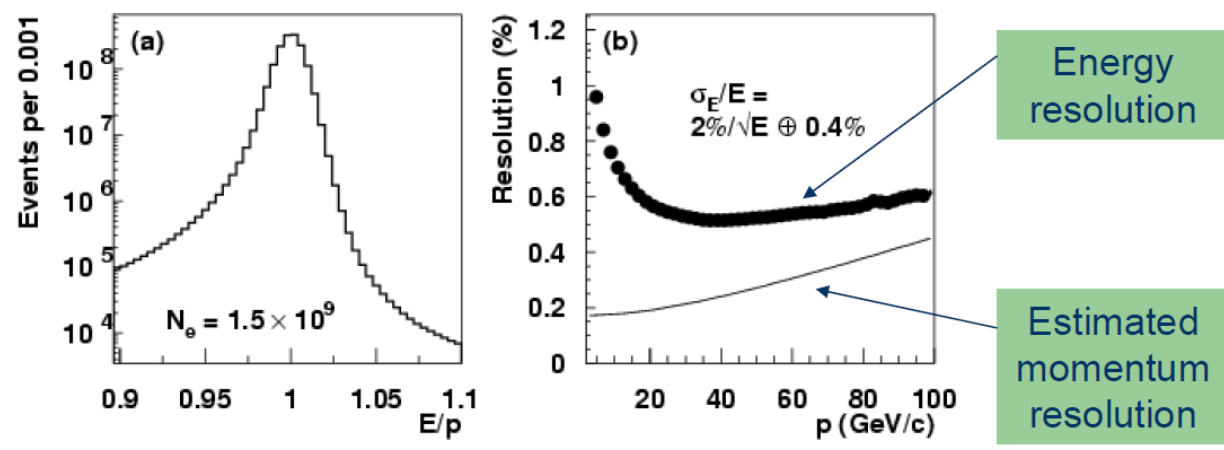

- Calibration based on 1.5 billion Ke3 electrons

- Final $\mathrm{E} / \mathrm{p}$ resolution after all corrections: $\sim 0.6 \%$

US-Japan 30th Anniversary Symposium October 20th 2010

R. Tschirhart - Fermila

US-Japan largely funded the visionary DAQ that was first in the field to filter out most events based on full reconstruction
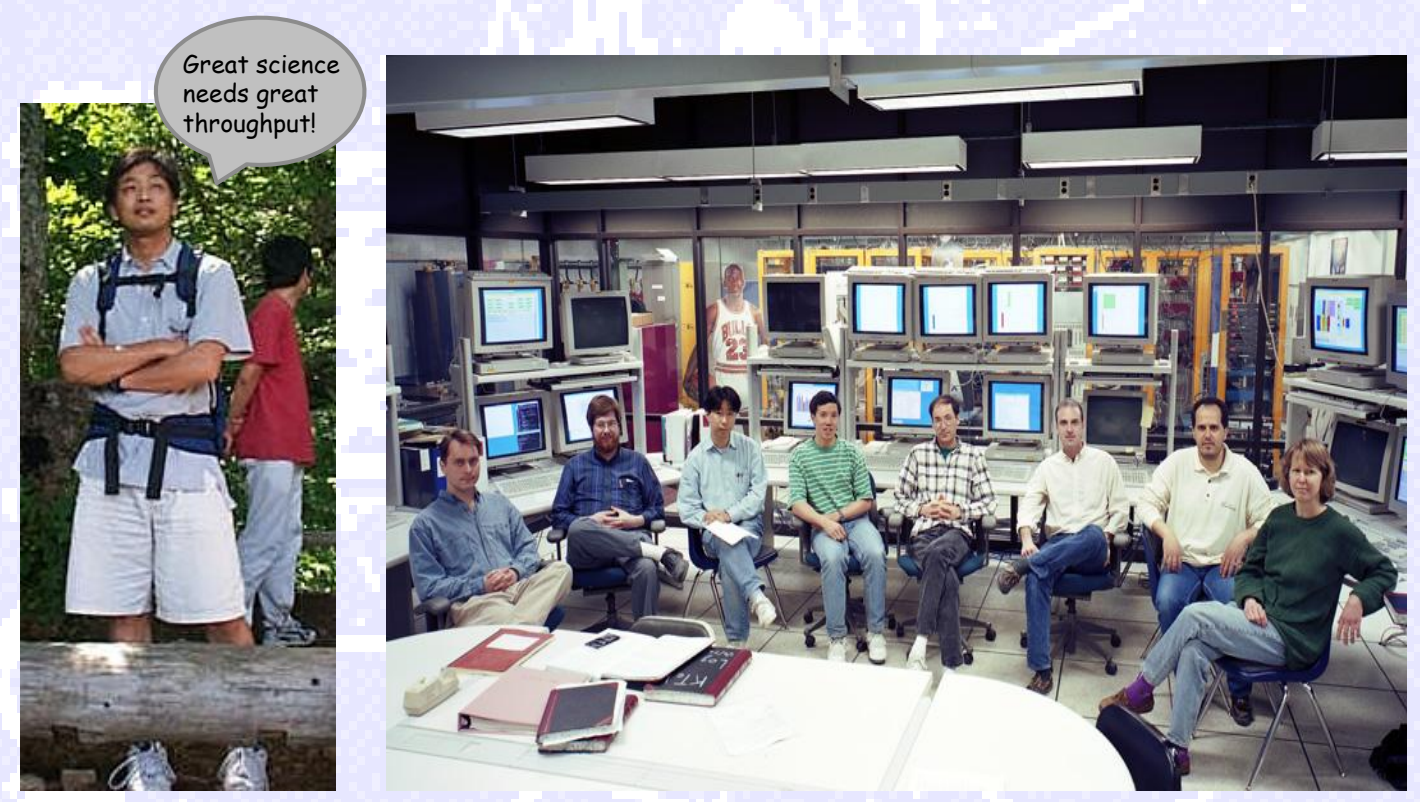

Visionary effort led by Yamanaka-san and Nakaya-san

US-Japan 30th Anniversary Symposium October 20th 2010

R. Tschirhart - Fermilab 


\section{The US-Japan@KTeV Legacy}

- Established decay-amplitude matter-antimatter asymmetries together with our CERN colleagues.

- Probed many, many rare-decays that have cranked up the tension on the "flavor problem".

- Substantially advanced the state of the art in precision calorimetry and data acquisition in High Energy Physics.

- Developed next generation leaders in flavor physics and elsewhere.

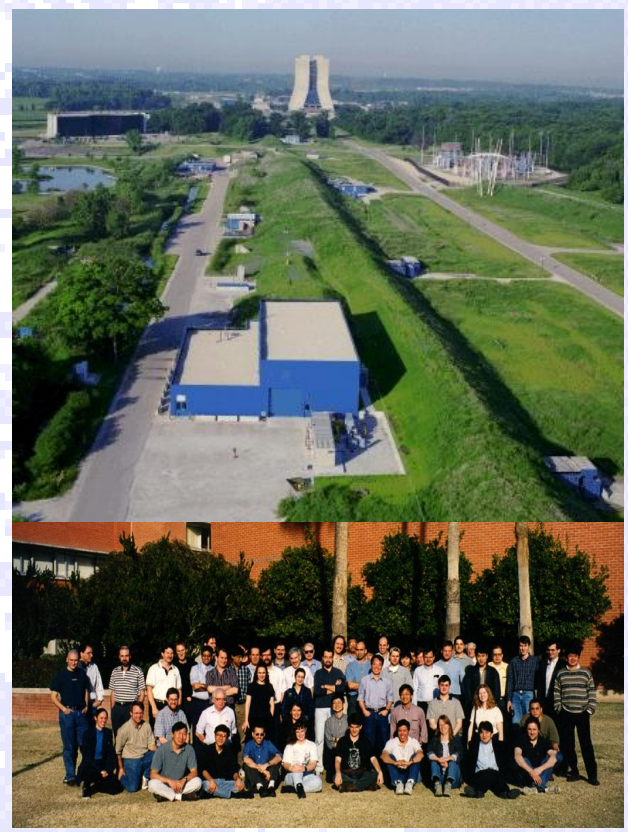

US-Japan 30th Anniversary Symposium October 20th 2010

R. Tschirhart - Fermilab 



\title{
The Rare K-decay Experiment at BNL and Its Accomplishments
}

\author{
Laurence Littenberg, BNL, Upton, NY
}

The Brookhaven National Laboratory rare kaon decay program at the AGS started in the early 1980's and by 1990 was in full swing. The most ambitious of these experiments was E787, a search for $\mathrm{K}^{+} \rightarrow \pi^{+} v \bar{v}$, which offered a unique window into short distance physics. But by 1991, although approaching the Standard Model predicted sensitivity, that experiment had reached a background limit. A strong Japanese group joined the effort in 1992 and both the beam and detector underwent major upgrades. As a result the experiment was able to observe two clean $\mathrm{K}^{+} \rightarrow \pi^{+} v \bar{v}$ events in the next three years. At that point, the switch over of the AGS to an injector to RHIC offered the possibility of using all its intensity in a single experiment. A further upgrade of the detector and the new running mode of the experiment, now called 'E949', allowed a total of 7 events to be observed. This yielded a branching ratio about twice that predicted by the Standard Model (although not statistically different from it). This success has inspired a subsequent round of rare $\mathrm{K}$ experiments. 


\section{A very interesting target}

- The kaon rare decay program at the BNL AGS was in full swing by 1990.

- One experiment, E787, stood out. It pursued $\mathrm{K}^{+} \rightarrow \pi^{+} v \bar{v}$, a very challenging signature.

- Why take this on?

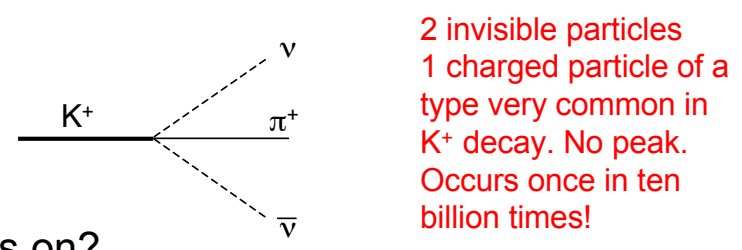

- Unlike the other rare decays under study, it had a Standard Model prediction that seemed to put it almost within reach.

- Because of the neutrinos in the final state and the close relation to the well-studied Ke3 decay, it was extremely clean from a theoretical point of view. It was firmly predictable in terms of the fundamental parameters of not only the SM, but almost ANY theory.

\section{Japan-US collaboration}

- E787 had reached a background limit at $\sim 10^{-9} \mathrm{evt}$

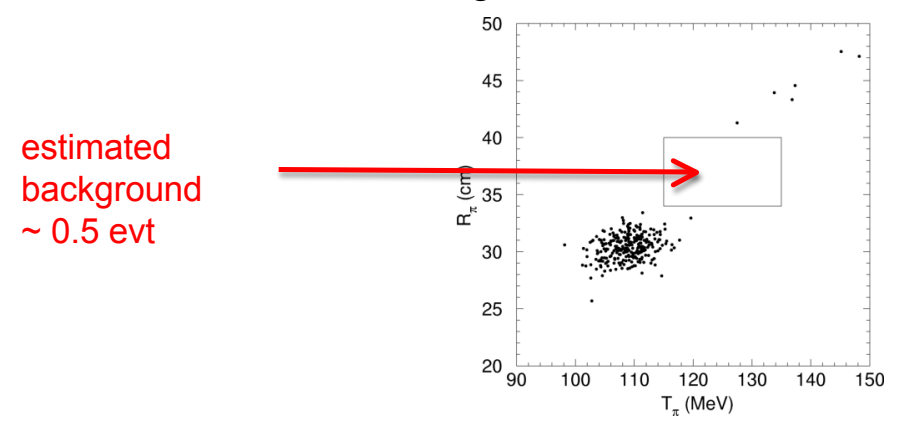

- To go further something major needed to be done.

- Japan could provide components and expertise for the upgrade not available in the US (e.g. high-field tolerant PMTs, YAIO calibration sources).

- Japan could also supply physicists skilled in rare-K experiments 


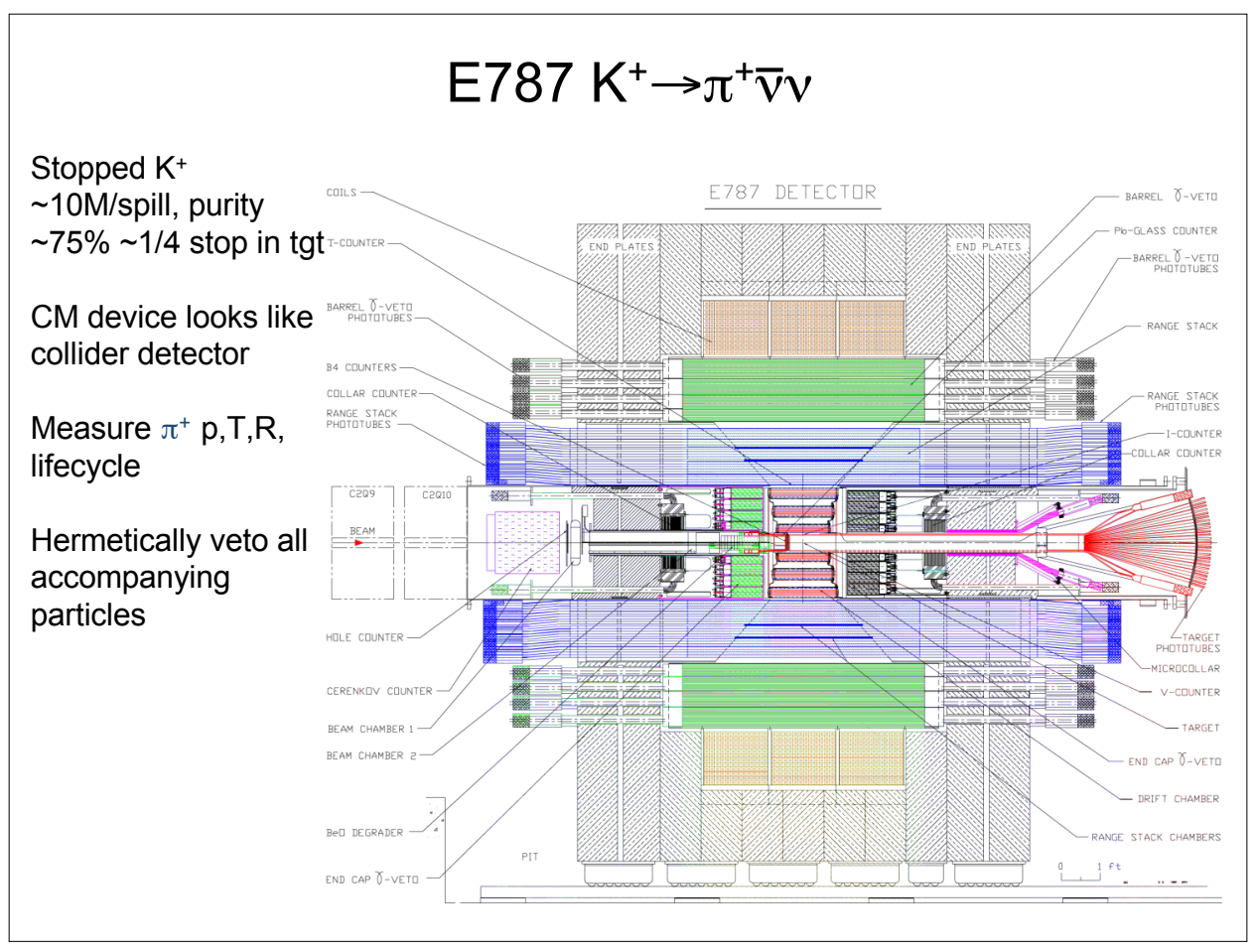

\section{Early history of the collaboration}

- The upgraded beam and detector were commissioned in 1994, in a short run whose main physics output was a measurement of $\mathrm{K}^{+} \rightarrow \mu^{+} v \gamma$.

- In 1995 the first extended run of the upgraded beam and detector took place. An very large data set was collected including the first example of $\mathrm{K}^{+} \rightarrow \pi^{+} v \bar{v}$.
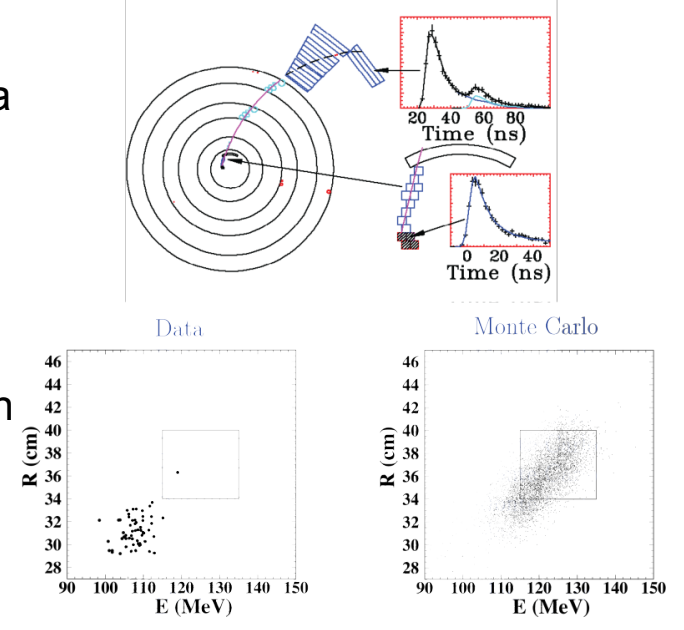


\section{The later runs of E787}

- Further data was collected in 1996, 7, and 8.

- A second event was found in the 1998 data.

- It excited much attention.

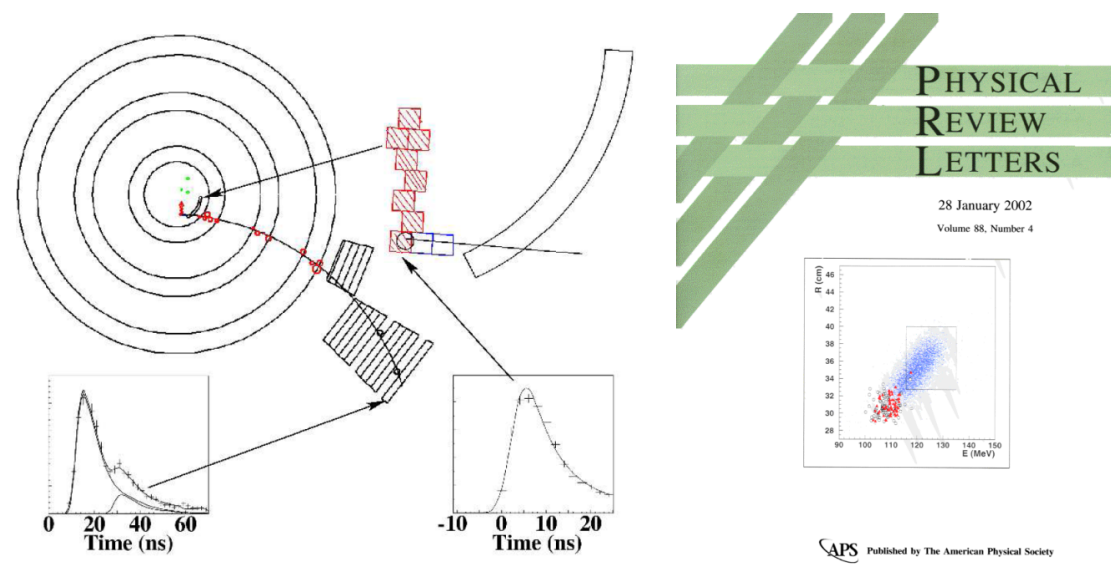

\section{The later runs of E787}

- One of the reasons for the excitement was that the measured rate was twice that predicted by the SM (although statistically consistent with it).

- Strong motivation for further running.

- But there was a big problem!

- With the advent of RHIC, the AGS was slated to become an injector, and DOE OHEP was no longer the landlord of BNL

- This meant many constraints on further high intensity proton running.

- Experiments would have to be approved by DOE on an individual basis. 


\section{The Miracle}

- Realization that since E787 was

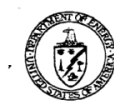

Department of Erergy

August 26, 1999

instantaneous rate-limited, if the AGS spill could be lengthened enough, we could use all the available beam

- The AGS was needed only a very small fraction of the time to feed RHIC

- If no other experiment competed with us for protons, we could gain sensitivity at an unprecedented rate.

- Fortuitously an idea developed for the KOPIO proposal allowed the AGS spill to be lengthened almost without limit.

- A proposal was written to use this idea along with modest improvements to the detector. This became E949.

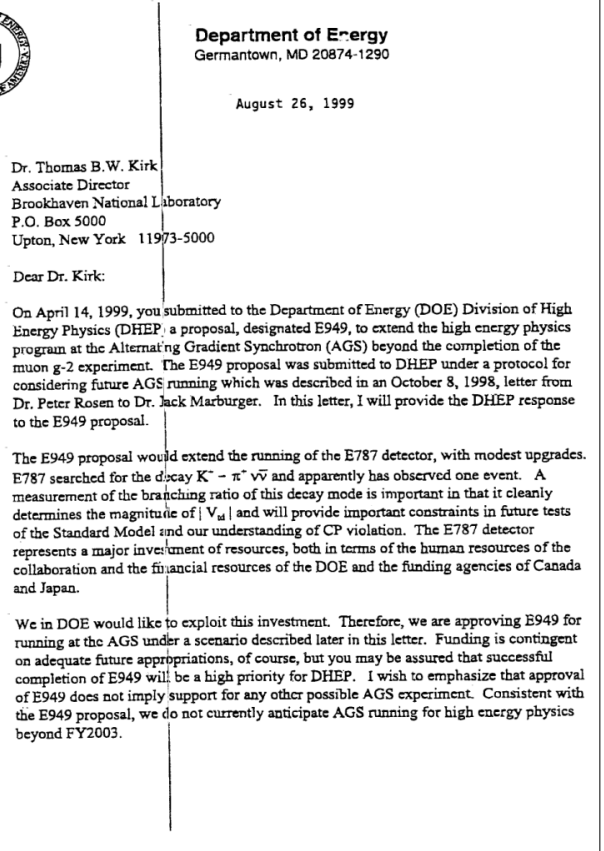

\section{E949}

- Unfortunately although E949 worked very well, it ran only in 2002 due to budget problems in High Energy Physics as a whole

- Still, in combination with E787, it produced a total of 7 events, including 4 in the difficult kinematic region in which the $\pi^{+}$ has less energy than that from the background reaction $\mathrm{K}^{+}$ $\rightarrow \pi^{+} \pi^{0}$.

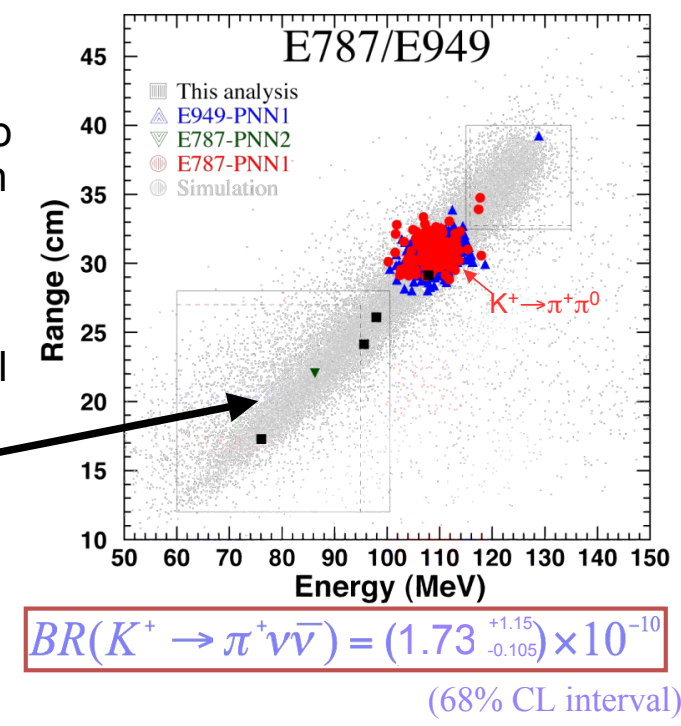

c.f. SM prediction: $(0.85 \pm 0.07) \times 10^{-10}$ 


\section{Products of the E787/949 Program}

- A hint of physics beyond the Standard Model

- 20 publications in peer-reviewed journals (so far)

- Several cited hundreds of times

- Three important new decay modes discovered

- Existence proof that one can access 2nd order GIMsuppressed decays with good $S: B$ even if they have rather poor signatures

- As a result both $\mathrm{K}^{+} \rightarrow \pi^{+} v \bar{v} \& \mathrm{~K}_{\mathrm{L}} \rightarrow \pi^{0} v \bar{v}$ are actively being pursued.

- New techniques developed that have been adopted by others

- Highly evolved blind analysis

- Fine-mesh phototubes

- Fiber stopping targets

- Several of the young people (students \& postdocs) who grew up on the experiment have gone on to be leaders in the field

\section{The Future}

- Encouraged by Professor Sugimoto, Takashi Nakano and others proposed to take the E949 detector to Japan

- Approved by the DOE in 2008

- This is underway at the moment. Some elements have already been shipped. The major work, disassembling the magnet iron and coils is about to start.

- It will be used first in photoproduction experiments at SPring-8.

- Eventually it will be taken to J-PARC where it will be used in various ways, including service as the basis of a new $\mathrm{K}^{+} \rightarrow \pi^{+} v \bar{v}$ experiment.

- Thus the cycle will be completed. 


\title{
The AMY Experiment at TRISTAN, KEK
}

\author{
Stephen Olsen, University of Hawaii
}

The detector used for the AMY experiment was based on a powerful superconducting solenoidal magnet that produced a central field of 3 Tesla, a field that was three or four times higher than those used by the other TRISTAN experiments. An advantage of the high field was that precision charged particle momentum measurements could be performed with shorter particle flight paths, which kept the overall size of the detector relatively small, thereby reducing costs. Other features unique to TRISTAN experiments was a photon detector located inside the magnet coil and space left for low-beta accelerator components that eventually produced improved luminosity. The AMY group was an international collaboration that involved groups from China, Japan, South Korea and the U.S. This was the first time that South Koreans and Chinese scientists did research at KEK (and with each other). The construction costs of the AMY detector were shared more-or-less equally between the U.S. and Japan. This was the first major U.S. investment in a high energy physics facility in Japan.
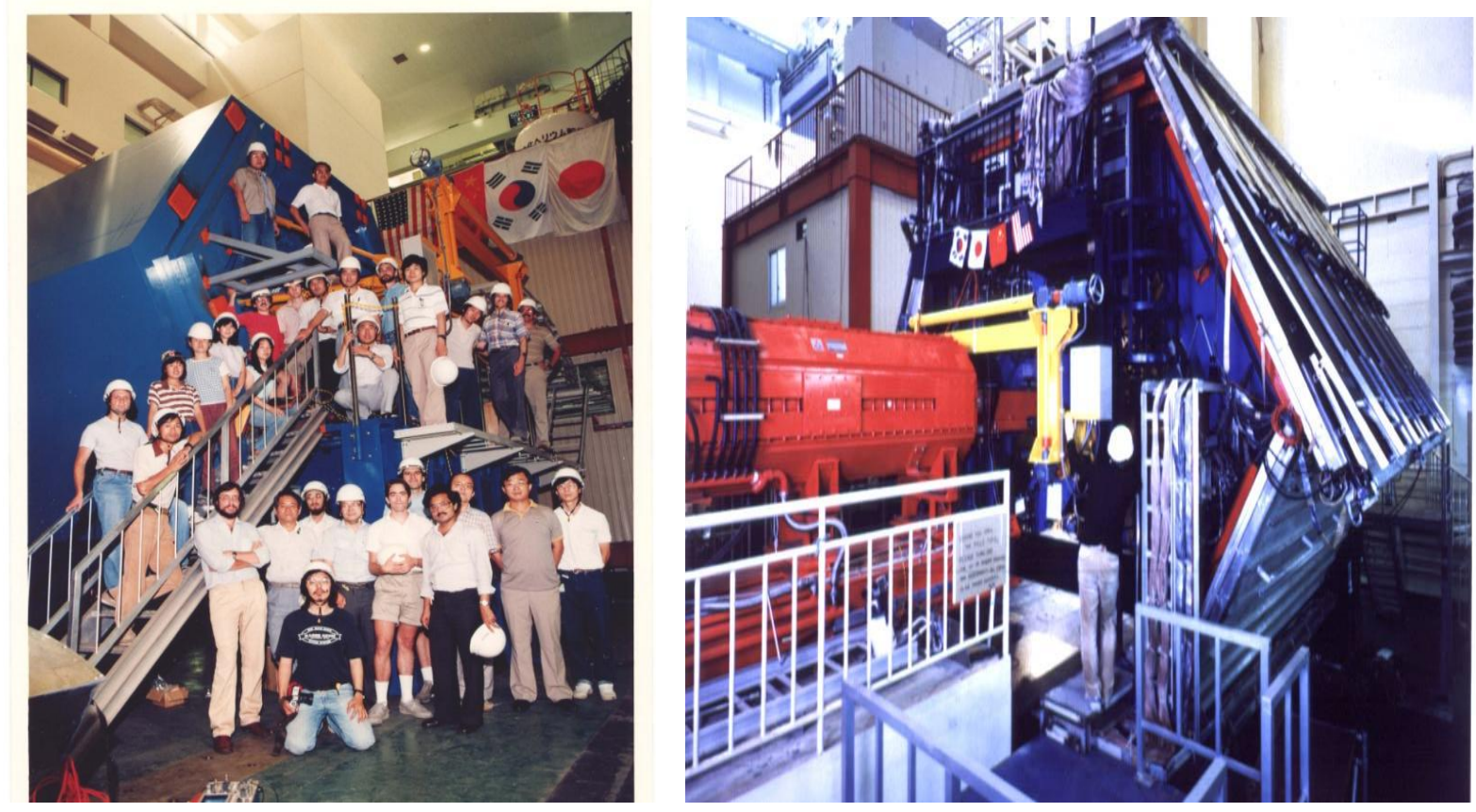

(Left) AMY iron structure with collaboration members. (Right) AMY detector in the beam line.

Although AMY was not able to accomplish its main scientific goal, the discovery of the topquark and studies of its properties -- we now know that the top-quark mass is far beyond the reach of TRISTAN - the group was still able to produce a number of interesting scientific results, including some pioneering analyses that continue to be cited in the high energy physics literature and have been used as a model for subsequent research at higher energy colliders. Among these were studies of the non-Abelian nature of Quantum Chromodynamics (QCD), and measurements of the hadronic structure of the photon. 
The formal structure of QCD, the theory that described the mutual interactions among quarks, is similar to that of the well established theory of Quantum Electrodynamics (QED) but with one major distinction. In QED, the quanta that mediate the forces between electrically charged particles, the photons, are electrically neutral and, thus do not interact directly with each other. In QCD, the corresponding quanta that mediate the forces between quarks, the so-called gluons, are also charged (i.e., carry color charge) and, therefore, interact with themselves. This gluon "self coupling" manifested itself in two ways in

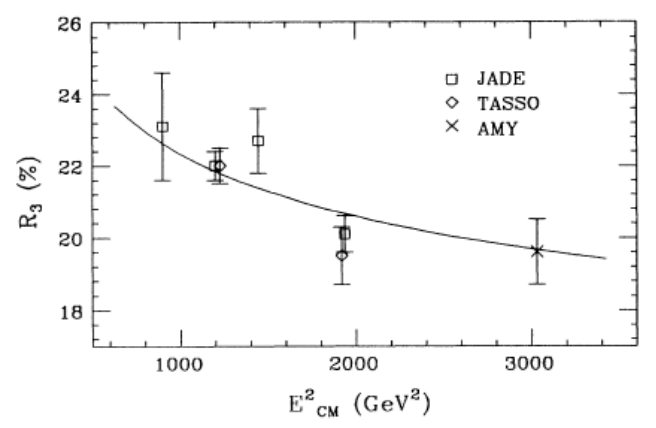

Measured values of the three-jet fraction $R_{3}$ vs center-of-mass energy squared $\mathrm{E}_{\mathrm{cm}}{ }^{2}$

AMY data: it resulted in a reduction of the rate for the production of three-jet events (events with three tightly collimated clusters of particles) in the AMY detector relative to that observed at lower energies; and it produced rather distinctive angular correlations between the individual jets in four-jet events. AMY measurements of the rate for three-jet events, coupled with those of the other TRISTAN groups and corresponding measurements by experiments at higher and lower energies, were prominently cited by the Nobel Committee in its announcement of the 2004 Physics Prize awards to theoretical physicists David Gross, David Politzer and Frank Wilczek. AMY's study of gluon self-coupling in four-jet events was a pioneering measurement that served as a model for a number of subsequent measurements at higher energy LEP collider at CERN.

AMY's measurements of the hadronic structure of the photon included studies of inelastic scattering of electrons from "target" photons radiated by the on-coming positrons, and the production of jets of hadrons by collisions of photons with other photons. In the former, when the scattered electron emerges at a large angle from the incident beam direction, these collisions probe the internal structure of the target photon at very small distance scales, revealing a very complex dynamical environment comprised of virtual quarks - quarks that spring in and out of existence due to quantum fluctuations. In principle, this structure can be calculated by the QCD theory; AMY's measurements provided sensitive tests of the theory. In the latter, AMY observed that when two high energy photons collide, the virtual particles in the photons scatter directly off each other, producing high energy jets of real particles in the detector. Photons that scatter in this way are called "resolved photons." Resolved photon processes are important complements of the electron-photon scattering measurements because virtual gluons fluctuating in and out of existence inside the photon can contribute to resolved photon scattering but, since gluons have no electric charge, not to the electron scattering from photons. Comparisons between the two measurements provide important information about the gluon influence on the photon's structure. AMY was the first experiment ever to detect and identify resolved photons. Studies of resolved photons were an important area of research at subsequent higher energy experiments, especially at the HERA high energy electron-proton collider at the DESY laboratory in Germany.

One of AMY's enduring legacies has been the subsequent careers of many of the AMY participants and the many interesting collaborations that ensued. Students and post-docs from AMY are now prominent leaders in the high energy physics communities in China, Japan, Korea and the US. In the intervening time since the end of AMY, collaborations between the four 
countries have flourished and have branched into many directions. The Institute of High Energy Physics in Beijing provided important components for KEKB as well as for the Pohang photon factory in Korea. In turn, KEK provided essential equipment for IHEP's new and successful BEPCII collider. Close ties between individuals and institutions in these countries continue to produce interesting scientific results. AMY alumni continue to play leading and essential roles in the extremely successful Belle experiment, which incorporated some of AMY's design features, such as a high field magnet, calorimeter inside the magnet coil, low-beta quadrupoles, etc. On a broader scale, the international user community at KEK, started by AMY, has exploded and continues to grow. 


\section{Quark jets vs gluon jets}

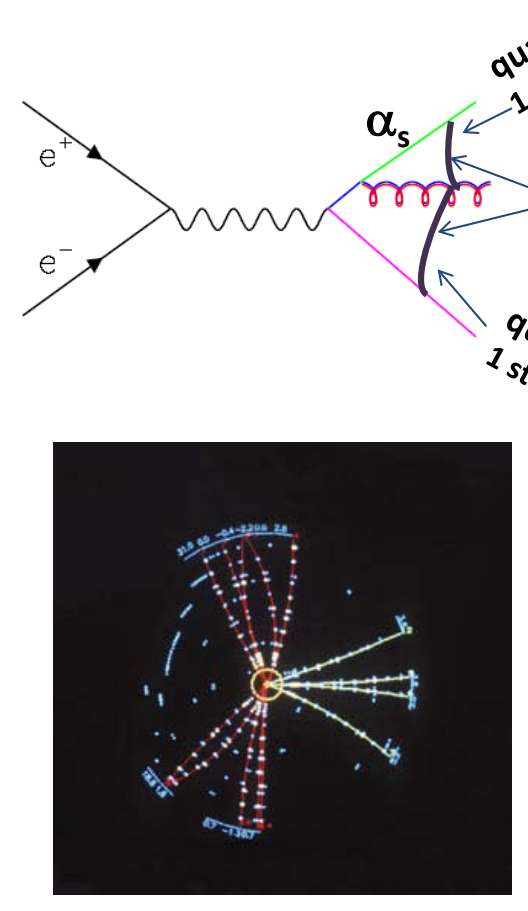

Y.-K. Kim et al., PRL 63, 1772 (1989)

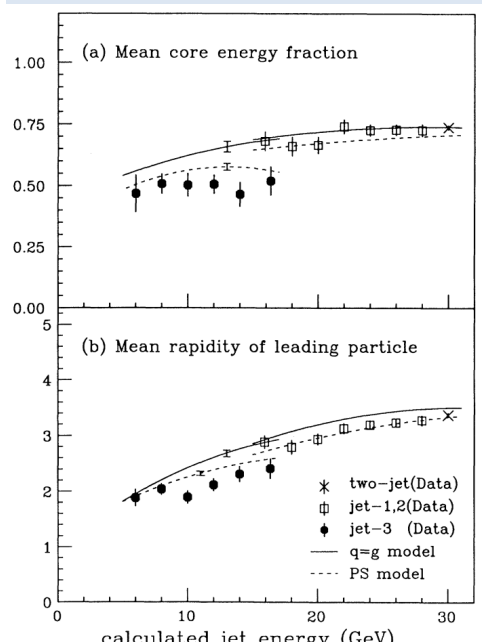

FIG. 1. (a) The mean energy fraction in a cone of half angle $60^{\circ} /\left(E_{\text {ig }}^{\text {eq }}\right)^{1 / 2}$ coaxial with the jet axis, $\xi$, and (b) the mean gle $60 \%$ (Eta) The coaxial wh the jet axis, $\xi$, and (b) the mean rapidity of the leading particle in the jet, $\eta$, as a fun of the calculated jet encrgy, Ekal. The sold poins are for the gluonenriched jet sample, the open points for the quarkentiched sample. The crosses indicale the results from the two-jet events. The solid cures (labeled $q-g$ ) indite the rosults from the $O\left(a_{s}\right)$ matrix-elennent model wS independent fragmentation, the dashed curves (labeld PS) are the results from the parton-shower model with string fragmentation. Error bars are statistical errors only; the error bar centered on each curve indicates the statistical uncertainty of the Monte Carlo simula-

\section{3-gauge particle coupling}

Electro-Weak

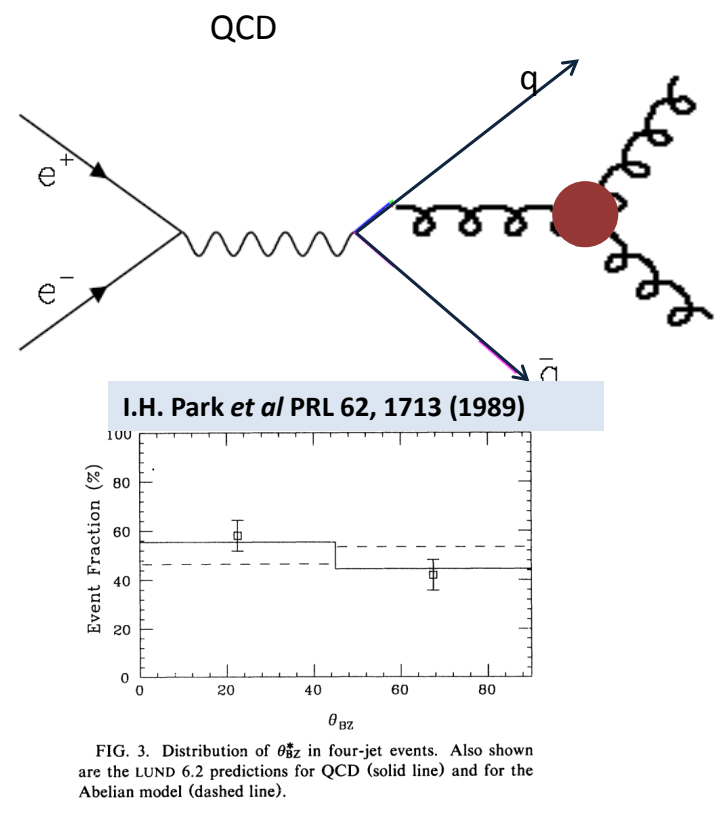




\section{Photon structure function}
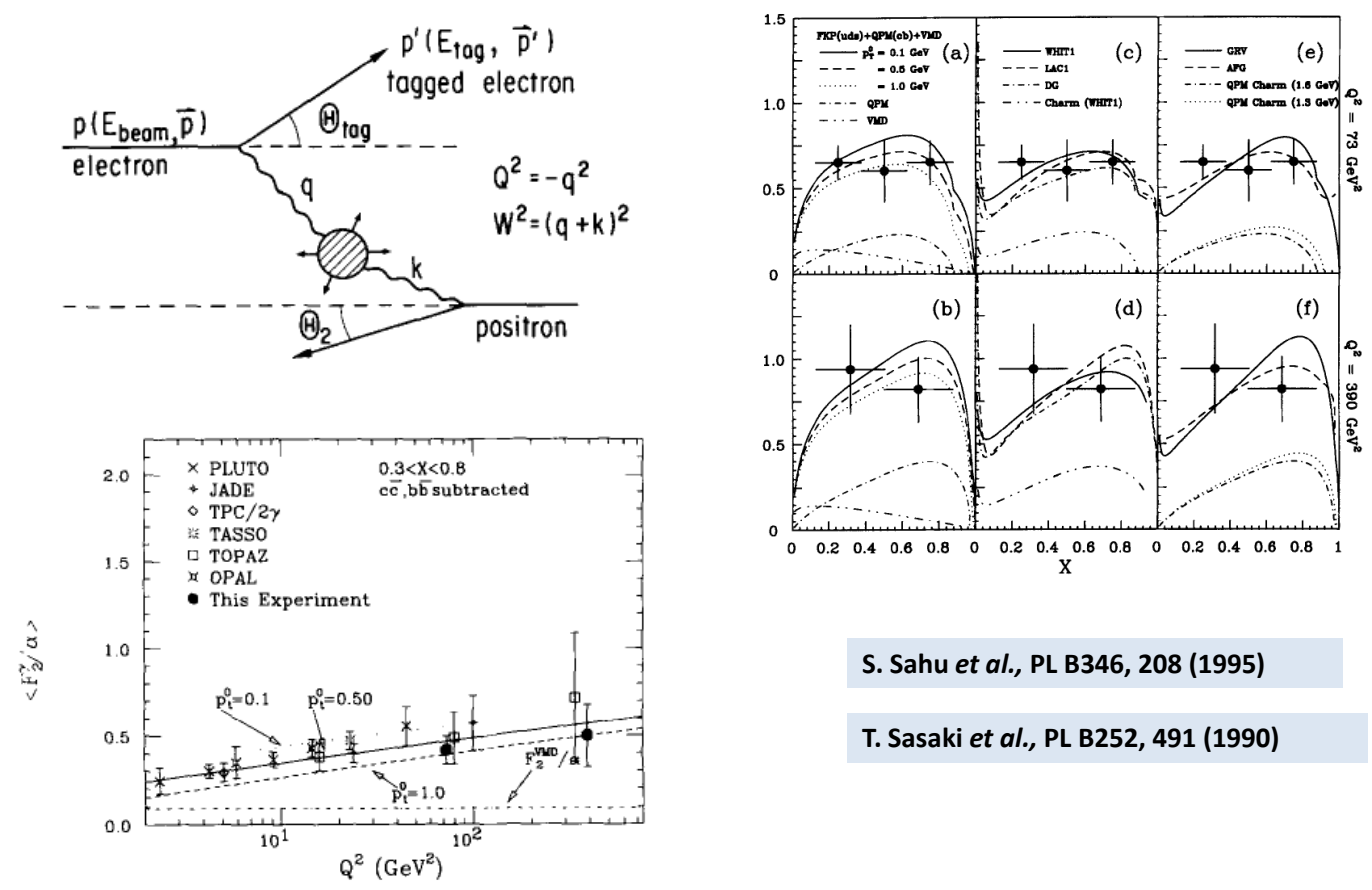

S. Sahu et al., PL B346, 208 (1995)

T. Sasaki et al., PL B252, 491 (1990)

\section{"Resolved" photon processes}
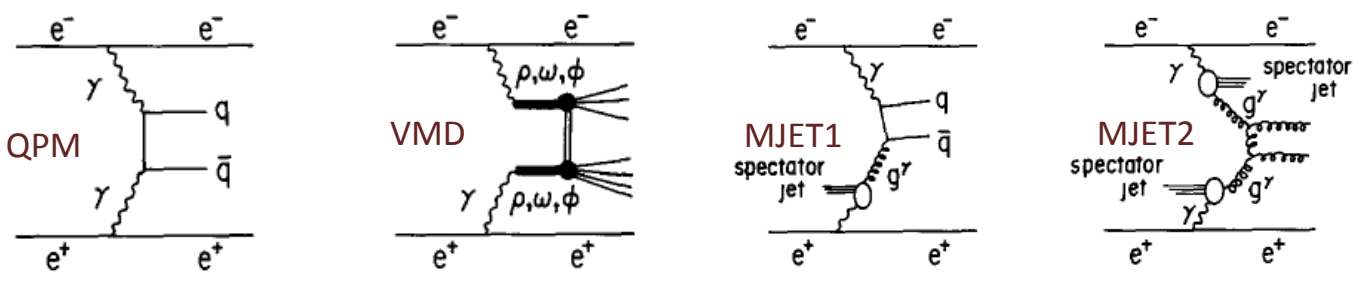

R. Tanaka et al., PL B277, 215 (1992)

QPM+VMD+MJET1\&2

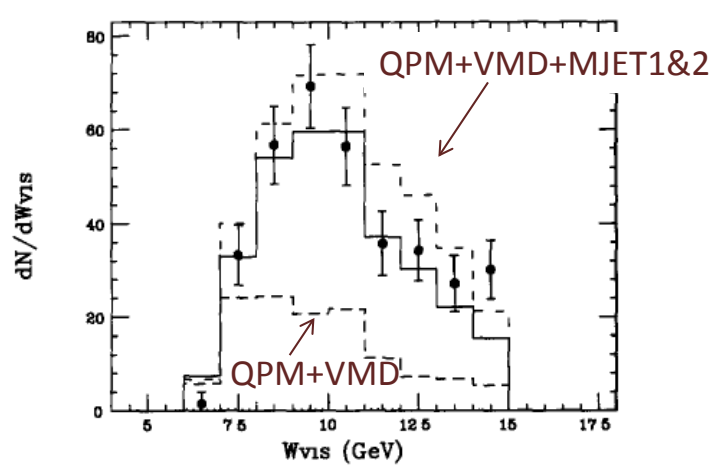
different gluon density params

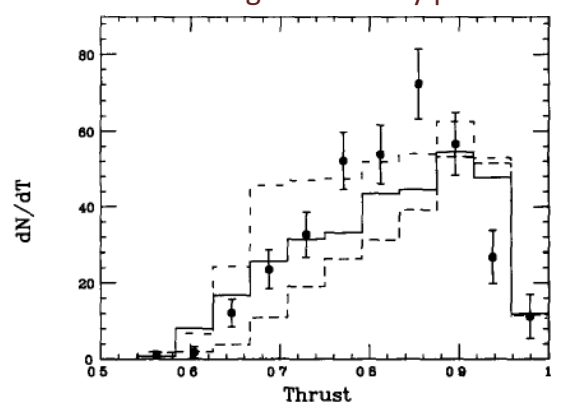





\title{
PHENIX Past, Present, and Future: QCD at large T and $\alpha_{\mathrm{s}}$
}

\author{
B. Jacak, Stony Brook University \\ And \\ K. Ozawa, University of Tokyo
}

Heavy-ion collisions at RHIC provide a way to study the structure and dynamics of a non-abelian system. A major goal of the PHENIX Experiment, achieved thanks to strong Japanese contributions supported by the US-Japan Cooperative Program, is to study QCD at high temperature, and test the duality of QCD-like theories with gravity which are based upon ideas from string theory.

We review some of the remarkable discoveries at RHIC. The hot medium reaches a temperature of 300-600 MeV, clearly putting it into the regime where hadrons melt into quark gluon plasma. The high opacity and nearly perfect collective flow provide striking evidence that this plasma is strongly coupled. Constraining hydrodynamics with data shows that the shear viscosity to entropy ratio is between 0.08 and 0.16 , very near to the quantum-mechanical lower bound of $1 / 4 \pi$, predicted from AdS/CFT correspondence.

Even more surprising is that heavy quarks lose nearly as much energy in the plasma as do gluons and light quarks. This cannot be purely via gluon radiation; collisions also must contribute. PHENIX has just installed a silicon vertex detector to separate B and D decays via displaced decay vertex reconstruction. This will show whether $b$ quarks also are stopped effectively ; such stopping would be a paradigm-changing discovery.

The discovery of strongly coupled quark gluon plasma led to a host of entirely new questions about the formation, properties, and dynamics of this new matter. RHIC offers a superb opportunity to address these questions, with its flexibility, dedicated running for this physics, enhanced luminosity, and sensitivity for probes in the $10-50 \mathrm{GeV}$ range. Jets and quarkonia are of particular interest, and have been key drivers for a major upgrade of PHENIX. We plan adding the first hadron calorimeter at RHIC, as well as low mass tracking, compact calorimetry, and a forward angle upgrade optimized for $\mathrm{d}+\mathrm{Au}, \mathrm{e}+\mathrm{p}$ and e+A physics. The upgrade concept is shown. 


\section{QGP at RHIC flows collectively, is opaque}

- Many particles $\rightarrow$ fast pressure build up

$\rightarrow$ strong collective, hydrodynamic flow interaction $\sigma$ is very large

data constrain hydro $\rightarrow$ fast thermalization

viscosity/entropy $(\eta / s)$ is small

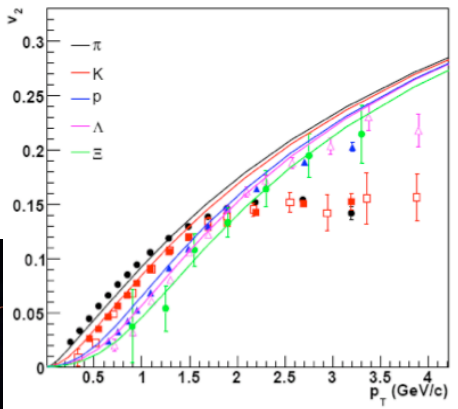

Huge energy loss by fast

partons traversing medium energy \& gluon density large medium is very opaque

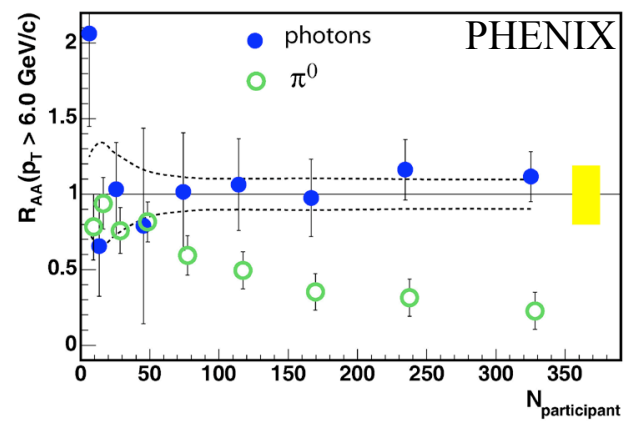

PH F

Quantify viscosity of QGP

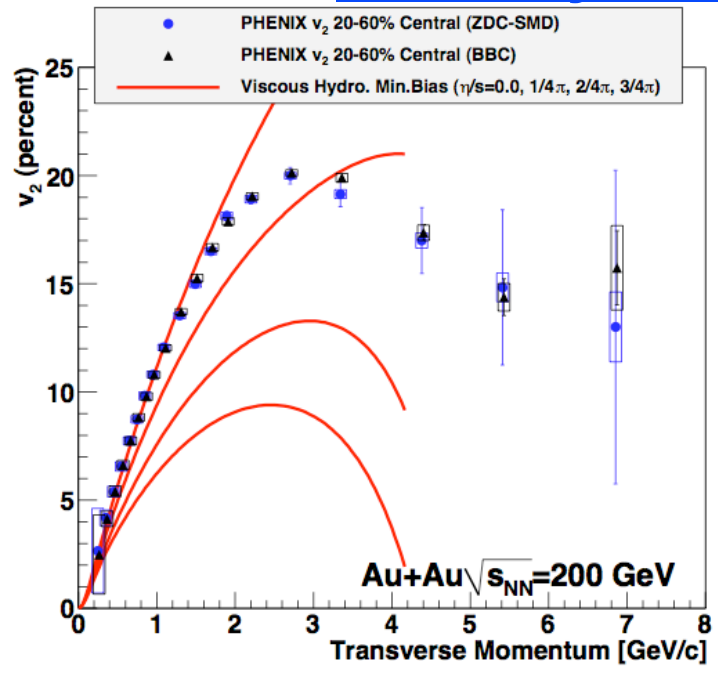

Data require low $\eta / s$ ! near quantum bound $1 / 4 \pi$ from AdS/CFT

$\eta / \mathrm{s}=\mathbf{0} .08-0.16$ from data

- Answer depends on initial condition: saturated gluon gas ("color glass") or NN collisions per Glauber?

At what scales is the coupling strong?

What are the initial conditions? Glauber, CGC? 


\section{QGP temperature? Thermal radiation}
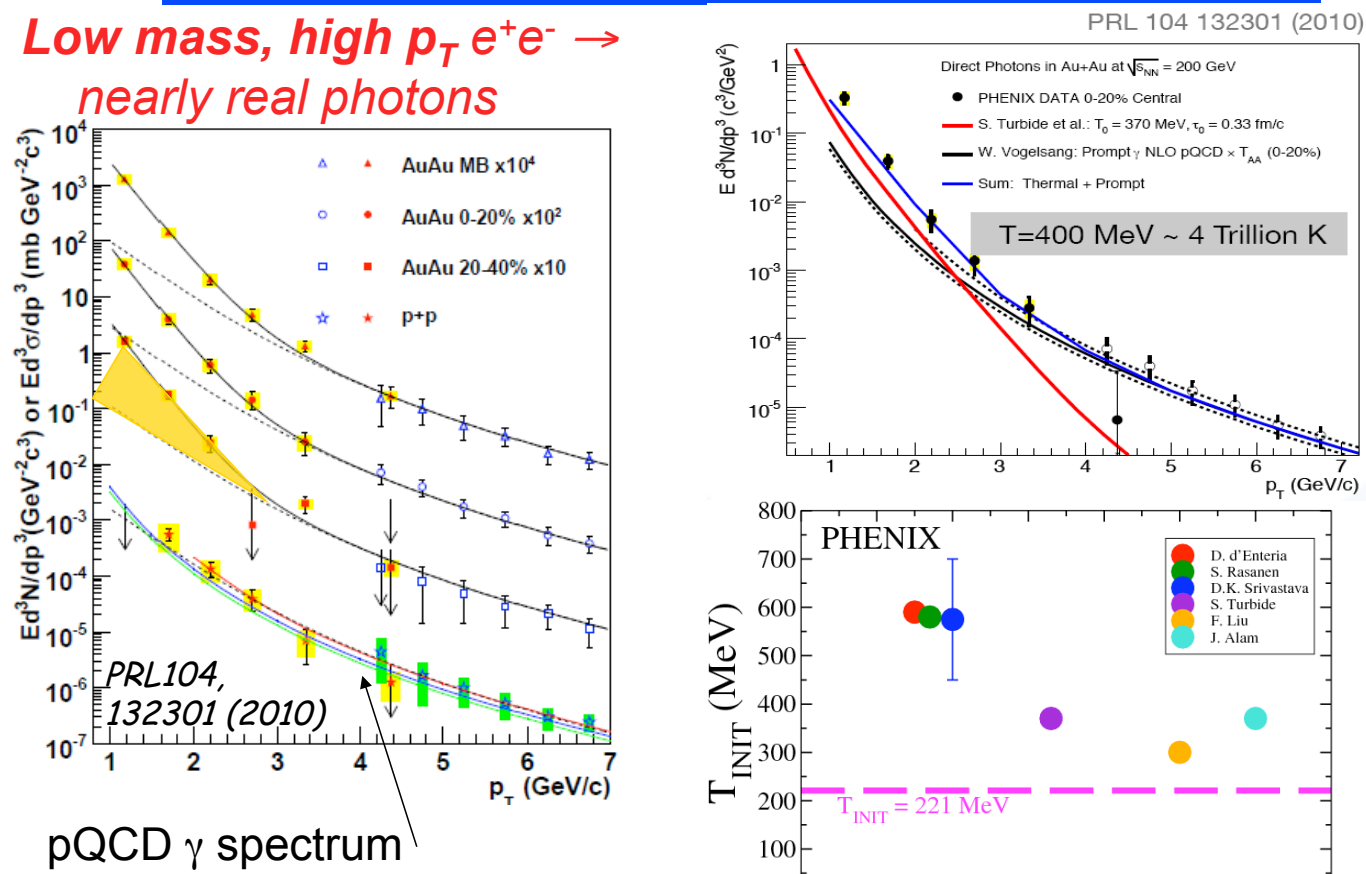

(Compton scattering @ NLO) agrees with $p+p$ data

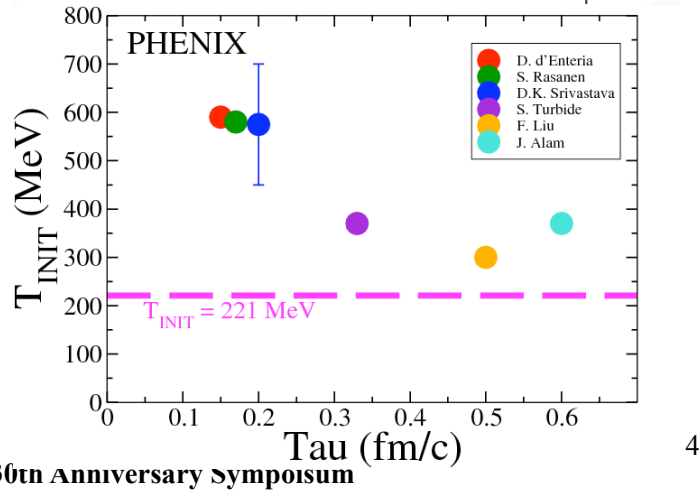

\section{Now we are on the map}

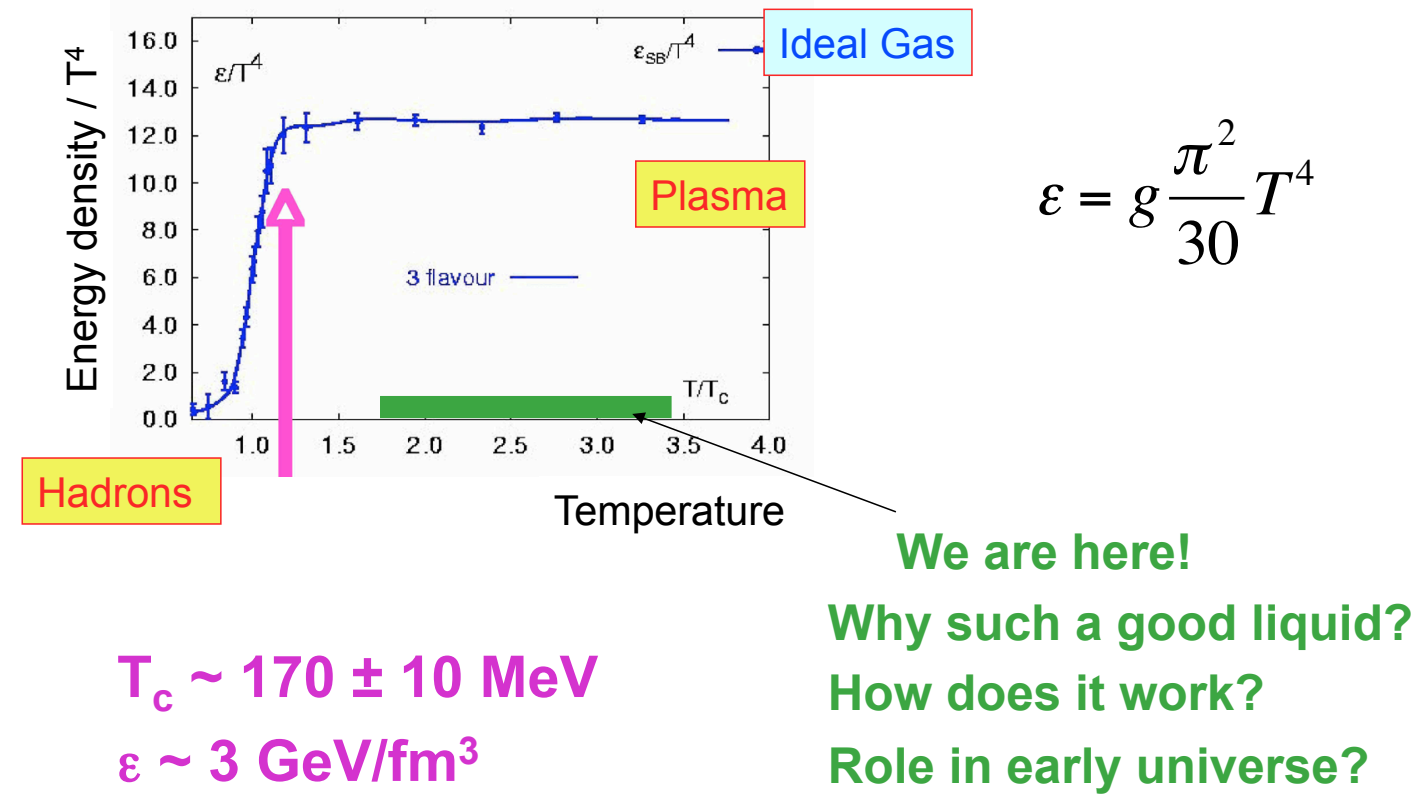

PH党 $\leqslant$ ENXX US/Japan Collaboration 30th Anniversary Sympoisum 


\section{Next Physics in 5 years}

Chiral symmetry restoration

- Measure

- Mass shift or modification is expected.

Current results has poor statistics due to a large background

- New detector for background rejection is already installed and operated in 2010.

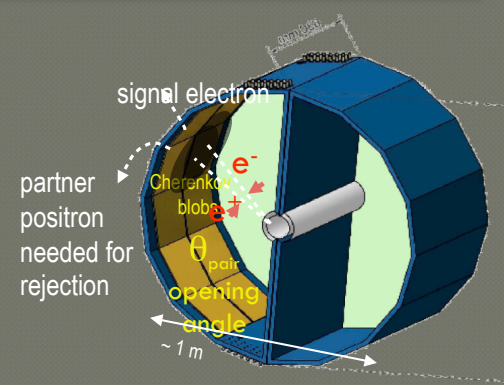

Heavy quarks (charm and beauty)

- Determine $\eta / s$ ratio, which is predictable by Ads/CFT

Current detector can't distinguish charm and beauty

- New vertex detector is being installed.

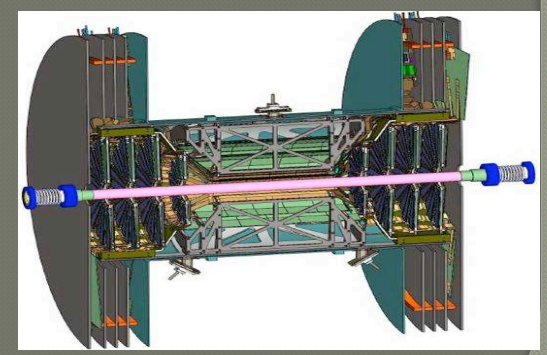

\section{Evidence of chiral symmetry restoration?}

Phys. Rev. C 81, 034911 (2010)

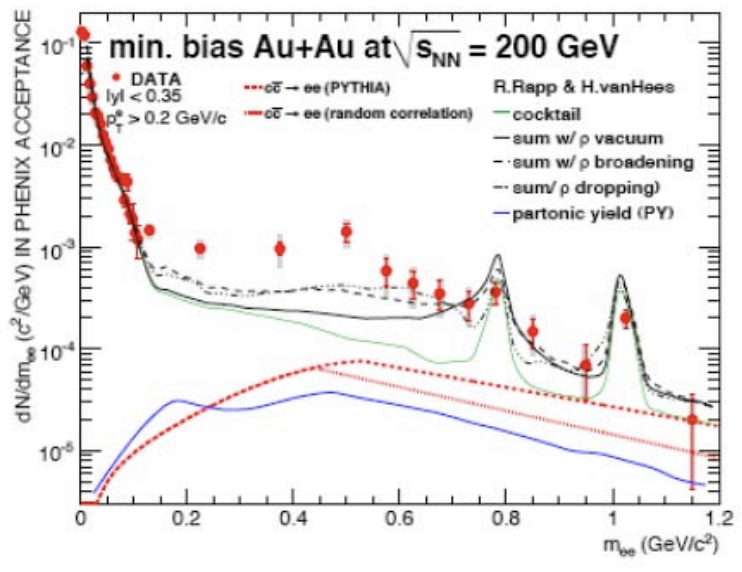

- Evidence for $\rho$ modification

- And low mass, low $p_{T}$ excess

Pre-equilibrium emission?

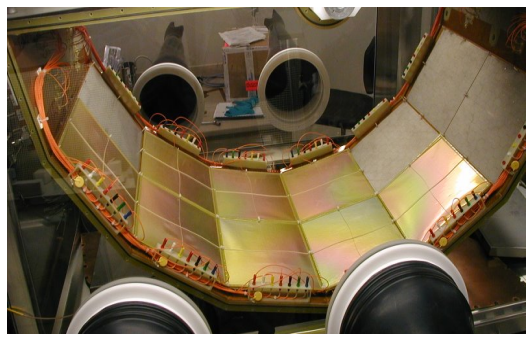

Just completed run with hadron blind detector. Expect $\sim 35$ times better effective statistical precision 


\section{Final Goal beyond 2015}

Understand QCD nature

Key theme: interplay between

perturbative and non-

perturbative physics in QCD
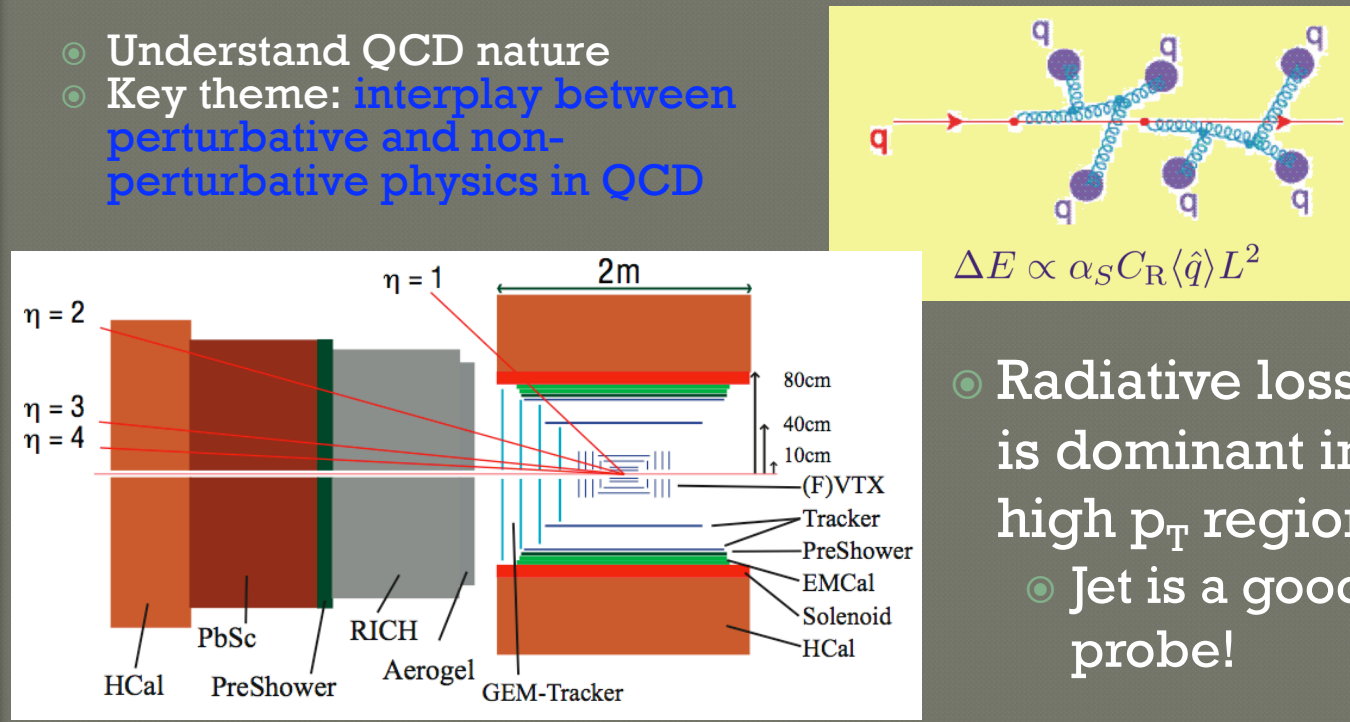

$\Delta E \propto \alpha_{S} C_{\mathrm{R}}\langle\hat{q}\rangle L^{2}$

SPHENIX

\section{Strong Japanese participation, leadership}

11 Institutions

CNS, Hiroshima, JAEA, KEK, Kyoto, Nagasaki IAS, RIKEN, Rikkyo, U. of Tokyo, Tokyo I. Tech, Tsukuba 87 scientists: 37 faculty, 12 postdoc 37 G.S. (1/2 US/J) 35 PHENIX Ph.D.'s in Japan to date

- Continuous Scientific Leadership by US/Japan Program Shoji Nagamiya - founding spokesperson Yasuyuki Akiba - current deputy spokesperson Many Executive Council, Detector Council members

- Detector subsystems RICH, TOF, Beam-Beam, Reaction Plane, Aerogel
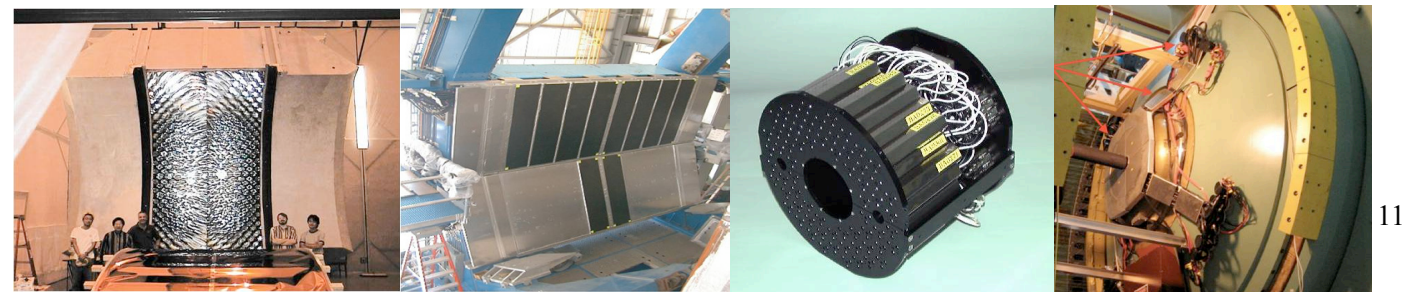



\title{
CDF Experiment at Fermilab
}

\author{
Fumihiko Ukegawa, \\ University of Tsukuba, Tsukuba, Ibaraki 305-8571, Japan
}

The Collider Detector at Fermilab (CDF) experiment at Fermilab's Tevatron proton-antiproton collider is one of the projects that started at the very beginning of the US-Japan collaboration, some thirty years ago. The experiment is still going on, though the detector has undergone vast upgrades while continuing to provide rich physics results through the years, including the discovery of the top quark in 1994. I review some of the achievements of the CDF experiment, concentrating more on those from the present running (Run II). Specifically, I describe the CDF Run-II detector and the B physics results.

\section{$\underline{\text { Some history }}$}

The initial work designing the original CDF detector was done in the late 1970s, and construction followed in the early 80s. By 1985, some of the detector's components were completed, including the superconducting solenoid, the central calorimeters, and the vertex TPC, leading to the observation of first proton-antiproton collisions in the fall of that year. In 1987, after completing the building of the detector, an engineering run took place, generating about $30 \mathrm{nb}^{-1}$ of data, so enabling CDF to perform the first physics measurements, such as the production of $\mathrm{W}$ bosons, QCD jet studies, and minimum bias physics. In 1988-89, CDF enjoyed the first official physics run (now called the Run 0), with a data size as much as $4.4 \mathrm{pb}^{-1}$. Using this data, CDF produced some significant physics measurements in the areas of electroweak physics, QCD, top quark, and exotics, including a (then) precise measurement of the $\mathrm{Z}$ boson mass, and specifying the lower limits on the top quark mass that greatly extended greatly the findings previously obtained at the CERN SPS collider. In addition, CDF demonstrated that its ability to detect B hadron decays, and to play a significant role there. In Run I, h spanning 1992 to 1996, CDF collected a total integrated luminosity of $110 \mathrm{pb}^{-1}$. Meanwhile, the detector continued to evolve, such as by the introduction of a silicon micro-strip detector to a hadron collider experiment, and the addition of extra absorber material and upgraded chambers for muon detection. This data sample produced rich physics results, including the discovery of the top quark $(1994,1995)$, at a mass of about $175 \mathrm{GeV}$. After Run I was completed, the CDF detector underwent a vast upgrade. New detectors replaced almost all of subdetectors inside the solenoid to cope with the anticipated increase in the accelerator's luminosity and a shortening of bunch spacing. The new main tracking chamber (COT), silicon detectors consisting of three subsystems, were installed. Also, the original gas calorimeters in the plug and forward regions were replaced with scintillator-fiber based devices to assure a ast time response. The Run-II experiment started in 2001, and it is still going on. CDF will continue to do produce rich physics results for a few more years, at least. 


\section{CDF Run-II detector}

Figure 1 shows the CDF Run-II detector. The superconducting solenoid, the central calorimeters and muon chambers, remain from the original detector. The tracking chambers were newly built, as were the plug calorimeters. The components contributed by Japanese institutions and physicists are as follows:
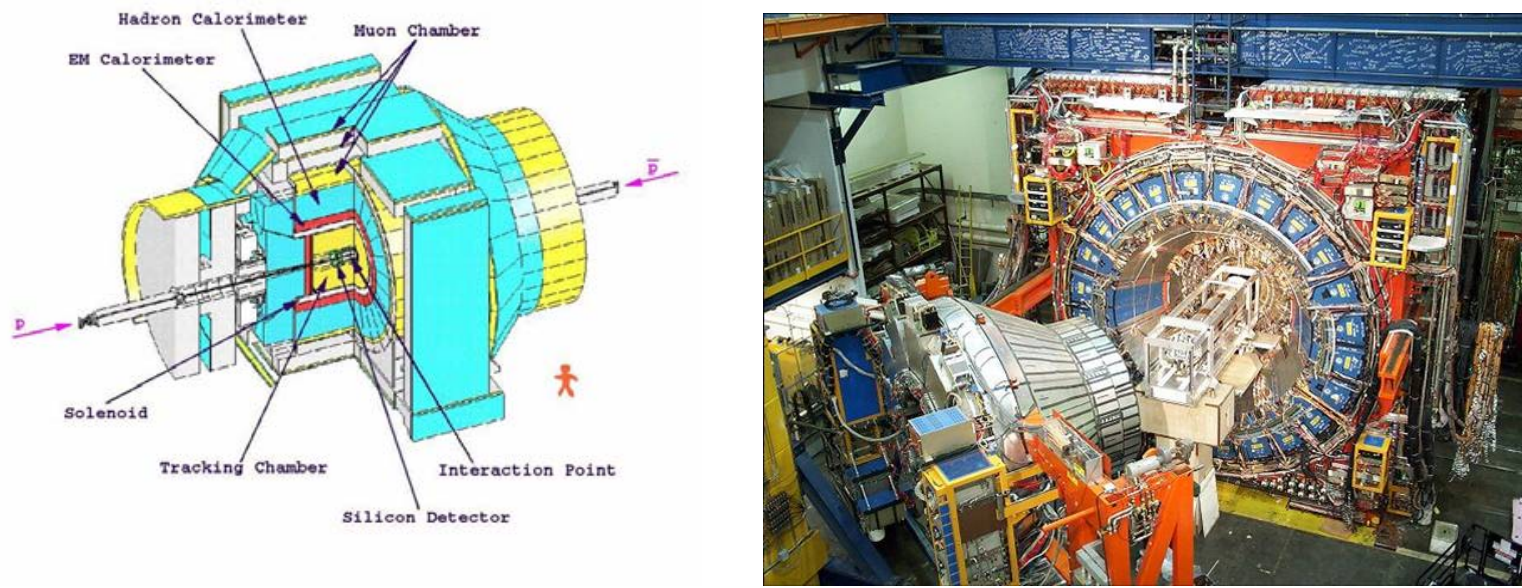

Figure 1: CDF Run-II detector.

- Plug electromagnetic calorimeter and pre-shower detector

The CDF's Run-II plug electromagnetic calorimeter (Figure 2) is a sampling calorimeter consisting of scintillator tiles with imbedded wavelength-shifting (WLS) fibers, through which light is read out with photomultiplier tubes (PMTs). It has a fast time-response while maintaining fine lateral segmentation with projected tower geometry. It also has excellent energy resolution, linearity, and uniformity with small inactive regions. The scintillators, WLS fibers, and PMTs (Hamamatsu R4125G) came from Japan. Physicists from Tsukuba University and Waseda University contributed to the detector's design, construction, cosmic-ray- and beam- tests, commissioning, and operations. .
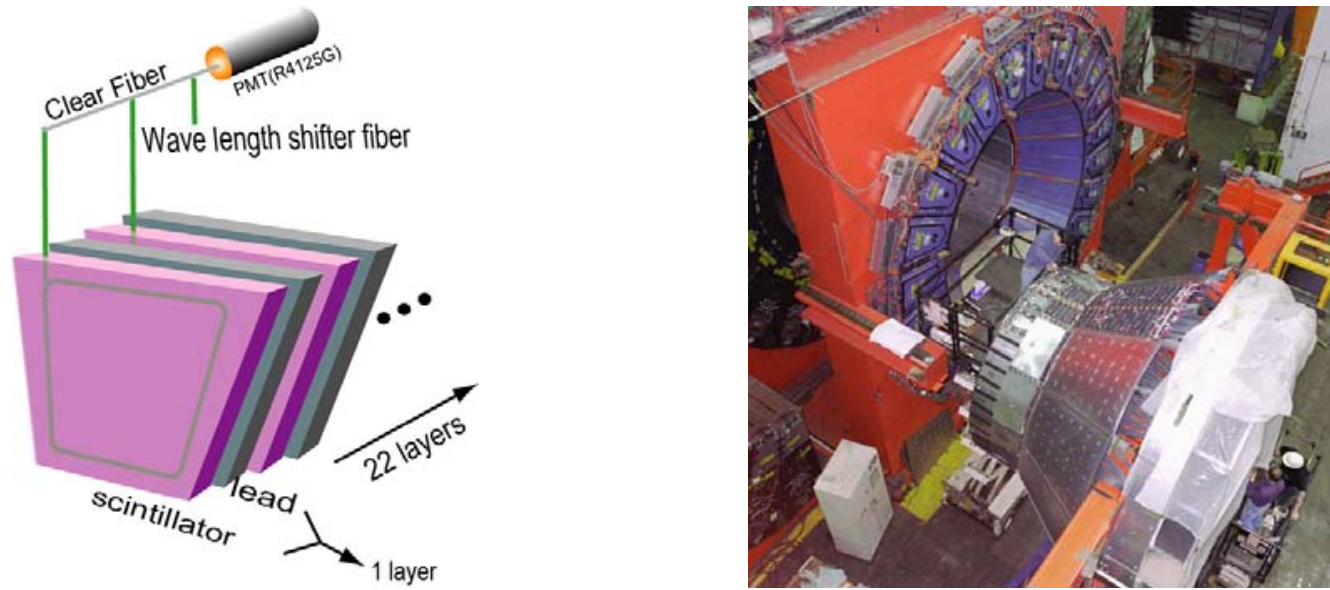

Figure 2: CDF Run-II plug electromagnetic calorimeter. 
- Silicon vertex detector (SVX-II), Intermediate Silicon Layers (ISL)

Silicon detectors play crucial roles for top quark- and B-physics; through precision tracking they identify and reconstruct secondary vertices. The basic design of the CDF Run-II silicon detectors employs double-sided silicon sensors to provide precise, three-dimensional position measurements of charged particles. The SVX-II detector (Figure 3 left) has five layers of sensors, of which layers 1, 2, and 4 were constructed in Japan by Hamamatsu Photonics, and are tested by physicists from Hiroshima- and Okayama- Universities. The ISL (Figure 3 right) has a similar construction to the SVX, but with three layers, the purpose of which is providing additional position measurements, in particular for tracks in the plug region. Hamaamtsu produced about $50 \%$ of the sensors. Physicists from the University of Tsukuba and Osaka City University have contributed. The sensors for the SVX-II and ISL subsequently were sent to FNAL for assembly and installation.
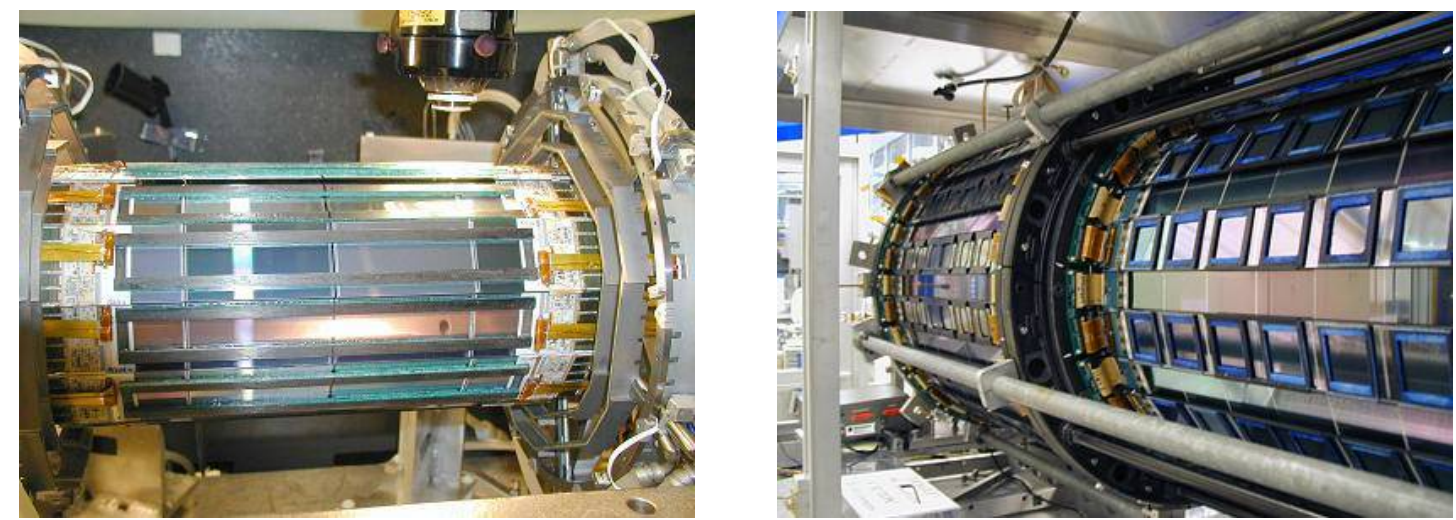

Figure 3: SVX-II (left) and ISL (right) silicon detectors for CDF Run II experiment.

- Time-of-Flight (TOF) counter

The TOF detector was later addition to the CDF Run-II upgrade, along with L00 silicon detector. The TOF's purpose is to enhance B physics capabilities with particle identification. It is sited on a radius of about $1.4 \mathrm{~m}$ from the bema line, and, with a resolution of about $100 \mathrm{ps}$, it provides a pion-kaon separation of more than 2-sigma significance for momenta up to 1.4 $\mathrm{GeV} / \mathrm{c}$. The scintillators and PMTs are located inside the 1.4-Tesla solenoid, so PMTs with a fine-mesh dynode structure (Hamamatsu R7761) are employed. They were constructed by Hamamatsu, and tested in a magnetic field by Tsukuba physicists before being sent to FNAL.

- Central pre-shower detector (CPR2)

The CPR detector identifies electrons and photons by detecting early portions of electromagnetic showers in front of the calorimeter. The CPR2 replaced the original CPR, a gas detector constructed for Run I. The CPR2 consists of scintillating tiles with a WLS fiber readout; it uses Hamamatsu H8711 multi-anode PMTs with 16 readout channels that were tested at Tsukuba, and then sent to FNAL for installation.

In addition adding in the construction of these detectors, collaborators from Japanese institutions participated in commissioning and operating them, and the experiment as a whole, including setting up the data-acquisition system, online monitoring, and the silicon vertex trigger (SVT). 


\section{$\underline{\text { CDF B physics }}$}

As I noted earlier, CDF B physics began in the "Run-0" period of the experiment, when some of the $\mathrm{B}$ hadron decays were reconstructed using samples of single electrons and di-muons. Initial measurements concerned the production of bottom quarks in hadron collisions. In Run I, a silicon vertex detector was added to $\mathrm{CDF}$, enabling the precise reconstruction if secondary vertexes. Researchers at the CDF published many significant measurements, including precise ones of individual $\mathrm{B}$ hadron lifetimes, and $\mathrm{B}_{\mathrm{d}}$ meson particle-antiparticle oscillations; they reported the discovery of the $B_{c}$ meson, by fully reconstructing $B$ decays using final states involving $J / \psi$, and by partially reconstructing semi-leptonic decays using correlated lepton-charm pairs. In Run II, the CDF's B physics capability is enhanced even more by adding the silicon vertex trigger (SVT). The SVT uses information from the silicon detectors on-line, and collects events with charged particles originating at secondary vertices. It enabled CDF' scientists to collect and reconstruct B hadron decays in a very inclusive way, including those with no leptons in the final states. An example is the $\mathrm{B}_{\mathrm{s}} \rightarrow \mathrm{D}_{\mathrm{s}}^{+}$pi mode that is used for measuring $\mathrm{B}_{\mathrm{s}}$ oscillations (Figure 4). A measurement of the particle-antiparticle oscillation frequency, $\Delta \mathrm{m}_{\mathrm{s}}$, used to determine the elements of the CabibboKobayashi-Maskawa matrix with small theoretical uncertainty, provided a stringent test of the Kobayashi-Maskawa theory of CP violation in weak interactions.
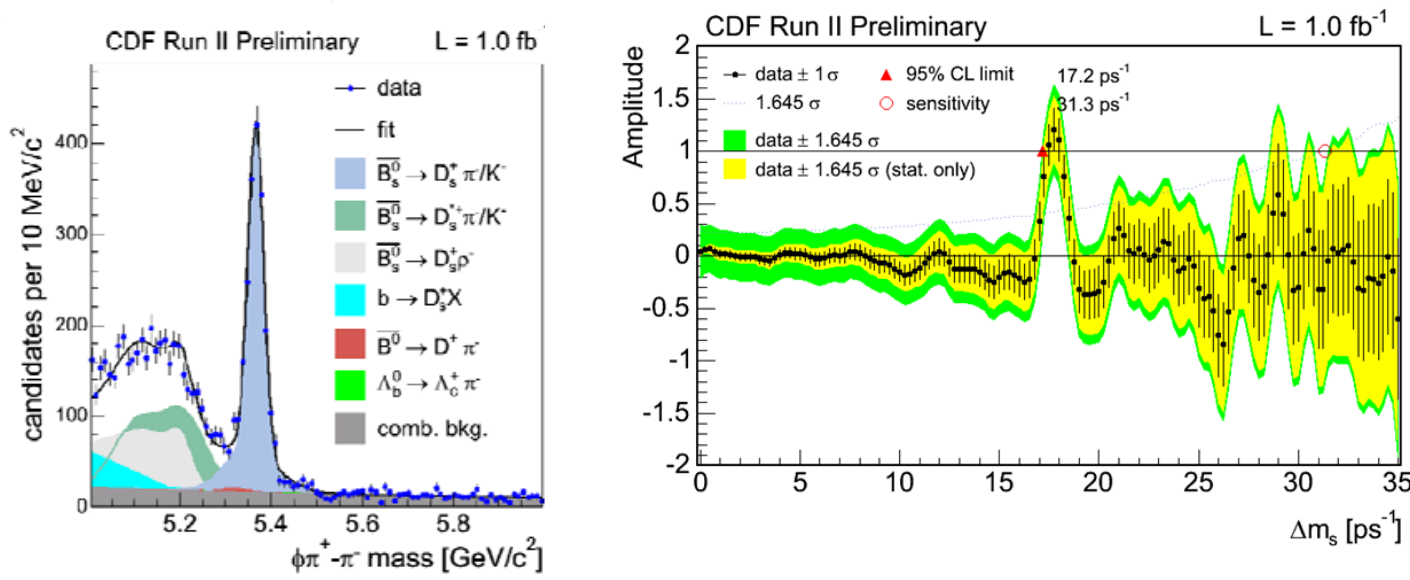

Figure 4: Signal for $\mathrm{B}_{\mathrm{s}} \rightarrow \mathrm{D}_{\mathrm{s}}^{+}$pi $^{-}$(left), and $\mathrm{B}_{\mathrm{s}}$ particle-antiparticle oscillation measurement (right).

Other recent $\mathrm{B}$ physics studies at $\mathrm{CDF}$ include the search for CP-violating phase in Bs meson mixing using the $\mathrm{J} / \psi \phi$ final state, and explorations of rare decays proceeding with the flavorchanging neutral current process $b \rightarrow s$. An example of the latter is a study of the decay mode $B_{d}$ $\rightarrow \mathrm{K}^{* 0} \mu^{+} \mu^{-}$(Figure 5), an interesting mode because of a possible hint of the new physics reported by the Belle experiment. 

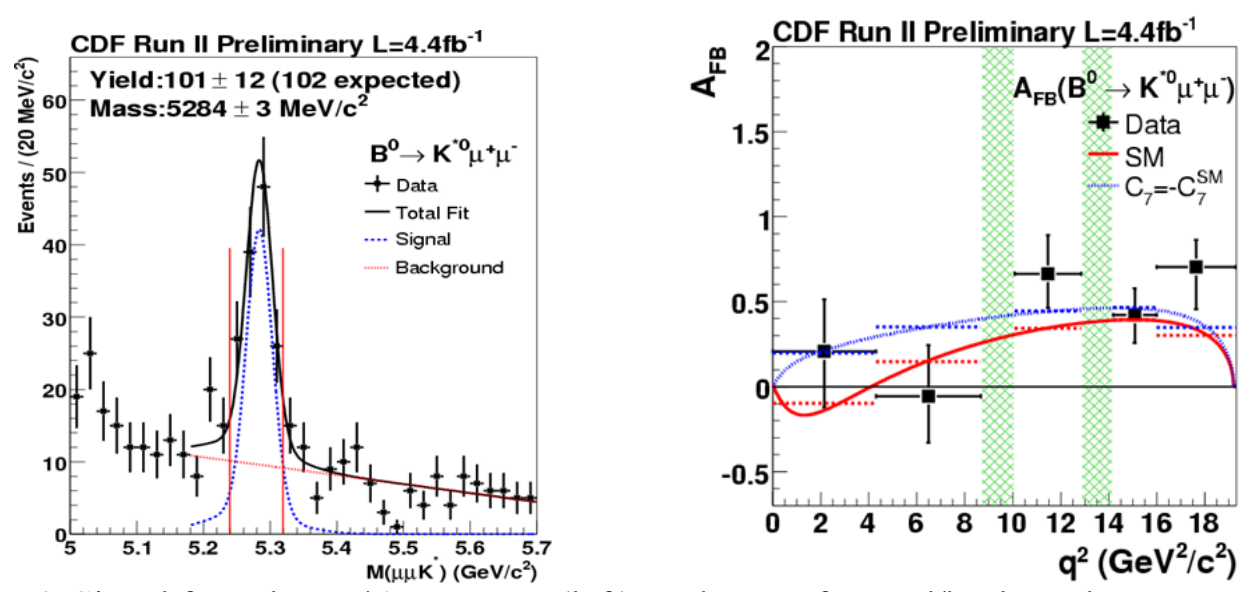

Figure 4: Signal for $\mathrm{Bd}->\mathrm{K}^{*} 0 \mathrm{mu}+\mathrm{mu}-$ (left), and muon forward/backward asymmetry (right).

These are only a few examples of CDF B physics findings; some results still limited statistically, and will benefit greatly from more data. Beyond those, we certainly are looking forward to a fruitful fourth decade of the US-Japan collaboration, and the CDF experiment. 



\title{
Exciting High Pt Physics Results and Opportunities in Run II
}

\author{
Robert Roser, Fermilab, Batavia, IL
}

The most important issue facing modern High Energy Physics is the origin of electroweak symmetry breaking and associated new physics at the electroweak scale. Other issues, such as "the origin of flavor", "the origin of mass patterns and mixing angles", "dark matter and dark energy", "is string theory the theory of everything?" are contingent on how electroweak symmetry breaking occurs. We cannot ascertain what the scientific handles on these latter problems are until we understand whether nature deploys an elementary Higgs boson, whether nature is super-symmetric, whether there are new strong forces, etc. Our entire philosophy of nature turns on the unknown physics at the electroweak scale. Moreover, the Standard Model itself points to a low mass for the Higgs boson, the simplest hypothetical agent of the origin of mass. Standard Model fits constrain the Higgs boson to the mass range $114 \mathrm{GeV}<\mathrm{MH}<145$ $\mathrm{GeV}$ at $95 \%$ C.L.

We are only beginning, with the Fermilab Tevatron, to reveal this layer of nature. It is important to realize that, while the Tevatron has the mass reach of the electroweak scale, it is now arriving at the required integrated luminosities to discover new objects produced by the electroweak scale interactions, such as the Higgs boson. This program is running spectacularly well and is beginning to probe possible realms of new physics.

The Tevatron collider has provided about $8 \mathrm{fb}-1$ per experiment of analyzable data to date, and is running extremely well. The Tevatron experiments are just now reaching the threshold of sensitivity to electroweak symmetry breaking effects. The low mass qq $\rightarrow \mathrm{W} / \mathrm{Z}+(\mathrm{H} \rightarrow \mathrm{bb})$ sensitivity is such that it is finally beginning to experimentally probe the Standard Model predictions for the Higgs boson. There is also an assortment of tantalizing excesses seen in various other search channels. These may be the first hints of new physics, expected to be revealed at the electroweak scale. The next few year's present opportunities for the Fermilab Tevatron to continue providing physics results at the frontier of knowledge, from its systematic multichannel program addressing the search for the Higgs as well as a variety of searches and other precision measurements in the Standard Model.

The Japanese physicists on CDF have played a driving role in this - picking physics topics such as the measurement of the top mass, discovery of single top quark production and the search for the Higgs Boson in the WH channel that were both important and appropriate for the data-set available at that time. The Tevatron will continue to hunt for the Higgs Boson and the Japanese physicists will play an important role in the collection, processing and analysis of this impressive dataset. 


\section{Japanese Run-Il physics analyses (grad students, * = postdocs+junior faculty)}

Higgs, SUSY, new particles

Higgs searches

Osaka City: Yamamoto*, Wakisaka, Hamaguchi

Tsukuba: Kobayashi, Ishizawa, Masubuchi, Nagai, Sudo, Kurata*

Waseda: Kusakabe, Ebina, Yorita*

SUSY, Leptoquarks, Z'searches

Top quark

Waseda: Ogawa, Ikado Tsukuba: Akimoto

Top mass Waseda: Yorita, Ebina, Tuchiya Tsukuba: $\underline{\text { Sato, Kubo, Tomura* }}$

Production and decay, spin correlations, single top

$B$ physics

Tsukuba: Takeuchi*, Kimura, Nakamura

Waseda: Naganoma

Bs, Bc lifetimes Tsukuba: Uozumi, Aoki

$J / \psi$ production Okayama: Yamashita

Rare $B$ decays Tsukuba: Naganoma*, Miyake*

W+photon production Okayama: Tanimoto Tsukuba: Nagano

W+jet production Tsukuba: Tsuno

Underlined: students who finished PhD's

Single Top Discovery!

\section{Optimized for s-channel}

- Multivariate Likelihood Function

- 7 input variables (kinematics,

kinematic solver and ANN b-tag outputs)

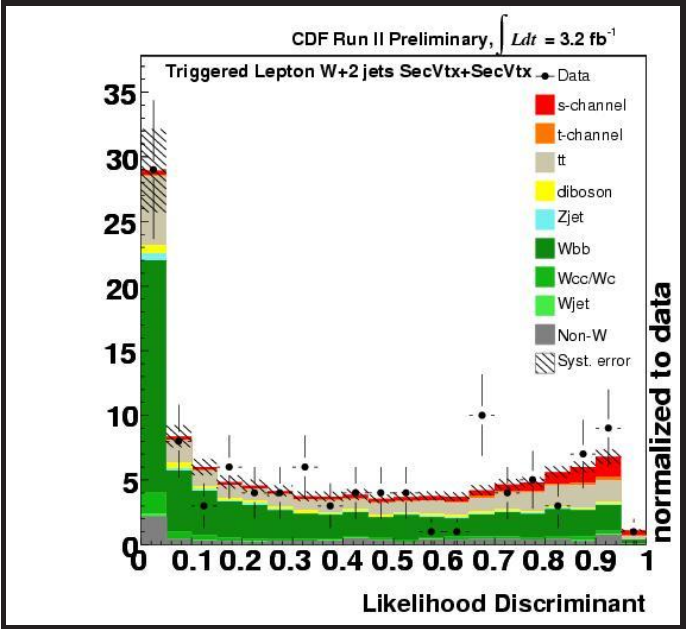

Koji Nakamura, PhD thesis (U of Tsukuba)

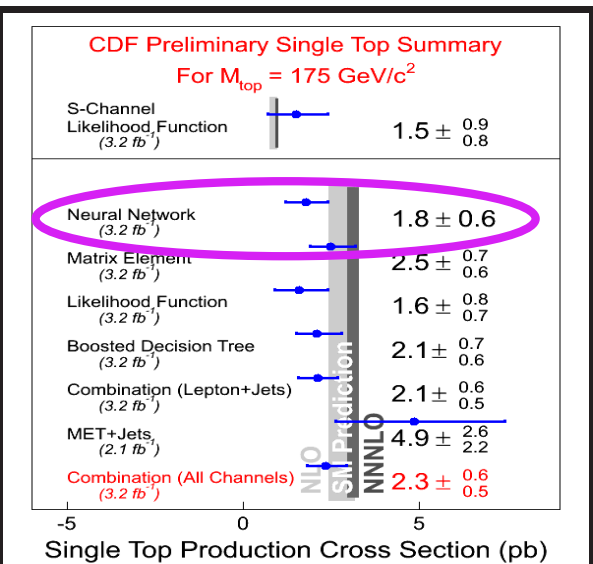

Single Top Production Cross Section (pb)

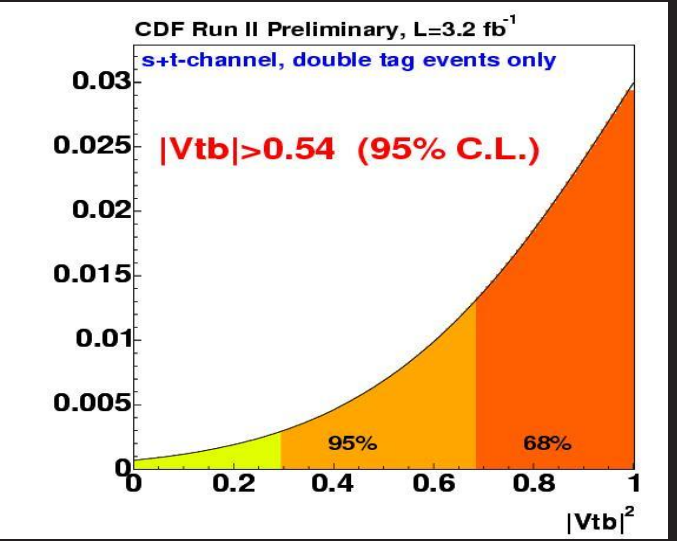




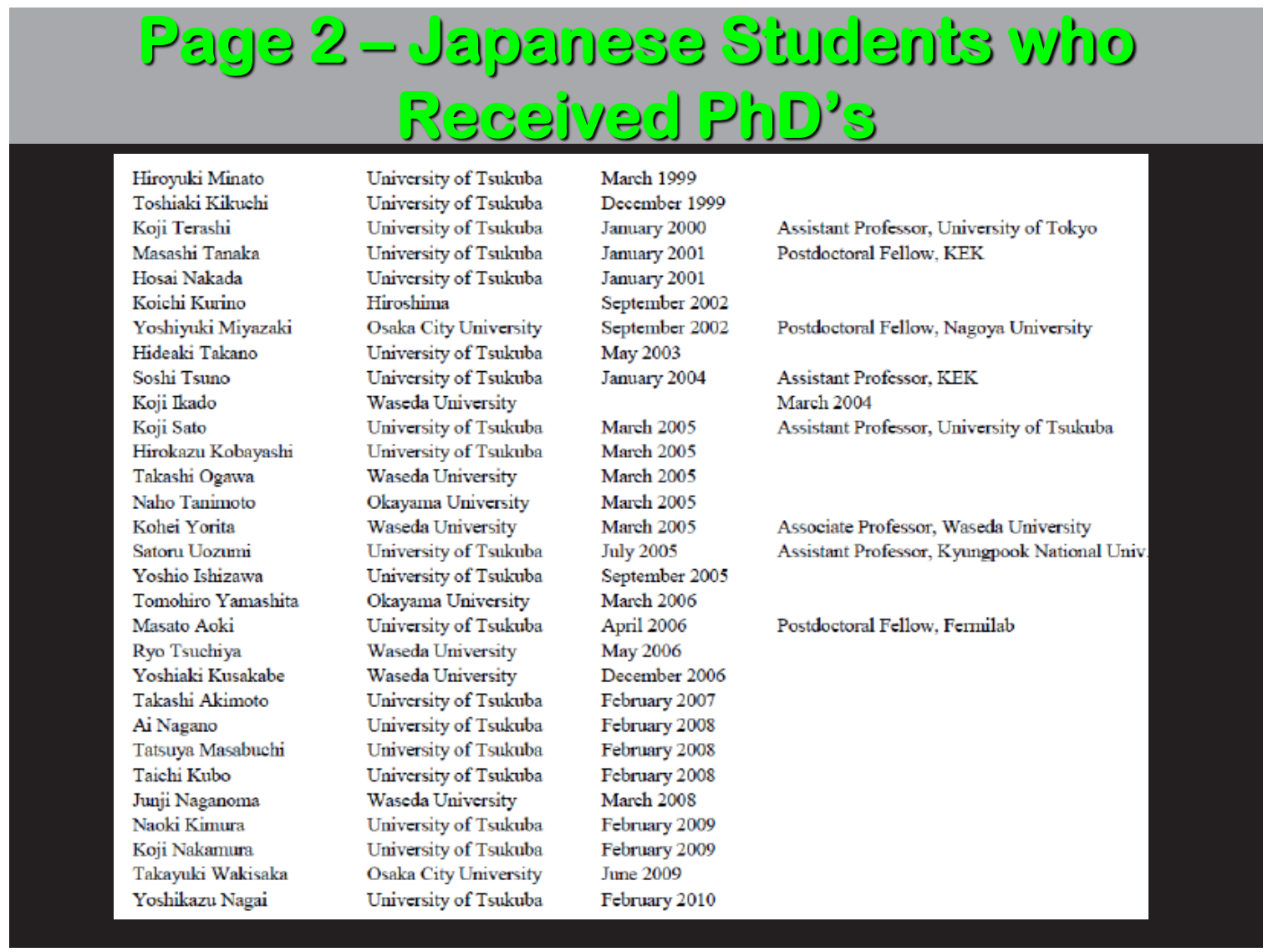

\section{Top mass measurement examples}

Run II, $162 \mathrm{pb}^{-1}$
Lepton + jet channel

\section{Template method}

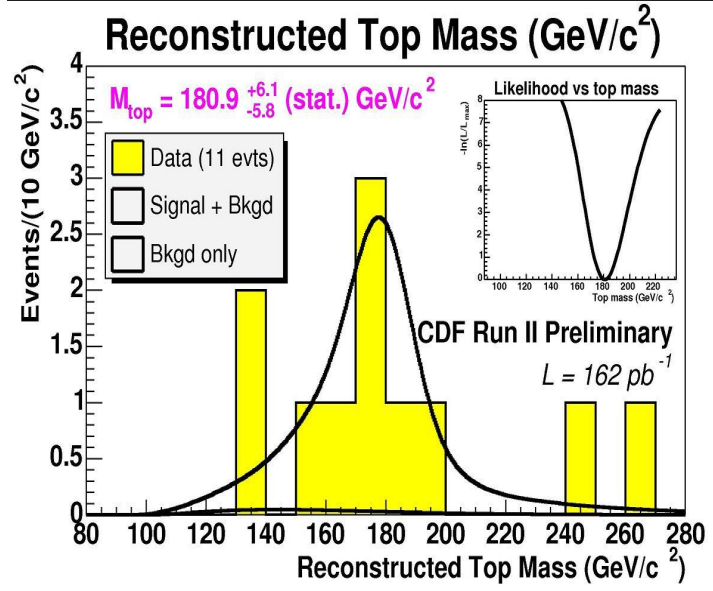

Dynamical likelihood method (K. Kondo, J.Phys.Soc.Jap. 프, 4126,1988)

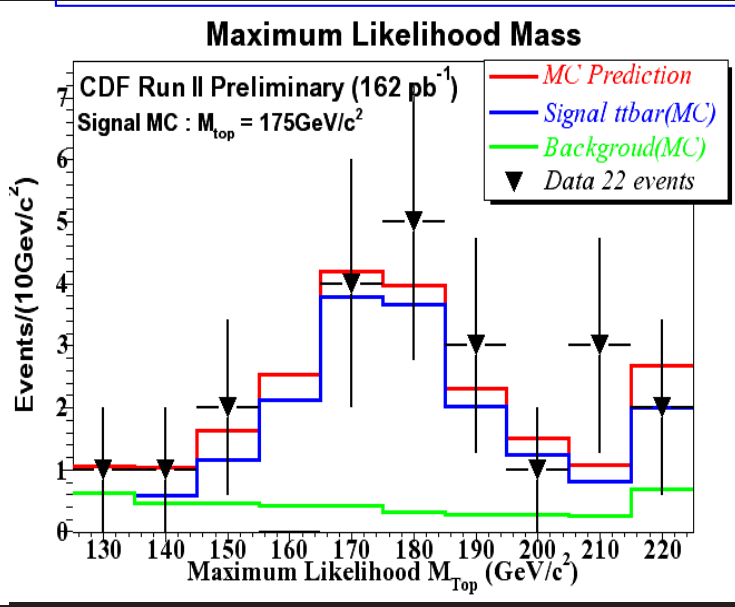

$$
m_{t}=177.8_{-5.0}^{+4.5} \pm 6.2 \mathrm{GeV} / c^{2}
$$

Kohei Yorita, PhD thesis, Waseda, 2005

Koji Sato, PhD thesis, Tsukuba, 2005

Also on top mass : Ryo Tsuchiya (Waseda, 2006), Taichi Kubo (Tsukuba, 2008) 

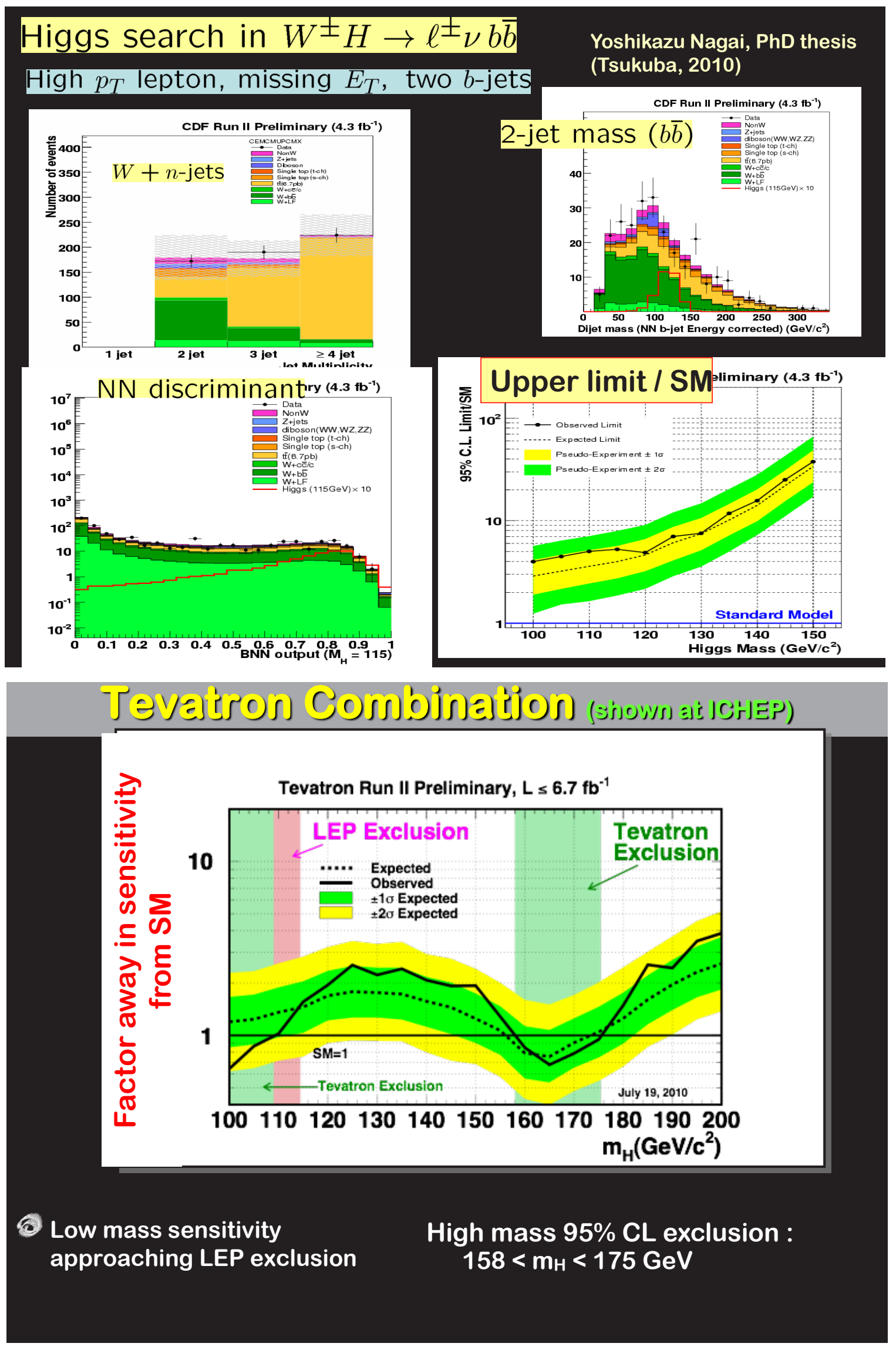


\section{Prospects for fljiggs Evidence}

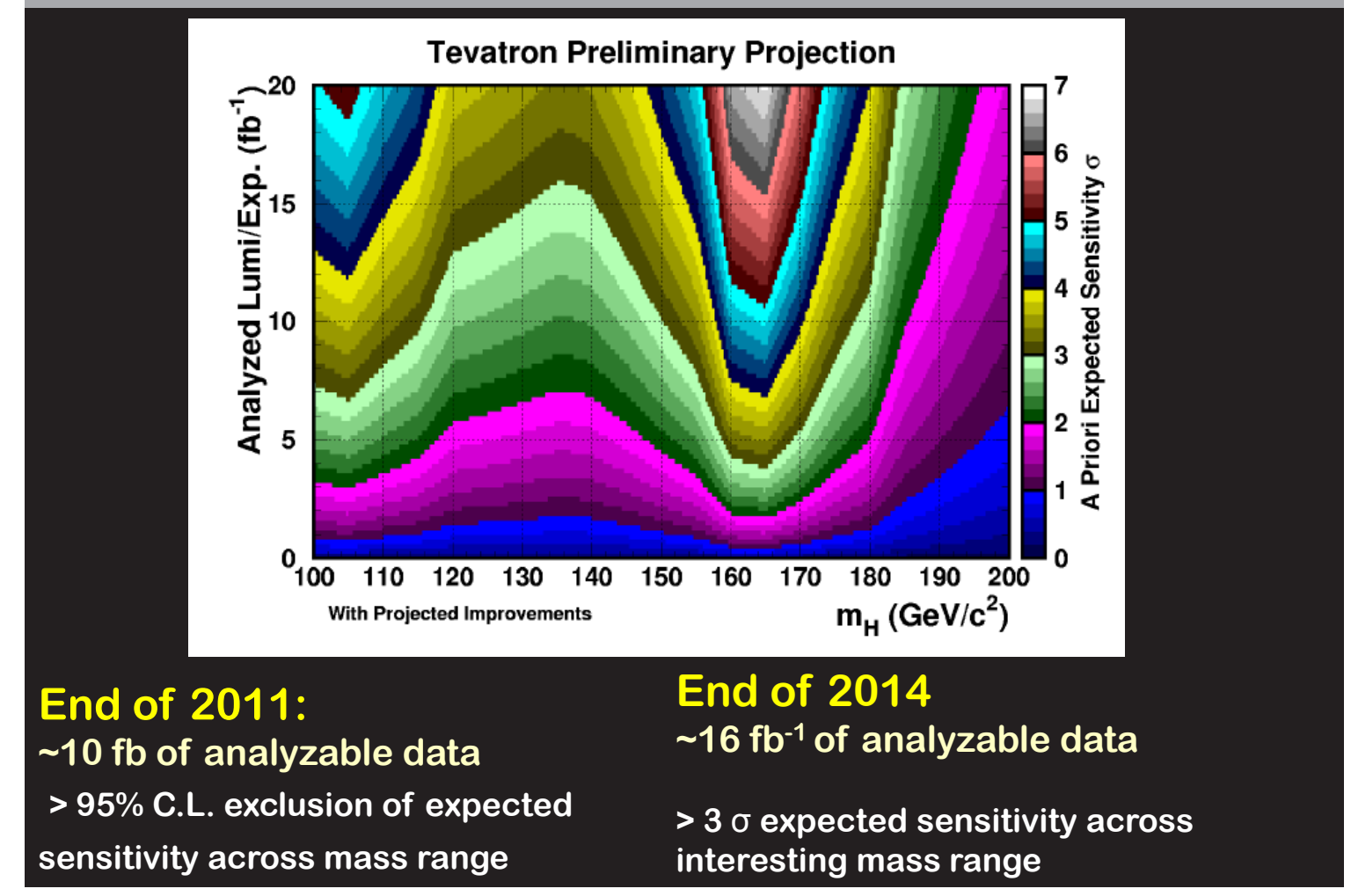





\title{
Fermi Large Area Telescope
}

\author{
Tsuneyoshi (Tune) Kamae
}

On Behalf of the participants in the US-Japan Fermi program

SLAC/KIPAC, Stanford University (formerly with Univ. of Tokyo)

Participating Japanese institutions: Hiroshima U., Tokyo Inst. of Tech, ISAS/JAXA, Nagoya U., U. of Tokyo, and Kyoto U.

\section{History of the program}

1995: GLAST - Large Area Telescope (LAT) coll. formed including Univ. of Tokyo.

1997: GLAST-LAT proposal to US-Japan approved: Japan took responsibility to design and supply the silicon strip detectors (Fig.1).

$\square$ 2001: The LAT coll. won the NASA Announcement of Opportunity.

- 2006: The LAT assembled and tested at SLAC, tested at Naval Research Lab.

June 11, 2008: The GLAST Observatory was launched into a Low Earth Orbit (Fig.2).

a August 2008: The mission was named after Enrico Fermi after a month-long verification phase.

August 4, 2008: Science operation started.

March 2010: Found 1400 point-like sources detected with $>4$ s significance (Fig.3).

口 October 2010: Surveyed the entire sky over $120 \mathrm{k}$ orbits and recorded $3.5 \times 10^{\wedge} 8 \gamma$-rays; the coll. has published 108 papers in refereed journals and collected about 3000 citations.

\section{Highlight of scientific results with a focus on particle astrophysics topics}

$\square$ Gamma-ray signals from Dark Matter (DM) annihilation and decay: many upper limits have been set including the one by line gamma-ray search (Fig.4).

$\square$ Gamma-ray and cosmic-ray lepton spectra measured by Fermi and PAMELA limit contribution of DM annihilation/decay to positron (Fig.5).

- Test on Lorentz invariance using a Gamma-Ray Burst exclude a quantum gravity model (Fig.6).

$\square$ Studies on particle accelerators in middle-aged supernova remnants and a AGN flare make important discoveries (Fig.7, 8).

$\square$ Fermi observation of near-by AGNs constraint the particle composition of UHECRs (Fig.9).

\section{Future prospects}

Fermi coll. is searching for particle DM in the Galactic Center and improving limits in other part of sky. We will keep setting the best limit for a range of spin-dependent interaction as well as excluding important parameter space of particle DM models (Fig.10).

$\square$ Fermi coll. has not yet fully explored the temporal domain of the sky survey. We expect to make interesting discoveries in this domain soon.

Many other studies relevant to particle physics are in progress and will be published soon. 

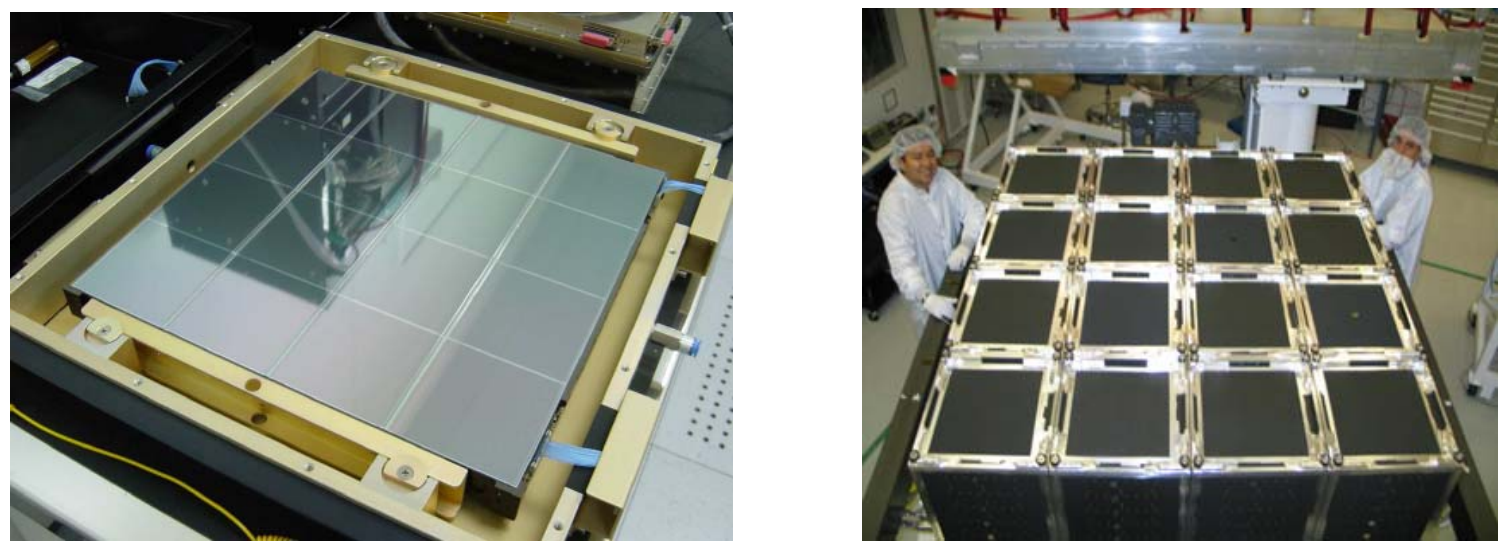

Fig.1: The GLAST-LAT silicon strip detectors (SSD) were designed and manufactured on the US-Japan. One Tracker plane consisting of 16 SSDs $\left(8.9 \times 8.9 \mathrm{~cm}^{\wedge} 2\right.$ each) is shown on the left panel. The 36 planes were assembled to one Tracker Tower in Pisa, Italy and shipped to SLAC. The 16 Towers were then integrated with the read-out electronics and the 16 Calorimeters at SLAC in 2006 as shown in the right panel.

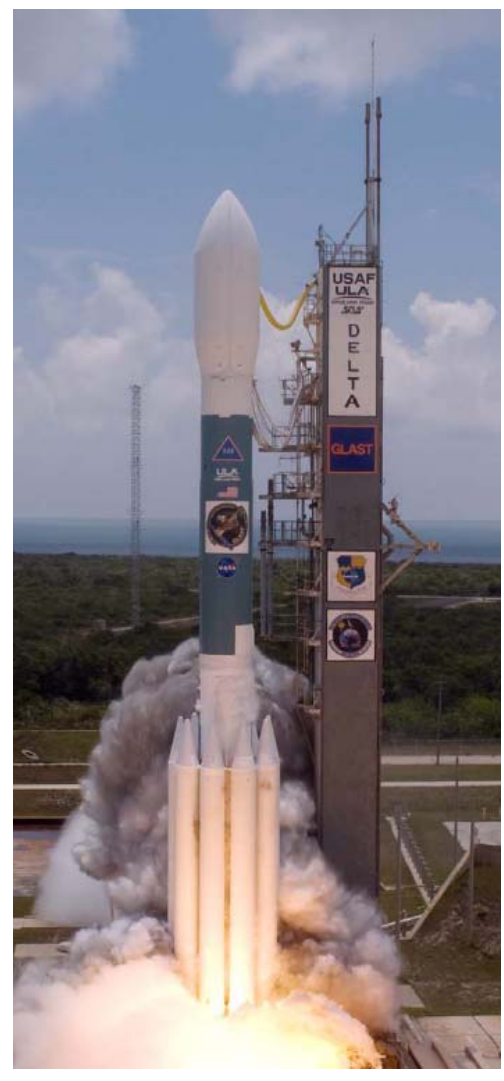

Fig.2: GLAST/Fermi was launched on June 11, 2008.

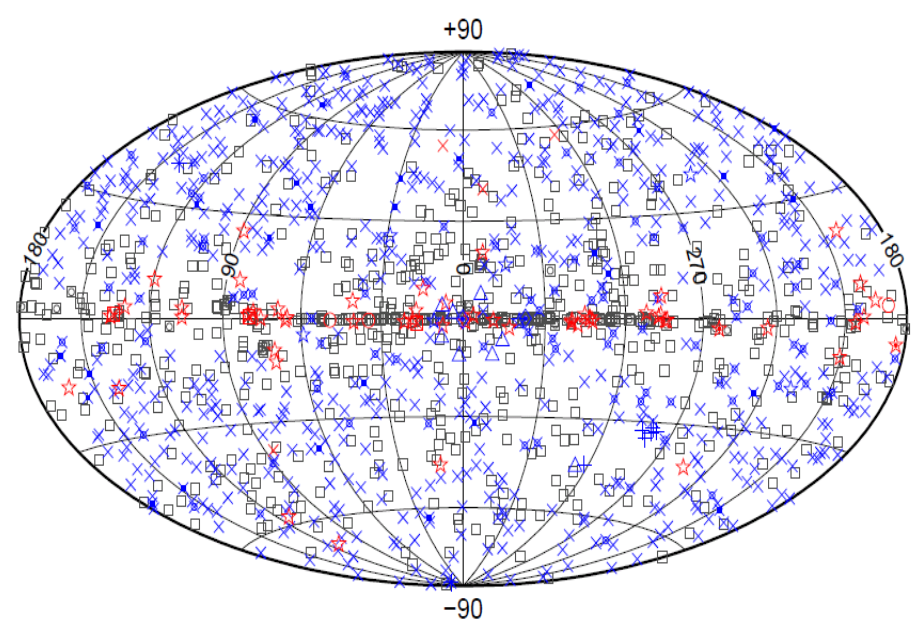

\begin{tabular}{|c|c|c|c|}
\hline$\square$ No association & Possible assoc & ation with & earby SNR or PWN \\
\hline$\times$ AGN - blazar & * Starburst Gal & « Pulsar & V/PWN \\
\hline$\times A G N-$ unknown & + Galaxy & $\diamond \mathrm{PWN}$ & $\Delta$ Globular cluster \\
\hline$\times A G N-$ non blazar & & O SNR & $\triangle \mathrm{XRB}$ or $\mathrm{MQO}$ \\
\hline
\end{tabular}

Fig.3: Fermi Collaboration published the First Fermi gammaray catalog (1FGL) in March 2010: Abdo, A. A. et al. 2010, ApJS, 188, 405. The catalog consists of 1451 sources of which about 820 are associated with known sources. They are broken down to 63 pulsars, 569 blazars, 120 non-blazar AGNs and 8 globular clusters. About 630 are not associated to known sources: some may lead to new discoveries. 


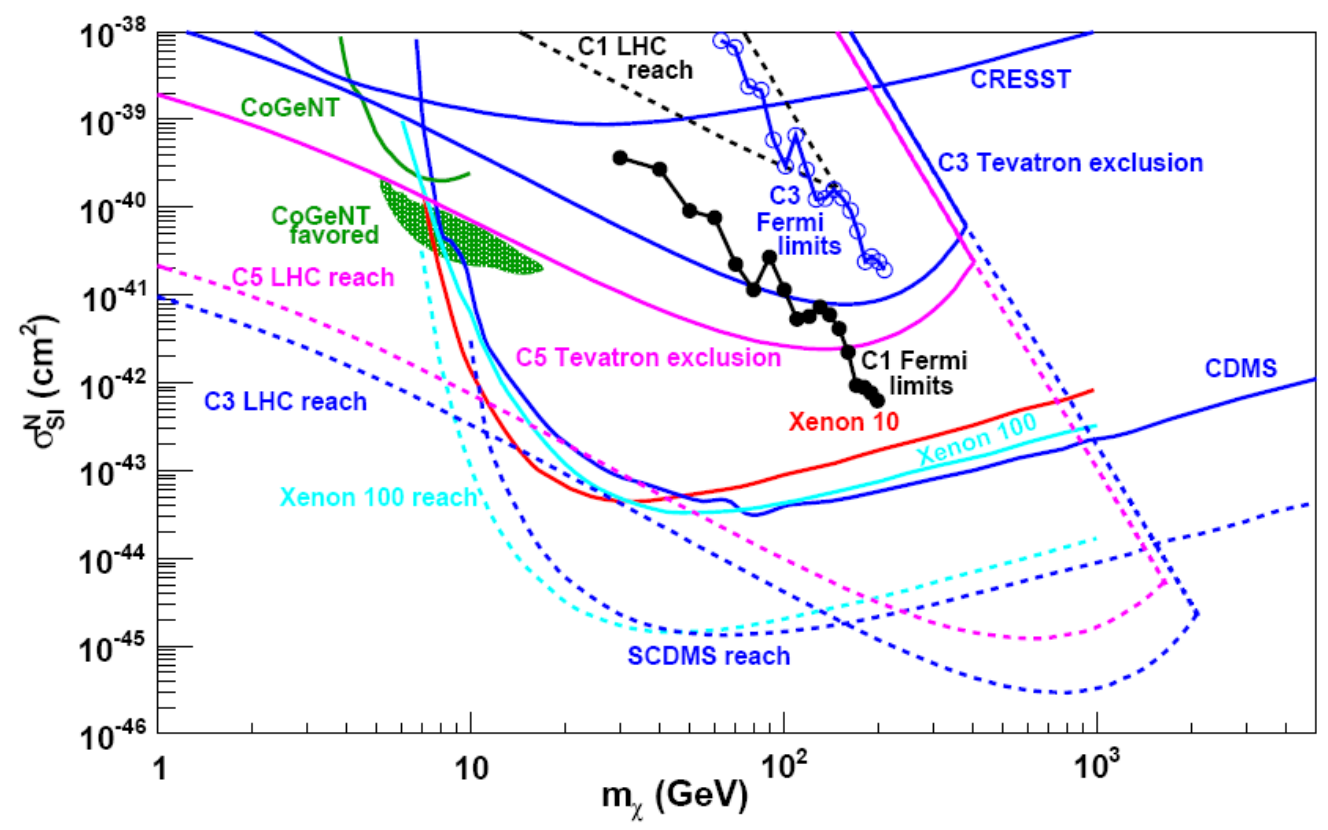

Fig.4: Upper limits on the particle dark matter (DM) on an effective interaction modeling by Goodman et al. arXiv:1009.0008v1. They compared the limits set by Fermi's line gamma-ray search (Abdo, A. A. et al. 2010, Phys. Rev. Lett., 104, 091302) with those by direct DM search experiments, Tevatron, and LHC for several spin-dependent interaction matrices.
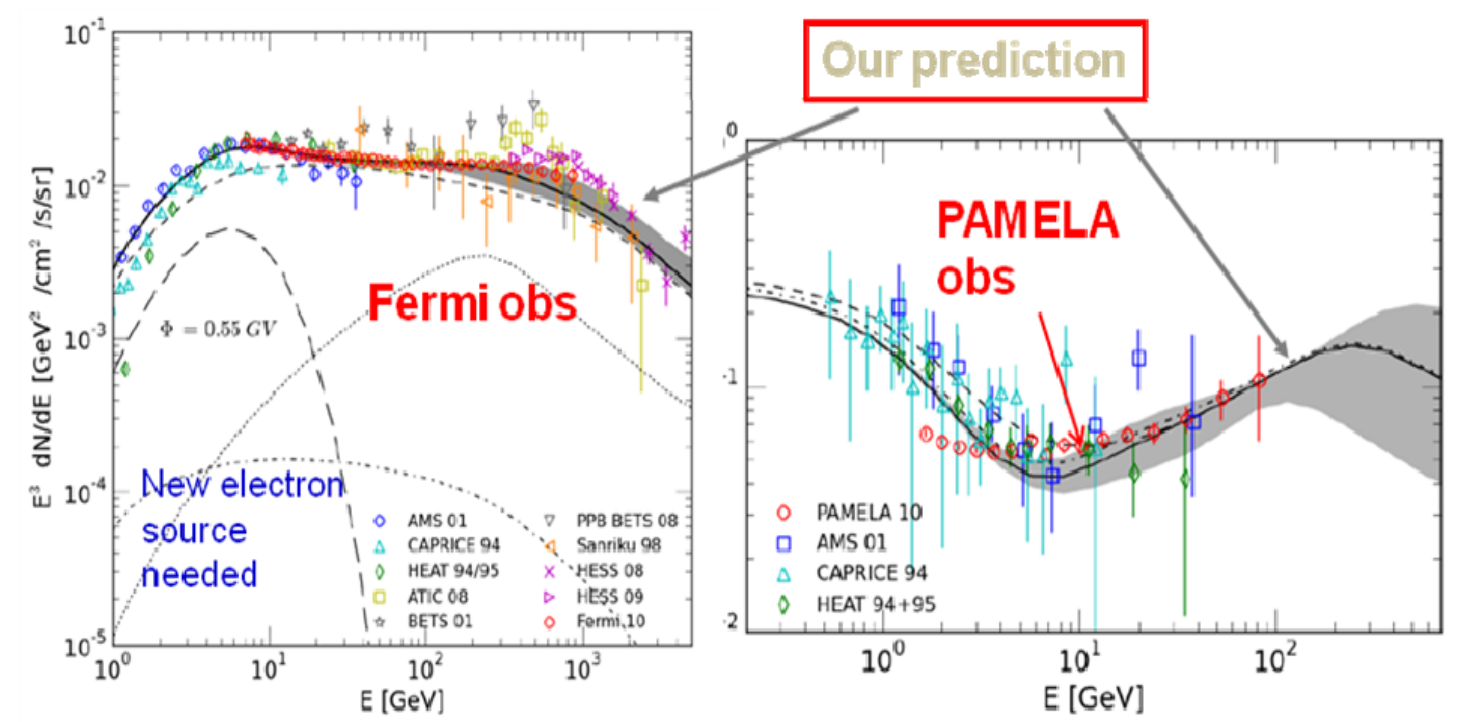

Fig.5: Analyses of cosmic ray (CR) $\mathrm{e}^{\wedge}++\mathrm{e}^{\wedge}$ - spectrum by Fermi (left, Ackermann, M. et al. 2010, Phys. Rev. D) and $\mathrm{e}^{\wedge}+/\left(\mathrm{e}^{\wedge}++\mathrm{e}^{\wedge}-\right)$ by PAMELA (right) assuming the $C R$ injection spectra deduced from Fermi and HESS gamma-ray observations. The predictions (gray bands) reproduce the Fermi and PAMELA results quite well (Kamae et al. arXiv1010.3477). 


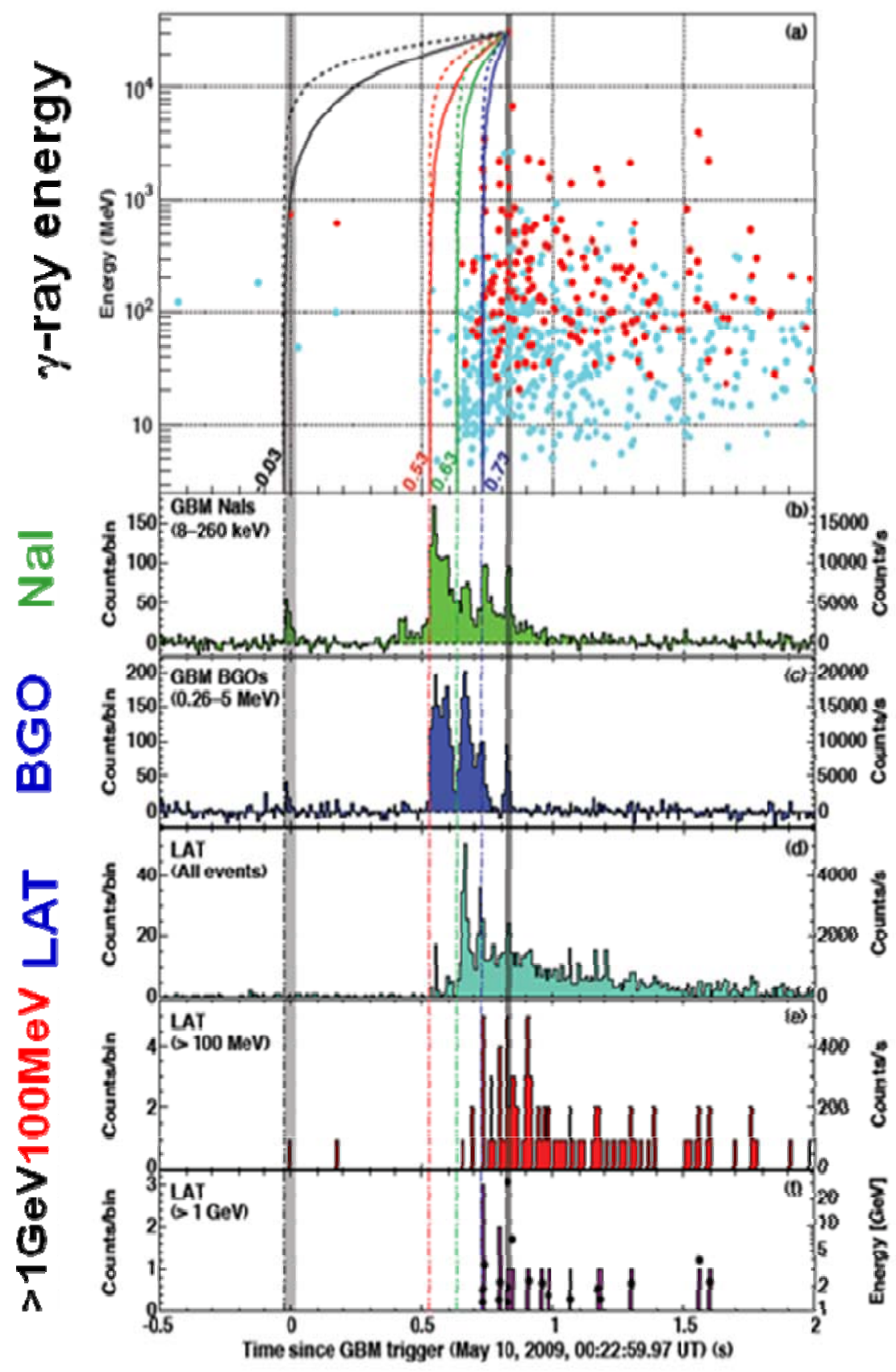

Fig.6: Arrival time dist. of gamma-rays in GRB090510 at $z=0.903$ : From top, all energy bans, 8$260 \mathrm{keV}, 0.26-0.5 \mathrm{MeV}$, the LAT band ( $>\mathrm{a}$ fewx10 MeV), $>100 \mathrm{MeV}$ and $>1 \mathrm{GeV}$. The arrival time of the highest energy gamma ray $(32 \mathrm{GeV})$ is shown by the vertical gray line on the right and the first photon detected on the left. The most conservative arrival time difference corresponds to M_QG M_Planck for a quantum gravity model predicting Lorentz Invariance Violation (Abdo, A. A. et al. 2009, Nature, $462,331)$. 


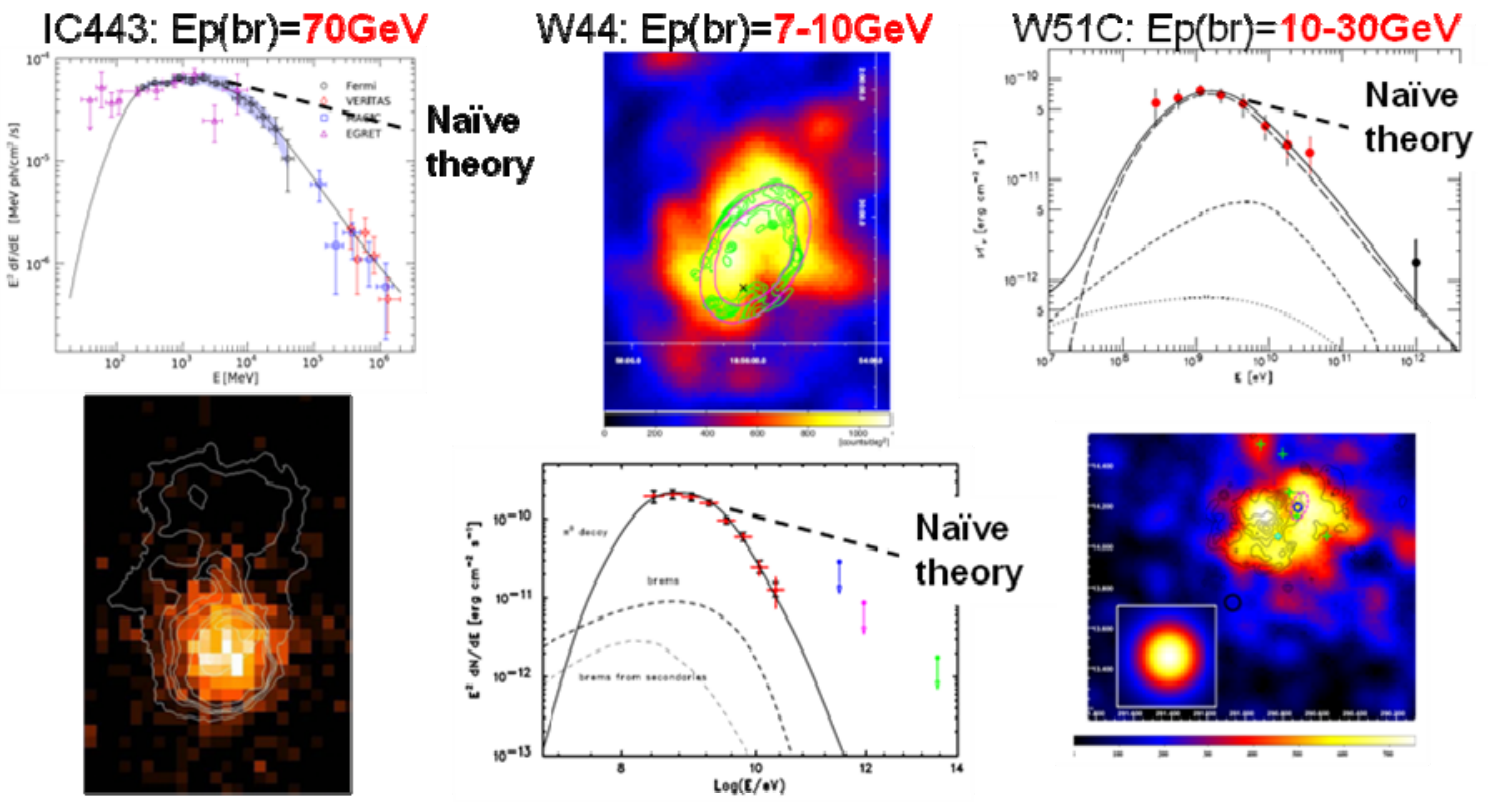

Fig.7: Gamma-ray spectra observed from middle-aged supernova remnants: IC443 (left, Abdo, A. A. et al. 2010, ApJ, 712, 459), W44 (middle, Abdo, A. A. et al. 2010, Science, 327, 1103) and W51C (right, Abdo, A. A. et al. 2009, ApJ, 706, L1). All show roll down at energies between $10-100 \mathrm{GeV}$ suggesting broken power-law spectra

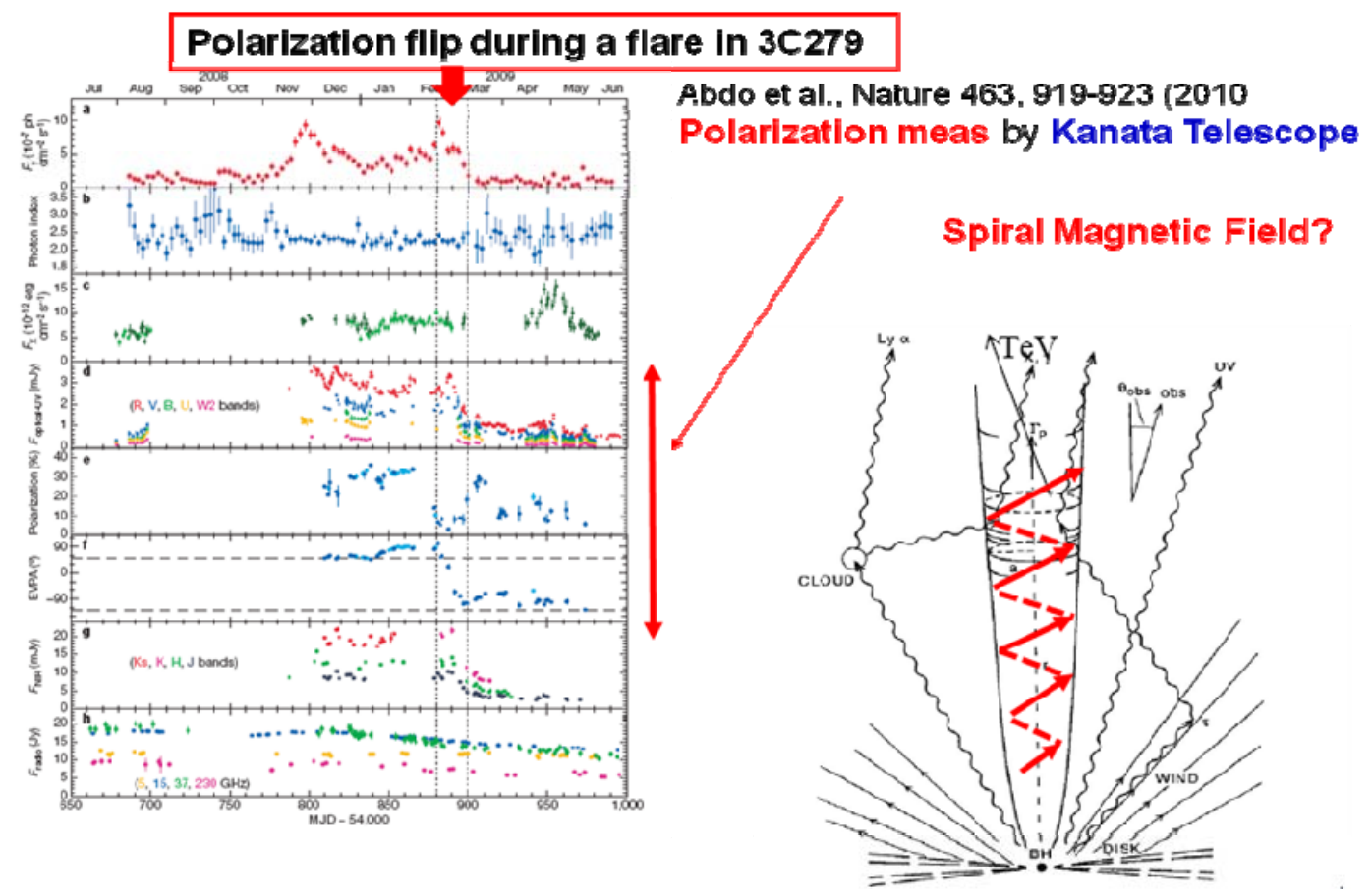

Fig.8: Light curves measured in different wave-bands at the time of a flare in 3C279. Polarization measurement by Kanata Telescope, Hiroshima Univ. (shown by the red arrow) suggests that magnetic field wraps around the jet in a spiral form (Abdo, A. A. et al. 2010, Nature, 463, 919). 
In the Internal shock scenarlo and $\%$ opacity argument, they derive following equation:

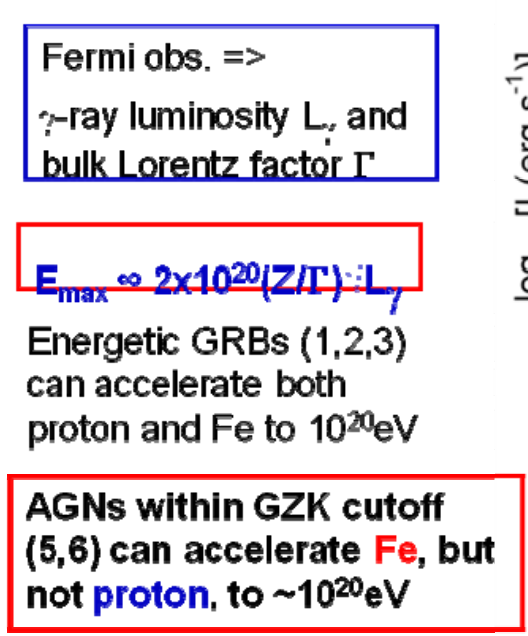

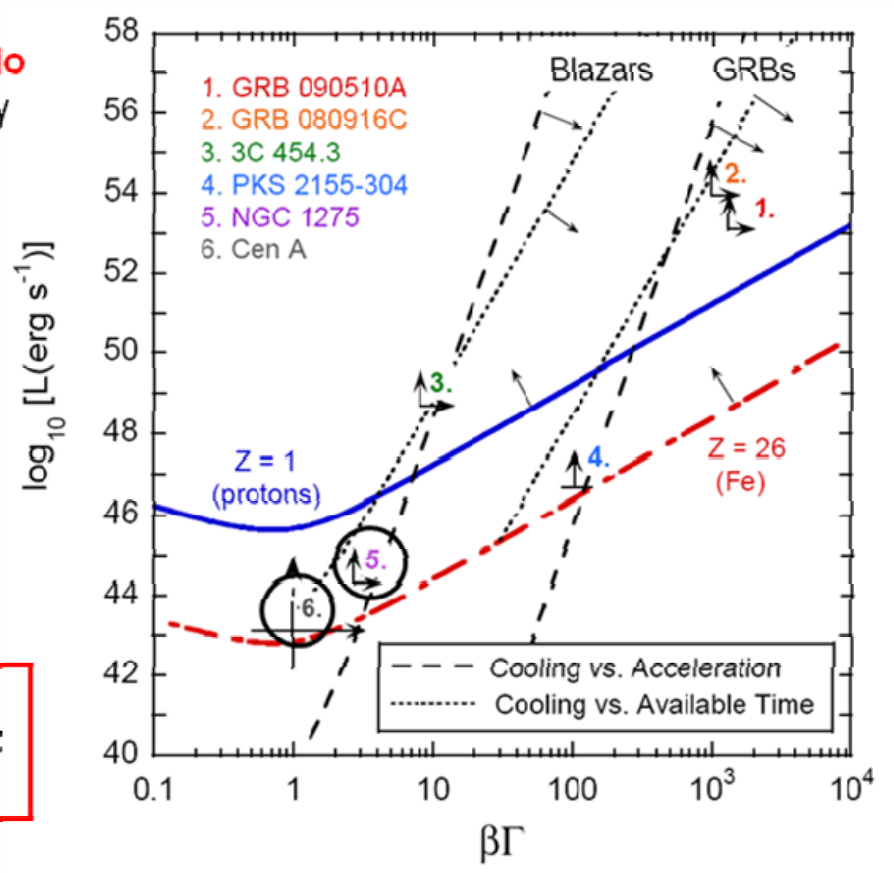

Fig.9: According to an analysis by Dermer \& Razzaque (arXiv:1004.4249), Fermi observations of flares in near-by AGNs constrain the maximum cosmic-ray energy reached in the internal shock acceleration scenario. The bulk Lorenz factor is determined on the gamma-ray spectra by HESS and those by Fermi constrain the Mach number and thickness of the shock. The authors calculate the maximum energy attained by particles with mass $A$ and charge $Z$. The authors conclude that an energy of $10^{\wedge} 20 \mathrm{eV}$ can be reached by $\mathrm{Fe}(\mathrm{Z}=26)$ but not by proton $(Z=1)$ at 2 candidate UHECR sources (Cen A and NGC 1275) within the Greisen-Zatsepin-Kuzmin radius.

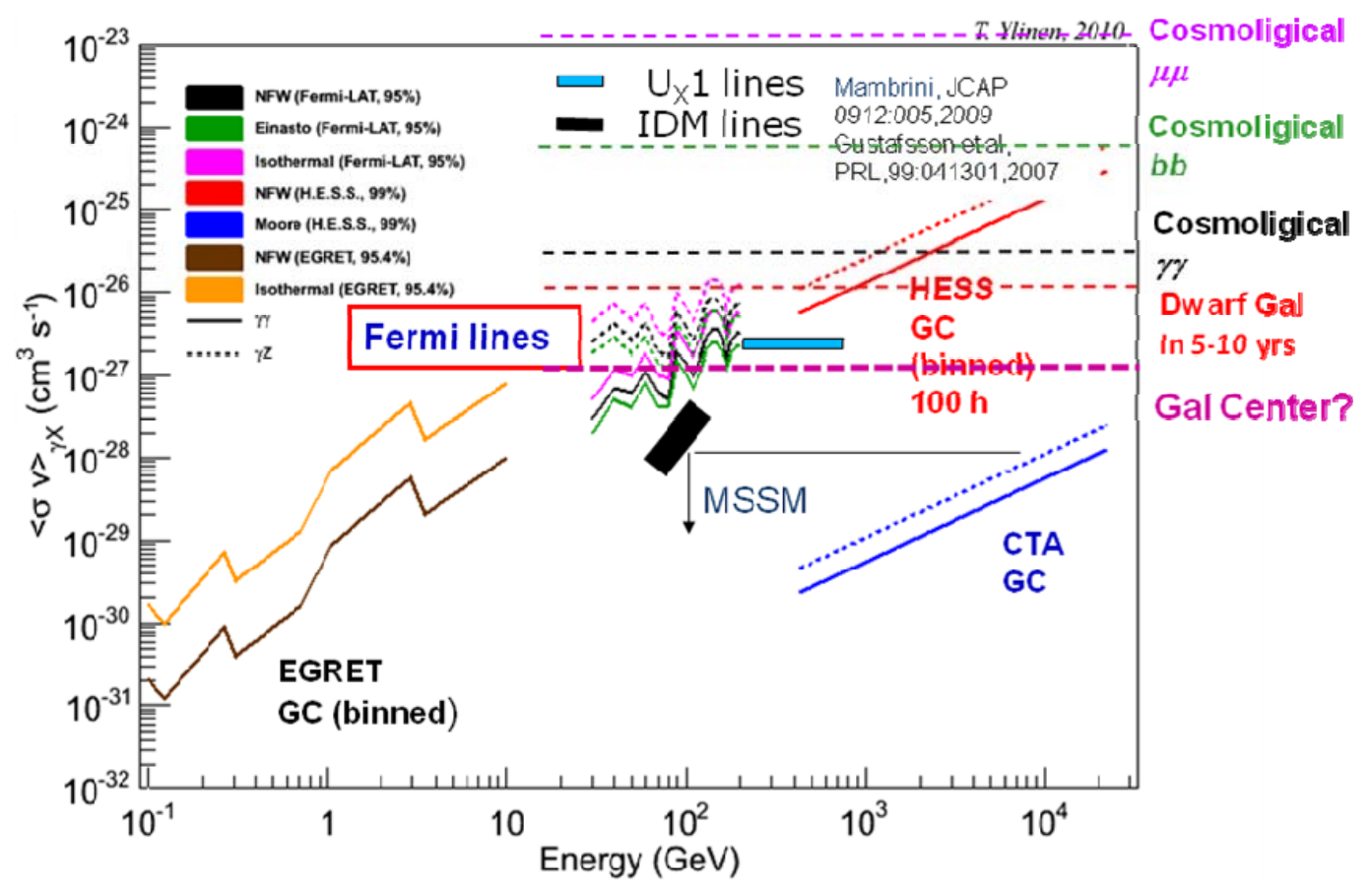

Fig.10: Expected upper limits for the DM by Fermi in the future. Search for annihilation signals in Dwarf Galaxies and in the Galactic Center is expected to limit significant portion of the DM model parameter space. 


\section{Belle and Belle-II}

Thomas E. Browder

University of Hawaii at Manoa, Honolulu, HI

The US-Japan HEP collaboration, also known as Nichibei, played a critical role in the construction of the Belle experiment at KEKB in Tsukuba, Japan. In particular, there were important contributions to the readout of the silicon detector, the time-of-flight (TOF) detector subsystem, and the K-long/muon (KLM) resistive-plate counters. Without Nichibei's support, US participation in this important experiment would have been very limited, or perhaps impossible. Belle, along with BaBar, detected the first signals of $\mathrm{CP}$ violation outside the strange quark sector, and verified the Kobayashi-Maskawa mechanism for CP violation. The 2008 Nobel Prize citation for Kobayashi and Maskawa noted and recognized the contributions of these two important experiments.

This year, on the $30^{\text {th }}$ anniversary of the Nichibei collaboration, the operation of Belle and KEKB have been shut down after integrating over $1000 \mathrm{fb}^{-1}$ of data. This sample forms the foundation of the on-going intense analysis phase at Belle. KEK also embarked on a new Super B factory project with an international detector-collaboration called Belle-II the goal of which is to discover new physics and new sources of CP violation in the quark sector. The new accelerator, SuperKEKB, will deliver 40 times more luminosity than the record-setting KEKB machine. With some support from Nichibei, US groups working closely with colleagues at Nagoya University and KEK are developing the high-momentum particle identification system for Belle-II called iTOP (imaging Time Of Propagation), as well as tools for improved muon detection and sophisticated beam monitoring. 


\section{Belle@KEKB, Belle-II@SuperKEKB \\ Tom Browder (University of Hawaii)}

On behalf of the Belle and Belle-Il collaborations as well as the US Belle I and Belle-II groups:

(Cincinnati, Hawaii, Princeton*, PNNL**, VPI and Wayne State)
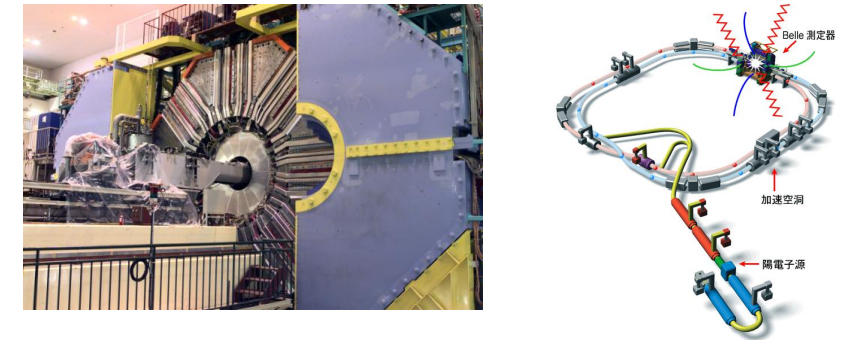

History + Physics + LHC Synergy

Nichibei 日米 (US-Japan) 30 th anniversary meeting, October 20, 2010

\section{Past and Present}

- In the 1990's the Belle experiment greatly benefited from Nichibei supported R+D on readout for silicon vertex detectors, high precision TOF counters and detection of muons and $\mathrm{K}_{\mathrm{L}}^{\prime}$ 's via RPC's.

- In 2010: we are conducting R+D for Belle-II with a strong collaboration involving a consortium of US universities, Nagoya University and KEK. The main focus is high momentum particle ID (with SLAC) and sophisticated beam monitoring. 


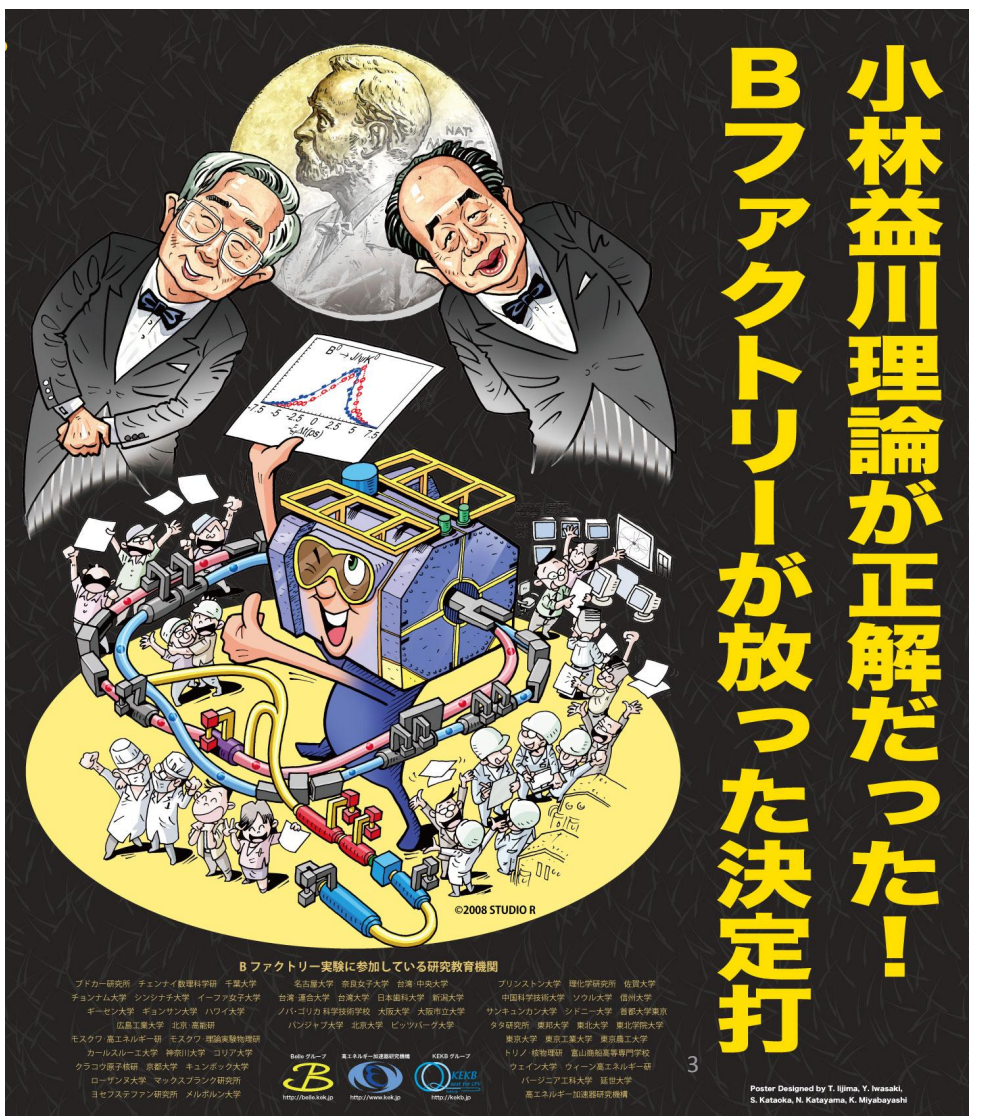

2008:

Critical Role of the B factories in the verification of the KM hypothesis was recognized and cited by the Nobel Foundation

A single irreducible phase in the weak interaction matrix accounts for most of the CPV observed in kaons and B's.

$\mathrm{CP}$ violating effects in the $\mathrm{B}$ sector are $\mathrm{O}(1)$ rather than $\mathrm{O}\left(10^{-3}\right)$ as in the kaon system.

The cartoon refers to Belle $B^{0} \rightarrow \mathrm{J} / \psi K^{0}$ data

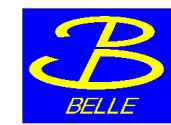

\section{M B $\bar{B}$ pairs}

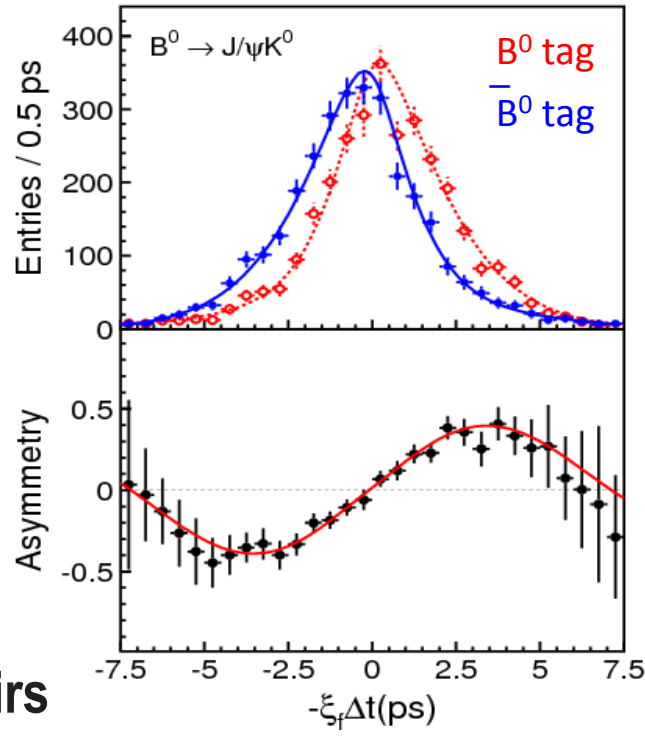

previous measurement $\sin 2 \phi_{I}=0.652 \pm 0.044$ (388 M B $\bar{B}$ pairs)

$$
\begin{array}{r}
\sin 2 \phi_{1}=0.642 \pm 0.031 \text { (stat) } \pm 0.017 \text { (syst) } \\
A=0.018 \pm 0.021 \text { (stat) } \pm 0.014 \text { (syst) }
\end{array}
$$

(An update with $50 \%$ more $L_{\text {eff }}$ coming soon)

hep-ex/0608039, PRL 


\section{Nobel Prizes from Surprising Discoveries about Weak}

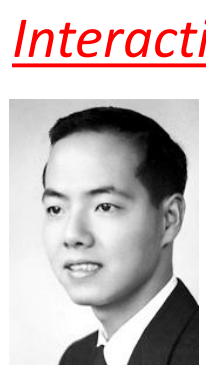

T.D. Lee

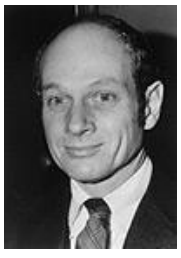

J. Cronin

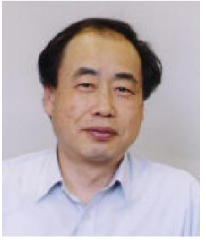

M. Kobayashi

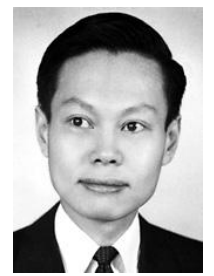

C.N. Yang

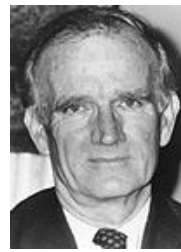

V. Fitch

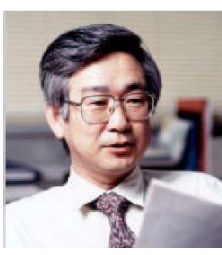

T. Maskawa

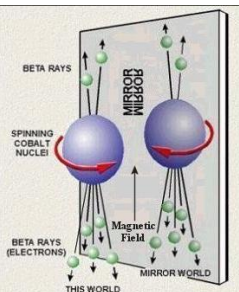

Maximal P

violation
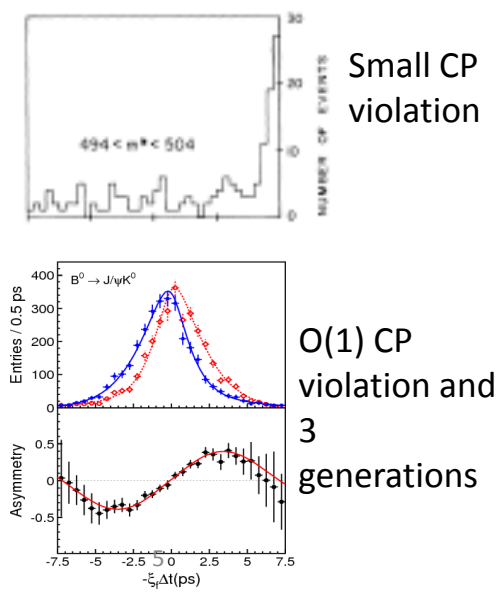

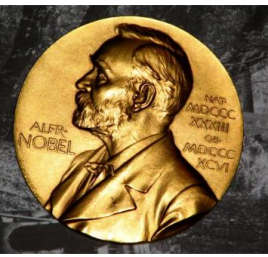

1957

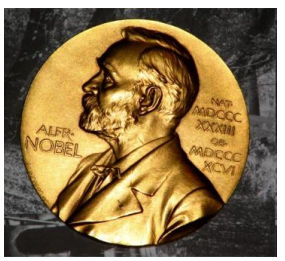

1980

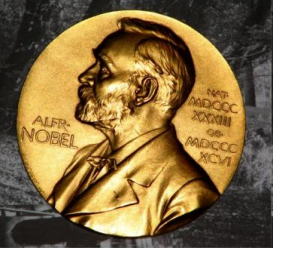

2008

In addition to observing $\mathrm{CP}$ violating phenomena and rare $B$ decay, Belle discovered a series of unexpected new particles.

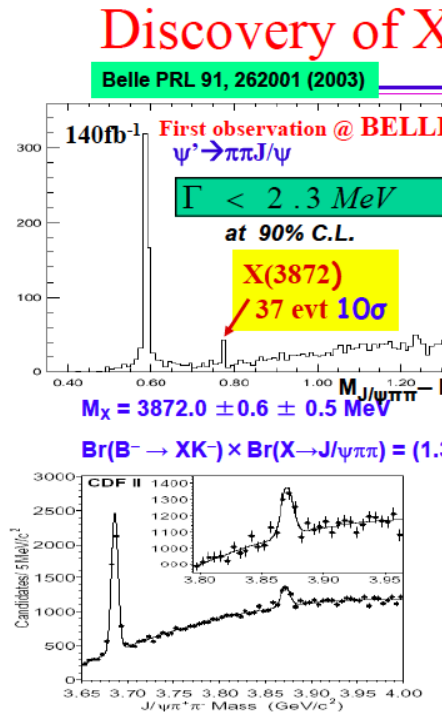

$\mathrm{M}_{\mathrm{X}}=3871.3 \pm 0.7 \pm 0.4 \mathrm{MeV}$

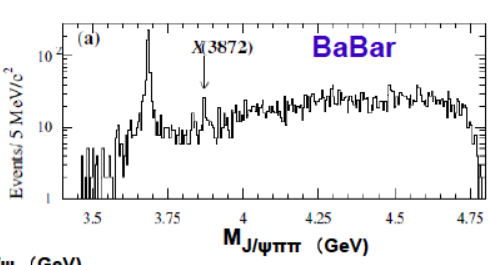

$3873.4 \pm 1.4 \mathrm{MeV}$

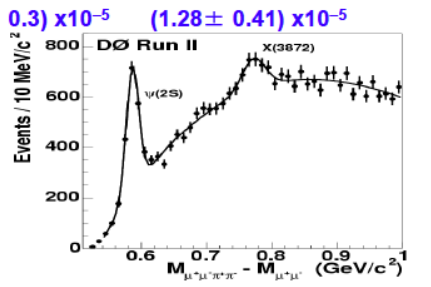

$\mathrm{M}_{\mathrm{J} / \psi \pi \pi}-\mathrm{M}_{\mathrm{J} / \psi}=774.9 \pm 3.1 \pm 3.0 \mathrm{MeV}$

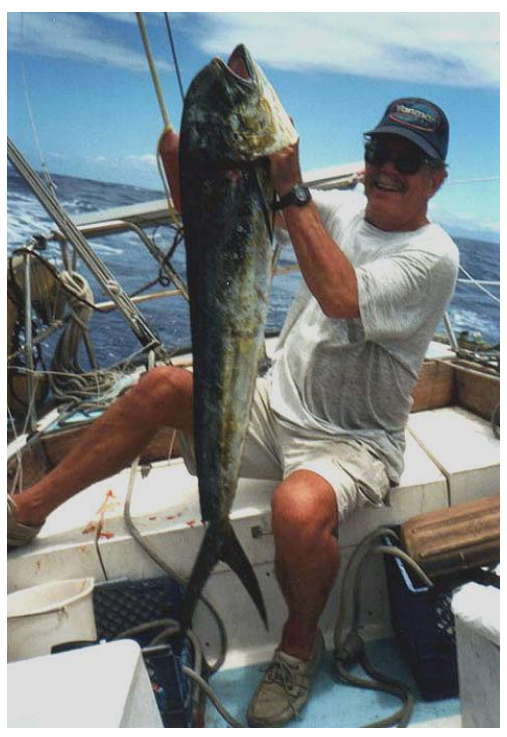

Followed by the discovery of the Y (3940) and a charged Z state. 
Belle/KEKB Integrated luminosity passed $1000 \mathrm{fb}^{-1}$

$\left(\rightarrow\right.$ have to switch to new units, $1 \mathrm{ab}^{-1}$ )

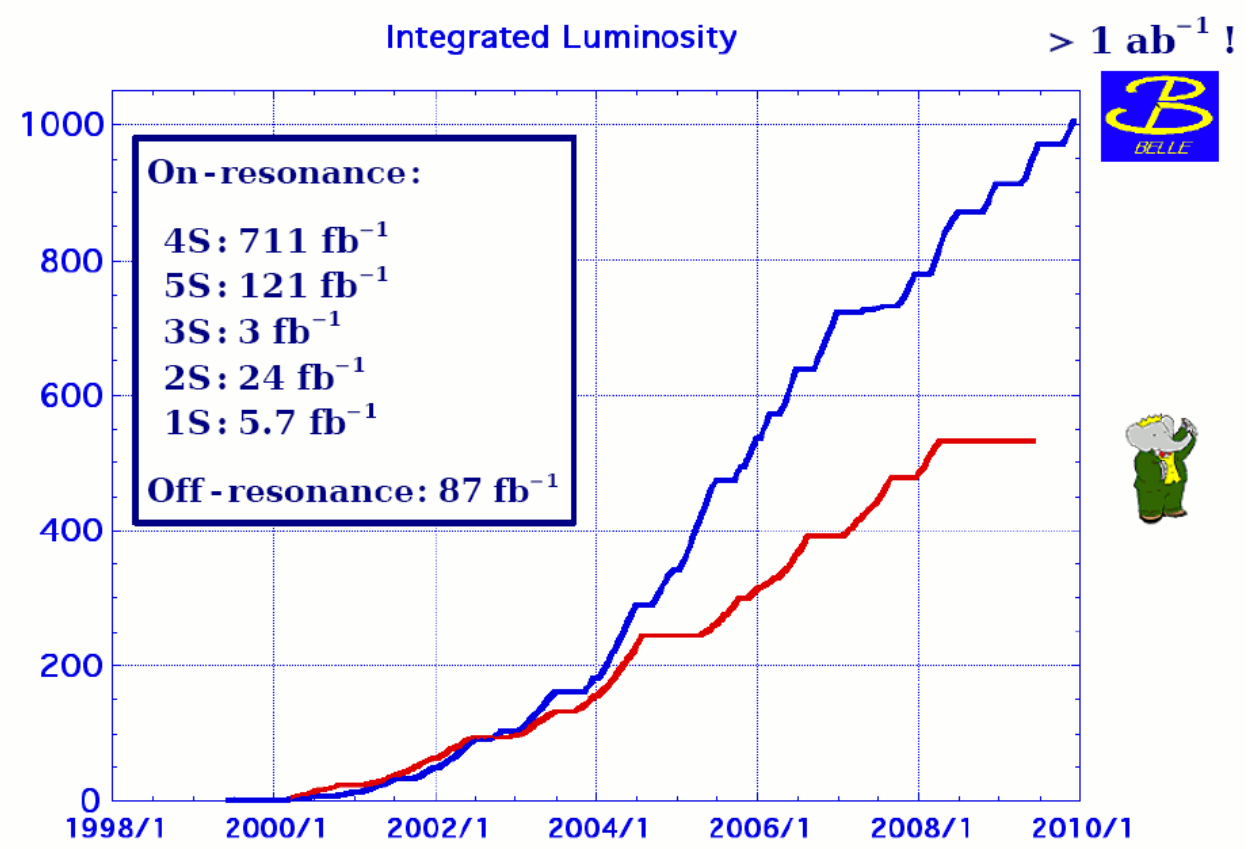

Peak lumi record at KEKB: L=2.1 $\times 1,0^{34} / \mathrm{cm}^{2} / \mathrm{sec}$ with crab cavities

KEKB Final Beam Abort Ceremony

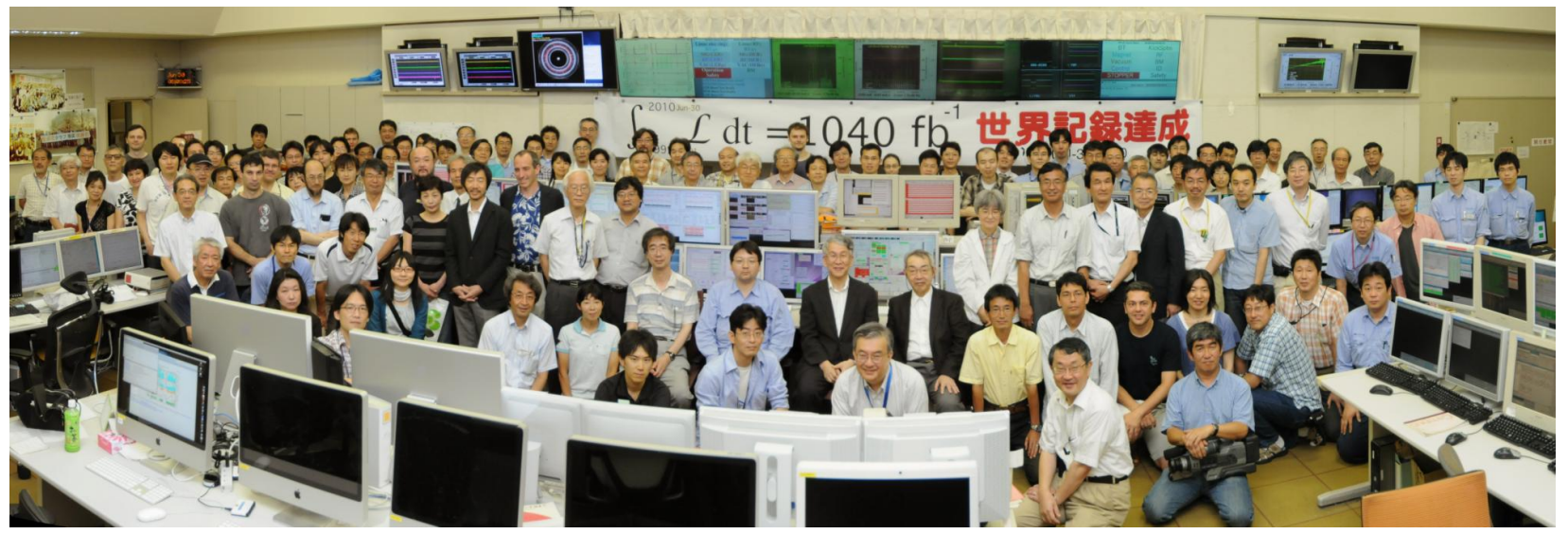

Belle実験グループ代表の一人、ハワイ大学のトム・ブラウダー教授は

「11年前にKEKBとBelleが実験を開始したとき、世界最高のルミノシテ イを達成すると外部の人は予想していなかった。ここに至るまでの道 のりは平坦ではなかったが、小林・益川両博士にノーベル賞をもたら した B 中間子のCP対称性の破れの確認など、世界各地の大学院生や研 究者が数多くの論文を執筆するための重要なデー夕を得ることができ た。これらのデータで得られた科学上の知見の大きさははかりしれな い。」と述べま

http://www.kek.jp/ja/news/topics/2010/KEKBfactory.html 


\section{Are we done ? (>335 Belle publications)}

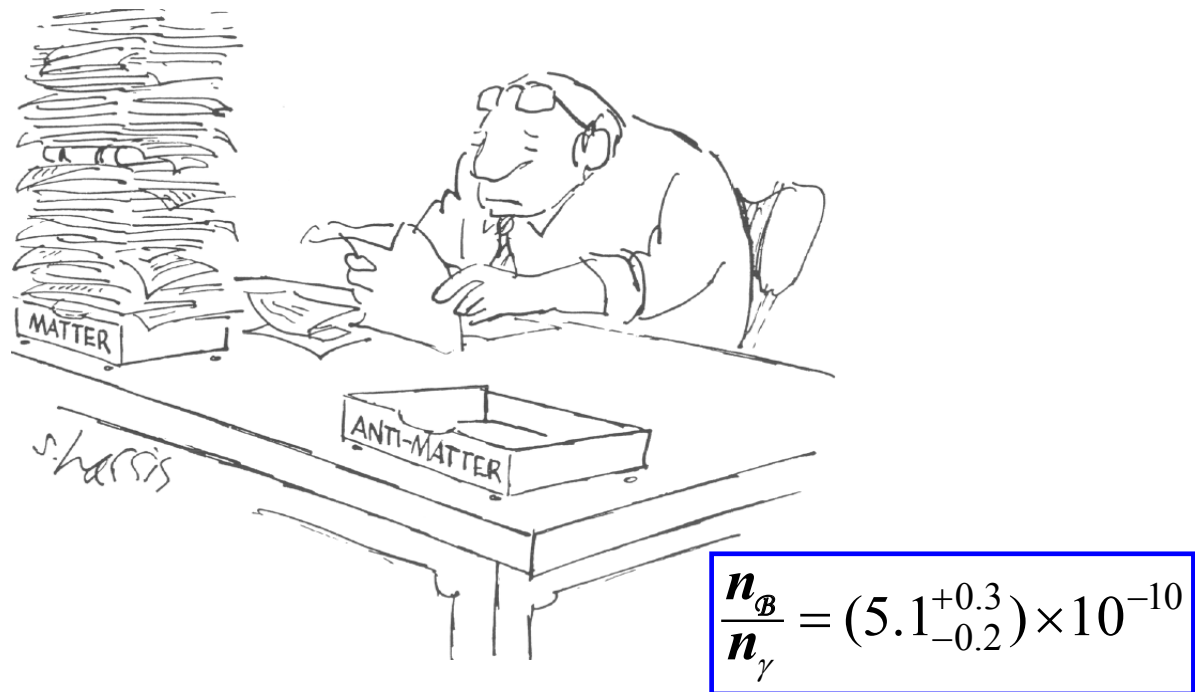

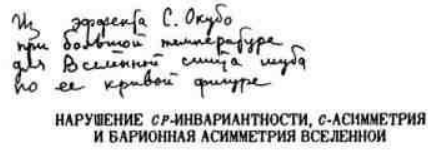

A.A.Cszapoe
$\mathrm{KM} \sim 10^{-20}$

Too small by 10 orders of magnitude in the SM

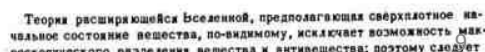

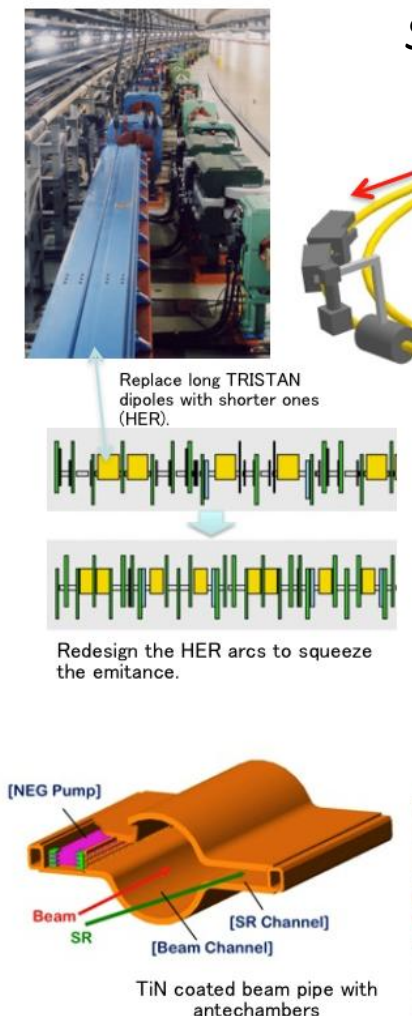

antechambers

\section{SFF Physics requires Luminosity}

Iit.

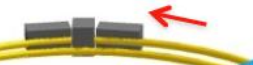

e-2.6 A $\longrightarrow$ New Superconducting /

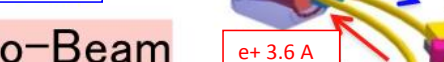

permanent final focusing

Nano-Beam

SuperKEKB
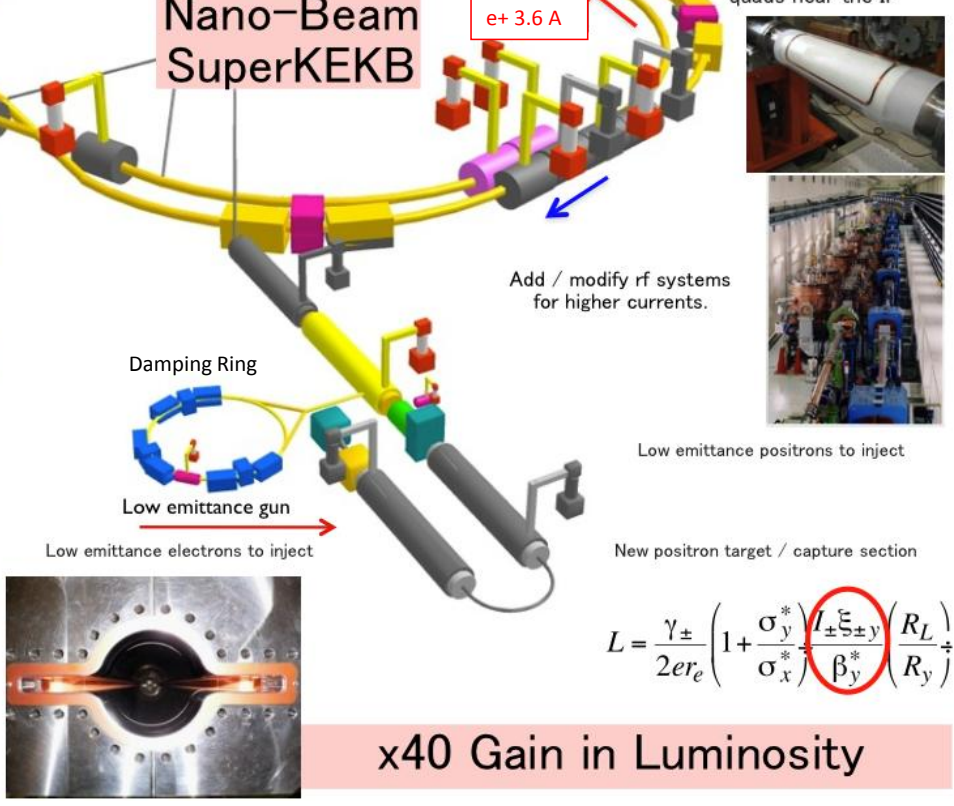

Low emittance positrons to inject

New positron target / capture section

$$
L=\frac{\gamma_{ \pm}}{2 e e_{e}}\left(1+\frac{\sigma_{y}^{*}}{\sigma_{x}^{*}}\right)\left(\frac{I_{ \pm} \xi_{ \pm}}{\beta_{y}^{*}}\right)\left(\frac{R_{L}}{R_{y}}\right)
$$

x40 Gain in Luminosity

(Must reduce $\sigma_{\mathrm{y}}$ from $1 \mu \mathrm{m} \rightarrow 59$ 


\section{High Momentum PID at Belle-II}

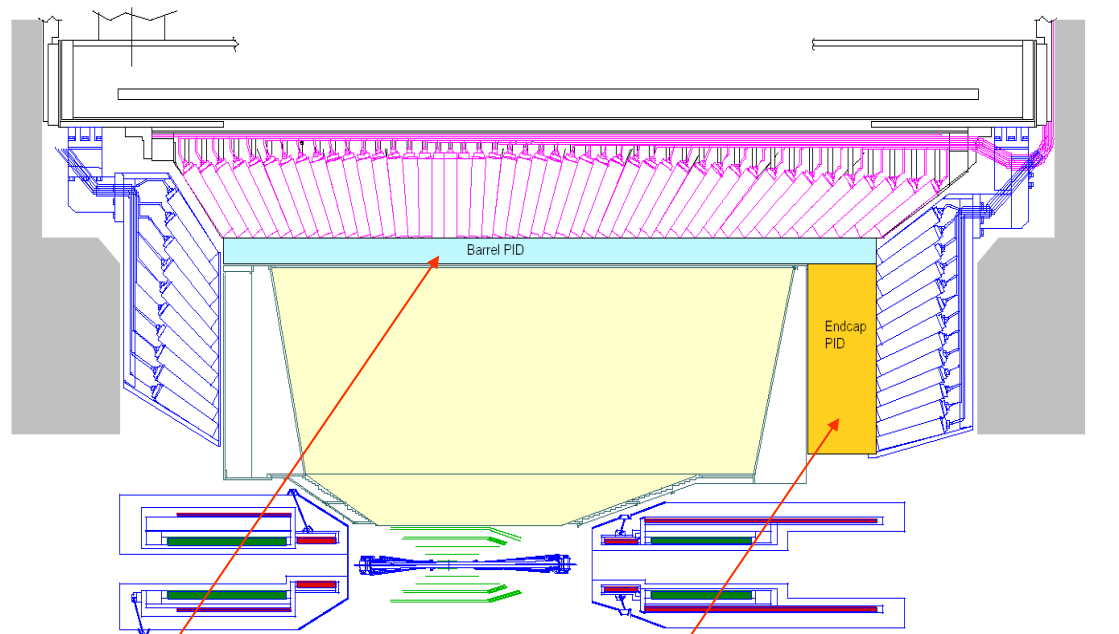

Two new particle ID devices, both using Cherenkov light:

Barrel: Time-Of-Propagation (iTOP) (baseline), (major US contributions to quartz, readout electronics, mechanics, optics), close collaboration with Nagoya and KEK

Endcap: proximity focusing aerogel RICH (Slovenia, KEK, Nagoya)

結論

SuperKEKB starts in 2014 with an international detector collaboration (Belle-II). [Talks by Yamauchi, Suzuki]

Belle-II TDR now available at $h t t p: / / x x x . l a n l . g o v / a b s / 1011.0352$

The project is designed to discover new FCNC and new sources of CPV. The physics program is deep, broad and should help elucidate new physics found at the LHC.

The US groups, SLAC, Nagoya and KEK are conducting R+D on the high momentum PID device as well as the scintillator based muon upgrade and beamstrahlung monitor.
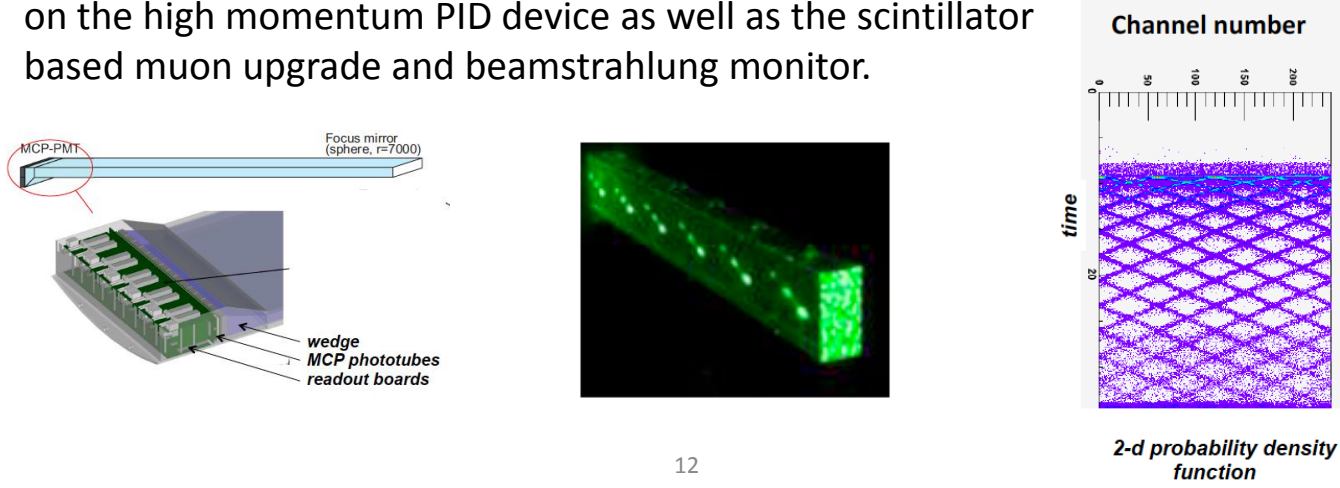



\title{
Super-Kamiokande
}

\author{
Y. Suzuki \\ Kamioka Observatory, Institute for Cosmic Ray Research, and \\ Kamioka Satellite, Institute for the Physics and Mathematics of the Universe, \\ The University of Tokyo
}

Super-Kamiokande (Super-K) has started to take data in 1996 primarily aiming to measure solar and atmospheric neutrinos precisely, and to look for neutrino bursts from supernovae. A search for proton decay is another important subject of the experiment.

In 1998, Super-K has discovered the neutrino oscillation in the study of atmospheric neutrinos, and following that discovery the long baseline neutrino oscillation experiment (K2K) where Super-K was used as a far detector, $250 \mathrm{~km}$ away from the neutrino source at KEK, has started and confirmed the atmospheric neutrino oscillation. In 2001, the combined data sets from SNO and Super-K have evidently shown that the solar neutrino also oscillates.

One of the remaining issues on the neutrino oscillation is to look for as yet undetermined parameter, $\theta_{13}$. The new generation long baseline oscillation experiment, T2K, shooting neutrino beam from JPARC to Super-K is under way for this purpose. If the experiment will be successful, we will open up a new horizon to unveil the secret of creation of matter in the early universe through the detailed study of $\mathrm{CP}$ violation in neutrino sector.

Though the Super-Kamiokande experiment is not a part of the US-Japan cooperative research program, but it is a successful example of the US-Japan collaboration. 


\section{Introduction}

1984

BNL E734 (200ton)

(US-Japan Cooperative RP)

YS analyzed and written

this oscillation paper

$v_{\mu} \rightarrow v_{\mathrm{e}}$ limit

$\mathrm{L}=96 \mathrm{~m},<\mathrm{E}_{v}>=1.2 \mathrm{GeV}$

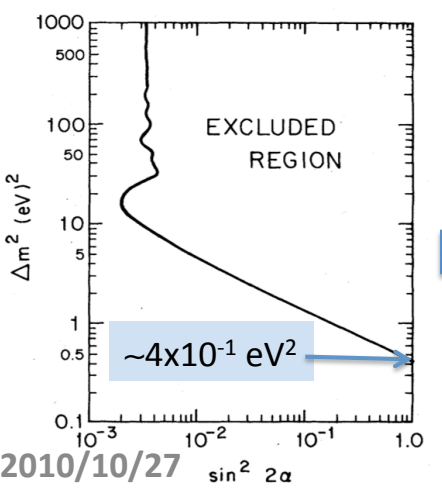

20 years

2004

K2K/SK (22.5kton)

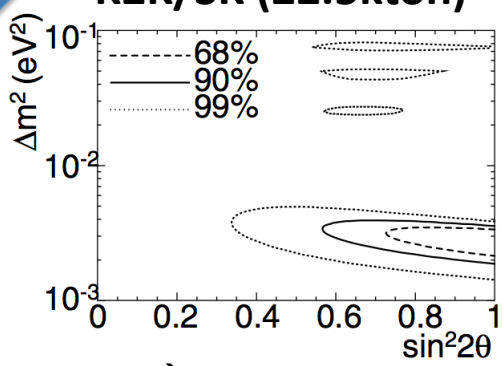

$v_{\mu} \rightarrow v_{\mu}$

$\mathrm{L}=250 \mathrm{~km},\left\langle\mathrm{E}_{v}\right\rangle=1.3 \mathrm{GeV}$

1000 times improvement

No body can predict experiments 20 years from now !

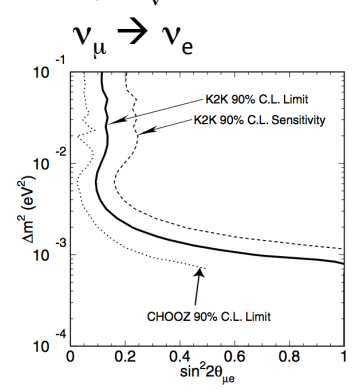

\section{Super-Kamiokande}

- Not a part of the US-Japan cooperative research program

- But a good example of successful US- Japan Collaboration

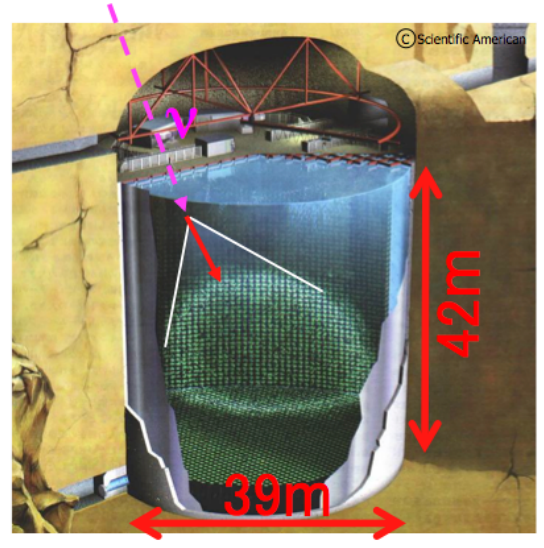

- 50,000 tons of Imaging Water Cherenkov Detector

- Inner: $\quad 32,000$ tons

(Outer Vol: $\sim 2.5 \mathrm{~m}$ thick)

- Fid. Vol: 22,500 tons

- 11,146 PMTs (ID)

$-50 \mathrm{~cm}$ in diameter

- $40 \%$ coverage

- 1,885 PMTs (OD)

$-20 \mathrm{~cm}$ in diameter

- $1,000 \mathrm{~m}$ underground

130 Collaborators from 36 inst. (6 countries) 


\section{US Joined}

- In 1992, US group joined the Super-Kamiokande experiment, one year after the start of the construction in 1991.

- Most of them were the former members of the IMB group who were the competitors of the Kamiokande experiment.

- Hank will discuss the international collaboration issues later

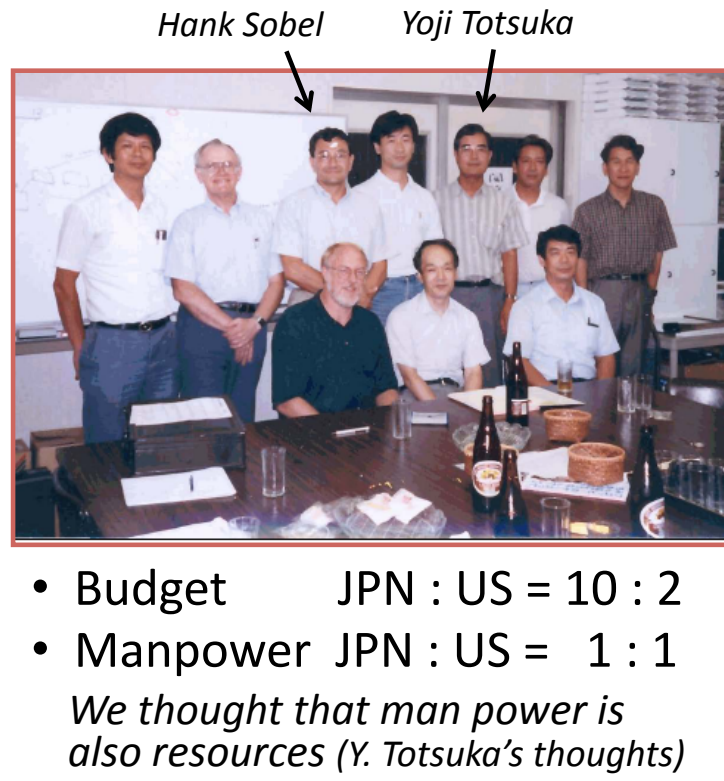

\section{Physics Objectives}

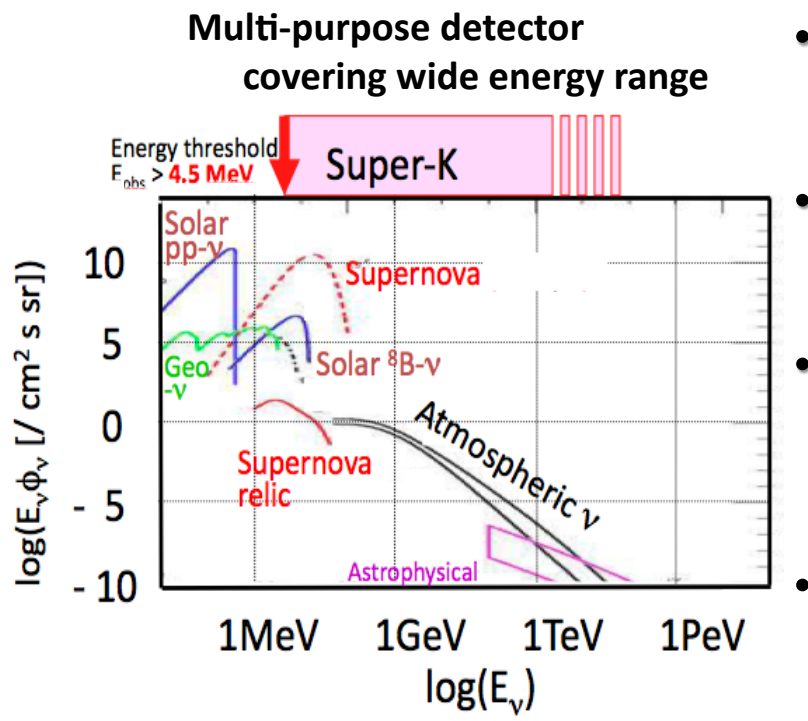

- Precise study of Atmospheric Neutrinos $>10$ events /day

- Precise study of solar neutrinos

$>15$ events /day

- Look for neutrinos from supernovae

$>8,000$ events for $\mathrm{SN}$ at $10 \mathrm{kpc}$ distance

Look for SN relic Neutrinos

- Look for Proton Decay

$$
\text { Up to } 10^{33} \sim 10^{34} \text { years } 10^{34} \text { yrs }
$$

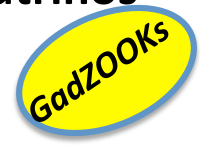

2010/10/27 Far Detector for Long baseline $\boldsymbol{v}$ Oscillation Experiments 


\section{Discovery of Atmospheric v Oscillation}

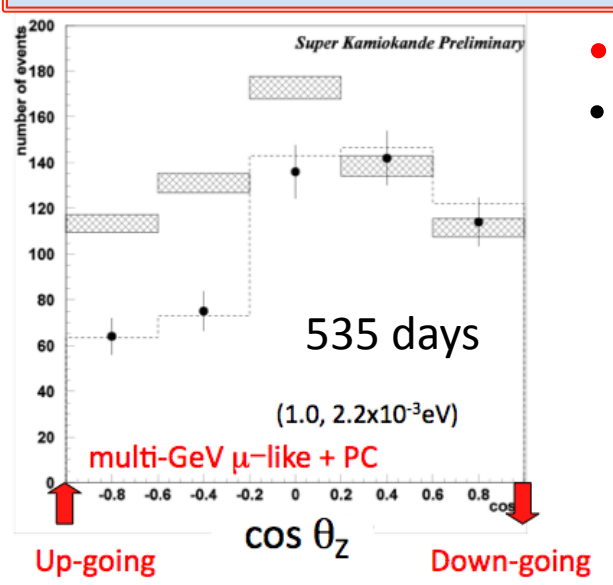

- In 1998

- SK observed

- Asymmetry of $\mu$-like events in the zenith angle distributions

$\rightarrow$ Definitive evidence of the neutrino oscillation independent of the flux calculations

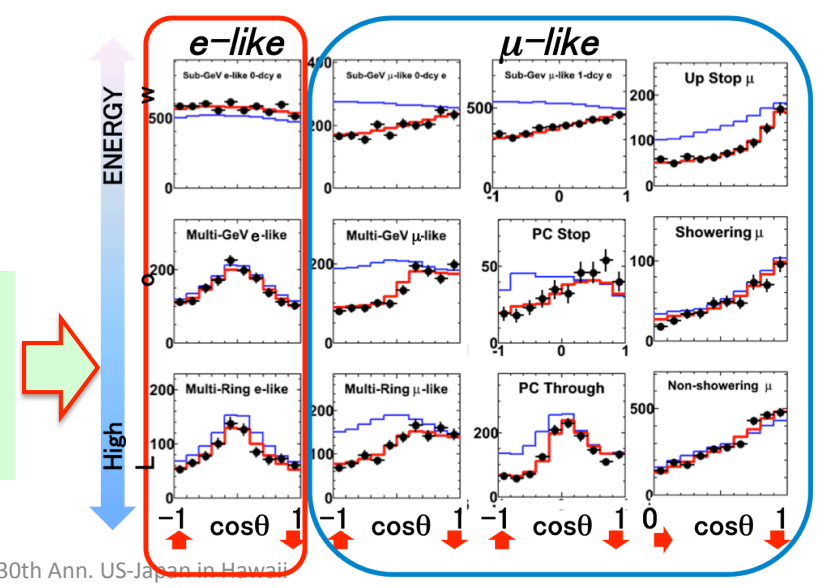

\section{Oscillation Parameters (3 flavor)}

\section{Normal Mass Hierarchy}

$$
\begin{aligned}
& \Delta m_{23}^{2}=2.11_{-0.12}^{+0.43} e V^{2} \\
& \sin ^{2} \theta_{23}=0.525_{-0.084}^{+0.072} \\
& \sin ^{2} \theta_{13}<0.066(90 \% \text { C.L. })
\end{aligned}
$$

Inverted Mass Hierarchy

$$
\begin{aligned}
& \Delta m_{23}^{2}=2.51_{-0.42}^{+0.13} e V^{2} \\
& \sin ^{2} \theta_{23}=0.575_{-0.074}^{+0.048} \\
& \sin ^{2} \theta_{13}<0.122(90 \% \text { C.L. })
\end{aligned}
$$

CP phase Inverted Mass Hierarchy

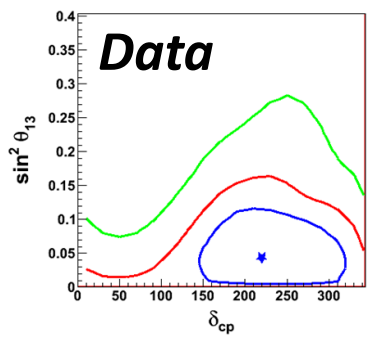

$2010 / 10 / 27$

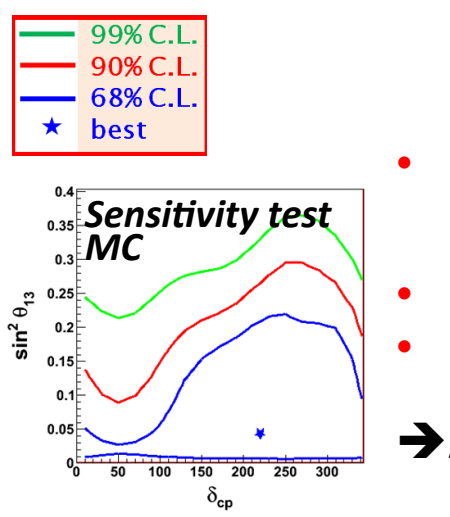

- Best fit (IMH)

- CP phase: $220^{\circ}$

- $\sin ^{2} \theta_{13}: 0.044$

- $1 \sigma$ level discussion

- Statistical fluctuation or may be something Atm- $v$ sensitivity to CPV 


\section{K2K First earth scale long baseline experiment}

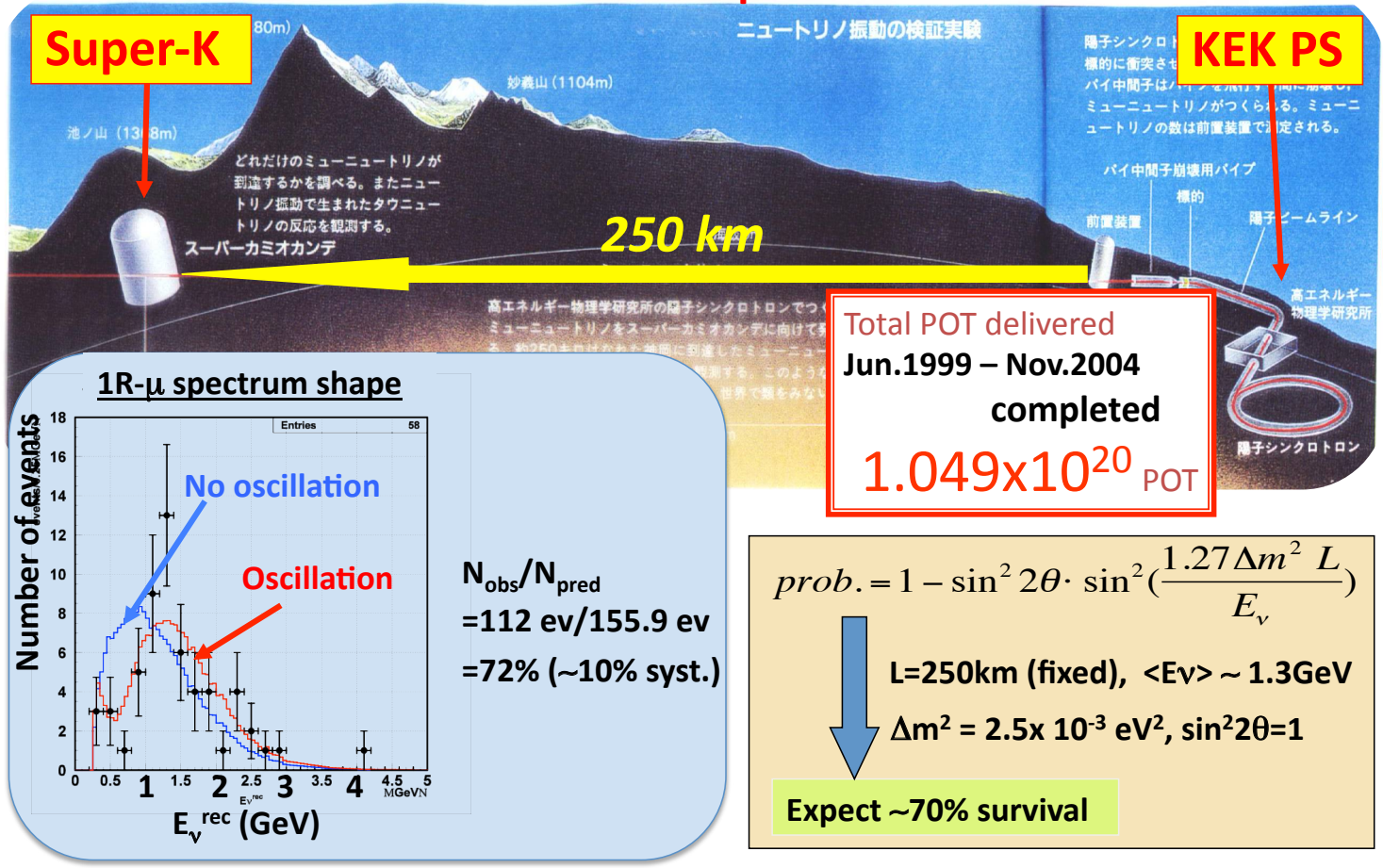

\section{SK Solar Neutrino Oscillation}

- SK 1258 days ES flux: PRL86 in June 18, 2001 issue $-v+e \rightarrow v+e \quad 2.35 \pm 0.02 \pm 0.08 \times 10^{6} \mathrm{~cm}^{-2} \mathrm{~s}^{-1}$

- SNO CC results: announcement in June 18, 2001

$-v_{\mathrm{e}}+\mathrm{d} \rightarrow \mathrm{p}+\mathrm{p}+\mathrm{e}^{-} \quad 1.76 \pm 0.06 \pm 0.09 \times 10^{6} \mathrm{~cm}^{-2} \mathrm{~s}^{-1}$

\section{Evidence of Neutrino Oscillation}

SNO $\left(v_{\mathrm{e}}\right)+\operatorname{SK}\left(v_{\mathrm{e}}+\left(v_{\mu}+v_{\tau}\right) \times 0.15\right)$ $4.3 \sigma$ effect of the existence of nonelectron neutrino components in solar neutrino on the earth
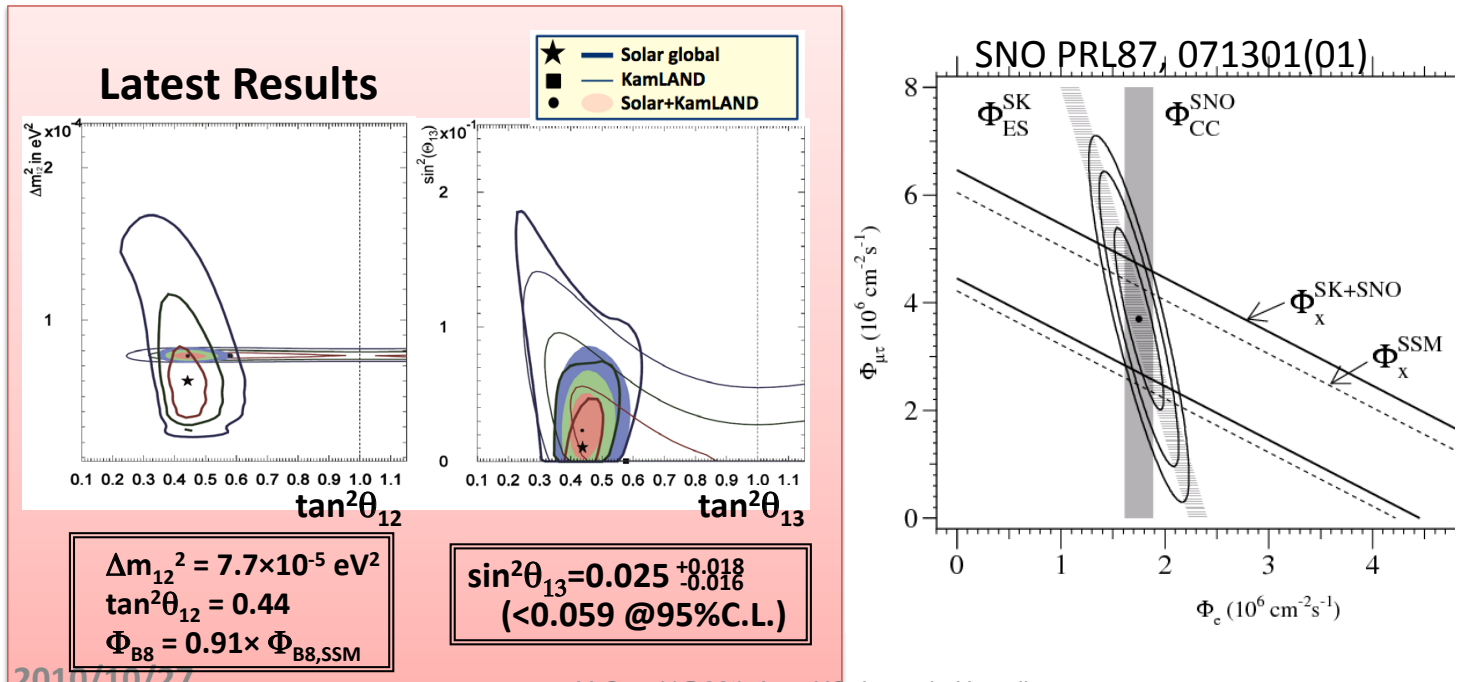


\section{$\mathrm{T} 2 \mathrm{~K}$}

- Look for $\theta_{13}$ : very small mixing angle $\left(v_{\mu} \rightarrow v_{\mathrm{e}}\right.$ appearance) $\rightarrow$ need statistics $\rightarrow$ higher beam intensity (K2K x 50)

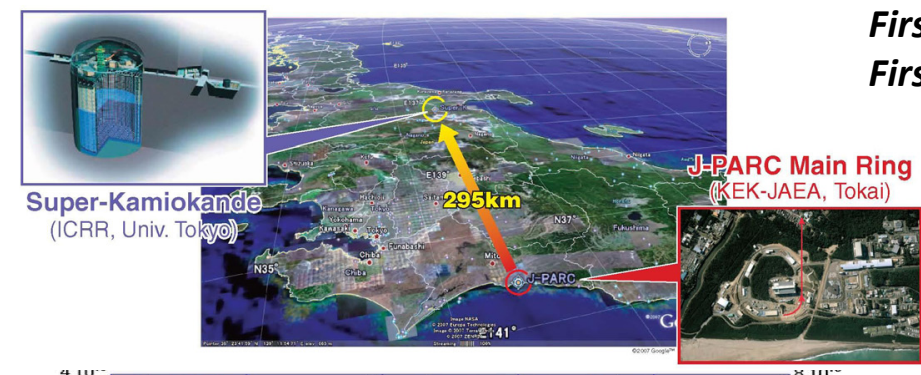

\section{First physics run}

Jan-23 $\rightarrow$ Jun-26, 2010

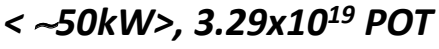
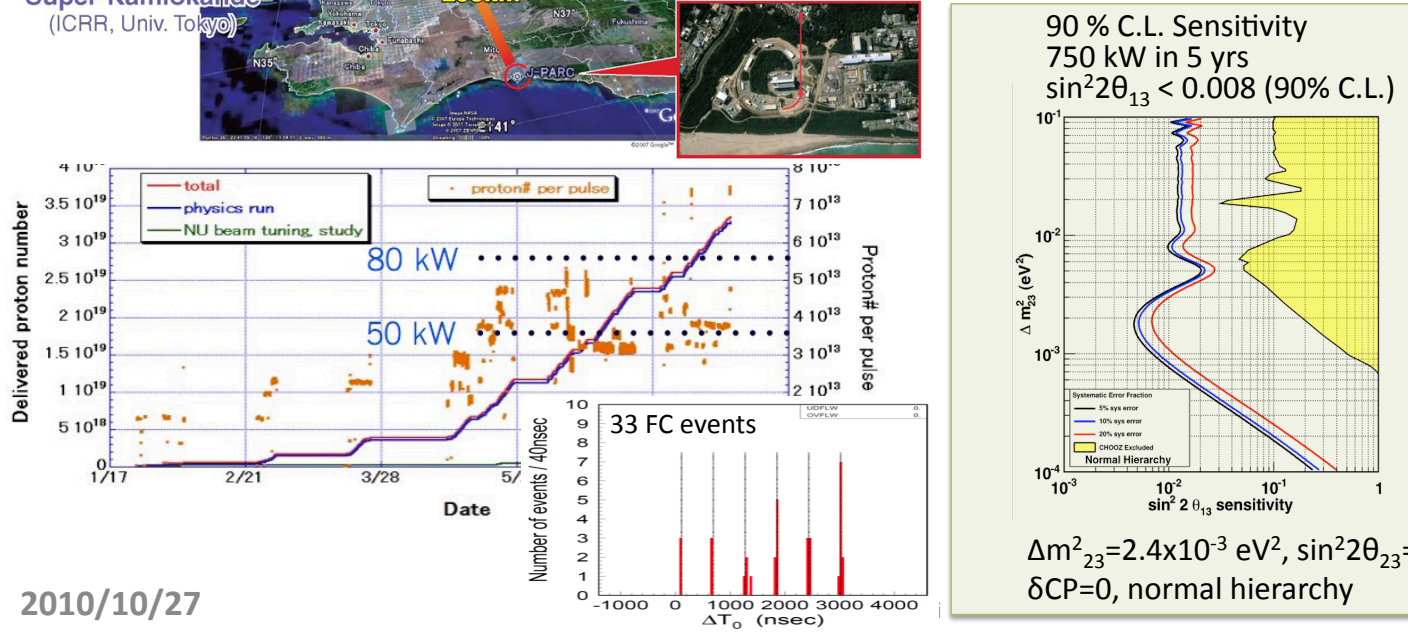

$\Delta \mathrm{m}^{2}{ }_{23}=2.4 \times 10^{-3} \mathrm{eV}^{2}, \sin ^{2} 2 \theta_{23}=1$, $\delta C P=0$, normal hierarchy

\section{The mission may continue to the Next generation detectors, Hyper-Kamiokandle and beyond}

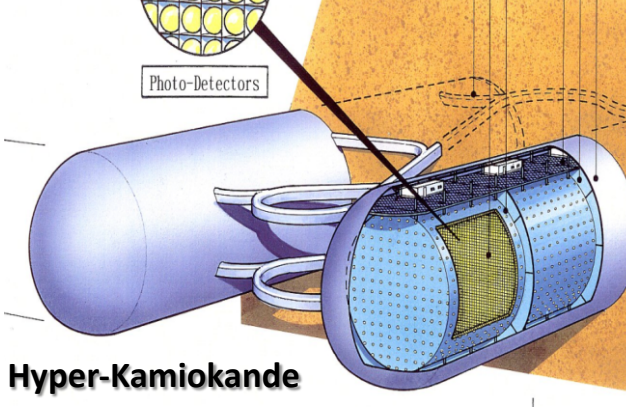

- Fiducial mass 540kt

$-\leftarrow 22.5$ kton: SK (x20)

- Precise study on solar and atmospheric neutrinos
- Tie up with the accelerator neutrino beam (a few MW)

- Study on CP Violation in neutrino sector

- Leads us to the origin of matter in the Universe

- Supernova neutrinos

- 200k neutrino events for SN at $8 \mathrm{kpc}$

- $250 v_{\mathrm{e}}$ from neutronization burst

- $\theta_{13}$ sensitivity to $\sin ^{2} \theta_{13}<10^{-4}$

- Proton decay

- $10^{35}$ yrs for $p \rightarrow e \pi^{0}$ mode

- Step into the discovery region 


\section{The Super-Kamiokande Collaboration \\ Henry W. Sobel \\ University of California, Irvine, CA 92697}

In the 1980's two large water Cherenkov detectors were built, one in Japan, the Kamiokande experiment, and one in the U.S., the IMB experiment. They were built to test the SU(5) Grand Unification Theory of Georgi, Glashow and Salam. This theory captured the imagination of the community and predicted that the proton would decay. Although the proton decay process had been search for previously, this theory gave the first definitive prediction of the lifetime and one could confidently design a detector that would be sensitive to it.

The Kamioka and IMB detectors were built and ran throughout the 1980's and though, unfortunately, they were unable to observe proton decay, thus definitively disproving SU(5), they were able to make other significant discoveries. Perhaps the most exciting discovery was the simultaneous observation of neutrinos from SN1987a in both detectors.

In 1991, Yoji Totsuka announced that he was successful in obtaining funding in Japan for a much larger version of the detector, Super-Kamiokande. At about the same time, the IMB Collaboration was turned down in the U.S. for the funding of their planned larger upgrade.

In 1992, the IMB Collaboration started discussions with Yoji Totsuka and Yoichiro Suzuki about the possibility of IMB joining forces to help build Super-Kamiokande. They proposed to use the IMB phototubes salvaged from the IMB detector to construct an outer anticoincidence for the detector and to bring essentially all of the IMB Collaboration to work on the construction and future operation. The proposal was accepted and in October 1992 an official collaboration agreement was signed.

The detector construction was completed in 1996 and the rest is history. It has been one of the most successful experiments and collaborations of the past decade. Our 1998 paper, which announced the discovery of neutrino oscillations, and therefore the existence of neutrino mass, is the fourth highest ranked experimental paper in Spires "Top Cites" of all time. As of 2009 it had over 3318 citations.

The detector productivity continues with the most recent inauguration of the T2K experiment which sends a neutrino beam from the new J-Parc facility to Super-Kamiokande $295 \mathrm{~km}$ away. 


\section{Kamiokande Detector circa 1980 built by ICRR University of Tokyo}

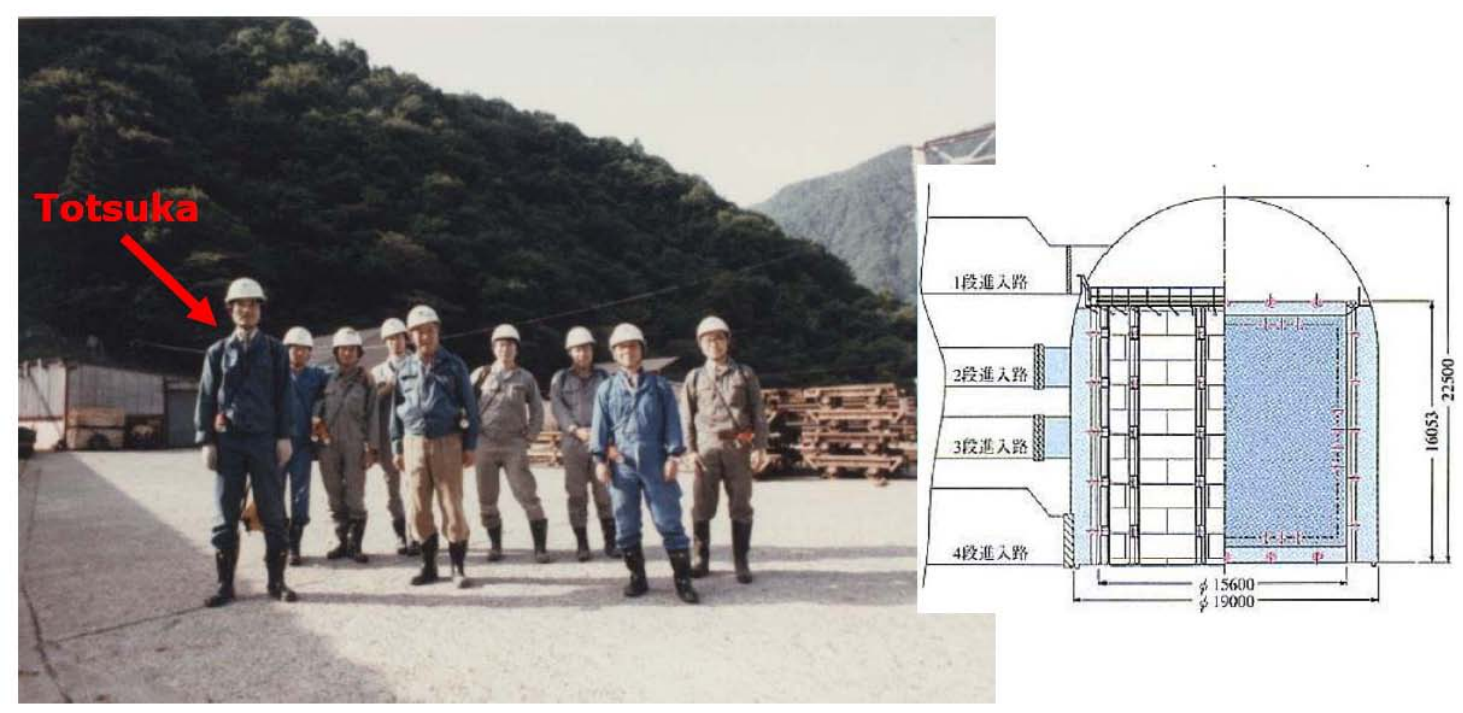

\section{IMB Detector Circa 1980 funded by US DOE}

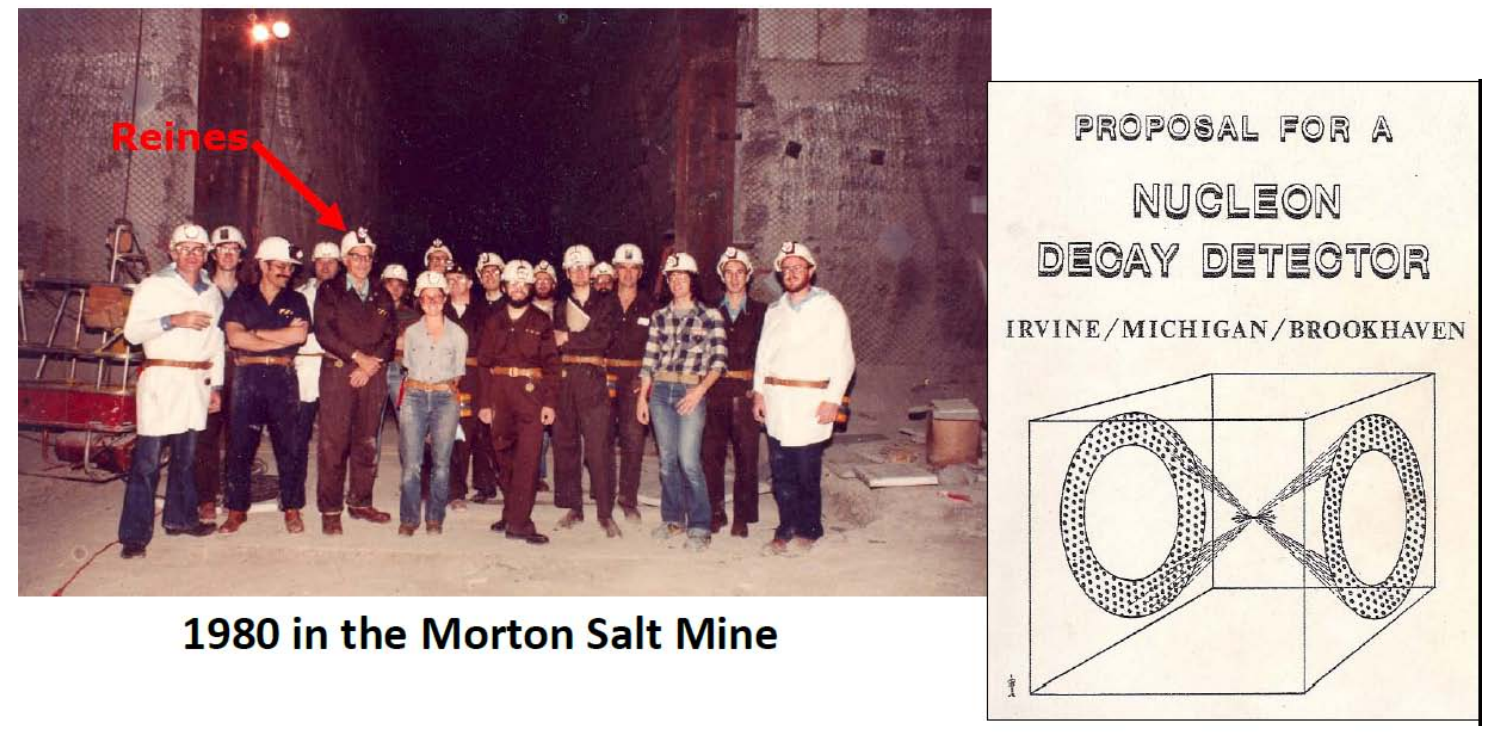




\section{Collaboration Agreement Signed October 18, 1992 in Takayama}
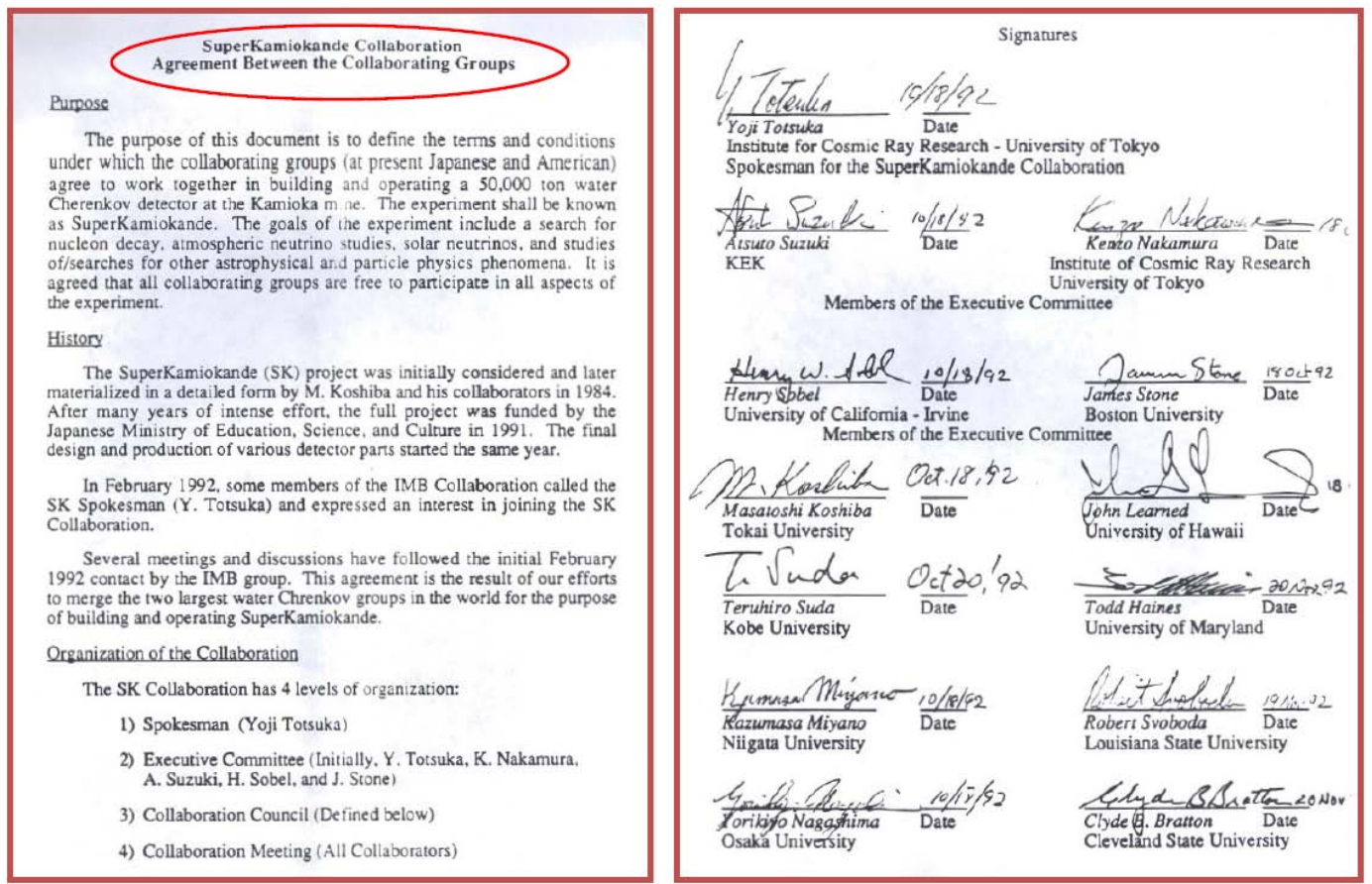

\section{Completion of Super-K I 1996}

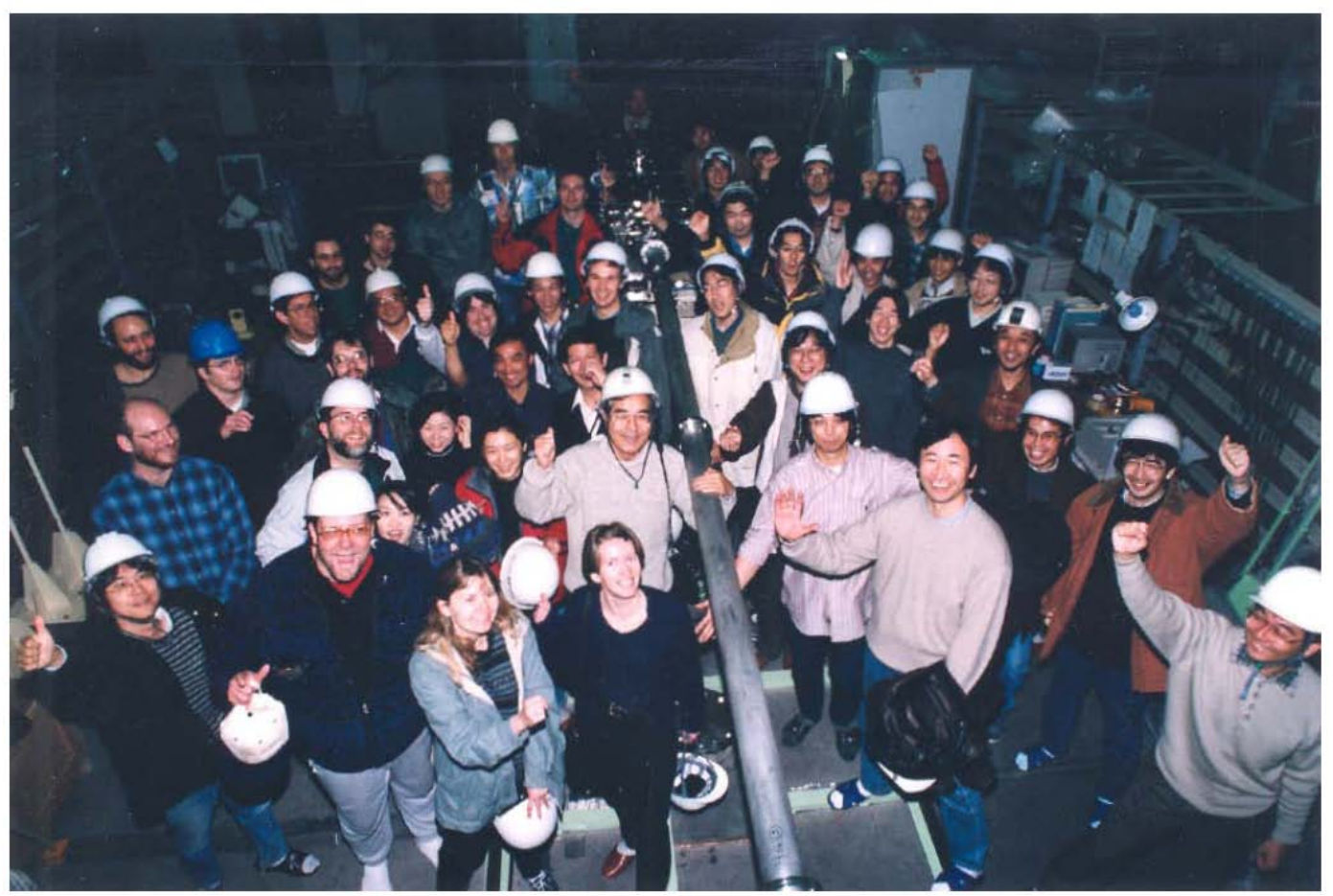




\section{Current View of Super-K}

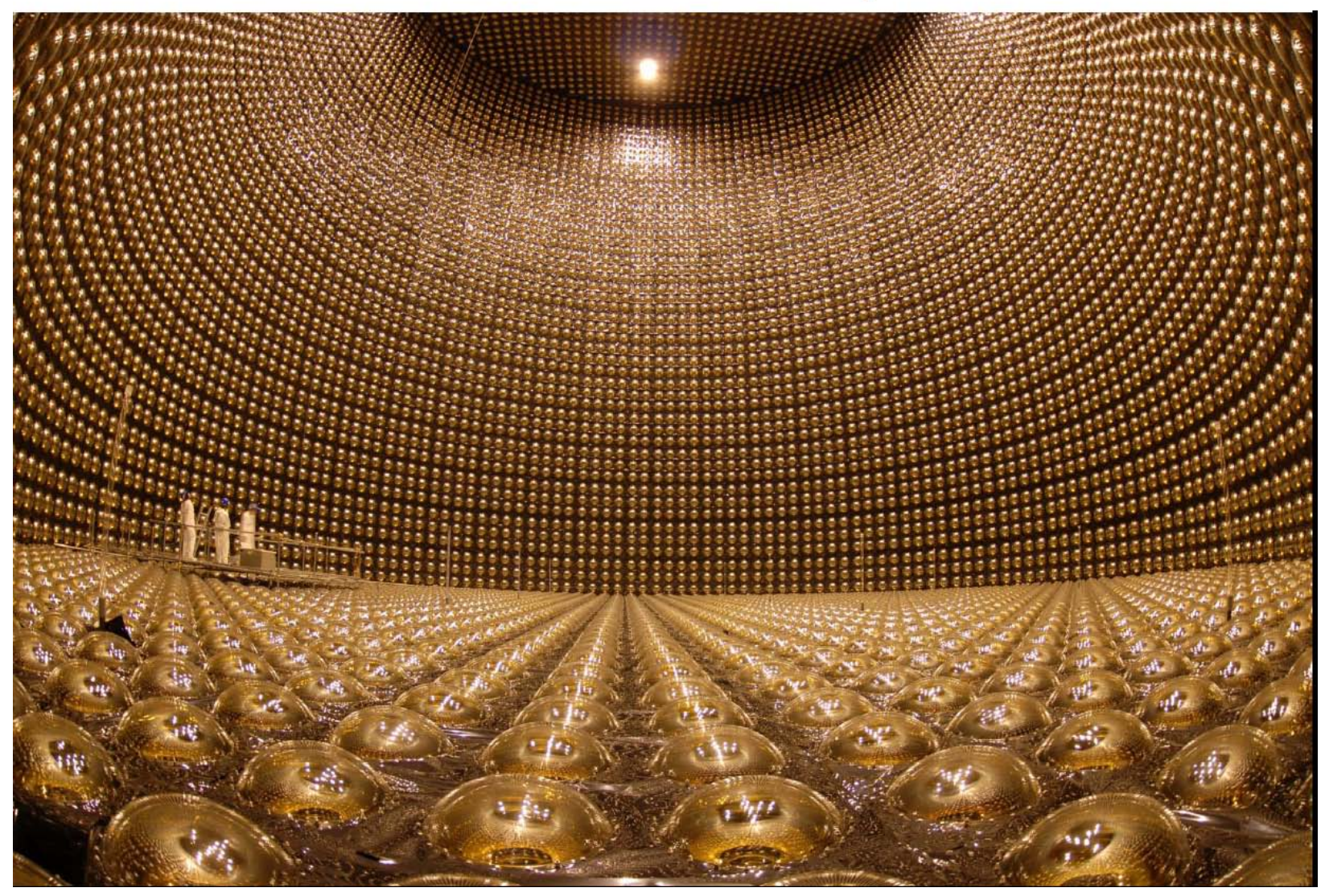




\title{
KamLAND
}

\author{
Kunio Inoue \\ Research Center for Neutrino Science, Tohoku University, Miyagi, Japan
}

\section{$\underline{\text { Neutrino Oscillation }}$}

KamLAND replaced the former Kamiokande detector and holds $1200 \mathrm{~m}^{3}$ ultra-pure liquid scintillator. Its primary target was to measure anti-neutrinos from surrounding nuclear power reactors, concerning the long-standing solar neutrino problem. The experiment started in 2002 and soon observed the evidence for reactor neutrino disappearance. It finally identified the large mixing angle neutrino oscillation as the solution for the solar neutrino problem through a process of elimination. And updated analyses with further data accumulation provided the apparent evidence of neutrino oscillation as two cycles of neutrino disappearance and reappearance, also resulted in the precise determination of a neutrino mass parameter.

\section{$\underline{\text { Neutrino Geophysics }}$}

This new understanding of neutrino propagation made it possible to utilize neutrinos as a tool to investigate optically opaque astronomical objects. KamLAND, at first, succeeded in detecting the geologically produced anti-neutrinos in 2005 and has opened the new interdisciplinary field "Neutrino Geophysics". Updated results in 2010 support the standard bulk silicate earth model and exclude a fully radiogenic model. Neutrino became a practical tool to discriminate geophysical earth models.

\section{Neutrino-less Double Beta Decay Search}

The ultra-low radioactivity environment achieved by KamLAND is adequate to search for rare phenomena. Xenon gas is soluble in the liquid scintillator more than $3 \mathrm{wt} \%$. Its isotopic enrichment and purification are already established. Very slow two-neutrino double beta decay of ${ }^{136} \mathrm{Xe}$ requires modest energy resolution. Thanks to these facts, the world most sensitive search for neutrino-less double beta decay can be performed by suspending a small balloon with xenon-loaded liquid scintillator at the center of KamLAND. It doesn't require a big modification to KamLAND and thus very cost effective. It will verify the degenerated hierarchy of neutrino masses with forthcoming KamLAND-Zen accommodating $400 \mathrm{~kg}$ of enriched xenon and will further survey the inverted hierarchy with the planned detector modification. 

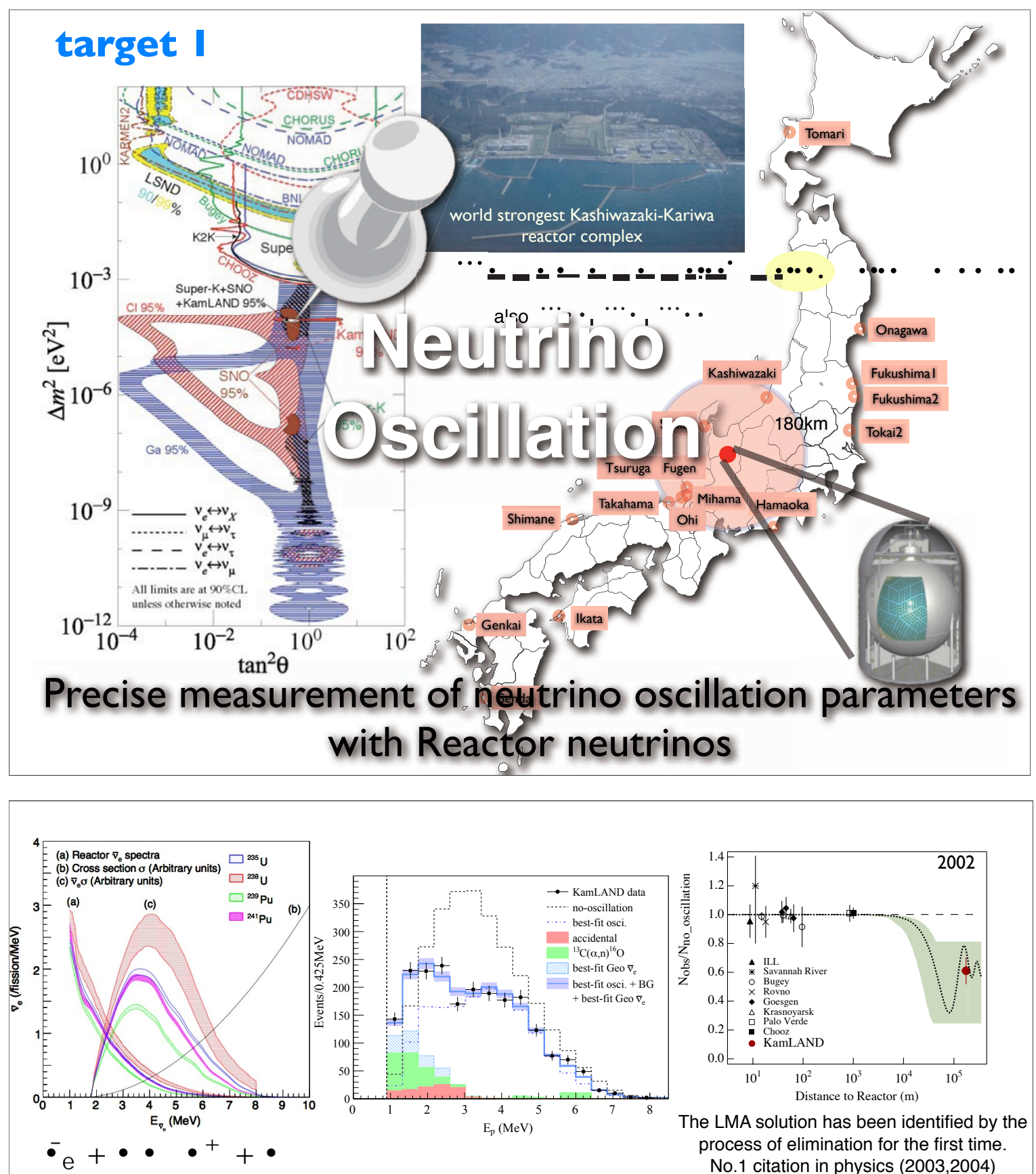

The LMA solution has been identified by the process of elimination for the first time. No. 1 citation in physics $(2003,2004)$

Two-fold delayed coincidence

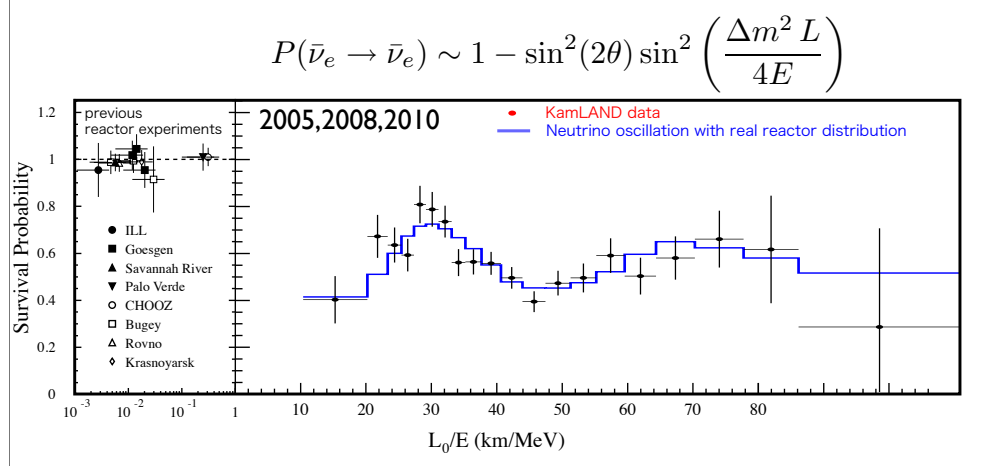

observed two cycles of oscillation

resulted in the precision measurement of $\Delta \mathrm{m}^{2}{ }_{21}$

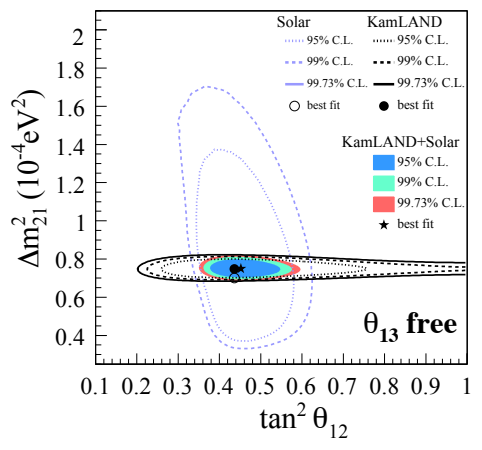

$\Delta m_{21}^{2}=7.49_{-0.20}^{+0.20} \times 10^{-5} \mathrm{eV}^{2}$ $2.7 \%$ precision ! 


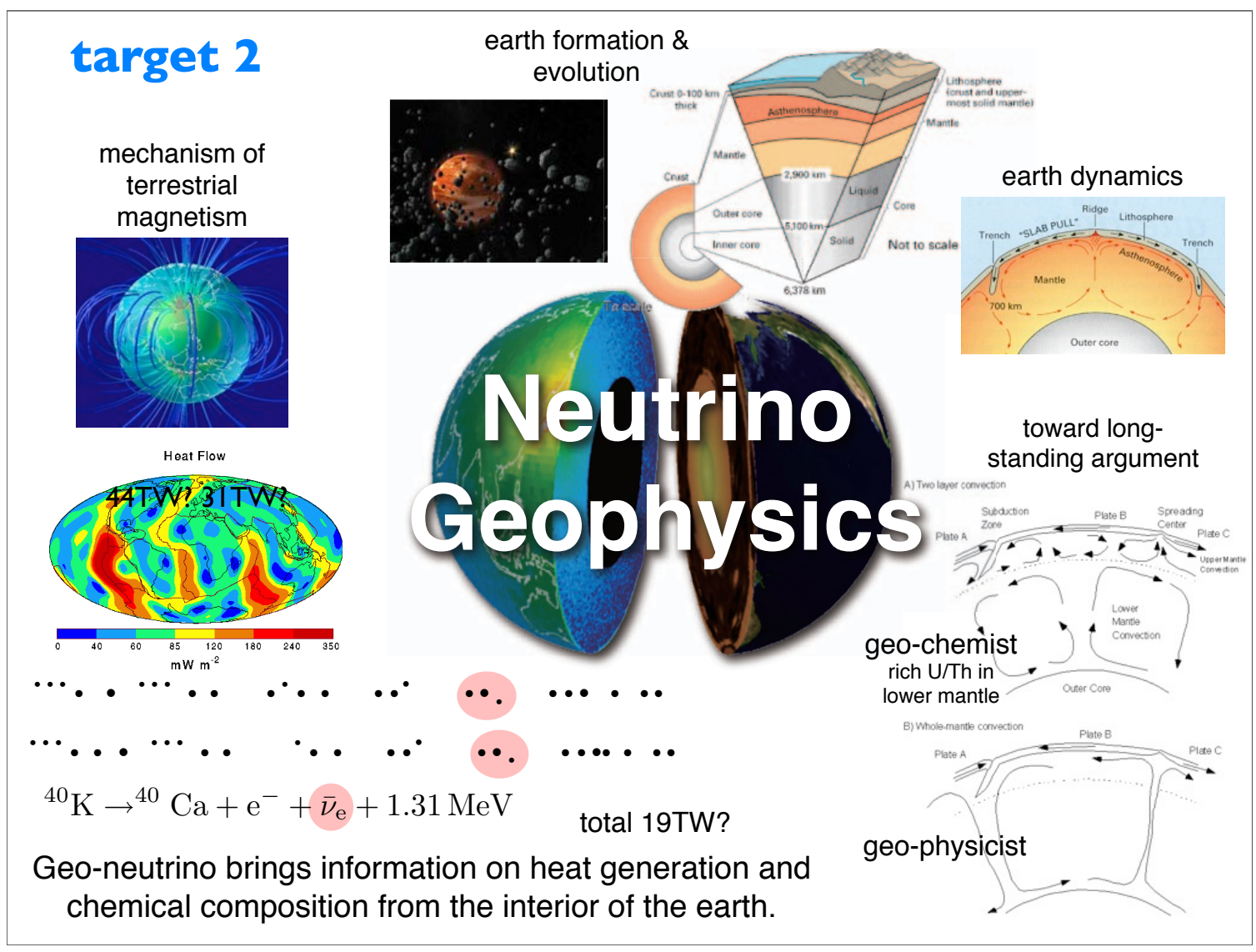

\section{Testing models of the Earth's composition}

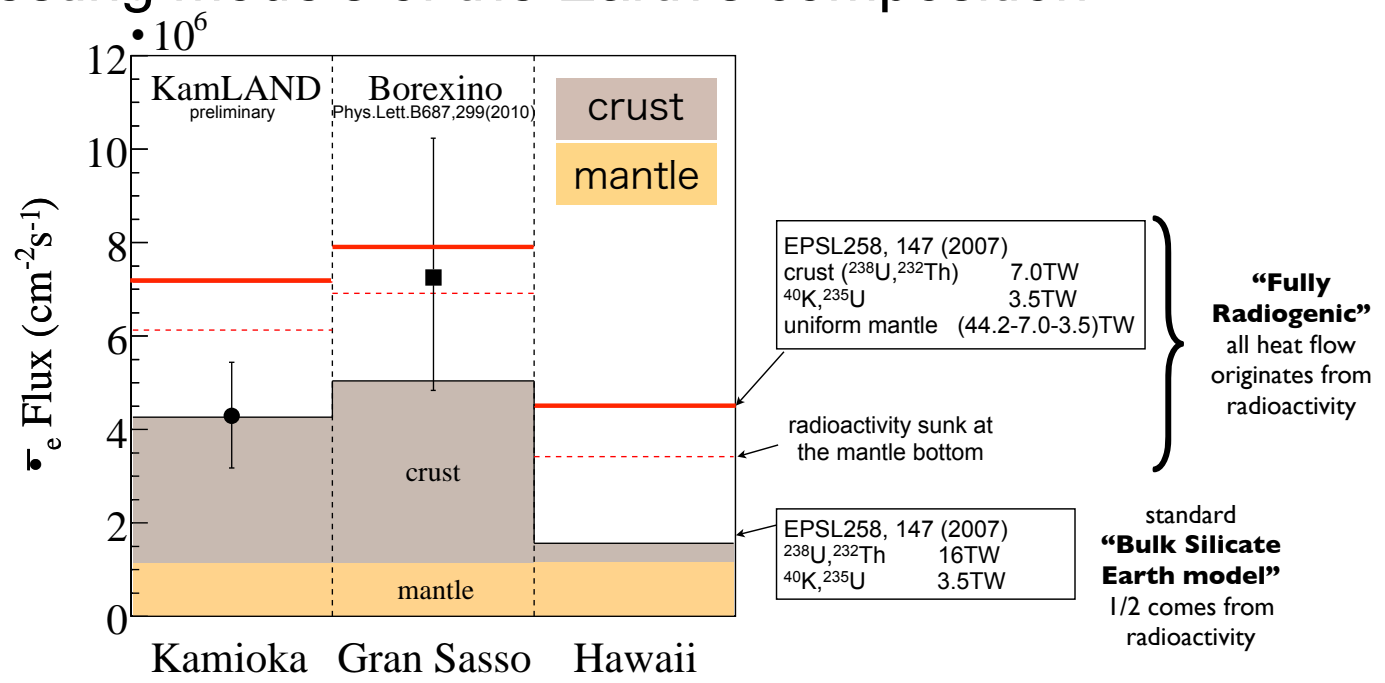

KamLAND pioneered "Neutrino Geophysics" and testing models of the Earth's composition has become possible. For the first time, fully radiogenic model (uniform mantle) was excluded at $2.4 \sigma$ level. The KamLAND result agrees well with the standard BSE model.

From a geophysical point of view, extracting the mantle contribution is very important. In the future, the combination of data from multiple sites and possible data from an oceanic experiment (where the crust is much thinner and so its contribution much smaller) will provide stronger constraints. 

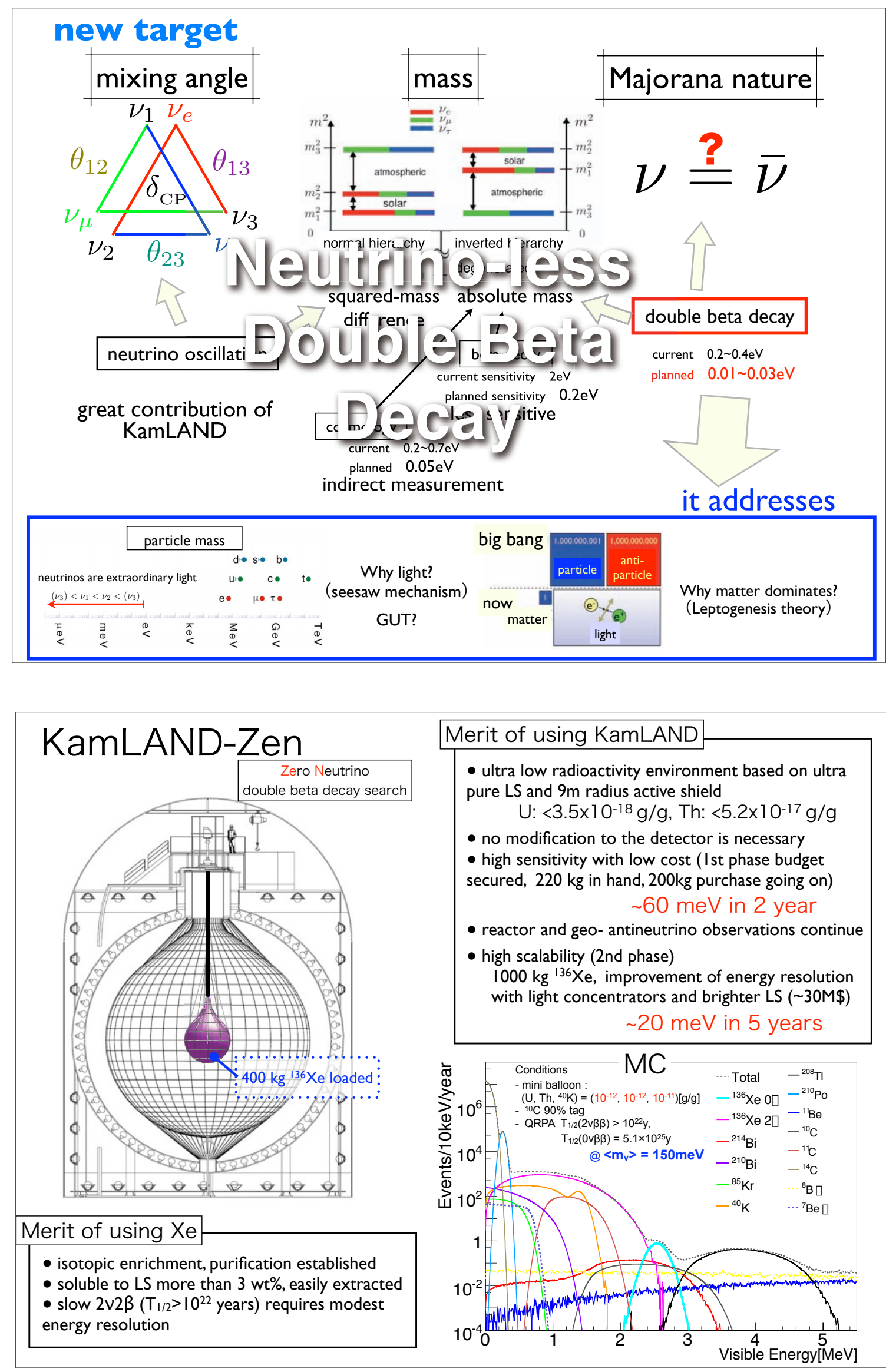


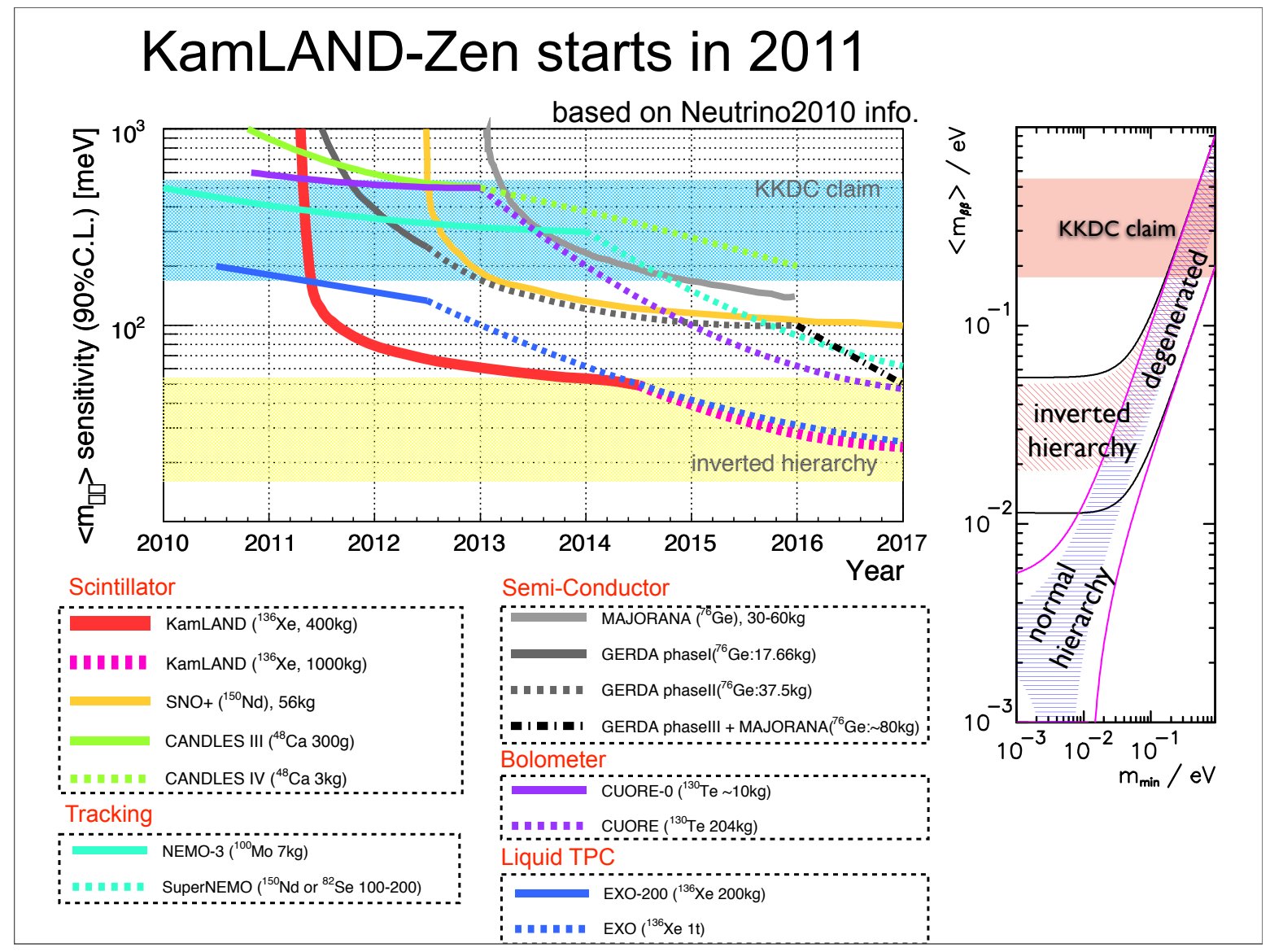

\section{KamLAND-Zen future prospects}

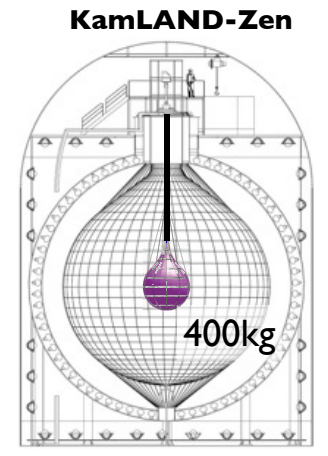

20II

covers

degenerated

$\sim 60 \mathrm{meV}$

forthcoming

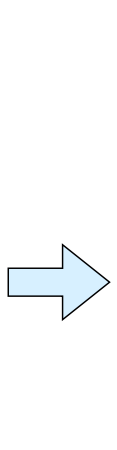

KamLAND2-Zen

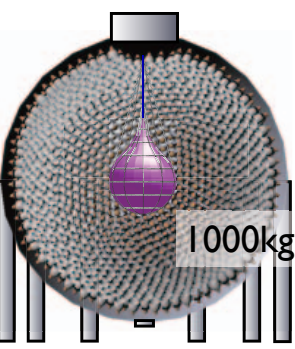

20|4 ?

covers inverted hierarchy

20meV

light-concentrator \& brighter LS

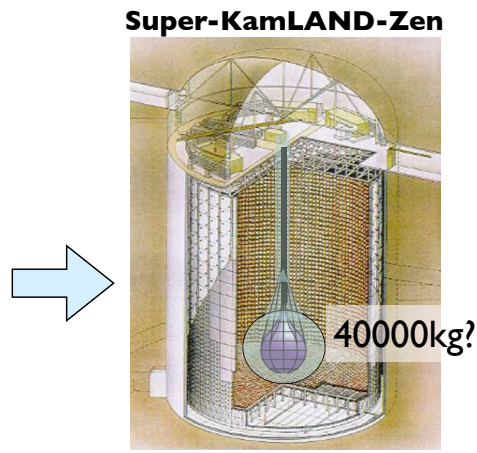

2020 ??

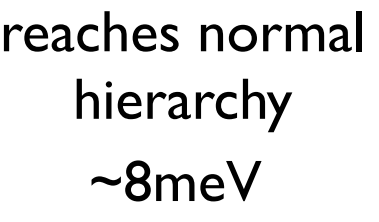

high pressure $\mathrm{Xe}$ imaging device $\mathrm{w} / \sim$ |\% coverage 



\title{
U.S./JAPAN Collaborative Accelerator R\&D: JLC/NLC/ILC
}

\author{
Gregory Loew \\ SLAC National Accelerator Laboratory, Menlo Park, CA
}

Accelerator R\&D under the Collaboration started in 1979 and evolved through two phases. The first phase lasted until 1985, and did not focus on any central topic. It involved studying RF superconductivity with $\mathrm{M}$. Tigner at Cornell University and Y. Kojima at KEK, developing a 150 MW S-Band klystron with G. Konrad at SLAC, J. Tanaka at KEK, and Mitsubishi/Toshiba, and exploring surface physics for RF sources and klystron windows at SLAC. The second phase from 1986 to the present addressed linear collider (LC) R\&D almost exclusively; from 1987, it involved many annual meetings and workshops. The KEK/SLAC LC workshop of March 1-4, 1988 at KEK played a seminal role (see photo and attendees). In June 1990, Directors H. Sugawara and B. Richter decided that these two institutes should focus on designing a 500 $\mathrm{GeV}$ c.m. LC. On June 23, 1994 the Collaboration was honored by the visit to SLAC of their Majesties, the Emperor and Empress of Japan (see photos). In 1995, the first ILC Technical Review Committee Report published the details of three JLC LC designs at S-Band, C-Band, and X-Band, and one NLC (SLAC) X-Band design (see schematics).

Between 1995 and 2004, the Collaboration was responsible for many technological advances. These included developing X-band klystrons with solenoidor permanent magnet focusing, $\mathrm{X}$-Band accelerator structures with wakefield suppression and up to $80 \mathrm{MV} / \mathrm{m}$ gradient, and $\mathrm{X}$-Band RF pulse- compression schemes using delay lines and reaching factors of 4 power gains with up to $600 \mathrm{MW}$ pulsed-peak output. An X-Band $350 \mathrm{MeV}$ test linac using these systems was built at SLAC. The SLAC Final Focus Test Beam was used to verify techniques to focus and measure ultra-small beams down to 70 nanometer vertical sigma. T. Shintake devised a revolutionary instrument to make these measurements using a laser-Compton profile monitor. By 2003, all these collaborative efforts converged on a common KEK-SLAC X-Band $500 \mathrm{GeV}$ c.m. LC design (see illustrations).

Following the August 2004 ICFA recommendation to concentrate on a single LC design using RF superconducting technology, the Collaboration broadened and now is working predominantly on the "cold" ILC design (as shown), although some X-Band work continues for possible applications to CERN's CLIC design. 


\section{KEK/SLAC LC WORKSHOP March 1-4, 1988 at KEK}

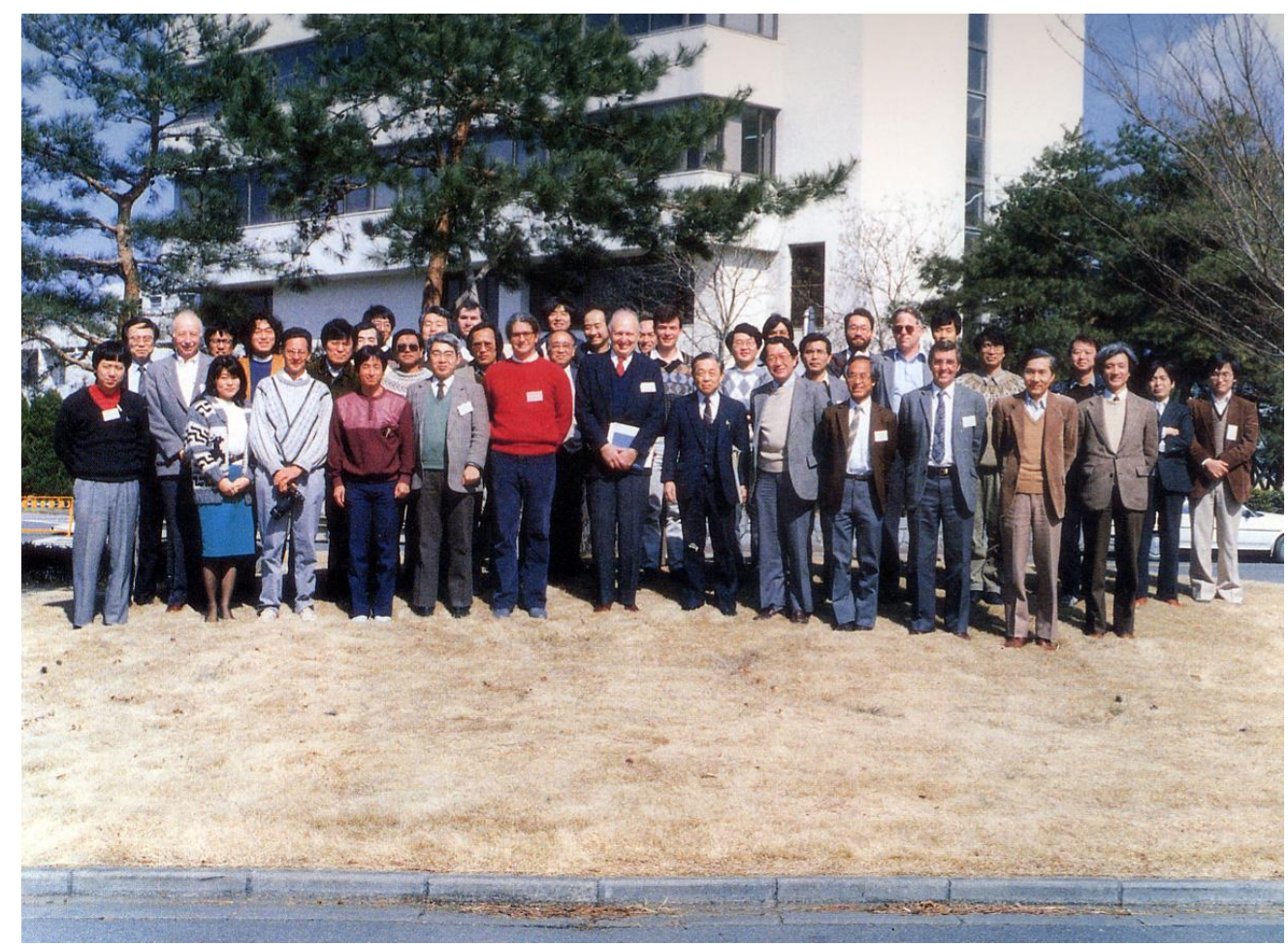

\section{Major Participants, starting at 1988 KEK Workshop}

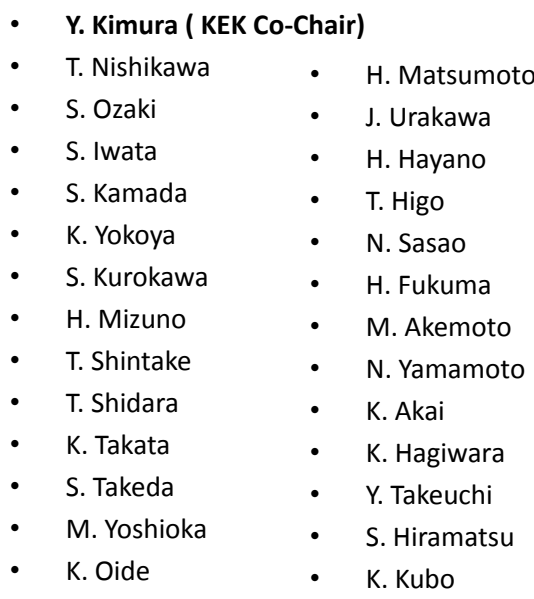

\begin{tabular}{|c|c|c|}
\hline \multicolumn{3}{|c|}{ G . Loew (SLAC Co-Chair) } \\
\hline B. Richter & & Later joined by: \\
\hline J. Paterson & • & D. Burke \\
\hline R. Ruth & • & T. Raubenheimer \\
\hline M. Allen & - & J. Wang \\
\hline J. Sheppard & • & G. Spalek \\
\hline N. Toge & • & T. Lee \\
\hline R. Palmer & • & T. Lavine \\
\hline & • & H. Hoag \\
\hline & • & J. Frisch \\
\hline & 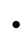 & A. Vlieks \\
\hline & $\bullet$ & G. Bowden \\
\hline & • & N. Phinney \\
\hline & • & M. Ross \\
\hline & & S. Tantawi \\
\hline & & D. McCormick \\
\hline & & K. Jobe \\
\hline & & T. Smith \\
\hline & & P. Tenenbaum \\
\hline & • & D. Yeremian \\
\hline & • & C. Adolphsen \\
\hline
\end{tabular}




\section{Their Majesties' Visit at SLAC June 23, 1994}
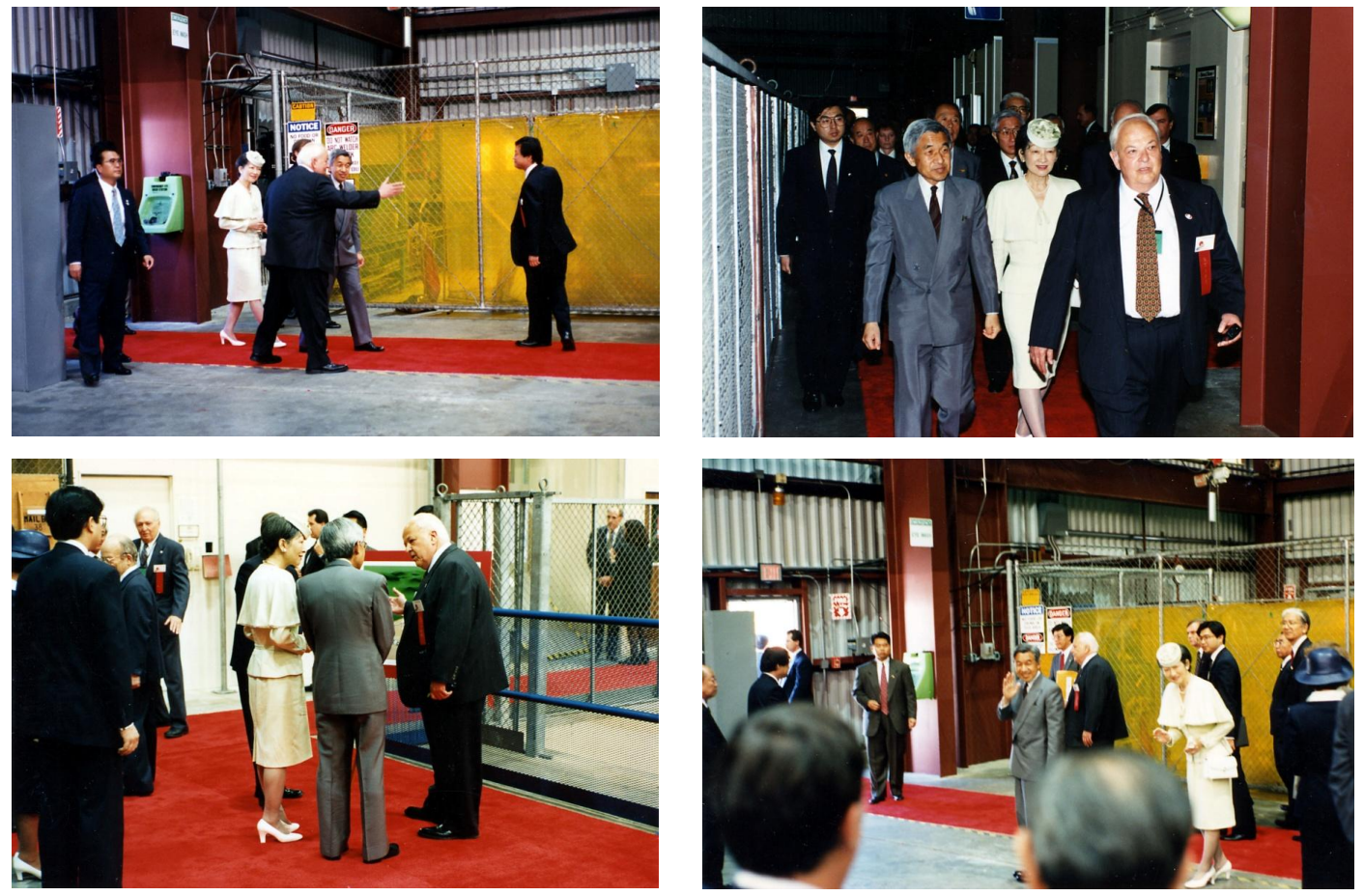

The Original S-Band, C-Band and X-Band JLC's (left) and X-Band NLC (right) in the 1995 ILC TRC Report
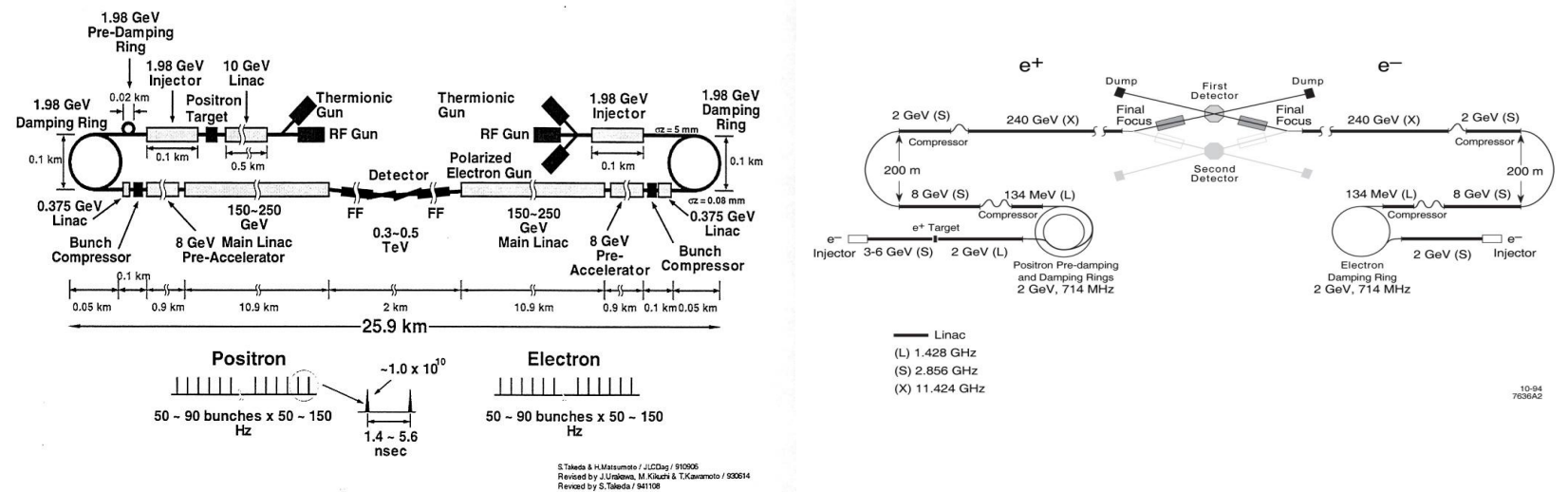
75 MW X-Band Klystron with PPM Focusing
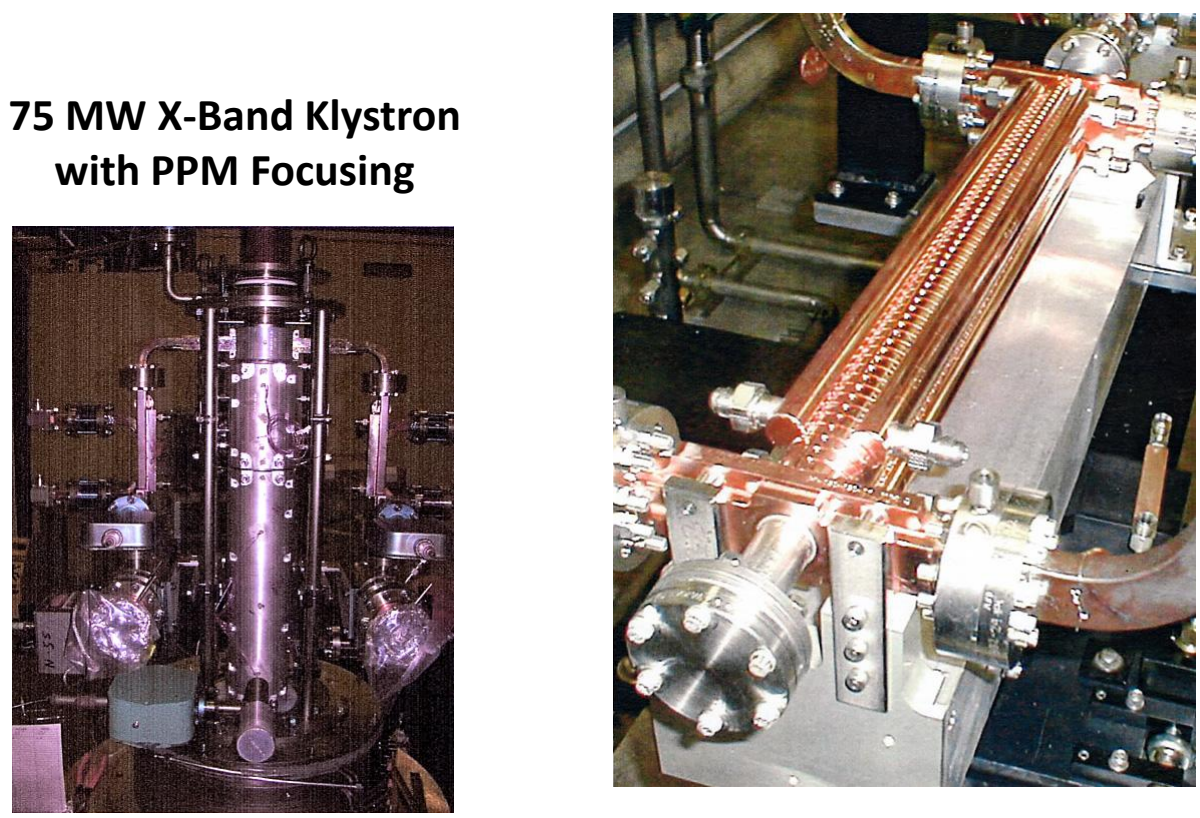

X-Band Linac Section with $80 \mathrm{MV} / \mathrm{m}$ gradient and wakefield suppression

RDDS Cutaway View

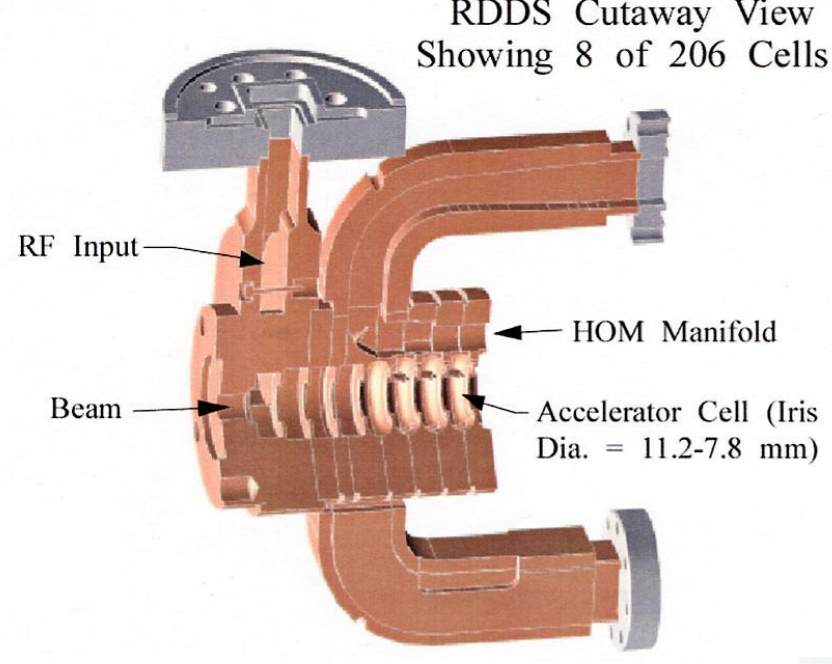

\section{Details of structure design}

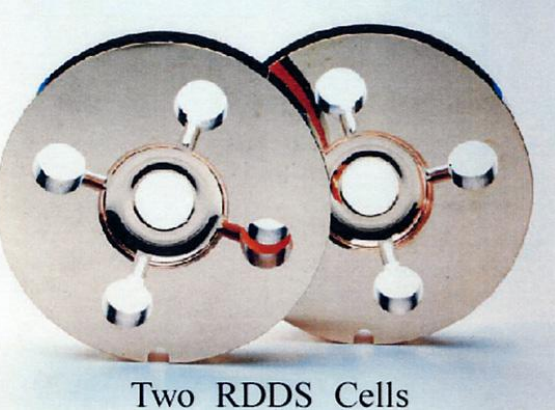




\section{Pulse Compression using Delay Lines producing 600 MW Peak X-Band Power}
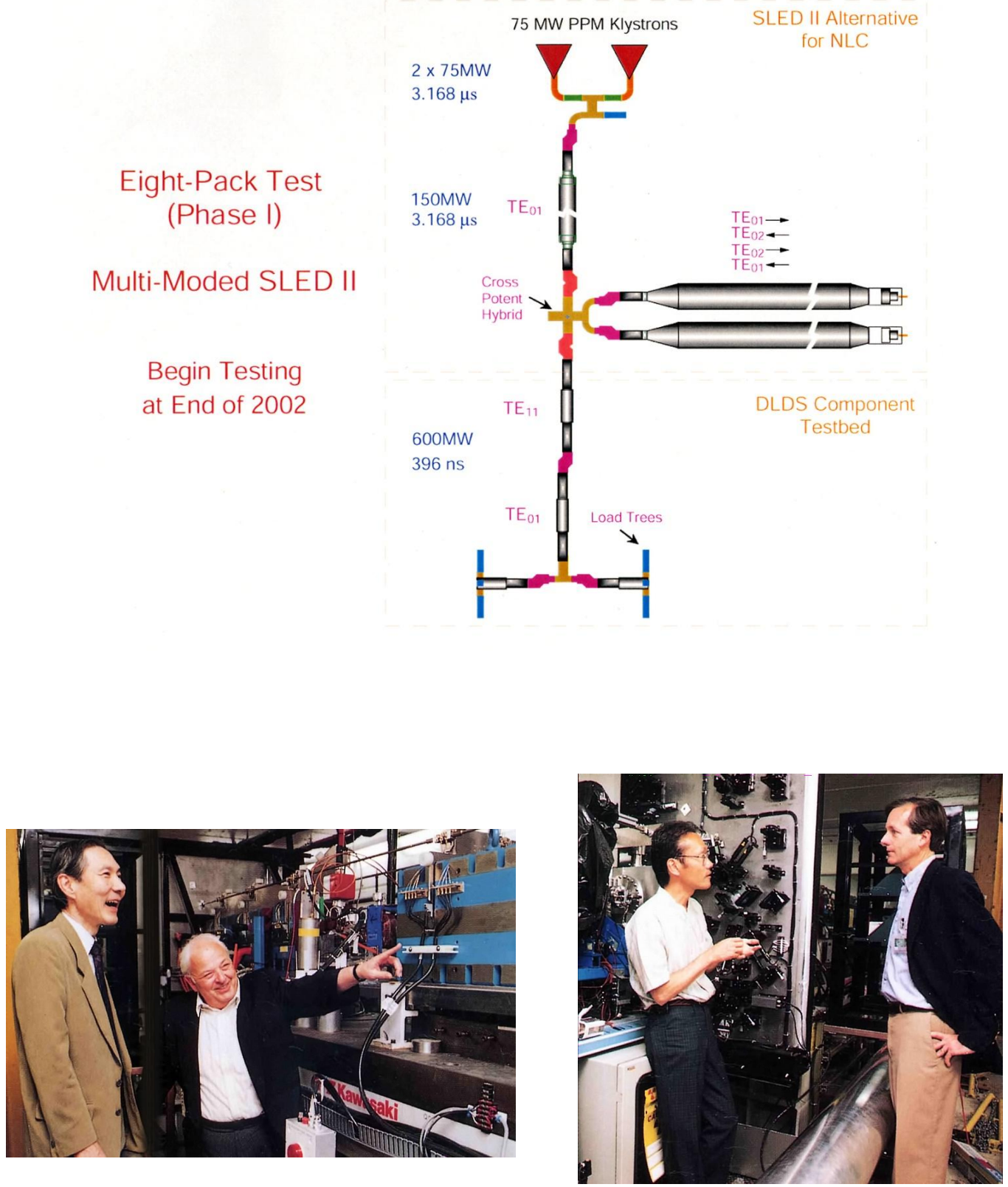

Final Focus Test Beam and Laser-Compton

Fringe Monitor

Measured sigma y of about $70 \mathrm{~nm}$ at $48 \mathrm{GeV}$

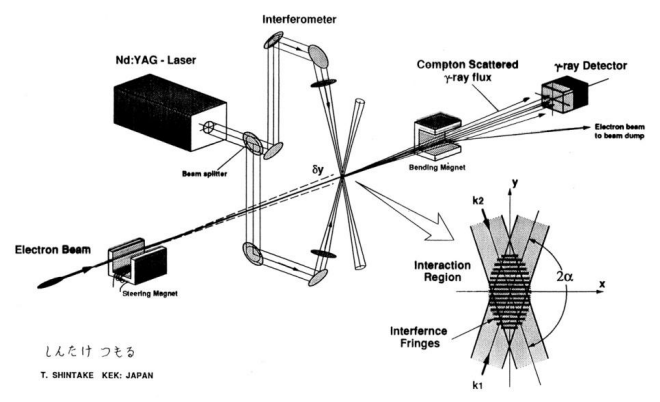




\section{JLC-X/NLC Common Design in 2003 ILC TRC Report}

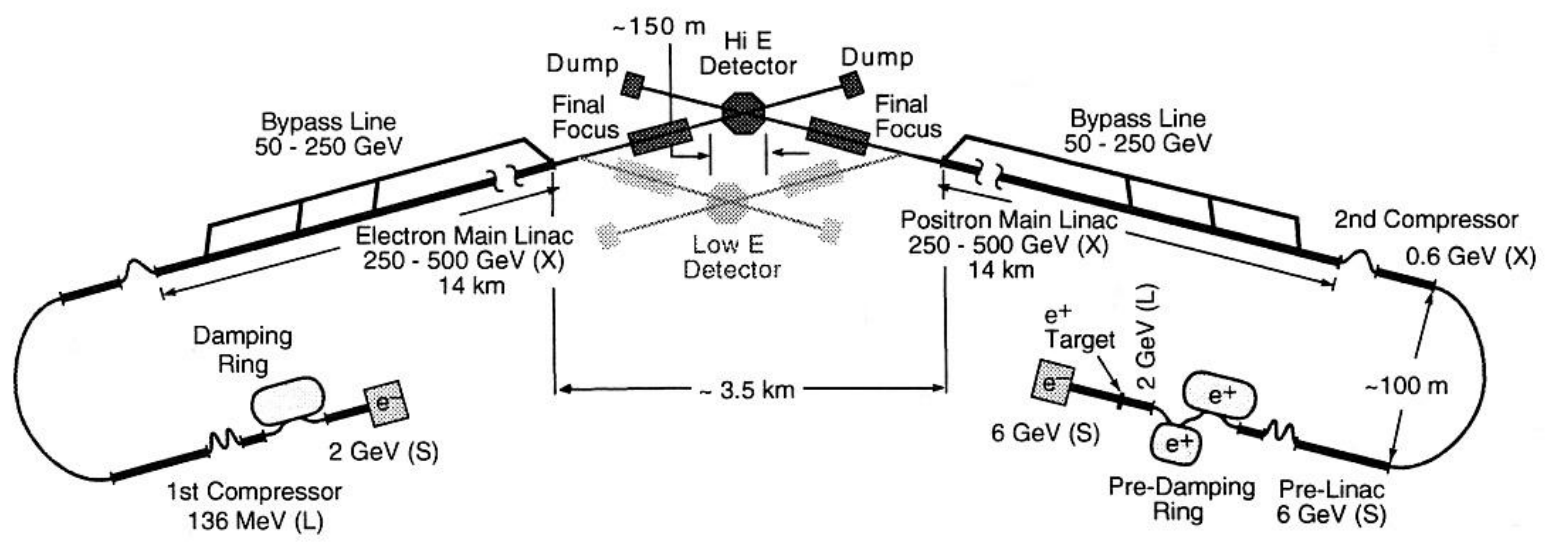

Components of the SB2009 ILC Design for Study and Review during 2010.

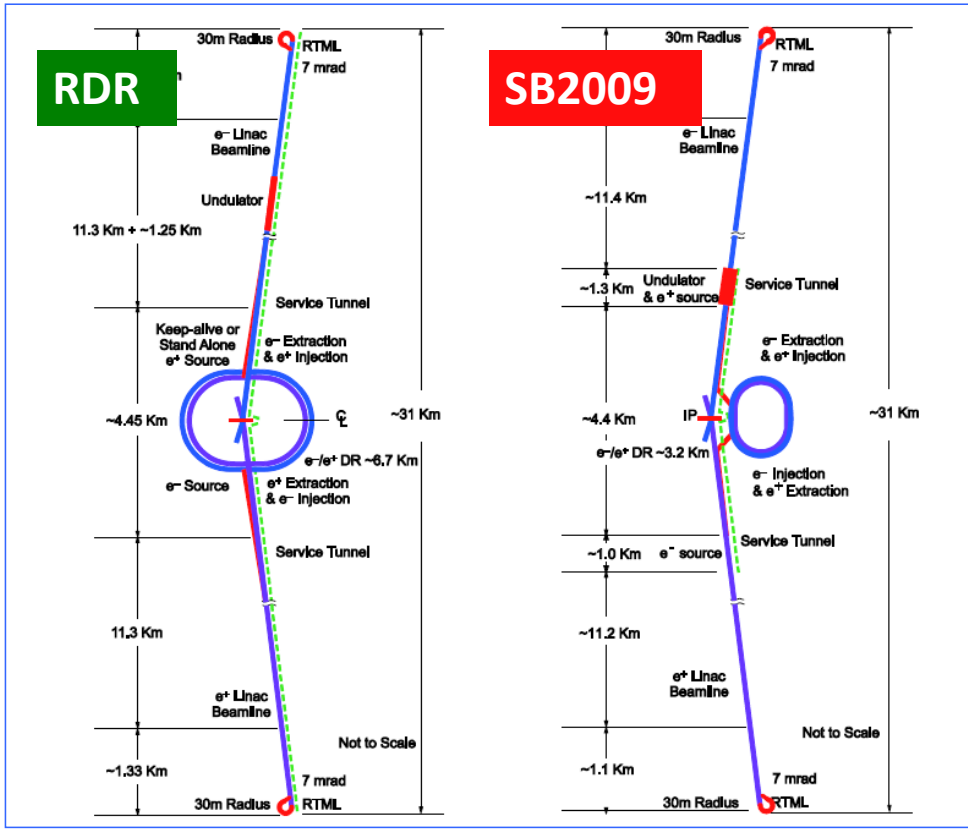

- Single Tunnel for main linac

- Move positron source to end of linac

- Reduce number of bunches factor of two (lower power)

- Reduce size of damping rings $(3.2 \mathrm{~km})$

- Re-evaluate optimum accelerating gradient

- Integrate central region

- Single stage bunch compressor

- Site dependent optimization 


\section{One Example of Site Dependent Optimization}

Process and Input to BAW-1

\section{A Review on Design Study of the ILC Conventional Facility in Mountain Region}

A Review on Design Study of the ILC Conventional Facility in Mountain Region
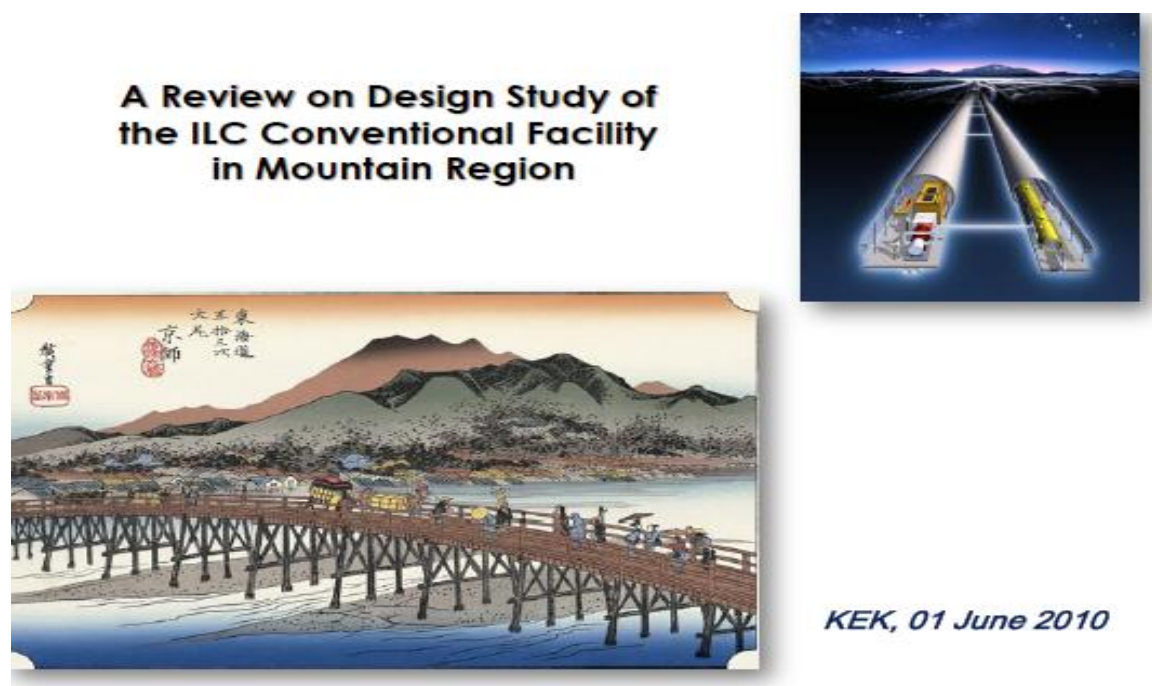

KEK, 01 June 2010 



\title{
Accelerator R\&D for LC - activities on ATF \& STF
}

\author{
Seiya Yamaguchi \\ High Energy Accelerator Research Organization, Tsukuba, Ibaraki, Japan
}

A number of research programs have been conducted at the Accelerator Test Facility (ATF) and Superconducting rf Test Facility (STF) for development of linear collider.

The ATF is a research center for studies on issues concerning the injector, damping ring (DR) and beam delivery system for the linear collider. The ATF is composed of $1.3 \mathrm{GeV}$ S-band linac, DR, and ATF2 beam line. The construction of linac, DR, ATF2 was completed in 1995, 1997 and 2008, respectively. Some research highlights at the ATF are summarized below.

- Interaction Point Beam Size Monitor (IP-BSM): The measurable beam size was improved from $70 \mathrm{~nm}$ to $35 \mathrm{~nm}$ by changing wavelength of laser from $1064 \mathrm{~nm}$ to $532 \mathrm{~nm}$.

- Fast Kicker: Beam extraction test from ATF DR to ATF2 beam line was performed with successful results.

- Feedback On Nano-second Time scale (FONT): The beam train (bunch spacing is $154 \mathrm{~ns}$ ) was successfully straightened by the FONT system.

The STF is a facility for R\&D of superconducting RF acceleration. The objectives of the STF are 1) to improve performance of superconducting cavity and 2) to demonstrate superconducting accelerator system. Main results are as follows.

- Development of high-gradient superconducting cavity: KEK's cavity was tested at JLAB with improvement of gradient. FNAL contributed to S1-global project, which is an international project conducted in the STF for demonstration of plug compatibility of accelerating module and operation of $31.5 \mathrm{MV} / \mathrm{m}$.

- Camera for observation of inner surface of superconducting cavity: This camera (developed by KEK and Kyoto University) is very useful tool to find defect or contamination in cavity. FNAL introduced the camera and KEK loaned the camera to JLAB.

- Pre-tuning machine: This pre-tuner was developed under collaboration between FNAL, DESY and KEK. KEK stuff visited FNAL to take training in July 2010 and the machine was delivered to KEK in Oct. 2010.

- RF-gun for ultra-low emittance beam: FNAL fabricated RF cavity and input coupler. KE fabricated photocathode and solenoid coil. The beam test is scheduled in 2011 at the STF. Using the ATF and STF, we established many advanced accelerator technologies for high quality beam and superconducting RF acceleration for linear collider. 


\section{Introduction}

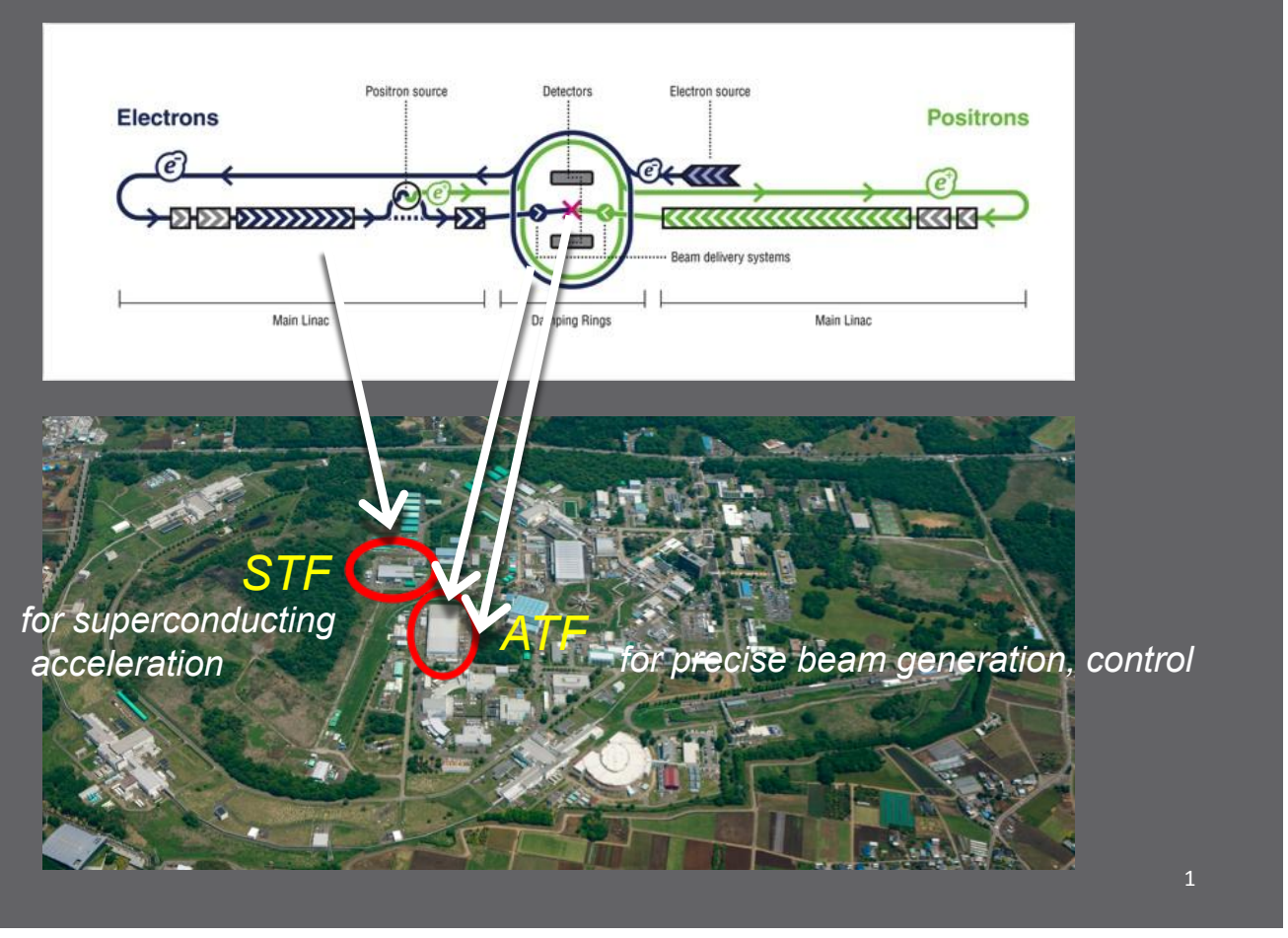

\section{Fast Kicker (KEK, SLAC)}

- rise/fall time less than $3 \mathrm{~ns}$.

- US team (SLAC) to develop high-speed pulsed power supplies. $(10 \mathrm{kV})$

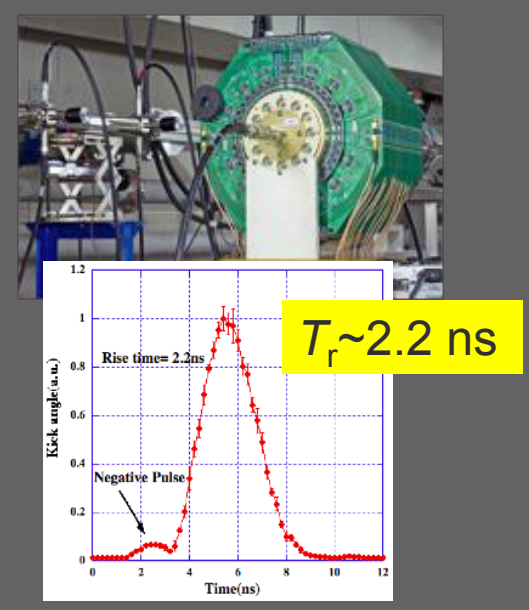

- KEK to develop strip-line electrode.
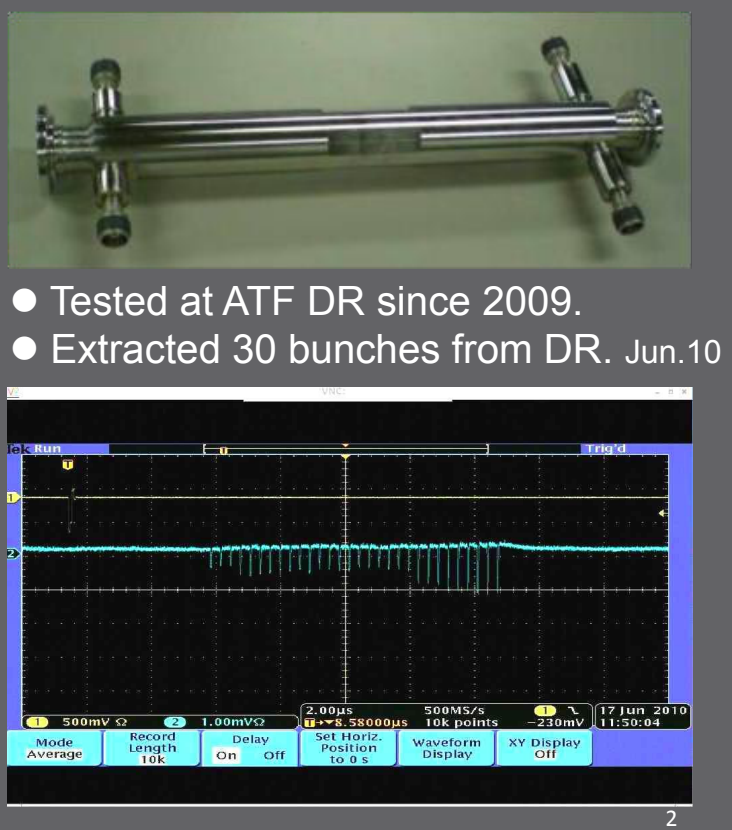


\section{Fast Feedback (KEK, SLAC)}

(FONT : Feedback On Nanosecond Time scale)

- Feedback and Feed forward will be used to straighten the train.

- FONT : latency estimate

- irreducible latency $=14 \mathrm{~ns}$

- electronics latency=118 ns

- total latency of FB loop

$$
=132 \mathrm{~ns}<154 \mathrm{~ns}
$$
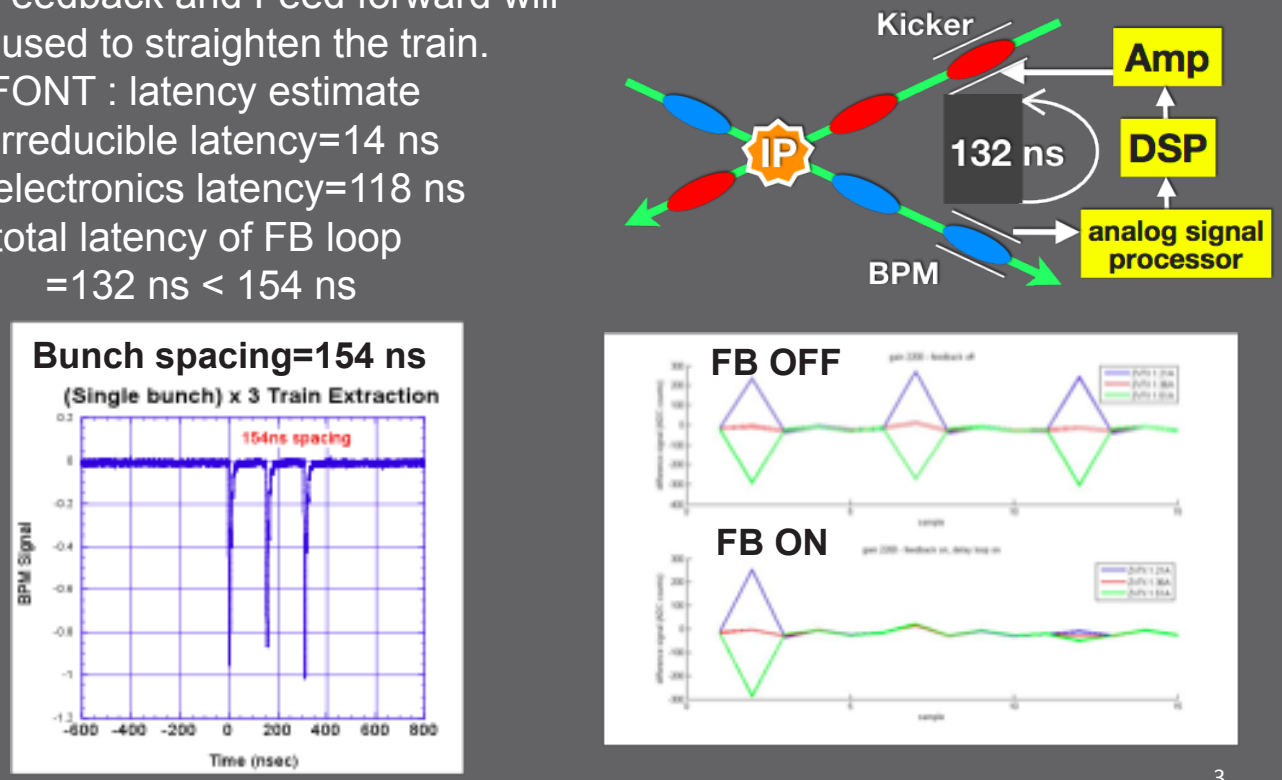

\section{High Gradient SC Cavity Development}

- VT at JLAB

- KEK cavity tested at JLAB

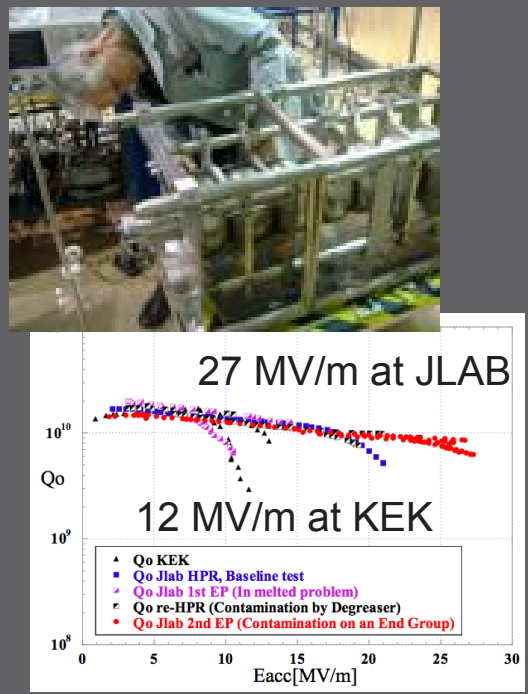

- S1-Global at KEK

- demonstrate plug compatibility

- operate at $31.5 \mathrm{MV} / \mathrm{m}$

- two cavities from FNAL, two from DESY KEK supplies 4 cavities
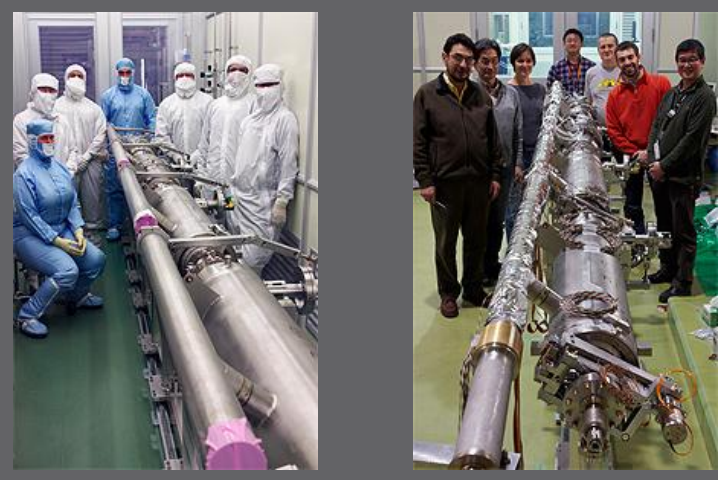

- assembly completed, RF test in CM on going. 


\section{Camera for observation of inner surface (KEK, Kyoto U, FNAL, JLAB)}

- To find defect, contamination in cavity

- FNAL introduced the inspection camera.

- KEK loaned the camera to JLAB for 1 year.

- Improvement of the camera for more bright image and high resolution
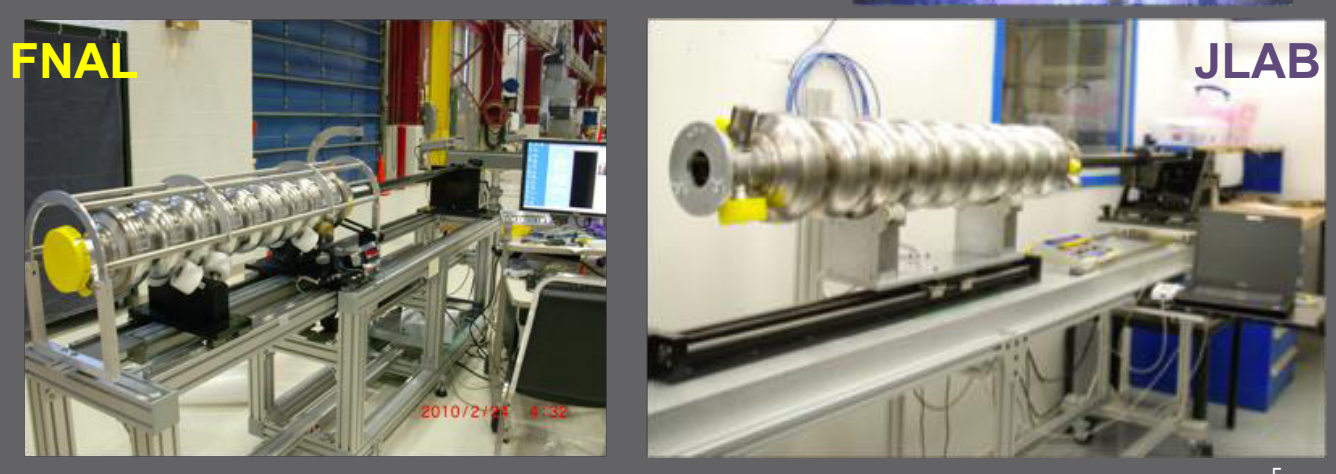

\section{Pre-tuning machine for 9-cell cavity}

- SC : deep drawing

$\rightarrow$ deform cavity shape

- FNAL-DESY-KEK collaboration

- Hardware and Software fabrication are complete.

- Training course at FNAL on Feb. 2010.

- Delivered to KEK Sep. 2010.

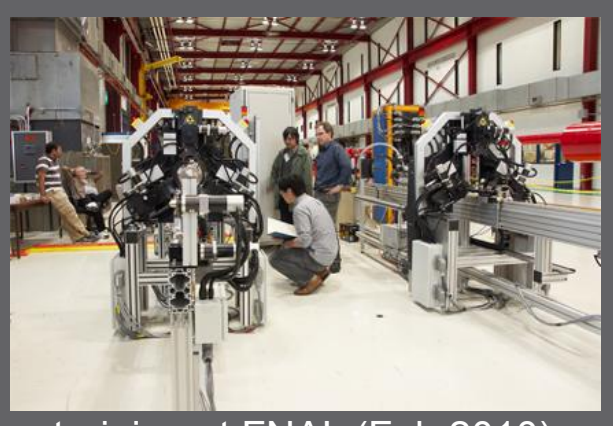

training at FNAL (Feb 2010)
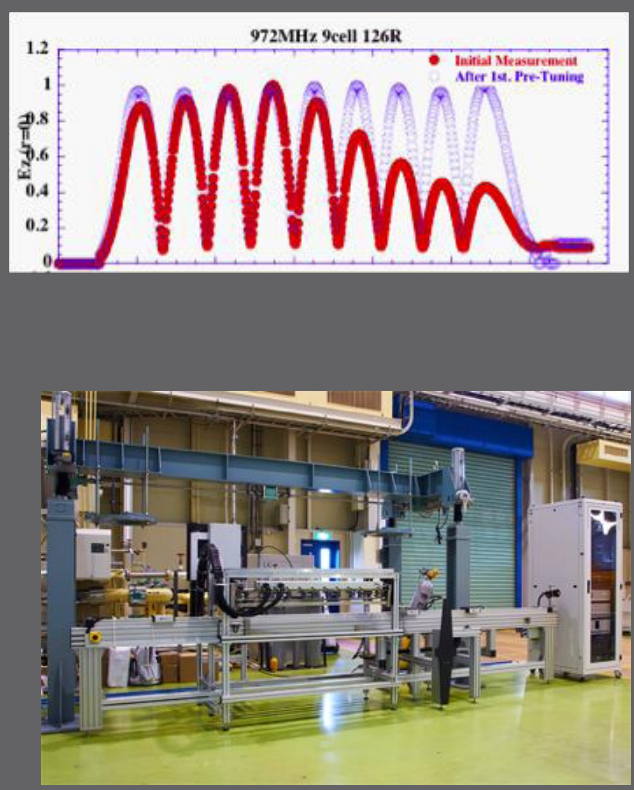

Delivered to at KEK (Sep.2010) 6 


\title{
Development of Superconducting Magnets for Particle Accelerators and Detectors
}

\author{
Takakazu Shintomi and Akira Yamamoto \\ KEK, Tsukuba, Ibaraki, Japan \\ On behalf of the US-Japan Superconducting Magnet Collaboration \\ KEK, Univ. of Tsukuba, FNAL, BNL, and LBNL
}

\section{Introduction:}

Superconducting magnets have been indispensable for high energy physics. The first practical application of superconducting magnets at KEK started with a secondary beam line so called, $\pi 1$, at the KEK $12-\mathrm{GeV}$ proton synchrotron in communication with the US laboratories [1]. On the basis of this experience, the first-stage program of the US-Japan superconducting magnet collaboration on High Energy Physics was performed since 1981 through 1996. The main activities focused on development of superconducting wires and magnets for particle accelerators and detectors. In the second stage, the technologies achieved in the first stage have been succeeded to the construction of the KEK-B insertion region quadrupoles (IRQ), the J-PARC neutrino beam line, and so on. Moreover, the collaboration program for the LHC insertion quadrupole (IRQ) magnet has been successfully accomplished on the basis of these technologies even though it was not directly funded but encouraged by the US-Japan Collaboration. Table 1 summarizes the activities from the US-Japan superconducting magnet collaboration.

Table 1 The projects developed by the US-Japan Collaboration for High Energy Physics

\begin{tabular}{|c|c|c|c|c|}
\hline Years & Projects & Contribution & Progress & Participation \\
\hline $\begin{array}{l}1981 \\
-1996\end{array}$ & $\begin{array}{l}\text { High field } \\
\text { dipole }\end{array}$ & $\begin{array}{l}10 \mathrm{~T} \text { dipole }[2] \\
\mathrm{Nb}_{3} \mathrm{Sn} \text { dipole }[3] \\
\text { Superconductors [4] }\end{array}$ & $\begin{array}{l}\text { Champion data } \\
\text { Improvement of } \mathrm{J}_{\mathrm{c}}\end{array}$ & $\begin{array}{l}\text { FNAL, BNL, } \\
\text { LBNL, KEK }\end{array}$ \\
\hline 1980 & CDF & $\begin{array}{l}\text { Central solenoid } \\
\text { (prototype) [5] }\end{array}$ & $\begin{array}{l}\text { Longest stable } \\
\text { operation }\end{array}$ & $\begin{array}{l}\text { FNAL, U. } \\
\text { Tsukuba, KEK }\end{array}$ \\
\hline $\begin{array}{l}1988 \\
-1994 \\
\end{array}$ & SSC-Acc. & $\begin{array}{l}\text { Accelerator magnets } \\
{[6],[7]}\end{array}$ & $\begin{array}{l}6.6 \mathrm{~T}, 1 \mathrm{~m} \text { and } 13 \mathrm{~m} \\
\text { dipoles }\end{array}$ & $\begin{array}{l}\text { SSC, FNAL, BNL, } \\
\text { LBNL, KEK }\end{array}$ \\
\hline 1989 & SSC-SDC & $\begin{array}{l}\text { Detector solenoid } \\
\text { (prototype) [8] }\end{array}$ & $2 \mathrm{~T}$, thin solenoid & FNAL, KEK \\
\hline $\begin{array}{l}1987 \\
-1993\end{array}$ & Muon G-2 & $\begin{array}{l}\text { Storage ring magnets } \\
\text { and inflector [9], [10] }\end{array}$ & $\begin{array}{l}\text { Al-stabilized } \\
\text { superconducting } \\
\text { magnets }\end{array}$ & BNL, KEK \\
\hline $\begin{array}{l}1995 \\
-2006\end{array}$ & LHC-IRQ & $\begin{array}{l}\text { Final focusing } \\
\text { quadrupoles [11] }\end{array}$ & $\begin{array}{l}70 \mathrm{~mm}^{\phi}, 215 \mathrm{~T} / \mathrm{m}, 7 \\
\mathrm{~m}\end{array}$ & $\begin{array}{l}\text { FNAL, KEK, } \\
\text { CERN } \\
\text { (not funded.) }\end{array}$ \\
\hline 2006- & \begin{tabular}{|l|} 
LHC \\
upgrade
\end{tabular} & $\begin{array}{l}\mathrm{Nb}_{3} \mathrm{Al} \text { conductor and } \\
\text { Rutherford cable [12] }\end{array}$ & $\begin{array}{l}\mathrm{Nb}_{3} \mathrm{Al} \text { cabling and } \\
\text { model coil } \\
\text { development }\end{array}$ & $\begin{array}{l}\text { NIMS, FNAL, } \\
\text { LBNL, KEK, } \\
\text { CERN (not } \\
\text { funded) }\end{array}$ \\
\hline $\begin{array}{l}2002 \\
-2004\end{array}$ & J-PARC & $\begin{array}{l}\text { T2K neutrino beam line } \\
\text { and corrector magnet } \\
{[13]}\end{array}$ & $\begin{array}{l}\text { Coil winding and } \\
\text { conductive cooling }\end{array}$ & BNL, KEK \\
\hline
\end{tabular}




\section{Development and achievement:}

The $\mathrm{Nb}-\mathrm{Ti}$ superconducting wire that was developed with industries showed high $\mathrm{J}_{\mathrm{c}}$ over the average level during the SSC and LHC projects as shown in Fig. 1. The development program of superconducting wire has been succeeded to that of $\mathrm{Nb}_{3} \mathrm{Al}$ wires which has the more mechanical toughness than $\mathrm{Nb}_{3} \mathrm{Sn}$ wires. The developed $\mathrm{Nb}_{3} \mathrm{Al}$ Rutherford cable has been motivated to be applied to the LHC upgrade program.

The development of superconducting magnets by the US-Japan Collaboration has focused on high magnetic field magnets. The $10 \mathrm{~T}$ dipole magnet with $\mathrm{Nb}$-Ti was developed. The coil was a block type with 8 racetrack coils and the end part of the four coils were bent for a beam aperture as shown in Fig. 2. The fabricated magnet was cooled to $1.8 \mathrm{~K}$, and excited to $10.4 \mathrm{~T}$ at the peak field in the coil winding. The value was the world highest achievement at that time.

A set of superconducting coils of the muon storage ring and the inflector magnet for the g-2 experiment at BNL were successfully developed by KEK. The coil was wound with a co-extruded aluminum stabilized $\mathrm{Nb}$-Ti wire developed for the program. The inflector was designed and fabricated with a sophisticated coil configuration not to leak the magnetic field outside as shown in Fig. 3. It took an essential role to obtain the most sensitive muon g-2 value ever achieved [14].
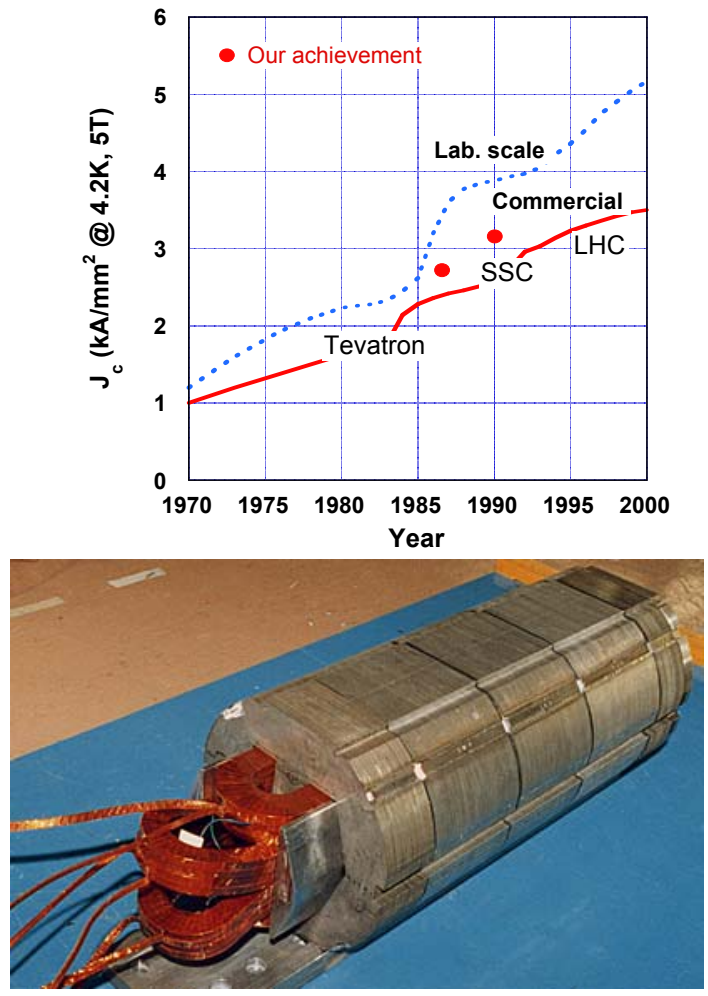

Fig. 2. The coil of the $10 \mathrm{~T}$ dipole magnet.

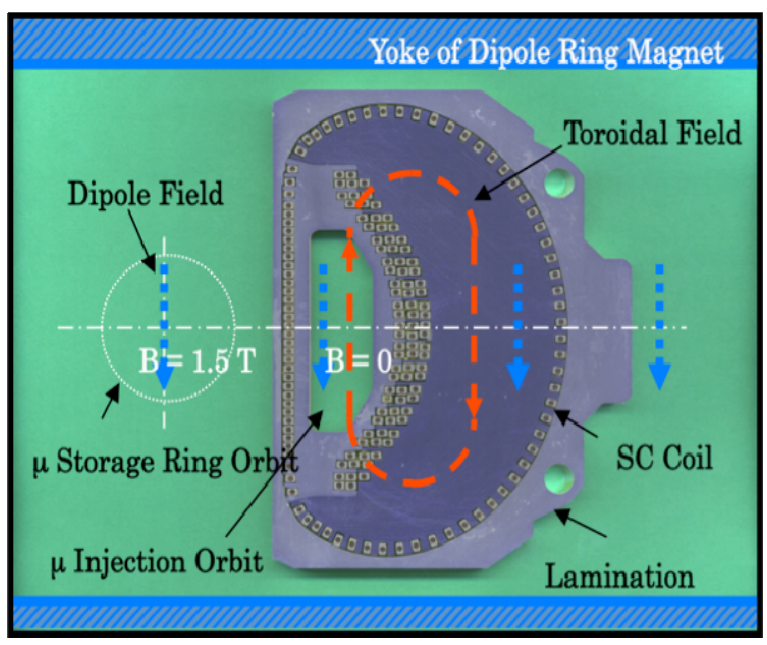

Fig. 3. The cross section and coil winding configuration of the inflector magnet. 
The SSC magnet R\&D were performed as eleven 1-m model dipoles and a 13-m long full-scale dipole were constructed and tested successfully. The training histories of the 1-m model magnets are shown in Fig. 4. It is important for accelerator magnets to be excited stably to the nominal magnetic field. All the magnets were excited over the nominal magnetic field after a couple of quenches.
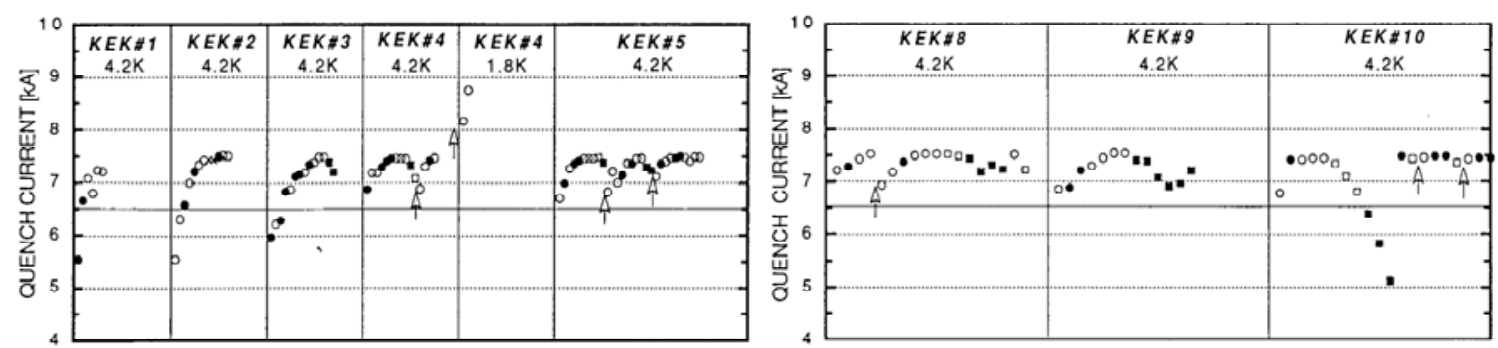

Fig. 4. Training quench histories of the SSC 1-m model dipole.

The prototypes of thin superconducting solenoids for particle detectors were developed. The prototype of the central solenoid for the CDF experiment was wound with a co-extruded aluminum stabilized $\mathrm{Nb}$-Ti wire, and the wound coil was inserted into an outside bobbin by a thermal shrink-fitting method. The prototype of the solenoid for SDC was fabricated by the inner winding method. The aluminum stabilized $\mathrm{Nb}-\mathrm{Ti}$ cable and the inner winding technology have been standard technologies for thin particle detector solenoids.

The LHC-IRQ collaboration program by KEK/FNAL/CERN was performed since 1995 to 2006. The program was to construct 16 IRQ magnets for the final focusing points. The magnet design and fabrication technologies were developed by KEK, and the technologies were transferred to an industry for fabrication. All the magnets were tested at KEK, and showed successful excitation performance and good field qualities as shown in Fig. 5. They were transported to FNAL and assembled into cryostats there. After that, they were installed into the CERN-LHC tunnel on time.
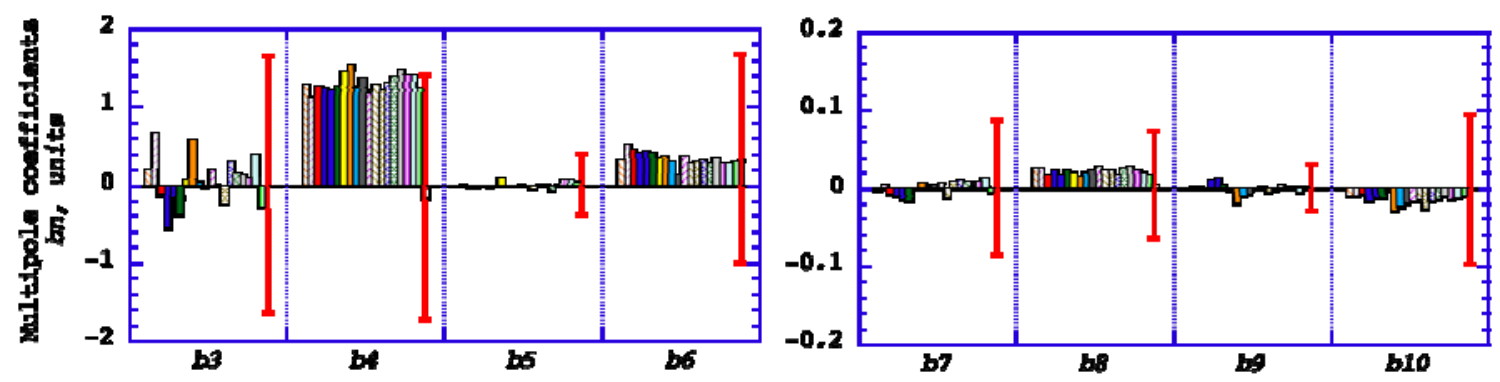

Fig. 5. Magnetic field characteristics and the higher harmonics of the LHC IRQ. 
The experience from the CERN-LHC interaction region quadrupole development has been well transferred to the $\mathrm{T} 2 \mathrm{~K}$ neutrino beam-line superconducting magnet system at the J-PARC program carried out in cooperation between KEK and BNL. A unique beam-line magnet designed with combined dipole and quadrupole field has been developed for the main magnet string and conduction-cooled corrector magnets have been realized with their cooperative effort supported by the US-Japan Collaboration program. Figure 6 shows the superconducting magnet string to transport the primary proton beam extracted from the J-PARC main ring to the neutrino production target station.

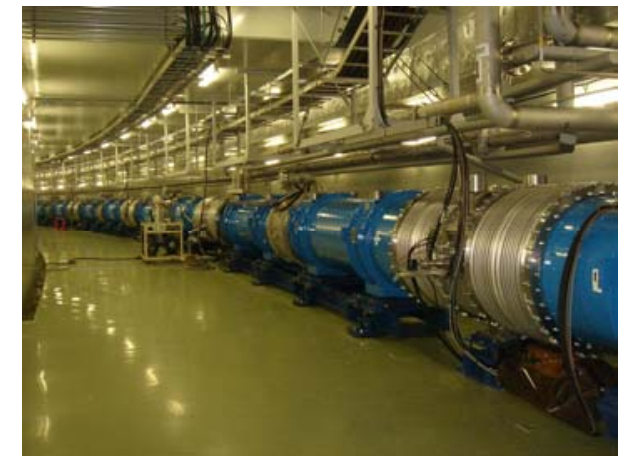

Fig. 6. The J-PARC superconducting proton beam line for the $\mathrm{T} 2 \mathrm{~K}$ neutrino experiment.

\section{Future program:}

KEK is contributing to superconducting magnets for the LHC luminosity upgrade, the high intensity muon beam source, the muon beam facility for the material and life sciences at J-PARC, the g-2 experiment at J-PARC and so on. These programs stand on technologies developed by the US -Japan Collaboration on High Energy Physics.

\section{$\underline{\text { References }}$}

1) A. Yamamoto et al., "A superconducting secondary beam line in the $12 \mathrm{GeV}$ proton synchrotron at KEK," Nucl. Instr. Meth., vol. A257, pp. 105-113, 1987.

2) T. Shintomi et al., "The construction and test results of a $10 \mathrm{~T}$ dipole magnet," IEEE Trans. Nucl. Sci., vol. NS-32, pp. 3719-3721, 1985.

3) L. Yan et al., Some results from the first test of a double-shell $\mathrm{Nb}_{3} \mathrm{Sn}$ dipole, KEK Report 87-14, September 1987.

4) M. Wake et al., " $1.8 \mathrm{~K}$ test of binary and ternary superconducting conductors," IEEE Trans. Magnetics, vol. MAG-19, pp. 552-555, 1983.

5) H. Hirabayashi, et al., "Cooling and excitation tests of a thin $1 \mathrm{~m}-\phi \times 1 \mathrm{~m}$ superconducting solenoid magnet”, Japanese J. of Appl. Phys., vol. 21, no. 8 pp. 1149-1154, 1982.

6) K. Hosoyama et al., "A status report on the development of 5-cm aperture, 1-m long SSC dipole magnet at KEK, Supercollider 5, pp. 229-232, Plenum Press, 1994.

7) K. Hosoyama et al., "Fabrication and testing of 13-m-long SSC model dipole magnets," Proc. of 16th Inter. Cryo. Engr. Conf./ Inter. Cryo. Materials Conf., pp. 851-854, 1997.

8) A. Yamamoto et al., "Development of a prototype thin superconducting solenoid magnet for the SDC detector", IEEE Trans. Appl. Supercond., vol. 5, no. 2, pp. 848-852, 1995.

9) G. T. Danby et al., "The Brookhaven muon storage ring magnet”, Nucl. Inst. Meth. Phys. Res., vol. A457, pp. 151-174, 2001.

10) A. Yamamoto et al., "The superconducting inflector for the BNL G-2 experiment", Nucl. Instr. Meth. Phys. Res., vol. A489, pp. 170-177, 2002.

11) Y. Ajima et al., "The MQXA quadrupoles for the LHC low-beta insertions," Nucl. Instr. Meth. Phys. Res., vol. A 550, pp. 499-513, 2005.

12) A. Kikuchi et al., "Strand and cable development for a high field $\mathrm{Nb}_{3} \mathrm{Sn}$ common coil manget," IEEE Trans. Appl. Supercond., vol. 20, no. 3, pp. 1428-1431, 2010.

13) T. Nakamoto et al., "Construction of superconducting magnet system for the J-PARC neutrino beam line," IEEE Trans. Appl. Supercond., vol. 20, no. 3, pp. 208-213, 2010.

14) G.W. Bennett et al., "Measurement of the positive muon anomalous magnetic moment to 0.7 ppm", Phys. Rev. Lett., vol. 89, no. 10, pp. 101804-1 101804-6, 2002. 


\title{
R\&D on the Detector Technology: Last decade and future
}

\author{
Junji Haba, KEK, Tsukuba, Japan
}

In the last decade, intense R\&D studies on the detector technologies have been pushed further ahead for the future HEP experiments. Among them, there are two distinct fields where number of important progresses have been achieved.

One is the efforts to develop the next generation particle identification system with novel photon sensors. Following the invention and the first realization of the innovative DIRC concept in BaBar at SLAC, various extensions of it have been proposed and studied in the framework of the US-Japan collaboration which includes a focusing DIRC and a TOP counter. Their concepts have been proven in the beam tests and several key components have newly been developed. Among them the novel photon sensors like a high-speed MCP PMT, a 144ch HPAD or a gas PMT are successfully developed.

The other is to develop several elemental technologies necessary for a light weight silicon tracker which is strongly required for the future collider experiments like a linear collider. Thanks to the outcome from the collaborative works among US and Japanese researchers including a thin flexible print cable, low-mass high-heat-conducting board or an assembly technology of silicon sensor with minimum material, very light weight tracker system were realized for the B-factories which outperform the impact parameter resolution demonstrated by the most modern LHC experiments.

Toward an ultimate silicon pixel sensor capable of tracking of the highest precision and sophisticated intelligence with the lightest material, the development of the next generation monolithic pixel device with a SOI technology has been started since 2006. The technology is so promising that the researchers of several institutes in US and Japan are working together on a single wafer (Multi Project Wafer; MPW run) to realize the ultimate goal for the coming decade.

In summary, the collaborations among US-J in the field of the detector technology R\&D have been very fruitful for the last decade. It should be much more important for the next decades because the applications of the most advanced industrial-level technology to the detector is almost impossible for any single institute/country to realize. 


\section{Contributions by US and JPN (Last decade)}

- Cherenkov PID \& advanced photon sensors

r High speed MCP PMT (SLAC, Nagoya)

- Focusing DIRC prototype (SLAC, Cincinati)

- GaAs photocathode, TOP (Nagaoya)

> 144 ch HPD for RICH counter(Nagoya)

- MPGD related technology

v GEM and MicroMEGAS for TPC read out (Saga,LBNL,KEK)

- Gas PMT with microMEGAS structure (TMU,SLAC)

- Silicon detector technologies for thin trackers

- Development of light weight silicon strip ladder, high heat conducting readout hybrid(Princeton)

Continuous Acquisition Pixel (CAP) sensor(Hawaii)

EMl from short bunch beam (Tohoku/KEK/SLAC)

Development of fine pitch flexible circuit (Niigata)

Striplet sensor (KEK)

Ladder assembly(KEK)

- SOl pixel

\section{Best timing sensors for single photon sensitivity even in a high B (SLAC,TMU)}

\section{MCP-PMT (Burle85011)}

It is possible to reach a resolution of $\sigma \sim 50 \mathrm{ps}$ at $15 \mathrm{kG}$.
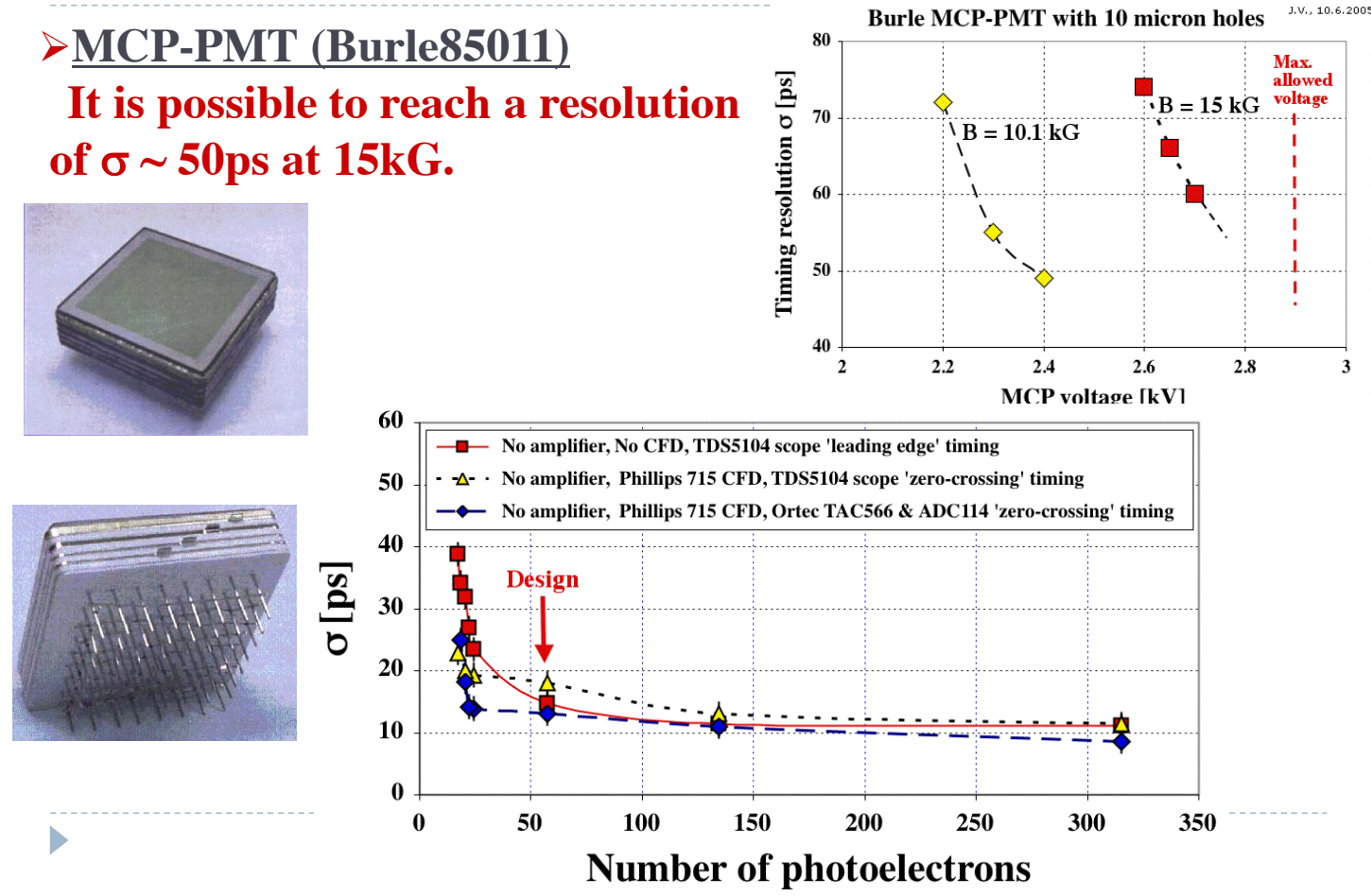


\section{AC RICH with 144ch HPD (Nagoya)}

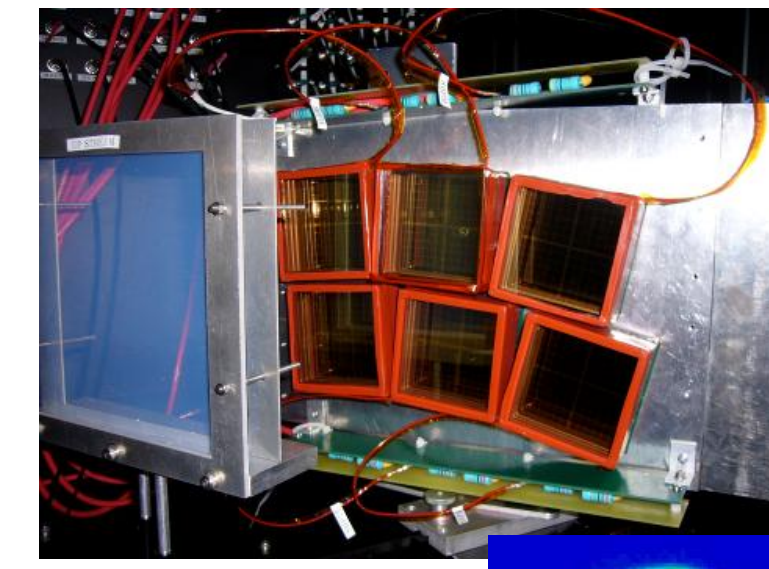

This corresponds to $144 \mathrm{ch}$ HAPD arrangement in inner layer of real ARICH (almost) Minimum distance between HAPDs $\sim 1.0 \mathrm{~mm}$

MultiAlkali

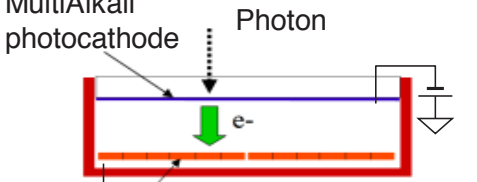

I Pixel APD

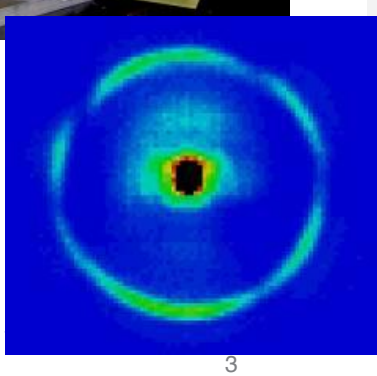

cangle $\{$ hithapd==0\}

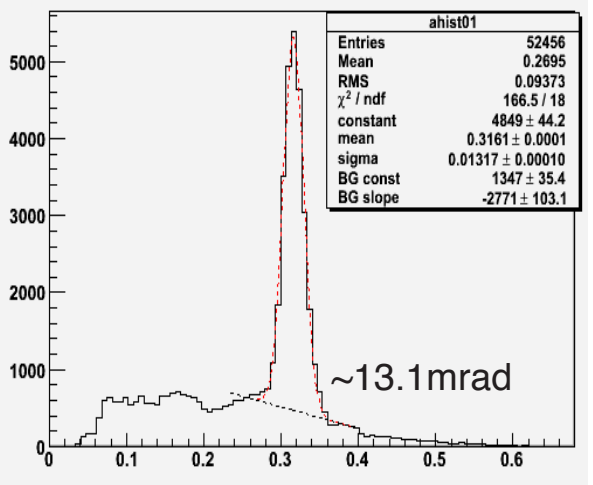

\section{Impact parameter resolution}

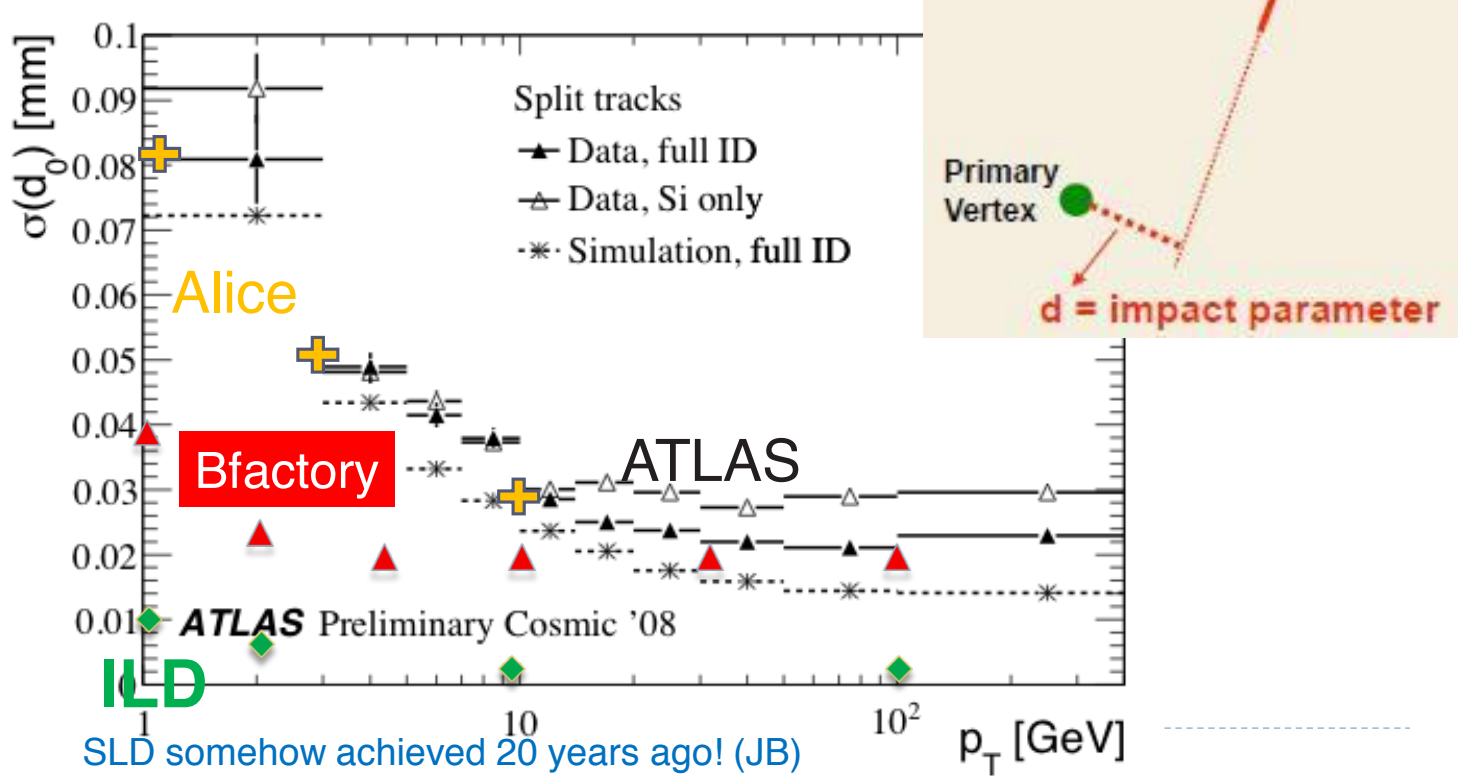




\section{Lightweight silicon strip tracker}

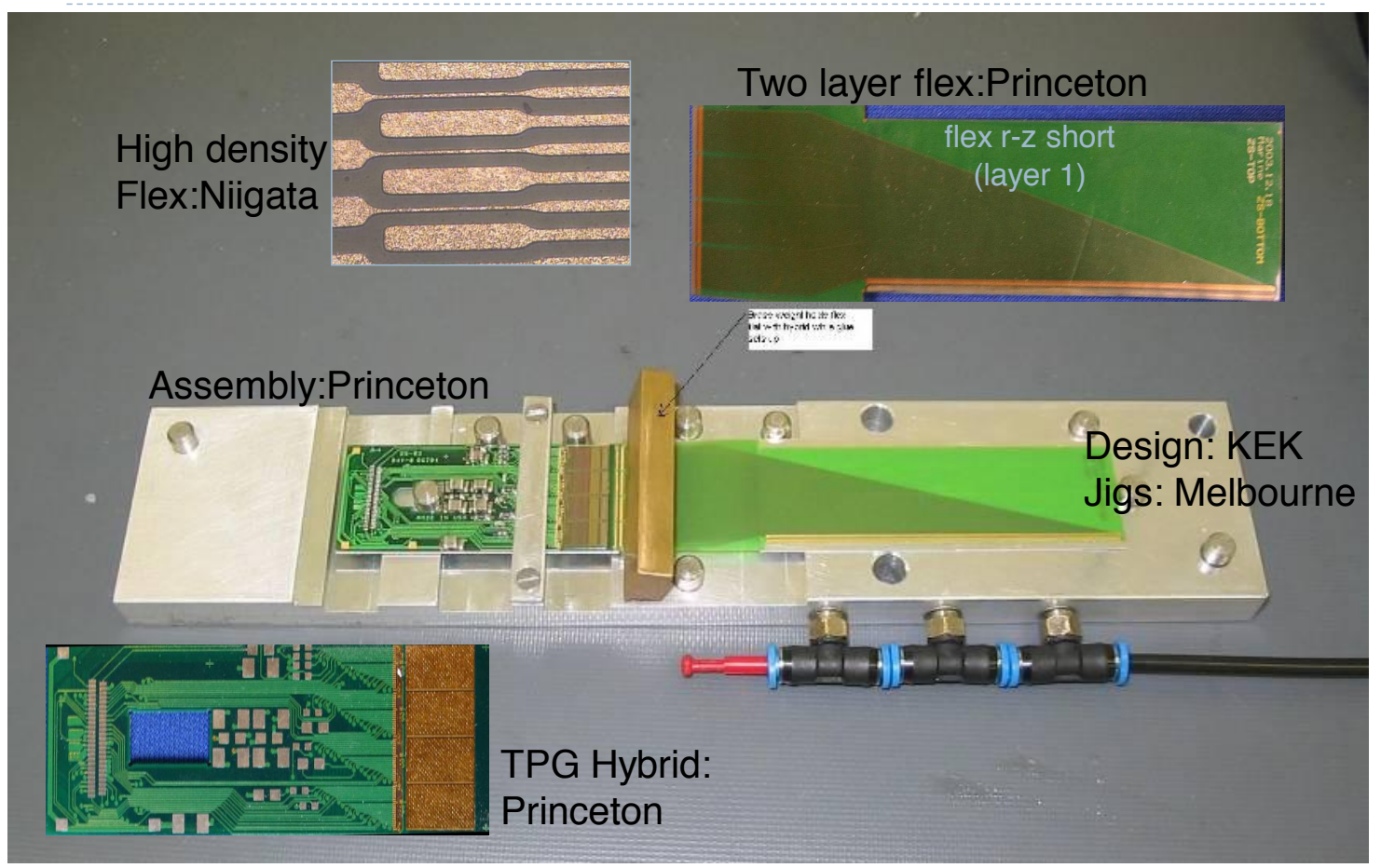

\section{SOl pixel comes next (KEK, FNL,LBNL $\square$ )}

Some SOI basics

- SOI technology integrates CMOS electronics on top of a Buried Oxide (SOI), ensuring full dielectric isolation, small active volume and low junction capacitance: latch-up immune, low power, high speed designs are thus favoured

- 0.15-0.20 $\mu \mathrm{m}$ Fully-Depleted (FD) SOI processes from OKI, Japan allow contacting a high-resistivity $(700 \Omega / \mathrm{cm})$ substrate through the BOX for pixel implanting and substrate reverse bias

- Possibility for small pitch pixel sensors with high density, full CMOS readout electronics integrated in the same device $\rightarrow$ SOI Monolithic Pixel Sensors
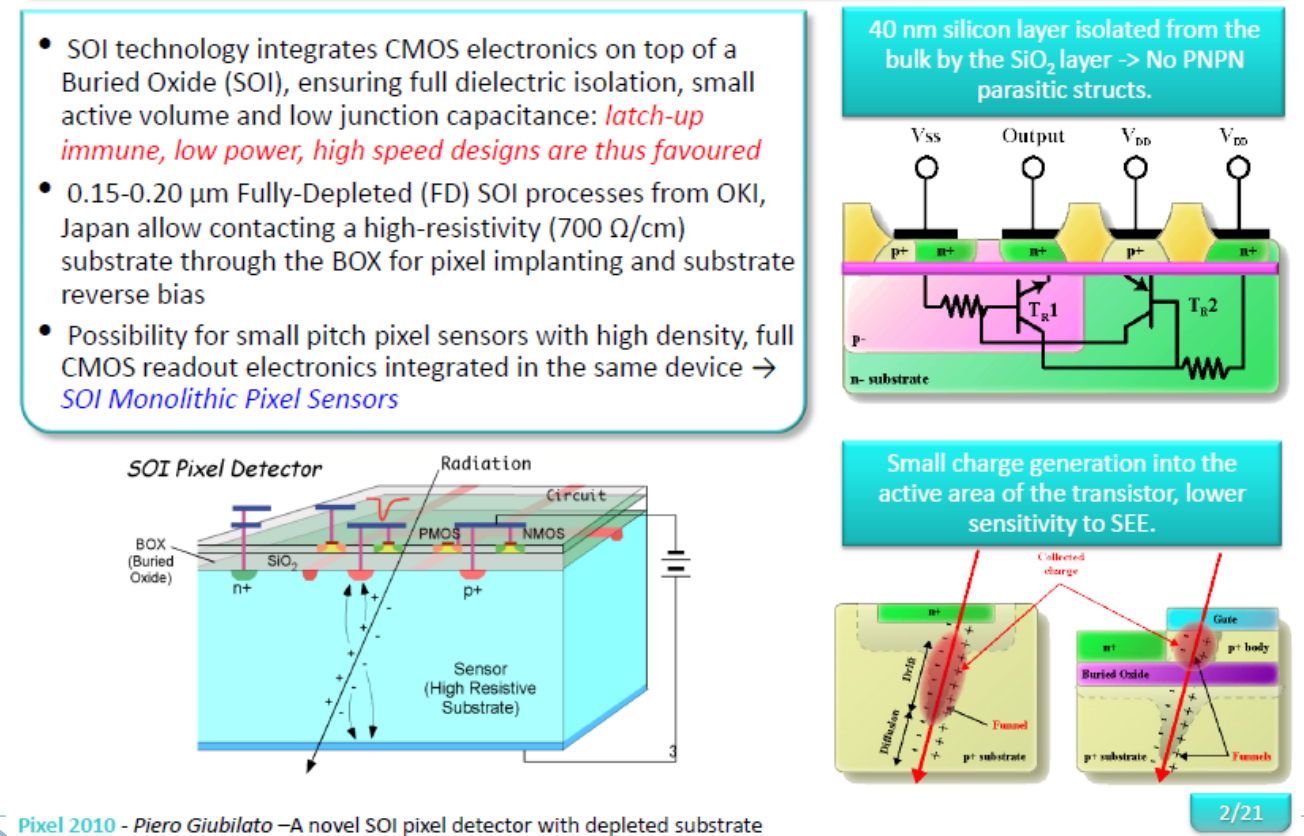

Slide presented at PIXEL2010 by the LBNL team 


\section{Perspective}

\section{Hitoshi Murayama}

Institute for the Physics and Mathematics of the Universe, University of Tokyo, Kashiwa, Japan

Department of Physics, University of California, Berkeley, CA 94720, USA

Physics Division, Lawrence Berkeley National Laboratory, Berkeley, CA 94720, USA

In this talk, I present my own perspective on what the last 30 years of Japan-US collaboration brought us, and to where it may be leading us. I first summarize the incredible success of the standard model that came from the last 30 years; we all should all be very proud of it. At the same time, the last 12 years opened our eyes to the irrefutable necessity of physics beyond the standard model, viz., non-baryonic dark matter, the finite mass of neutrinos, the accelerating expansion of the Universe, the apparently acausal density fluctuations in the Universe, and the cosmic baryon's asymmetry.

In addition, we are poised to reach the energy scale to study the weak interaction, while similar milestones have been reached only twice in this century ( 1900 for electromagnetism, and $\sim 1970$ for the strong interaction). Given this unique opportunity, I discuss the two most likely outcomes of the next 30 years, namely understanding the mechanism behind the electroweak symmetry breaking, and the nature of dark matter. "I consider the following topics in detail".

\section{T-shirt ready}

11 Post-Higgs Problem

12 Once upon a time, there was a hierarchy problem

13 Anti-matter comes to the rescue by doubling the number of particles

15 History repeats itself?

20 Need for precision measurements

24 What dark matter is not

25 MACHOS $\rightarrow$ WIMPs

28 Dark Matter Concordance

29 History of the Universe 


\section{T-shirt ready}

BERKELEY CENTER FOR THEORETICAL PHYSICS

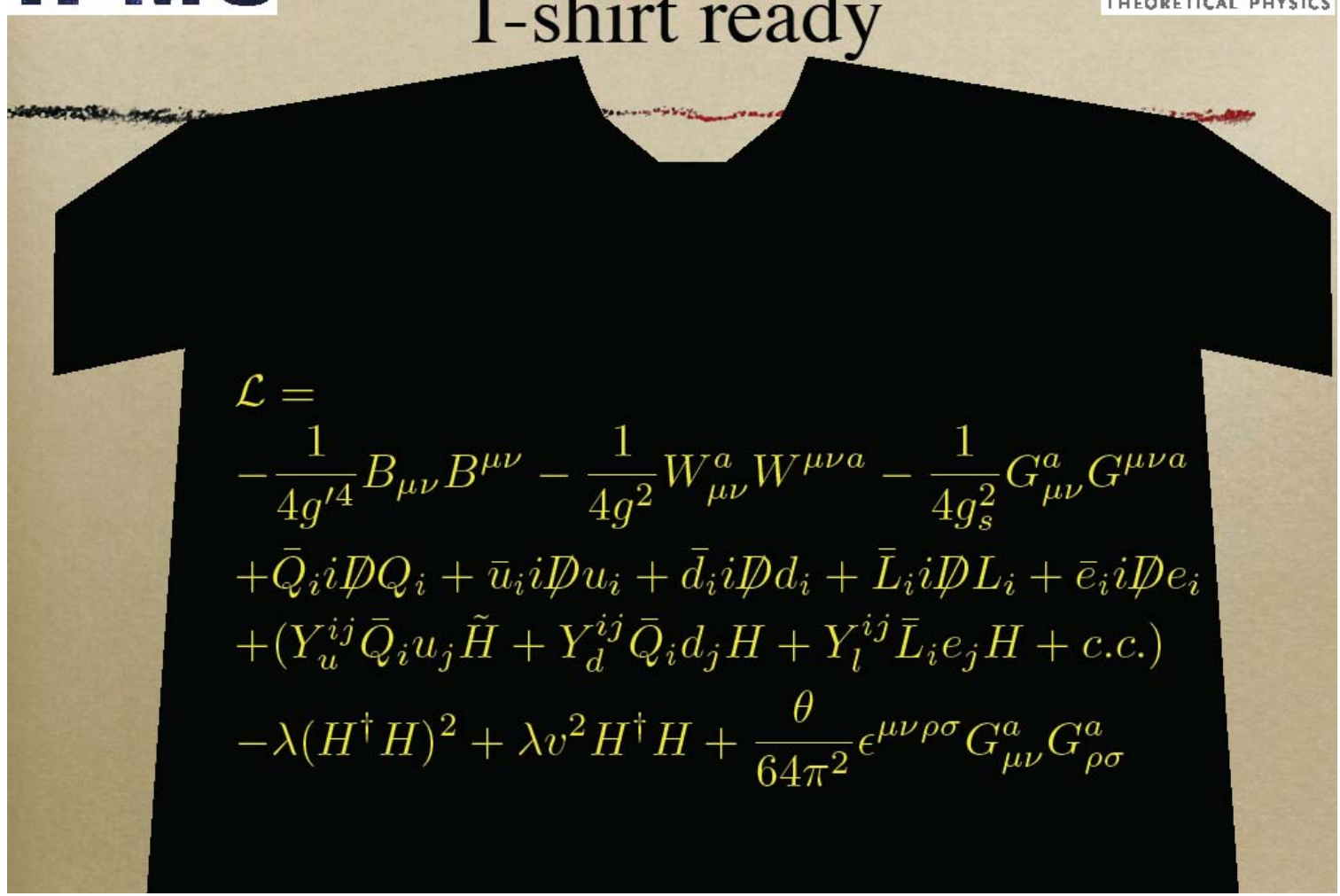

iPMU

\section{Post-Higgs Problem}

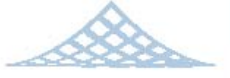

BERKELEY CENTER FOR THEORETICAL PHYSICS

- We see "what" makes up the Dark Field

- But we still don't know "why" it is there

- Two problems:

- Why anything is condensed at all

- Why is the scale of condensation $\sim \mathrm{TeV} \ll M_{P l}=10^{15} \mathrm{TeV}$

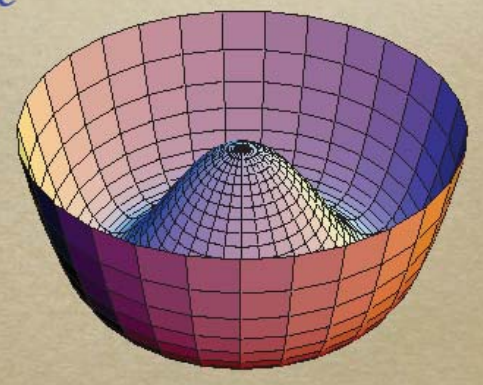

- Explanation to be at $\sim T e V$ scale because this is the relevant energy scale, of. BCS 


\section{IPMU Once upon a time, there was a hierarchy problem...}

- At the end of 19th century: a "crisis" about electron

- Like charges repel: hard to keep electric charge in a small pack

- Electron is point-like

- At least smaller than $10^{-17} \mathrm{~cm}$

$\circ$

$$
\Delta m_{e} c^{2} \sim \frac{\alpha}{r_{e}} \sim \mathrm{GeV} \frac{10^{-17} \mathrm{~cm}}{r_{e}}
$$

- Correction $\Delta m_{e} c^{2}>m_{e} c^{2}$ for $r_{e}<10^{-13} \mathrm{~cm}$

- Breakdown of theory of electromagnetism

$$
\Rightarrow \text { Can't discuss physics below } 10^{-13} \mathrm{~cm}
$$

\section{IPMU Anti-Matter Comes to \\ Rescue by Doubling of \#Particles}

BERKELEY CENTER FOR THEORETICAL PHYSICS

$\circ$

- Vacuum bubble of matter anti-matter creation/annihilation

- Electron annihilates the positron in the bubble

$\Rightarrow$ only $10 \%$ of mass even

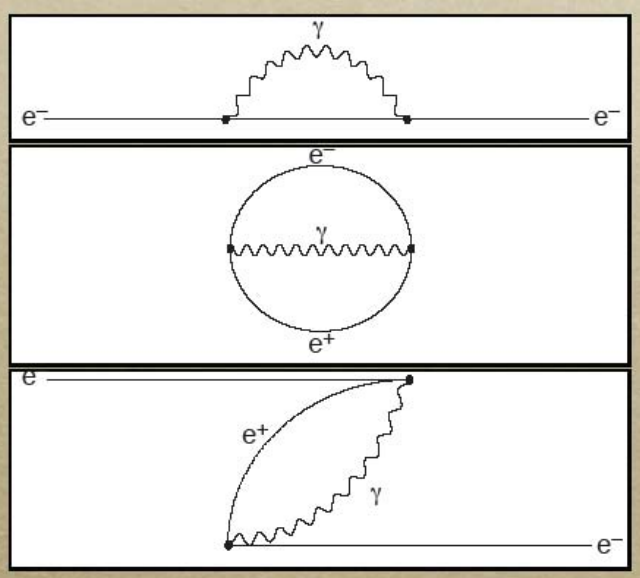

for Planck-size electron

$$
\frac{\Delta m_{e}}{m_{e}} \sim \frac{\alpha}{4 \pi} \log \left(m_{e} r_{e}\right)
$$




\section{History repeats itself?}

o

- "Vacuum bubbles" of superpartners cancel the energy required to contain Higgs boson in itself

- Standard Model made consistent with whatever $\Delta m_{H}^{2} \sim \frac{\alpha}{4 \pi} m_{S U S Y}^{2} \log \left(m_{H} r_{H}\right)$ physics at shorter distances

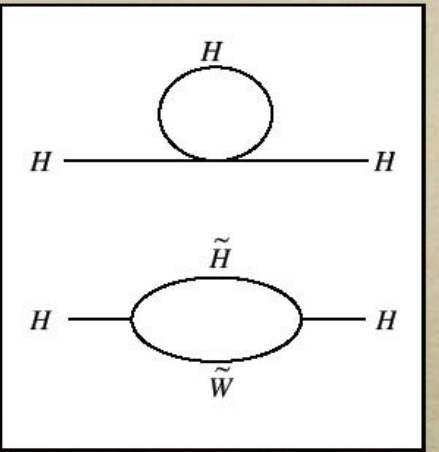

\section{need precision measurements}

- SUSY spectroscopy

- kinematic fits, partial wave analysis, Dalitz analysis, etc

- precision mass, BR measurements

- all techniques from current hadron spectrocopy!

\section{Squarks}

$$
\text { 」=0? PDG } 2016
$$

The following data are averaged over all light flavors, presumably u, d, s, c with both chiralities. For flavor-tagged data, see listings for Stop and Sbottom. Most results assume minimal supergravity, an untested hypothesis with only five parameters. Alternative interpretation as extra dimensional particles is possible. See KK particle listing.

\section{SQUARK MASS}

\begin{tabular}{|c|c|c|c|}
\hline VALUE $(\mathrm{GeV})$ & DOCUMENT ID & TECN & COMMENT \\
\hline $538 \pm 10$ & OUR FIT & & $\overline{\text { mSUGRA }}$ assumptions \\
\hline $532 \pm 11$ & ${ }^{2} \mathrm{ABBIENDI} 11 \mathrm{D}$ & CMS & $\begin{array}{l}\text { Missing ET with } \\
\text { mSUGRA assumptions }\end{array}$ \\
\hline $541 \pm 14$ & ${ }^{2}$ ADLER 110 & ATLAS & $\begin{array}{l}\text { Missing ET with } \\
\text { mSUGRA assumptions }\end{array}$ \\
\hline \multicolumn{4}{|c|}{. . We do not use the following data for averages, fits, limits, etc . . } \\
\hline $652 \pm 105$ & ${ }^{3} \mathrm{ABBIENDI} 11 \mathrm{~K}$ & CMS & $\begin{array}{l}\text { extended mSUGRA } \\
\text { with } 5 \text { more parameters }\end{array}$ \\
\hline
\end{tabular}

${ }^{2} \mathrm{ABBIENDI} 11 \mathrm{D}$ assumes minimal supergravity in the fits to the data of jets and missing energies and set $A_{0}=0$ and $\tan \beta=3$. See Fig. 5 of the paper for other choices of $A_{0}$ and $\tan \beta$. The result is correlated with the gluino mass $M_{3}$. See listing for gluino.

gluino.
${ }^{2}$ ADLER 110 uses the same set of assumptions as ABBIENDI 11D, but with $\tan \beta=5$. ${ }^{3}$ ABBIENDI $11 \mathrm{~K}$ extends minimal supergravity by allowing for different scalar masses squared for $\mathrm{Hu}, \mathrm{Hd}, 5^{*}$ and 10 scalars at the GUT scale.

SQUARK DECAY MODES

$\begin{array}{lllll}\frac{M O D E}{j+m i s s} & \frac{B R(\%)}{32 \pm 5} & \text { DOCUMENT ID } & \text { TECN } & \text { COMMENT } \\ \mathrm{j} 1+\text { miss } & 73 \pm 10 & \text { ABE 10U } & \text { ATLAS } & \\ \mathrm{j} e+\text { miss } & 22 \pm 8 & \text { ABE } 10 U & \text { ATLAS } & \text { lepton universality } \\ \mathrm{j} \mu+\text { miss } & 25 \pm 7 & \text { ABE 10U } & \text { ATLAS } & \\ q \chi^{+} & \text {seen } & \text { ABE } 10 U & \text { ATLAS } & \end{array}$




\section{iPMU ${ }_{\text {What dark matter is not }}$}
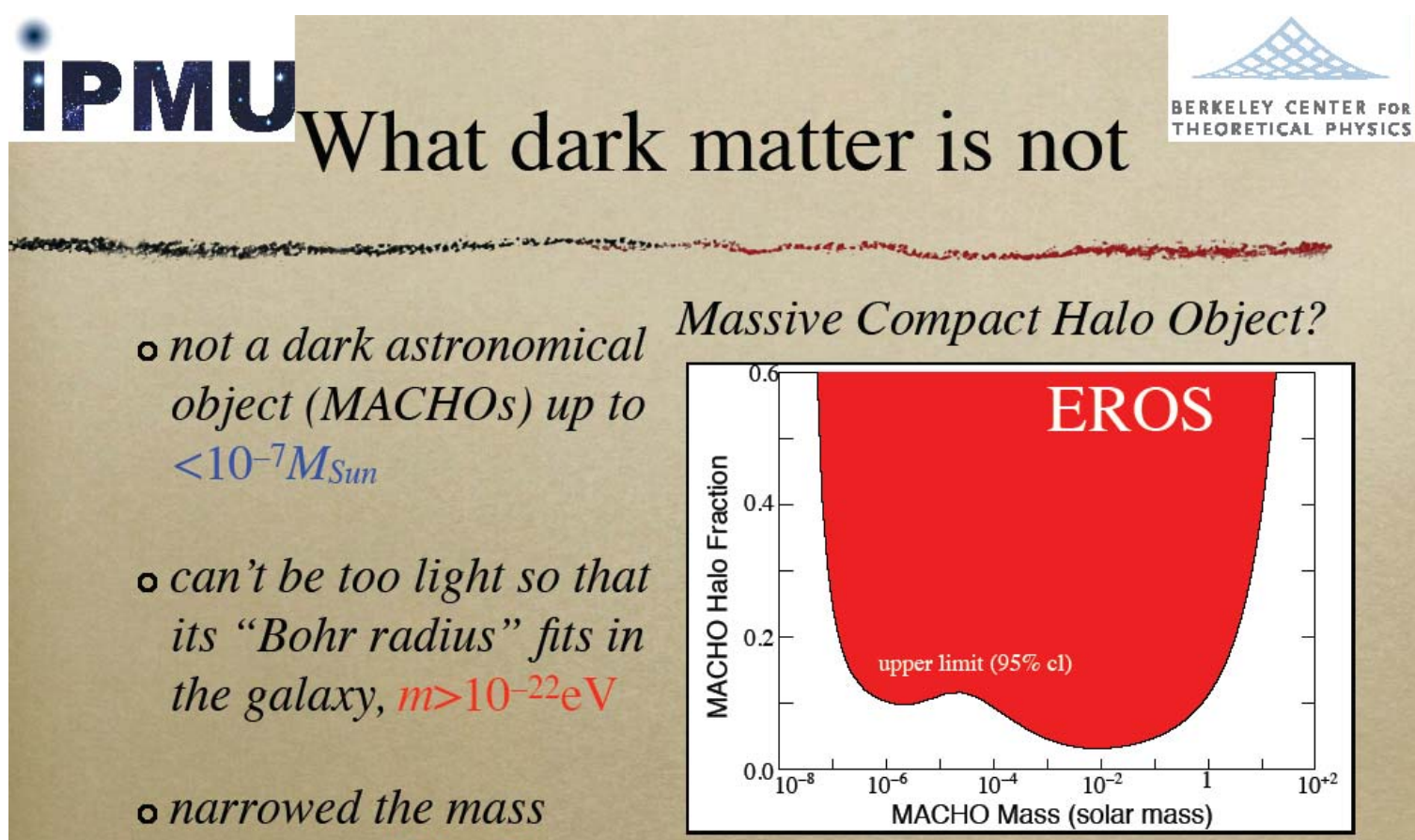

range to

iPMU ${ }_{\text {MACHOs }} \Rightarrow$ WIMPs

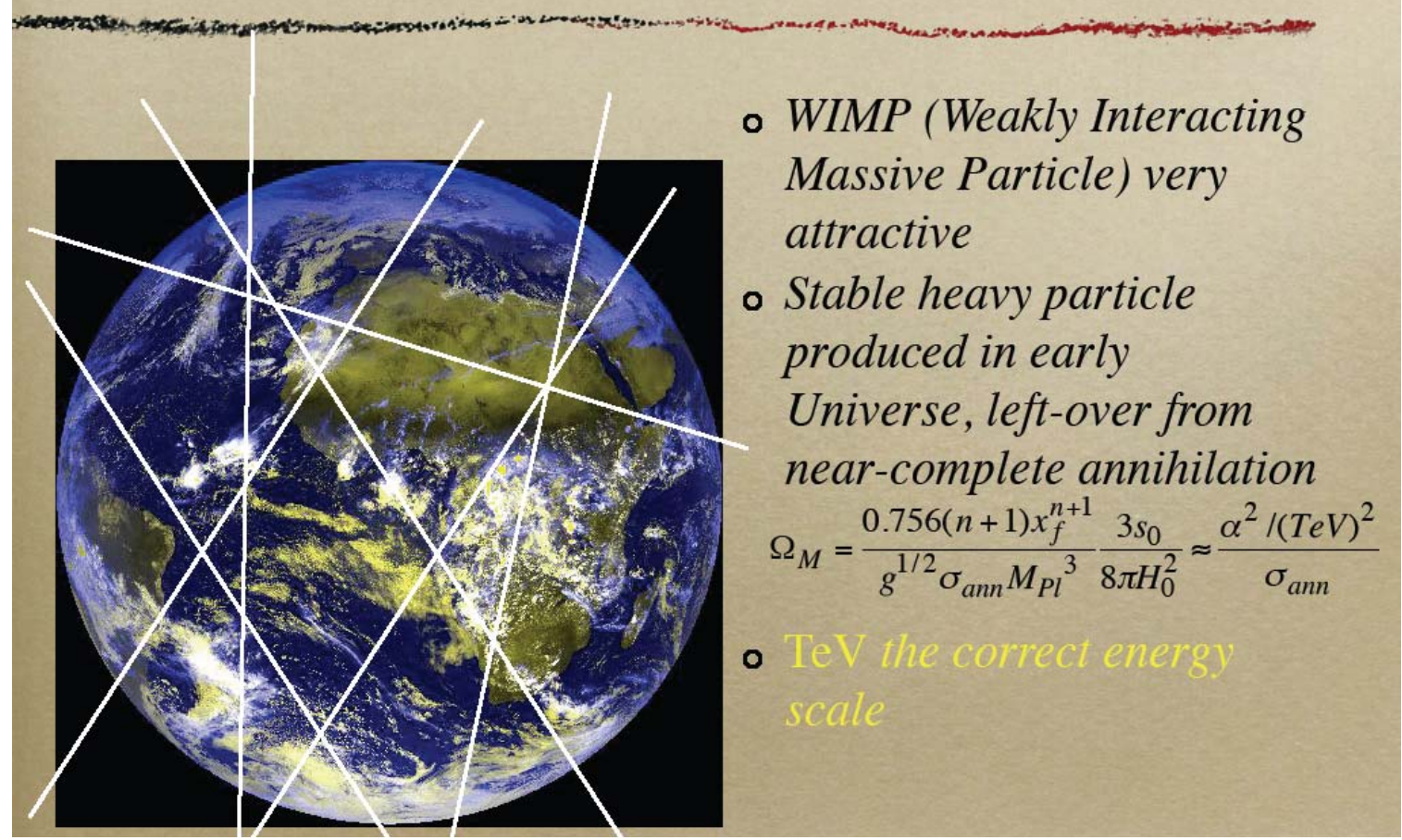



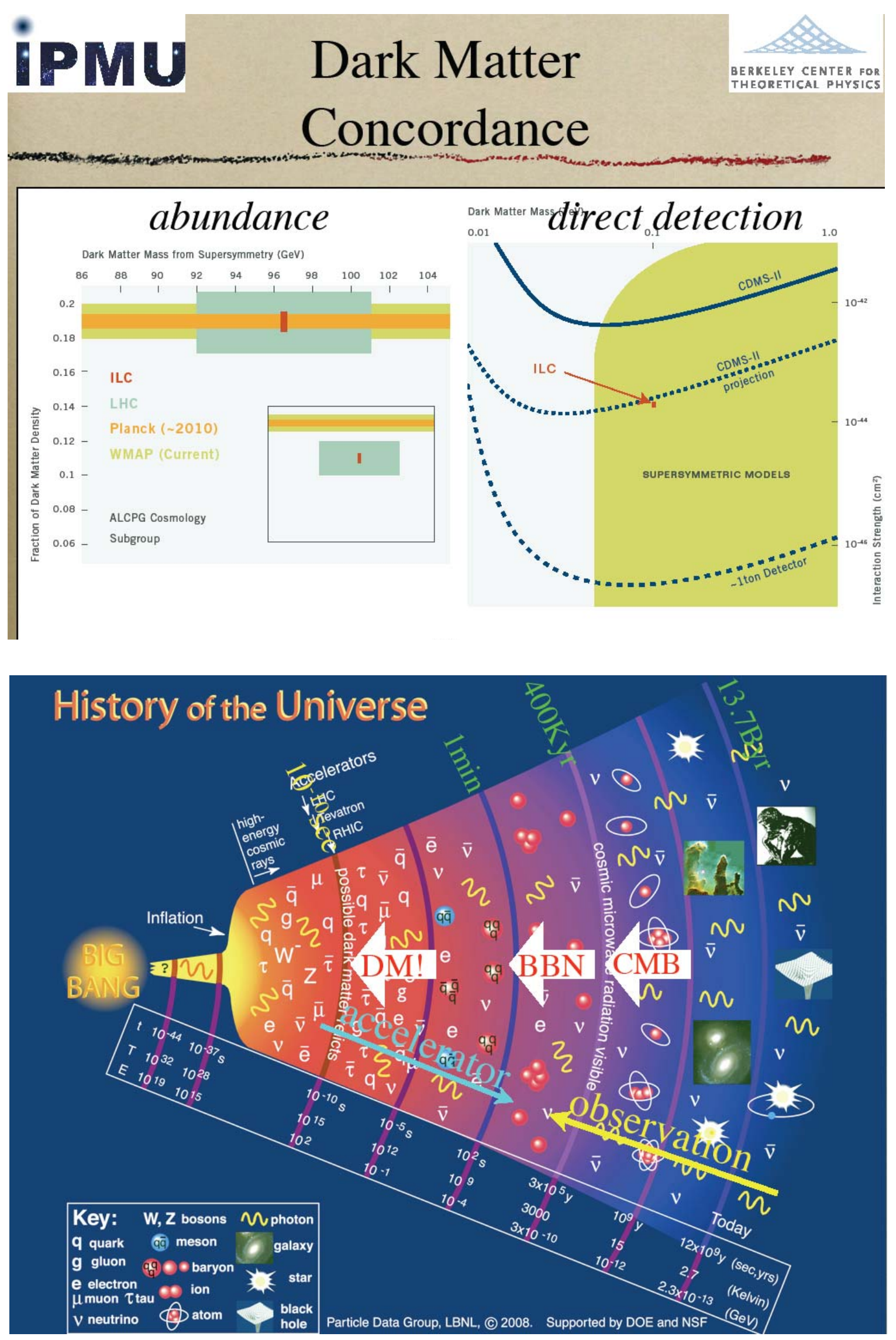
The Future of the US Program

P. Oddone, Fermilab, Batavia, IL

Drivers for the Program

- Drivers for the US program are simple and universal: the quest to solve the profound mysteries that surround us.

- The ability to bring together the teams to solve these mysteries:

$\circ$ The next generation of accelerators

- The next generation of detector technology

○ New simulation, computation and analysis techniques 


\title{
The Future of the US program
}

\author{
Pier Oddone \\ Symposium for the $30^{\text {th }}$ anniversary \\ celebration of the US/Japan \\ Agreement on High Energy Physics
}

October $21^{\text {st }}, 2010$

\section{Drivers for the program}

- Drivers for the US program are simple and universal: the quest to solve the profound mysteries that surround us

- The ability to bring together the teams to solve these mysteries:

- The next generation of accelerators

- The next generation of detector technology

- New simulation, computation and analysis techniques 


\section{and the mysteries}

- Mystery one: why are we here? From what we learned we should be a soup of photons!!!

- Matter and antimatter annihilation.

- We need to violate symmetry!

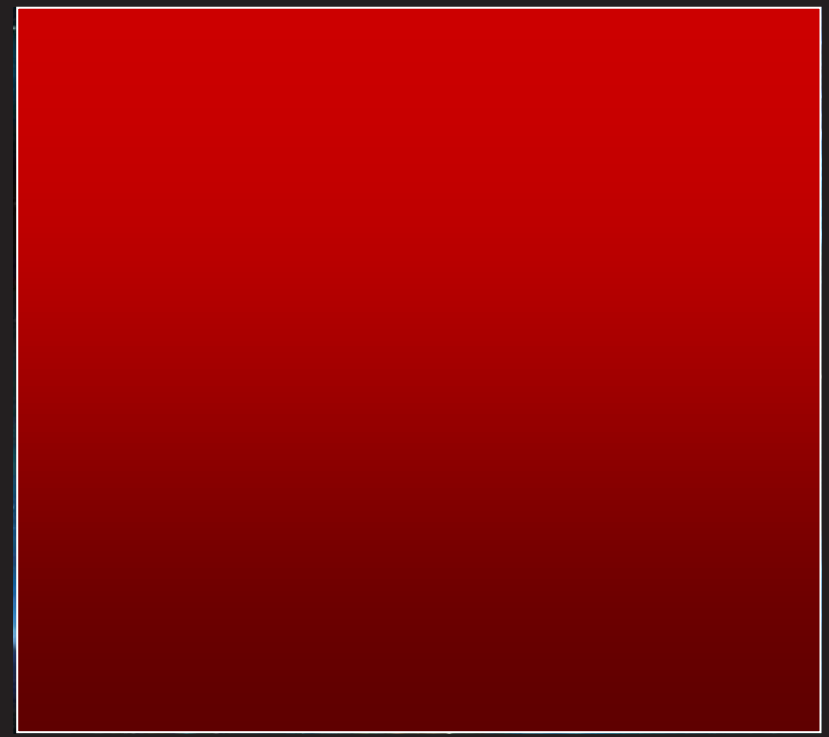

\section{Is it quarks? neutrinos? higgses?}
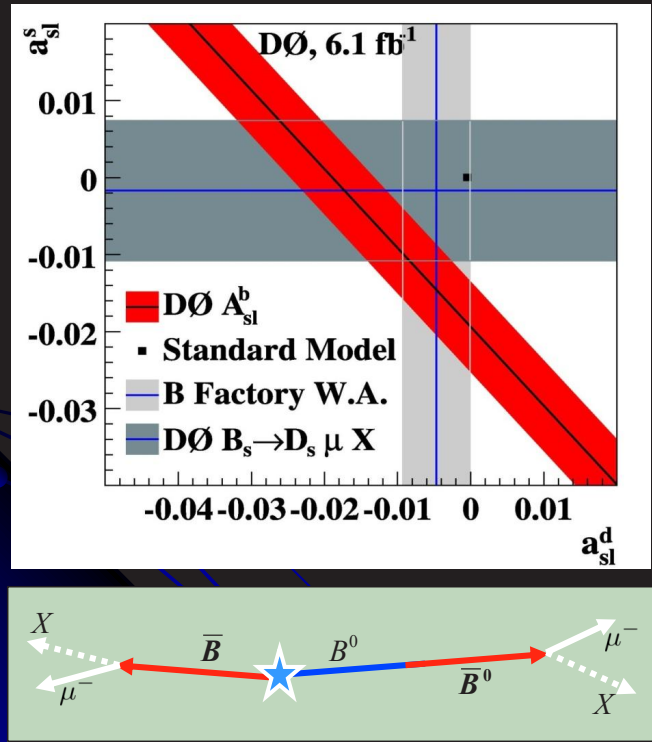

- Quark sector also studied at Tevatron and $\mathrm{LHCb}$

- Are we starting to see "anomalous" CP violation in $B_{s}$ decays?

- To be followed up with Tevatron extension, $\mathrm{LHCb}$, and Super-B factories 
Is it neutrinos? Ultimate Goal

multi MW beam

large detector (a few 100 kton)

long distance (> 1,200 km)
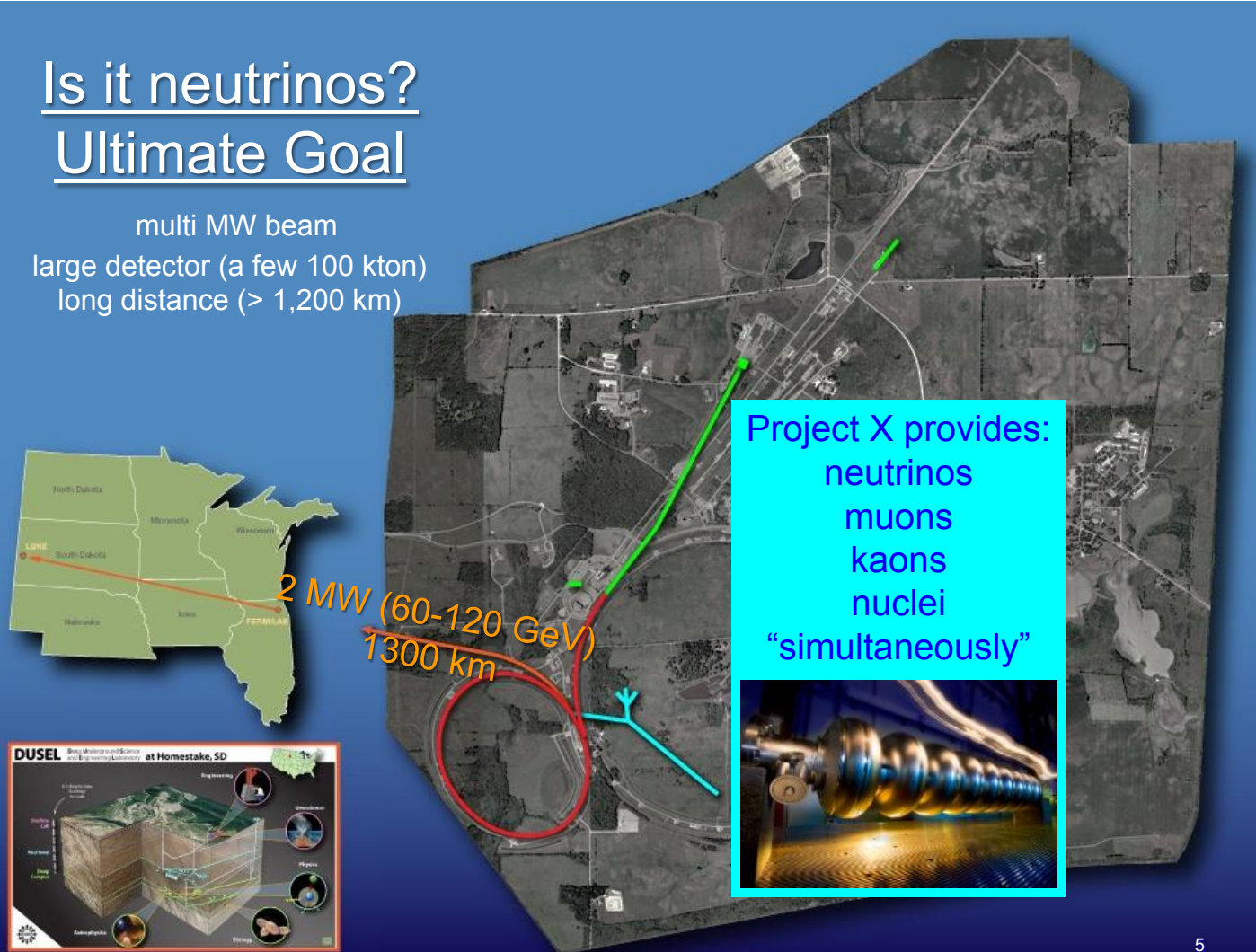

\section{Project $X$ provides:} neutrinos muons kaons nuclei

\section{More surprises in neutrinos?}
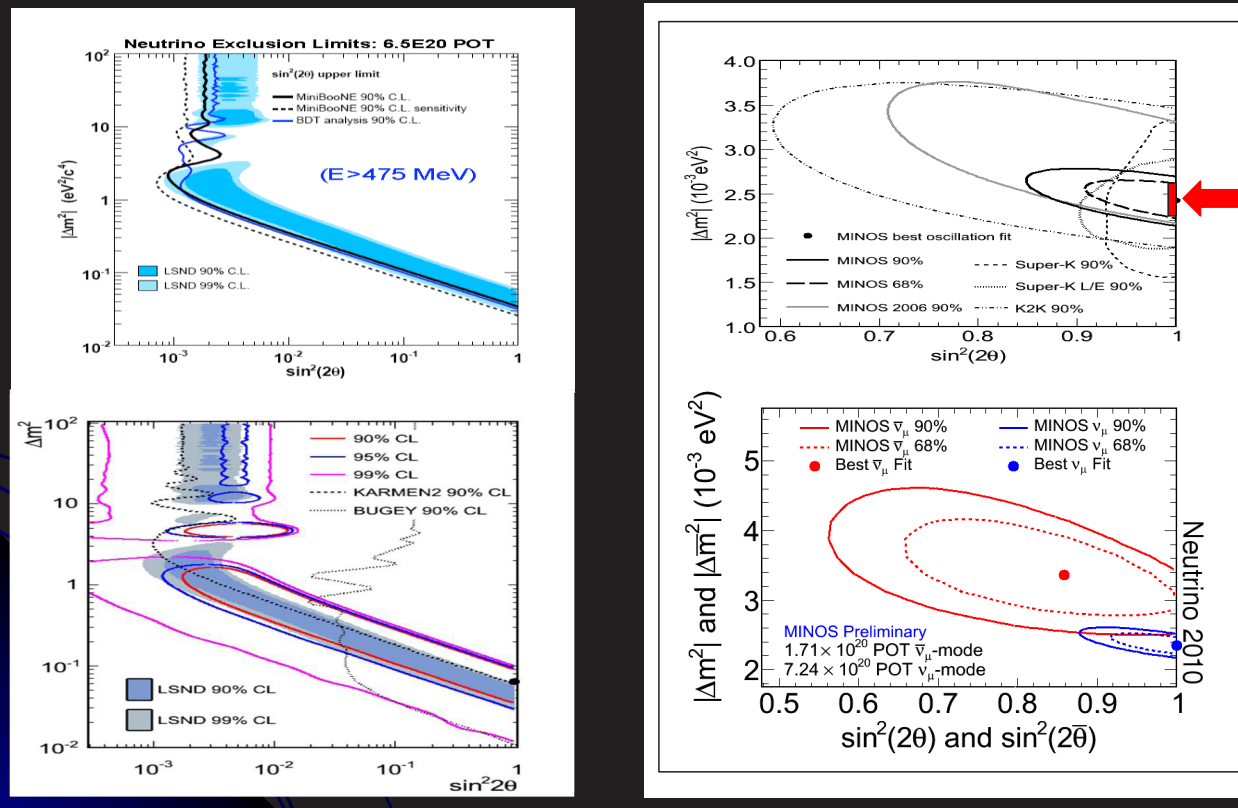


\section{Is it neutrinos?}

- The attack on neutrino parameters is broad:

- Fermilab (MINOS, MINERvA, MiniBooNE and MicroBooNE, NOvA, LBNE) and JPARC beams (T2K, HyperK): different baselines, different energies

- Reactor experiments: Double Chooz and Daya Bay

- Neutrino-less double B decay: Majorana and EXO

- New technologies: scale up of liquid argon TPCs to many kilotons

- R\&D on neutrino factory

\section{Higgs searches}
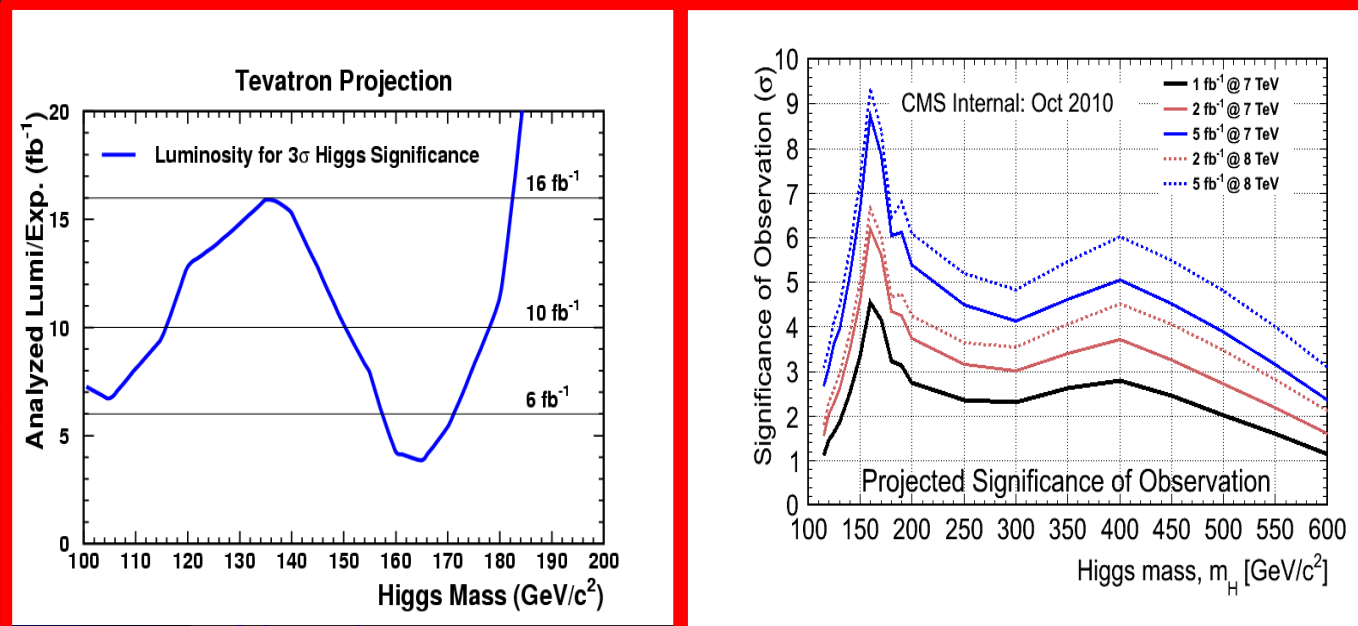

For very light Higgs, Tevatron is competitive through 2014 in the principal decay mode to the b-bbar - LHC needs about $30 \mathrm{fb}^{-1}$ to get results for b-bbar mode 


\section{Biggest decision of the decade !}

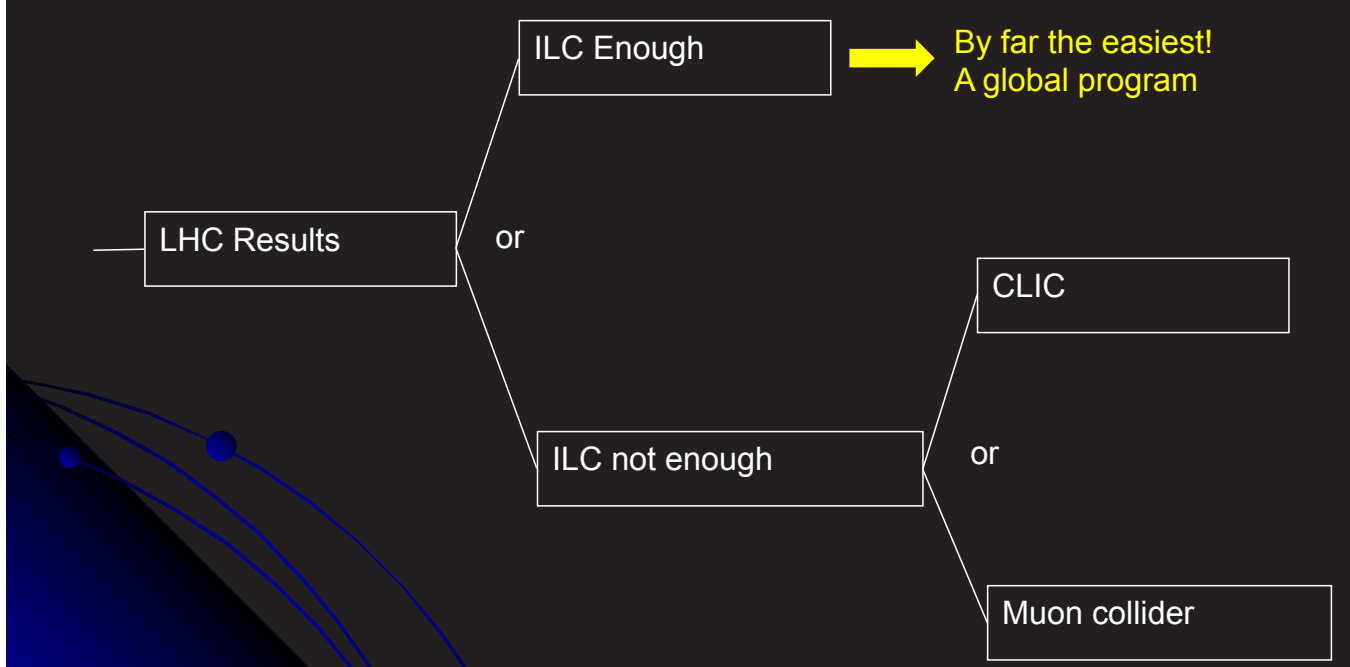

\section{Comparison of Particle Colliders}

To reach higher and higher collision energies, scientists have built and proposed larger and larger machines.

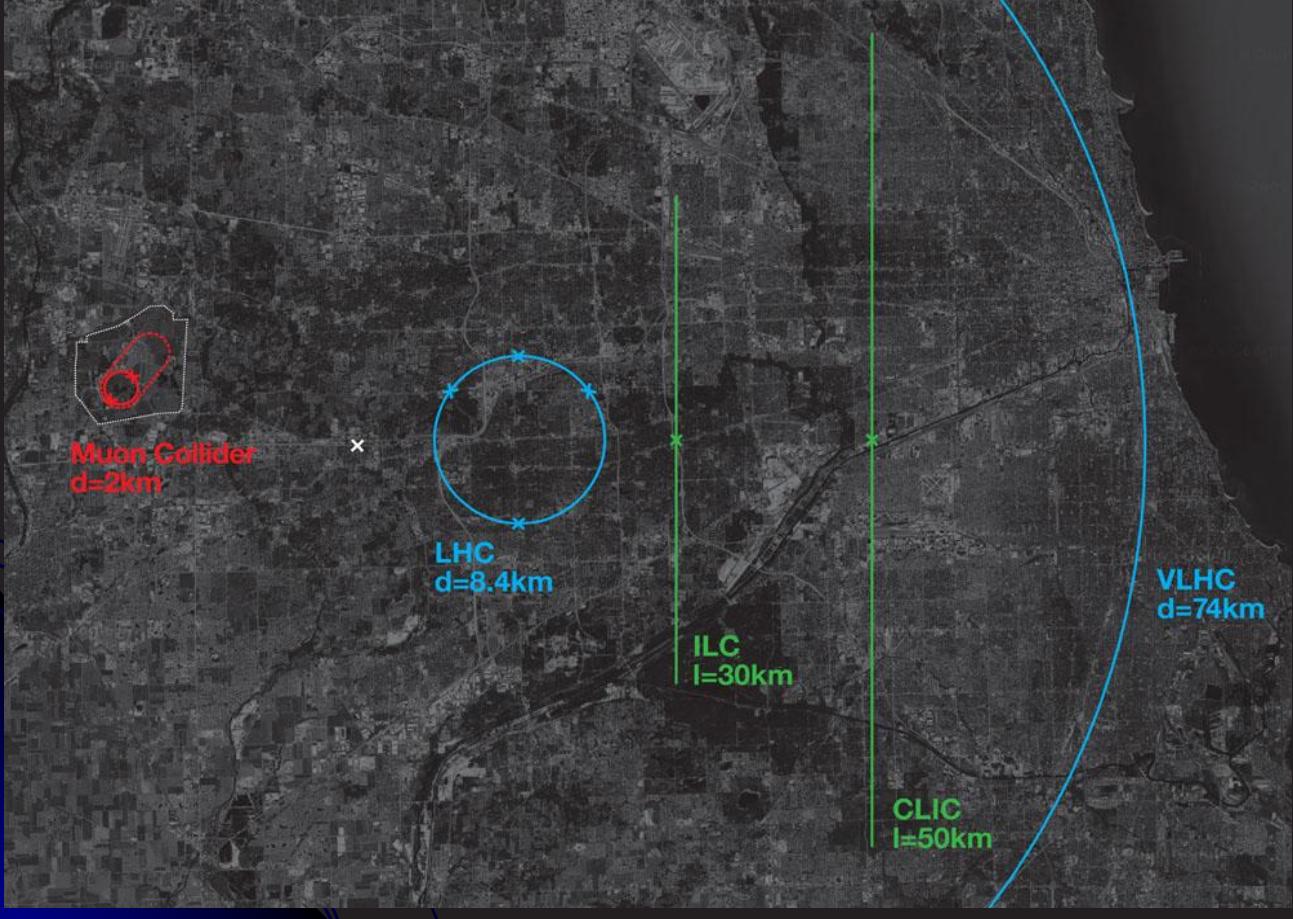




\section{Mu2e can probe $10^{3}-10^{4} \mathrm{TeV}$}

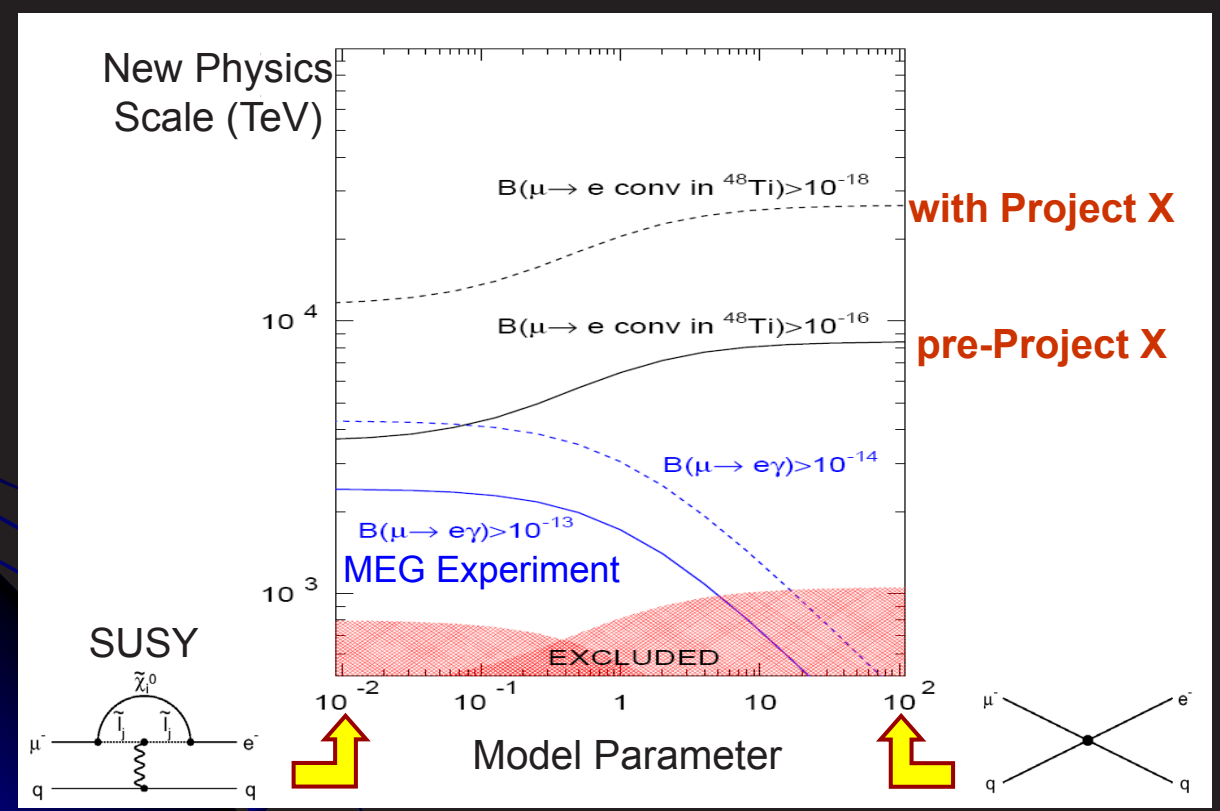

The search for dark matter
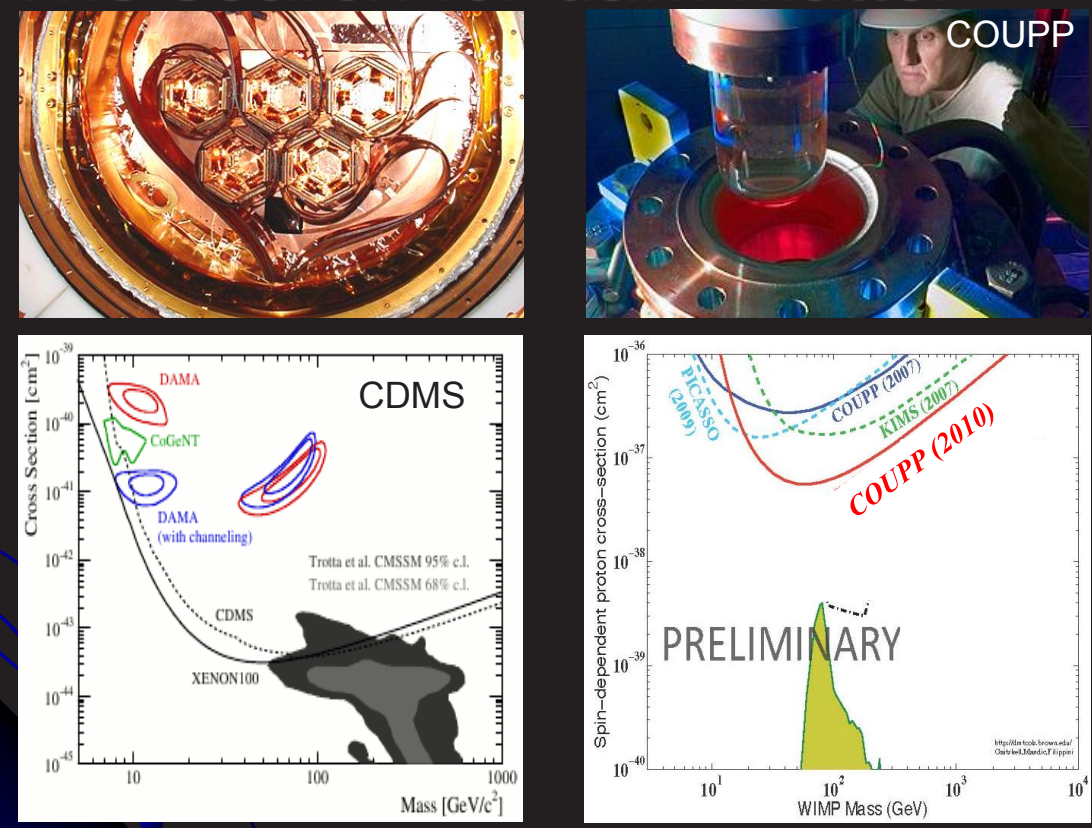


\section{Dark Energy}

1. SDSS (Sloan Digital Sky Survey)

- Ranks as highest impact facility in astronomy for the $4^{\text {rd }}$ year in a row.

- Baryon acoustic oscillations

2. DES (Dark Energy Survey)

- 4 meter telescope in Chile

- DES Camera under construction

- Operation: 2011 - 2016

3. LSST

- Important DOE role

- DOE/NSF collaboration

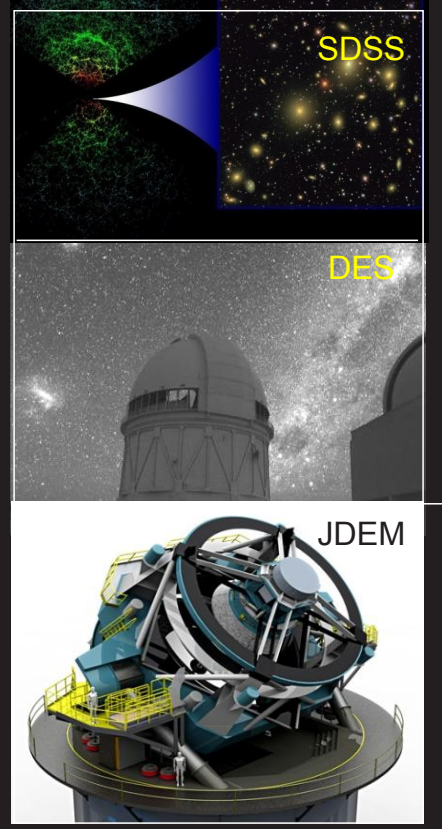

\section{Collaborative efforts at Fermilab}

International

collaborations

for our programs

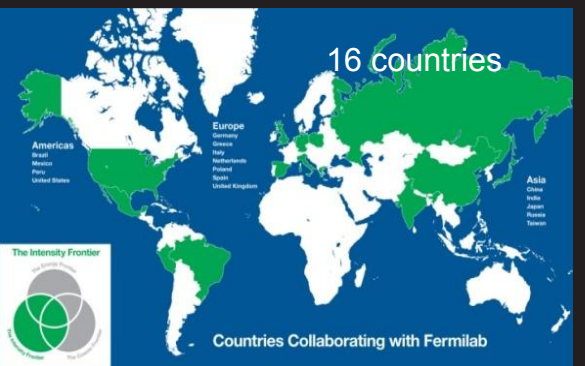

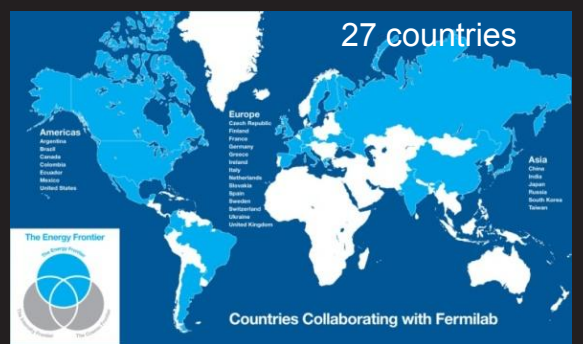

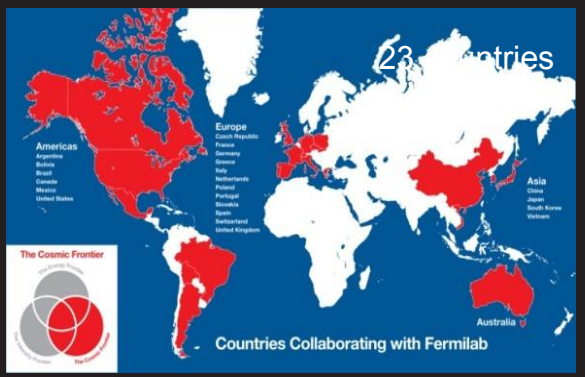




\title{
Future Accelerators - ICFA Chair' View
}

\author{
Atsuto Suzuki \\ KEK, High Energy Accelerator Research Organization \\ Tsukuba, Ibaragi, JAPAN
}

In the middle of 2000s, the world's high-energy physics community got down to developing a strategic plan for particle physics over the next ten years. In 2005, the US NRC (National Research Council) formed the committee EPP2010, charging members with recommending a 15-year implementation plan with realistic, ordered priorities.

In 2007, the P5 (Report of the Particle Physics Project Prioritization Panel) submitted strong, integrated research programs at the energy frontier, the intensity frontier, and the cosmic frontier; Slide 1 shows these detailed projects.

In 2006, the CERN council approved the European strategy for particle physics. The recommended scientific activity comprises eight courses with Research at the LHC and Upgrade, Accelerator R\&D of CLIC, Advance of ILC Design, Neutrino Programs, Flavor Studies, Astrophysics Research, Developing New Theories, and Work with Nuclear Physicists (Slide 2). It was recognized that the Japanese HEP projects would stand at a crossroads around 2010. Against this backdrop, the HEP project's roadmap was forged in 2008. Three approaches were proposed for new physics in the next decade: Search for new particles and new interactions through energy-frontier experiments (LHC, ILC); lepton physics through neutrino experiments, lepton flavor violations, and muon g-2 at J-PARC; and, Quark Flavor Physics at SuperKEKB and JPARC (Slide 3). Slide 4 gives the timelines of the recommended projects. Possible future world HEP facilities at the frontiers of energy and luminosity are summarized in Slide 5, from a presentation given by J.P. Delahaye at ICHEP (International Conference on High Energy Physics) 2010 held in Paris.

In particular, the lepton collider facilities are depicted in Slide 6-8. Previously, ICFA generally has only been involved in global projects, not local ones, but since particle physics is an international endeavor, ICFA perhaps should look at the complete picture, even though it has not done so in the past. In ICHEP at Paris, the ICFA reached a consensus that there would be value in writing a document coherently describing opportunities in particle physics throughout the world. Accordingly, a steering committee was established. There was also an extensive discussion of the governance for future large collider projects. Among several governance models, the multinational laboratory model shown in Slide 9 is found, in principal, to lead any future lepton collider that physics case justifies. In summary, for implementing future HEP projects, we are facing several difficulties arising from the increasing size of projects, their higher costs, and their much longer time span. The essential ways to overcome these problems are to globalize projects to avoid duplicating expenses, to assure adequate human- and technology- resources, and to secure strong public support through technological innovations benefitting society (Slide 10). 
Energy Frontier:

-- Continuation of Tevatron

-- LHC with the $1^{\text {st }}$ priority

-- R\&D for the next lepton

collidereve gis:
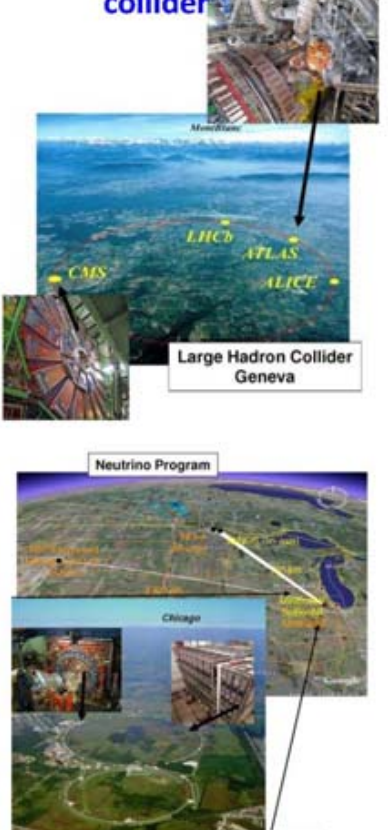

\section{US}

P5 : a strong, integrated research program at three frontiers
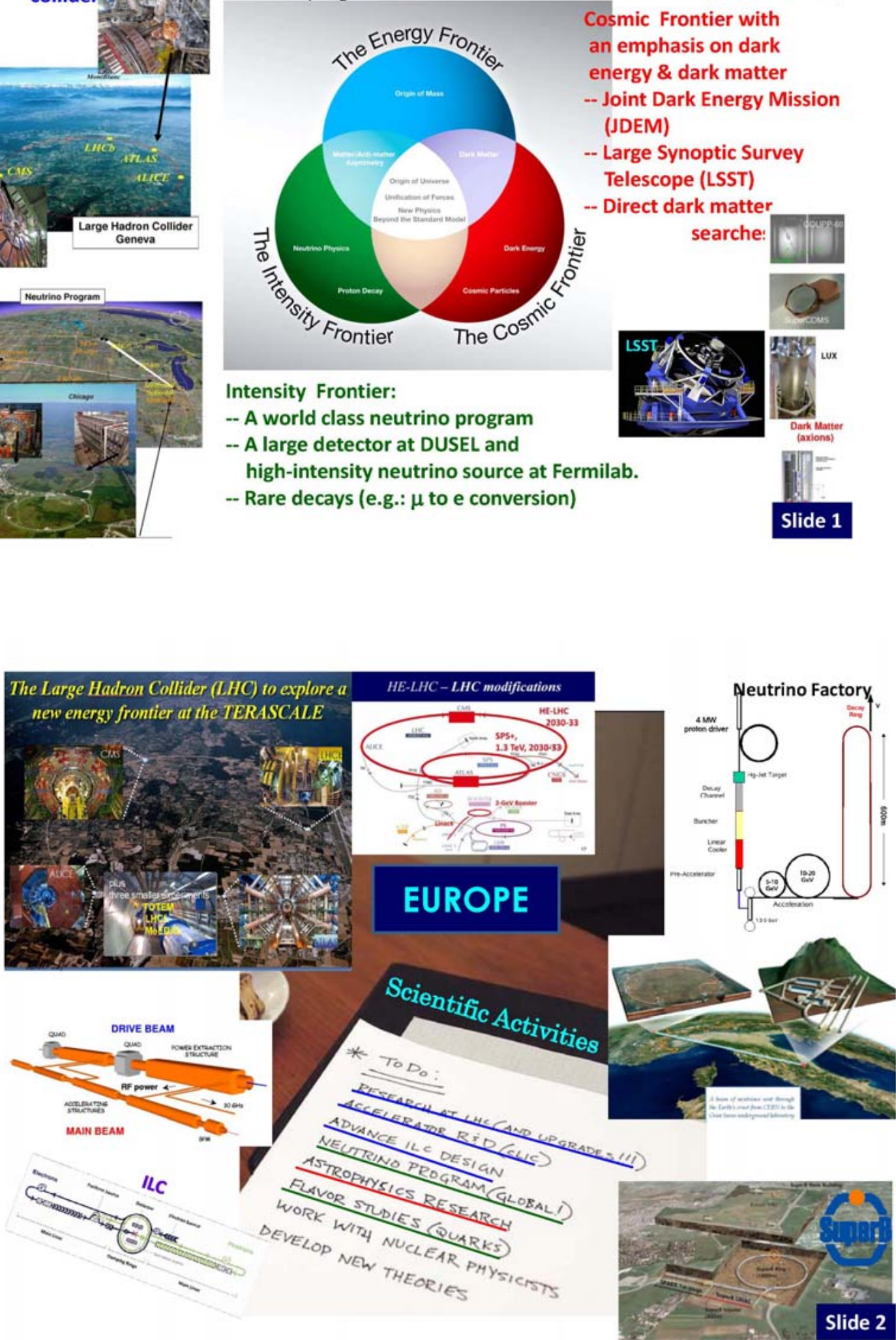


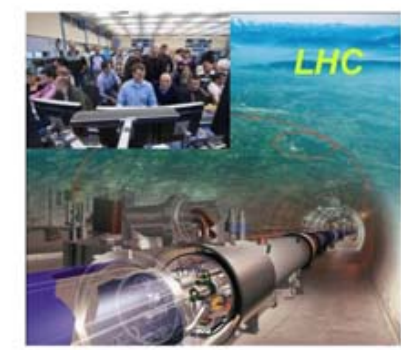

\section{Japan}

Energy frontier experiments LHC, ILC,

Higgs, SUSY, Dark matter, New understanding of space-time...
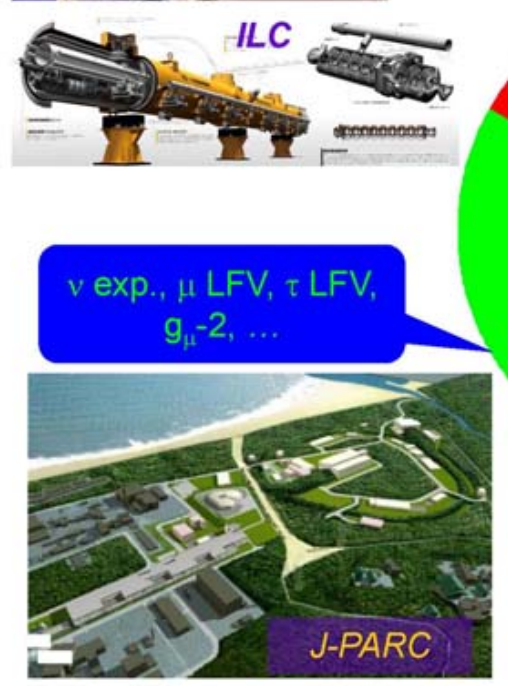

New particles and new interactions

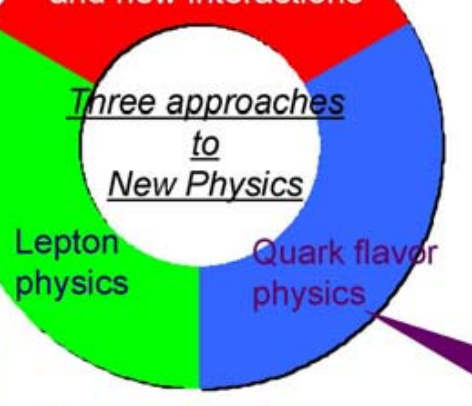

Neutrino mixing/masses, Lepton number nonconservation...

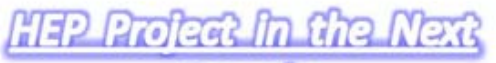
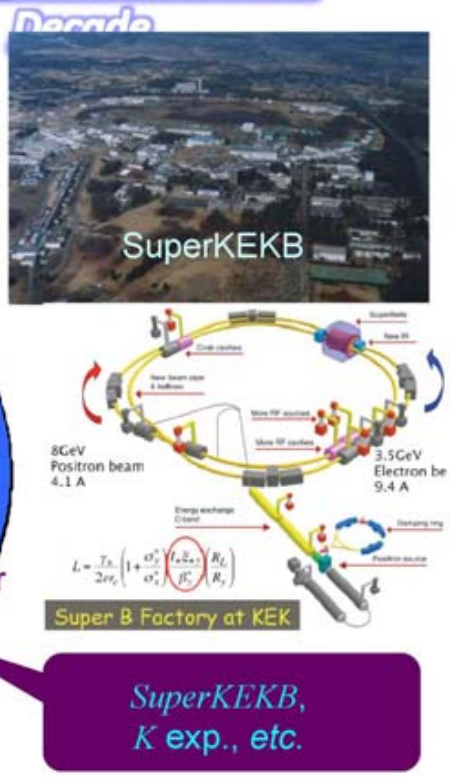

$\mathrm{CP}$ asymmetry, Baryogenesis, Left-right symmetry, New sources of flavor mixing...

Slide 3

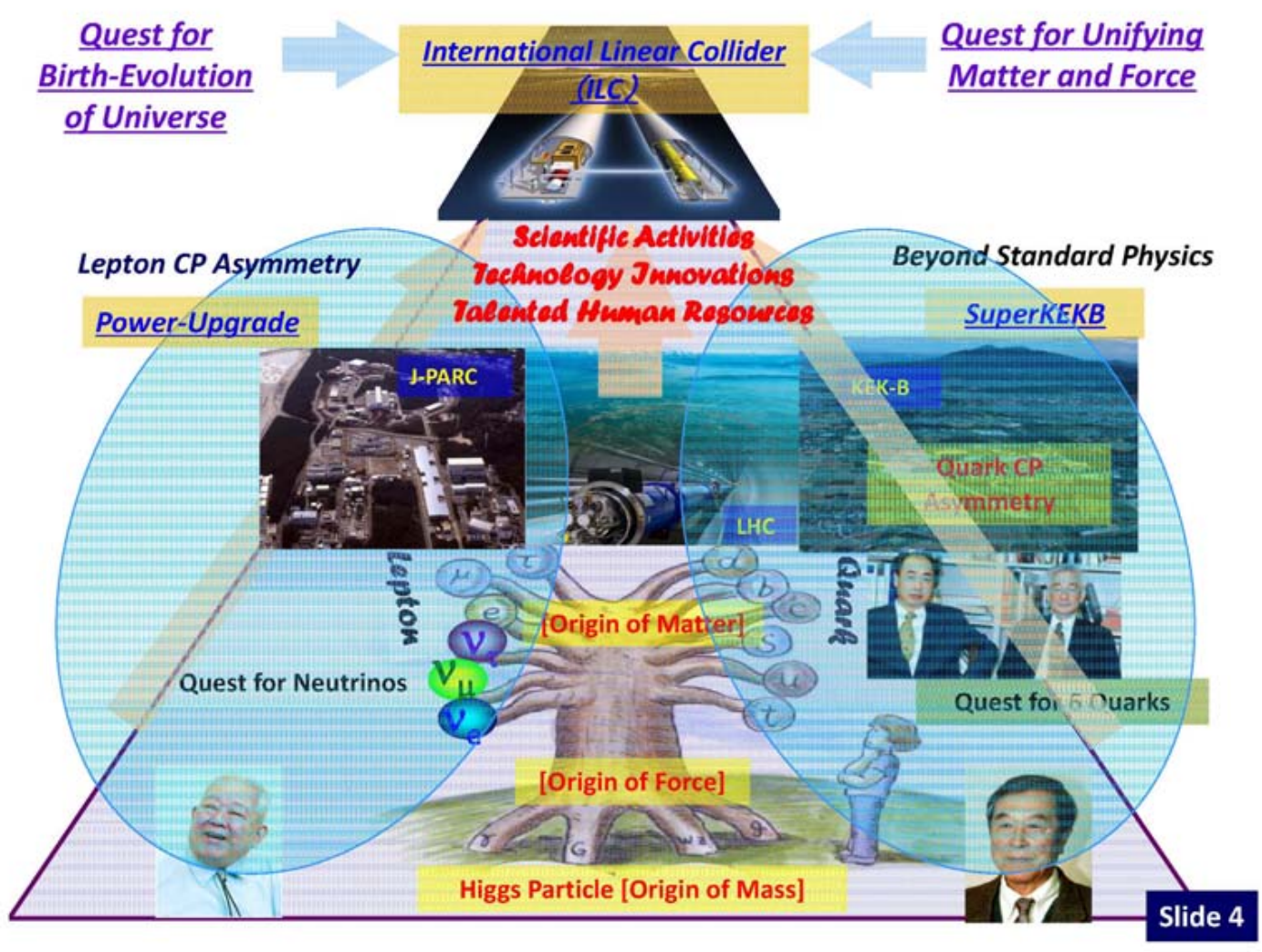




\section{Possible future HEP facilities at Energy/Luminosity frontier}

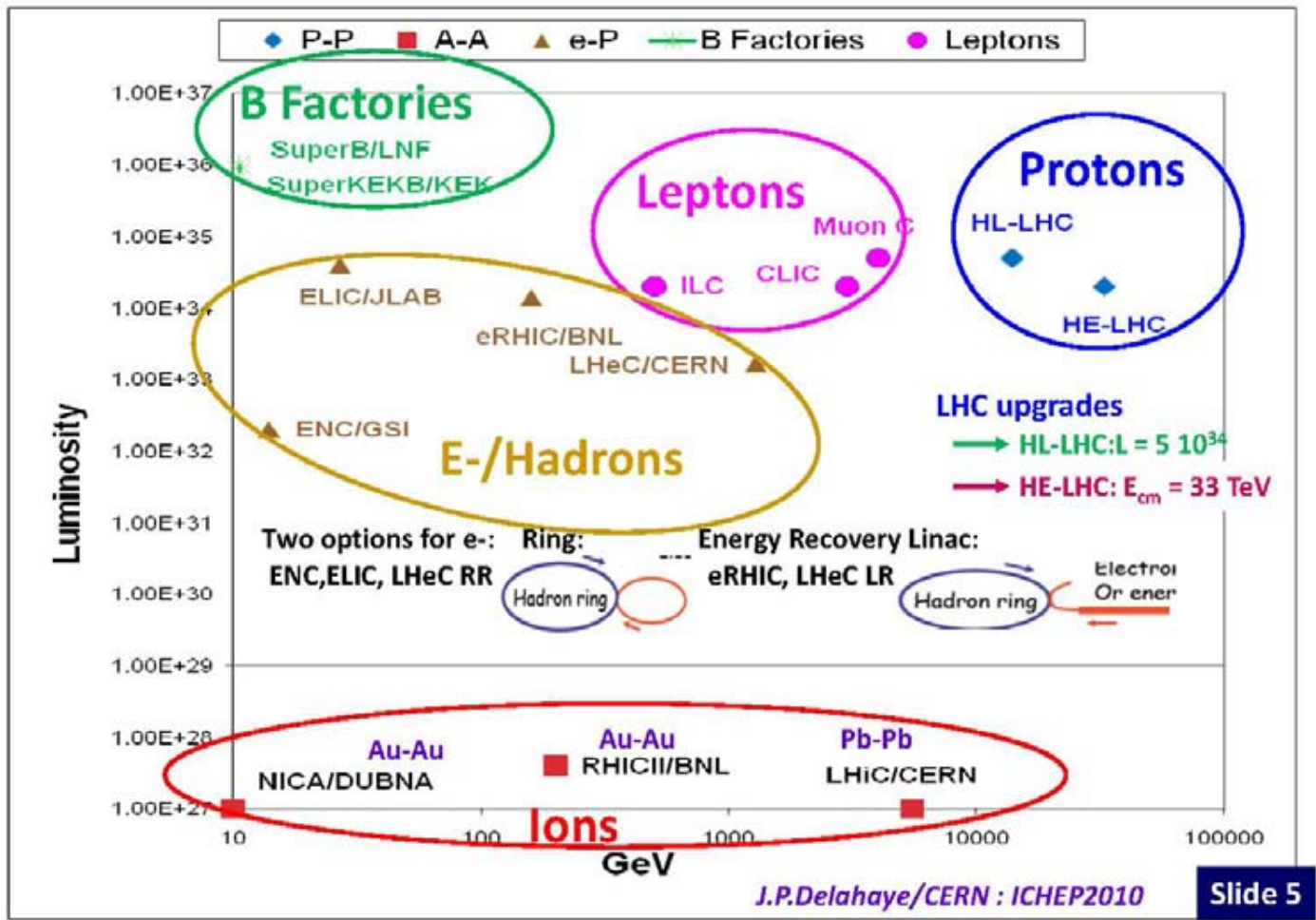

$\underline{\text { Lepton Colliders }}$

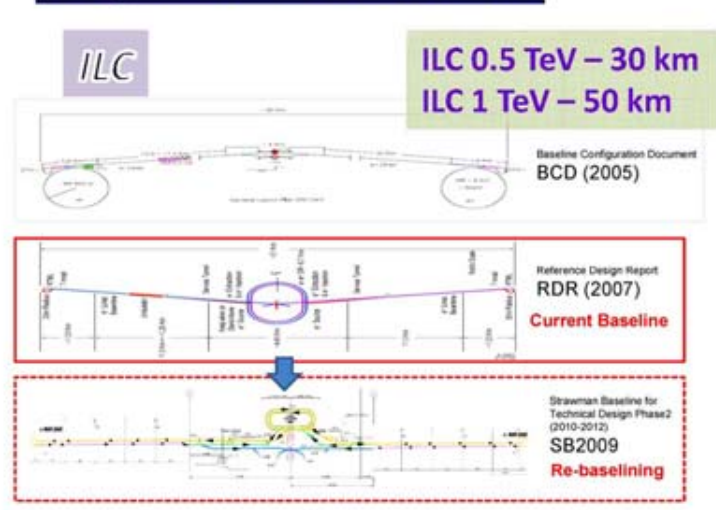

[ Cost containment]

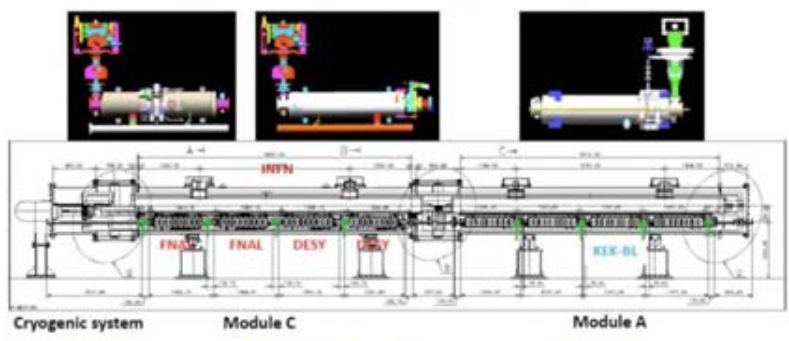

Plug-compatibility to be examined
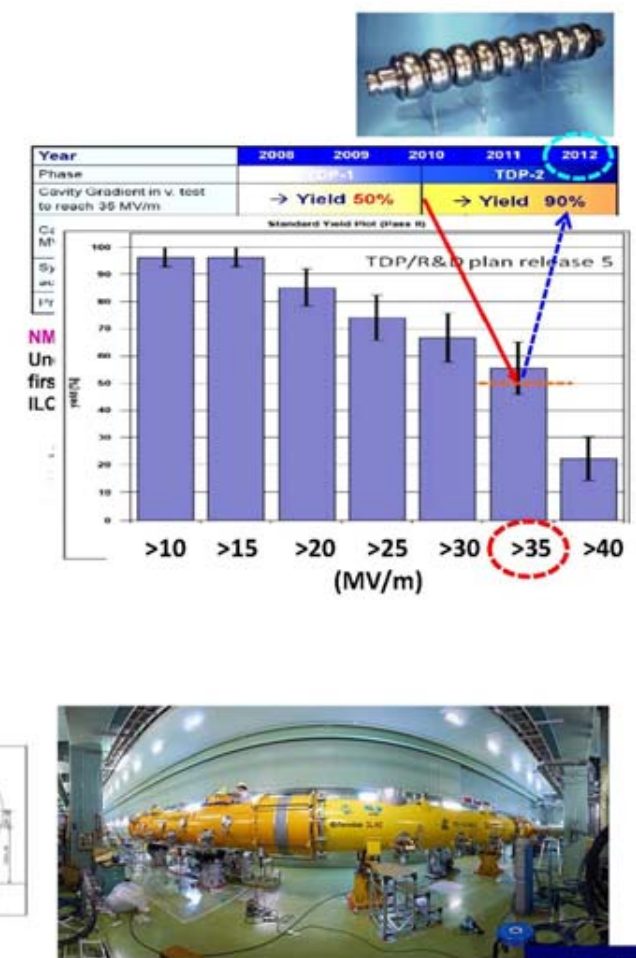

Slide 6 


\section{Muon Collider possible alternative}

for Multi-TeV Lepton Collider ?

Limited synchroton radiation and beamstrahlung due to high mass: $\left(m_{\mu} / m_{e} \sim 207\right)$

J.P.Delahaye/CERN

: ICHEP2010

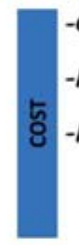

OMPACT

Fits on FNAL laboratory site -MULTI-PASS ACCELERATION Cost Effective -MULTIPASS COLLISIONS IN A RING ( 1000 turns)

Relaxed emittance requirements $\&$ hence relaxed tolerances

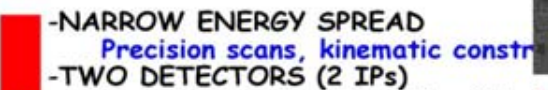
TWO DETECTORS (2 IPs)

y - $\Delta \mathrm{T}_{\text {bunch }} \sim 10 \mu \mathrm{s} \ldots$ (e.g. $4 \mathrm{TeV}$ collider) Lots of time for readout Backgrounds don't pile up $-\left(\mathrm{m}_{\mu} / \mathrm{m}_{\mathrm{e}}\right)^{2}=\sim 40000$

Enhanced s-channel rates for Higgs-like particles
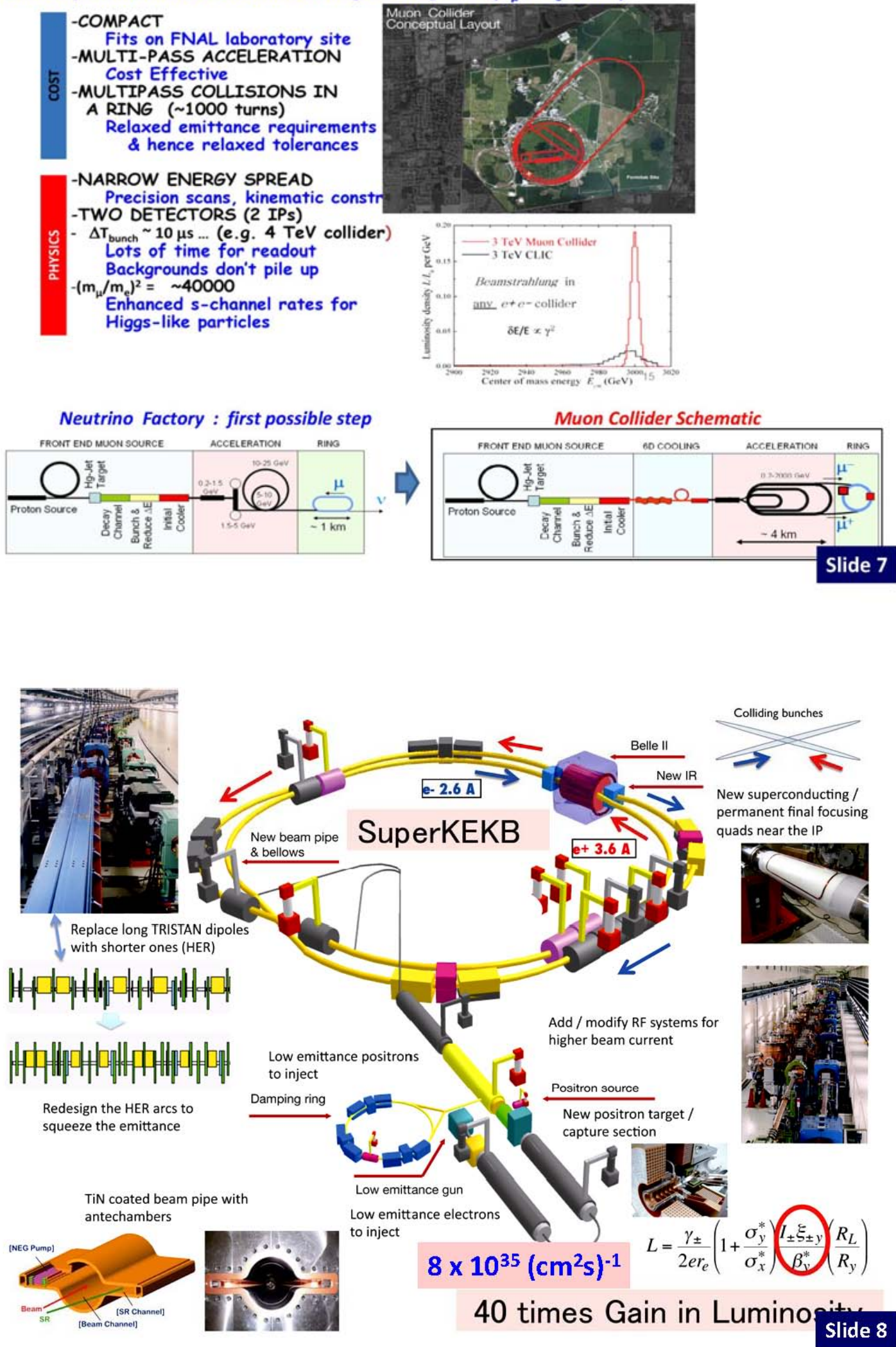
Possible Model of Global Governance for ILC
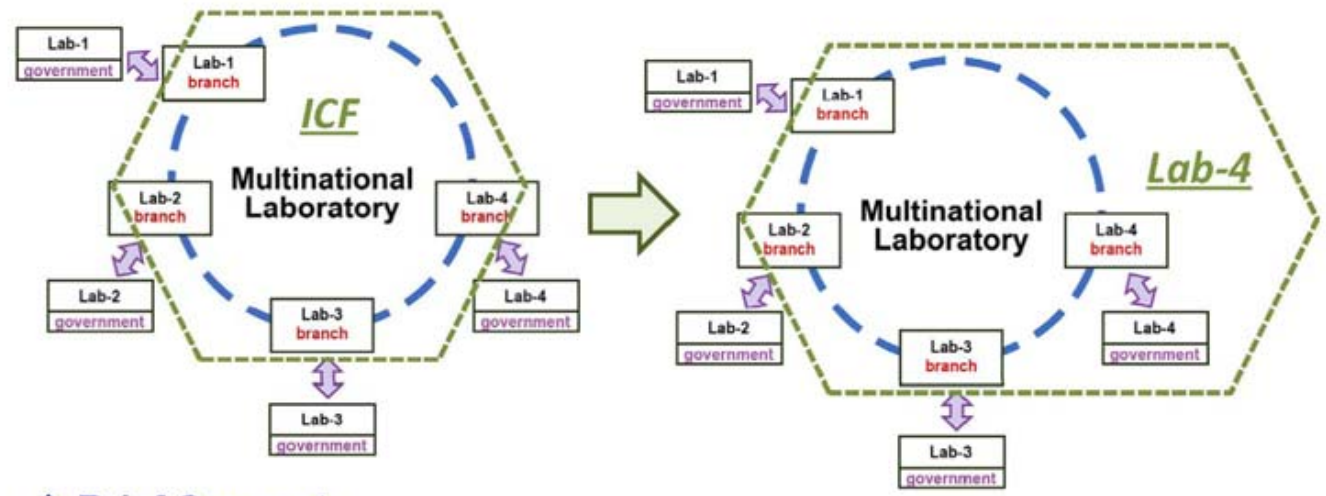

Brief Concept:

※ Each of world HEP-labs, which wishes to participate, sets up its branch within Multinational-Lab. These participating HEP-labs are called member-labs.

※ The member-labs contribute in sharing the human and financial resources with in-kind and common fund issues.

※ This Multinational-Lab is virtually built first in ICFA, and inside the host laboratory after the host-site selection.

\section{Summary}

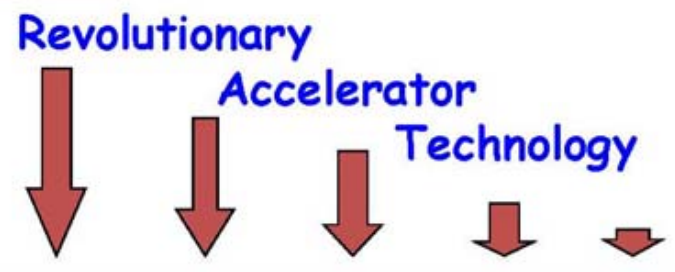

\section{Project Trends in High Energy Physics}

- Project Size

- Project Cost

- Project Time Span :

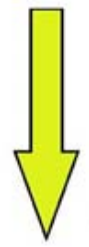

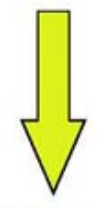

to Society
Bigger and Bigger Higher and Higher longer and longer

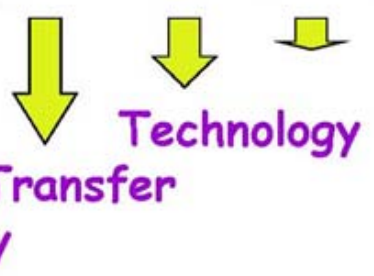




\title{
Future Plans at SLAC
}

\author{
David B. MacFarlane \\ SLAC National Accelerator Laboratory, Menlo Park, CA
}

In recent years, the scientific direction of the SLAC National Accelerator Laboratory broadened from its original focus on high-energy physics, to a mission centering on internationally-leading $\mathrm{x}$-ray light source facilities. The future development of these facilities is supported by an ongoing premier accelerator research-and-development program encompassing cutting edge research for Free Electron Lasers (FEL), high-gradient x-band technology, and novel acceleration mechanisms. SLAC also is pursuing selected strategic initiatives in particle physics, particle astrophysics, and cosmology. The program has benefited from decades of close cooperation, collaboration, and healthy competition with our Japanese colleagues. We anticipate that these important connections will continue in future. A particularly compelling example of close cooperation between SLAC and KEK, although not formally a part of the US-Japan Cooperation Agreement, was the closely tied development, operation, and scientific exploitation of the PEP-II and KEKB asymmetric-energy $e^{+} e^{-} B$ Factories. Both $B$ Factory programs benefited enormously from these valuable interactions, and the exchange of ideas and technical innovations in advancing the science of heavy-flavor physics and $C P$ violation.

My presentation briefly reviews the status of, and future upgrade plans for the photon-science facilities operating at SLAC, namely the Linac Coherent Light Source (LCLS), the world's first $\mathrm{x}$-ray FEL, and the SSRL's third-generation synchrotron light source. I discuss the existing and planned high-energy physics program, focusing on plans for accelerator R\&D and the major new projects we will pursue to address physics questions at the energy-, intensity-, and cosmologyfrontiers. Accelerator R\&D includes a major effort to enable the next energy-frontier linear collider, studies on the cutting edge of $\mathrm{x}$-band high gradient research, and novel plasma wakefield acceleration techniques with the FACET facility. Our new initiatives in particle physics and particle astrophysics include the Enriched Xenon Observatory (EXO), the Large Synoptic Survey Telescope (LSST), and the next-generation implementation of the Cryogenic Dark Matter Search (SuperCDMS) experiment.

\section{Outline}

- Current and future SLAC photon science facilities

- Current and future High Energy Physics program

- Accelerator research and development directions

○ Intensity Frontier: EXO

- Cosmic Frontier: LSST, SuperCDMS

- Conclusions 

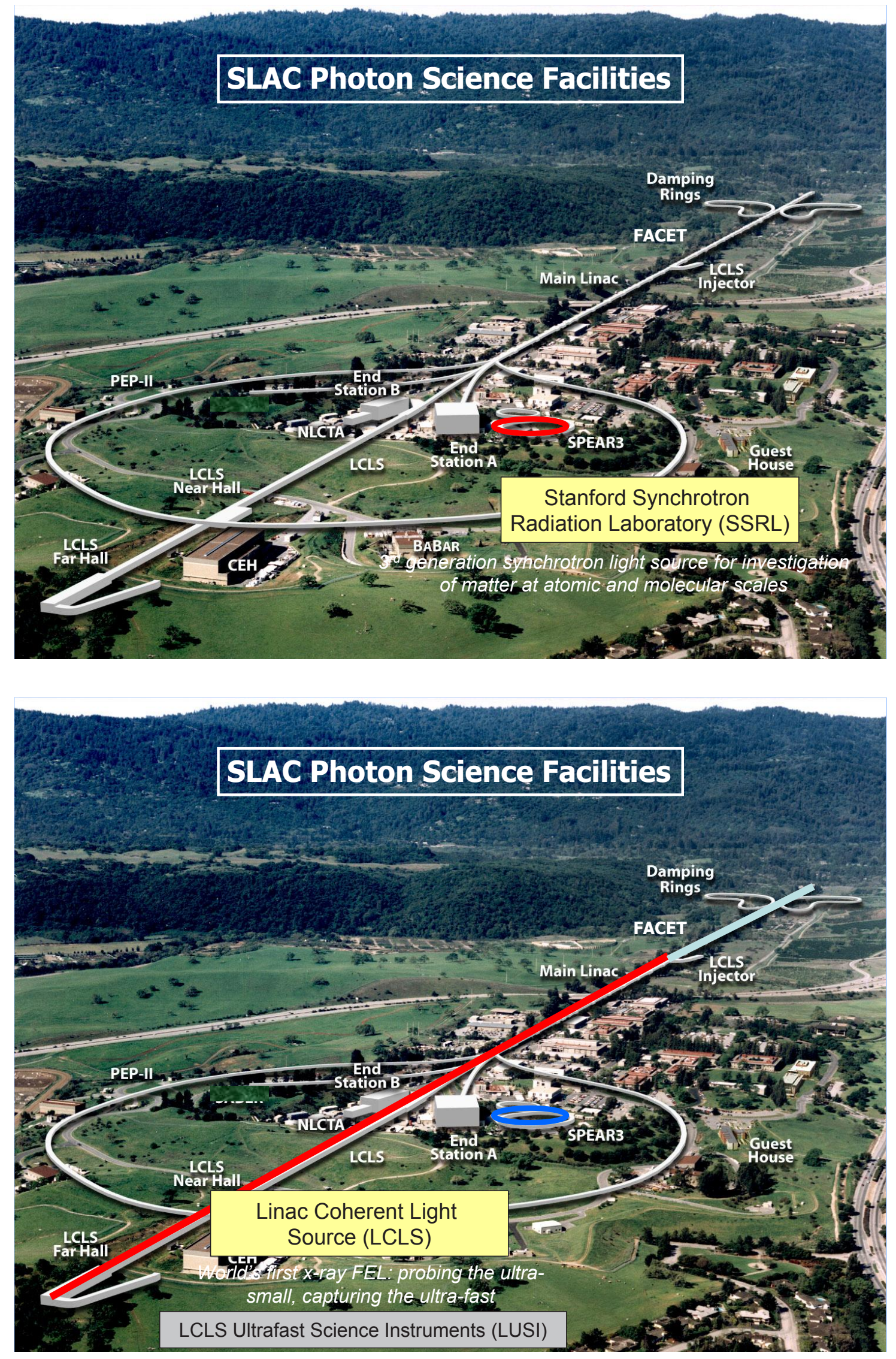


\section{Future of SLAC x-ray Facilities}

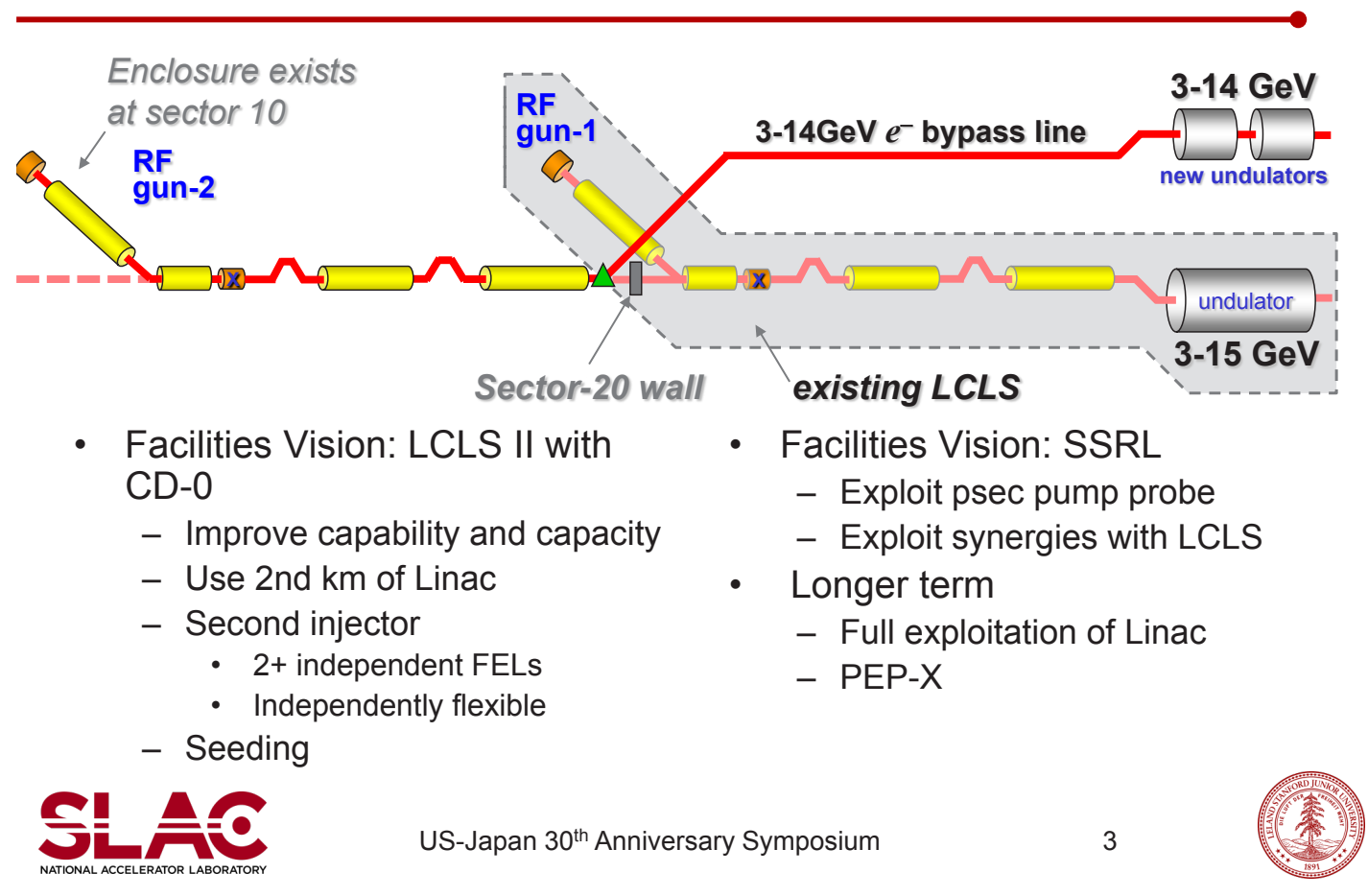

\section{Accelerator R\&D Directions}

- SLAC Accelerator research key to future of the laboratory

- Focused on advancing operating facilities and next generation of HEP and BES accelerators

- Supports all labwide objectives

- Goals of accelerator R\&D Initiative

- Maintain world-leading XFEL program with innovation and new concepts

- Be the world-leader in high power rf systems and high gradient rf linacs

- Be a world-leader in advanced accelerator R\&D with focus on e+/e-

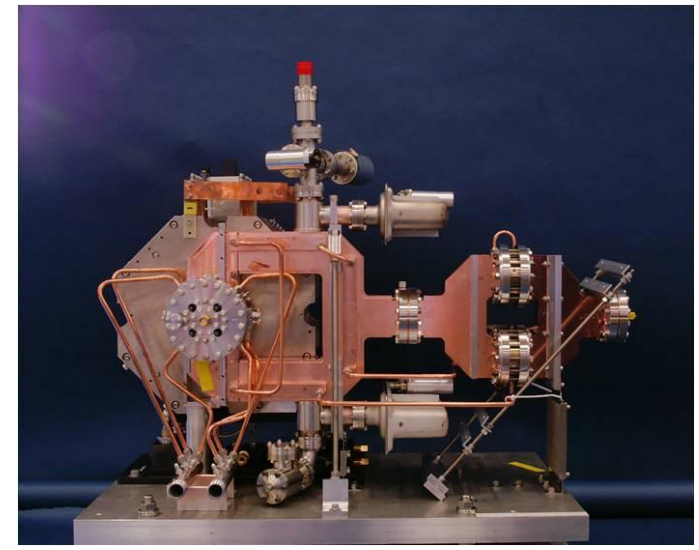

- Associated benefits

- Support ongoing accelerator-based laboratory program

- Accelerator education program 


\section{Strategy: Next Energy Frontier machine}

- Strong partner in International Linear Collider (ILC) R\&D

- GDE plan for R\&D and TDR development by 2012

- Reduced longer-term R\&D effort thereafter, unless project launched

- High gradient research pursued in conjunction with CERN's Compact Linear Collider (CLIC) effort

- Collaboration with GDE on many systems \& ILC detector community

- Participant in Muon Collider R\&D program led by Fermilab

- Part of Fermilab's long-term strategy building on high-power proton sources: Project- $X$, neutrino factory, and muon collider

- Major technical challenges to be addressed

- Explore other warm rf strategies with CERN, KEK

- Physics or cost may drive international community to consider a broader approach to energy frontier lepton colliders

\section{Plasma Acceleration: 1000x present gradients}

- FACET

- Demonstrate feasibility for controlled electron/positron bunch acceleration

- Future

- $50 \mathrm{GV} / \mathrm{m}$ linear collider or compact radiation source

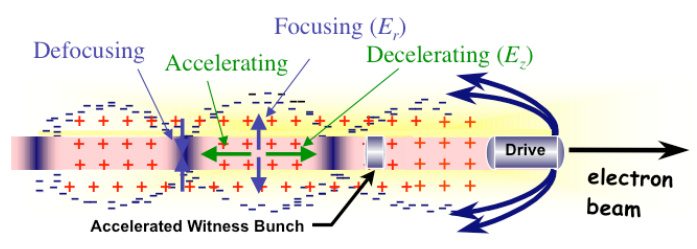

Simulation of $25 \mathrm{GeV}$ PWFA stage

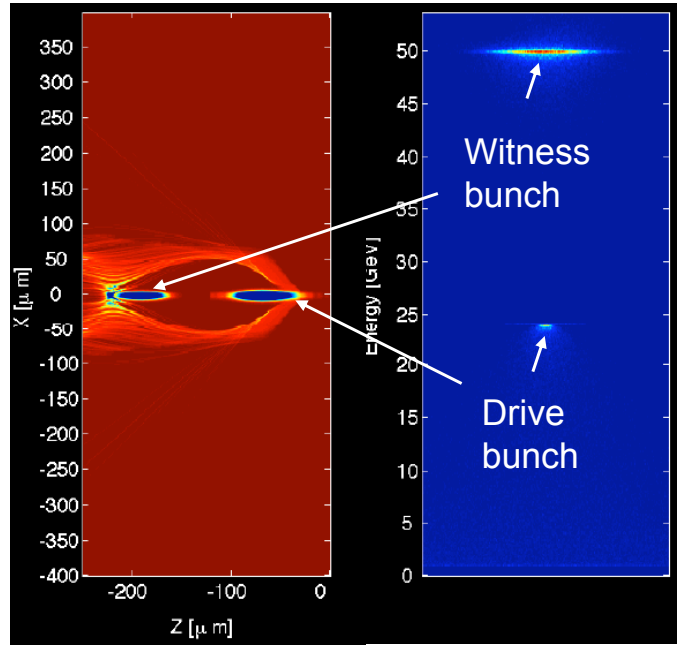




\section{Enriched Xenon Observatory (EXO-200)}

- Search for neutrinoless double beta decay in $200 \mathrm{~kg}$ of ${ }^{136} \mathrm{Xe}$

- Occurs if neutrinos are Majorana \& lepton number violated

- Rate is proportional to $<m_{v}>2$

- EXO-200 currently being set up at WIPP

- Should be taking data with natural xenon by fall of 2010

- SLAC plan

- EXO-200 data taking; R\&D for tonne-scale EXO over next 5 years
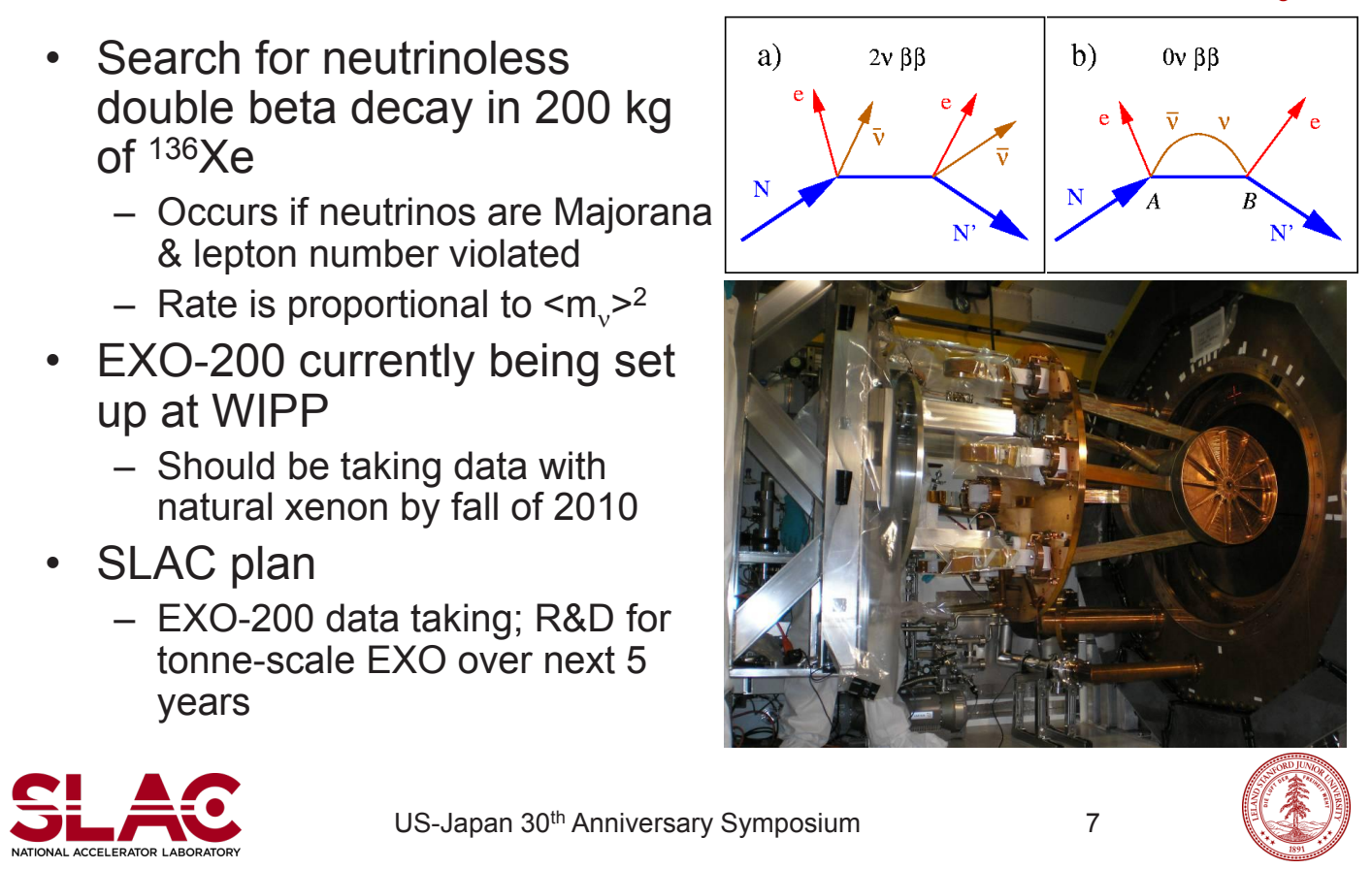

\section{Large Synoptic Survey Telescope}

- Provide a sensitive survey of the entire sky at visible wavelengths every few nights

- Tight constraints on cosmic expansion history, and thus on the nature of Dark Energy

- Envisioned as a collaborative NSF and DOE-HEP project

- NSF: telescope \& data system

- DOE: 3.2 Gigapixel camera

- SLAC plan

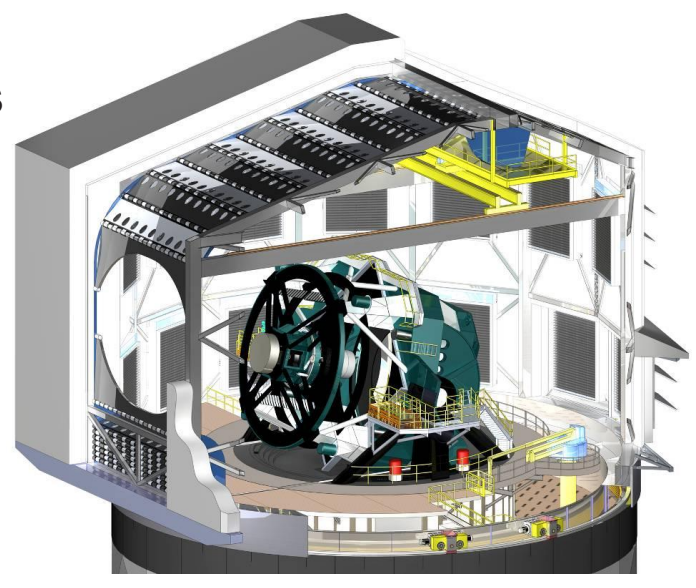

- Lead consortium to complete camera design and construction 


\section{Direct dark matter search: SuperCDMS}

- Direct search for relic dark matter

- Cross sections $\sim 10^{-46} \mathrm{~cm}^{2}$ for $100 \mathrm{GeV}$ WIMP masses

- SNOLAB project aims at $100 \mathrm{~kg} \mathrm{Ge}$ detector mass

- 6-fold increase over current Soudan project

- SLAC plan

- Partner with Fermilab on SNOLAB project, with focus on large $\mathrm{Ge}$ sensors and towers

Next generation sensors:

$100 \mathrm{~mm}$ diameter $\times 33 \mathrm{~mm}$ thick

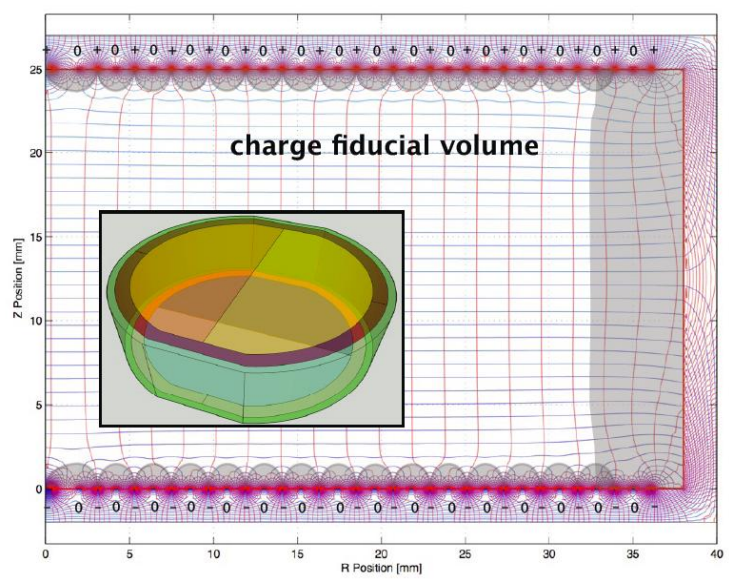

9

\section{The Future of SLAC}

- A new scientific frontier is being opened at LCLS

- Biggest surprises are yet to come!

- Strategic programs in particle physics and astrophysics

- Energy frontier with LHC

- Cosmic frontier with Fermi, LSST, and SuperCDMS

- Accelerator Research is a cornerstone of science programs

- We look forward to a continuing rich collaboration in years to come!

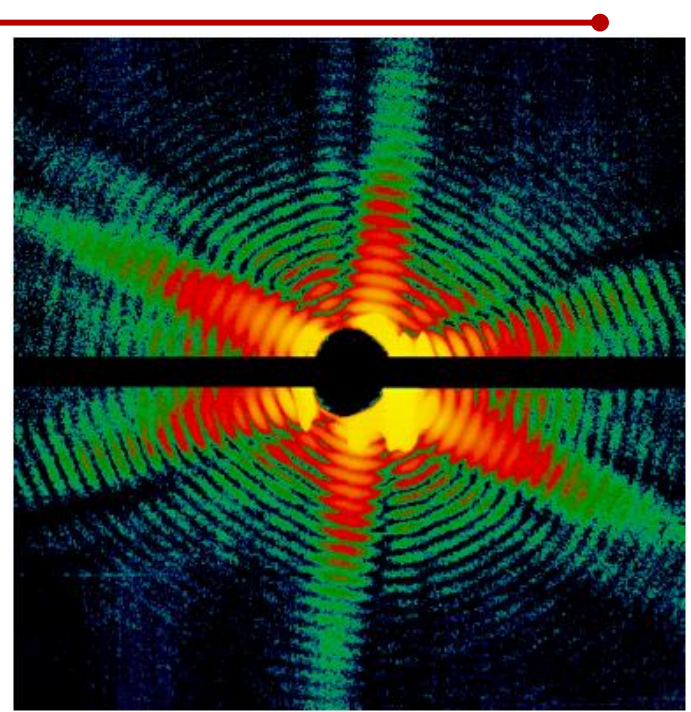

Single Shot Image from LCLS 


\title{
Future Plans at Brookhaven National Laboratory
}

\author{
Samuel Aronson \\ Brookhaven National Laboratory, Upton, NY 11973, US
}

I discuss present activities and our plans in Nuclear Physics, Particle Physics, and Accelerator Science and Technology in the context of the very successful ongoing US-Japan Collaboration in High Energy Physics. I highlight the contributions to this research by Japanese laboratories and universities.

Following the fixed target relativistic heavy ion studies at the BNL AGS, Nuclear Physics research at BNL and in the US-Japan Collaboration focused on the PHENIX experiment at BNL's Relativistic Heavy Ion Collider. The Japanese institutions have had a major impact on PHENIX, providing scientific leadership, constructing its hardware, processing the data, and analyzing the physics. Many Japanese researchers were trained at PHENIX (and in other BNL experiments) through the US-Japan Collaboration, and have gone on to occupy the leadership ranks of Japanese nuclear- and particle physics-research. Expectedly, the heavy ion- and spinprograms at RHIC and PHENIX will run productively for up to 10 more years, thereby elucidating the significant and unexpected findings at RHIC so far. During this period, the LHC heavy-ion program will begin to operate, affording us complementary data. BNL's longer range planning is aimed at adding a high-energy electron linac to RHIC (eRHIC) to allow e+A- and polarized $e^{+} p$-collisions at high energy and very high luminosity. Significant upgrades to the PHENIX detector currently under discussion for eRHIC will provide a future path for the USJapan effort in PHENIX.

The US-Japan Collaboration enabled the participation of Japanese research institutions in several high-impact experiments in Particle Physics at BNL, including the electron-neutrino elastic scattering experiment (E734), and the ultra-rare K-decay experiment (E787/E949). Currently, and into the coming decade or more, BNL and Japanese institutions are participating in ATLAS at the LHC. In addition to working on ATLAS and its upgrades, BNL's plans in Particle Physics include neutrino oscillation experiments and particle astrophysics and cosmology.

Collaborative work on Accelerator Science and Technology at BNL included R\&D on superconducting RF and other technologies. BNL is designing a muon collider/neutrino factory, as well as energy-recovery linacs and high-brightness electron guns for eRHIC and X-ray free electron lasers. Advanced acceleration concepts are being pursued at the BNL Accelerator Test Facility, a unique, peer-reviewed facility. 


\section{BNL's Overall Future Plan}

- Multi-program lab

- Discovery to Deployment

- Activities

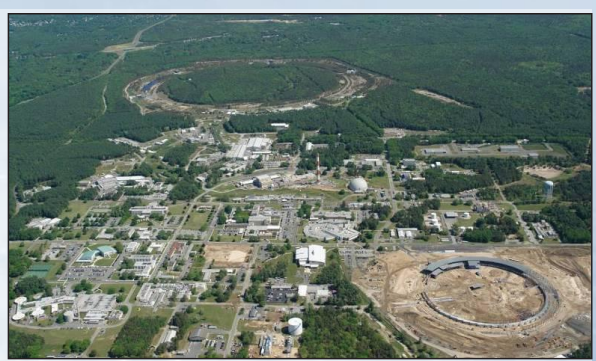

- Nuclear \& Particle Physics

- Photon Sciences

- Energy \& Nanoscience

- Climate \& Life Sciences

- Nuclear Nonproliferation \& National Security

- A Core Capability

- Accelerator Science \& Technology

\section{Activities in Nuclear Physics}

- RHIC

$-10^{\text {th }}$ run completed in July

- Au-Au, Cu-Cu, d-Au, p-p, ${ }_{s_{\mathrm{NN}}}$ from 7.7 to $500 \mathrm{GeV}$

- Results (some unexpected)

- Strongly coupled (!) QGP

$-\mathrm{T}>\mathrm{T}_{\text {crit }}$

- Collective flow $\rightarrow$ "Perfect Liquid"

RHIC Spin

- Gluon contribution to nucleon spin

- $\mathrm{W}^{ \pm}$production to flavor-separate

$q, \bar{q}$ helicity distributions
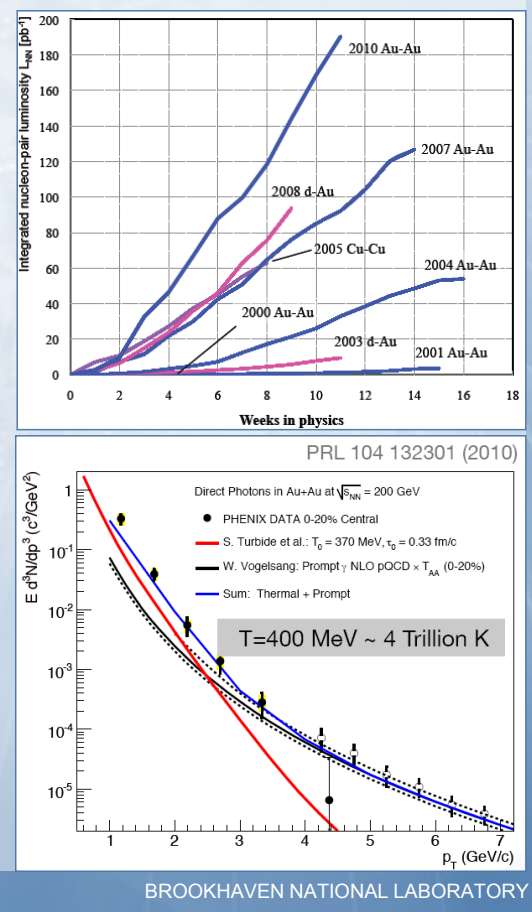


\section{RHIC Physics, Impact}

- RHIC has turned out to be a powerful and flexible tool:

- Structure and dynamics of a non-abelian gauge theory

- Phase diagram of QCD

- Spin of the proton

- Relationship to other strongly coupled systems

- Cold atomic gases

- Strongly correlated electron systems

- Relationship of QCD to semi-classical gravity and black holes (AdS/CFT)
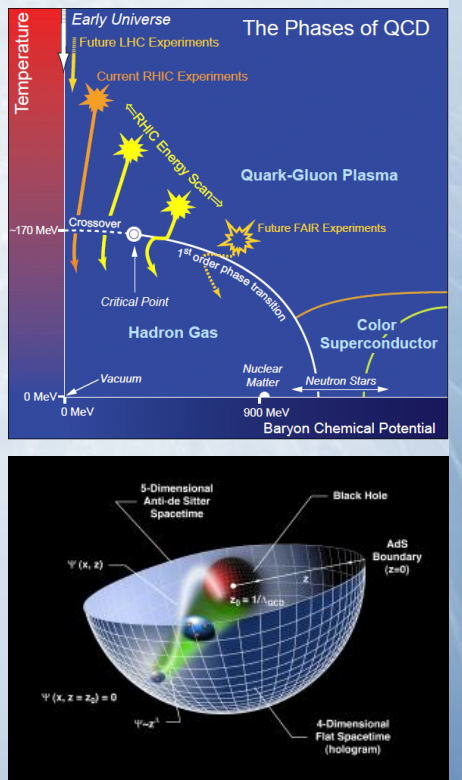

\section{Long-Term Strategic Timeline for the RHIC Facility}

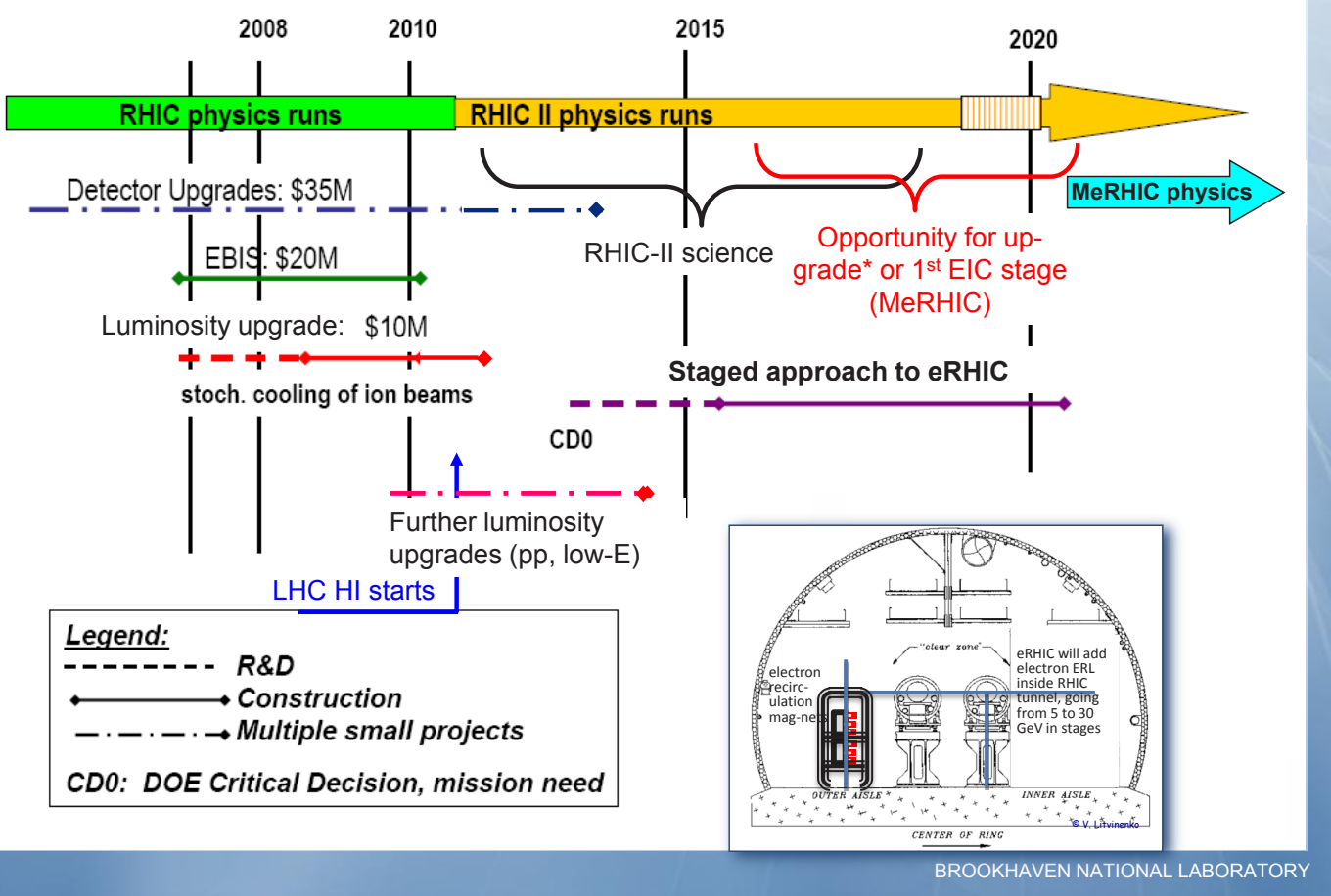


Midterm ( $\leq 2015$ ) and Long-Term (>2015) Plans in Nuclear Physics

- RHIC-II

- Luminosity (with stochastic cooling)

- Detector upgrades under way, some online in 2011

- RHIC-II Physics

- Is there a critical point in the QCD phase diagram?

- How are QCD jets affected by the medium?

- Mechanisms of energy loss, light \& heavy flavor jets

- What are the properties of the SQGP?

- Chiral symmetry restoration?

- Ultimate RHIC Physics Questions

- Detailed mechanisms for parton-QGP interactions?

- Quasi-particles in the medium? What are $m$ and $\Gamma$ ?

- Is there a relevant color screening length in the QGP?

- How are rapid equilibration and entropy production achieved?

- Gluon saturation/shadowing effects in A+A collisions?

- RHIC Requirements

- Further $\mathcal{L}$ enhancements

- Significant further detector upgrades
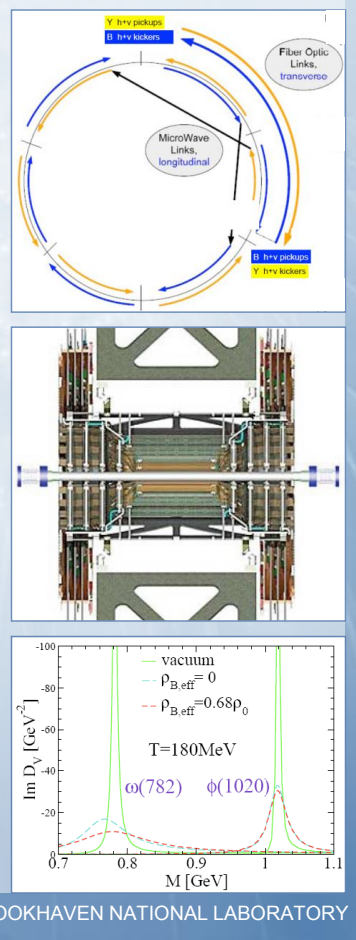

\section{Long-term Plans in Nuclear Physics (>2015) continued}

- eRHIC Science (e+A)

- Do gluon self-interactions $\Rightarrow$ "universal" saturated gluonic matter?

- eRHIC probes weak coupling regime of very high gluon density, where boson occupancy $\gg 1 \Rightarrow$ classical force field

- Can we

- Demonstrate its universal behavior?

- Track transition from parton gas to CGC?

- "See"confinement reflected in soft-gluon spatial distributions inside nuclei?

- eRHIC Science $(\overrightarrow{\mathrm{e}}+\overrightarrow{\mathrm{p}})$

- Low-x helicity structure of the nucleon

- Proton "tomography;" exclusive reactions $\rightarrow$ constrain GPDs

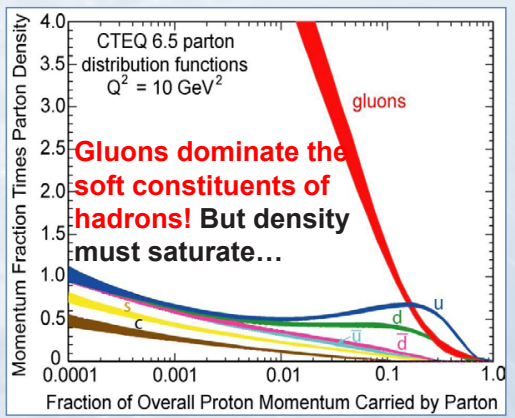

Fraction of Overall Proton Momentum Carried by Parton

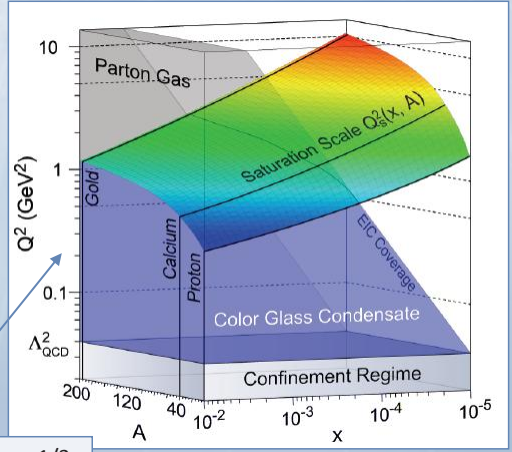




\section{Impact of RHIC on Nuclear and Particle Physics in Japan}

- The US-Japan program, and later RIKEN, contributed significantly to PHENIX and RHIC

- Scientific leadership, hardware, computing, analysis

- S. Nagamiya - founding spokesperson

- Y. Akiba - current deputy spokesperson

- RICH: (Hamagaki), TOF, Aerogel (Miake), Beam-Beam Counters (Sugitate)

- 10,000 citations to 100 published PHENIX papers

- Scientific manpower

- Currently 87 from Japan (out of about 500 total)

- About half from US-Japan program

- 37 Grad students, 12 Post-docs, 37 Faculty, 1 support

- PHENIX and precursor AGS experiments have so far produced 37 advanced degrees in Japan (35 PhD, 2 MS)

- 26 working in HEP and NP experiments, 11 in other fields, industry

- 14 have tenure or equivalent positions

PH労ENIX

\section{Activities and Plans in Particle Physics}

- ATLAS

- US-ATLAS host; Tier-1 data center

- Daya Bay

- Good construction progress; $1^{\text {st }}$ runs early 2011

- LBNE

- Intellectual leadership, Detector Design, R\&D

- LSST

- Focal plane detector

- Long-term Goal: Continued technical and intellectual leadership at the energy, intensity, cosmology frontiers

- 2010-16 physics payoff with ATLAS, Daya Bay

- LBNE and ATLAS upgrades fuel physics beyond 2018

- Major ongoing roles in LARP, APUL

- Particle astrophysics group established, with roles in DES, BOSS and LSST

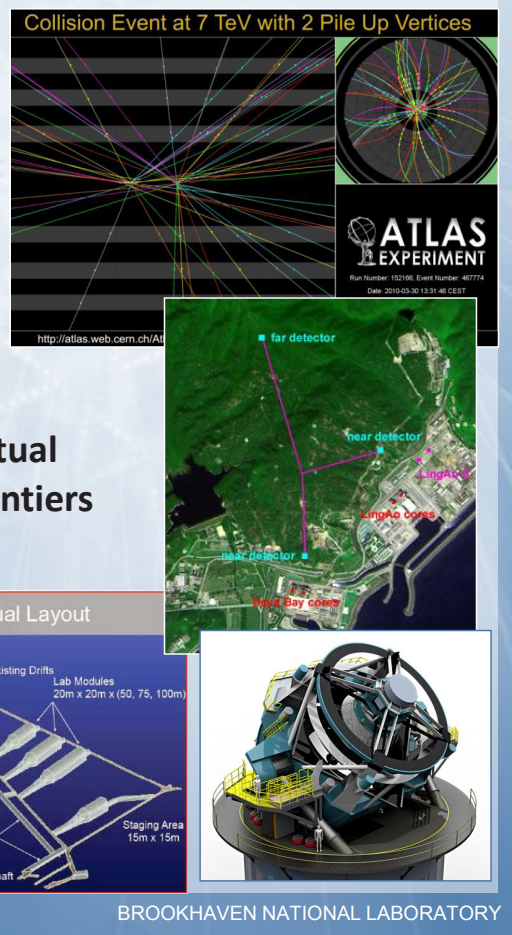




\section{Activities in Nuclear, Particle and Accelerator Physics}

- Stochastic Cooling at RHIC

- First working design for high-energy bunched beams

- EBIS - e-Beam Ion Source

- $\mathrm{eRHIC}$

- Design advancing

- Multi-pass ERL, SRF, CeC

- Muon Collider

- XFEL

- eRHIC ERL as driver
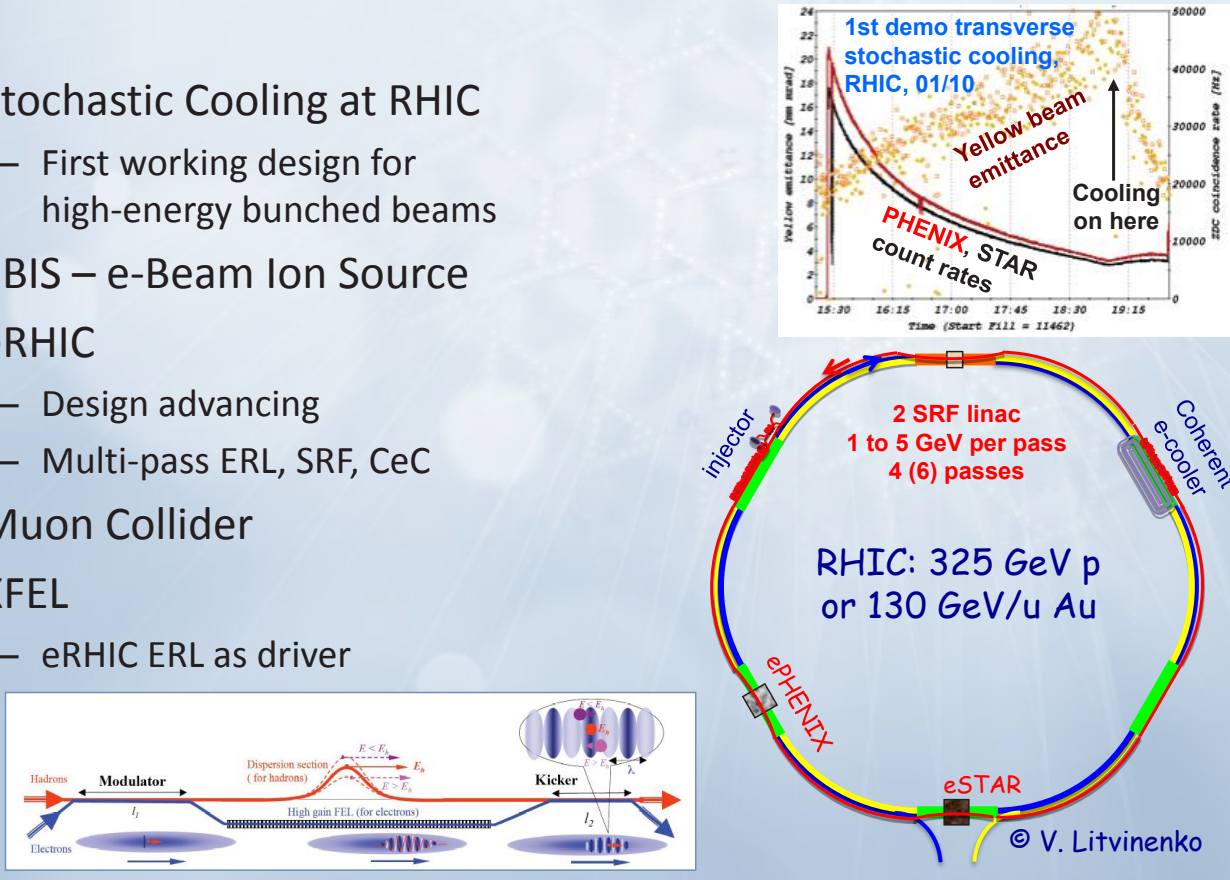

\section{Summary}

- BNL is currently active in - and has future plans in Nuclear Physics, Particle Physics and Accelerator S\&T

- BNL has benefitted from the US-Japan Collaboration in these areas and continues to do so

- PHENIX

$-v$ elastic scattering, rare $K$ decays, relativistic heavy ions, SRF and other accelerator R\&D, Detector/DAQ R\&D

- Japanese Institutions have benefitted from these joint activities as well - both in scientific advance \& impact and in the training of scientists

- BNL looks forward to the next 30 years of US-Japan Collaboration! 


\section{Fermilab: Now and Future}

Y-K. Kim, Fermilab, Batavia, IL 


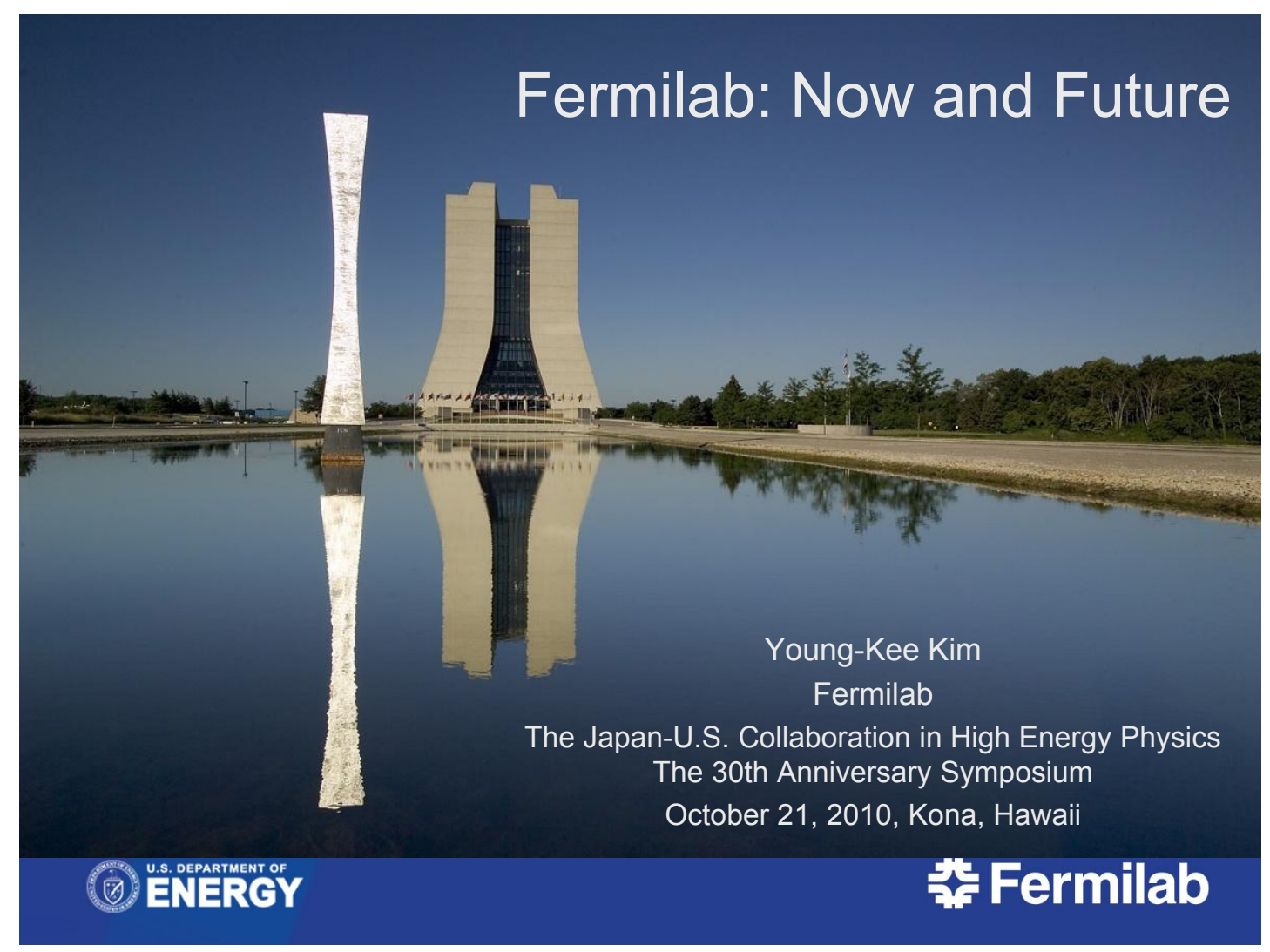

\section{Current Collaborative Efforts at Fermilab}

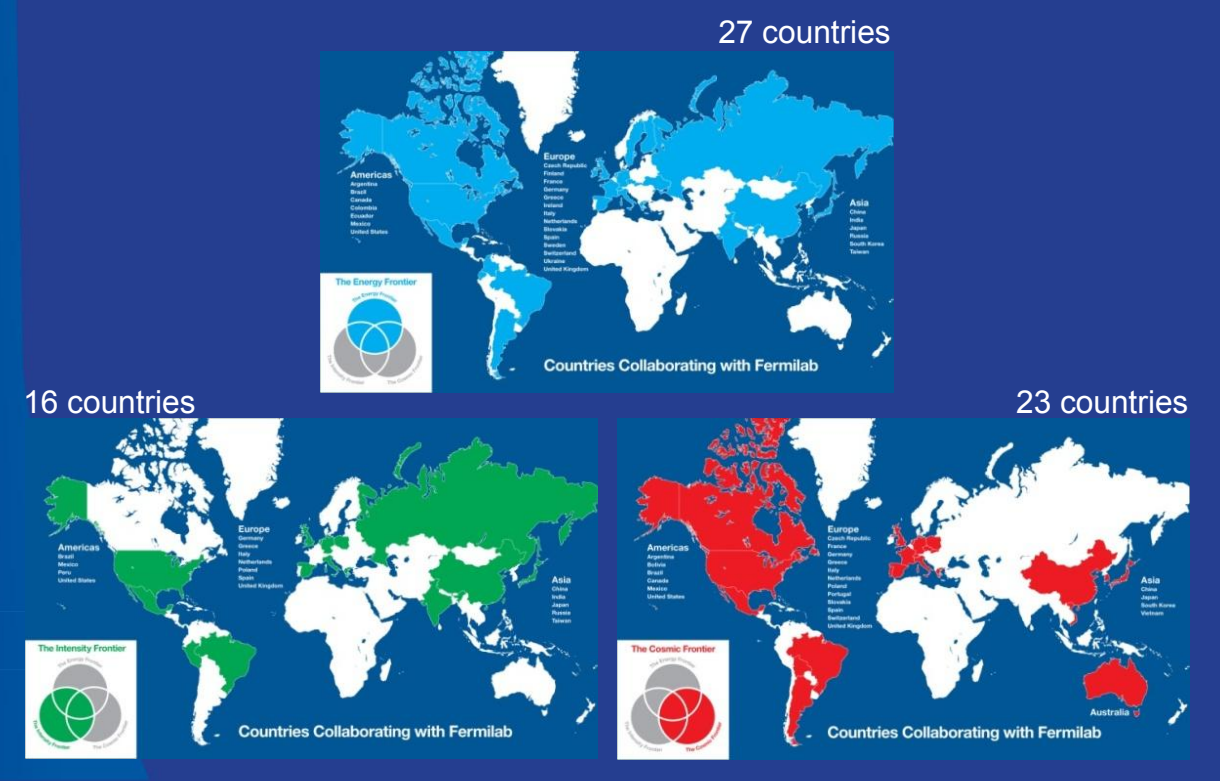




\section{Cosmic Frontier Strategy}

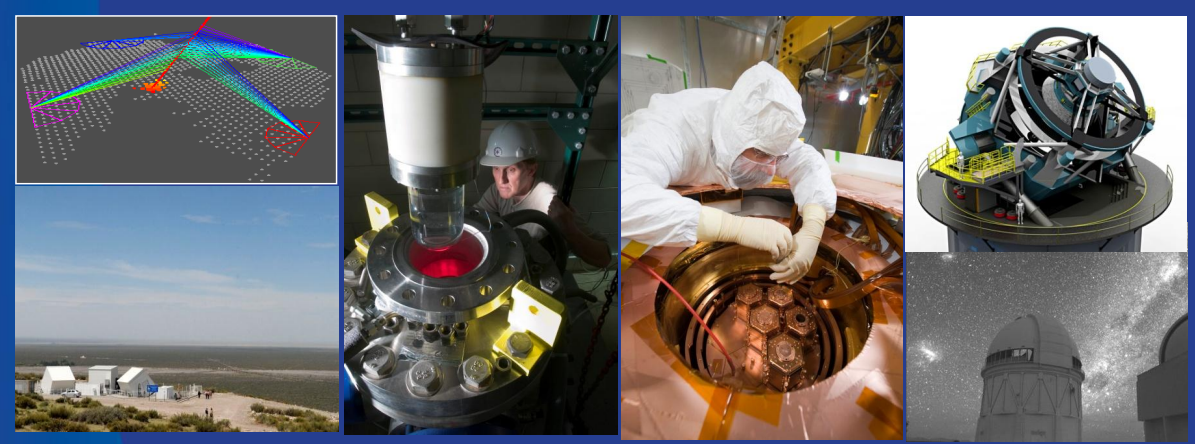

\begin{tabular}{|l|l|l|l|}
\hline & DM: $100 \mathrm{~kg}$ & DM: 1 ton & \\
DM: $10 \mathrm{~kg}$ & DE: DES & DE: LSST & DE: LSST \\
DE: SDSS & P. Auger & WFIRST?? & WFIRST?? \\
P. Auger & Holometer? & BigBOSS?? & \\
\hline
\end{tabular}

Fermilab Accelerator Complex

Operating Simultaneously 


\section{Tevatron (CDF \& DZero)}

\section{$>9 \mathrm{fb}^{-1} /$ experiment}

152 abstracts submitted to ICHEP2010
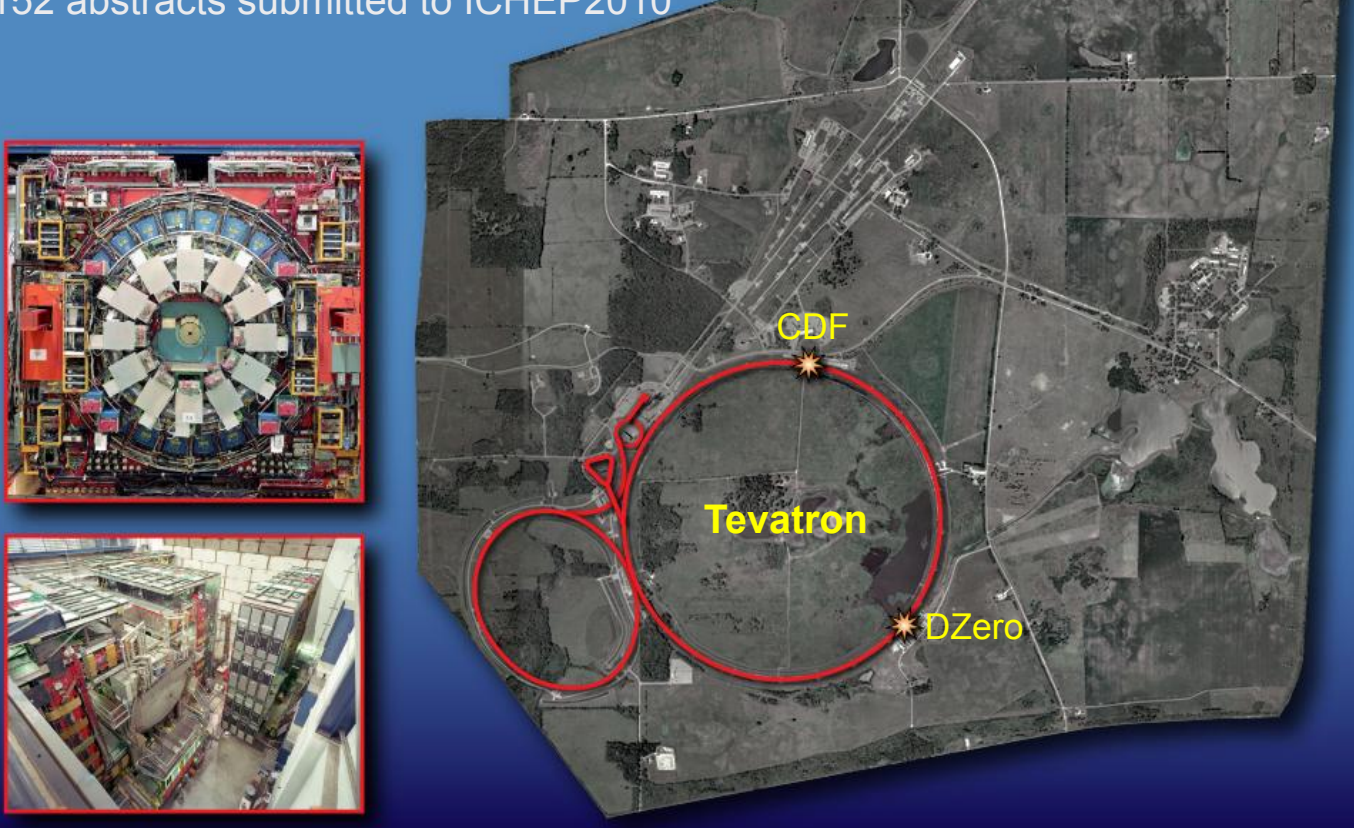

$25^{\text {th }}$ anniversary of the first collisions at Tevatron (Oct.13, 2010)

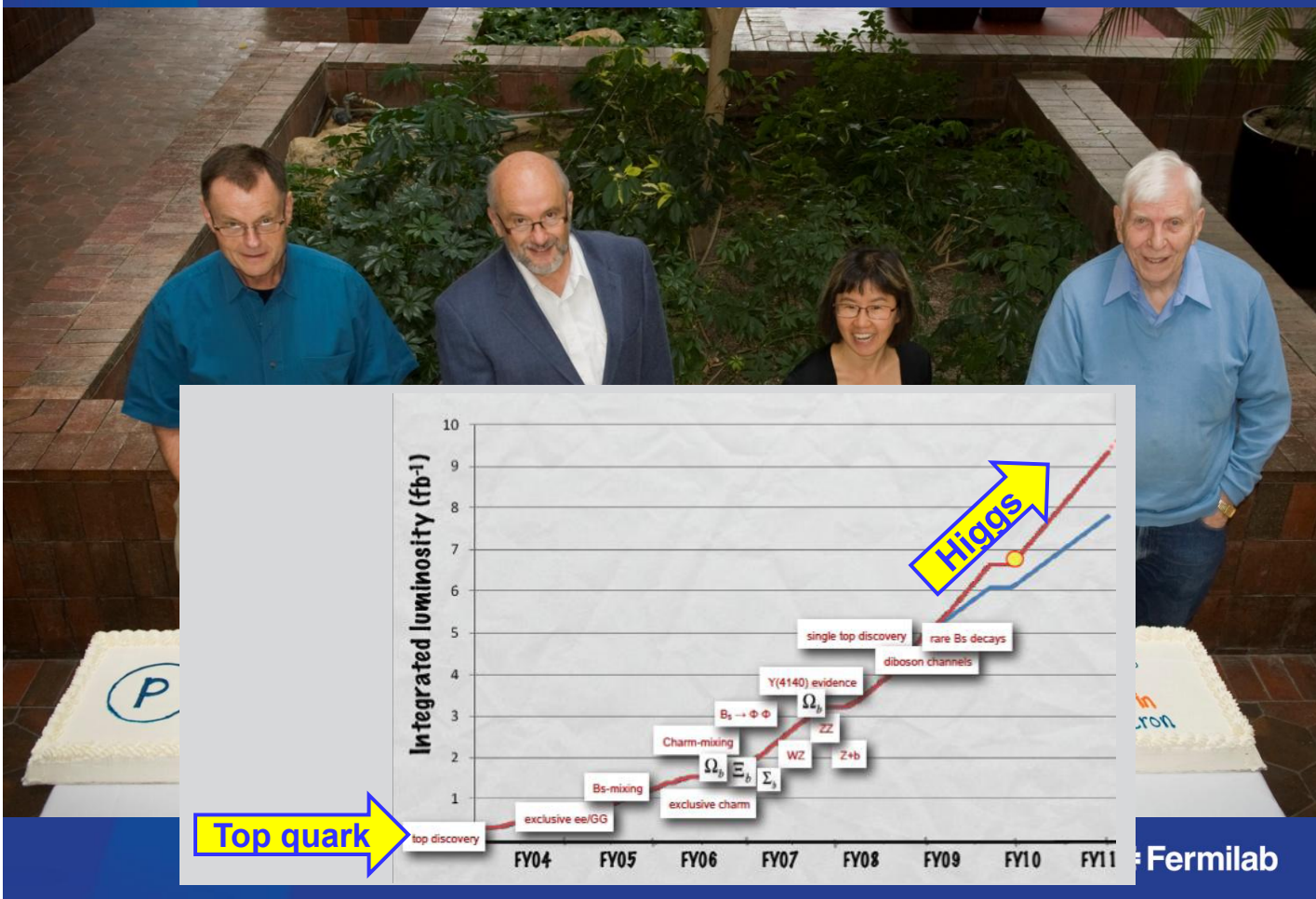




\section{Energy Frontier Strategy}

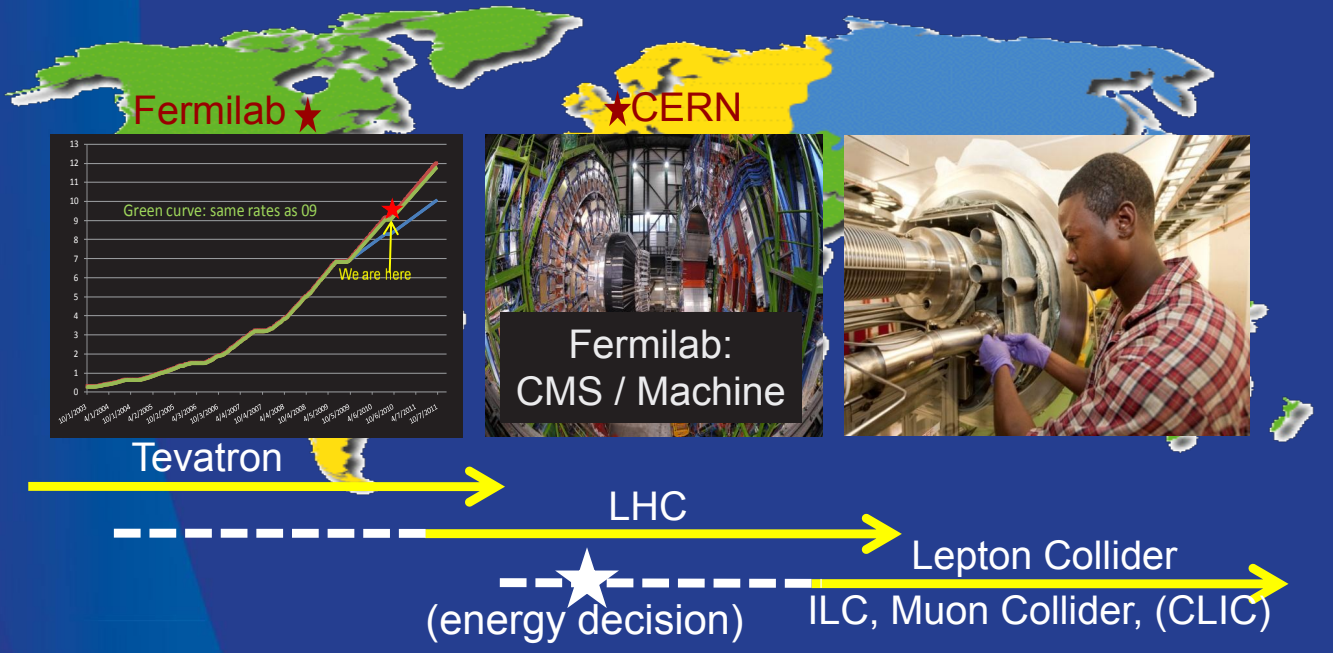

SCRF: ILC, Muon Collider, Project X

High field magnets: LHC upgrades, Muon Collider

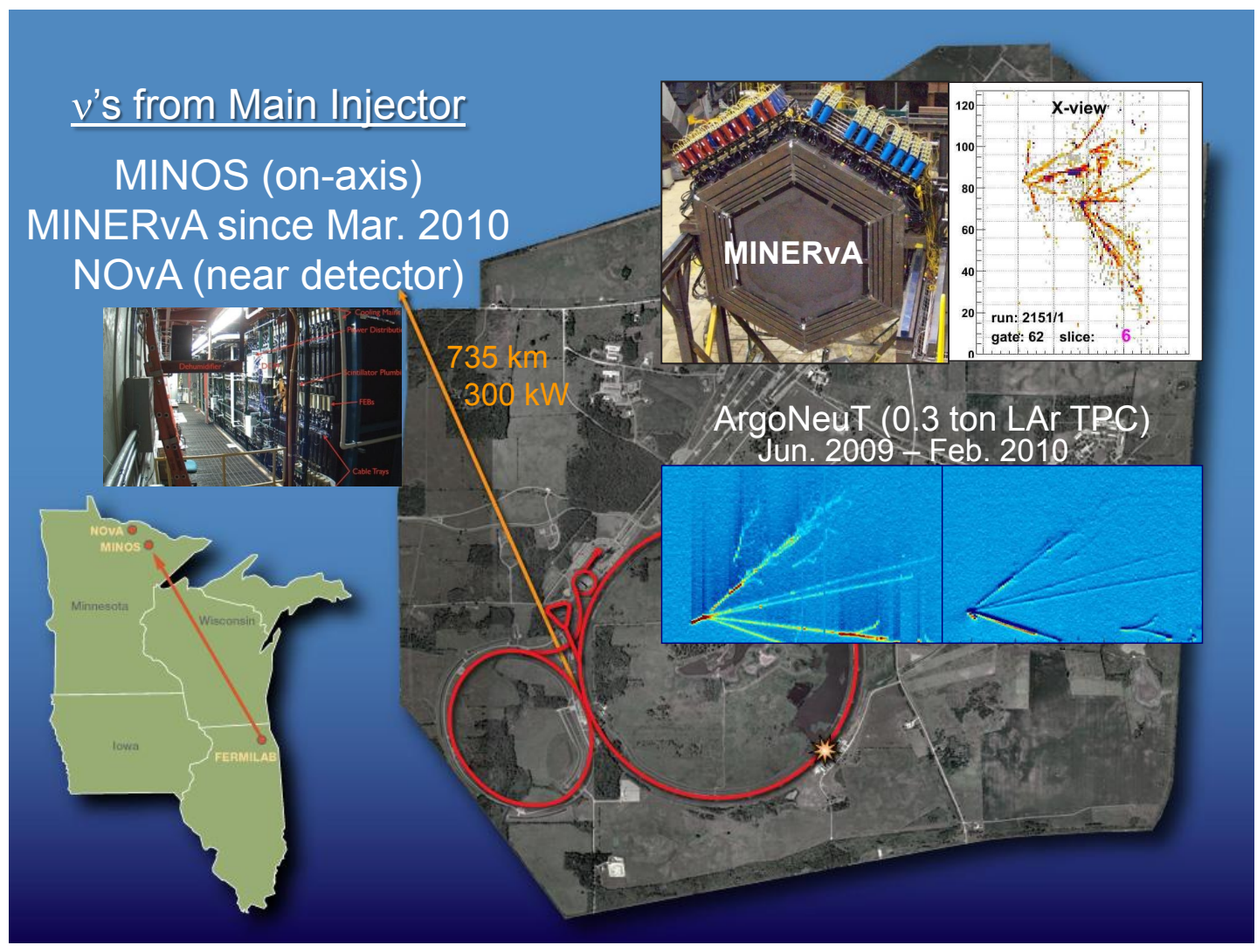




\title{
MINOS and MiniBooNE
}

\author{
neutrino behaviors = anti-neutrino behaviors ??
}

MINOS: $v$ vs anti- $v$

MiniBooNE: $v$ vs anti- $v$
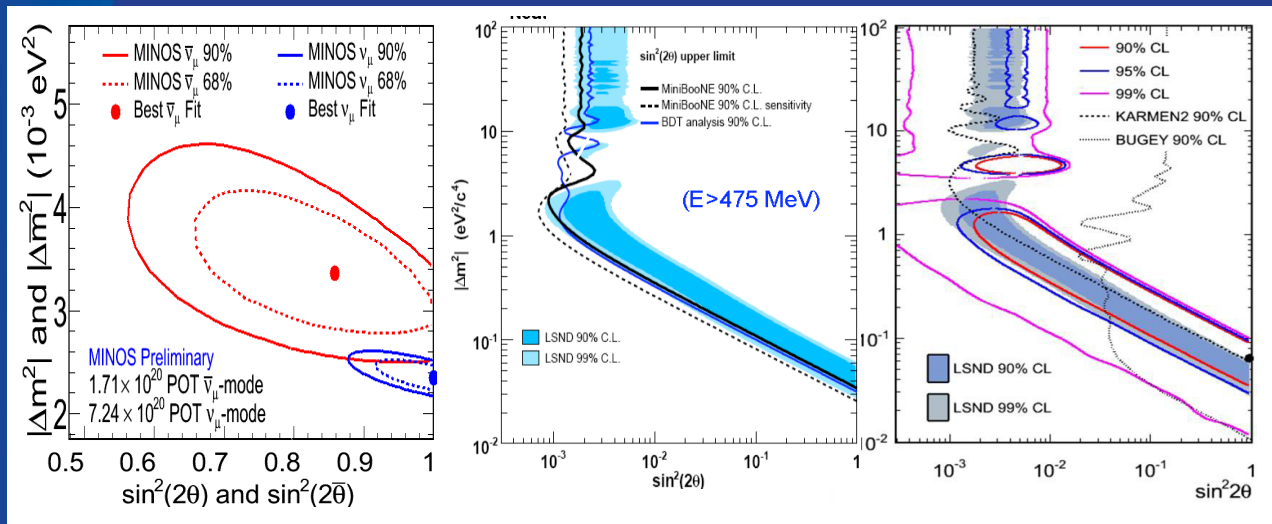

more anti-neutrino data

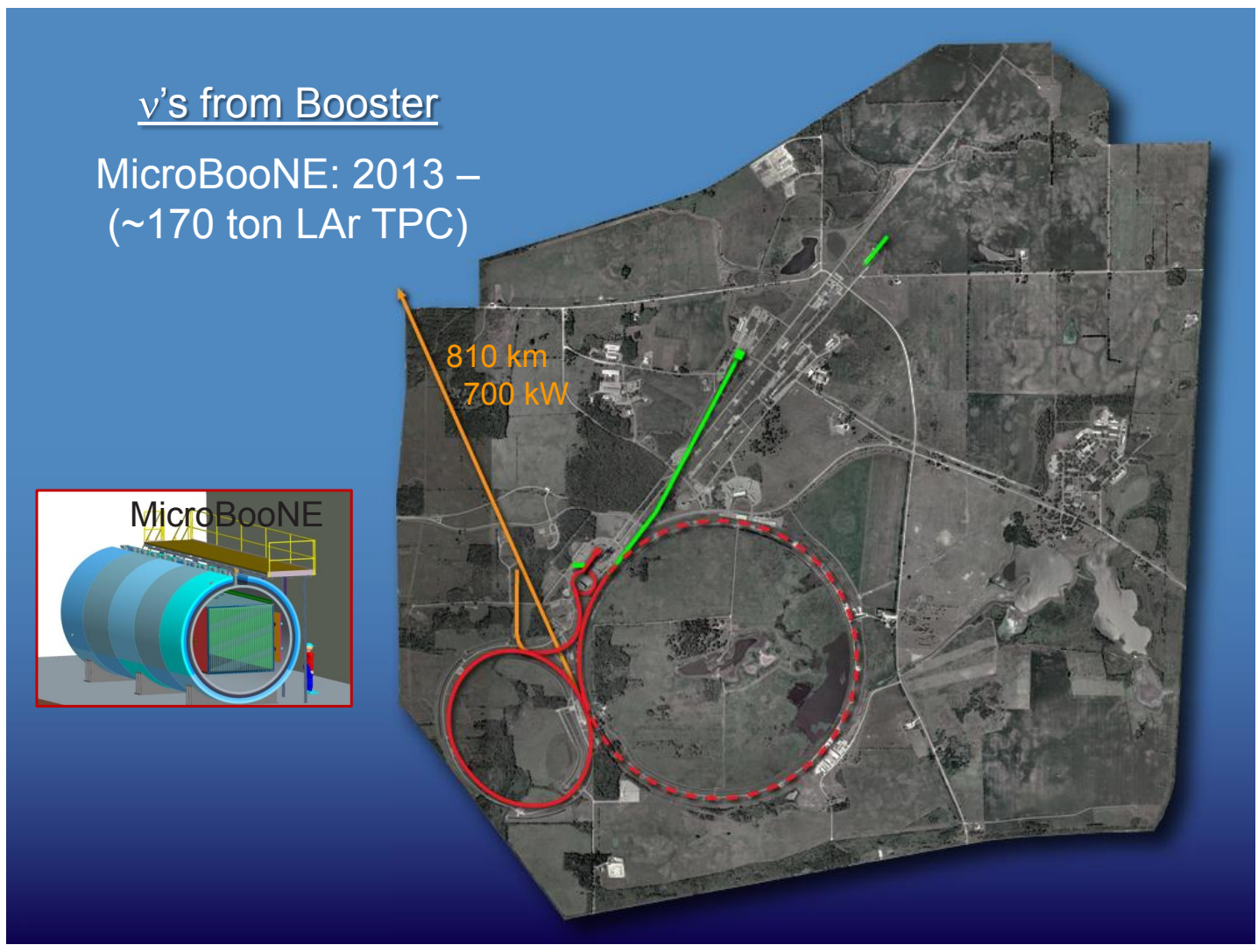




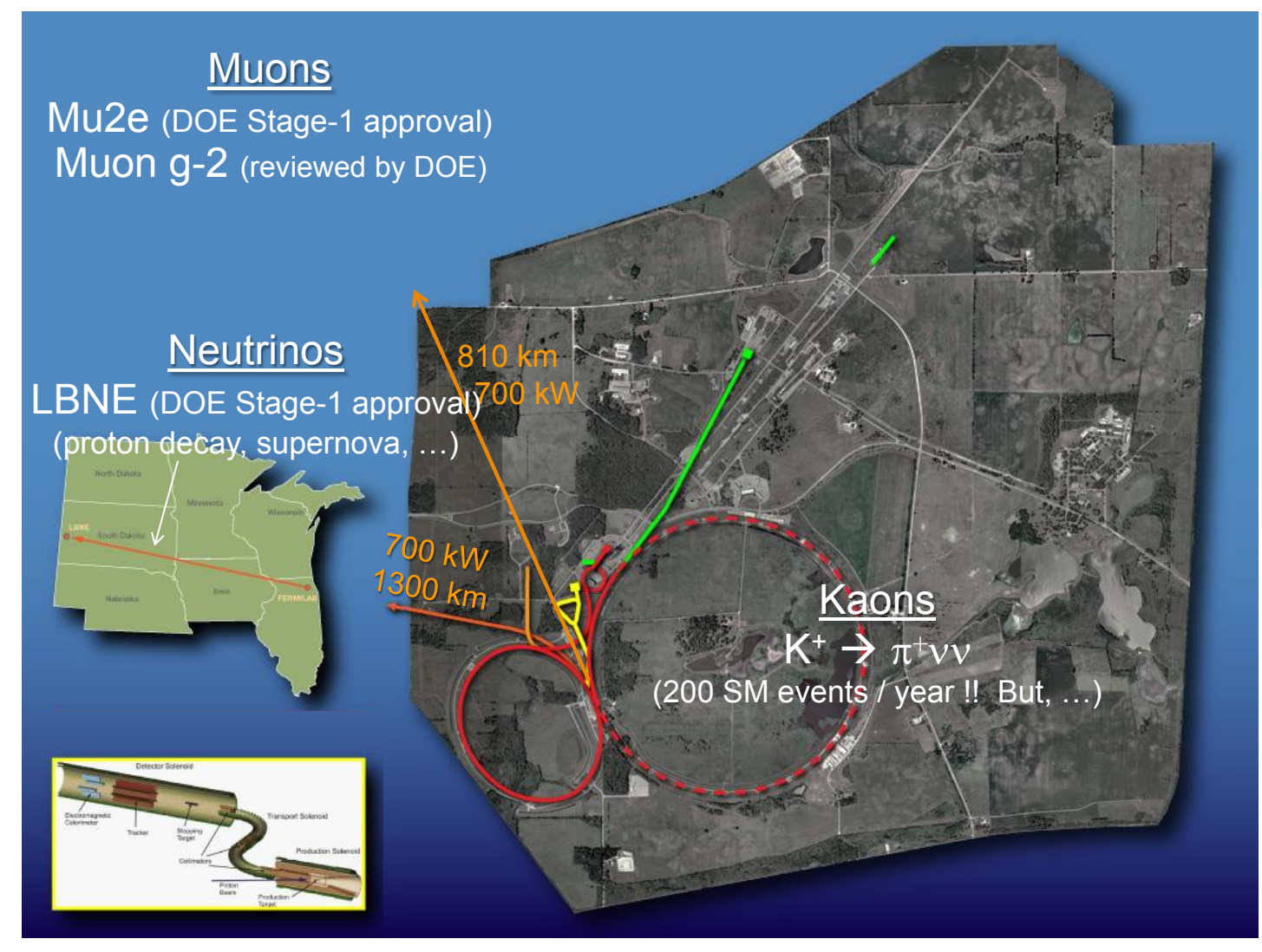

\section{Intensity Frontier Strategy}
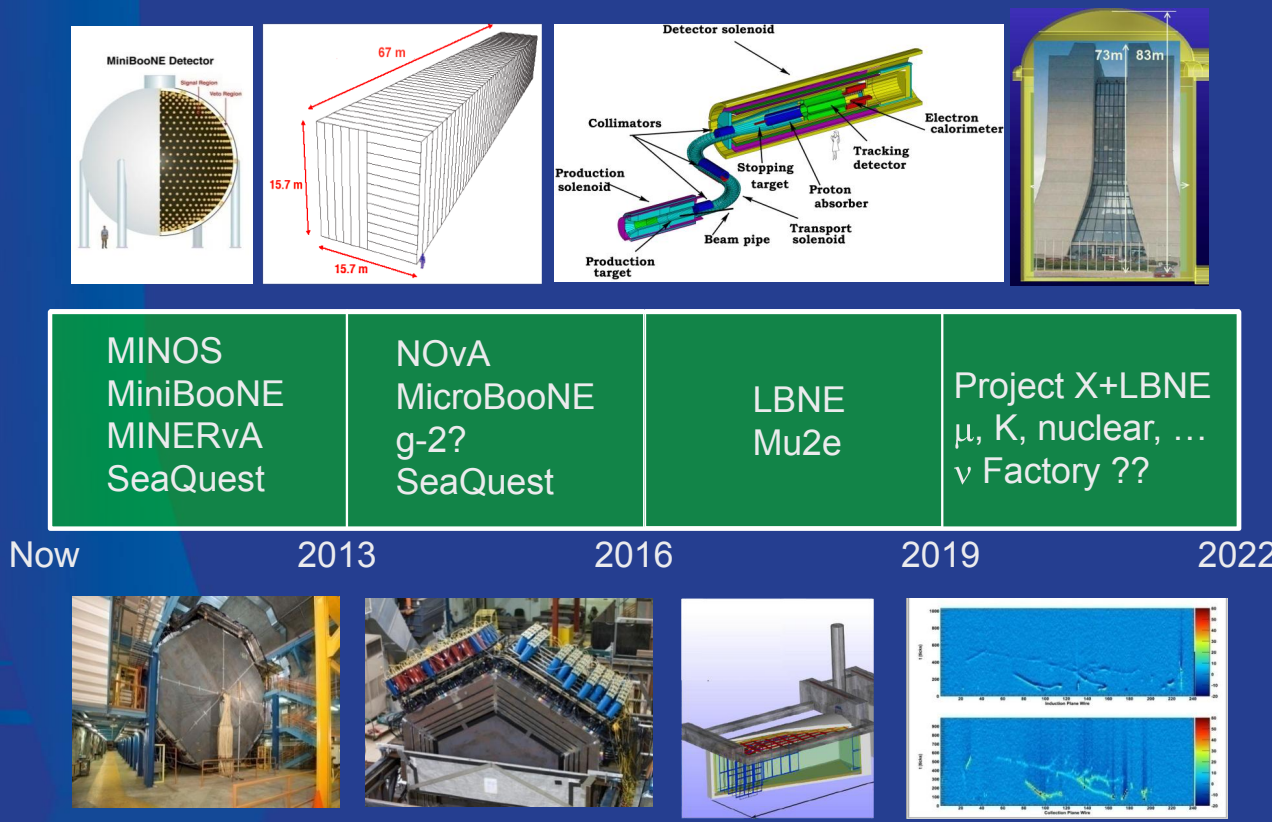


\section{Future of Fermilab}

\begin{tabular}{|c|c|c|c|}
\hline $\begin{array}{l}\text { Tevatron } \\
\text { LHC }\end{array}$ & LHC & $\begin{array}{l}\text { LHC Upgrades } \\
\text { ILC?? }\end{array}$ & $\begin{array}{l}\text { LHC } \\
\text { ILC, CLIC or } \\
\text { Muon Collider }\end{array}$ \\
\hline $\begin{array}{l}\text { MINOS } \\
\text { MiniBooNE } \\
\text { MINERvA } \\
\text { SeaQuest }\end{array}$ & $\begin{array}{l}\text { NOvA } \\
\text { MicroBooNE } \\
\text { g-2? } \\
\text { SeaQuest }\end{array}$ & $\begin{array}{l}\text { LBNE } \\
\text { Mu2e }\end{array}$ & $\begin{array}{l}\text { Project } \mathrm{X}+\mathrm{LBNE} \\
\mu, \mathrm{K} \text {, nuclear, } \ldots \\
\nu \text { Factory ?? }\end{array}$ \\
\hline $\begin{array}{l}\text { DM: } \sim 10 \mathrm{~kg} \\
\text { DE: SDSS } \\
\text { P. Auger }\end{array}$ & $\begin{array}{l}\text { DM: } \sim 100 \mathrm{~kg} \\
\text { DE: DES } \\
\text { P. Auger } \\
\text { Holometer? }\end{array}$ & $\begin{array}{l}\text { DM: 1 ton } \\
\text { DE: LSST } \\
\text { WFIRST?? } \\
\text { BigBOSS?? }\end{array}$ & $\begin{array}{l}\text { DE: LSST } \\
\text { WFIRST?? }\end{array}$ \\
\hline
\end{tabular}




\title{
Future Plans at KEK
}

\author{
Masanori Yamauchi \\ Institute of Particle and Nuclear Studies, KEK, Tsukuba, Ibaraki, Japan
}

In this article, I review the future plans for KEK's Tsukuba campus, emphasizing the highenergy physics program. In 2008, KEK released its five-year roadmap in high-energy physics and related research fields, wherein the neutrino program at J-PARC, the luminosity upgrade of KEKB, and the commitment to the LHC experiment are listed as three major projects of the accelerator-based particle-physics program.

The B factory at KEK, KEKB, has operated successfully for 11 years, and the Belle experiment collected more than $1000 \mathrm{fb}^{-1}$ data at $\Upsilon$ region. Using this, the world's largest data sample, diverse important results were obtained, notably the discovery of CP violation in the B meson system, quantitative confirmation of the Kobayashi-Maskawa model, and discoveries of exotic hadron states. Among the new measurements, some results are not fully comprehensible with the framework of the Standard Model. For example, the magnitude of the direct $\mathrm{CP}$ asymmetry in $\mathrm{B} \rightarrow \mathrm{K} \pi$ decays differs between charged and neutral $\mathrm{B}$, and the rate of $\mathrm{D}$ and anti-D mesons was measured as approximately $1 \%$, while it is predicted to be smaller than $10^{-4}$ in most model calculations based on the Standard Model. One possible interpretation of these phenomena is that new physics beyond the Standard Model is generating deviations from its predictions in some observables sensitive to the new physics. To clarify this, KEK decided to upgrade to SuperKEKB, a new B factory with 40 times higher luminosity by replacing the beam pipes, the magnets' configuration, the final focusing scheme, the injection system and so on. Planned upgrades of the Belle detector will assure better performance in the high background environment.

KEK also made a strong commitment to the energy-frontier program at CERN, and tto R\&D for the future linear collider. At the interaction points of LHC, triplet quadrupole magnets focus the beam. Two types of superconducting magnets separately are developed and manufactured at KEK and Fermilab. Both magnets were assembled with a common cryostat at Fermilab, and then were shipped to CERN. Detector R\&D for the ILD experiment at ILC includes TPC, using GEM to detect drift electrons, and a precision calorimeter based on tungsten-scintillator sandwich read out by MPPC. Both of these R\&D efforts are conducted by large international collaborations comprising physicists from twelve countries.

KEK launched an astrophysics group in 2007 to expand its research field, and started its commitment to the QUIET experiment in the Atacama Desert in Chile. The purpose of this experiment is to measure anisotropy in the polarization of the cosmic microwave background by employing coherent receivers at $43 \mathrm{GHz}$ and $95 \mathrm{GHz}$. The combination of these frequencies confers sensitivity to foreground contributions from diffuse Galactic synchrotron-radiation. 

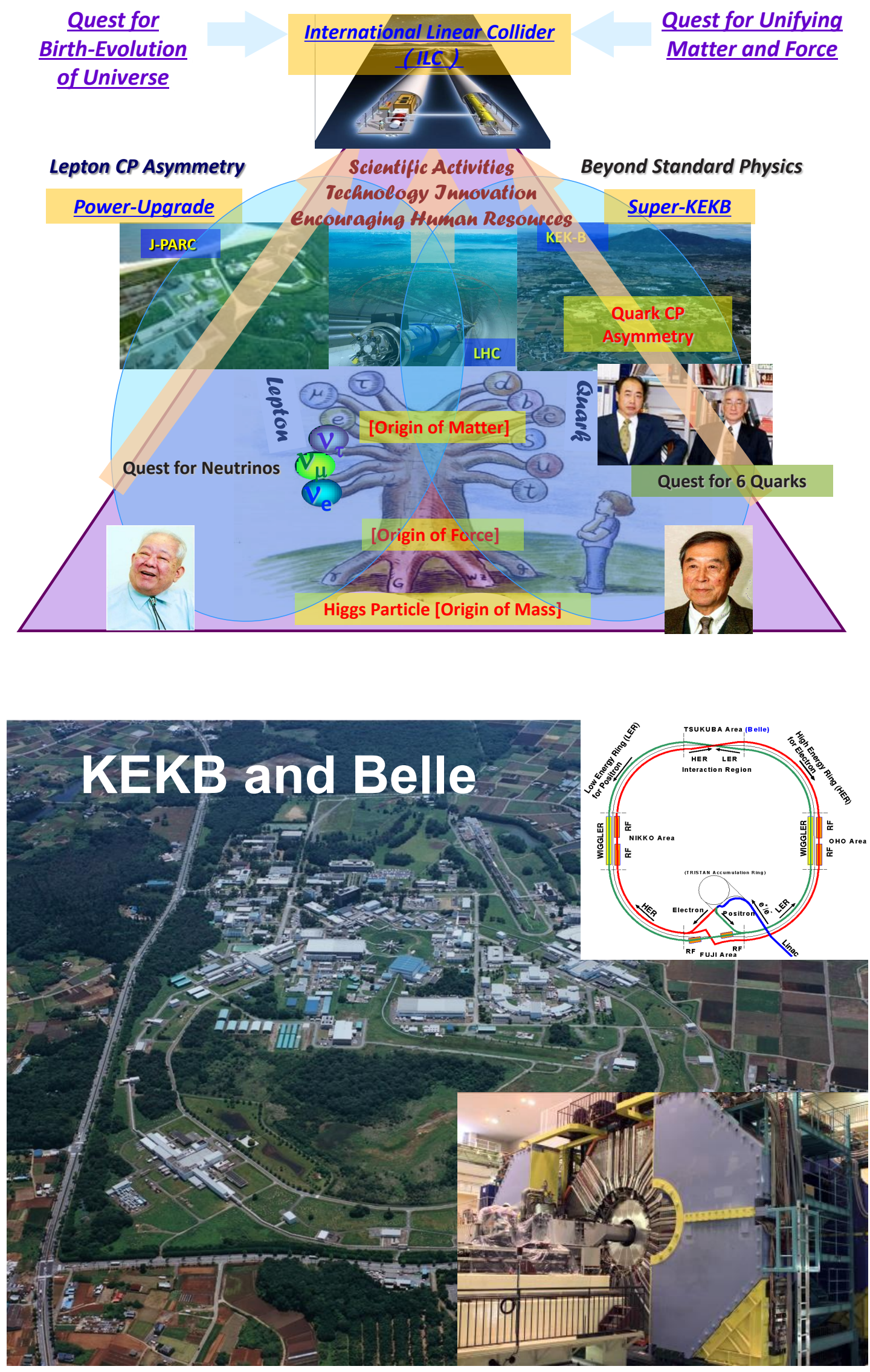


\section{Major achievements at Belle}

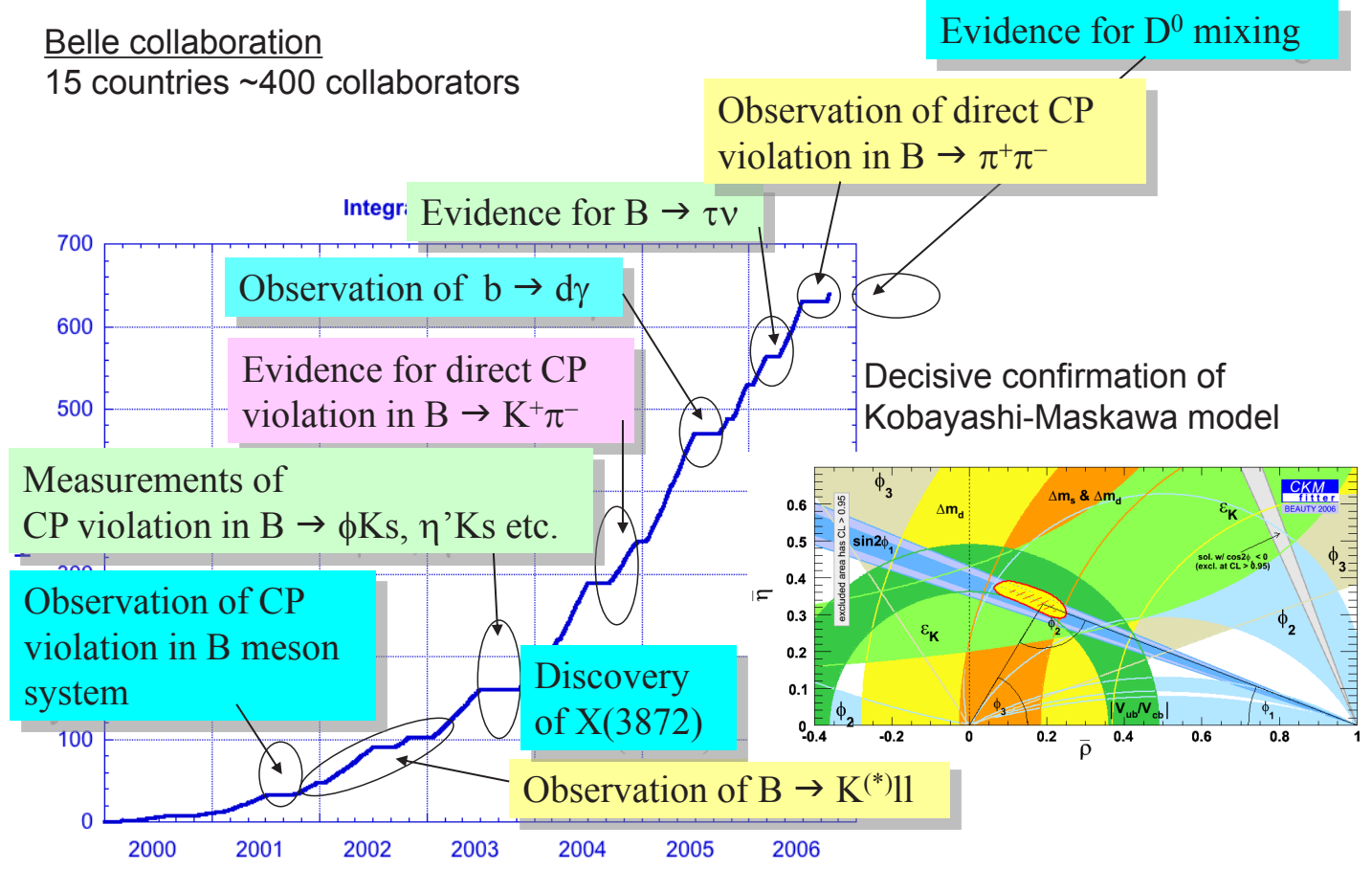

\section{Possible hints for NP?}

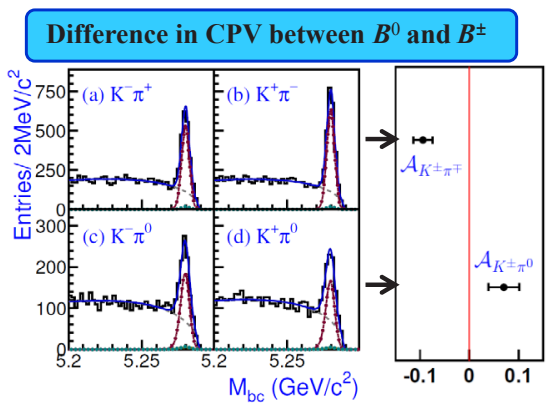

Inconsistency in unitarity triangle?

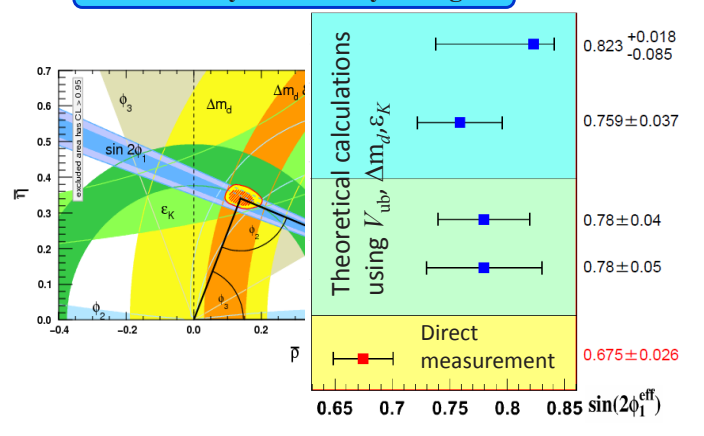

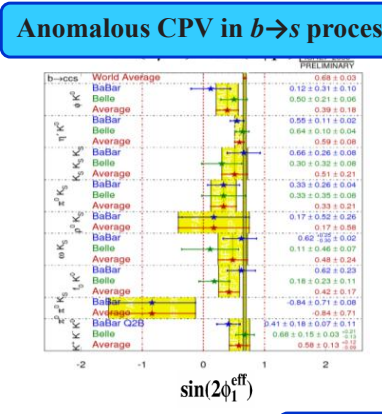

Anomaly in $B \rightarrow K * I l$ decay?

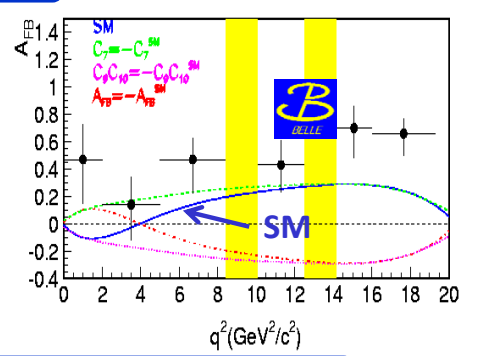

Unexpectedly large $\overline{D^{0} \bar{D}^{0}}$ oscillation

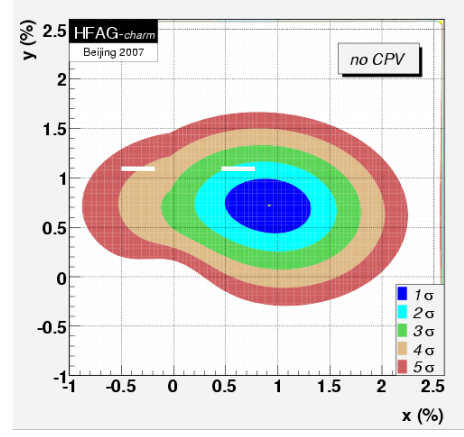




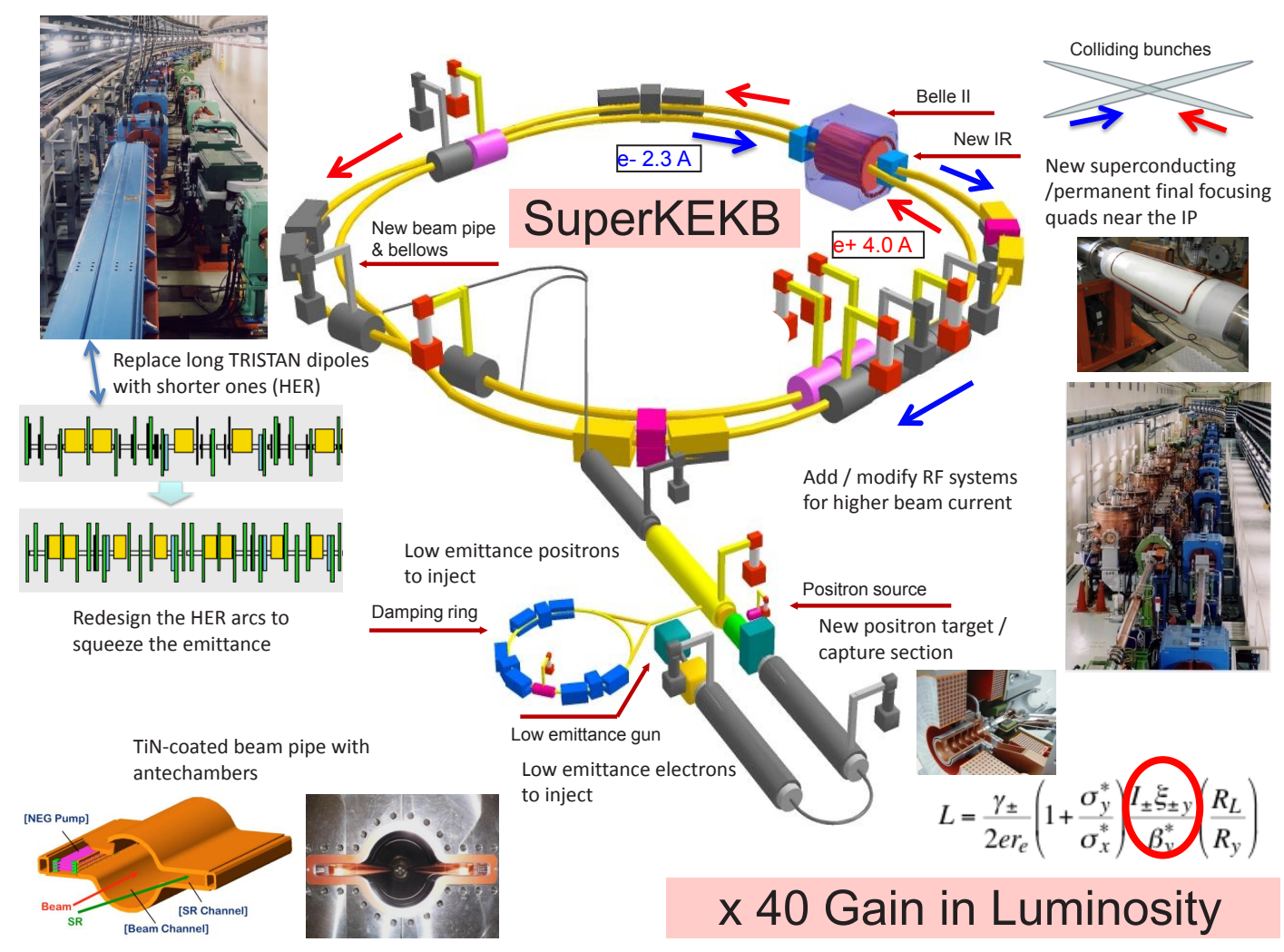

\section{Belle Upgrade}

Better background tolerance Better performance

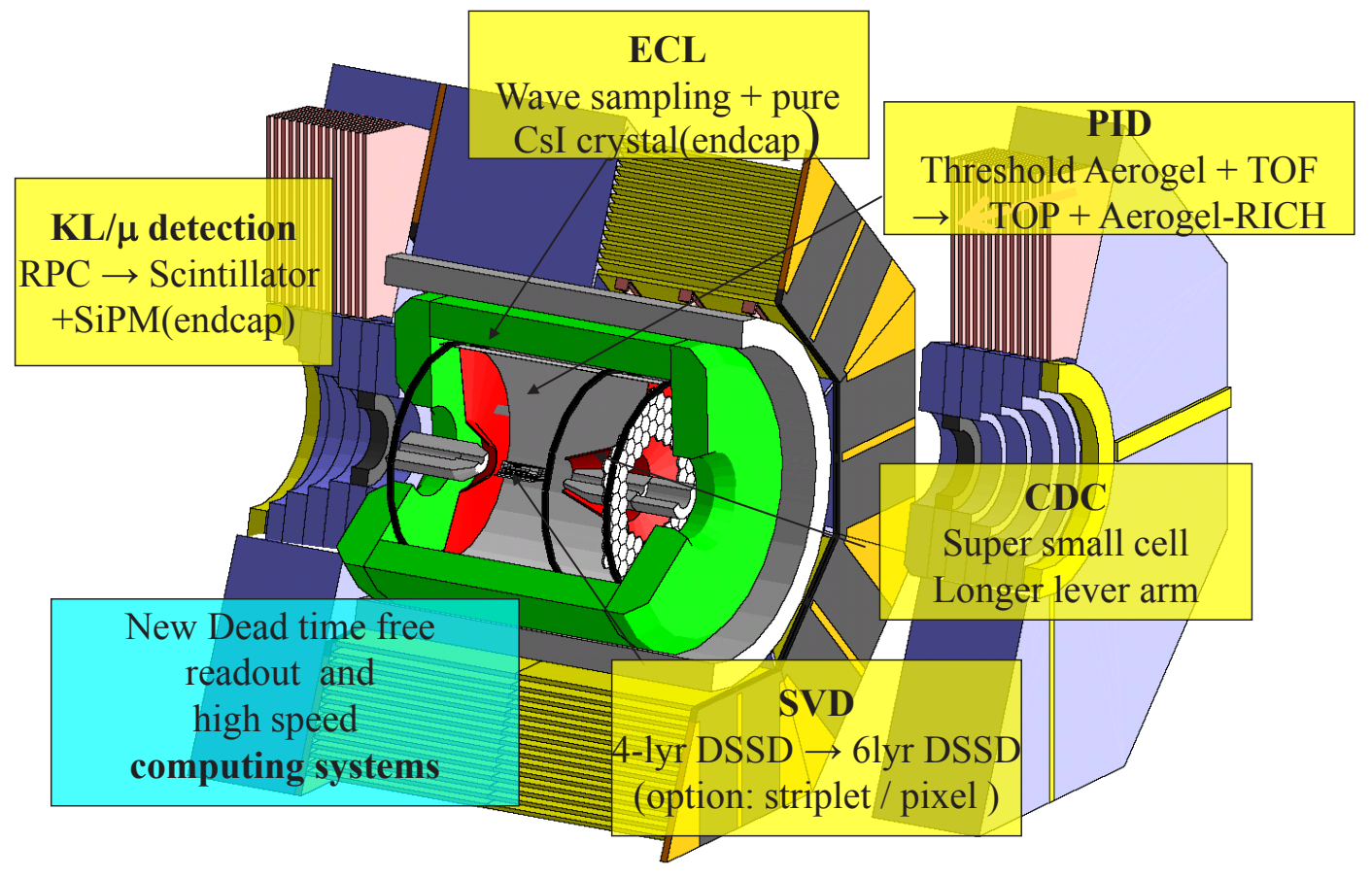




\section{Inner Triplet Quadrupoles}

Near the interaction points, triplet quadrupole magnets focus the beam. Two types of superconducting magnets are separately developed and manufactured at KEK and Fermilab. Both magnets were assembled with common cryostat at Fermilab and then shipped to CERN.

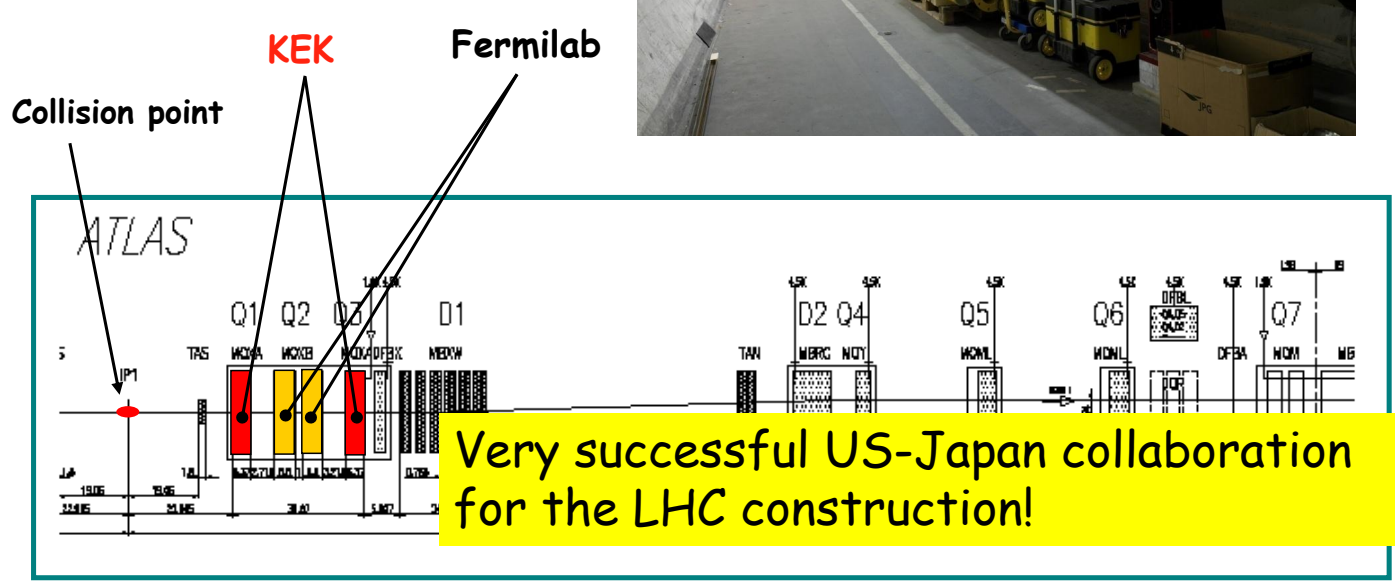

\section{Toward the LHC upgrade}

Recently the design group for High-Luminosity LHC is formed. KEK is contributing to the R\&D for the two key technologies:

- High-field magnet $\left(\mathrm{Nb}_{3} \mathrm{Al}\right)$ and

- Crab cavity, in close collaboration with CERN and the other labs

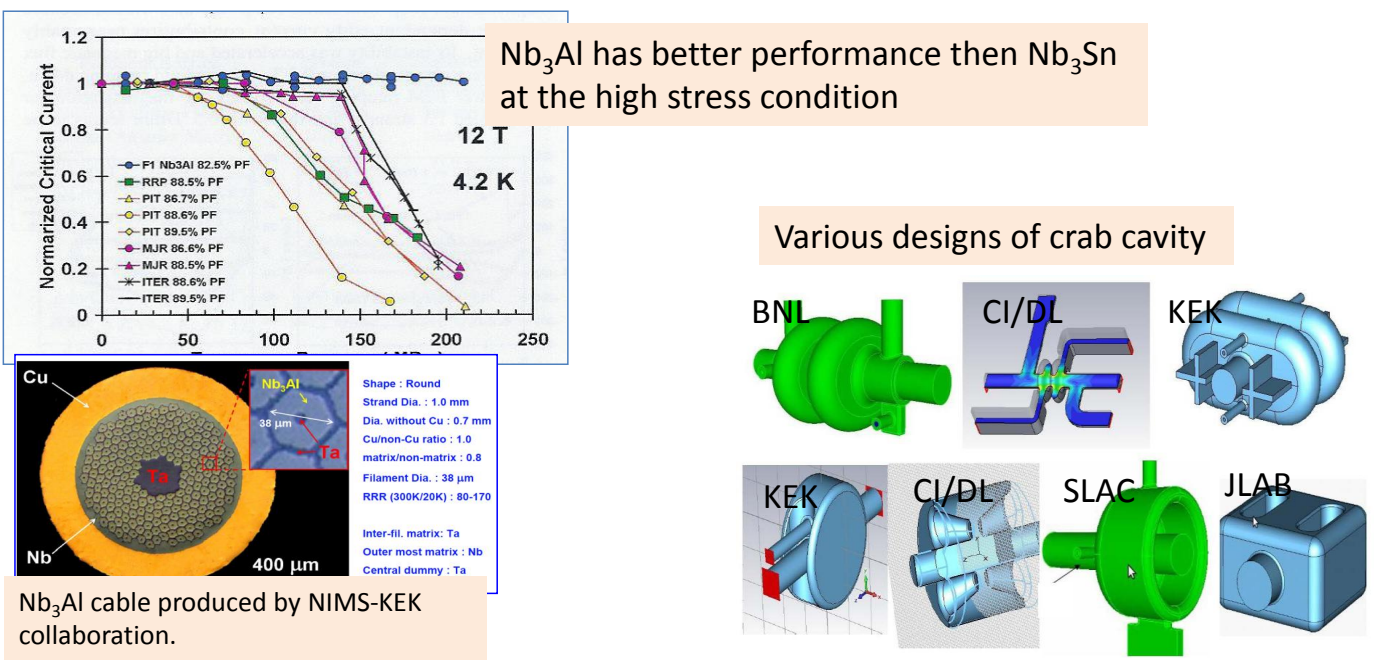




\section{KEK-related Activities for ILD}
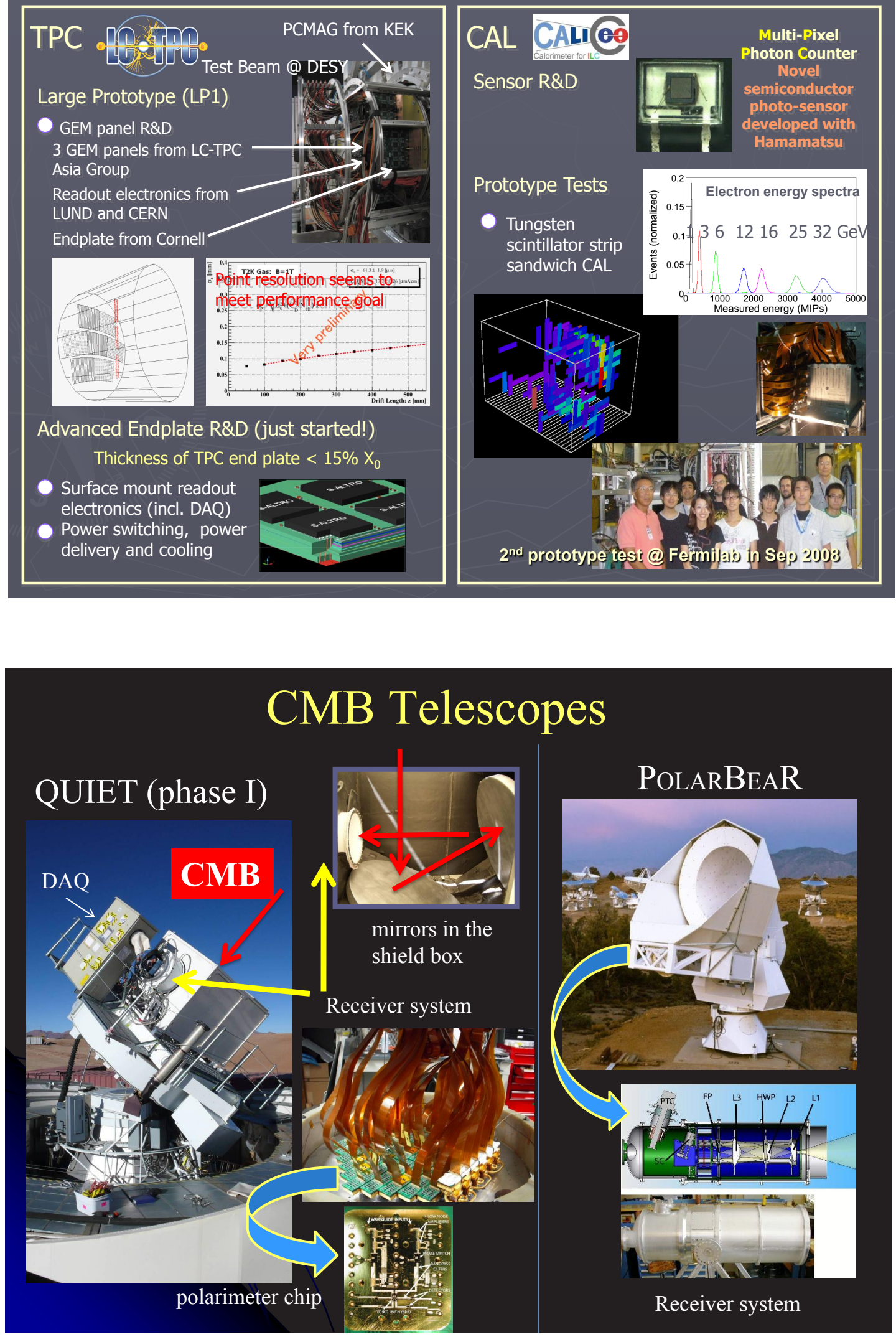


\title{
Future Plan at J-PARC
}

\author{
Koichiro Nishikawa \\ High Energy Accelerator Research Organization (KEK), Tsukuba, Ibaraki, Japan
}

It has been a pleasure collaborating with the US physicists in various occasions. In Japan, I am working with a US groups in the Super-Kamiokande, K2K long baseline-neutrino experiment and now starting up a new neutrino experiment at J-PARC (Japan Proton Accelerator Research Complex).

J-PARC is a joint project between KEK and JAEA (Japan Atomic Energy Agency) to study a wide range of sciences, encompassing from life sciences to neutrino physics. The accelerator facility consists of a $180 \mathrm{MeV}$ LINAC, a $25 \mathrm{~Hz} 3 \mathrm{GeV}$ rapid cycling synchrotron (RCS), and the main ring (MR), operating at $30 \mathrm{GeV}$. The construction started in 2001 and finished in 2008.

The RCS has experimental facility for research on muons, and a spallation neutron source for lifeand material-sciences. The MR has two beam facilities: One is with a resonant slow-extraction (SX) for particle and nuclear physics; the other is with a single-turn fast-extraction (FX) for neutrino physics. The beams were commissioned in 2009. The design goal of the RCS is 1MW with the LINAC's energy upgraded to $400 \mathrm{MeV}$ and the design power of MR at $750 \mathrm{~kW}$.

The experimental program for particle and nuclear physics includes a long baseline neutrino oscillation experiment (T2K) with the fast extracted beam, and searches for the source of $\mathrm{CP}$ violations in rare kaon decays,. Nuclear physics research will focus on the strangeness. All experiments require excellent accelerator performance.

At first, two main issues limited the integrated beam power of the FX. The number of protons per pulse was confined by the slow rise time of the fast-extraction kicker that limited the usable number of bunches to six. Also, a beam-loading effect was observed at about $10^{13}$ protons / bunch. This summer, a new kicker was installed. The beam time needed for acquiring data also posed a problem. . We modified various components of the accelerator that required maintenance and repair. Thus, the repetition rate of the accelerator will increase by the modification of the power supplies of the MR. We expect $150 \mathrm{~kW}$ continuous operation. The next critical path is a MR $300 \mathrm{~kW}$ trial, corresponding to $2 \cdot 10^{13}$ protons / bunch with an eight- bunch operation. The immediate goal is a continuous operation with greater than $150 \mathrm{~kW}$ starting in November 2010. Thereafter, we aim to reach more than $300 \mathrm{~kW}$, starting in the fall of 2011 , and an accumulation of integral power of $1 \mathrm{MW}^{*} 10^{7}$ by the summer of 2014. For further long-term improvements of the accelerator, R\&D efforts on basic technologies started for attain faster cycling. In parallel, we began R\&D work on the LAr detector for a future neutrino /proton decay experiment after T2K.

Presently, the slow-extracted beam presents two problems. One is the spiky spill-structure, principally caused by the ripple in the MR power supply. Various hardware was installed during its summer shutdown, such as a feed-back system with a RF noise- and ripple-cancellation system. Some improvement in the accidental rates was observed, but further improvement is needed to utilize the full design power. Another problem is beam loss. A dynamic bump scheme was installed this summer that led to a substantial improvement. The radiation level must be measured before deciding upon the next operational mode.

In summary, J-PARC has started to operati. For the running period from October 2010 to June 2011, the goal is to assure the FX beam continuously reaches $150 \mathrm{~kW}$ for 7 months. For the SX beam, various improvements were realized, and we will try to further improve the spill structure and beam loss. Many R\&D efforts for mu-e conversion, muon g-2/EDM, nEDM, LiqAr TPC detector continue. Intensive R\&D efforts on accelerator started for future multi-MW operations. 


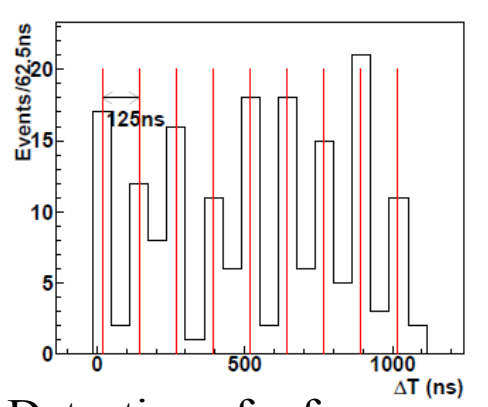

Detection of $v$ from accelerator $250 \mathrm{~km}$ away

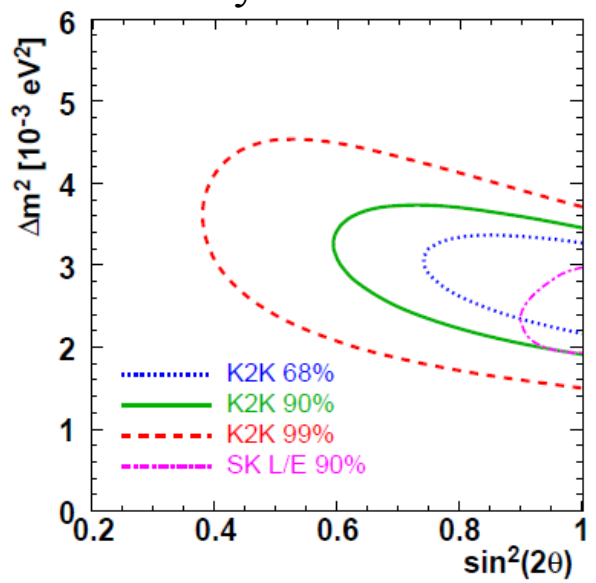

K2K Experiment 1999-2005 Started as Japan-US-Korea collab.

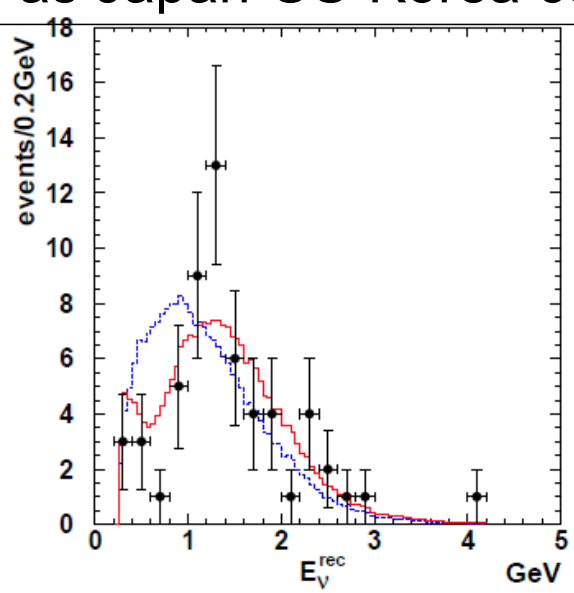

\begin{tabular}{lc}
\hline \hline & K2K-I+II \\
\hline Shape + Norm. & $\mathbf{0 . 0 0 1 5 \% ( 4 . 3 \sigma )}$ \\
\hline Shape only & $0.42 \%(2.9 \sigma)$ \\
Norm. only & $0.06 \%(3.4 \sigma)$ \\
\hline \hline
\end{tabular}

Existence of $v$ oscillation

\section{Present and near future of J-PARC}

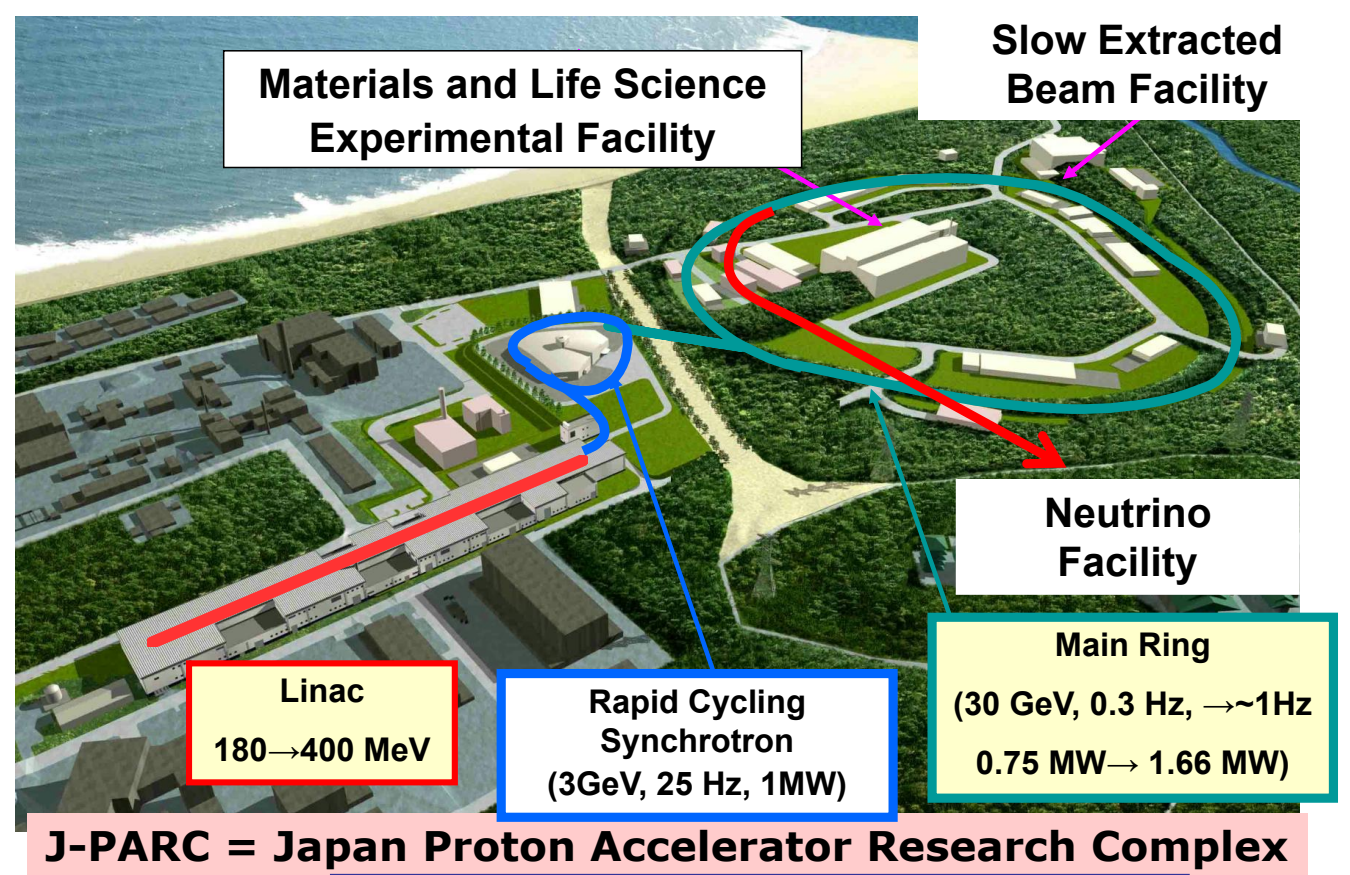

Joint Project between KEK and JAEA 


\section{T2K (Tokai to Kamioka) experiment}

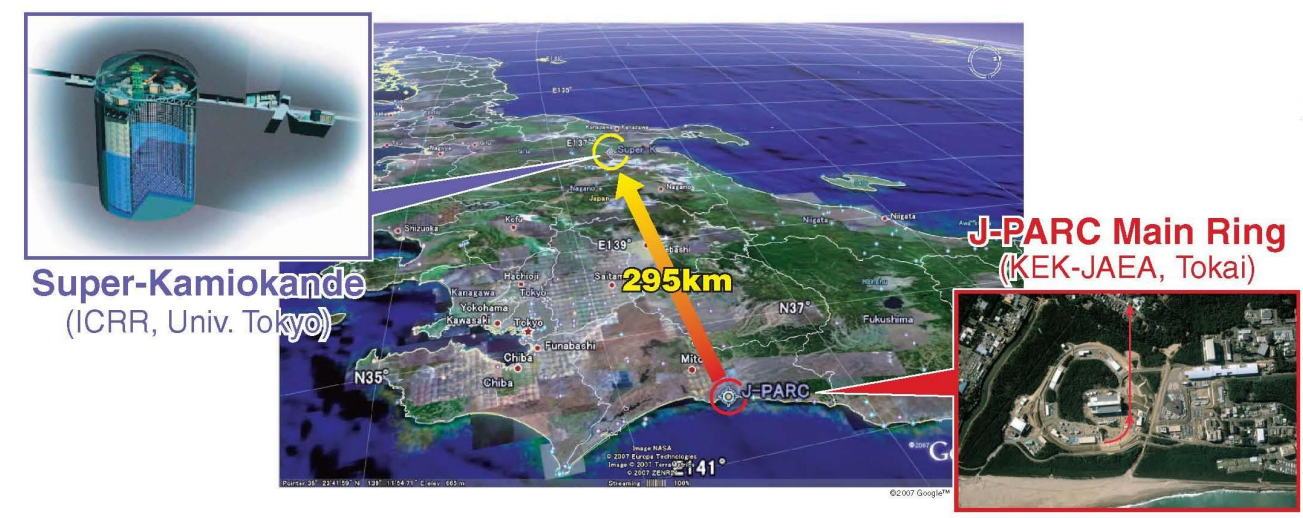

- High intensity $v_{\mu}$ beam from

J-PARC MR to Super-Kamiokande @, 295km

- Discovery of $v_{\mathrm{e}}$ appearance $\rightarrow$ Determine $\theta_{13}$

- Last unknown mixing angle

- Open possibility to explore CPV in lepton sector

- Precise meas. of $v_{\mu}$ disappearance $\rightarrow \theta_{23}, \Delta \mathbf{m}_{23}{ }^{2}$

- Really maximum mixing? Any symmetry?
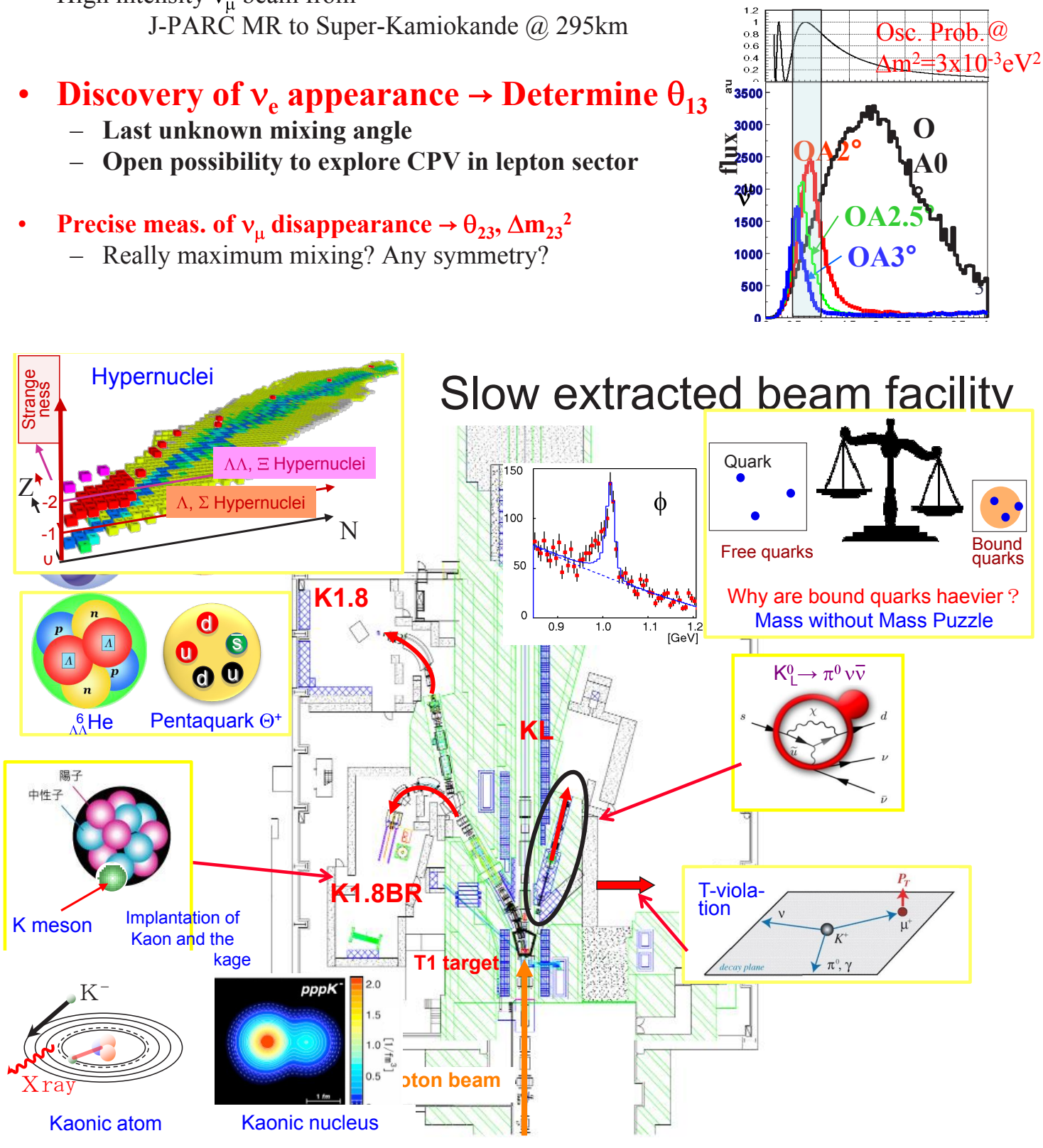


\section{Critical path in neutrino program}

- Beam power - Immediate goal

- RCS kicker repaired (operation time)

- New FX kicker installed this summer ( $6 \Rightarrow 8$ bunches, aim for $>1.2 \cdot 10^{13}$ protons $/$ bunch)

- Improvement of MR magnet power supply and RF

- Repetition rate from $3.52 \mathrm{sec} . \Rightarrow 3.22 \mathrm{sec}$

- in a few years $<2.64 \mathrm{sec}$

- RCS 500kW (MR 300kW) trial $\left(2 \cdot 10^{13}\right.$ protons/bunch and 8 bunches operation)

- Medium period goal

- Continuous run with $>300 \mathrm{~kW}$ starting 2011 fall

- $\mathrm{MW}^{*} 10^{7}$ sec integral power by 2014

- Further improvement by faster rapid cycling

\section{Quest for the Origin of Matter Dominated Universe}

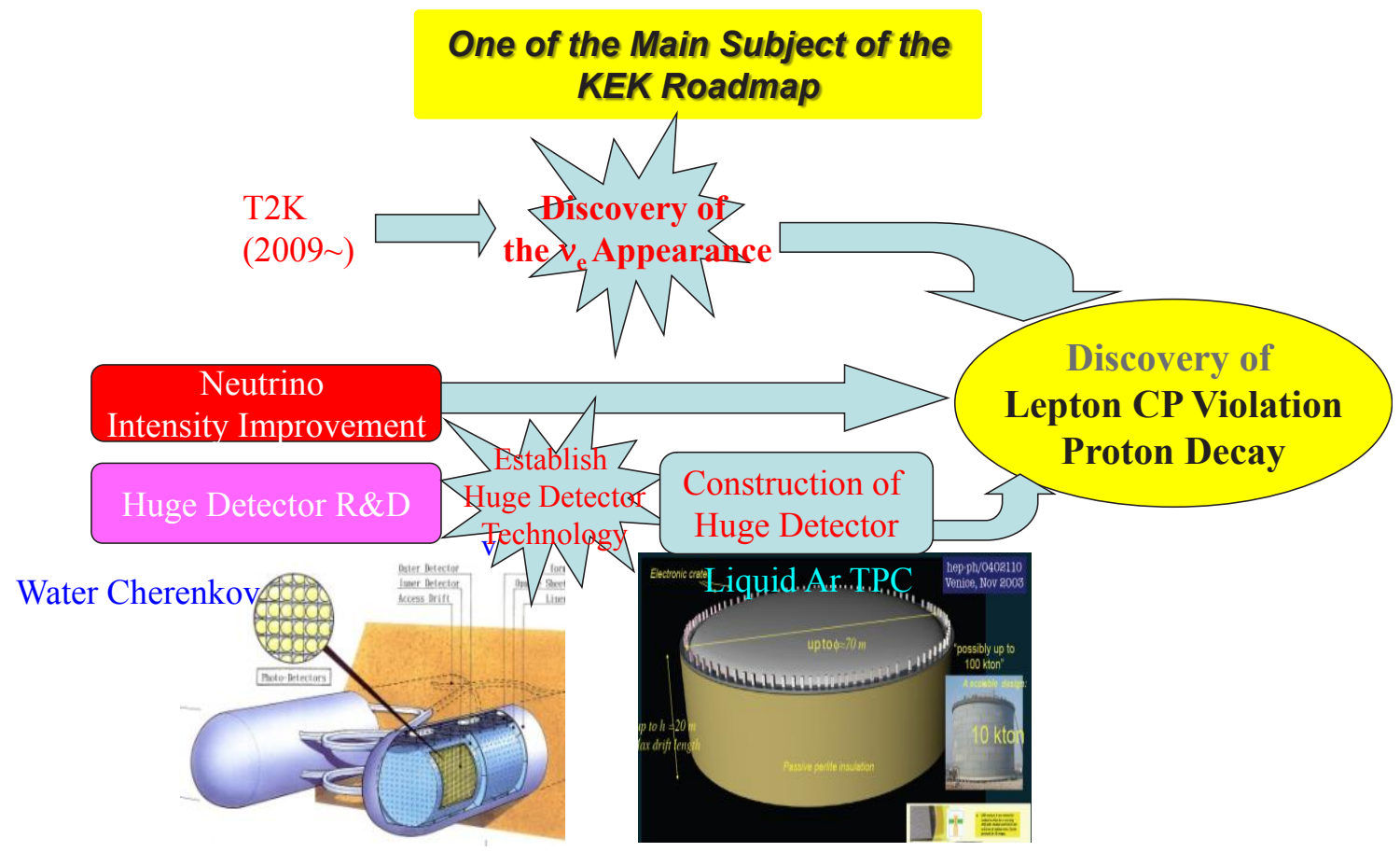




\section{Slow Extraction}

Spill structure Radioactivity by beam loss
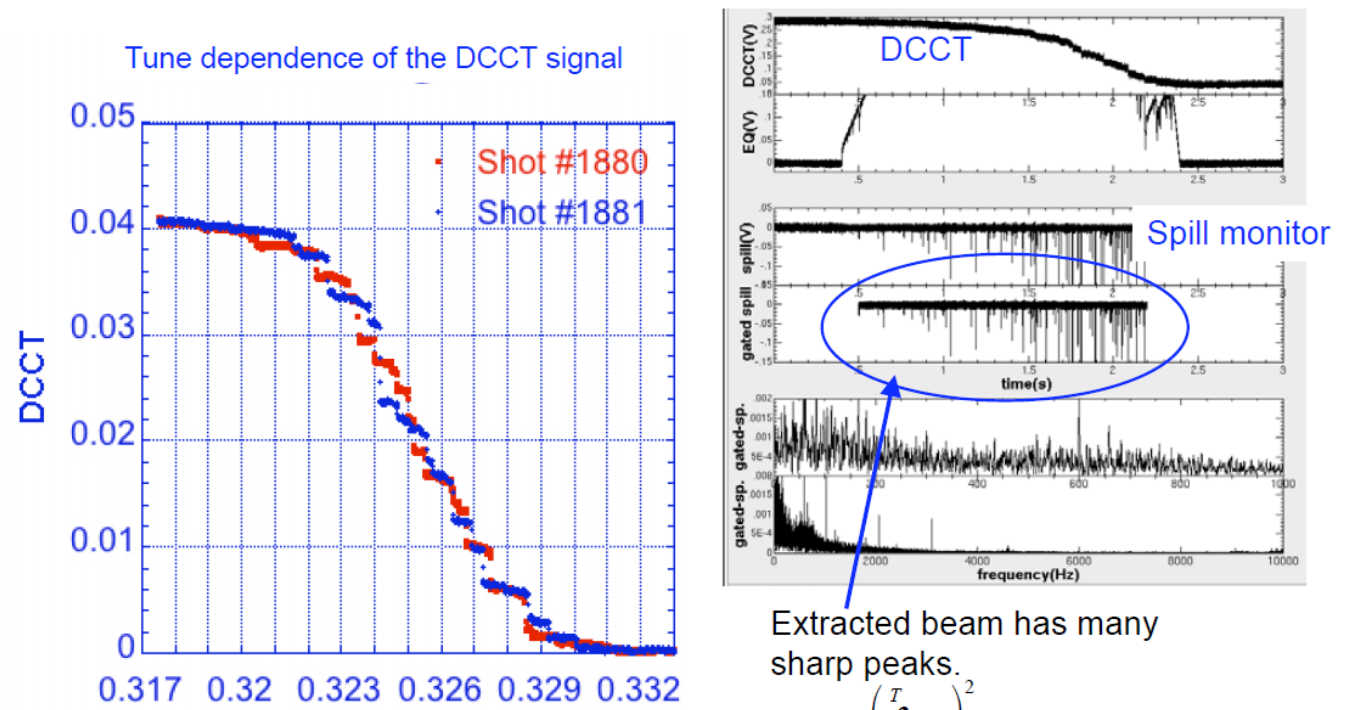

Extracted beam has many sharp peaks.

Because of the tune fluctuation, the circulating beam decreases in the step-like shape

\section{Program at slow extracted beam facility starting now}

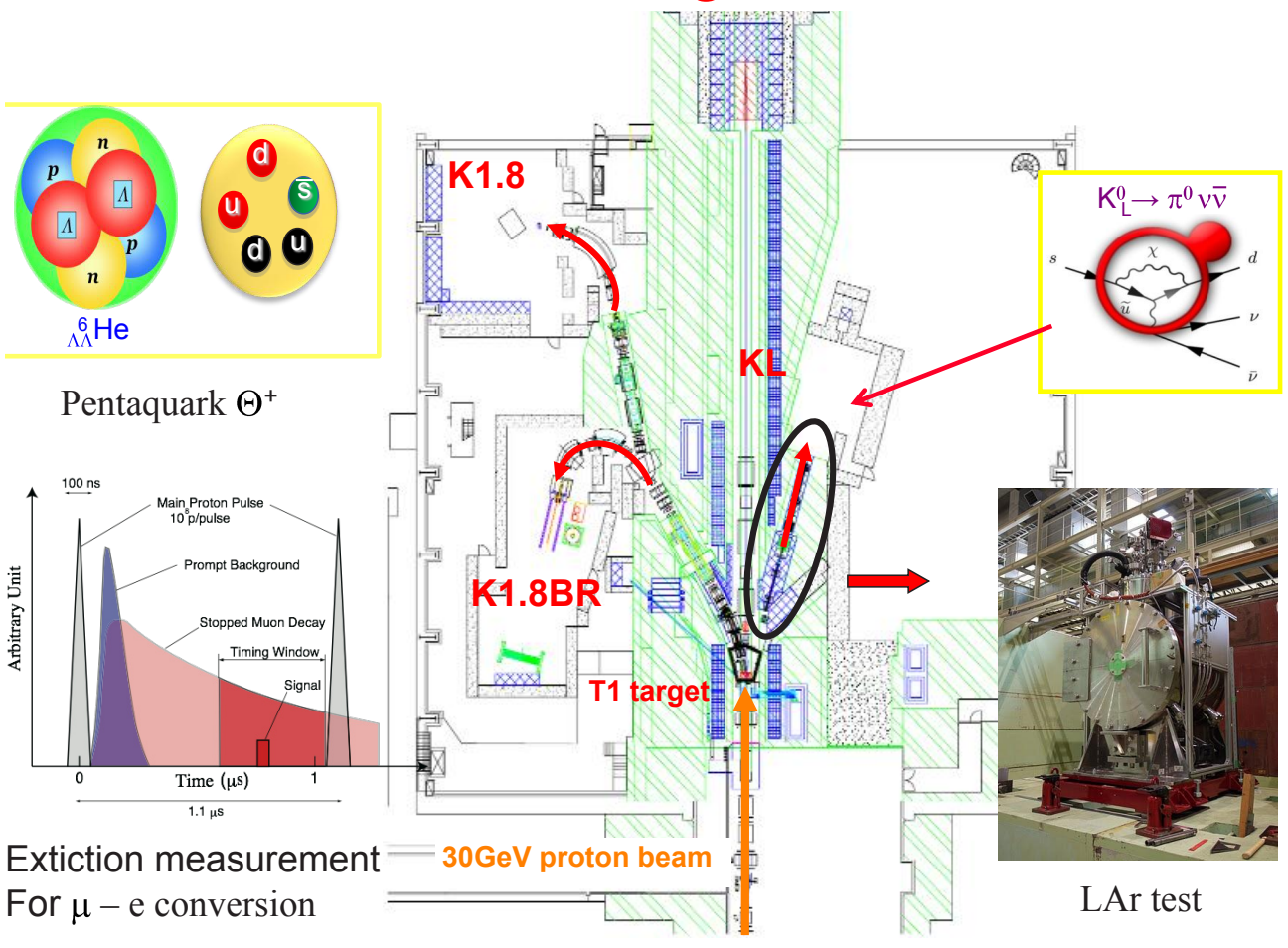




\section{Summary}

- All J-PARC started operation

- Beam power

- FX(Neutrino): $50 \mathrm{~kW}$ stable (100kW trial) realized, aim for $150 \mathrm{~kW}$ and continuous physics run starting in Nov.

- SX: $2.6 \mathrm{~kW}$ realized, tuning for $5 \mathrm{~kW}$ continuous operation with new additional hardware

- Particle physics program:

- T2K: Started Data taking!

- KOTO: Commissioning of beam done, further beam study with calorimeter installed

- Penta-quark search data taking starting up

- Intensive work on preparation and R\&D on

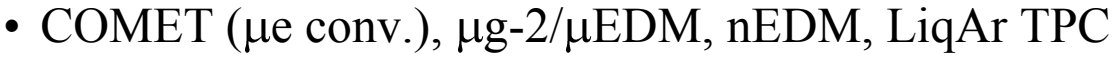

- Beam power will be increased in stages toward realizing

- Design power of $750 \mathrm{~kW}$

- Then 1.7MW (KEK roadmap) 


\title{
Proposal for Japan-US collaboration on New Test Facility for Novel Accelerator R\&D (FACET at KEK)
}

\author{
M. Yoshida, K. Nakajima, M. Sato, S. Matsumoto, M. Nozaki, S. Yamaguchi \\ KEK Novel Accelerator Promotion Office
}

The construction is being considered of a new test facility for the novel accelerator at the end of the KEK $8 \mathrm{GeV}$ LINAC. With it, we aim to obtain the ultra-high-gradient accelerating field induced by the beam-driven plasma or the dielectric wakefield similar to FACET at SLAC. Further, we are debating whether the laser plasma's self-injection electron beam will be a good candidate as the witness bunch that is a probe of the femtosecond-order wakefield.

There are many future targets for the beam-driven novel accelerator; one of them is the so-called "after burner" for the ILC. For this, we have to think carefully about how to make the drive beam, and how to accelerate the long bunch inside the wakefield. For example, expectedly, the small vertical emittance will convert to the bunch length. Our target is not only to obtain the high gradient, but also to study those solutions.

The KEK $8 \mathrm{GeV}$ LINAC already successfully injected into three rings, viz., KEKB-LER/HER and PF under continuous operation using the fast beam switch. It has been undergoing in the four years an upgraded construction of a new RF-Gun and a damping ring. The key technologies for the novel accelerator-test facility are the fast beam-switch to operate this facility during injection, the high-charge, low-emittance RF-Gun, and the higher RF frequency unit for harmonic compensation and bunch compression.

The fast beam-switch assuring continuous injection into the three rings already is working. This switch consists of a new event system, which selects each beam mode at anytime, and a new pulse bend to kick the $2.5 \mathrm{GeV}$ electron beam for PF. We plan to use this pulse bend to extract the test beam into an iron beam pipe to avoid the subsequent bend magnet. Thus, this new facility for the novel accelerator can be operated continuously as pulse-to-pulse until the shutdown of Super-KEKB shutdown well into the future.

We plan to install a few types of new RF-Guns since a low- emittance RF-Gun is required for Super-KEKB ring. One of them is a velocity bunching DAW RF-Gun that can produce a highly charged electron bunch for FACET. Additionally, this RF-Gun easily can generate a complex bunch with a picosecond-order structure that entails a femtosecond-order structure after bunch compression. We anticipate that this bunch structure will attain a higher transformer ratio.

Further, the higher RF frequency leads very efficient harmonic compensation and bunch compression that are affected linearly to both the frequency and the accelerating gradient. The X-band technology, originally developed for a previous linear-collider project based on Japan-US collaboration, can be applied for harmonic compensation inside the electron bunch, and effective bunch compression.

Aspects of this FACET at KEK are different than those at the FACET at SLAC, and already we can offer unique abilities for some researches. Thus, we expected that KEK's FACET will become a good complementary facility to that at SLAC. 


\section{FACET at SLAC}

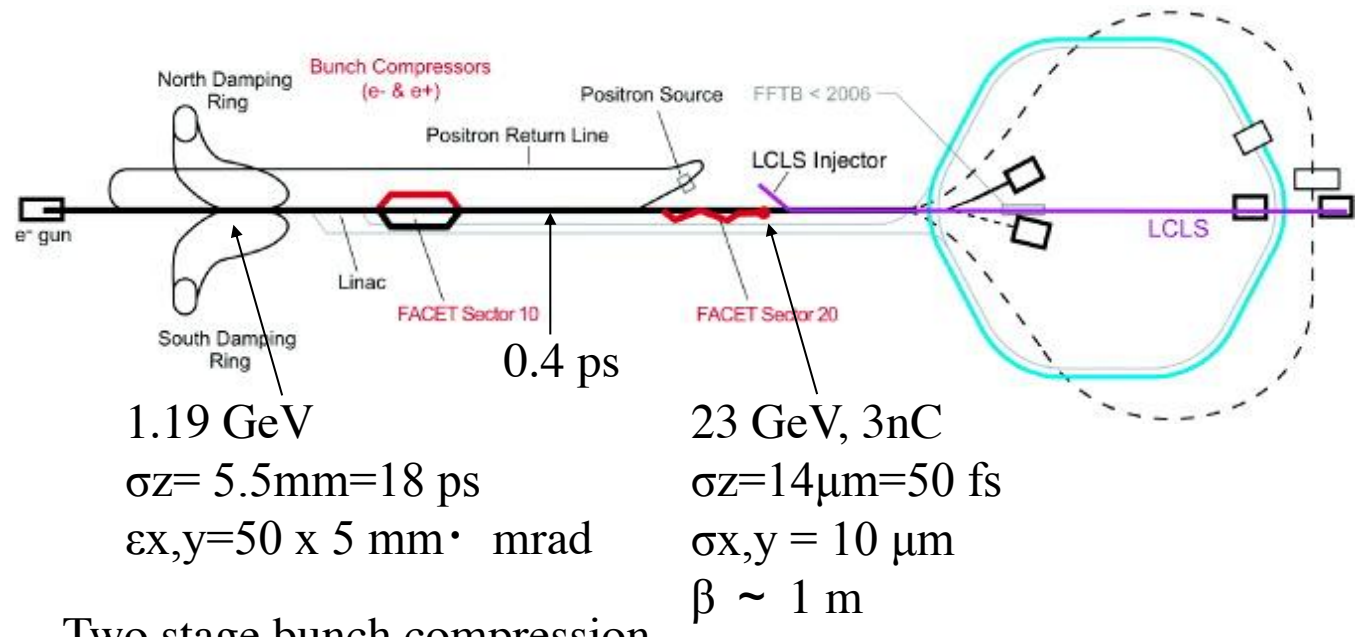

Two stage bunch compression.

At FFTB experiment :

Gradient : PWFA(>30GV/m), DWA( $>16 \mathrm{GV} / \mathrm{m})$.

Total acceleration voltage : $>$ a few $\mathrm{GeV}$.

We are discussing with Dr. Mark Hogan.

\section{FACET at KEK}

$\underline{8 \mathrm{GeV} \text { LINAC from } 1998}$

Install Photocathode RF-Gun

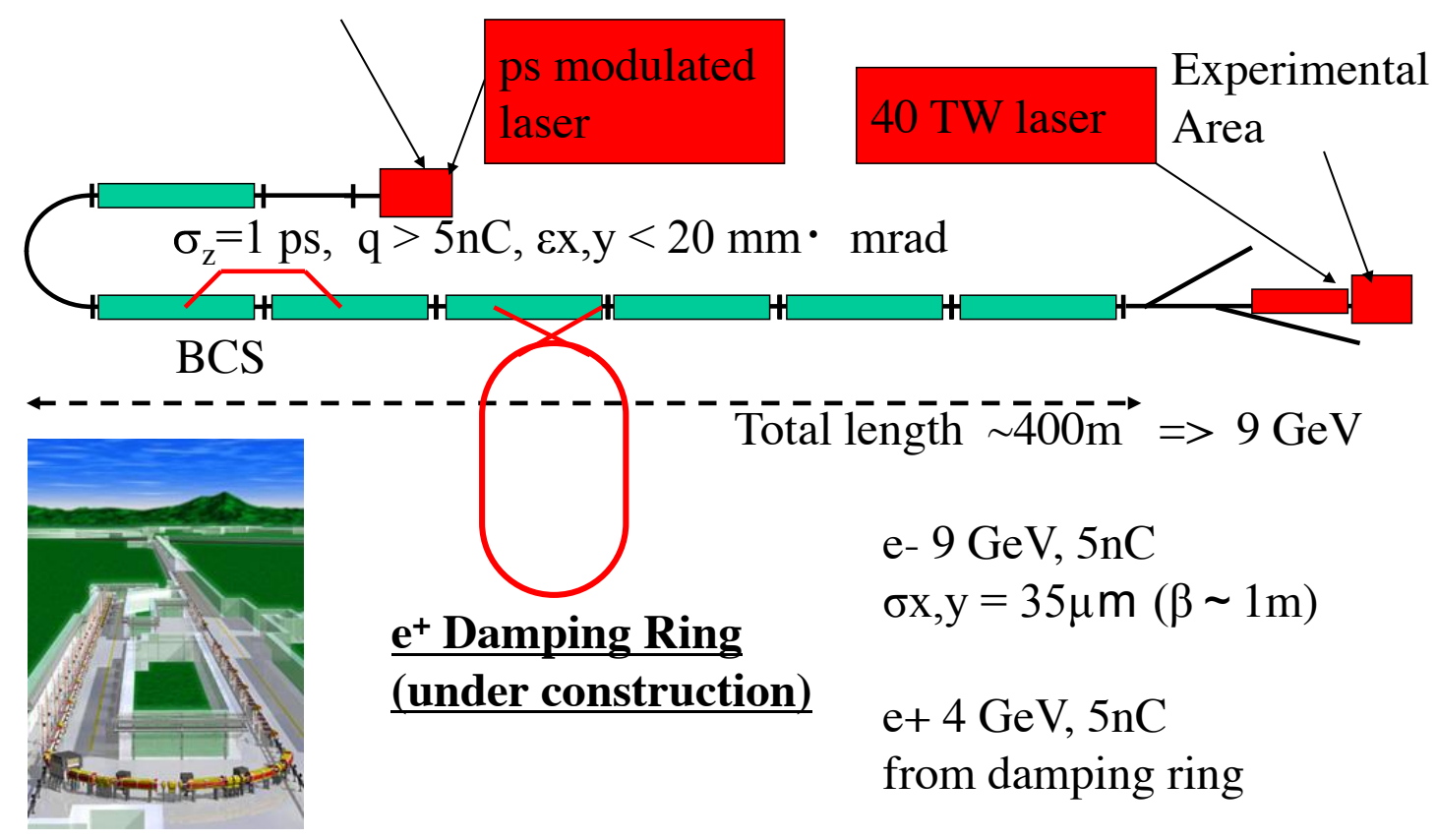




\section{Target for FACET in KEK}

- Continuous novel accelerator facility until SuperKEKB shutdown 2025?

- Short pulse laser complex facility :

Laser modulated photo cathode RF-Gun and

LWFA injector for femtosecond witness beam.

- User facility for University etc.

LWFA

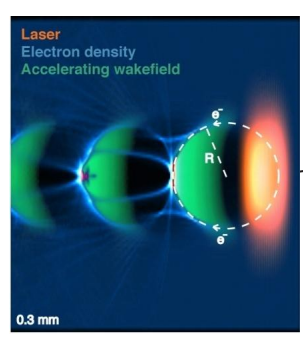

$\sim 100$ fs electron bunch

\section{Ex. DWA}

DWA experiment is just started using another low energy fs beam.

Single-wall DWA:

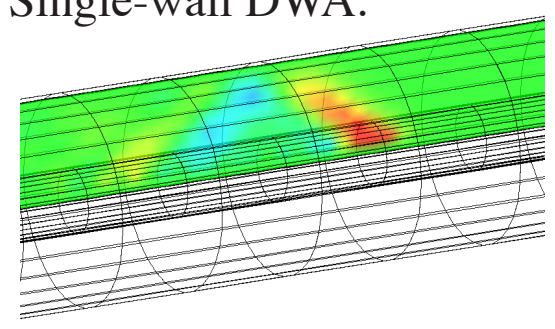

$$
\begin{aligned}
e E_{z, d e c} \approx & \frac{-4 N_{b} r_{e} m_{e} c^{2}}{a\left[\sqrt{\frac{8 \pi}{\varepsilon-1}} \varepsilon \sigma_{z}+a\right]}=2 G V / m\left(\sigma_{t}=0.1 p s\right) \\
& \times \text { Transformer Ratio }(\sim 3)
\end{aligned}
$$

Multi-wall or photonic DWA with multi femtosecond bunch:

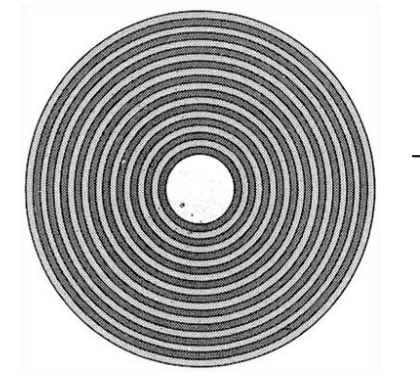
$\begin{aligned} & \text { Laser modulated } \\ & \text { micro bunch structure }\end{aligned} \Rightarrow \begin{aligned} & \text { Higher } \\ & \text { Transformer ratio }\end{aligned}$ $+0000$

For microwave:

Klystron : $350 \mathrm{kV}$

Accelerator : $35 \mathrm{MV} / \mathrm{m}$

$=>$ transformer ratio $=100$ 

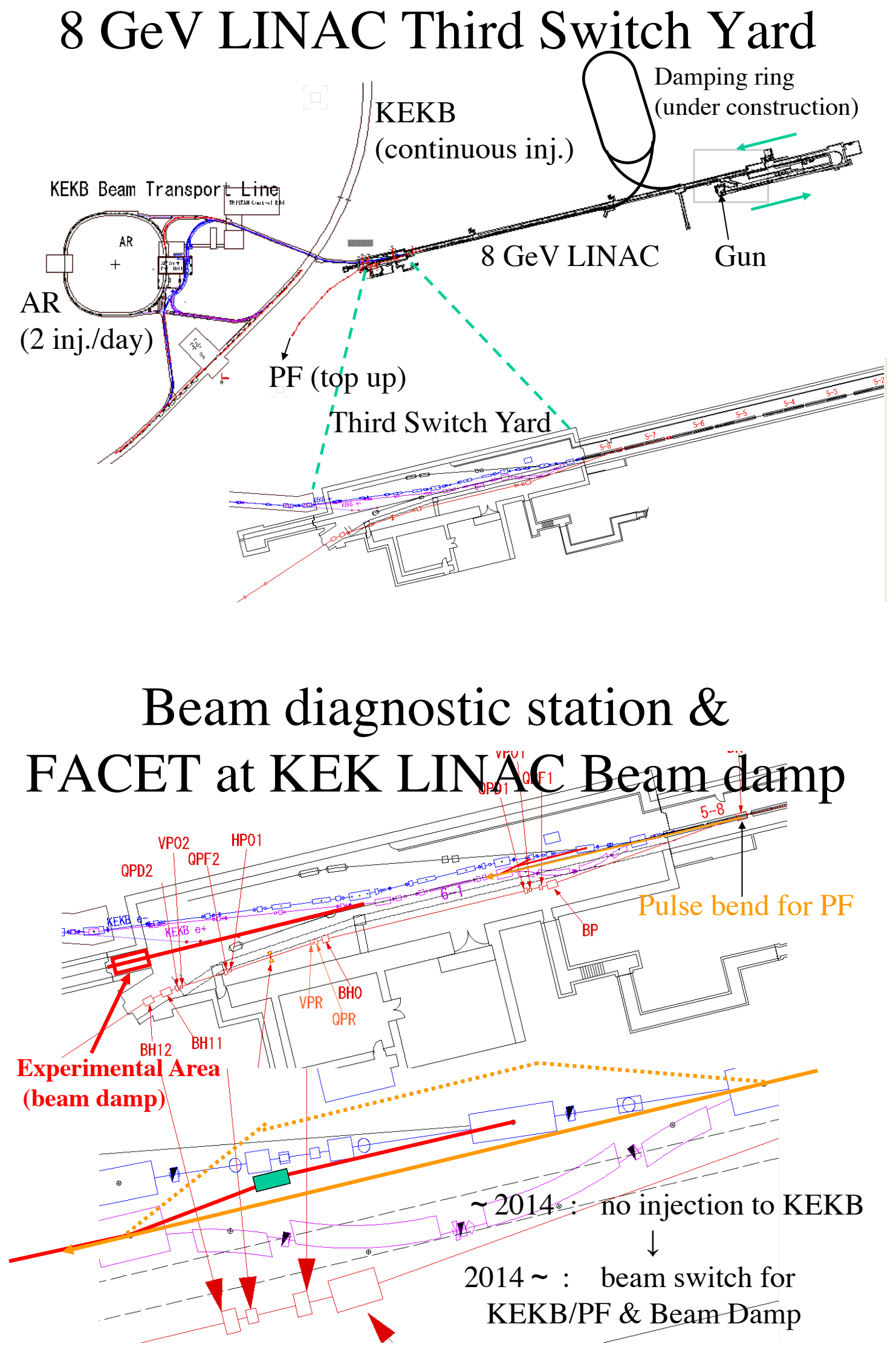


\section{Key technologies}

- Laser plasma injector

$\square$ The witness beam.

$\square$ Sub-fs beam.

- Fast beam switch (completed)

Event system

Pulse bend

- RF-Gun (will be installed soon)

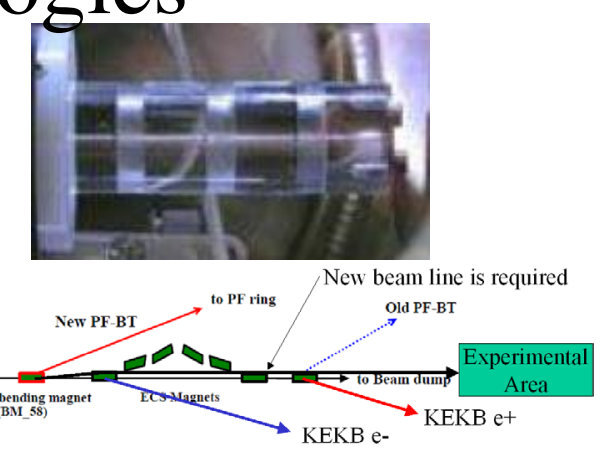

$\square$ Velocity bunching DAW => high charge \& low emittance

$\square$ Modulated laser $=>$ micro bunch structure.

- X-band for harmonic compensation and bunch compression.

Also use for Beam Diagnostic Station for LINAC Upgrade :

Bunch Length / Sliced Emittance

\section{Summary}

- FACET at KEK is considered to construct the beam driven and short laser complex facility, it has some different aspects compared with FACET at SLAC.

- KEK $8 \mathrm{GeV}$ LINAC has unique ability for novel accelerator test facility except for SLAC in the world.

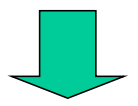

- It is expected to become a good complementary project to FACET at SLAC. 



\title{
Laser Plasma Accelerators for Future Colliders and Light Sources
}

\author{
Wim P. Leemans \\ Lawrence Berkeley National Laboratory, Berkeley, CA
}

Accelerators, x-ray light sources, and lasers have been essential tools for advancing science and technology, and provided the foundation for a vast number of industrial ventures and societal benefits in the $20^{\text {th }}$ century. As we enter the second decade of the $21^{\text {st }}$ century, resolving key challenges in science and technology will require our having next-generation accelerators, light sources, and lasers that far exceed today's capabilities.

My colleagues and I presented a vision for building accelerators for high-energy physics and light sources rooted in the concepts of laser plasma acceleration [1,2]. Progress in laser plasma accelerators (LPAs) in the last five years included the demonstration of narrow energy-spread beams [3], and $\mathrm{GeV}$ electron- beams from cm-scale plasma-based accelerating structures powered by intense lasers [4]. This important progress resulted in these LPAs being considered for driving hyperspectral compact light sources, such as free electron lasers (FELs) operating in the soft x-ray regime, or intense gamma-ray sources for security- and medical-applications. Experiments are underway for demonstrating a 10-GeV, meter- scale LPA module using the BELLA facility under construction at LBNL. Such a module might well be the building block for a LPA-based collider, as well as for hard x-ray FELs.

A key aspect for ensuring the continued success of LPA-based technologies will be the development of peak-power laser systems with much higher average power levels than today's systems. Modern accelerators have become increasingly dependent on laser technology, ranging from the production and manipulation of electron beams, to novel acceleration techniques and advanced light sources. The high average power demands imposed by today's accelerators on lasers rapidly are exceeding their state-ofthe-art abilities. To bridge the gap between what exists today and what is needed in the future, a Joint Taskforce (JTF) was formed, endorsed by the International Committee for Future Accelerators (ICFA) and the International Committee for Ultra-intense Lasers (ICUIL), to develop a roadmap for laser technology for future accelerators.

For light sources and colliders, the required lasers must operate at power levels two-to-four orders of magnitude greater than the petawatt-class laser that will drive the BELLA facility. This necessitates formulation of an aggressive R\&D strategy that will leverage investments made in developing systems that are relevant to defense and industry, as well as for laser-fusion-based power plants. Some important aspects of the short-pulse lasers suitable for accelerators do however differ from those systems and dedicated funding in support of research on high average peak power ultra-fast lasers will be essential. Such research is underway in Europe through the Extreme Light Infrastructure project, in support of the planned Hungarian, Czech Republic and Romanian installations that have recently been awarded 790 M€. Additional support has been made available towards Exawatt systems through the ILE project in France, which is a precursor to the "fourth pillar" of ELI. This work was supported by the US Department of Energy under contract DE-AC02-05CH11231. The author gratefully acknowledges valuable input and contributions from all LOASIS and BELLA team members, as well as the members of the JTF.

\section{$\underline{\text { References }}$}

[1] W. Leemans and E. Esarey, Physics Today 62, p.44-49 (2009)

[2] E. Esarey, C.B. Schroeder and W.P. Leemans, Rev. Mod. Phys. 81, 1229 (2009).

[3] S. Mangles et al., Nature 431, p.535-538 (2004); C.G.R. Geddes et al., ibid, p. 538-541; J. Faure et al., ibid, p. 541-544.

[4] W.P. Leemans et al., Nature Physics 2, p.696-699(2006). 


\section{Laser plasma accelerators}

\section{Wim Leemans}

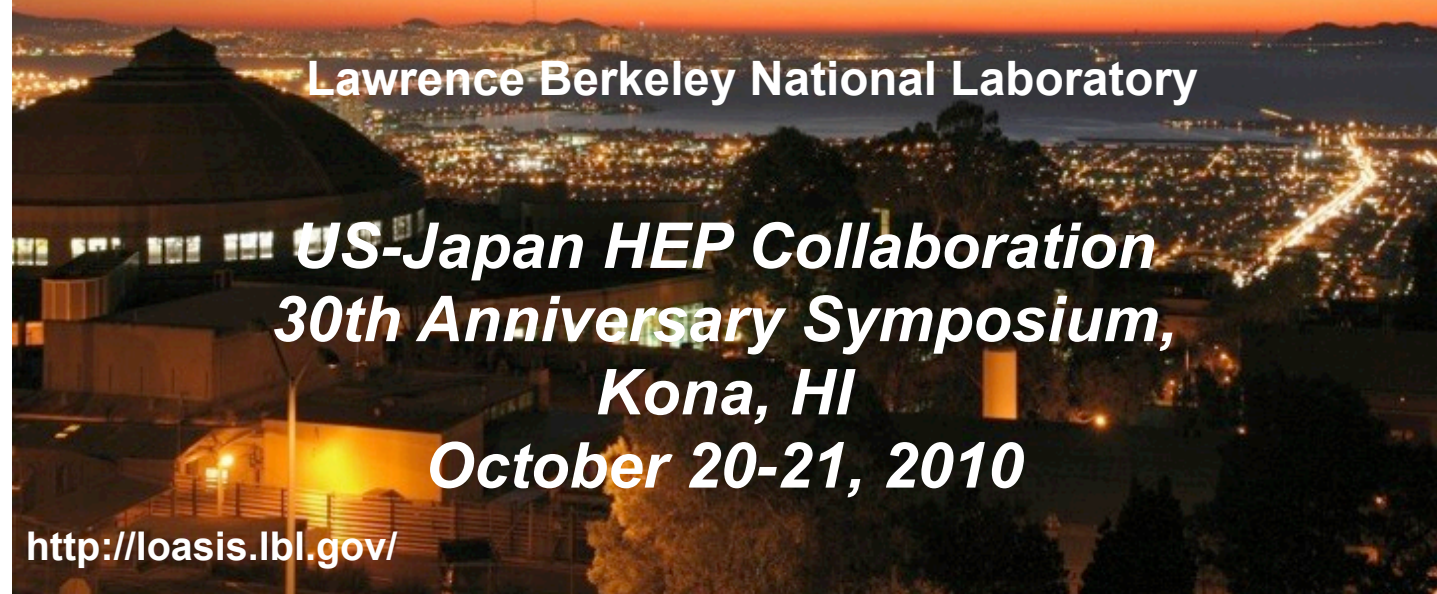

Wednesday, December 8, 2010

\section{Building a laser plasma accelerator following conventional linac paradigm}

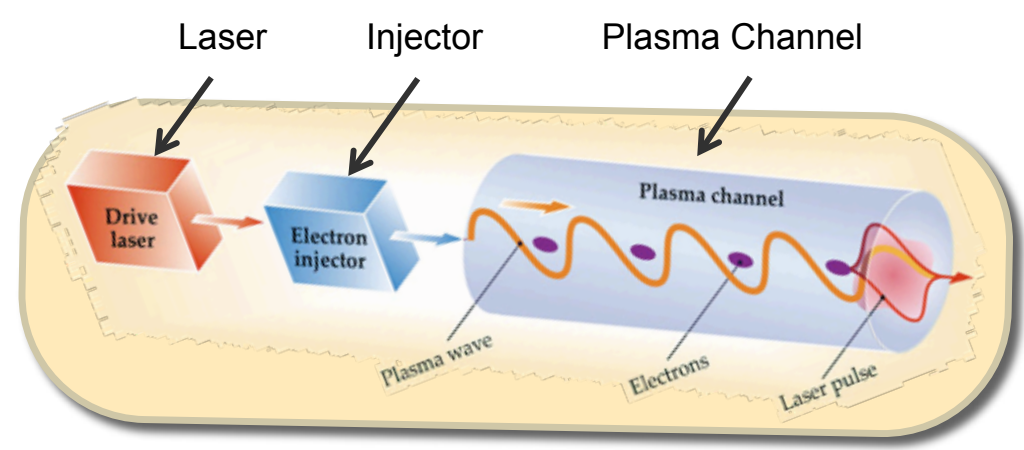




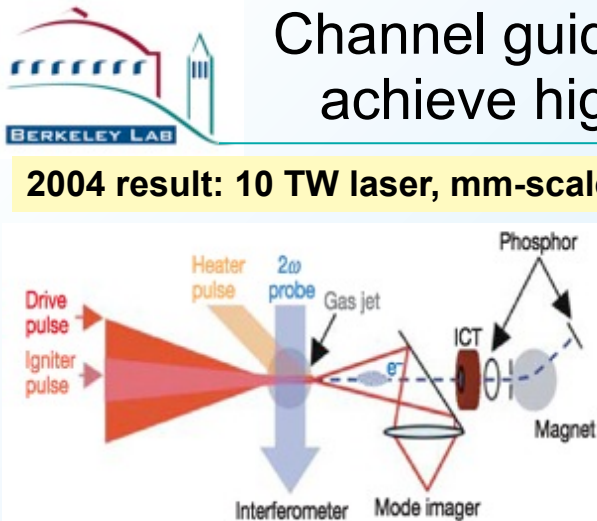

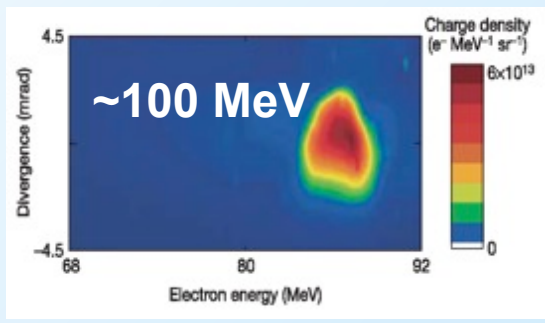

C. G. R. Geddes,et al, Nature,431, p538 (2004)

S. Mangles et al., Nature 431, p535 (2004)

J. Faure et al., Nature 431, p541 (2004)

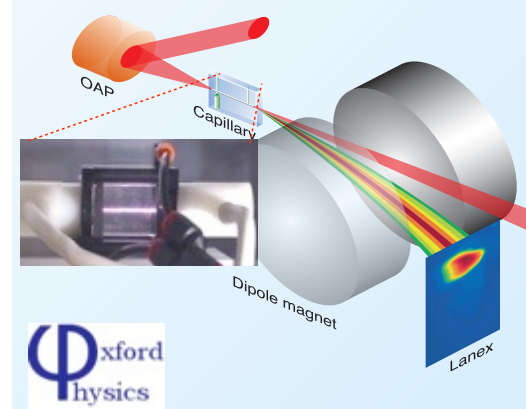

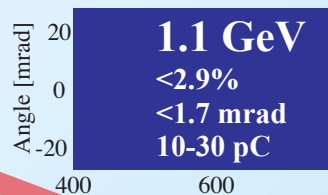

W.P. Leemans et. al, Nature Physics 2, p696 (2006) K. Nakamura et al., Phys. Plasmas 14, 056708 (2007)

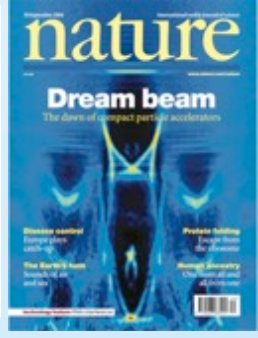




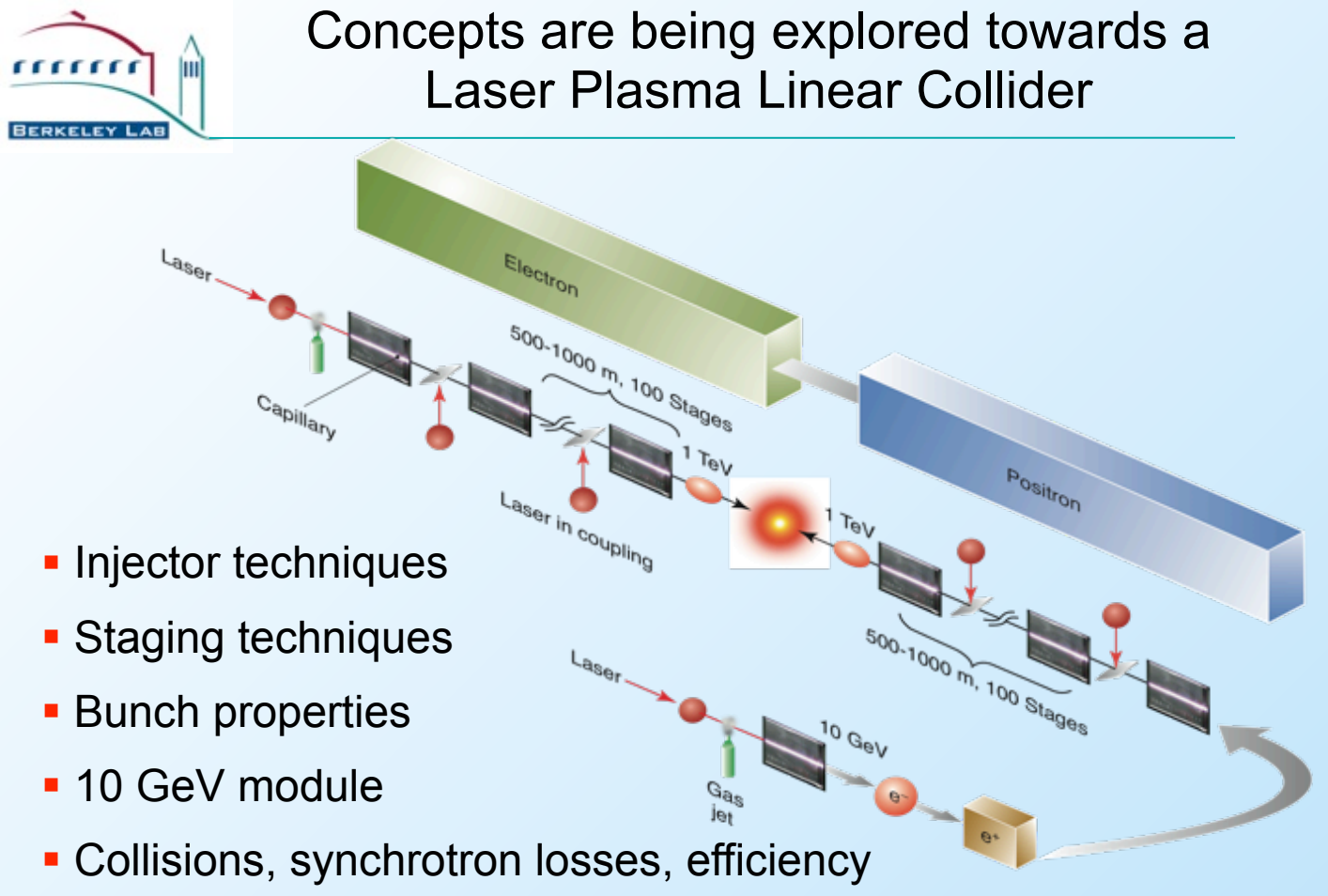

W. Leemans and E. Esarey, Physics Today (2009); C.B. Schroeder et al., PRST-AB 2010

Wednesday, December 8, 2010

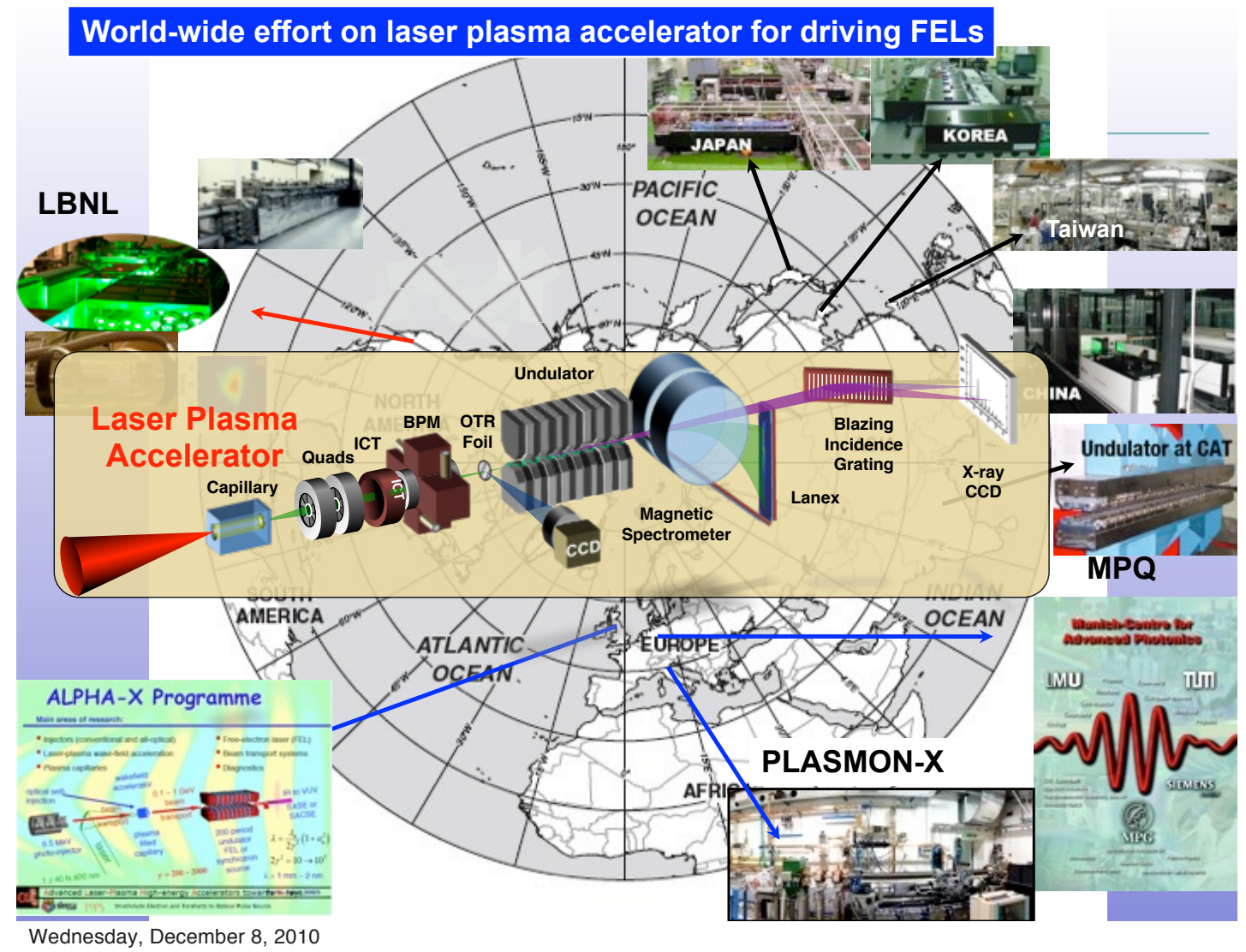




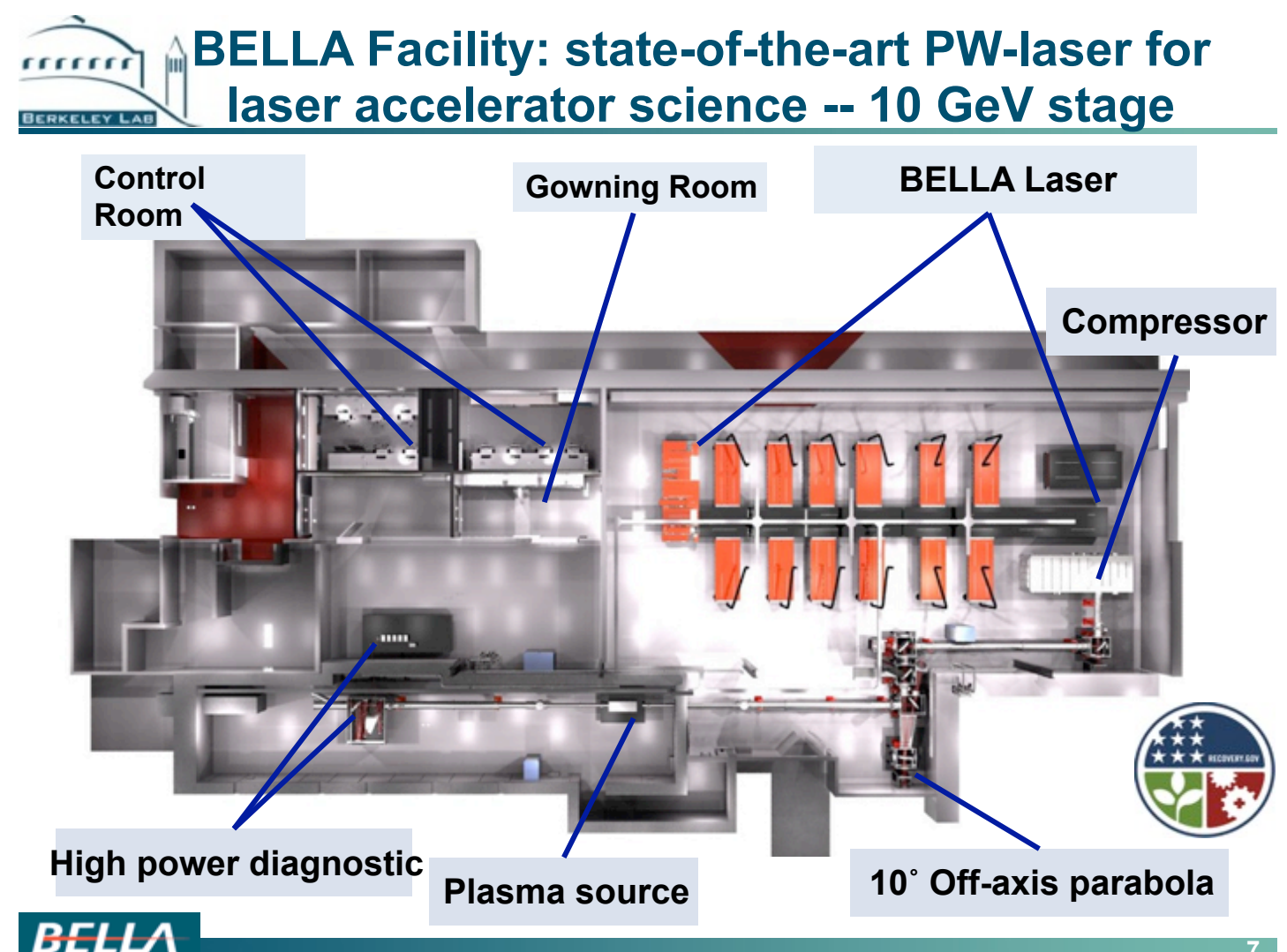

Wednesday, December 8, 2010

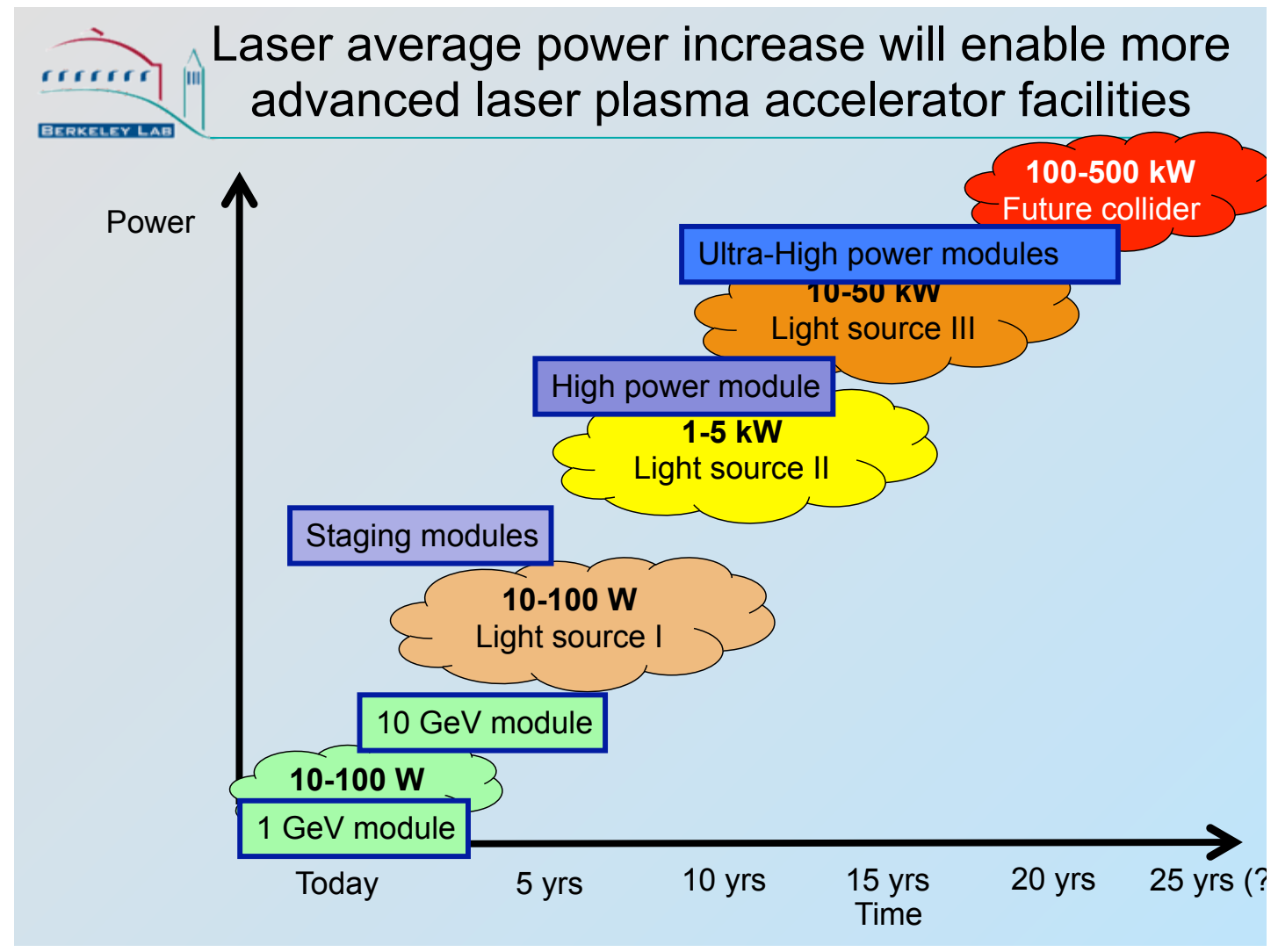




\section{ICFA-ICUIL Joint Task Force for Laser Technology: engaging two communities}

- Joint ICFA-ICUIL taskforce on "Roadmap for high average power laser technology for future accelerators"

- $\quad$ Leadership: Chou (ICFA-BD), Uesaka (ICFA-ANA), Leemans (JTF Chair, ICFAANA\&ICUIL), Barty (ICUIL), Sandner (ICUIL)

- First Workshop by JTF held @ GSI, Darmstadt, April, 2010

- 47 experts from accelerator and laser communities

- Requirements on lasers for colliders, light sources, medical applications

- Identifying promising laser technologies and bottlenecks

- Developing strategic roadmap

- Report in progress

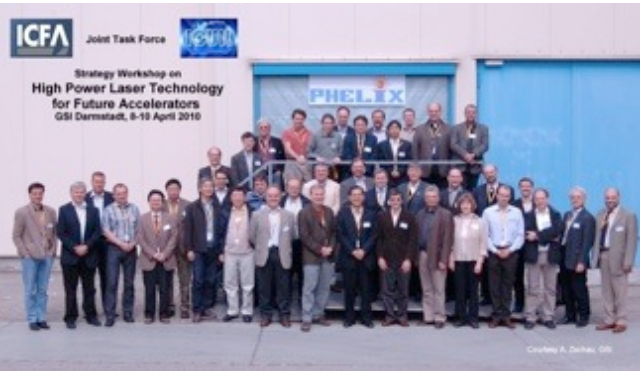

Wednesday, December 8, 2010

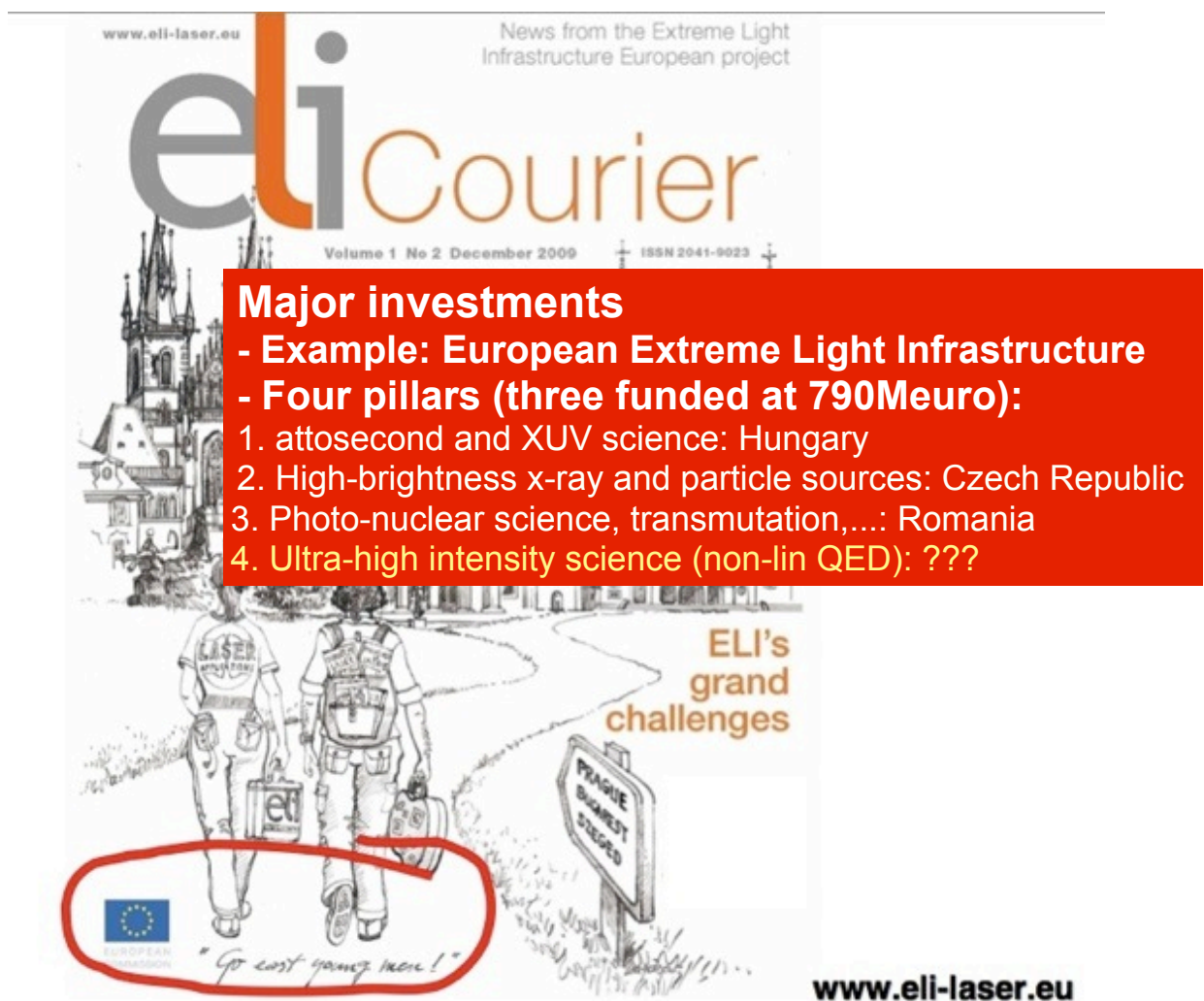

Wednesday, December 8, 2010 


\section{Conclusion}

- Laser plasma accelerator science is vibrant

- $10 \mathrm{GeV}$, high quality beams towards collider applications

- FEL proof-of-principle experiments towards Light Source Facility

- Gamma-ray sources

- Medical and other applications

- Attracting many students, postdocs into field

- Very significant investments being made around world:

- Example: USA, Europe, Japan, China, Korea,...with collaborations

- "Big science" apps will require major investment in high average power laser technology

- multi-kW (light sources, medical) to 100's of kW (colliders), Petawatt lasers needed

- Sustained, long range R\&D needed for accelerator relevant lasers -similar to klystron effort, 50 yrs ago

- Opportunities for US-Japan collaborative efforts

Wednesday, December 8, 2010 



\title{
Advanced and Innovative Accelerators
}

\author{
Mitsuru Uesaka, Nuclear University of Tokyo, Japan
}

Under the US/Japan high-energy physics collaboration, several long-term researches were undertaken on advanced accelerators, involving staff from KEK/U.Tokyo/LBNL/BNL/SLAC /UCLA /ANL. Many paths forward were discussed and developed for advanced high-field acceleration, such as RF-source-driven microwave structures, and beam-driven microwave structures for the accelerating field of $\sim 100 \mathrm{MV} / \mathrm{m}$. Another notable area was laser-driven and beam-driven dielectric structures for $\sim 1 \mathrm{GeV} / \mathrm{m}$, and laser/beam-driven plasma acceleration for $\sim 10 \mathrm{GeV} / \mathrm{m}$. Derived from the Linear Collider study were the X-band $(9.3-11.424 \mathrm{GHz})$ linacs for cancer therapy $(6 \mathrm{MeV})$, and the on-site inspection technologies for petrochemical complexes $(950 \mathrm{keV})$ and bridges $(3.95 \mathrm{MeV})$. Especially, the very early stages of the beam/laser-driven plasma-electron acceleration experiment was carried out using the S-band twin linacs (35, 18 $\mathrm{MeV}$ ) and 3 TW 100 fs Ti:Sapphire laser by KEK/JAEA (Japan Atomic Energy Agency)/U. Tokyo under the US/Japan collaboration. Furthermore, BNL introduced the laser photocathode RF-electron gun that now is used for electron sources for X-ray FELs as was the generation of femtoseconds Compton-scattering X-rays.

Many researchers continue to contribute to the ICFA panel on Advanced and Novel Accelerators, chaired by Dr. Wim Leemans from 2000 to 2007, and by me thereafter. Recently, collaborative work started between the ICFA and the ICUIL (International Committee for Ultra Intense Lasers); a task force was established, and the first joint workshop was held at GSI, Germany, in April 2010. It consists of the four working groups; viz., laser development, high-energy applications, light-source applications, and medical applications. I was delighted to chair the last one. We discussed the following issues: Starting with current clinical facilities as a reference for comparison, we assumed the requirement for maximum flexibility to enable treatment of small in-depth tumor volumes, as well as large ones, so requiring the maximum energy of $250 \mathrm{MeV}$ for protons, and $400 \mathrm{MeV} / \mathrm{u}$ for carbon. We discussed the prospects of requirements some 10-20 years later. Especially, we emphasized the necessity of developing and distributing advanced solid-state stable lasers, such as diode-pumped lasers and fiber lasers.

In summary, many advanced and innovative accelerators have been/will be realized under the US/Japan collaboration. The achievements are applicable to not only high-energy physics but also in the medical- and industrial-fields uses. We expect closer collaborations on laser plasma acceleration with the development of sophisticated optics. 


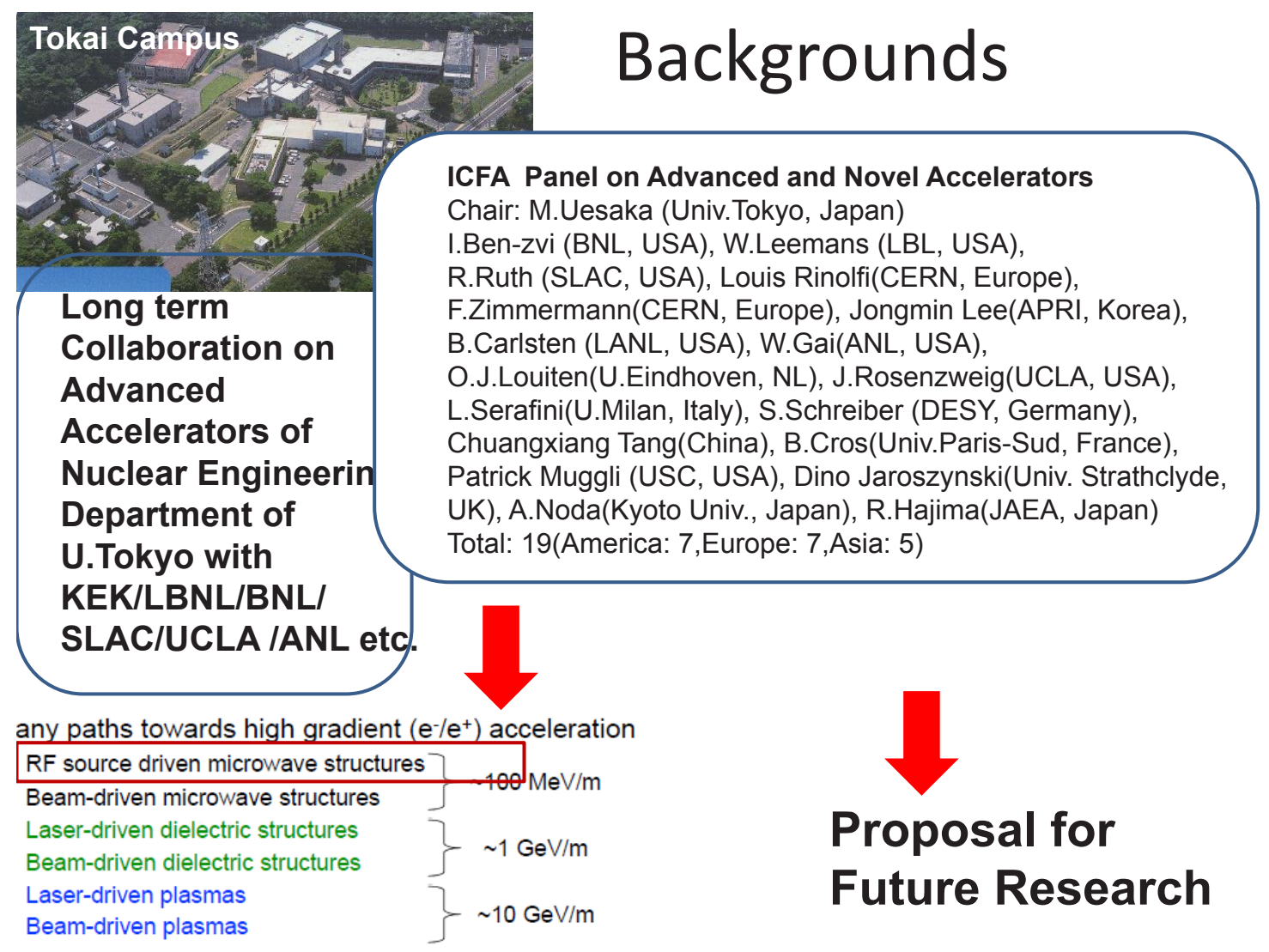

\section{Compact Compton Scattering Monochromatic X-ray Source based on $\mathrm{X}$-band(11.424GHz) Linac and YAG laser}

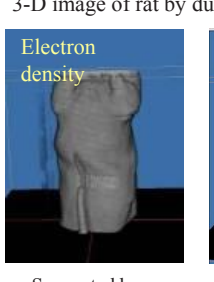

Supported by Japanese Ministry of Education, Culture, Sports, Sciences, and Technology and Japanese Ministry of Health and Welfare from 2007.
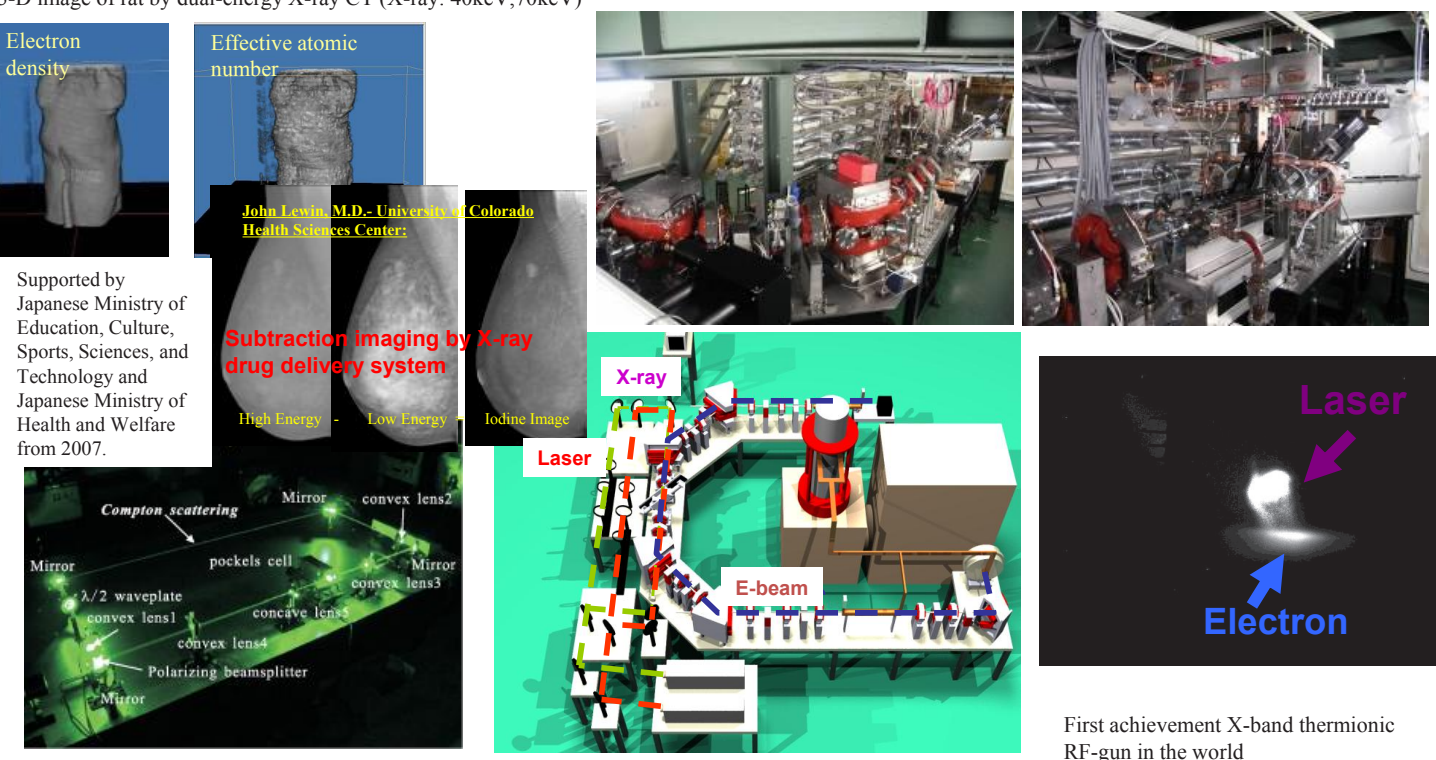

Electron beam: $30 \mathrm{MeV}, 20 \mathrm{pC} / \mathrm{bunch}, 10^{4}$ bunches/RF pulse, $10 \mathrm{pps}$ Laser: Q-switch Nd:YAG $1064 \mathrm{~nm}, 2.5 \mathrm{~J}, 10 \mathrm{pps}$

X-ray : $\quad 21.9 \mathrm{keV}, 1.7 \times 10^{9}$ photons $/ \mathrm{s}$ $532 \mathrm{~nm}, 1.4 \mathrm{~J}, 10 \mathrm{pps}$ X-ray $42.9 \mathrm{keV}, 1.0 \times 10^{9}$ photons $/ \mathrm{s}$

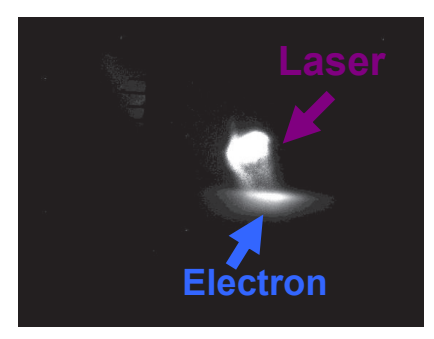

First achievement X-band thermionic RF-gun in the world

A. Fukasawa et. al., Nucl. and Meth. B 241, p.921 (2005) K. Dobashi et. al., Jpn.J.Appl.Phys., 44, p.1999 (2005)

F. Sakamoto et. al., J. Korean Phys. Soc. 49, p.286 (2006) 


\section{X-band Linacs for Industrial and Medical Applications}

1. $950 \mathrm{keV}$ Linac X-ray Source for On-site Inspection

- $0.2 \mathrm{~Gy} / \mathrm{min}$ at $1 \mathrm{~m}$

- Erosion of outdoor metal pipes of petrochemical complex

- Material evaluation by 2 colored X-ray transmission measurement
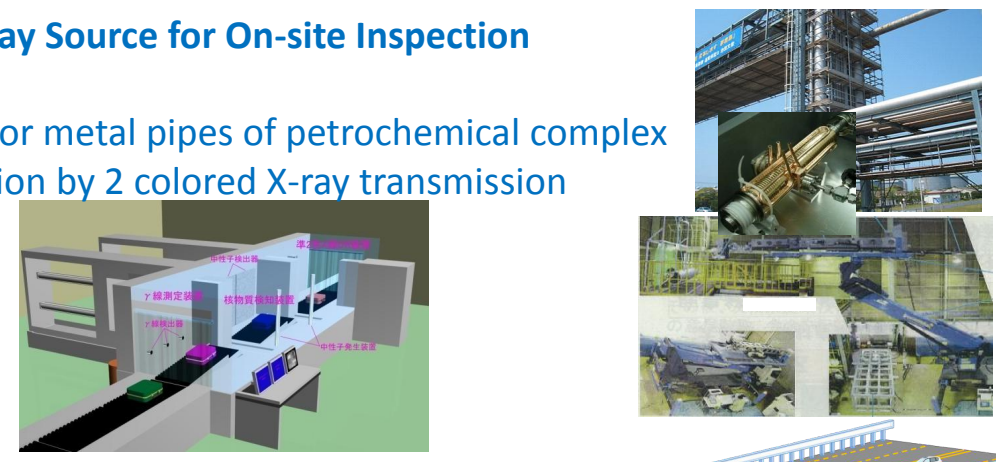

2. $3.95 \mathrm{MeV}$ Linac $X$-ray Source for On-site Bridge Inspection $5 \mathrm{~Gy} / \mathrm{min}$ at $1 \mathrm{~m}$

3. $6 \mathrm{MeV}$ Linac X-ray Source for Cancer Therapy

- $10 \mathrm{~Gy} / \mathrm{min} @ 1 \mathrm{~m}$,

- X-ray spot :1mm at cancers

- Stereotactic therapy

- Dynamic tracking therapy

- for moving lung cancers
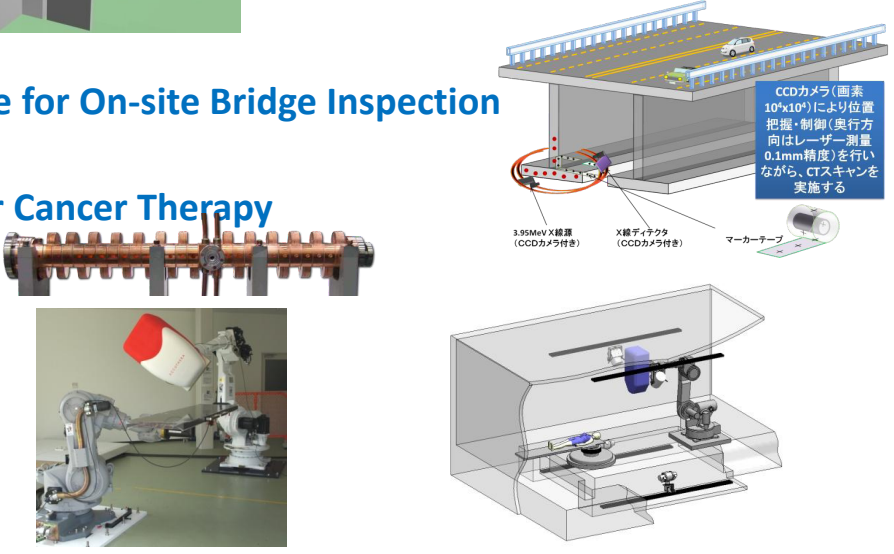

\section{Laser Driven Dielectric Structures(E.Colby) -Optical Fiber Accelerator?-}

V., Technion-Isra

1D Photonic Xtal: Optical Bragg Acceleration Structure
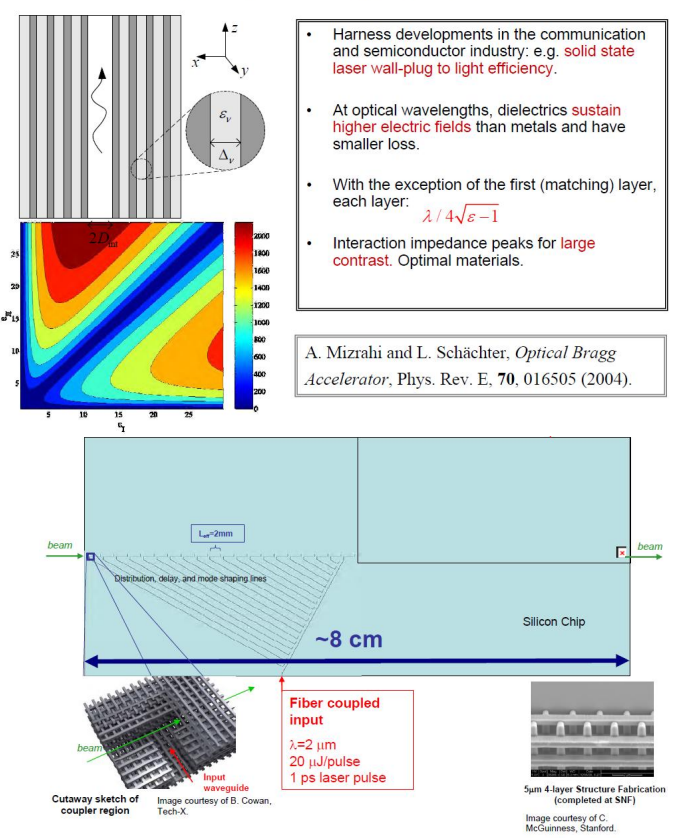

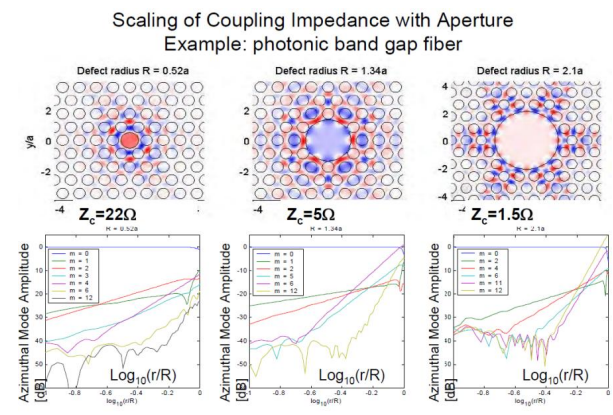

Beam Tests of Microstructures
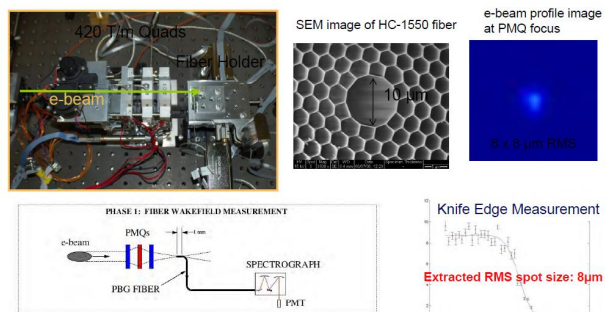

"Knife Edge Measurement Extracted RMS spot size: 8um

E163 Facility at SLAC See Joel England's Talk, WG3, Friday 1:30pm 
Laser Plasma Acceleration Work under the US/Japan Collaboration was initiated by Profs.A.Ogata and K.Nakajima (KEK) from the Japanese side in 1993

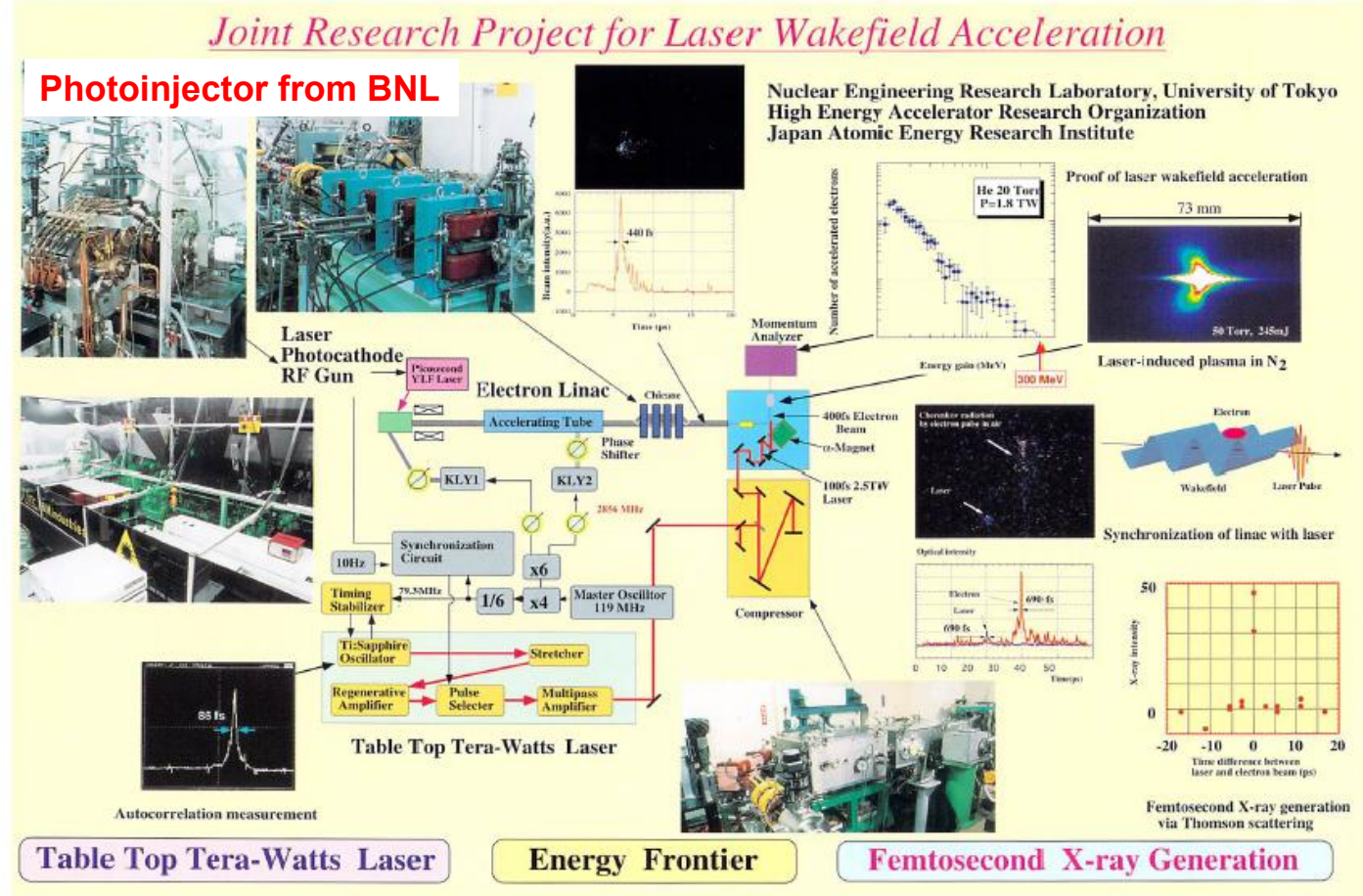

\section{History and Updates of Photoinjectors(J.Power)}

Properties of Photocathodes

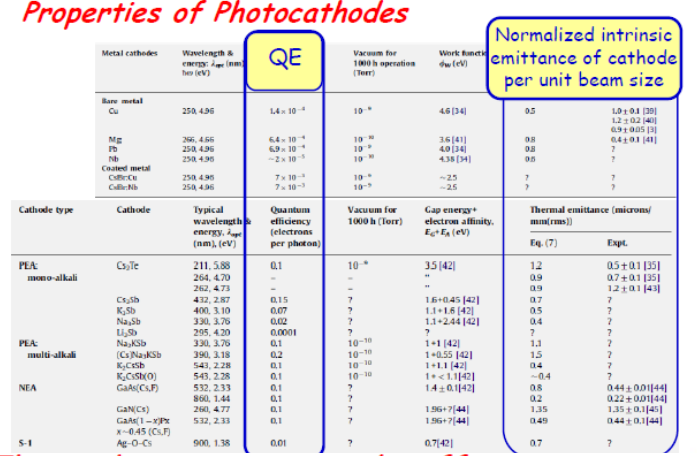

The peak vs average trade-off

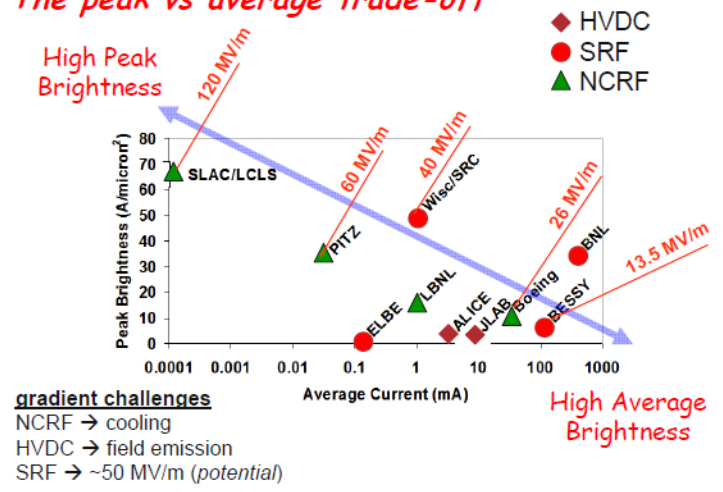

The trade-off: cathodes and lasers

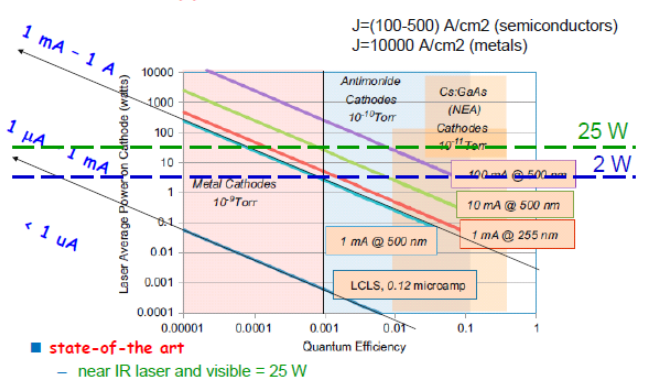

near IR laser an

UV laser $=2 \mathrm{~W}$

laser pulse shaping

Generated ellipsoidal be in blowout regime
$Q=20 \mathrm{pC}(35 \mathrm{fs} \rightarrow 300$ .

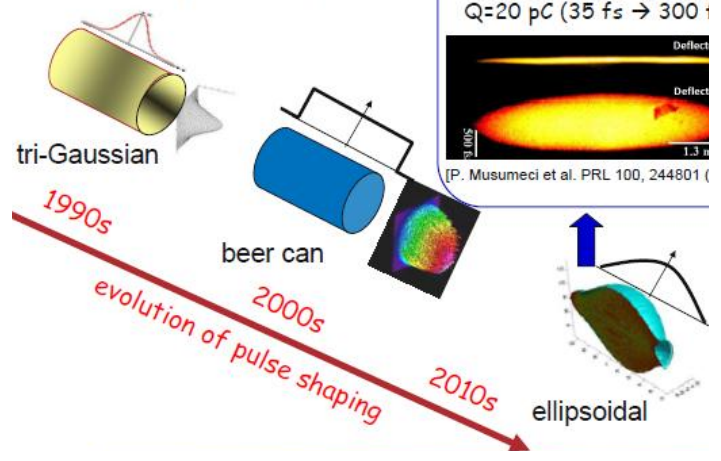




\section{Femtosecond Electron Linac@U.Tokyo}

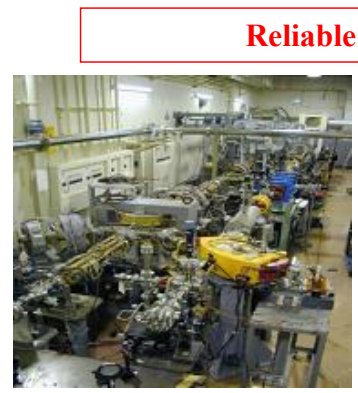

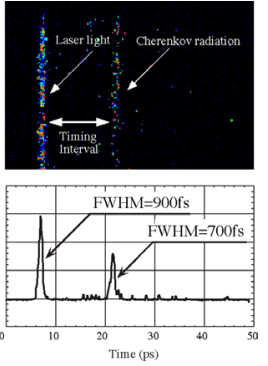

Cart

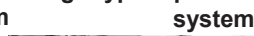

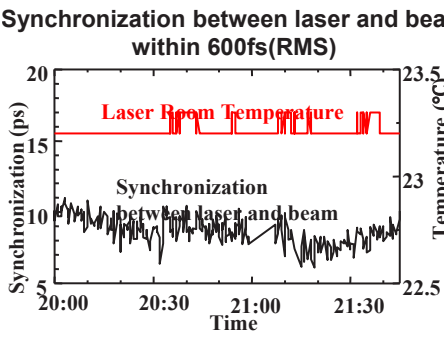

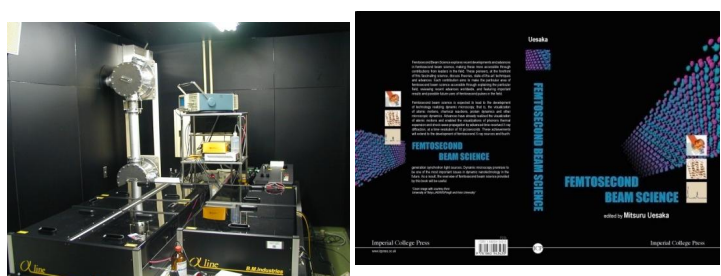

Femtosecond Beam Science, Imperial College Press(2005)

Quantum Efficiency and charge of several cathode material system

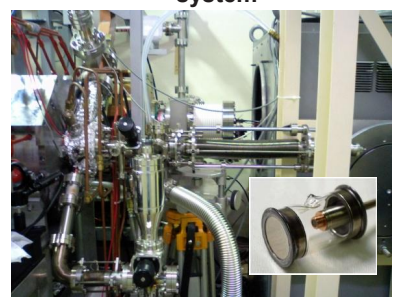

\begin{tabular}{|c|c|c|c|}
\hline Cathode & & $Q E$ & Charge (max) \\
\hline \multirow{3}{*}{$\mathrm{Cu}$} & $B N L$ & $1.4 \times 10^{-4}$ & $10 \mathrm{pC}$ \\
\hline & SLAC & $3 \times 10^{-5}$ & $15 \mathrm{pC}$ \\
\hline & U-Tokyo & $1.4 \times 10^{4}$ & $7 \mathrm{nC}(@ 250 \mu \mathrm{J})$ \\
\hline \multirow{3}{*}{$\mathrm{Mg}$} & $B N L$ & $5 \times 10^{-4}$ & $4 \mathrm{nC}(@ 70 \mu \mathrm{J})$ \\
\hline & SHI & $1 \times 10^{-3}$ & $<2 \mathrm{nC}$ \\
\hline & U-Tokyo & $1.3 \times 10^{4}$ & $4 \mathrm{nC}(@ 70 \mu \mathrm{J})$ \\
\hline \multirow{3}{*}{$\mathrm{Cs}_{2} \mathrm{Te}$} & CERN & 0.015 & $2.4 \mathrm{nC}$ \\
\hline & KEK & $<0.01$ & $1.6 \mathrm{nC}(@$ several $\mu \mathrm{J})$ \\
\hline & U-Tokyo & $<0.01$ & $9 \mathrm{nC}(@$ several $\mu \mathrm{J})$ \\
\hline $\mathrm{Na}_{2} \mathrm{KSb}$ & U-Tokyo & $<0.01$ & 4nC (@several $\mu \mathrm{J})$ \\
\hline
\end{tabular}

World record of femtosecond beam generation, $700 \mathrm{fs}$ )

M.Uesaka, et al., Trans. Plasma Science, 28,4(2000),p.1133 (High quality 200 fs beam)

M. Uesaka et al.,Nucl. Instr. Meth. B241 p.880 (2005)

(Synchronization system)

\section{Target tumors for laser driven $80 \mathrm{MeV}$ proton}

\section{Shallow tumors which exist within $5 \mathrm{~cm}$ below the skin}

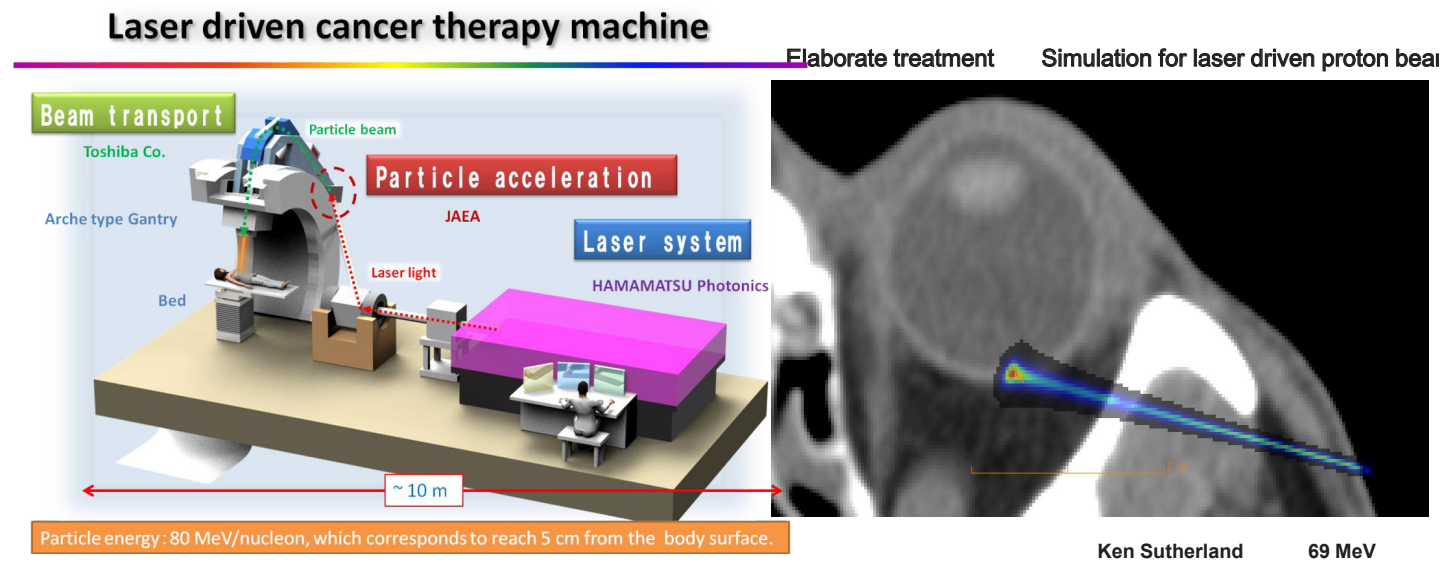

Japan Atomic Energy Agency

By Prof. Murakami (HIBMC) 
Table 1: Parameters of Laser-Acceleration for Medical Applications

\begin{tabular}{|c|c|c|c|}
\hline Parameter & $\begin{array}{c}\text { HIT } \\
\text { (proton/carbon) }\end{array}$ & laser carbon & laser proton \\
\hline Comments: & $\begin{array}{l}\text { synchrotron based } \\
\text { slow extraction }\end{array}$ & & $\begin{array}{c}\text { RPA, SOBP, } \\
\varepsilon \text { chromatic limit }\end{array}$ \\
\hline Energy of protons $(\mathrm{MeV})$ & $50-250$ & 400 & 250 \\
\hline Ions per fraction & $\sim 1 \times 10^{11} / 2.5 \times 10^{9}(5 \mathrm{~min})$ & $\sim 2.5 \times 10^{9}$ & $\sim 1 \times 10^{11}(5 \mathrm{~min})$ \\
\hline Voxels per fraction & $20 \mathrm{k}$ & $2-4 k$ & $\begin{array}{c}2-4 \text { k(assume high puls } \\
\text { reproducibility }<5 \%)\end{array}$ \\
\hline Laser rep rate $(\mathrm{Hz})$ & & 10 & 10 \\
\hline Max. ions per voxel $\left(\times 10^{8}\right)$ & $<0.4$ & 0.01 & $<4$ \\
\hline $\begin{array}{l}\text { Max. ion/s }\left(\times 10^{9}\right) \text { used for } \\
\text { therapy }\end{array}$ & $4 / 0.1$ & 0.1 & 4 \\
\hline Energy steps & $\sim \mathbf{5 0}$ & $5-10$ & $5-10$ \\
\hline Energy width & $<0.005$ & $\begin{array}{c}+/-0.05 \text { (except rear } \\
\text { edge) }\end{array}$ & $+/-0.05$ \\
\hline $\begin{array}{l}\text { Bunch/laser repetition rate } \\
(\mathrm{Hz})\end{array}$ & & 10 & 10 \\
\hline $\begin{array}{l}\text { Emittances }(<\text { window })(\mathrm{mm}- \\
\text { mrad) }\end{array}$ & $2-3$ & 2 & 2 \\
\hline Max ion beam power $(W)$ & 0.16 & $\sim 0.08$ & 0.16 (used) \\
\hline Max production power (W) & $\sim 0.16$ & ( target dependent) & $\begin{array}{c}\sim 4 \quad\left(10^{10} \mathrm{p} / \mathrm{shot}\right) \\
\text { ( target dependent) }\end{array}$ \\
\hline Laser W/cm ${ }^{2}$ & & $1-3 \times 10^{22}$ & $1-3 \times 10^{21}$ \\
\hline Pulse duration (fs) & & $50-150$ & $50-150$ \\
\hline contrast (at 5ps- 500ps) & & $10^{-9}\left(10^{-13}\right)$ & $10^{-8}\left(10^{-12}\right)$ \\
\hline pulse rise time(fs) & & $<20$ & $<20$ \\
\hline Spot radius $\mu \mathrm{m}$ & & $\begin{array}{l}5 \text { (pulse shaping, flat } \\
\text { top to prevent the } \\
\text { instabilities) }\end{array}$ & 5 \\
\hline Laser power & & $10-30 P W$ & 1-3 PW \\
\hline Laser pulse energy & & $1500 \mathrm{~J}$ & $150 \mathrm{~J}$ \\
\hline Laser average power & & $15 \mathrm{~kW}$ & $1.5 \mathrm{~kW}$ \\
\hline
\end{tabular}

\section{Proposal for US/Japan Collaboration on Laser Plasma Acceleration by developing more stable/reliable/high- average-powered Lasers}

\section{DPSS (Diode-pumped Solid-state) LASER}

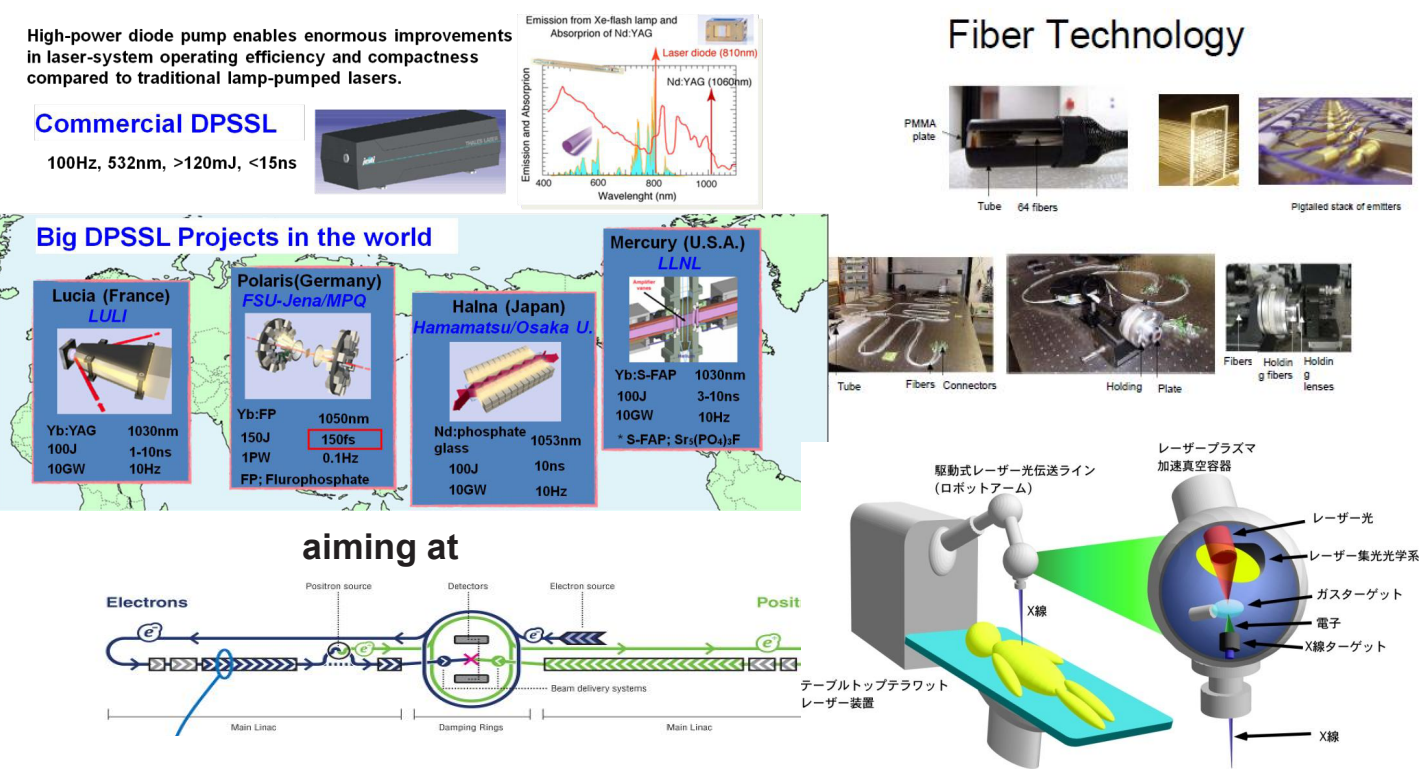




\title{
Proposal for US-Japan Collaboration for Precision Muon Physics g-2 and EDM Measurements at J-PARC and Fermilab
}

\author{
Naohito Saito \\ High Energy Accelerator Research Organization (KEK), Tsukuba, Japan
}

We propose to start the collaborative activities between the US and Japan on the muon g-2/EDM experiments. Activities would include both experiments at J-PARC and at Fermilab. Fermilab experiment continues the precision measurement of the muon $g-2$ at magic momentum with the storage ring from the previous experiment at BNL, E821. J-PARC experiment is to employ an ultracold muon beam to measure g-2 and EDM of the muon. There are many areas in both experiments to be benefited from the mutual collaboration. Those areas include:

- beam injections

- ultra-precision field measurements

- improving Muonium hyper-fine splittings to determine $\mu_{\mu} / \mu_{p}$, and

- better understanding of vacuum polarization

The latest and the best determination of the muon g- 2 was obtained by BNL E821 at the level of $0.54 \mathrm{ppm}$ precision. Their result exhibits a significant deviation from the standard model prediction as large as 3.4 standard deviation. Obviously it requires further exploration, preferably with a new method.

Fermilab experiment is aiming for an improvement in both statistics and systematic uncertainties by a factor 5. J-PARC experiment is also aiming the similar precision, however with a completely new technique. Since they have quite different systematics, two experiments are complimentary.

The new experiment at J-PARC utilizes the ultra-cold muon (UCM) beam, whose transverse dispersion of the muon beam relative to the longitudinal momentum, $\sigma\left(p_{\mathrm{T}}\right) / p_{\mathrm{L}}<10^{-5}$. Such a beam can be stored in a static magnetic field long enough to measure its precession frequency without focusing field. With the primary proton beam of $3 \mathrm{GeV}$ at the Materials and Life Science Facility (MLF) and the carbon graphite target, a large number of secondary particles are produced; among them the positive pion stops at the surface of the target material provides monochromatic positive muon $(\mu+)$ through its two body decay. Such a low-energy monochromatic $\mu+$ beam is transported to the experimental hall to produce a muonium $(\mathrm{Mu})$, a bound state of a $\mu+$ and an electron. $\mathrm{Mu}$ can be produced at the room temperature, which corresponds to a kinetic energy of $25 \mathrm{meV}$. This thermal process is essential to cool down the muon beam. Those Mu' s evaporated into a vacuum are ionized by resonant laser ionization. The resulting $\mu+$ is moving in any directions with the kinetic energy of $25 \mathrm{meV}$, which is $2.3 \mathrm{keV} / \mathrm{c}$ in momentum. Those muons are accelerated to $300 \mathrm{MeV} / \mathrm{c}$ with muon LINAC without any additional transverse momentum, therefore the UCM beam is realized. The UCM beam is injected into the ultra-precision field of $3 \mathrm{~T}$ to measure the precession frequency vector. It will be measured by detecting the decay positrons by a silicon tracker inside the solenoid magnet. Since the rotation due to g-2 and EDM are orthogonal each other, we can separate them by detecting the positron trajectories. We intend to start the experiment in five years. It is essential to continue the US-Japan collaboration to realize this kind of high precision and technically challenging experiment. 


\section{Particle Dipole Moments}

- Magnetic and Electric Dipole Moments are related to Spin of the Particle: axial vector

$$
\begin{array}{r}
\vec{\mu}=g\left(\frac{e}{2 m}\right) \vec{s} \quad \vec{d}=\eta\left(\frac{e}{2 m c}\right) \vec{s} \\
a=\frac{g-2}{2} \\
H=-\vec{\mu} \cdot \vec{B}-\vec{d} \cdot \vec{E}
\end{array}
$$

MDM (Magnetic Dipole Moment) EDM (Electric Dipole Moment) Contains contributions from ALL PHYSICS!

- EW, QCD, and New Physics

$\Rightarrow$ precision test of the SM

$\Rightarrow$ the most precise

determination of $\alpha_{E M}$ from

electron $\mathrm{g}-2(0.37 \mathrm{ppb})$

\section{SM Contribution to $a \neq 0$}

Any particle which couples to muon/photon would contribute : QED $\gg$ > Hadron > Weak

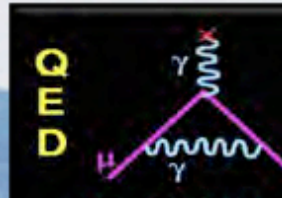

$\sim 1.2 \times 10^{-3}(\sigma \sim 1 \mathrm{ppb})$

$11658470.57(.29) \times 10^{-10}$
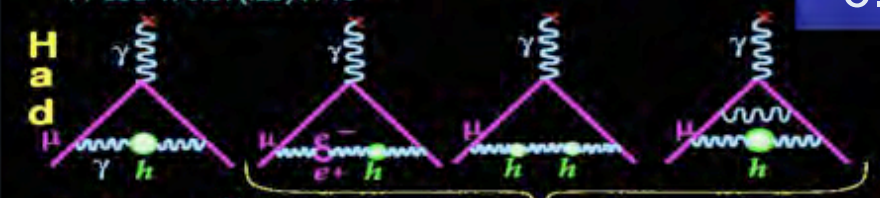

$\sim 6.9 \times 10^{-8}(\sigma \sim 0.41 \mathrm{ppm})$

$692.4(6.2) \times 10^{-10}$
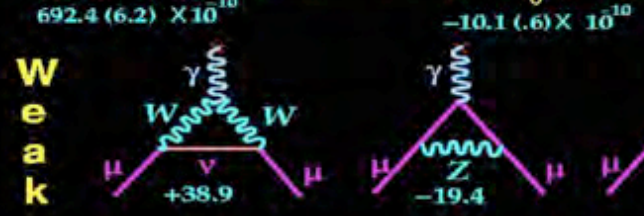

+ higher order terms

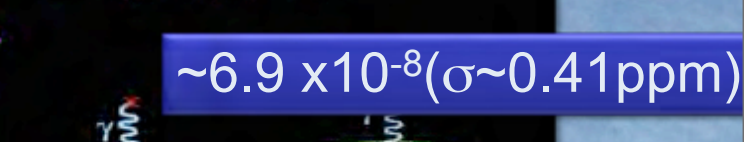

1 st +2 nd Order Weak $=$

15.1 (.4) $\times 10^{-10}$
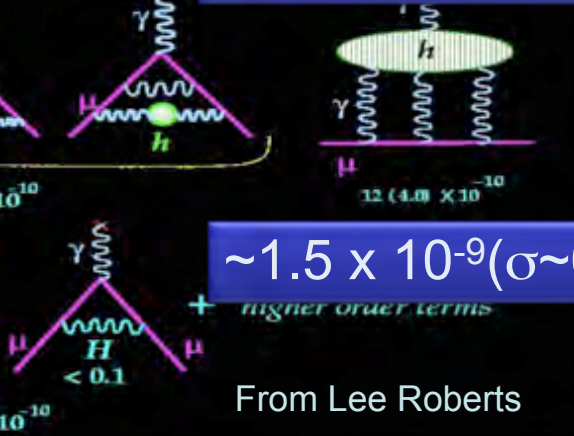

$\sim 1.5 \times 10^{-9}(\sigma \sim 0.02 \mathrm{ppm}$

From Lee Roberts 


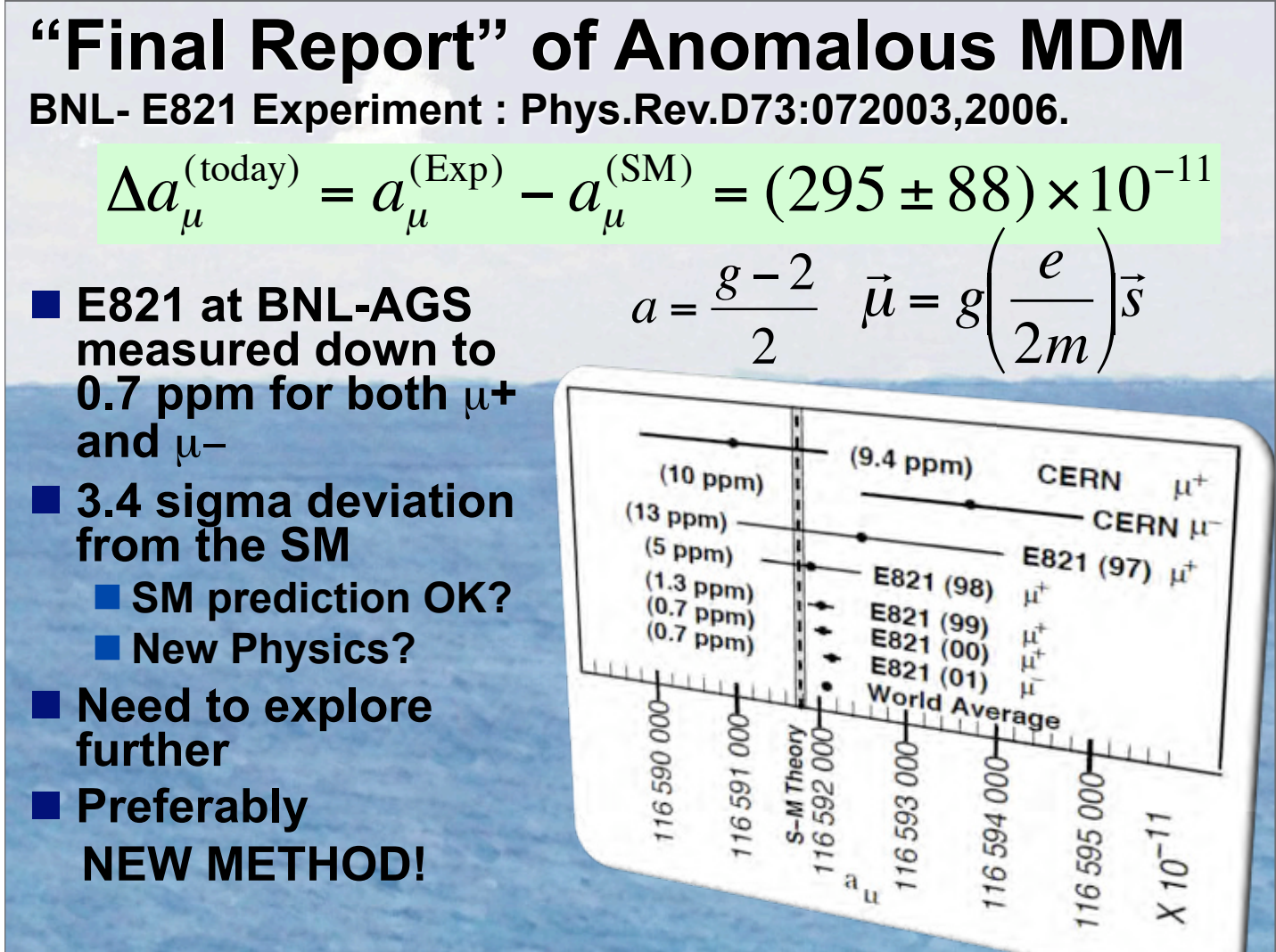

2010年11月28日日曜日

\section{Muon g-2 in the LHC era}

Even the first SUSY discovery was made at LHC, the muon g-2 measurement remains unique to determine SUSY parameters:

$\mu$ and $\tan \beta$

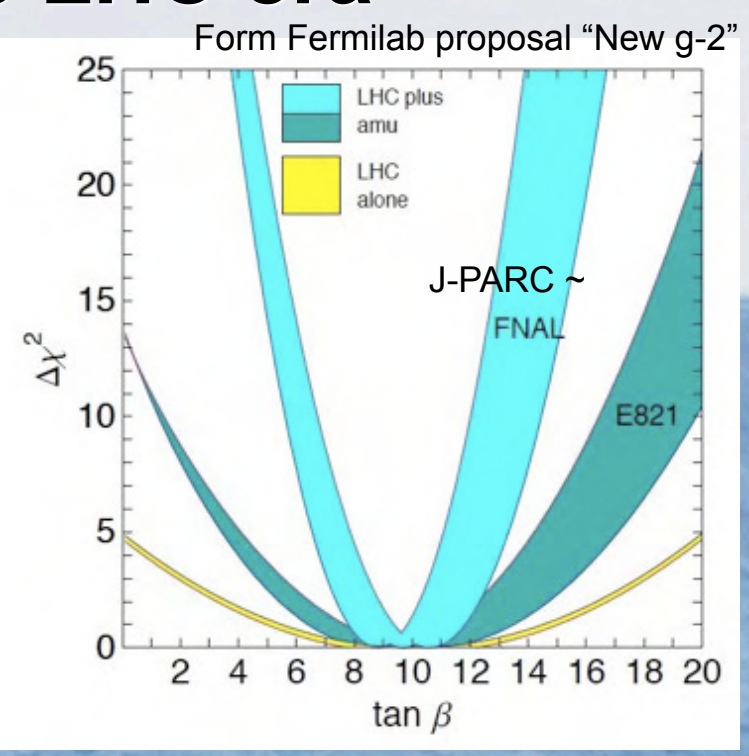

$$
a_{\mu}(\mathrm{SUSY}) \approx(\operatorname{sgn} \mu) 13 \times 10^{-10} \tan \beta\left(\frac{100 \mathrm{GeV}}{\tilde{m}}\right)^{2}
$$




\section{g-2, EDM and cLFV}

\section{- Large g-2 $\rightarrow$ Large cLFV $\rightarrow$ Large EDM}

G. Isidori, F. Mescia, P. Paradisi, and

D. Temes, PRD 75 (2007) 115019

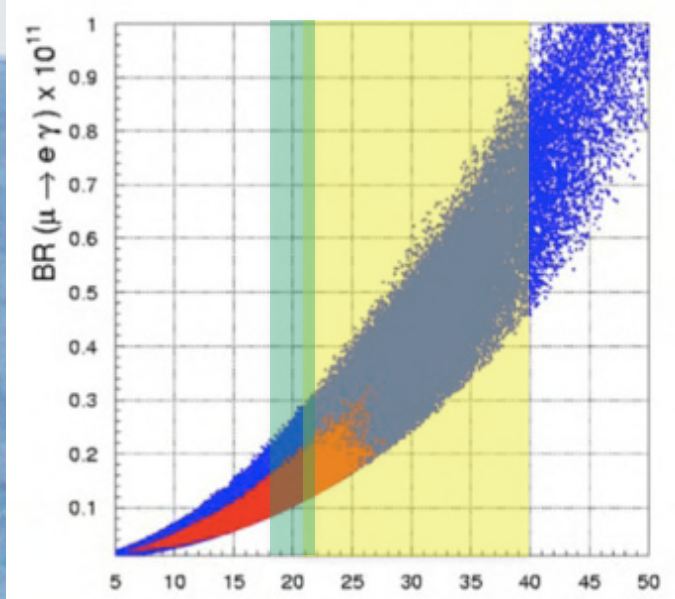

Current limit by MEGA $1.2 \times 10^{-11} \Delta \mathrm{a}_{\mu} \times 10^{10}$

To be superseded by MEG soon
J. Hisano, Nagai, Paradisi

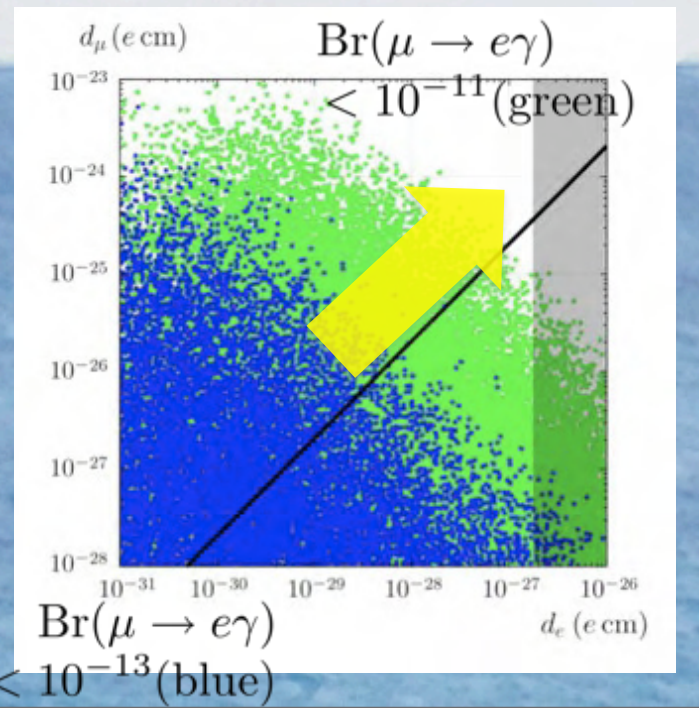

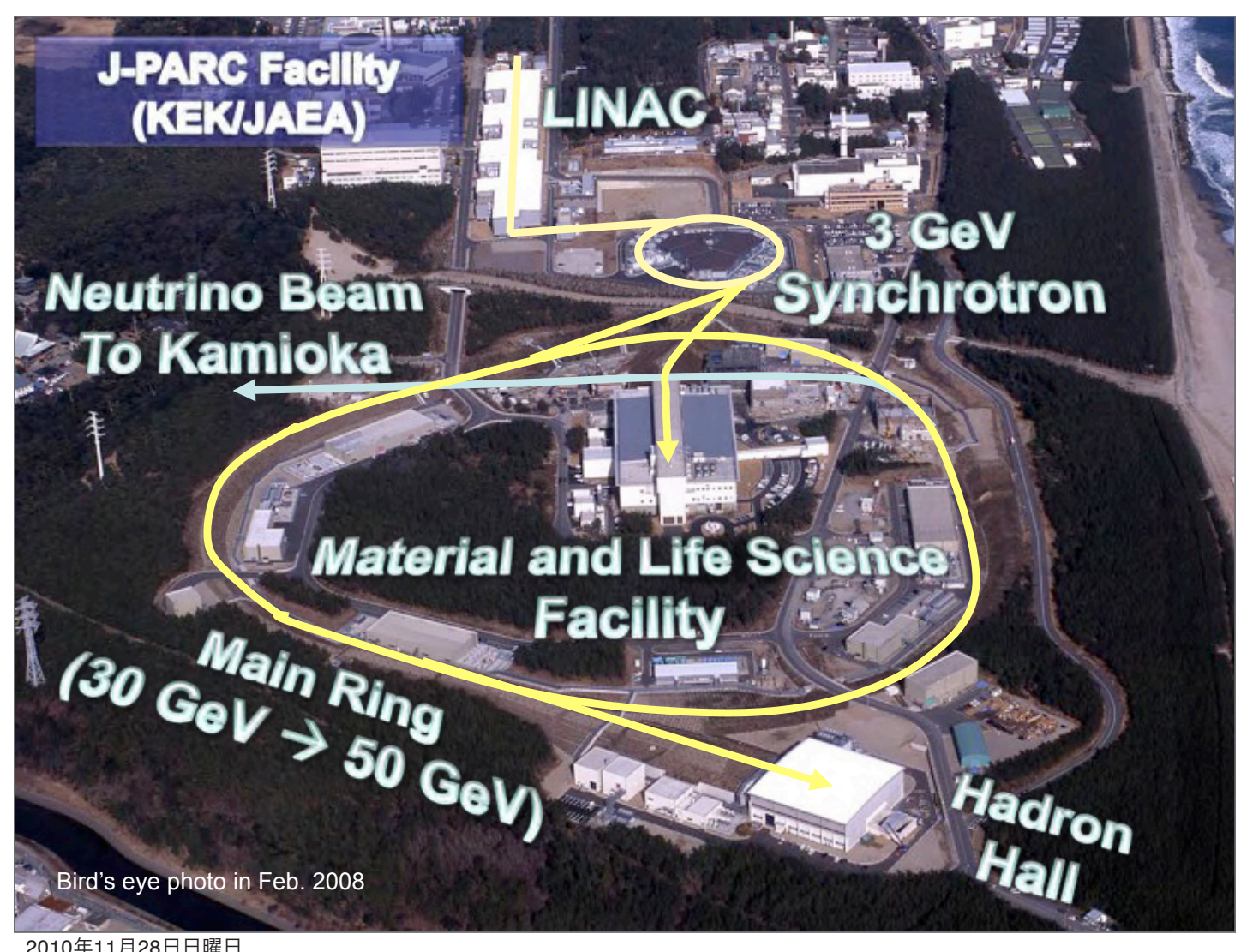

2010年11月28日日曜日 


\section{Off Magic Momentum?}

- Tertiary Muon Beam

Widely spread over phase space

- Contamination of pion

\section{Electric Field for Focusing $\Rightarrow$ Magic Momentum}

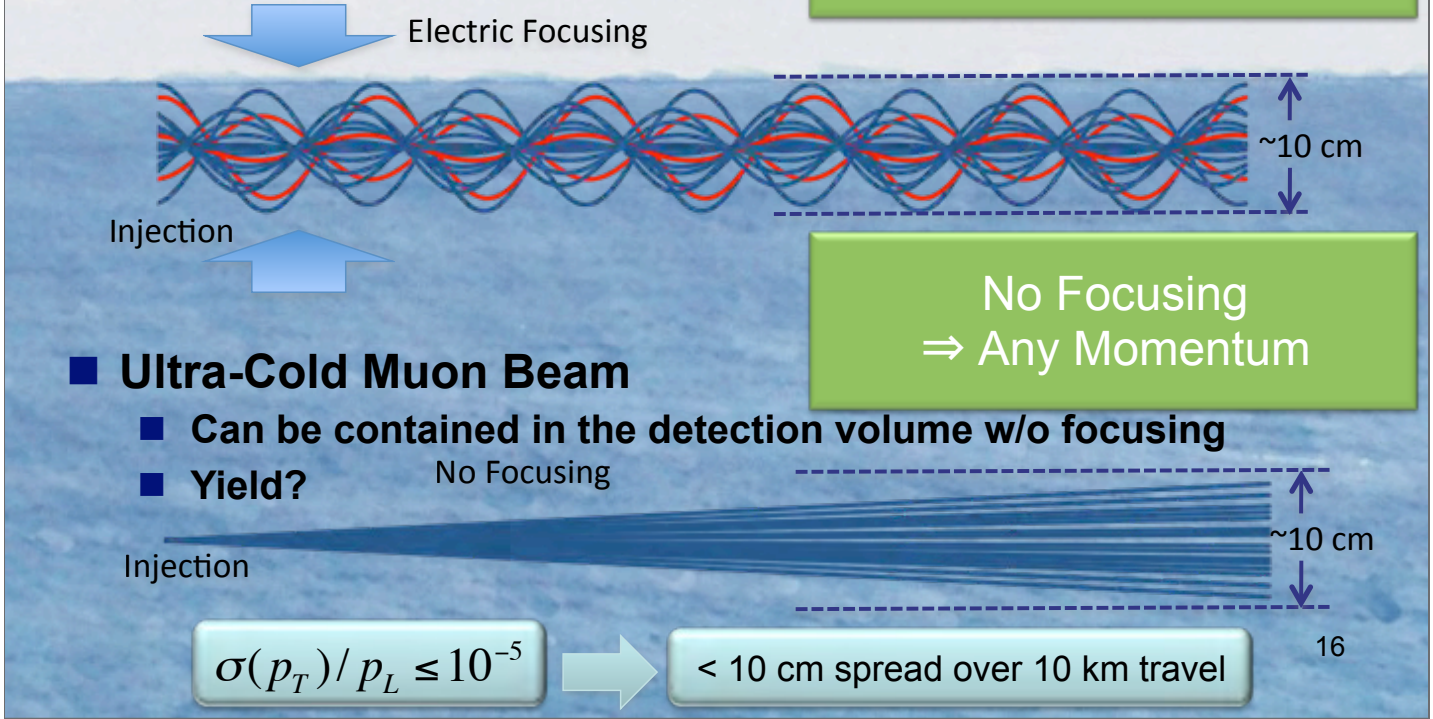

2010年11月28日日曜日

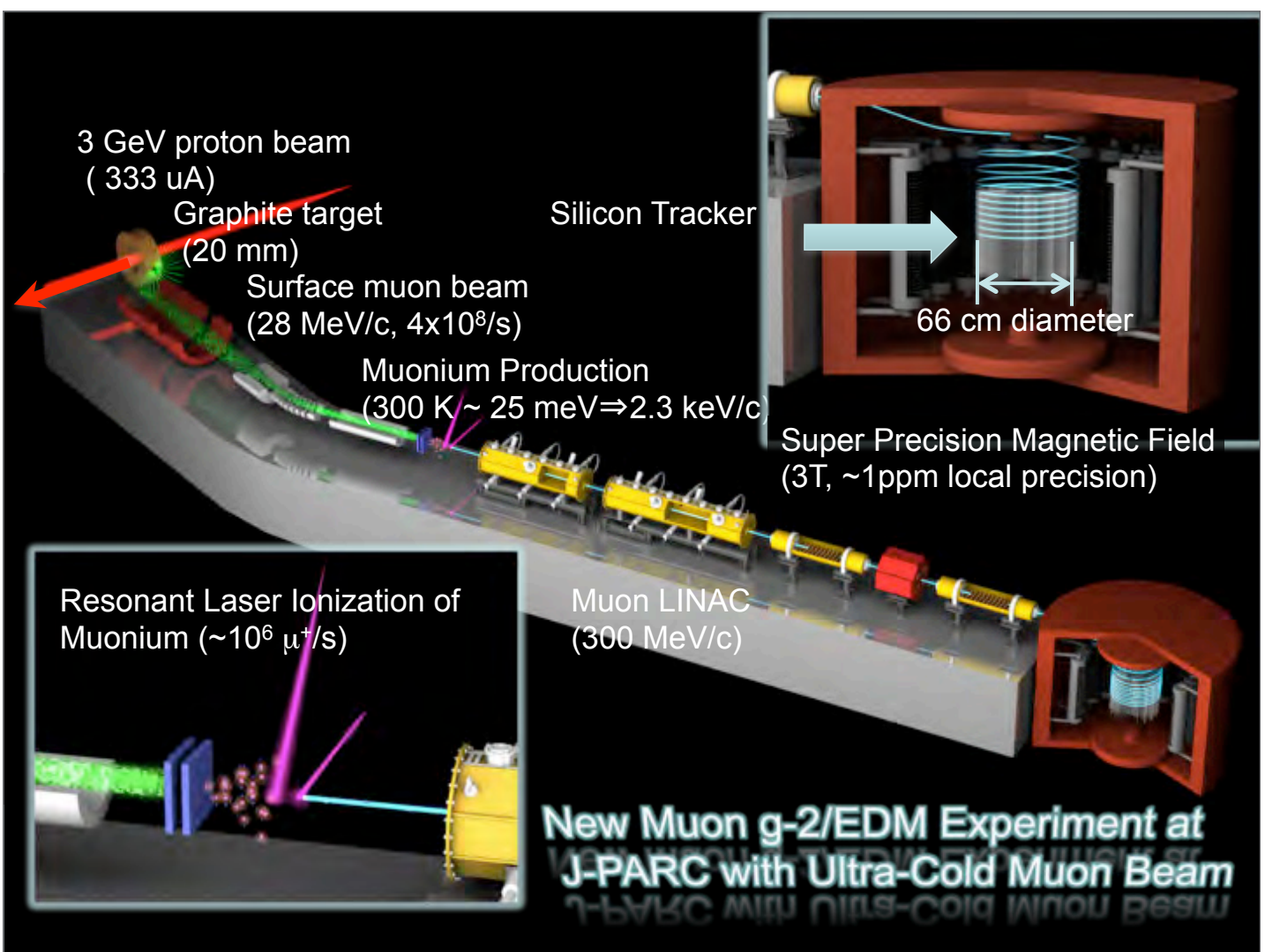

2010年11月28日日曜日 


\section{Magic vs "New Magic"}

\section{Complimentary!}

$$
\vec{\omega}=-\frac{e}{m}\left[a_{\mu} \vec{B}-\left(a_{\mu}-\frac{1}{\gamma^{2}-1}\right) \frac{\vec{\beta} \times \vec{E}}{c}+\frac{\eta}{2}\left(\vec{\beta} \times \vec{B}+\frac{\vec{E}}{c}\right)\right]
$$

BNL/Fermilab Approach

$$
a_{\mu}-\frac{1}{\gamma^{2}-1}=0 \quad \eta \approx 0
$$
J-PARC Approach

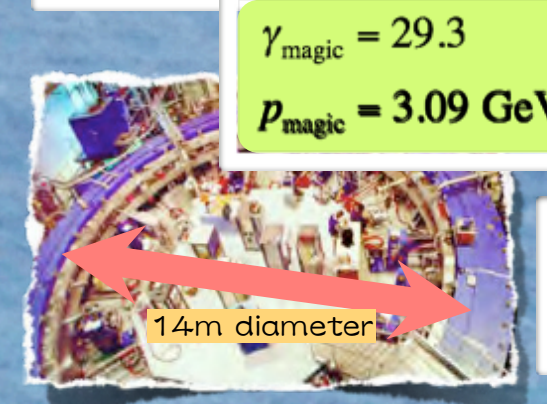

$$
\vec{\omega}_{a}=-\frac{e}{m} a_{\mu} \vec{B}
$$

2010年11月28日日曜日

\section{Magic vs "New Magic"}

\section{Complimentary!}

$$
\vec{\omega}=-\frac{e}{m}\left[a_{\mu} \vec{B} . \quad+\frac{\eta}{2}(\vec{\beta} \times \vec{B})\right]
$$

BNL/Fermilab Approach

$$
a_{\mu}-\frac{1}{\gamma^{2}-1}=0
$$

$$
\eta \approx 0
$$

$$
\gamma_{\text {magic }}=29.3
$$

$$
\text { 27y } p_{\text {magic }}=3.09 \mathrm{GeV} / \mathrm{c}
$$

$$
\vec{\omega}_{a}=-\frac{e}{m} a_{\mu} \vec{B}
$$




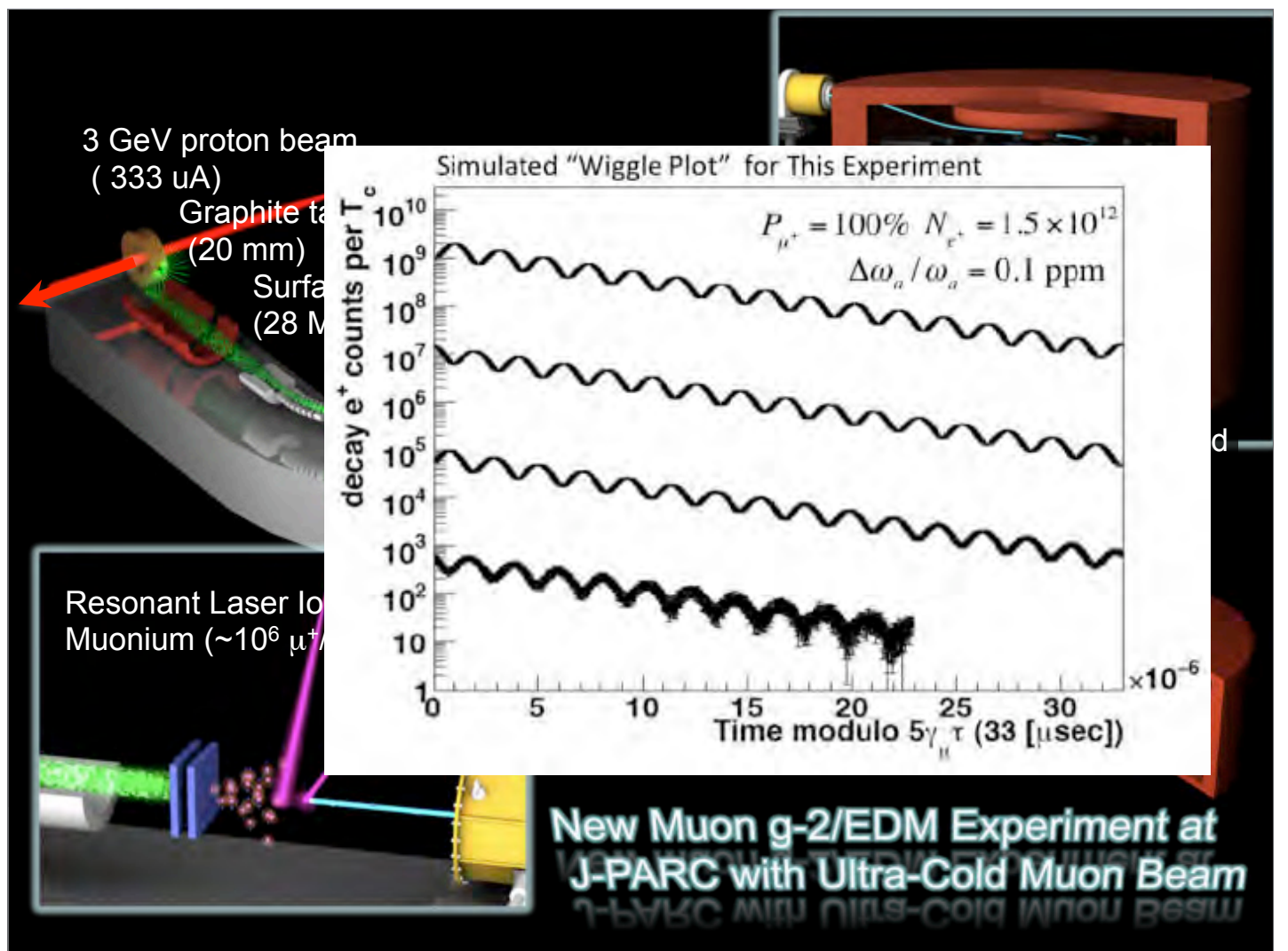

2010年11月28日日曜日

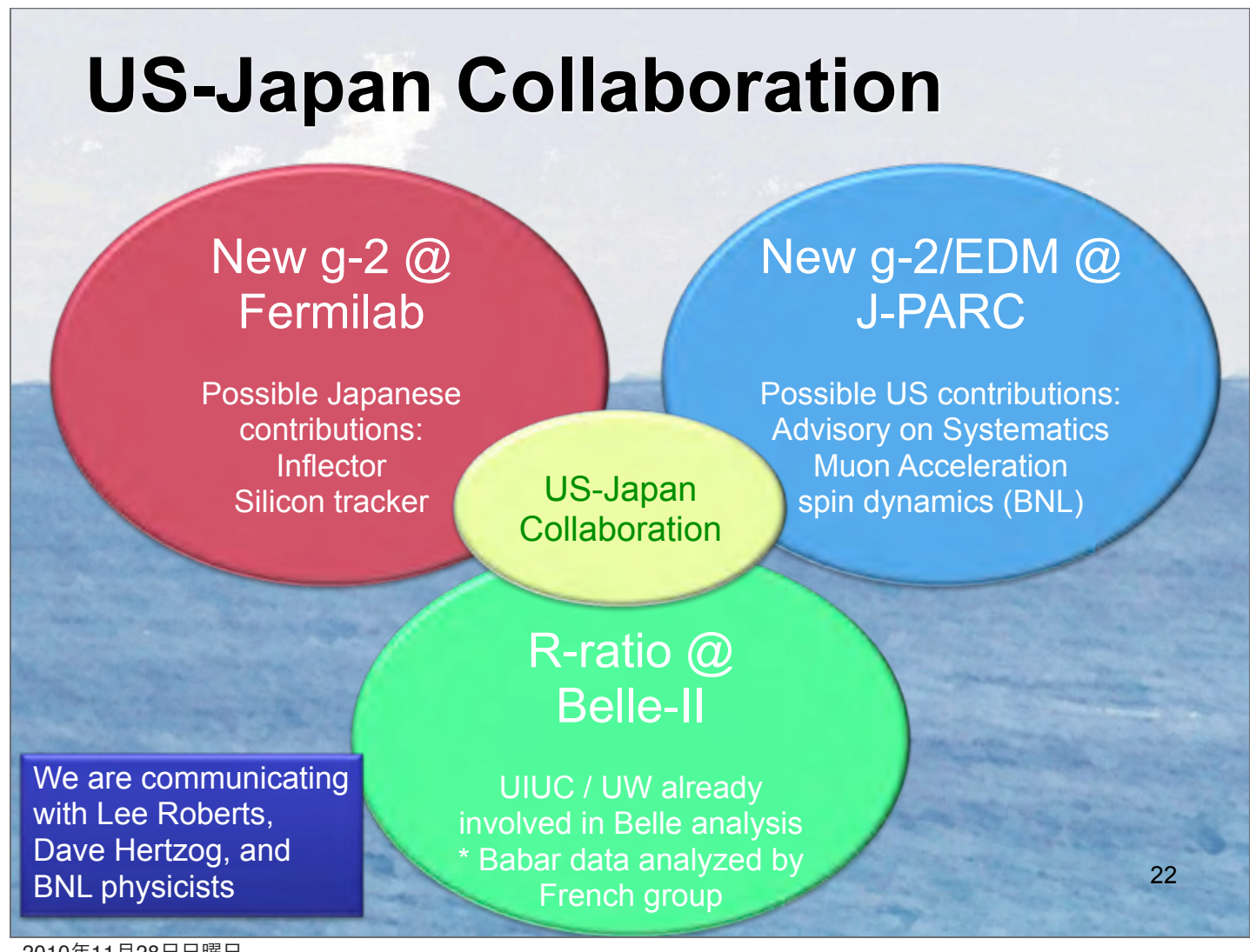

2010年11月28日日曜日 



\title{
Muon Collider: R\&D Status
}

\author{
Robert Palmer \\ Brookhaven National Laboratory, Upton, NY
}

A high-energy muon collider can be circular and compact [1], whereas an electron collider must be linear and long. Muon acceleration can be attained in multiple turns, using much less rf. Interactions occur in multiple turns in a ring, allowing larger bunches and easier tolerances. It appears likely that a muon collider would use less power and cost less than an electron collider of the same energy and luminosity. All components of a complete scheme [2] have been simulated at some level. Muons are made from pions generated in a mercury jet target intersected by a 4-MW proton beam. The muons are 'phase rotated' into interleaved trains of bunches, and then charge- separated into distinct trains of each sign. These trains are ionization-cooled [3] in all 6 dimensions until they are small enough to be merged into single bunches of each sign. Then, they are cooled further, first in $6 \mathrm{D}$, then only in the transverse coordinates in very high field (approximately $40 \mathrm{~T}$ ) solenoids. We then accelerate these muons in a sequence of linacs, RLAs, and pulsed-magnet synchrotrons, and finally inject them, in opposite directions, into a single collider ring. Most work was done on a $1.5 \mathrm{TeV}$ ( $\mathrm{c} \mathrm{of} \mathrm{m}$ ) collider with a luminosity of 1 $10^{34} \mathrm{~cm}^{-2} \mathrm{sec}^{-1}$. A design for $3 \mathrm{TeV}$ with a luminosity of $410^{34}$ is being studied.

An R\&D program demonstrated (MERIT [4] at CERN) the concept of a mercury jet target. An experiment (MICE [5] at RAL) will demonstrate ionization cooling. The MuCOOL collaboration [6] is developing the rf components, magnets, and hydrogen absorbers needed for 6D ionization cooling. Several ideas are being explored [7] to overcome observed rf breakdowns in the required magnetic fields. A BNL/PBL SBIR [8] collaboration is building the approximately $40 \mathrm{~T}$ super-conducting solenoid needed for final 4D cooling.

So far, the DOE-funded Neutrino Factory and Muon Collider Collaboration (NFMCC), and the Fermi Lab-supported Muon Collider Task Force (MCTF) have funded this work. Now, a single Muon Accelerator Program (MAP) [9] is being formulated. . By 2013, it should generate a Muon Collider Feasibility Study including the approximate costs; by 2016, we anticipate having an RDR, and a CDR by 2019. 


\section{Why a Muon Collider?}

- Point like interactions as in linear $e^{+} e^{-}$

- Negligible synchrotron radiation:

Acceleration in rings Small footprint Less rf Hopefully cheaper

- Collider is a Ring

$\approx 1000$ crossings per bunch Larger spot Easier tolerances 2 Detectors

- Negligible Beamstrahlung Narrow energy spread

- 40,000 greater S channel Higgs Enabling study of widths

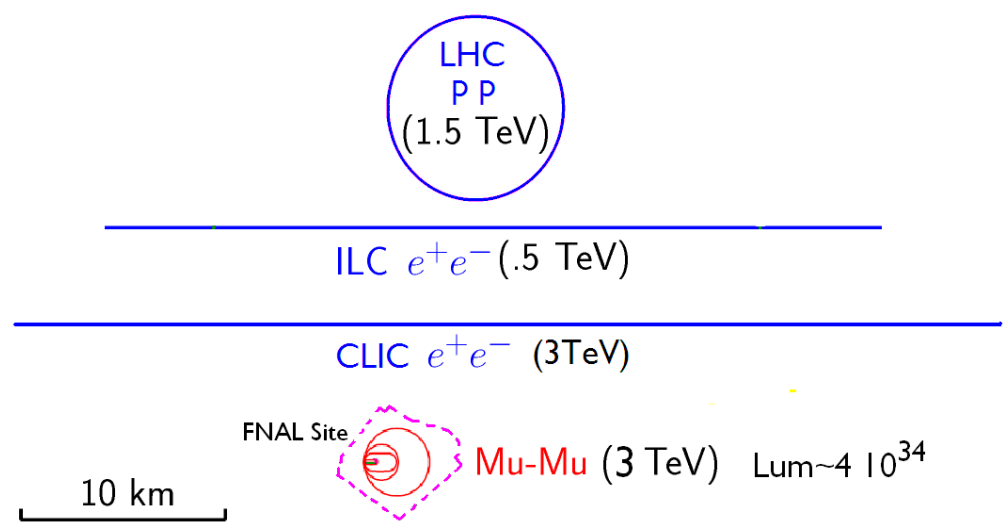

Scheme

a)<smiles>C1=COC1</smiles>

品<smiles>O[Hg]</smiles><smiles>O[Hg]</smiles>

$\mu_{\mathrm{O} 日 \mathrm{O}}^{+} \mu^{-}$

日早

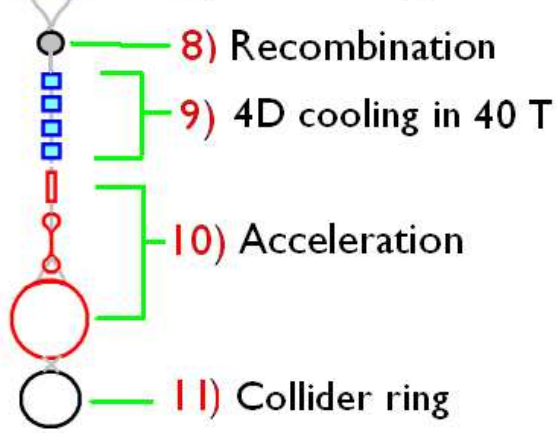

\& Emittances vs. Stage

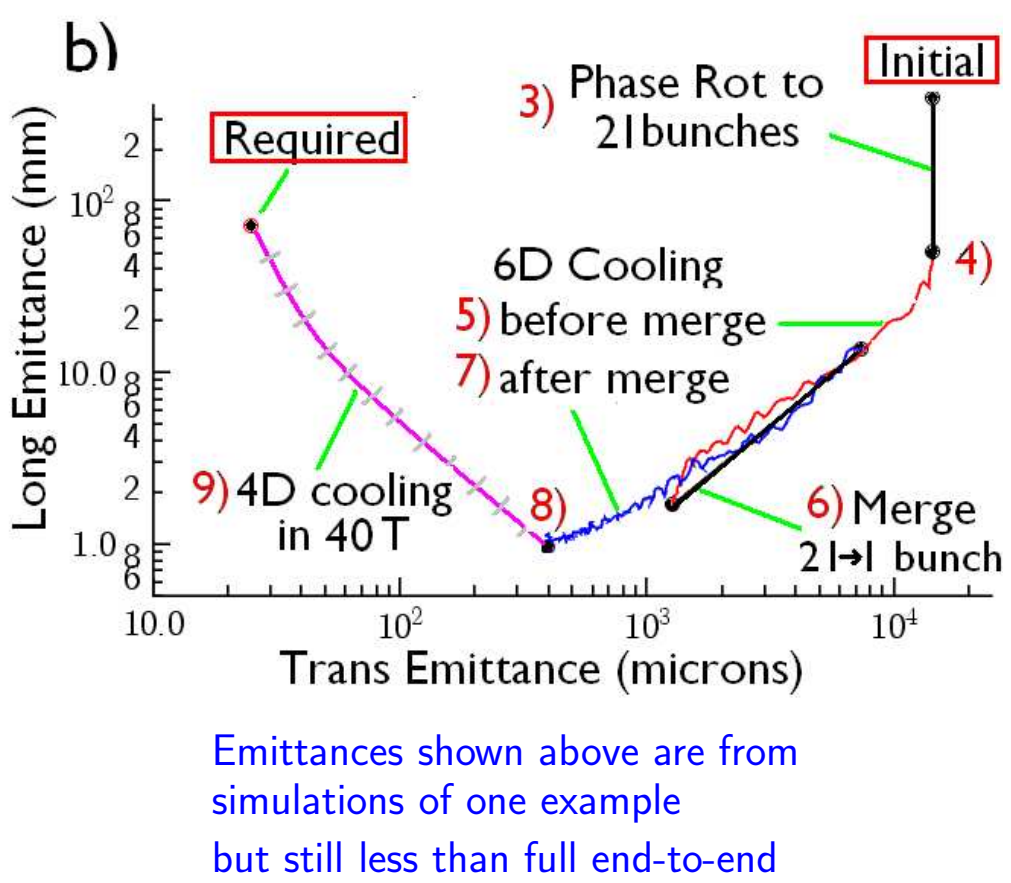




\section{Ionization Cooling}
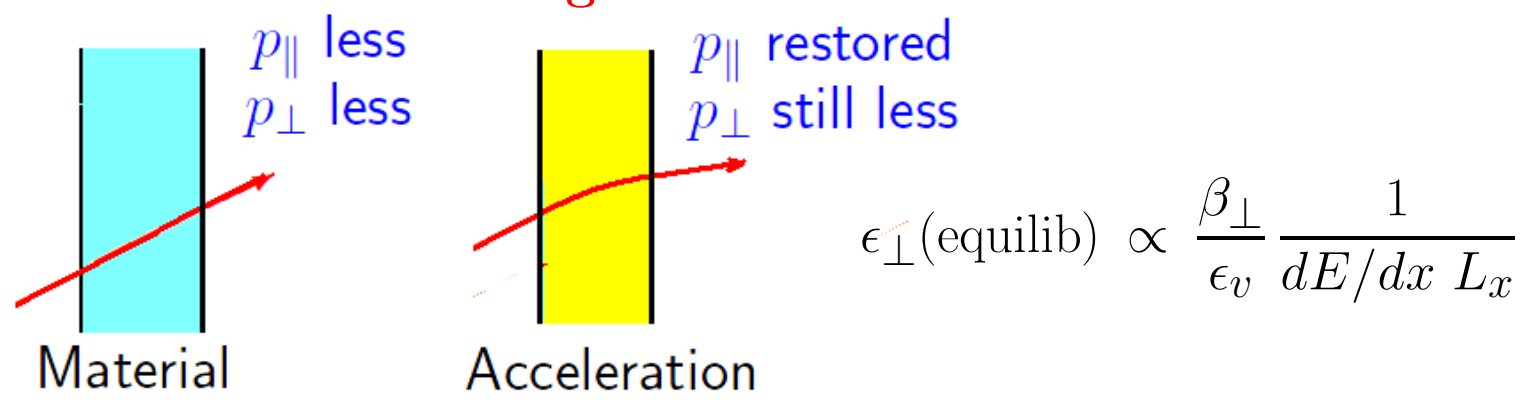

- Best Material is Hydrogen

- For 6D cooling we require Emittance Exchange

- Best energy to avoid blow up of $\mathrm{dp} / \mathrm{p} \approx 130 \mathrm{MeV}$

- But for final cooling, best energy $\approx 6 \mathrm{MeV}$

Dispersion and wedge absorber for 'Guggenheim'

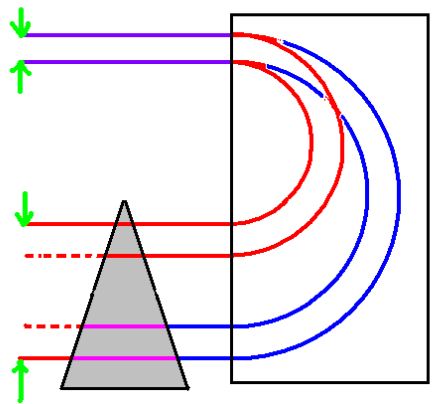

Path differences in gas absorber for HCC

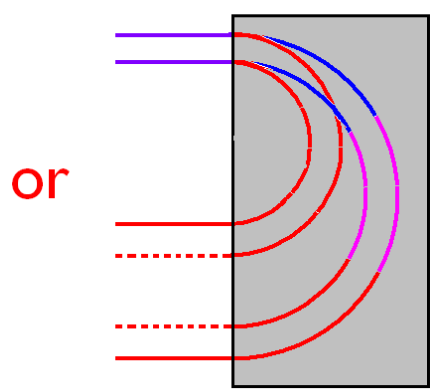

\section{R\&D}

\section{1) MERIT Experiment}

(Harold Kirk, Kirk McDonald)

- MERIT demonstrated liquid mercury target for multi-megawatt beams

- Splash velocities moderate and reduced by magnetic field

- Remaining need to improve jet quality
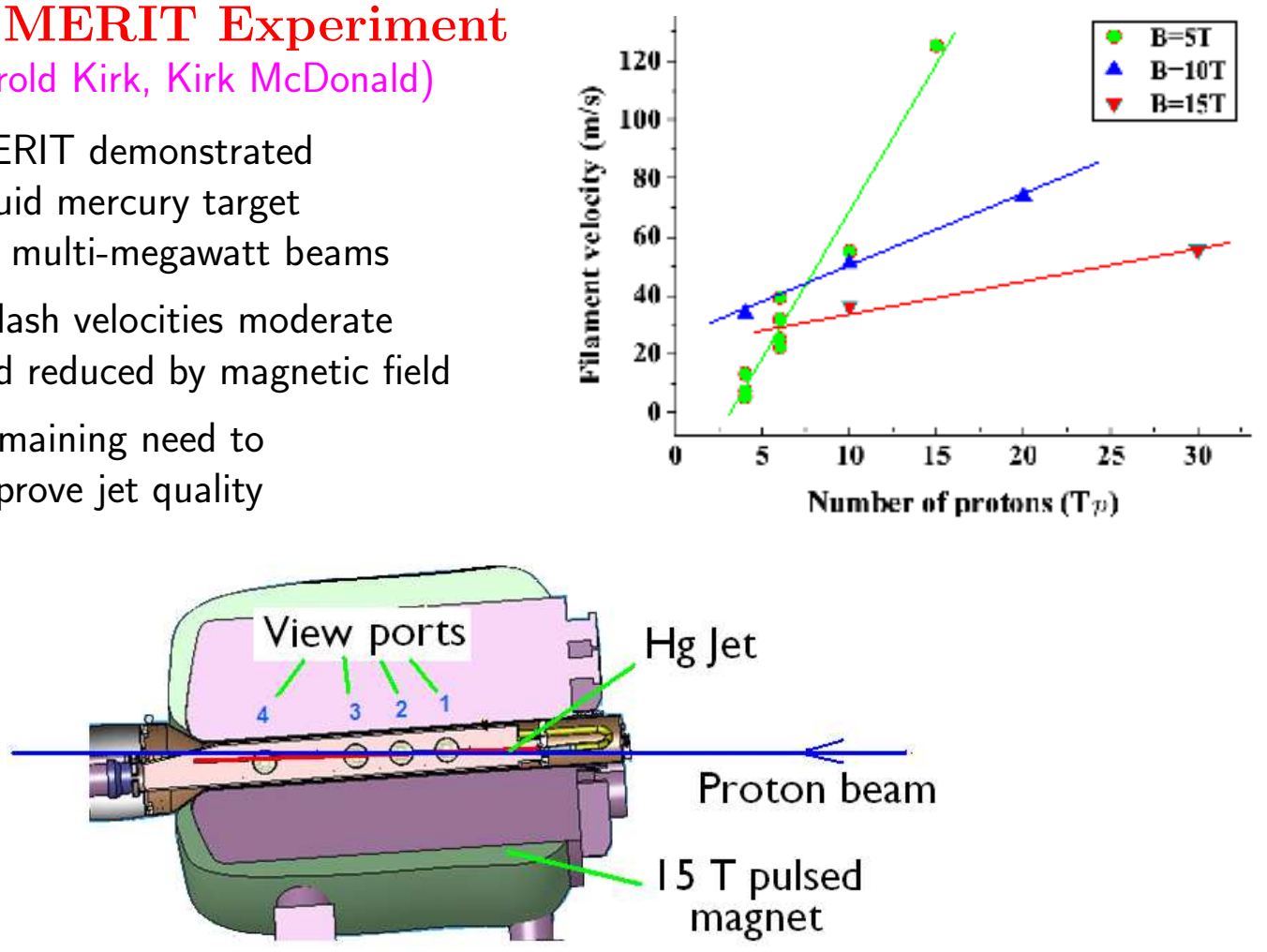
2) Muon Ionization Cooling Experiment (MICE) International collaboration at RAL, US, UK, Japan (Blondel)

- Will demonstrate transverse cooling in liquid hydrogen, including $r$ re-acceleration

- Uses a different version of 'Guggenheim' lattice

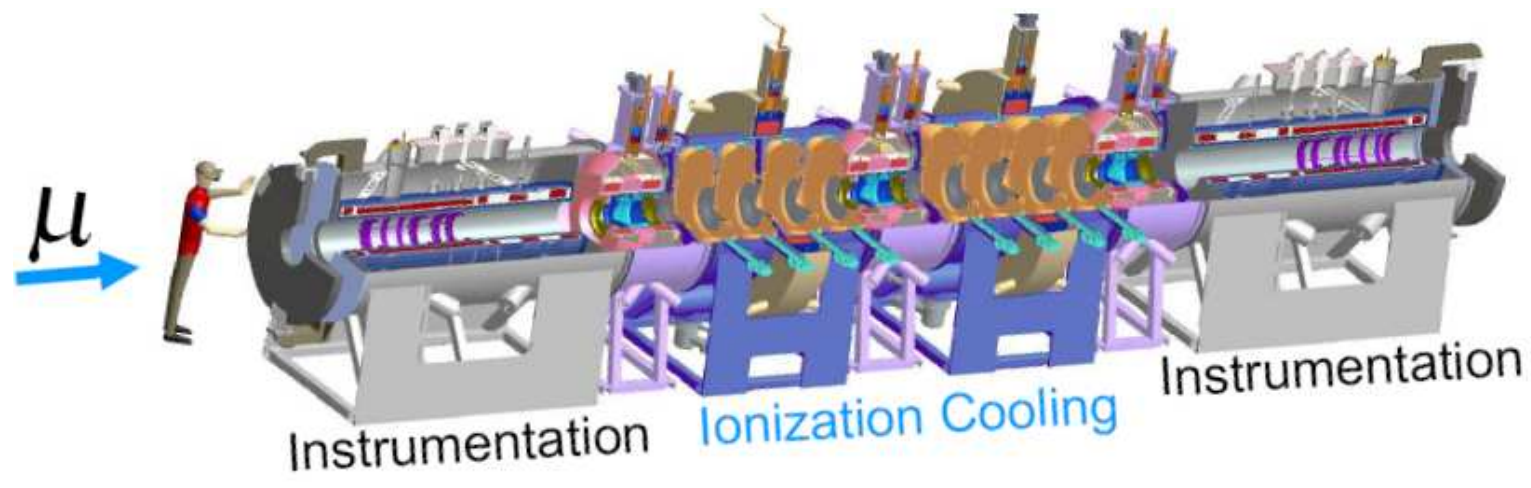

- Early Experiment to demonstrate Emittance Exchange

- Dispersion by weighting

- Cooling in all dimensions

- But no re-acceleration

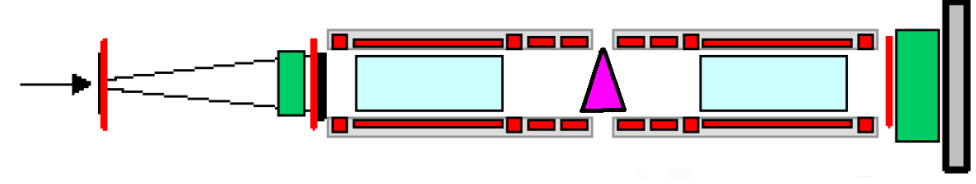

3) MuCool, and MuCool Test Area (MTA) at FNAL International collaboration US, UK, Japan (Bross)

- Liquid hydrogen absorber tested Supported by US-Japan Funds

- Open \& pillbox $805 \mathrm{MHz}$ cavities in magnetic fields to $4 \mathrm{~T}$

- $201 \mathrm{MHz}$ cavity tested in stray magnetic field of $0.7 \mathrm{~T}$ Later, with coupling coil, to $2 \mathrm{~T}$

- High pressure H2 gas $805 \mathrm{MHz}$ pillbox cavity tested

- Soon: $805 \mathrm{MHz}$ gas Cavity with proton beam

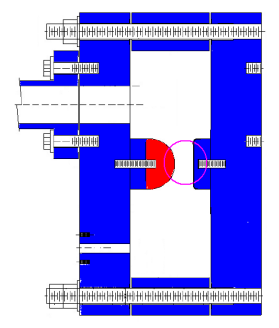

HP Gas cavity

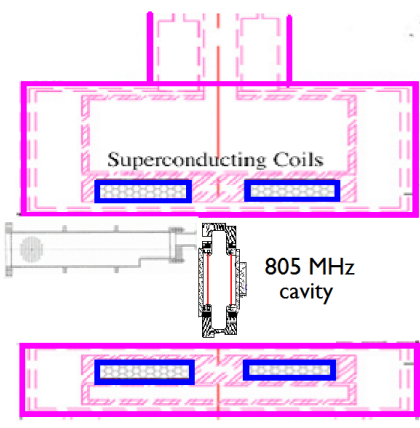

$805 \mathrm{MHz}$ in $4 \mathrm{~T}$ magnet

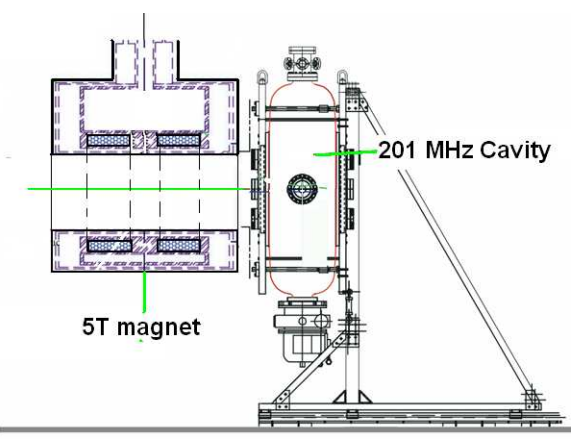

$201 \mathrm{MHz}$ next to magnet 

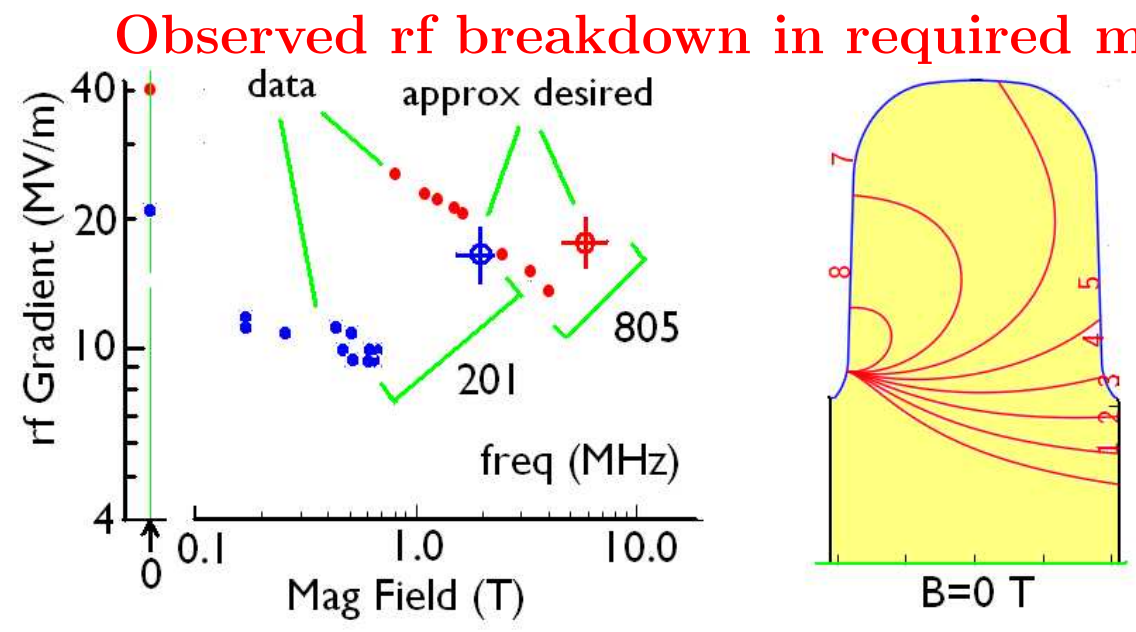

- Theory:

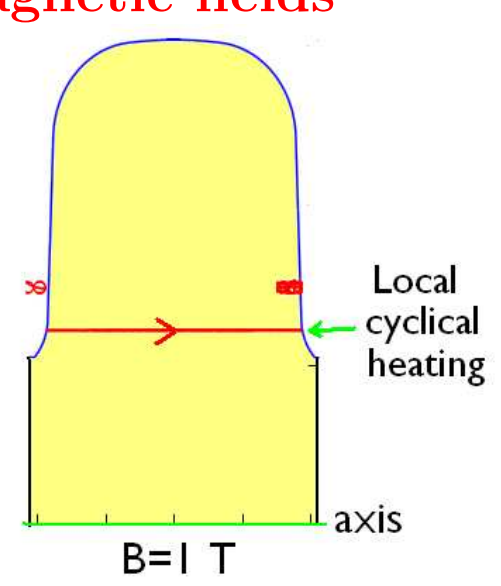

- Electrons from field emission accelerated to $\approx 1 \mathrm{MeV}$

- Focused by field $\rightarrow$ fatigue damage from cyclical heating of $\Delta T \approx 50 \mathrm{deg}$.

- Solutions ?

- Magnetically insulation, by crossing $E$ \& $B$, reduces effect

- High pressure gas shows no B effect. Possible rf losses with beam tested soon

- Beryllium surfaces should suffer smaller $\Delta \mathrm{T}$. To be tested soon

4) HTS R\&D towards a $40 \mathrm{~T}$ solenoid

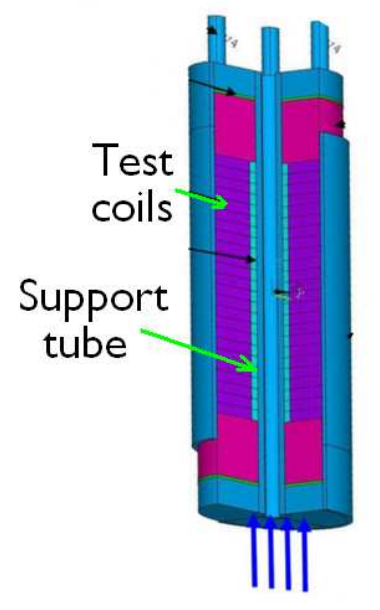

- FNAL program

- Testing multiple small coils in existing $12 \mathrm{~T}$ facility

- Fields up to $25 \mathrm{~T}$

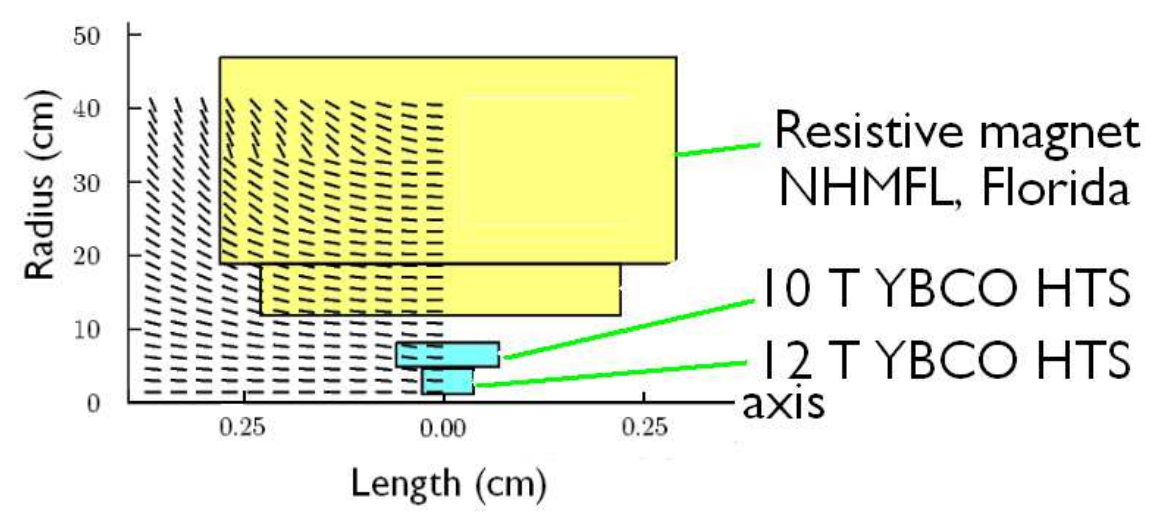

- BNL/PBL Program (SBIR)

- Nested YBCO HTS coils under construction

- $12+10 \mathrm{~T}=22 \mathrm{~T}$ stand alone

- $40 \mathrm{~T}$ in $19 \mathrm{~T}$ NHMFL magnet

- Design for $19 \mathrm{~T} \mathrm{NbTi}+\mathrm{Nb}_{3} \mathrm{Sn}$ design is straightforward 
Muon Accelerator Program (MAP) submitted to DoE Administered by FNAL, but National Program, with International Collaboration (Interim Directors: Steve Geer, Mike Zisman)

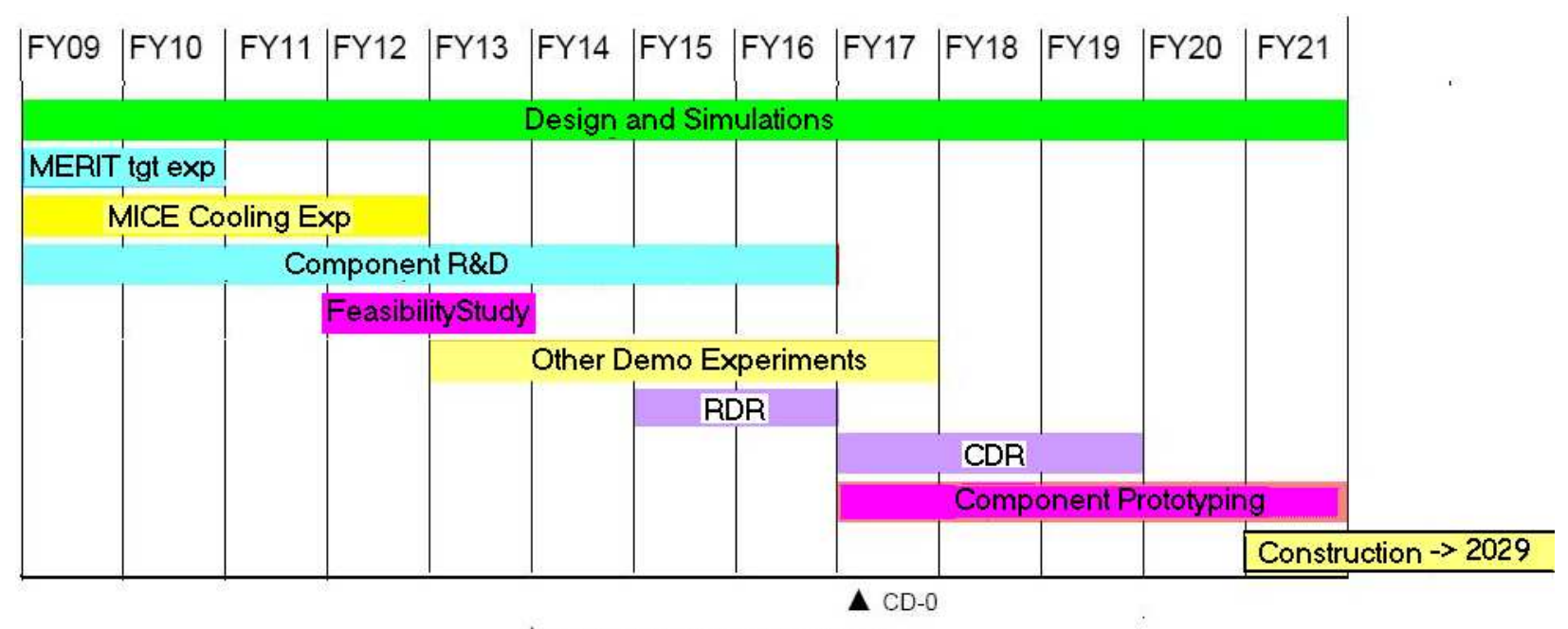

$\mathbf{\Delta}$ Choice of staged or direct path

\begin{tabular}{l|l|l|l|l|l|l|l|l|l|l}
\hline 8 & II & 13 & 20 & 25 & 25 & 25 & 35 & 40 & 40 & R\&D Funds M\$/year \\
\hline
\end{tabular}

Delayed 1 year from P5 presentation 


\title{
"Value of the US/Japan Collaboration in the Past and Future"
}

\author{
Summary of Round Table Discussions
}

As part of the Closing Session we held Round Table Discussions on the "Value of the US/Japan Collaboration in the Past and Future". The Round Table Discussions were led by Pier Oddone and Atsuto Suzuki, including Yorikiyo Nagashima, Tsuyoshi Nakaya, David MacFarlane and Barbara Jacak as panel members. In addition, many other Symposium attendees also participated.

Pier Oddone began by suggesting that we organize the Round Discussions based on topics that we think might be important, especially when we look at the future, how we move forward, and how we can make the next 30 years better. There were active discussions on subjects that related to the past and future collaboration, including lessons learned. Although the round table discussions did not reach definite conclusions on specific topics raised, these opinions and comments would be very valuable input to the US/Japan Task Force that is deliberating the future of this successful program.

In summary, the agreement was unanimous that the Collaboration had been an outstanding success up to now, and an excellent example of scientific cooperation between international partners. Some possible future modifications were suggested, but overwhelmingly the opinion was that the Collaboration was extremely valuable to both sides, and should definitely be continued. Several attendees expressed their anticipation of a second Anniversary Symposium celebrating another 30 successful years of the Collaboration. 



\title{
Value of the US-Japan Collaboration in the Past and Future Closing Address
}

\author{
Takayuki Fujiyoshi, MEXT (Office for Quantum Radiation Research)
}

Thank you for your attention. So, we have reached the conclusion to this symposium.

On behalf of MEXT, let me first congratulate this successful symposium which marks the 30th year anniversary of US-Japan collaborations on high energy physics.

Since its start in 1979, (through the period of early development of Japanese high energy physics), the US-Japan Collaboration on high energy physics has allowed Japanese researchers to join the world's frontier of science,( contributed to internationalization of their activities,) and played a vital role in the development of this area in Japan.

The level of Japanese high energy physics, which was still developing in the beginning, now reaches the world's premier level after 30 years. I believe this is the result of our collaboration and each researcher's effort.

This collaboration created many scientific developments which contributed to the progress of global physics, such as the discovery of top-quark and Tau neutrino by FNAL-TEVARON, the research of Quark Gluon Plasma by BNL-RHIC, and the development of advanced accelerators at SLAC. Also, a number of Japanese researchers who were fostered by this collaboration are now leaders in Japanese physics society and achieved great progress in their research, such as in B-factory programs which have the highest performance in luminosity, and in experiments at Super-Kamiokande that opened a new era in neutrino physics. Thus our collaborations made innumerable direct and indirect contributions to the science.

Looking back to the past 30 years, I highly value the US-Japan collaboration on high energy physics which has created so many achievements. I believe that there have been not only indescribable efforts on behalf of researchers in both countries, but also strong support and understanding of DOE and US laboratories. I would like to salute the contributions of everyone involved in this collaboration.

Originally, this collaboration was started for the purpose of Japan to join in US experiments which were world-leading at the time. Japanese levels eventually improved, and, in recent years, we have been able to collaborate as partners, which has become fruitful for both countries. I believe this system worked because of the selection and evaluation of projects by strict peer review, and also because of the long-term stable and flexible funding.

I would also like to mention another thing that supported this fruitful relationship. This collaboration was carried out under the "Agreement between US and Japan on Cooperation in Research and Development in Energy and Related Fields" and the "DOE-MEXT Implement agreement" started in 1979, which gave the collaboration a kind of flame and support. However, this Agreement ended in 2005, and since then we have been discussing and trying to coordinate a new scheme. On top of the close communication between scientists like in this symposium, I think we should reconstruct the official framework to promote smooth collaboration at the governmental level. 
In this symposium, we discussed future collaboration schemes that acknowledge each-others research status and our future collaborations. It is hopeful that we geographically extend the research sites to both countries and encourage mutual cooperation. I understand the situation of research funding is difficult throughout the world these days, yet we still would like to support this collaboration in many aspects. I hope the US will also succeed in their efforts to contribute the necessary resources for this collaboration too.

I would like to close by saying that the Japanese Ministry wishes this cooperative relationship between the US and Japan strengthen and that our research collaboration contribute to the progress of research in this field and keep producing many more outcomes.

Thank you. 


\title{
US-Japan HEP Collaboration 30 $^{\text {th }}$ Anniversary Symposium Closing Address
}

\author{
D. Kovar, DOE
}

The goals of this Symposium were the following:

- To look back on the past activities and accomplishments of the US/Japan Collaboration

- To discuss ongoing programs

- To look forward to future prospects

In looking back on the close experimental collaborations over the last thirty years, I see the following:

- These collaborations have mapped well on the major accomplishments of particle physics over this period

- The Collaboration has been mutually beneficial, wherein participation

$\circ$ allowed and enhanced the proposed- and planned-activities

$\circ$ involved students and young researchers from both countries, whose careers and perspectives, I believe, were positively affected

- allowed both countries to justifiably share in the success and accomplishments of the joint ventures

- assured that the collaborations have been compatible with the programs of both nations, and indeed, enhanced and enabled them

- I agree with the assessments made by the presenters and participants at the symposium that the Collaboration has been a fruitful, successful one.

In looking at the ongoing programs, I see the following:

- Our collaborations have focused on several activities that have the promise of making significant discoveries (CDF, LHC (ATLAS/CMS), T2K, S-K, Fermi (GLAST))

- Our Collaboration remains mutually beneficial, where:

- participation is making possible and enhancing the programs of both countries

- but I see that more resources are flowing to Japan than in the past

$\circ$ participation involves students and young researchers

- but again, I see a larger flow of these from the United States to Japan

- participation is such that it makes these collaborations a true partnership

- where the problems and successes are shared

○ the collaborations are compatible with the programs of both nations

- I believe that the ongoing programs are full of promise, and fully expect that they will successfully deliver exciting, vital results

- I believe that the evolution of the US-Japan Collaboration to the point where now research and the R\&D capabilities within the two countries are more equal is

$\circ$ an indication of its success for the last thirty years

- a projection of a sound foundation for a relationship in the future that will benefit both of our programs 
In looking to the future, I believe that we should do the following:

- We should continue those practices that have proved successful in past: that is;

- assure that we choose projects that will deliver significant science

- make sure the collaborations remain mutually beneficial to both programs

- where participation supports and/or enhances programs

- where participation involves students and young researchers

- where participation is such that these activities are a partnership

- where the collaborations are compatible with the programs of both nations

- While we have a solid foundation built on thirty years of collaboration, there will be challenges because of some realities:

- First, all next-generation research capabilities are larger and more complex, cost more, and take longer to implement

- Second, particle physics has expanded beyond being entirely an accelerator-based science; for example, the cosmic frontier addresses some important questions. However, next-generation cosmic-frontier observatories also will be larger, more expensive, and take longer to implement.

$\circ$ Third, there are national considerations; for the US program, an operating US HEP accelerator facility and a strong accelerator R\&D program are priorities for two related reasons:

- First, I believe that a complement of research facilities will be needed for delivering timely scientific breakthroughs for particle physics

- Second, I believe that such research facilities are needed to be the core of national accelerator R\&D programs (along with adequate human resources and physical infrastructures) to both address the needs of the field (viz., higher energy, more intense, less expensive accelerators for the future), while fulfilling our responsibilities for developing accelerator technology/competency for our nations.

- I should also add that I believe that accelerator R\&D research needs to be both globally diverse and globally coordinated to meet the challenge of the field. I hope that Japan would continue to be interested in participating in this effort.

- Fourth, the near-term prospects for increased funding to implement new HEP research facilities will be constrained, given the financial state of countries globally, and the challenges faced by and the resulting priorities of the United States and many other countries

Given these realities, it is clear that HEP globally needs to collaborate, coordinate, and leverage resources if it hopes to make the discoveries and answer the questions that were discussed this morning

I would like to congratulate all who participated in the very successful US-Japan collaboration. You have provided a model and a sound foundation for meeting the challenges of the future. 


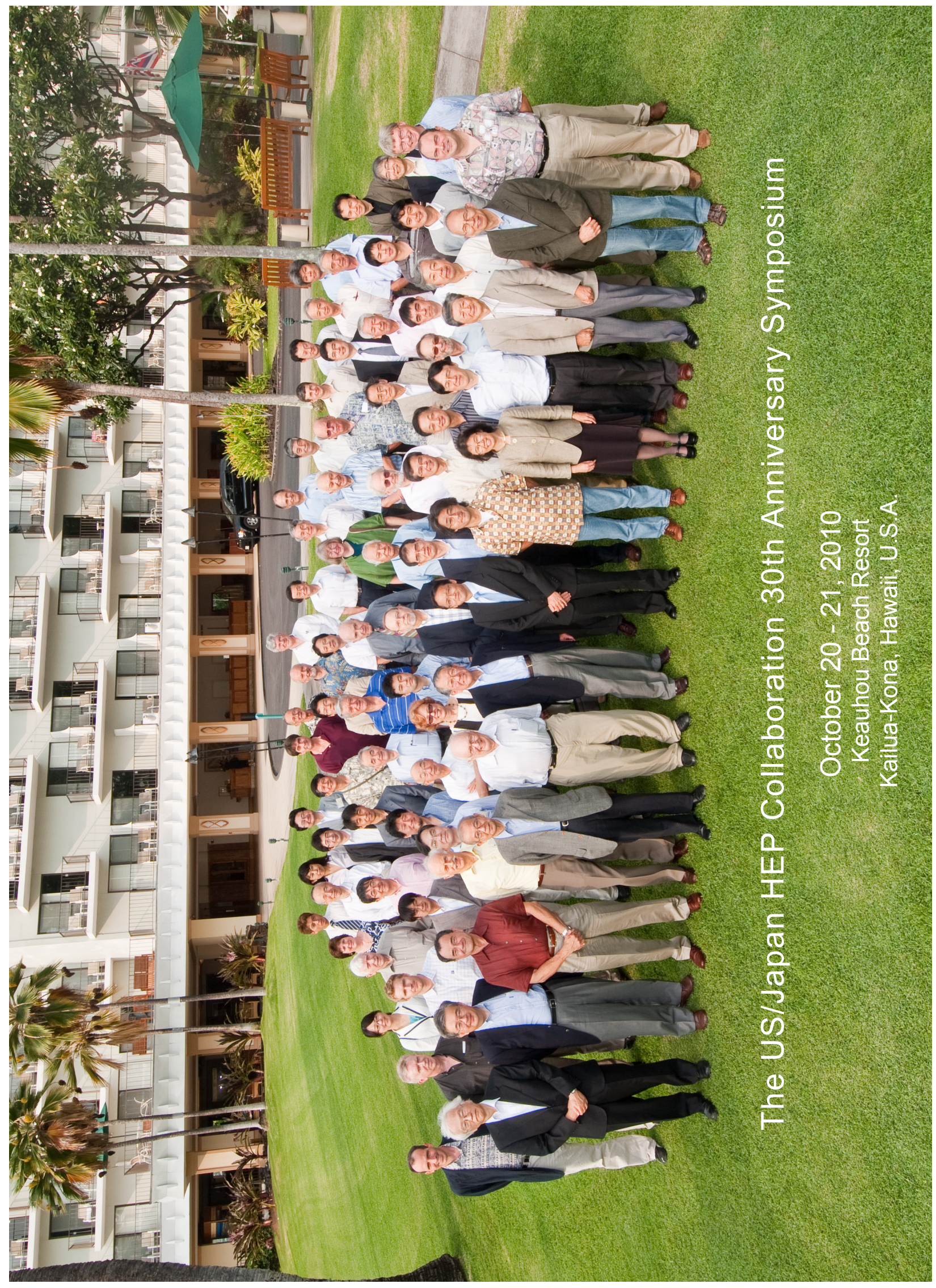




\section{AGENDA}

Wednesday, October 20, 2011

9:00 - 12:35 Session 1: Opening Session

9:00 Organization

9:05 Welcome Greeting

9:10 Congratulatory Remarks

9:15 Welcome to Hawaii

9:20 At the Beginning

9:35 Prehistoric Anecdotes

9:45 Status of HEP in Japan in the late 1970s

10:00 Status of HEP in the US in the late 1970s, and CDF as an Initial Pillar of the Collaboration

10:25 Program and Funding History

10:35 - 11:00 Morning Break

11:00 - 12:45 Session 2: Science Highlight I (Completed Experiments)

Chairperson: A. Masaike, Kyoto University.

Scientific Secretary: U. M. Yokoyama, University of Tokyo

11:00 PEP4 Program at LBNL and SLAC

11:15 Neutrino Scattering Experiment at BNL

11:30 Tau-neutrino Experiment at Fermilab

11:45 LASS at SLAC

12:00 K-TeV at Fermilab

12:15 Rare K Decay at BNL

12:30 AMY at TRISTAN, KEK

12:45 - 14:00 Lunch Break

14:00 - 15:55 Session 3: Science Highlight II (On-going Experiments)

Chairperson: Roy Rubinstein, Fermilab*

Scientific Secretary: E. O’Brien, $B N L$

14:00 PHENIX at RHIC, BNL

14:20 CDF at Tevatron, Fermilab

14:40 GLAST/Fermi Observatory, SLAC

14:50 Belle at KEK-B

15:05 Super Kamiokande

15:25 KamLAND

15:35 Discussions

15:55 - 16:20 Afternoon Break

16:20 - 17:20 Session 4: Science Highlight III (R\&D on Acc. \& Det.)

Chairperson: TBD (Japan Side)

Scientific Secretary: K. Shigaki, Hiroshima University

16:20 JLC/NLC/ILC R\&D

16:40 KEK ATF Report

16:50 LHC Quadrupole Magnets Experiences

17:05 Detector R\&D Summary Talk
Chairperson: Satoshi Ozaki, $B N L$ Scientific Secretary: E. O'Brien, $B N L$

S. Ozaki, $B N L$

F. Takasaki, KEK

Y. Kamo, Consulate General of Japan, Honolulu

A. Teramura, University of Hawaii

B. Hildebrand, $D O E$

W. Wallenmeyer, $D O E$

K. Kondo, University Tsukuba

H. Sugawara, JSPS-Washington DC

A. Tollestrup, Fermilab

K. Takahashi, KEK

\footnotetext{
H. Aihara, University of Tokyo Y. Nagashima, Osaka University M. Nakamura, Nagoya University D. Leith, $S L A C$

B. Tschirhart, Fermilab

L. Littenberg, $B N L$

S. Olsen, Hawaii
}

B. Jacak, Stony Brook University / K. Ozawa, University of Tokyo F. Ukegawa, University of Tsukuba/ R. Roser, Fermilab

T. Kamae, SLAC and University of Tokyo

T. Browder, University of Hawaii

Y. Suzuki, ICRR, University of Tokyo/H. Sobel, UC Irvine

K. Inoue, Tohoku University

All

G. Loew, SLAC

S. Yamaguchi, $K E K$

T. Shintomi, $K E K$

J. Haba, KEK 
Thursday, October 21, 2011

\author{
9:00 - 10:30 Session 5: "Future Prospects for High Energy Physics and Accelerators" \\ Chairperson: T. Browder, University of Hawaii* \\ Scientific Secretary: Y. Takeuchi, University of Tsukuba \\ 9:00 Physics Prospect \\ 9:30 Future of High Energy Physics in the US \\ 10:00 Future Accelerators: ICFA Chair' View \\ H. Murayama, IPMU, University Tokyo \\ P. Oddone, Fermilab \\ A. Suzuki, $K E K$ \\ 10:30 - 11:00 Morning Break \\ 11:00-12:30 Session 6: “Future Plan of Laboratories" \\ Chairperson: H. Sugawara, JSPS-Washington DC \\ Scientific Secretary: K. Sato, University of Tsukuba \\ 11:00 Future Plans at SLAC \\ 11:20 Future Plans at BNL \\ 11:40 Future Plans at Fermilab \\ 12:00 Future Plans at KEK \\ 12:15 Future Plans at J-PARC \\ D. MacFarlane, $S L A C$ \\ S. Aronson, $B N L$ \\ Y-K Kim, Fermilab \\ M. Yamauchi, $K E K$ \\ K. Nishikawa, $K E K$
}

12:30 - 14:00 Lunch Break

14:00 - 15:30 Session 7: "Research Topics for Future"

Chairperson: G. Loew, SLAC*

Scientific Secretary: T. Tomura, University of Tsukuba

14:00 New Concept in Acceleration

M.Yoshida, $K E K$

14:15 Laser Acceleration

14:30 Advanced and Innovative Accelerator

14:45 Muon g-2

15:00 Proton EDM

15:15 Muon Collider R\&D

15:30 Group Photo Session

W. Leemans, $L B N L$

M. Uesaka, University of Tokyo

N. Saito, $K E K$

Y. Semerizidis, $B N L$

R. Palmer, $B N L$

15:40 - 16:10 Afternoon Break

16:10 - 17:30 Session 8: Closing Session

Chairperson: F. Takasaki

Scientific Secretary: K. Yorita, Waseda University

16:10 Round Table Discussions: "Value of the US/Japan Collaboration in the Past and Future"

Discussion Leaders: P. Oddone, Fermilab, A. Suzuki, KEK

Panelist: Y. Nagashima, Osaka University, N. Sasao, Okayama University,

T. Nakaya, Kyoto University, D. MacFarlane, SLAC, B. Jacak, Stony Brook University

17:00 Closing Address

17:10 Closing Address

T. Fujiyoshi, $M E X T$

17:30 Adjourn

D. Kovar, $D O E$ 


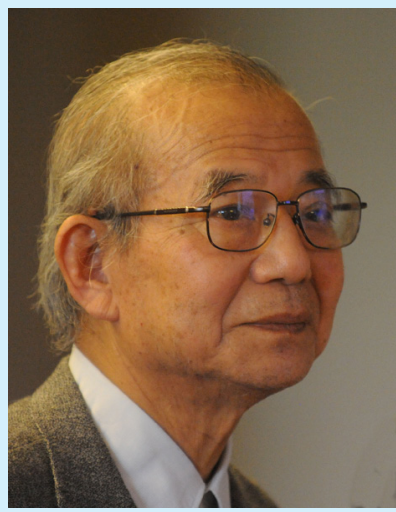

Kunitaka Kondo

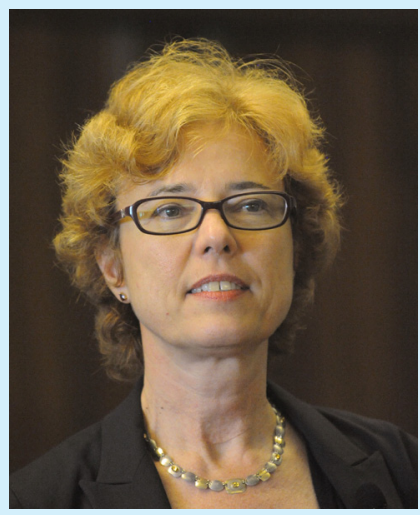

Barbara Jacak

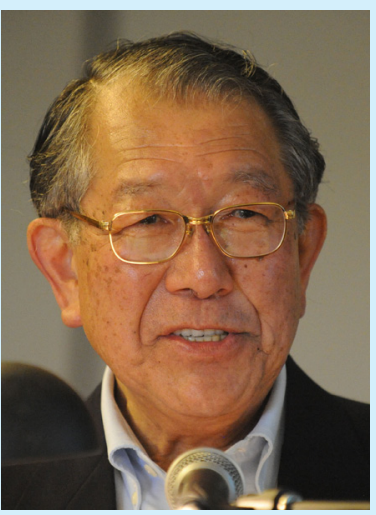

Satoshi Ozaki

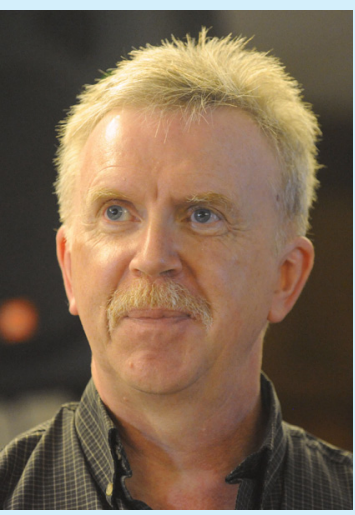

David B. MacFarlane

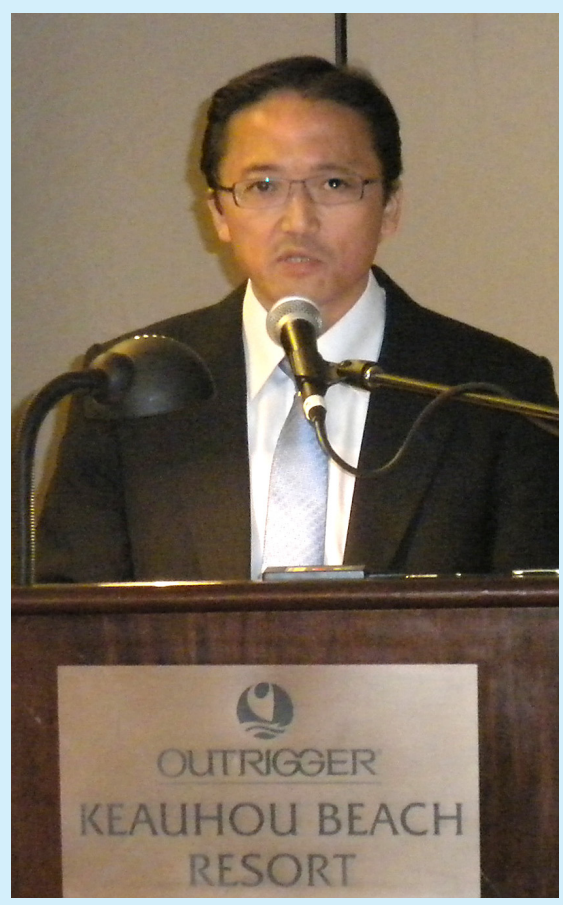

Takayuki Fujiyoshi

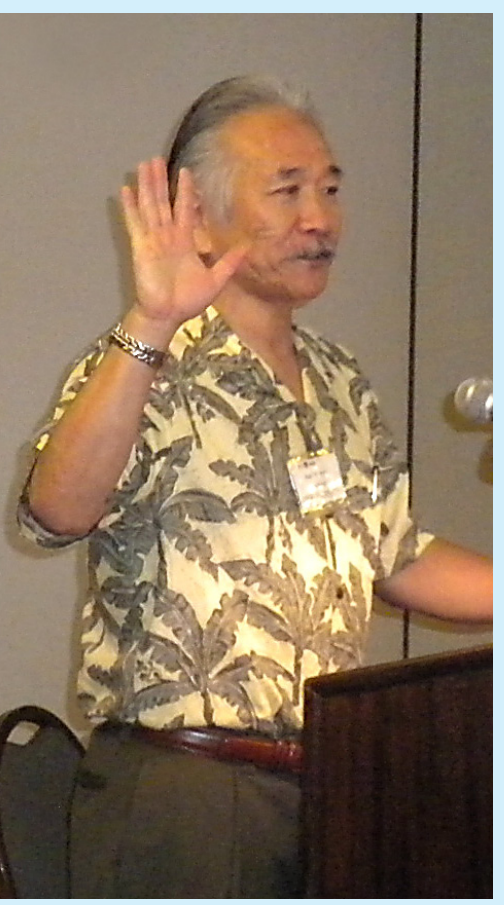

Alan Teramura

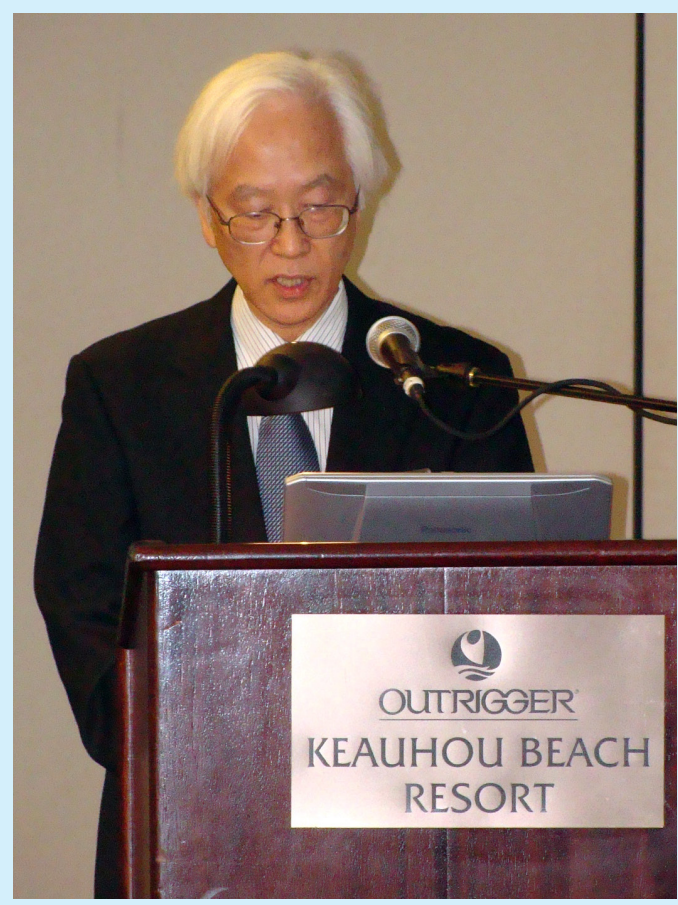

Fumihiko Takasaki

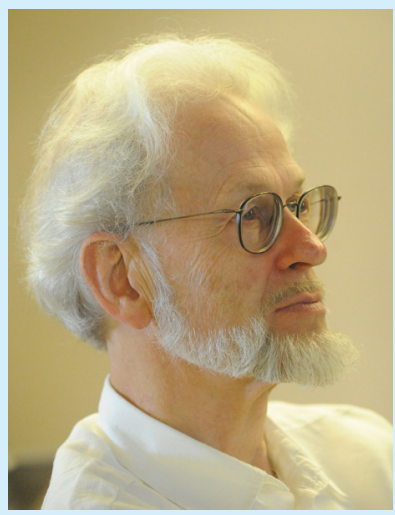

David Nygren

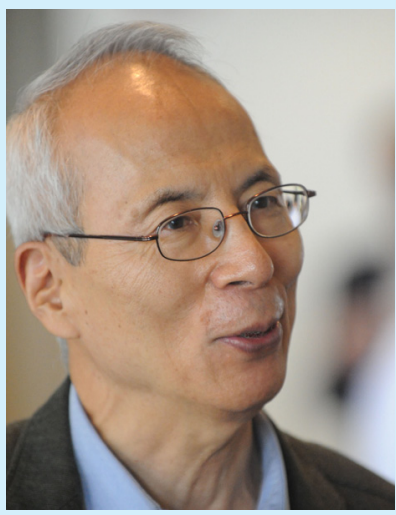

Tsuneyoshi (Tune) Kamae

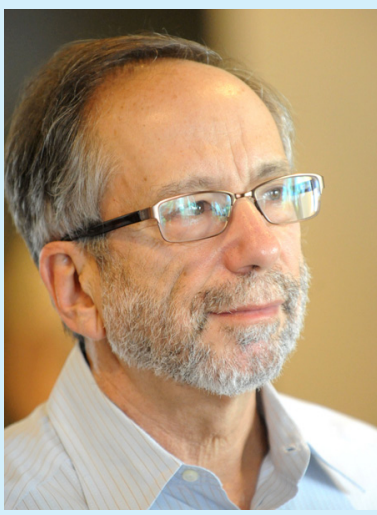

Laurence Littenberg

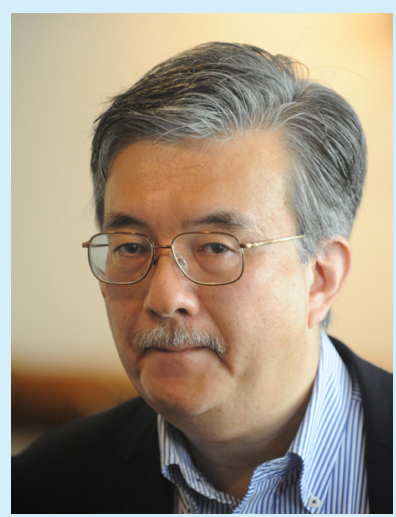

Masanori Yamauchi 


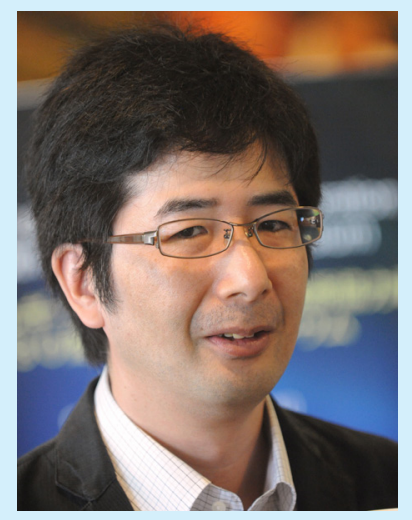

Kunio Inoue

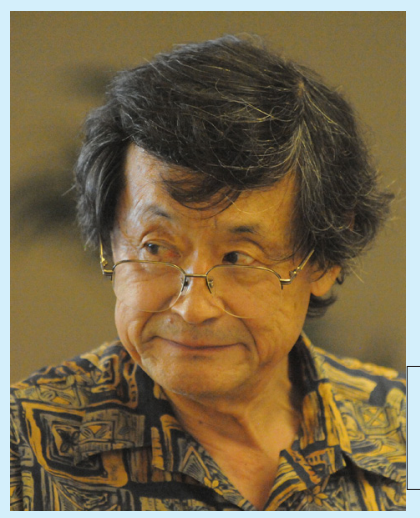

Hirotaka Sugawara

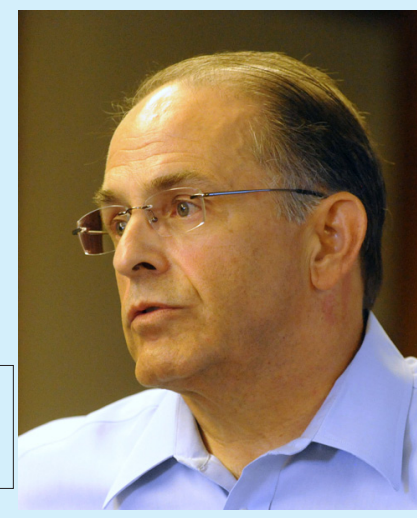

Samuel Aronson

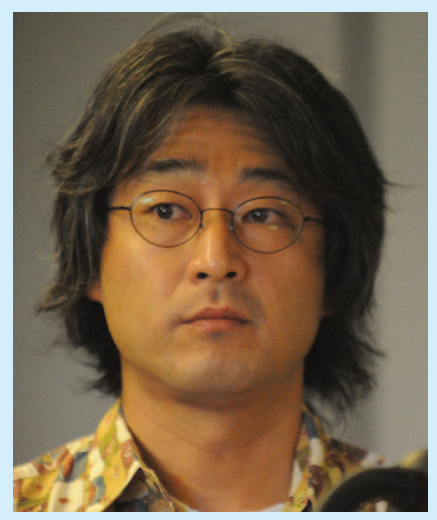

Hitoshi Murayama

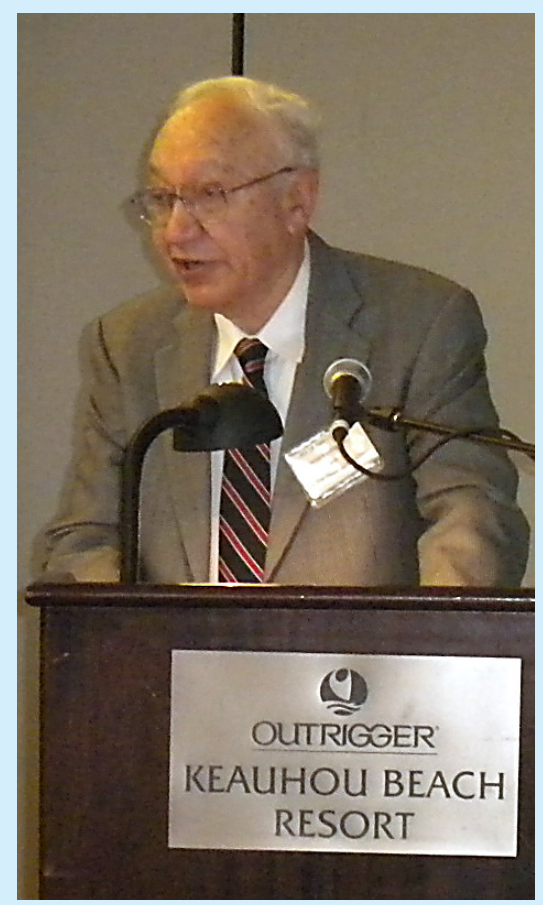

Bernard Hildebrand

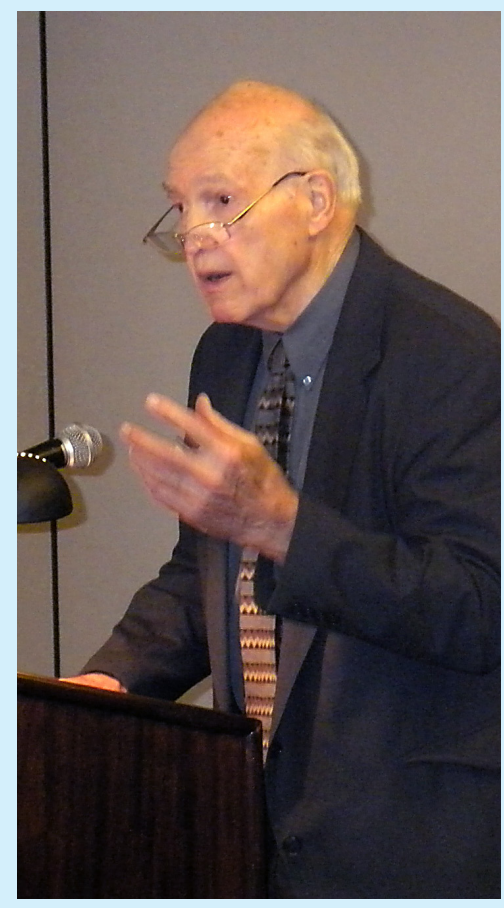

William Wallenmeyer

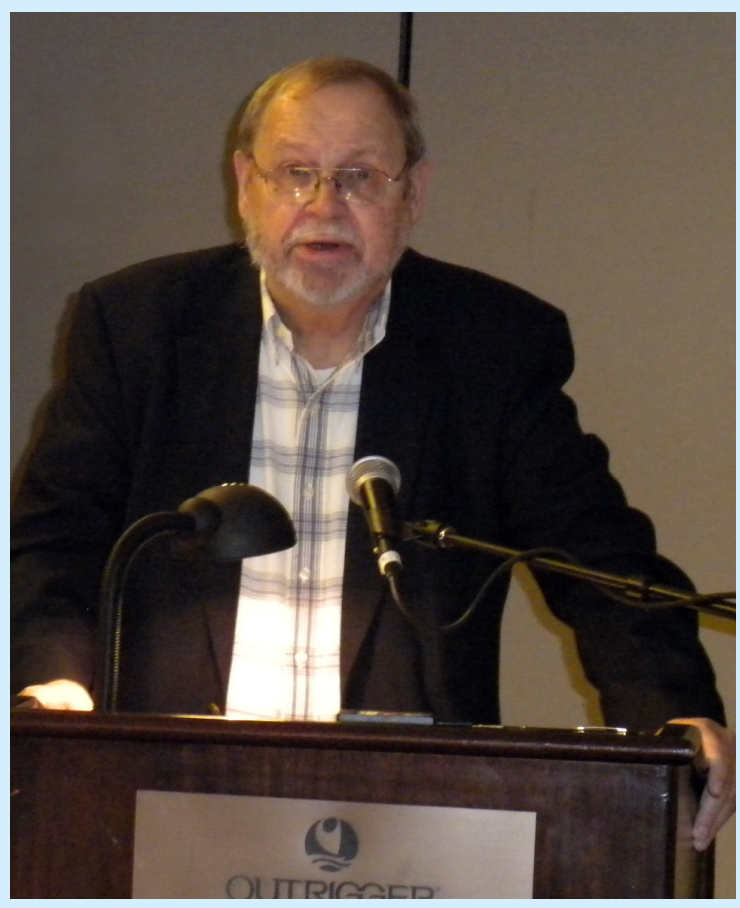

Dennis Kovar

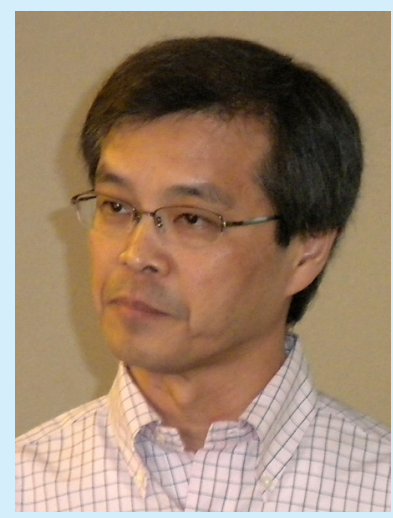

Hiroaki Aihara

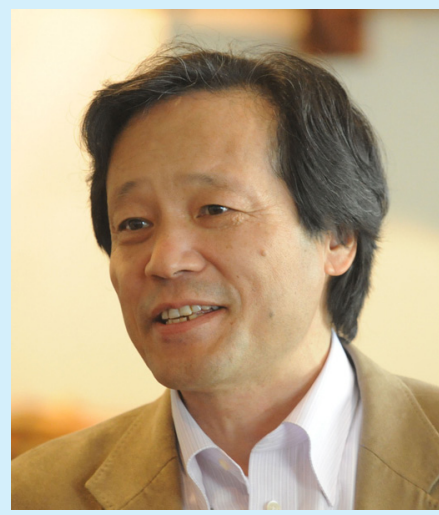

Junji Haba

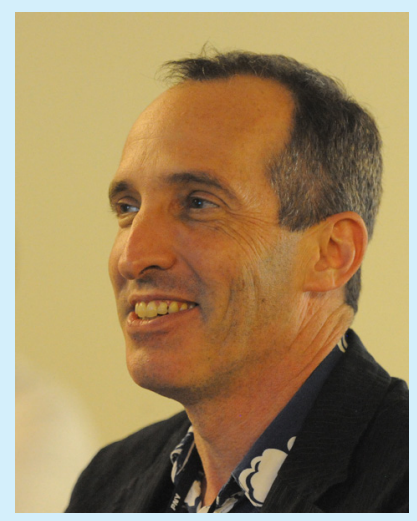

Thomas Browder

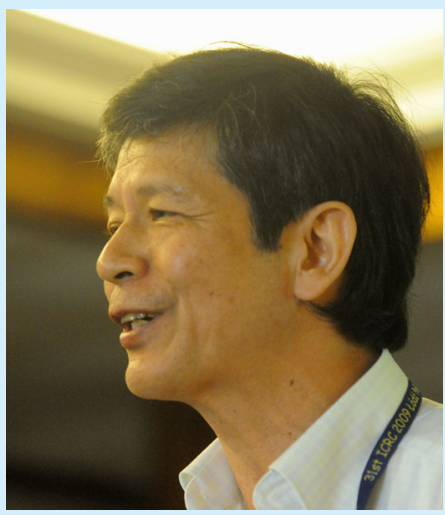

Yoichiro Suzuki 


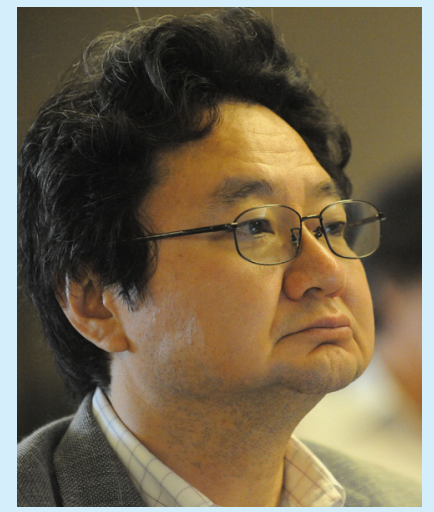

Mitsuru Uesaka

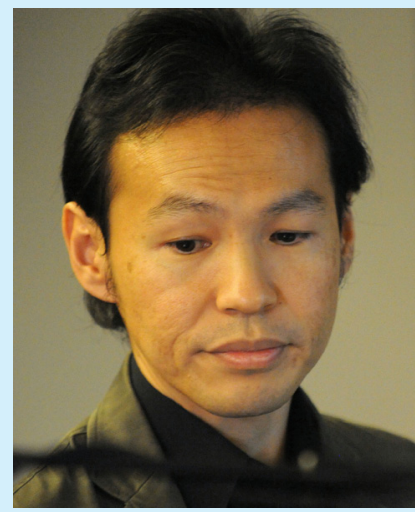

Mitsuhiro Yoshida

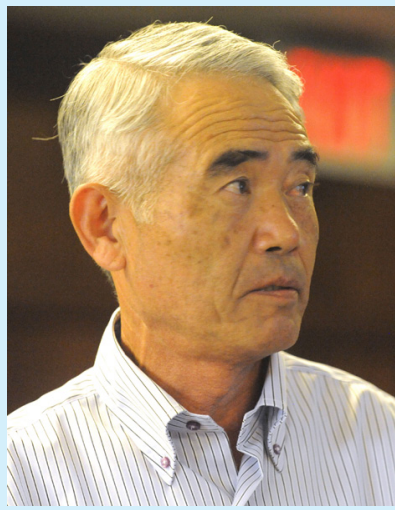

Takakazu Shintomi

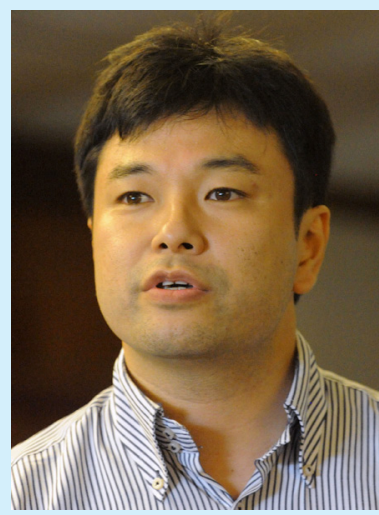

Kyoichiro Ozawa

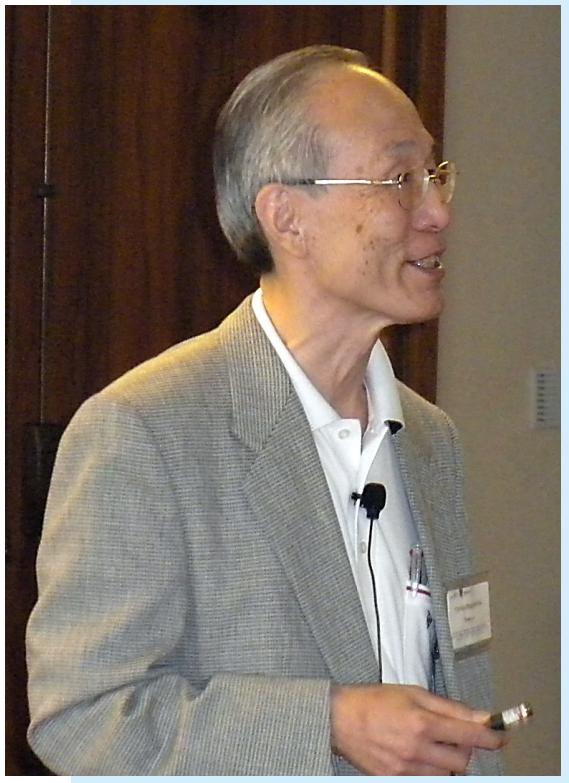

Yorikiyo Nagashima

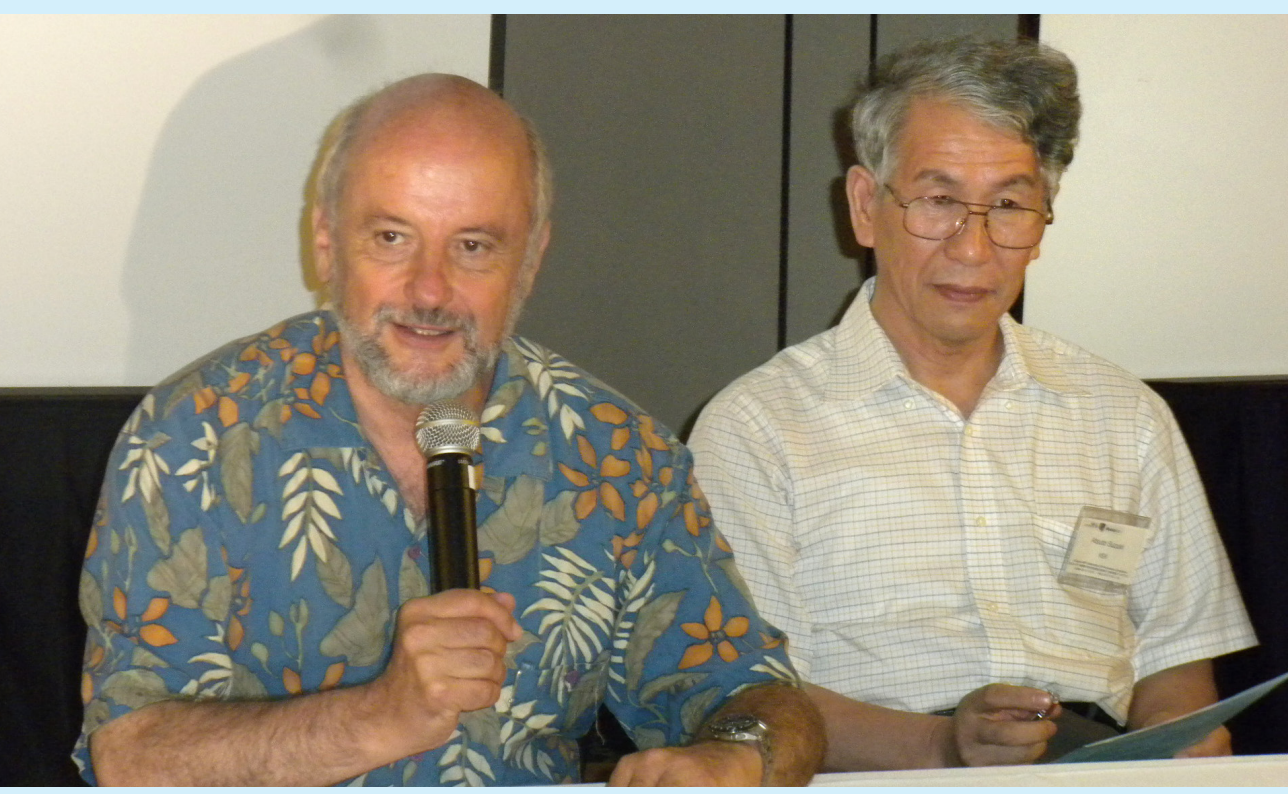

Pier Oddone and Atsuto Suzuki

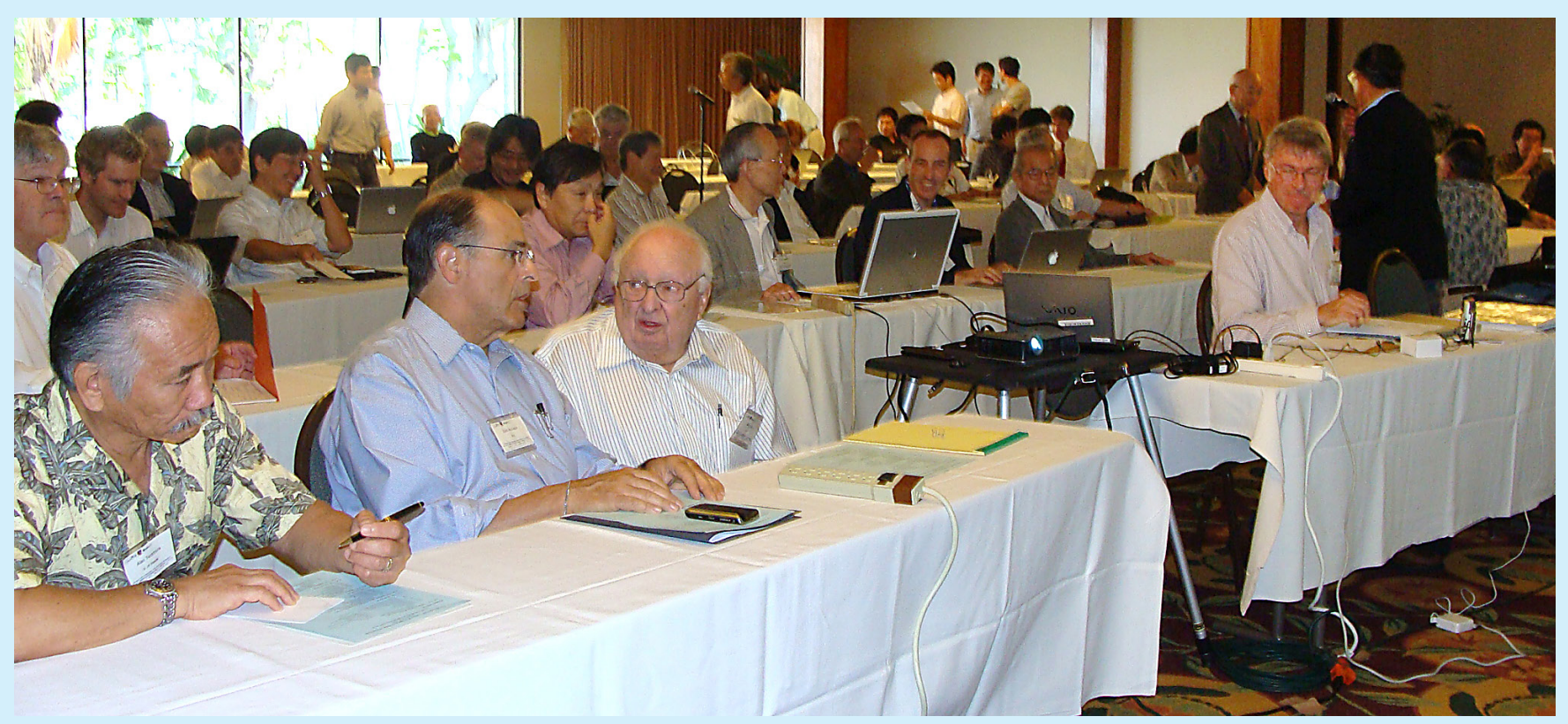




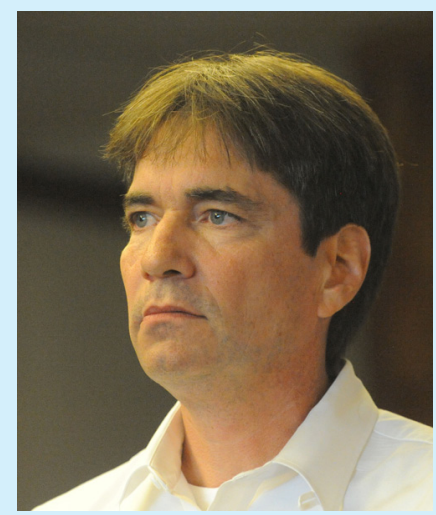

Robert Tschirhart

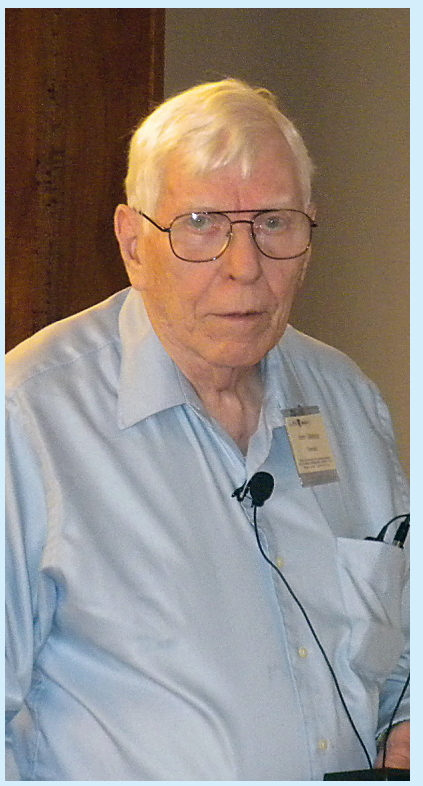

Alvin Tollestrup

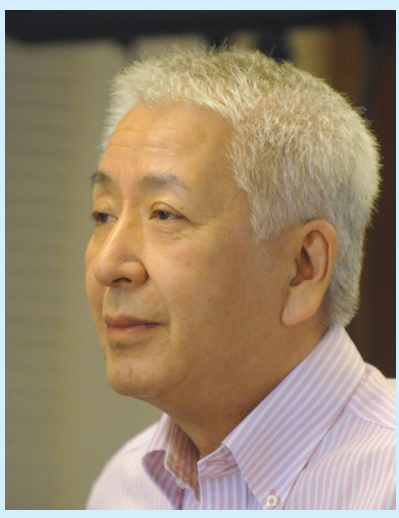

Koichiro Nishikawa

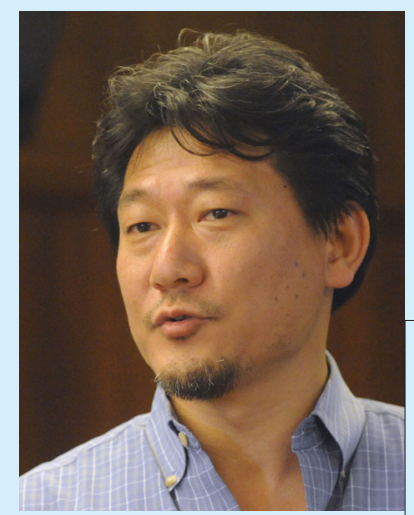

Naohito Saito

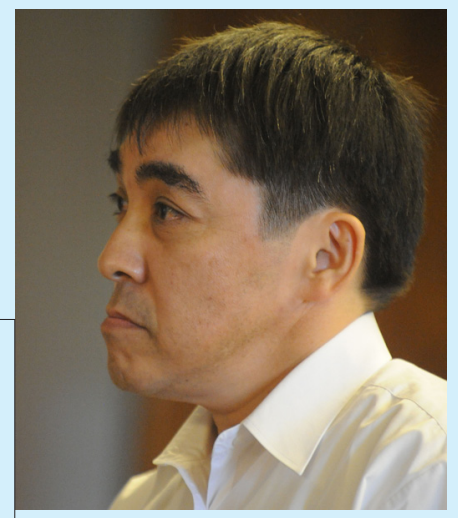

Mitsuhiro Nakamura

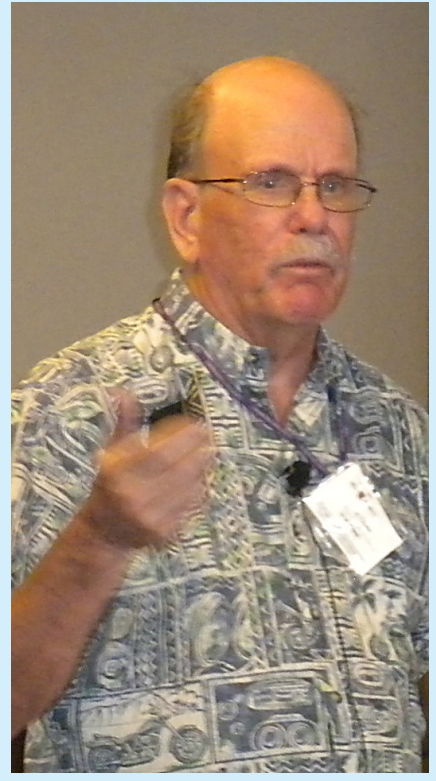

Stephen Olsen

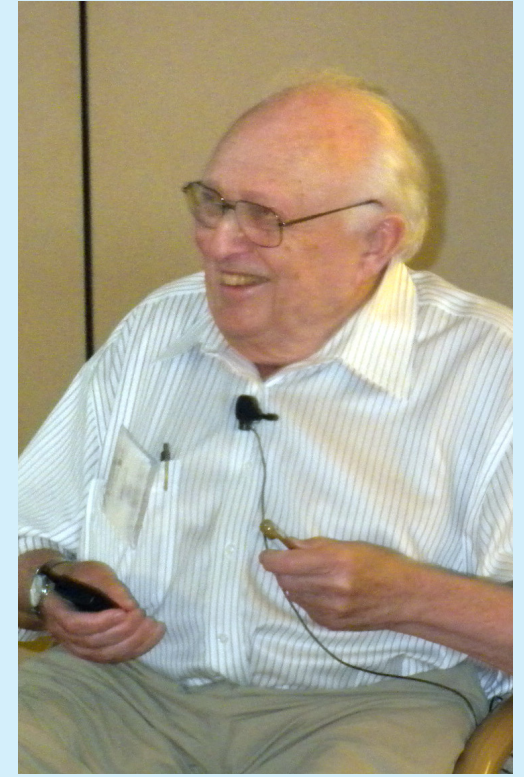

Gregory Loew

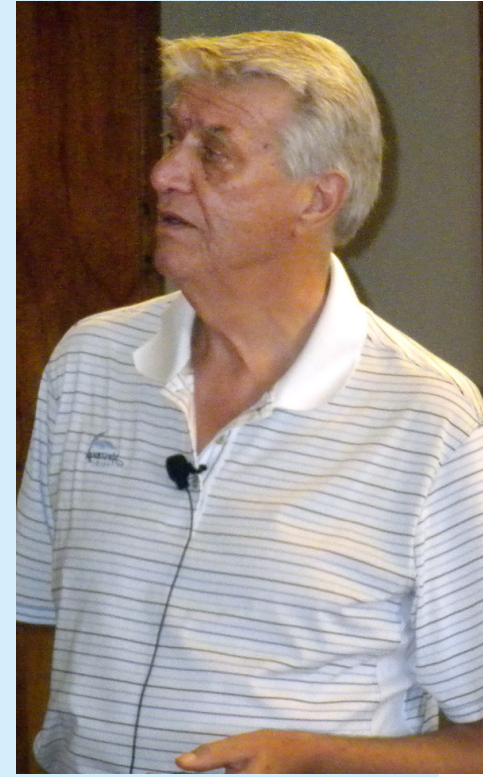

David Leith
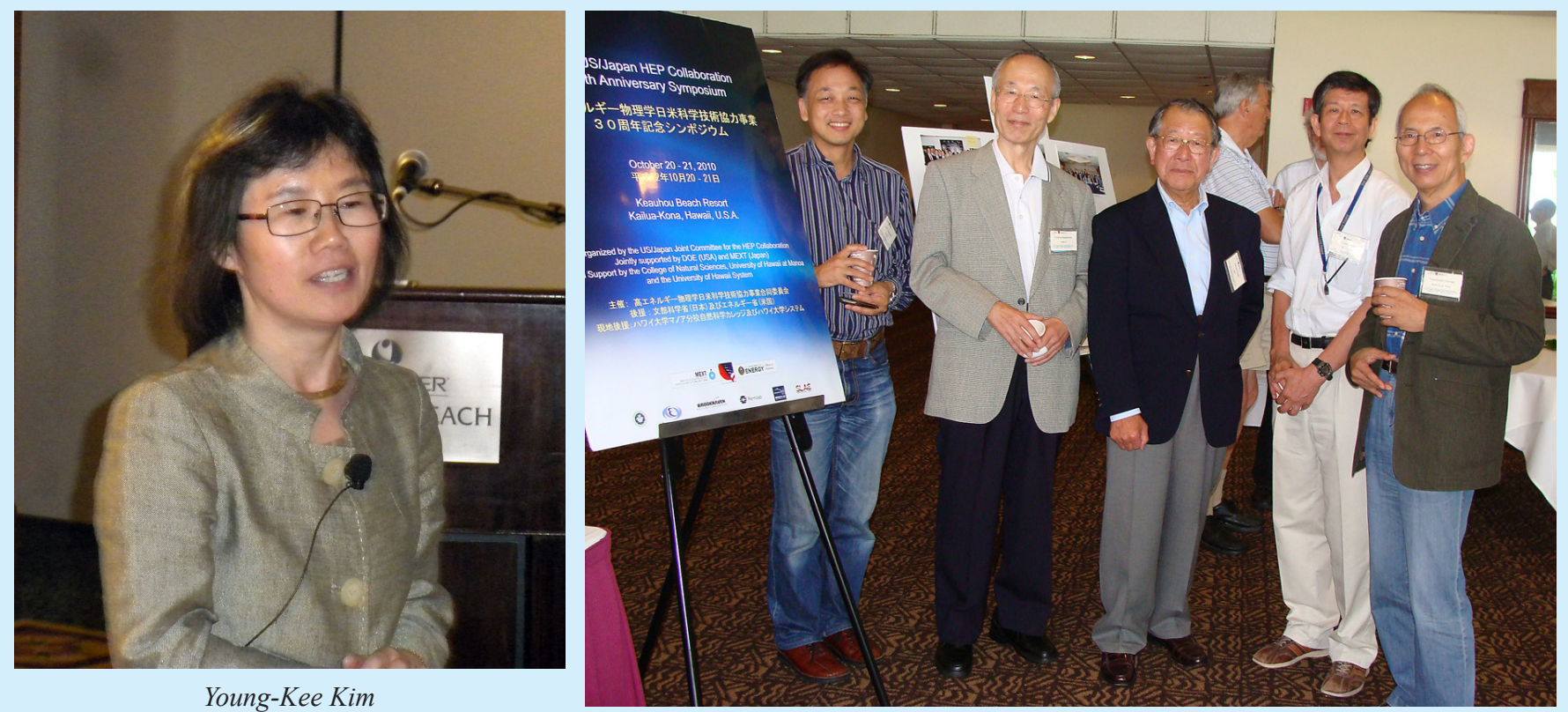
Q Bnisto-ustis?
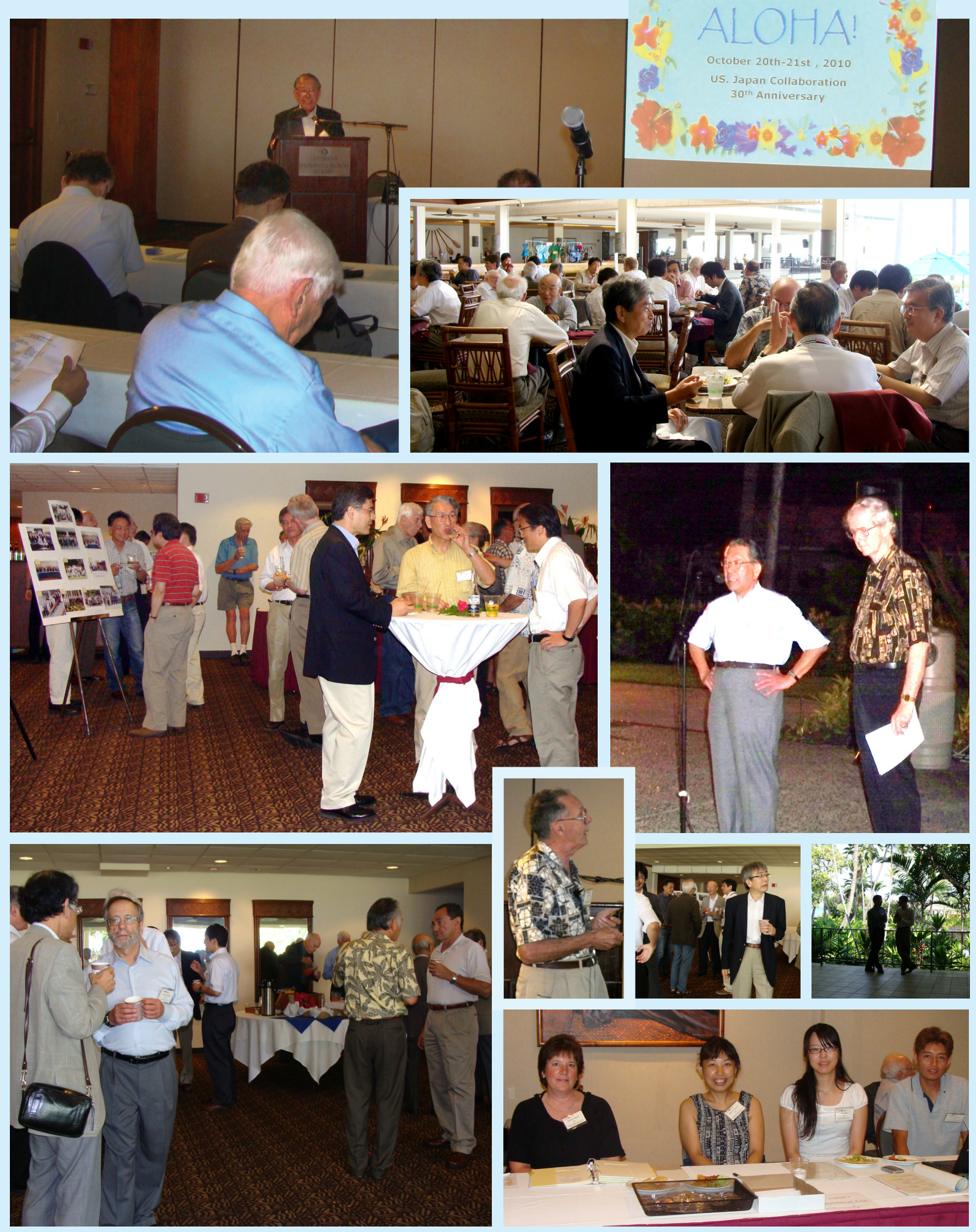


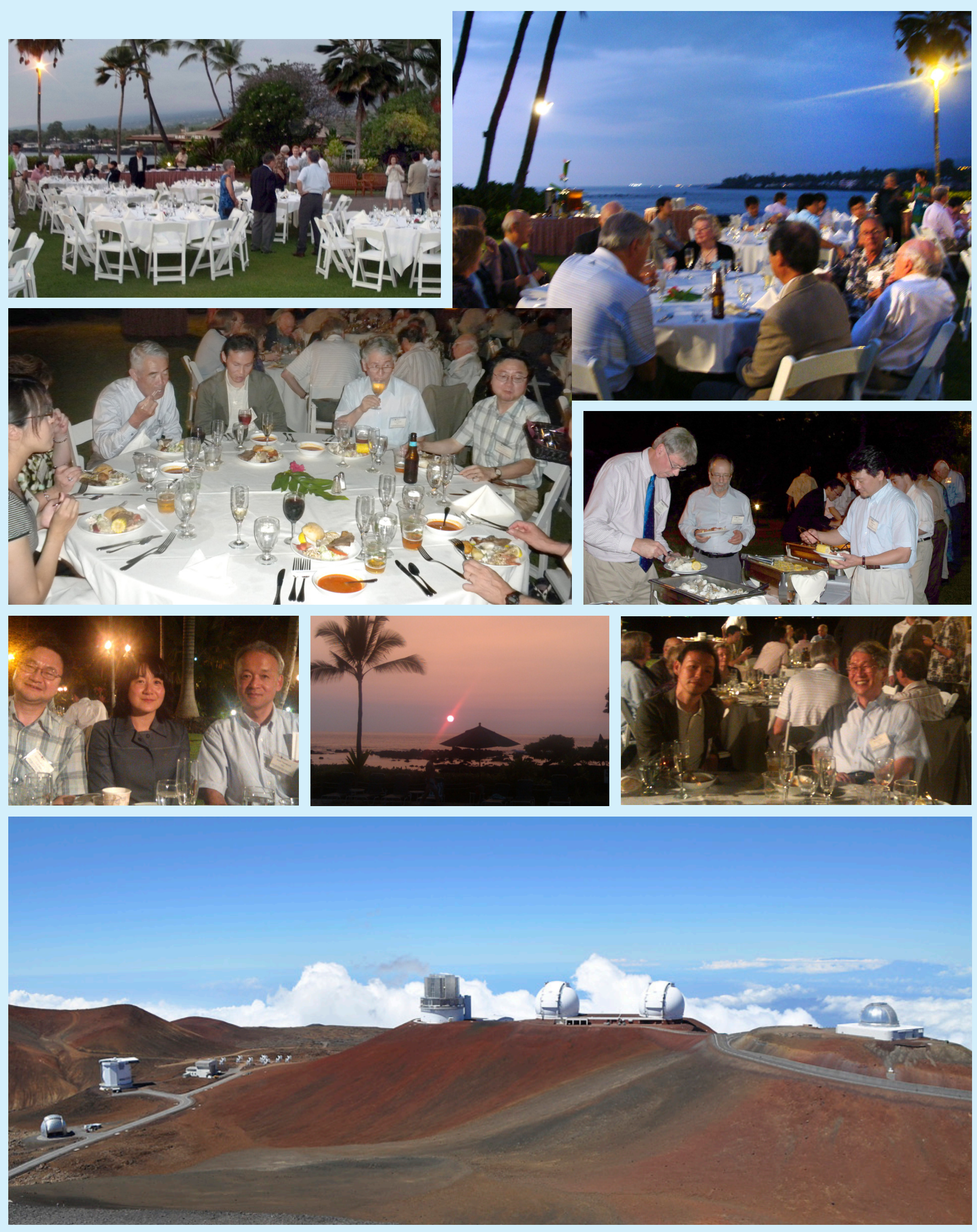







\section{LIST OF PARTICIPANTS}

Hiroaki Aihara, University of Tokyo

Sam Aronson, $B N L$

Thom Browder, University of Hawaii

Takayuki Fujiyoshi, $M E X T$

James R. Gaines, University of Hawaii Systems

Junji Haba, KEK

Bernard Hildebrand, $D O E$

Kunio Inoue, Tohoku University

Barbara Jacak, SUNY Stony Brook

Ryoichi Kajikawa, Nagoya University

Tsuneyoshi Kamae, SLAC/University of Tokyo

Yoshihiko Kamo, Consulate General of Japan

Fumiyo Kaneko, JSPS Washington Office

Young-Kee Kim, Fermilab

Shinhong Kim, University of Tsukuba

Kunitaka Kondo, University of Tsukuba

Dennis Kovar, DOE

Wim Leemans, $L B N L$

David Leith, SLAC

L.K. Len, $D O E$

Laurence Littenberg, $B N L$

Greg Loew, SLAC

David Brian MacFarlane, SLAC

Akira Masaike, Kyoto University

Masanor Mishina, KEK

Misa Miyai**, KEK

Barbara Moebes**, $B N L$

Toshinori Mori, University of Tokyo

Shigeki Mori, University Tsukuba

Hitoshi Murayama, IPMU, University Tokyo

Yorikiyo Nagashima, Osaka University

Mitsuhiro Nakamura, Nagoya University

Tsuyoshi Nakaya, Kyoto University

Koichiro Nishikawa, KEK

Mitsuaki Nozaki, $K E K$

David Nygren, $L B N L$

Ed O'Brien*, BNL

Pier Oddone, Fermilab

Steve Olsen, University of Hawaii

*Scientific Secretary

**Administrative
Satoshi Ozaki, BNL

Kyoichiro Ozawa, University of Tokyo

Robert Palmer, BNL

Rob Roser, Fermilab

Roy Rubinstein, Fermilab

Naohito Saito, KEK

Michael Salamon, $D O E$

Noboru Sasao, Okayama University

Koji Sato*, University of Tsukuba

Kenta Shigaki*, Hiroshima University

Takakazu Shintomi, KEK

Henry Sobel, UC Irvine

Hirotaka Sugawara, JSPS Washington Office

Atsuto Suzuki, KEK

Yoichiro Suzuki, ICRR, University of Tokyo

Kasuke Takahashi, KEK

Fumihiko Takasaki, KEK

Hiroshi Takeda, Kobe University

Yuji Takeuchi*, University of Tsukuba

Alan Teramura, University of Hawaii

Alvin Tollestrup, Fermilab

Tomonori Tomura*, University of Tsukuba

Sharon Traweek, UCLA

Robert Tschirhart, Fermilab

Mitsuru Uesaka, University of Tokyo

Fumihiko Ukegawa, University of Tsukuba

Sven Vahsen, University of Hawaii

Gary Varner, University of Hawaii

William A. Wallenmeyer, DOE

Seiya Yamaguchi, $K E K$

Akira Yamaguchi**, KEK

Taku Yamanaka, Osaka University

Masanori Yamauchi, KEK

Masashi Yokoyama*, University of Tokyo

Masami Yokoyama**, KEK

Kohei Yorita*, Waseda University

Mitsuhiro Yoshida, KEK

Keisuke Yoshio, KEK 
